\title{
DETECTING CHANGE IN CENTRAL CALIFORNIA COAST COHO SALMON \\ HABITAT IN SCOTTS CREEK, CALIFORNIA, FROM 1997 -2013
}

\author{
A Thesis \\ presented to \\ the Faculty of California Polytechnic State University, \\ San Luis Obispo
}

\author{
In Partial Fulfillment \\ of the Requirements for the Degree \\ Master of Science in Forestry Sciences
}

by

Ashley Brubaker Hillard

April 2015 
(C)2015

Ashley Brubaker Hillard ALL RIGHTS RESERVED 
TITLE:

AUTHOR:

DATE SUBMITTED:

COMMITTEE CHAIR:

COMMITTEE MEMBER:

COMMITTEE MEMBER:
Detecting Change in Central California Coast Coho Salmon Habitat in Scotts Creek, California, from 1997-2013

Ashley Brubaker Hillard

April 2015

Brian Dietterick, Ph.D., P.H.

Director, Swanton Pacific Ranch; Professor of Hydrology and Watershed Management, Natural Resources Management and Environmental Sciences Department

Joseph D. Kiernan, Ph.D.

Research Ecologist, NOAA Fisheries Southwest Fisheries Science Center, Fisheries Ecology Division

Rene Henery, Ph.D.

California Science Director, Trout Unlimited 


\section{ABSTRACT \\ Detecting Change in Central California Coast Coho Salmon Habitat in Scotts Creek, California, from 1997-2013}

Ashley Brubaker Hillard

Scotts Creek, in Santa Cruz County, Calif., supports the southernmost extant population of Coho Salmon (Oncorhynchus kisutch) in North America. In 1997, the California Department of Fish and Game (now Fish and Wildlife) conducted an extensive habitat typing survey of mainstem Scotts Creek, describing all habitat units from the top of the estuary to the limit of anadromy approximately $12 \mathrm{~km}$ upstream. I repeated this survey in 2013 to (1) assess changes in the quantity and quality of instream habitat, (2) compare the current condition to goals and standards established in the federal Central California Coast (CCC) Coho Salmon Recovery Plan, and (3) identify opportunities for possible future restoration. A comparison of the two surveys revealed an overall increase in mean canopy cover, mean bank vegetation, mean percentage instream cover, pool depth diversity, and percentage riffles since 1997, and decreases in mean residual pool depth, percentage flatwater, and number of primary pools. Overall, the percentage of the total mainstem classified as pool habitat did not change between the two survey periods. Results for individual habitat metrics were more variable when the stream was broken into discrete reaches delineated by major tributary junctions. Although a large woody debris (LWD) survey was not conducted as part of the 1997 survey, contrasting our results with data collected during intervening years indicated that instream LWD has become more abundant, primarily due to increases in hard-wood species (i.e., red alder [Alnus rubra] and California bay [Umbellularia californica]). When compared to habitat goals established in the federal CCC Coho Salmon Recovery Plan, Scotts Creek has adequate canopy cover and percentage pools, but is lacking in percentage riffles, instream cover, key pieces of LWD per100 m, and percentage primary pools.

Keywords: Habitat Assessment, Salmon Recovery, Habitat Restoration, Habitat Typing 


\section{ACKNOWLEDGMENTS}

Many people were instrumental to the completion of this project. Al Smith's generous gift of Swanton Pacific Ranch to Cal Poly made this study possible. I would like to thank my committee, Brian Dietterick, Joe Kiernan, and Rene Henery for all of the effort, advice, and time they put into this project. The three Swanton Pacific Ranch summer interns that participated in the stream survey were instrumental to the project, Kira Thornley, Adrian Broz, and James Raney. It was thanks to Mark Shelton and his personal interest in fly fishing that I became connected with this project in the first place. And finally, my family, Sandy and Bill Brubaker, and Jim Hillard, provided indispensable support and applied just the right amount of pressure throughout the project. 


\section{TABLE OF CONTENTS}

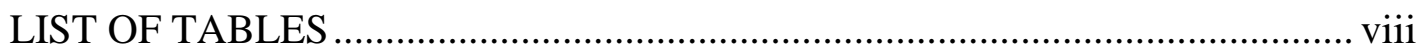

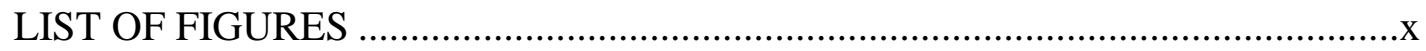

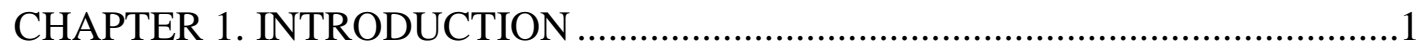

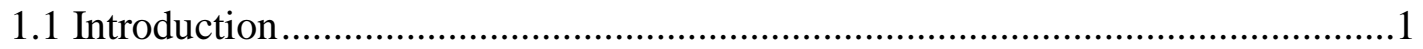

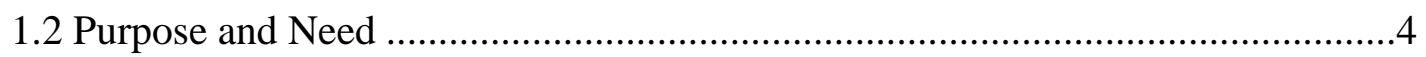

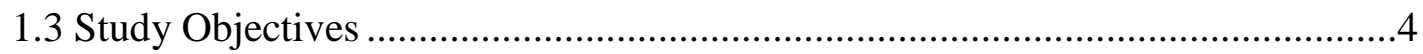

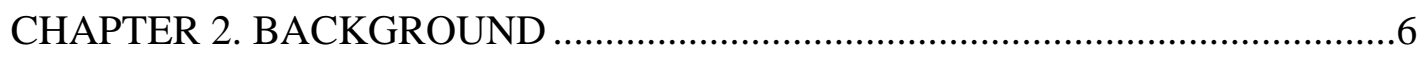

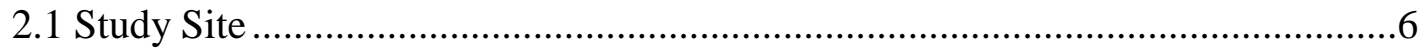

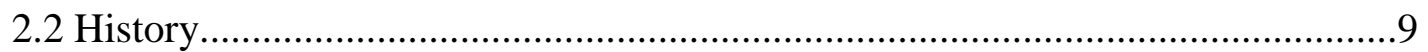

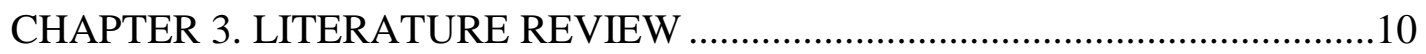

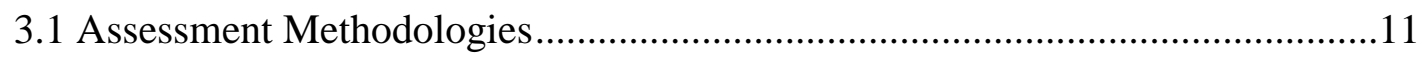

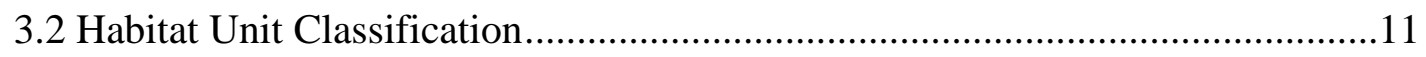

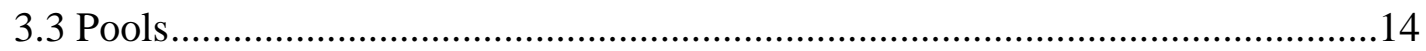

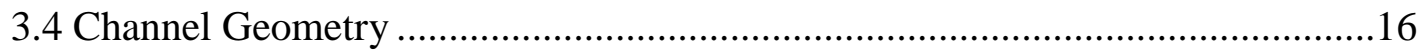

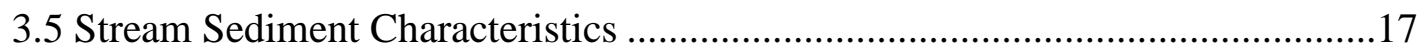

3.6 Streambanks and Vegetation.......................................................................

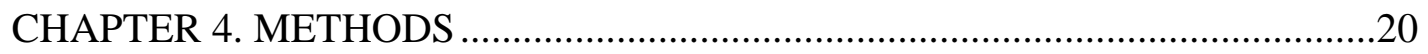

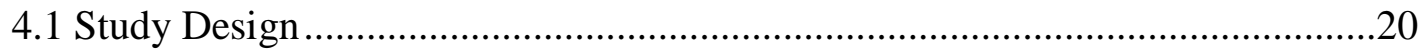

4.1.1 2013 Parameters and Survey ...................................................................22

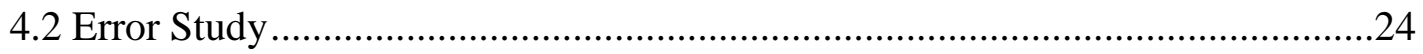

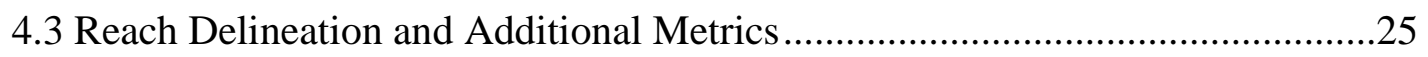

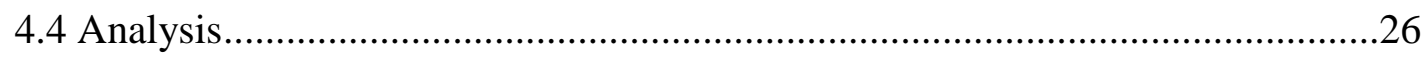

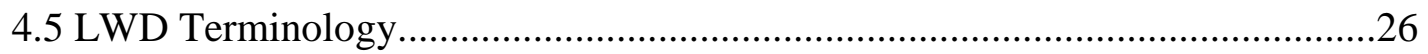

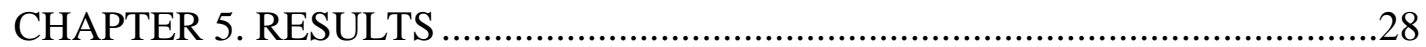

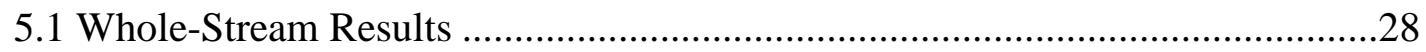

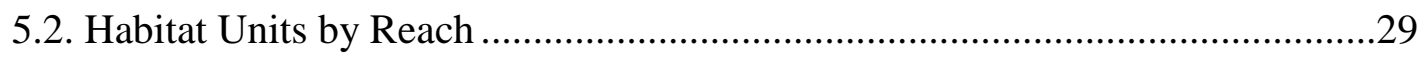

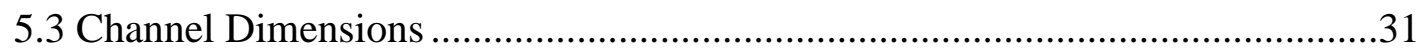

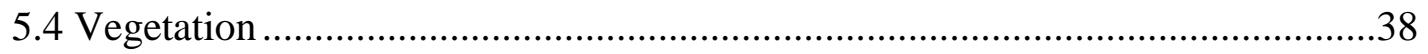

5.5 Instream Cover and Substrate ......................................................................40

5.6 Large Woody Debris...................................................................................

5.6.1 Temporal Trends in LWD .........................................................................4 


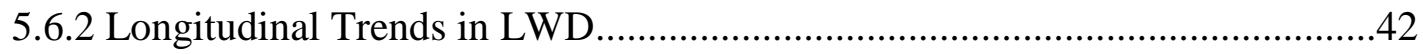

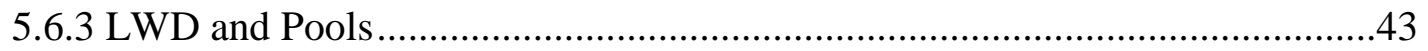

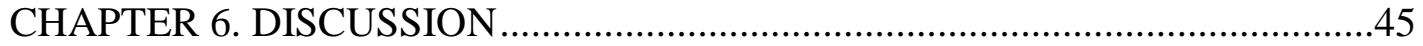

6.1 Constraints and Drivers of Change from 1997-2013 _...................................45

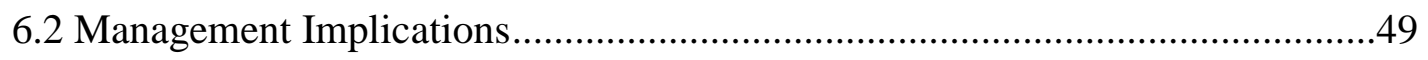

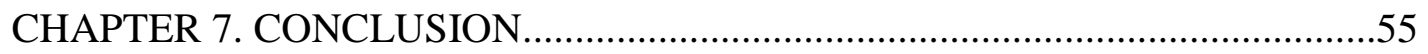

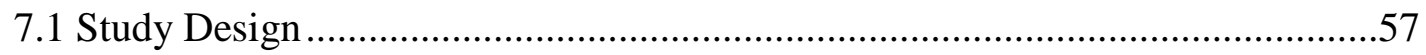

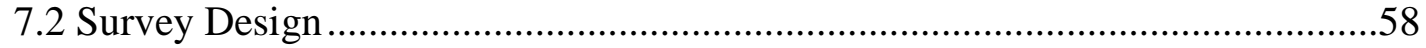

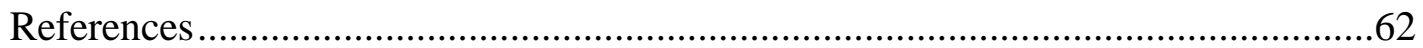

APPENDICES

Appendix A: Survey Methods from the California Salmonid Stream Habitat Restoration Handbook, Part III, Habitat Inventory Methods.................................66

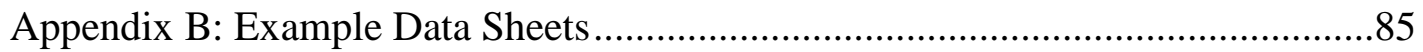

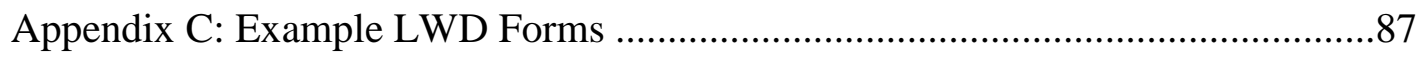

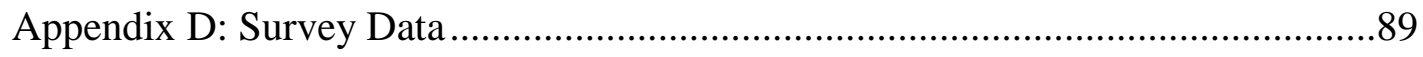




\section{LIST OF TABLES}

Table 3.1: The four levels of habitat units defined by California Department of Fish and Wildlife.

Table 4.1.1: Stream habitat measurements taken by each surveyor (A and B) during the 2013 habitat survey of Scotts Creek (Santa Cruz County, CA).

Table 5.1.1: Whole stream habitat related values for 1997 and 2013 28

Table 5.1.2: Whole stream channel dimension values for 1997 and 2013. Standard deviation values are in parenthesis.

Table 5.1.3: Mean canopy, vegetation, and instream cover values for the entire survey length for 1997 and 2013. Standard deviation values are in parenthesis.

Table 5.2.1: Habitat units and pool units for each reach. Values in bold text indicate significant change between survey years using a Bonferroni adjusted alpha $(\alpha)$ level of $0.01(0.05 / 5)$. Standard deviation values are in parentheses.

Table 5.2.2: Riffle units for each reach 30

Table 5.2.3: Pool-Riffle-Flatwater ratio for each reach. The federal CCC Coho Salmon Recovery Plan deems $>30 \%$ pools and $>20 \%$ riffle suitable for Coho Salmon.

Table 5.3.1: Channel dimension values for each reach. Values in bold text indicate significant change between survey years using a Bonferroni adjusted alpha $(\alpha)$ level of $0.01(0.05 / 5)$. Standard deviation values are in parentheses. An asterisk (*) indicates change in pool depth greater than that expected by surveyor error $( \pm 8.0 \mathrm{~cm})$.

Table 5.3.2: Primary pool ( $\geq 91 \mathrm{~cm}$ deep) values for each reach. Percentage primary pools is relative to the total number of pools in each reach, standard deviation values are in parentheses.

Table 5.4.1: Mean percentage canopy by reach. Values in bold text indicate significant change between survey years using a Bonferroni adjusted alpha $(\alpha)$ level of $0.01(0.05 / 5)$. Standard deviation values are in parentheses.

Table 5.4.2: Mean percentage bank vegetation for each reach, right and left banks combined. Values in bold text indicate significant change between survey years using a Bonferroni adjusted alpha $(\alpha)$ level of $0.01(0.05 / 5)$. Standard deviation values are in parentheses. 
Table 5.5.1: Mean percentage of instream cover, measured as the percentage of each unit covered, per reach. No significant change was detected between survey years using a Bonferroni adjusted alpha $(\alpha)$ level of $0.01(0.05 / 5)$. Standard deviation values are in parentheses.

Table 5.2.2: The dominant substrate by reach, sand $<2.03 \mathrm{~mm}$, gravel $2.03-63.5 \mathrm{~mm} . .40$

Table 5.6.1: Temporal trends in LWD using results from surveys in 2001-2002 (Leicester, 2005), 2004-2005 (Dodson, 2005), 2010-2011 (Monteverde et al., 2011) and 2013.

Table 5.6.2: Longitudianl trends in LWD for 2013. Values are for lowflow/pool and bankfull/backwater pieces only

Table 6.1.1: Peak flows and return periods for the San Lorenzo River at Big Trees (USGS gauge 11160500), long-term average flow is $224 \mathrm{~m}^{3} / \mathrm{s}$. The three years preceding the first survey were included due to the impact they could have had on instream habitat.

Table 6.2.1: Instream habitat parameter goals as set by the Final Recovery Plan for Central California Coast Coho Salmon Evolutionarily Significant Unit (NMFS, 2012), and actual values as determined by the 2013 Scotts Creek survey. 


\section{LIST OF FIGURES}

Figure 1.1: Estimated annual escapement of Coho Salmon to Scotts Creek (Santa Cruz County, California), 2004-2014. Source: NOAA Fisheries Southwest Fisheries Science Center, unpublished data.

Figure 2.1.1: The Scotts Creek watershed (drainage area $=78 \mathrm{~km}^{2}$ ) is located $19 \mathrm{~km}$ north of the City of Santa Cruz, CA. The inset map depicts mainstem Scotts Creek and its tributaries, from south to north, Queseria Creek, Archibald Creek, Winter Creek, Little Creek, Big Creek, Mill Creek and Bettencourt Gulch.

Figure 3.1: An illustration of residual pools, the shaded areas demonstrate the area contained within the residual pool measurement, which remains consistent across different flows. Source: Mossop and Bradford, 2005. 14

Figure 4.1.1: Scotts Creek survey extent and reaches, delineated by tributary confluences (minus Archibald Creek and Winter Creek, which are often dry at the confluence in the summer), including the location of the streamgage below Archibald Creek.

Figure 5.3.1: Residual pool depths and distribution for Reach 1, below Little Creek...... 33

Figure 5.3.2: Residual pool depths and distribution for Reach 2, Little Creek to Big Creek.

Figure 5.3.3: Residual pool depths and distribution for Reach 3, Big Creek to Mill creek

Figure 5.3.4: Residual pool depths and distribution for Reach 4, Mill Creek to Bettencourt Gulch.

Figure 5.3.5: Residual pool depths and distribution for Reach 5, above Bettencourt Gulch

Figure 5.3.6: Histograms showing the frequency distribution of residual pool depths in 1997 and 2013 for each reach.

Figure 7.1: A photograph of a logjam in the lower section of Scotts Creek forming a mid-channel pool. 
Figure 7.2: A difficult pool to classify under the current habitat unit classification system because it occupies greater than $60 \%$ of the channel, but is obviously enhanced by a root wad 


\section{CHAPTER 1. INTRODUCTION}

\subsection{Introduction}

In 1996, the National Marine Fisheries Service (NMFS) listed the Central California Coast (CCC) Evolutionarily Significant Unit (ESU) of Coho Salmon (Oncorhynchus kisutch) as a federally-threatened species under the U.S. Endangered Species Act, and in 2005 upgraded the status of the species to endangered. Reasons for the decline of CCC Coho Salmon are many and include inadequate streamflows and passage, overfishing, negative interactions with hatchery-origin salmon, and habitat degradation and loss (Nehlsen et al., 1991; NMFS, 2012). The loss of high-quality freshwater habitat, influenced by natural and anthropogenic factors, leads to decreased resiliency to stressors such as disease, predator pressure, climatic and environmental fluctuations, and poor ocean conditions. In undisturbed rivers and streams, habitat type and availability depend largely on the nature and physical features of the channel, which both interact with and are shaped by flow regime, sediment supply, streamside vegetation, and topography (Montgomery and Buffington, 1998).

Critical elements of riverine habitat for Coho Salmon generally include cool, clean water; deep, complex pools; access to off-channel pools and floodplain habitat; clean, loose gravel; and adequate flows to permit migration at key life stages (NMFS, 2012). Riparian vegetation plays an important role in providing and maintaining many of these habitat requirements. Streamside vegetation shades the stream and helps to maintain low water temperatures. Moreover, streamside vegetation stabilizes banks, decreases erosion and allows the formation of undercut banks that provide cover for aquatic organisms. Within the stream channel, large wood contributes to pool-forming scour, provides cover to protect 
from predators, and sorts sediment (NMFS, 2012). Historically, the loss of large woody debris (LWD) caused by clearing forests for timber, agriculture, and urbanization has been a major cause of decline in habitat complexity (Reeves et al., 1993). Additionally, channels were routinely cleared to prevent debris blockages and avoid exacerbating localized flooding and prevent bank erosion and channel avulsion.

The North American range of Coho Salmon extends from Point Hope, Alaska, south to Monterey Bay, California. The historical range of Coho Salmon within the CCC ESU extends from Punta Gorda in the north to Aptos Creek in the south (NMFS, 2012). Scotts Creek, the focus of this study, is located in northern Santa Cruz County near the southern end of the CCC ESU, and supports the southern -most extant population of Coho Salmon in North America.

Although there is a dearth of long-term data on Coho Salmon abundance in individual streams in California, statewide estimates suggest that populations have decreased from 200,000-500,000 fish in the 1940s, to less than 3,000 wild adults in 2011 (NMFS, 2012). Within the CCC ESU, total adult escapement has declined from approximately 56,000 fish in 1963 to 6,000 in the 1990s (NMFS, 2012). The Scotts Creek Coho Salmon population has followed a slightly different trend. In 1991 the estimated number of returning adult Coho Salmon was as low as 30-40 fish (Brown and Moyle, 1991), however, in 2004 and 2005 escapement numbers rose above 250 fish, due to supplementation from Kingfisher Flat Hatchery, then dropped below 20 in 2007-2012 (Figure 1.1; NOAA Fisheries Southwest Fisheries Science Center, unpublished). The National Marine Fisheries Service (NMFS) has set annual target recovery goals for Scotts 
Creek of 255 returning adult spawners to downlist the species to threatened, and 510 for full recovery (NMFS, 2012).

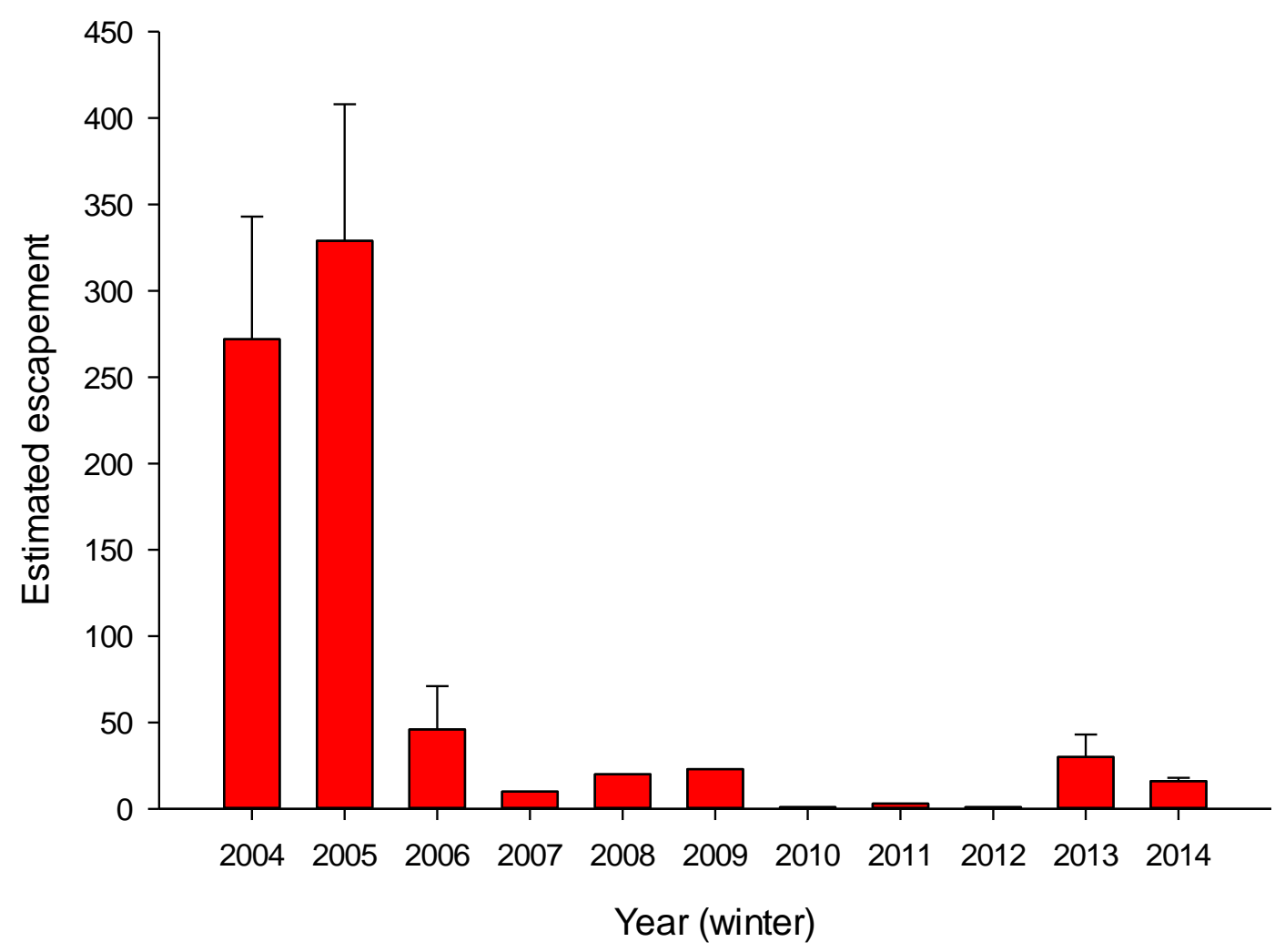

Figure 1.1: Estimated annual escapement of Coho Salmon to Scotts Creek (Santa Cruz County, California), 2004-2014. Source: NOAA Fisheries Southwest Fisheries Science Center, unpublished data.

An estimate by Moyle and others (2008) puts the population of CCC Coho Salmon at $5 \%$ of its historical abundance and the decline of streams containing Coho Salmon at $50 \%$ of historical numbers. Among the major factors responsible for the loss or degradation of Coho Salmon habitat in California are dams, logging, urbanization, agriculture, and mining (Brown et al., 1994). Habitat degradation likely began with wide-spread timber harvest in the mid-1800s through the early 1900s. Practices associated with logging, urbanization, agriculture, and mining, such as road and railroad construction and vegetation removal, decreased habitat complexity and increased sedimentation. Often, these practices 
left few to no trees for future LWD recruitment, causing a scarcity of LWD at present even in streams surrounded by healthy forests (Moyle et al., 2008).

\subsection{Purpose and Need}

As the loss of physical habitat is a major cause in the decline of CCC Coho Salmon, physical habitat condition plays an important role in stream and land management, policy, and restoration decisions in watersheds that support Coho Salmon. Scotts Creek is the site of many ongoing salmonid and hydrological studies, due to a) the existence of the Kingfisher Flat Hatchery on one of the tributaries, which is under the operation of Monterey Bay Salmon and Trout Project in association with NOAA Fisheries; b) the stream's use as a living laboratory by Cal Poly students; and c) its proximity and accessibility for NOAA researchers at the Southwest Fisheries Science Center. However, prior to this study, the only extensive physical habitat survey in Scotts Creek was conducted by the California Department of Fish and Wildlife (CDFW; then the California Department of Fish and Game) in 1997. Consequently, the lack of contemporary information concerning habitat status and trend were identified as critical knowledge gaps limiting effective conservation and management of the Scotts Creek Coho Salmon population (NMFS, 2012).

\subsection{Study Objectives}

The objectives of this study were - to:

1) determine the amount and direction of change to the instream habitat since the previous (1997) habitat survey conducted by $\mathrm{CDFW}^{1}$, and

\footnotetext{
${ }^{1}$ Data were obtained from CDFW and NMFS.
} 
2) lay the foundation for a long-term habitat monitoring study in Scotts Creek that a) investigates the rate, direction, and primary drivers of change in the Scotts Creek watershed, b) identifies key measurements for comparison and c) streamlines survey protocols.

Whereas more than two years of data are necessary to assess trends in habitat change, at least one more survey must be conducted in the future to accurately determine habitat trends. This study therefore aimed to provide a foundation for a long-term trends investigation, in the process identifying which parameters are most feasible for use in a long-term study, as well as shortcomings in the existing survey methods and opportunities to improve the methods without making past survey data unusable. 


\section{CHAPTER 2. BACKGROUND}

\subsection{Study Site}

Scotts Creek is a $5^{\text {th }}$ order coastal stream (based on LiDAR-derived drainage network using $1 \mathrm{~m}^{2}$ resolution LiDAR) draining $78 \mathrm{~km}^{2}$ of the Santa Cruz Mountains and emptying into the Pacific Ocean approximately $19 \mathrm{~km}$ north of the City of Santa Cruz, California (Figure 2.1.1). From source to sea, the mainstem of Scotts Creek measures approximately $19 \mathrm{~km}$, and is fed by 7 named tributaries (Queseria Creek, Archibald Creek, Winter Creek, Little Creek, Big Creek, Mill Creek, and Bettencourt Gulch) and many small intermittent streams and agricultural drainage ditches (Figure 2.1.1). The Scotts Creek watershed is characterized by major periodic disturbances such as fire, mass wasting, intense floods, and periods of prolonged drought. Land use in the watershed includes scattered houses, timber management, organic agriculture, cattle grazing, and forest, with supporting infrastructure consisting of a network of paved and unpaved roads and numerous horse and hiking trails. The outlet of Scotts Creek is a small estuary which is closed off from the ocean by a sandbar during the summer low (base) flow period, and opens with the first sizeable winter storm. The mouth of the estuary is confined by the Highway 1 Bridge, and levees bound both sides of the lowermost $500 \mathrm{~m}$ and extend another $1.5 \mathrm{~km}$ upstream along the eastern side of the channel. The functional condition of the estuary is being intensively studied (by multiple research groups and resource agencies) and the HWY 1 bridge crossing is expected to be replaced within the next 10 years. It is anticipated that the bridge size and location will be changed and that additional restoration of the estuary will occur.

The Santa Cruz Mountains have a Mediterranean climate with cool, wet winters, and foggy, dry summers. Almost all of the precipitation falls as rain between October and 
April. Consequently, streamflow in Scotts Creek during the summer months is very low $\left(<1.0 \mathrm{~m}^{3} / \mathrm{s}\right)$ and slowly decreases until the first significant rain event. In the winter, Scotts Creek usually exhibits a number of peak flows (ranging between 600-2000 $\mathrm{m}^{3} / \mathrm{s}$ ) resulting from heavy rains fueled by tropical moisture and enhanced by local orographic influences. The Coho Salmon spawning run in Scotts Creek typically begins in late-November or early-December following the first heavy rain event that initiates the flows necessary to breach the sandbar at the mouth of the estuary (Moyle, 2002).

The Scotts Creek watershed is situated on the Pacific Plate, just west of the San Andreas Fault. The watershed is located on the southwest tilted Ben Lomond Mountain tectonic block, comprised of granitic and metamorphic rock overlain by sedimentary rock (Scotts Creek Watershed Assessment, 2005). The overlaying Santa Cruz mudstone dominates the surface geology, but quartz diorite is evident in the upper reaches of the tributaries and main stem (Brabb, 1989). 


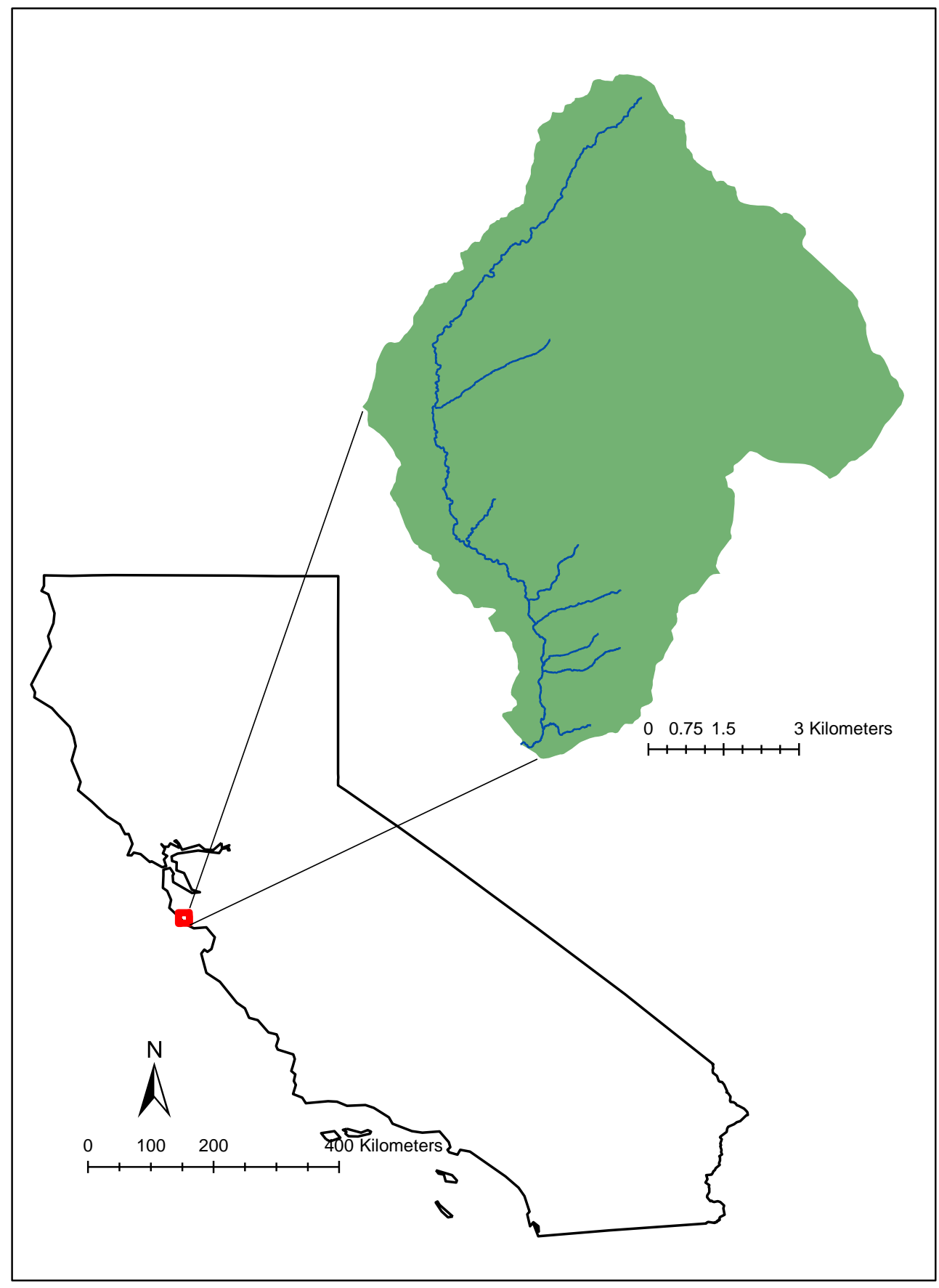

Figure 2.1.1: The Scotts Creek watershed (drainage area $=78 \mathrm{~km}^{2}$ ) is located $19 \mathrm{~km}$ north of the City of Santa Cruz, CA. The inset map depicts mainstem Scotts Creek and its tributaries, from south to north, Queseria Creek, Archibald Creek, Winter Creek, Little Creek, Big Creek, Mill Creek and Bettencourt Gulch. 


\subsection{History}

The Ohlone people are the first known residents of the Scotts Creek watershed. Evidence of bones found in midden piles indicates that steelhead trout (Oncorhynchus mykiss) and Pacific salmon (Oncorhynchus spp.) were an important part of their diet. The Ohlone people routinely burned large portions of land in order to increase the availability of choice food plants. In the late 1700s, with the introduction of the Spanish Missions, it is likely that the Ohlone people were moved out of the watershed and the land was primarily used for cattle grazing (SCWA, 2005).

Early settlers began logging the Scotts Creek watershed in the 1800s, but the pace of timber harvest increased rapidly after the 1906 San Francisco earthquake. By the mid1920s most of the watershed had been clearcut. The timber operations required roads and a railroad, camps, mills, and skid trails.

In 1938, the Scotts Creek outlet was relocated and confined by the Highway 1 bridge opening and the estuary and channel upstream was leveed. The lower section of stream, including the estuary, was straightened and the Army Corps of Engineers built additional levees to protect agricultural fields below the confluence with Archibald Creek (Figure 4.1.1). Channel dredging and levee maintenance continued until 1982 (SCWA, 2005).

In 1927, the California Department of Fish and Game began hatchery operations on Big Creek, which continued until 1940 when heavy flooding damaged the facility beyond repair. In 1982, the hatchery was re-opened under the operation of Monterey Bay Salmon and Trout Project, and currently operates in collaboration with NOAA Fisheries (SCWA, 2005). 


\section{CHAPTER 3. LITERATURE REVIEW}

An inventory of stream physical habitat attributes, or "habitat typing," is a common method for surveying and assessing the quantity and quality of riverine habitat. Organizations including the U.S. Department of Agriculture, Forest Service (USDA, USFS), U.S. Environmental Protection Agency (EPA), California Department of Fish and Wildlife (CDFW), and private researchers and non-governmental organizations employ many different methods of habitat typing (Flosi et al., 2004; Moore et al., 1999; USFS, 2010; Kaufmann et al., 1999). Habitat typing can be used for stream monitoring, impact assessment, habitat quality assessment, restoration planning, species management plans, identification of limiting factors, cumulative effects assessment, and land management planning (Bain and Stevenson, 1999; USFS, 2010; Flosi et al. 2004). Stream monitoring often occurs after a large disturbance event such as a flood (Myers and Swanson, 1996; Newman and Swanson, 2008), to detect land use impact (Kershner et al, 2004), to assess the impact of a stream restoration project (Opperman and Merenlender, 2004), or to detect trends in stream habitat condition (Anlauf et al., 2011).

Although many studies have been conducted to evaluate the effectiveness of different stream monitoring techniques, there is no standardized set of metrics to detect stream change (Roper et al., 2002; Poole et al., 1997). Appropriate metrics depend on stream characteristics, the type of impact (e.g., flood, timber harvest, cattle grazing, general trend), the extent of pre-existing data, and the principal research questions or management objectives. When pre-event/land use change data is not available, comparisons are often made between a similar but un-impacted stream in a "space for time" study. Due to the difficulty in predicting episodic disturbance events (e.g., fires or floods) and of conducting surveys that span decades, "space for time" studies are common in determining the effect 
of land use change or a major natural event. This section will review commonly used habitat assessment metrics and their efficacy and sensitivity in characterizing status and trend over space and time.

\subsection{Assessment Methodologies}

Habitat surveys typically consist of data collected at two scales: the broader channel-scale portion and the narrower habitat-scale portion. Broad-scale data collection can often be conducted prior to going into the field using geographical information systems, remote sensing information and aerial photographs, and topographical maps. Specific parameters vary across methodologies but frequently include sinuosity, channel gradient, drainage area, and stream length. Basin-wide attributes can often account for differences in habitat characteristics between similar streams. For example, Kershner et al., (2004) reported that average precipitation and gradient consistently described a large amount of variability in habitat characteristics, bank descriptors, and channel substrate in interior Columbia River Basin streams. Typical information collected during the narrower focused, habitat-scale portion of a habitat survey includes habitat unit type and length, wetted width and depth, bankfull width and depth, water temperature, bank and riparian vegetation information, size and position of large woody debris (LWD), and substrate type (i.e., particle size distribution). Methods for identifying or collecting these data may vary, thus influencing the accuracy of the data and limiting the ability to compare results across surveys.

\subsection{Habitat Unit Classification}

The identification and description of individual habitat units is fundamental to the habitat survey; however, multiple systems of naming habitat units are employed by 
different resource agencies. The most basic habitat units, upon which most classification systems are based, are pools, riffles, and runs. The CDFW system of habitat typing is based on the system proposed by Bisson (1982) and designed to describe 100 percent of the wetted channel. The CDFW survey contains four different levels of habitat description, with level one being the most basic and each additional level describing the habitat units with increasing complexity (Table 3.1). At the most complex level (Level IV), CDFW identifies 28 unique habitat units (Flosi et al., 2004). In contrast, the most complex level of characterization employed by USFS only recognizes 13 different habitat types (USFS, 2010). Furthermore, Oregon Department of Fish and Wildlife identifies 26 distinct habitat types (Moore et al., 2006), and the United States Geological Survey (USGS) acknowledges the existence of multiple types of pools, but for recording purposes only identifies habitat units to be pool, riffle, or run (Fitzpatrick et al., 1998).

Since physical habitat units are relatively stable over time compared to riverine biotic assemblages, they may be used to monitor change and provide a time-integrated picture of conditions upon which management decisions may be based (Bain et al, 1999). However, the use of habitat units as a monitoring tool is sometimes viewed as ineffective and imprecise. Habitat units are flow dependent, thus measurements for comparison need to be made at similar streamflow levels (Armour et al., 1983; Kaufmann et al., 1999) and total area for a given habitat unit type may vary significantly with flow. Moreover, individual surveyors may differ in their delineation of habitat units; a survey conducted by Ralph and others (1991) of 11 stream reaches by two different survey teams yielded two reaches for which the habitat units could not be matched at all, and nine with an $85-88 \%$ match (as cited in Poole et al., 1997). Poole et al. (1997) reported observer agreement to 
be $29-56 \%$ better than random unit classification agreement. Although unit aggregation into fewer, broader habitat types would increase observer agreement, aggregation also limits the unit's sensitivity to change and, "even larger shifts in channel morphology are necessary to evoke an unequivocal response in the data," (Poole et al., 1997 p.888). Nonetheless, with sufficient sample size (e.g., whole watershed surveys) habitat units may be adequate to detect change (Poole et al., 1997).

Table 3.1: The four levels of habitat units defined by California Department of Fish and Wildlife.

\begin{tabular}{|c|c|c|c|}
\hline Level I & Level II & Level III & Level IV \\
\hline \multirow[t]{2}{*}{ Riffle } & Riffle & $\begin{array}{l}\text { Riffle } \\
\text { Cascade }\end{array}$ & $\begin{array}{l}\text { Low Gradient Riffle } \\
\text { High Gradient Riffle } \\
\text { Cascade } \\
\text { Bedrock Sheet } \\
\end{array}$ \\
\hline & Flatwater & Flatwater & $\begin{array}{l}\text { Pocket Water } \\
\text { Glide } \\
\text { Run } \\
\text { Step Run } \\
\text { Edgewater }\end{array}$ \\
\hline \multirow[t]{3}{*}{ Pool } & Pool & Main Channel Pool & \multirow{2}{*}{$\begin{array}{l}\text { Trench Pool } \\
\text { Mid-Channel Pool } \\
\text { Channel Confluence Pool } \\
\text { Step Pool } \\
\text { Corner Pool } \\
\text { Lateral Scour Pool - Log Enhanced } \\
\text { Lateral Scour Pool - Root Wad Enhanced } \\
\text { Lateral Scour Pool - Bedrock Formed } \\
\text { Lateral Scour Pool - Boulder Formed } \\
\text { Plunge Pool } \\
\text { Secondary Channel Pool } \\
\text { Backwater Pool - Boulder Formed } \\
\text { Backwater Pool - Root Wad Formed } \\
\text { Backwater Pool - Log formed } \\
\text { Dammed Pool }\end{array}$} \\
\hline & & Backwater Pool & \\
\hline & & Additional Units & $\begin{array}{l}\text { Dry } \\
\text { Culvert } \\
\text { Not Surveyed } \\
\text { Not Surveyed due to a marsh }\end{array}$ \\
\hline
\end{tabular}




\subsection{Pools}

Pool habitat units are of particular interest in habitat assessment and monitoring for Coho Salmon because they serve as critical habitat for all life stages (Nickelson et al., 1992). Consequently, many physical aspects of pool habitat are considered for monitoring, and pool quantity and quality are often assessed. Unlike other habitat units, pool depth can be measured independently of flow by using residual pool depth, defined as the maximum pool depth minus the depth of the downstream riffle (or pool tail crest) (Lisle, 1987) (Figure

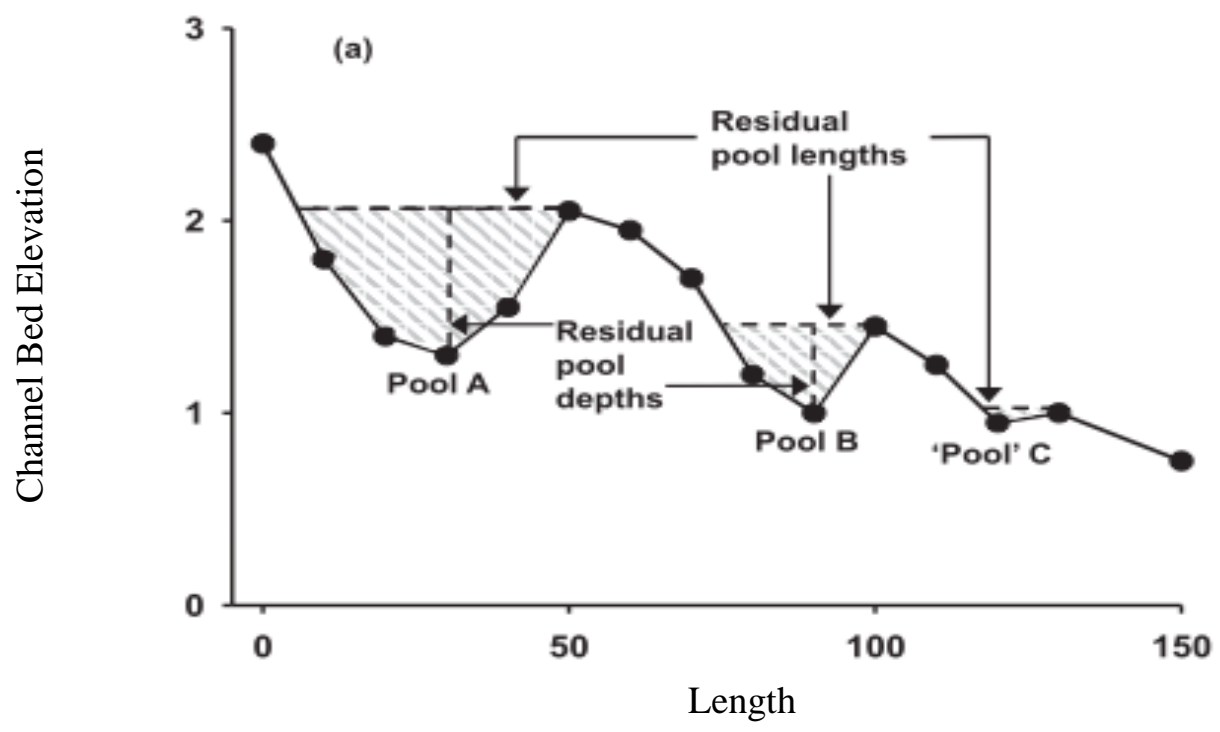

Figure 3.1: An illustration of residual pools, the shaded areas demonstrate the area contained within the residual pool measurement, which remains consistent across different flows. Source: Mossop and Bradford, 2005.

Pool depth is often considered a quantitative surrogate for pool quality (e.g., Madej, 1999; Mossop and Bradford, 2005; McIntosh et al., 2000). McIntosh and others (2000) used a pool depth of $1.8 \mathrm{~m}$ or greater to represent higher quality habitat (McIntosh et al., 
2000). Newman and Swanson (2008) defined "quality" pools as being either at least $0.3 \mathrm{~m}$ deep with cover of vegetation or undercut banks or $0.6 \mathrm{~m}$ deep if no cover was available.

Unlike many pool attributes, pool depth is a fairly robust measurement containing a relatively small amount of observer and measurement error. Roper et al. (2002) compared residual pool measurements made on six stream reaches by six or seven different teams of observers and found that stream heterogeneity accounted for $80 \%$ of the total variance, while difference between observers accounted for $20 \%$ of the total measurement variance. The authors determined a sample size of 102 pools was needed to detect a $10 \%$ change in mean residual pool depth with a $90 \%$ confidence interval (Roper et al., 2002). A study to quantify variance associated with several common habitat survey parameters in Pacific Northwest streams found the variability due to different survey crews accounted for approximately $20 \%$ of the total variability (Archer et al., 2004). In a study of the precision of commonly used physical habitat assessment metrics, Kaufmann et al. (1999) reported residual pool depth to be one of the most precise metrics. Keim and Skaugset (2002) examined the variance associated with residual pool measurement using topographic surveys and found reach level residual pool measurements to be more accurate than individual pool measurements.

Pool quantity, or abundance, has been described various ways in the literature including the percentage of pools per reach (Anlauf et al., 2011), length in residual pool (Mossop and Bradford, 2006), and pool riffle ratio (Bauer and Ralph, 2001). Many of the same drawbacks previously outlined for habitat types also apply to pool abundance. Chiefly, pool abundance metrics may not be responsive to short-term impacts and can potentially be confounded due to habitat identification discrepancies (Bauer and Ralph, 
2001; Poole et al., 1997). However, like habitat units, pool abundance may be a useful indicator of large-scale management impacts (Bauer and Ralph, 2001). Roper and others (2002) did not find percentage pool habitat to be a useful measurement in detecting difference between streams, as the percentage variance due to the observer $(56 \%)$ was greater than that due to stream heterogeneity (44\%). Kaufmann and others (1999) rated percentage pool habitat as having moderate to low precision and labeled pools as, "visual determinations of flow sensitive channel unit classes" (p. 78). Moreover, Kaufmann and others (1999) found percentage of pools per reach measurements to have twice as much variance due to difference between streams as difference in measurements from repeat visits to the same stream.

\subsection{Channel Geometry}

Channel dimensions are commonly measured in stream surveys and can be used to assess differences between streams or change over time. Width-to-depth ratio is an especially common metric, however, the type of width (i.e., bankfull or wetted) and depth measured and reported are often inconsistent across studies. Nonetheless, width-to-depth ratio can characterize habitat quality; deeper, narrower streams tend to provide higher quality habitat, expose less of the water surface to solar radiation, and promote undercut banks more than shallow, wide streams (Foster et al., 2001). Width-to-depth ratio is also sensitive to changes in discharge and sediment load (Knighton, 1998). Variance due to observer in width and depth measurements is often low. For example, it accounted for $12 \%$ of total variability in a survey of 26 interior Columbia River basin reaches (Archer et al., 2004), and $15 \%$ of total variability in a survey of six Idaho streams (Roper et al., 2002). Width-to-depth ratio significantly decreased during post-flood years of low to normal flow in Nevada streams with various degrees of degradation due to cattle grazing (Myers and 
Swanson, 1996). In a separate survey in Nevada, width-to-depth ratio did not change between surveys that were conducted 4 to 8 years apart (with a difference between means of 0.9), though all other indicators and metrics contrasted exhibited change. In the same study, significant improvement in width-to-depth ratio was detected over a 21-year interval. The shorter time period may not have been long enough for this metric to detect change, or the use of wetted- as opposed to bankfull widths and depths may have been inadequate to capture change (Newman and Swanson, 2008). Kershner and others (2004) found width-to-depth ratio was only a robust indicator of change for streams with a bankfull width greater than $5.0 \mathrm{~m}$.

\subsection{Stream Sediment Characteristics}

Salmon require channel substrate of a size small enough to be mobilized during redd building, but not so fine as to clog interstitial spaces and block water and oxygen flow to eggs incubating in the streambed. Common sediment metrics include percent fines (i.e., substrate < 6 mm; Archer et al, 2004; Roper et al, 2002) and particle size descriptors such as $\mathrm{D}_{16}, \mathrm{D}_{50}$, and $\mathrm{D}_{86}$. Sediment size measurements can be taken by conducting a pebble count (Wolman, 1954), percentage estimates of each substrate size class, or identification of dominant and secondary substrate types (Flosi et al., 2004). In Nevada streams, percentage fines (in Rosgen -B type channels) initially decreased significantly due to flooding, then increased significantly during a subsequent series of low -flow years (Myers and Swanson, 1996). In the Columbia River basin, pool tail fines on reference streams were $3.1 \%$ less than on managed streams, while riffle fines were $1.8 \%$ greater on reference streams than managed streams. It is instructive to note, however, that neither difference was of sufficient magnitude to be statistically significant (Kershner et al., 2004). In the 
same study, $\mathrm{D}_{50}$, (i.e., median particle size) was significantly smaller in reference streams than managed streams (29.7 mm versus $38.7 \mathrm{~mm}$, respectively). Likewise, $\mathrm{D}_{16}$ (particle size for which $16 \%$ of the substrate is smaller) was smaller for reference reaches, but not significantly so (9.3 $\mathrm{mm}$ vs. $11.5 \mathrm{~mm}$ for managed streams). $\mathrm{D}_{84}$ (the size for which $84 \%$ is smaller) was significantly different between reference and managed reaches only in streams greater than $5.0 \mathrm{~m}$ wide, and the difference in $\mathrm{D}_{84}$ size increased as stream width increased (Kershner et al., 2004). Variability due to observer has been found to account for $20 \%$ of total variability for pool tail fines and $30.5 \%$ for riffle fines (Archer et al., 2004). If the limit of observer variability for a useful metric is set at $20 \%$, percentage riffle fines are not adequate, whereas percentage pool tail fines are just barely suitable (Roper et al., 2002). In the same studies, the observer variability for $D_{50}$ accounted for $9.6-11 \%$ of total variability, making this substrate measurement more robust than percent fines (Archer et al., 2004; Roper et al., 2002).

\subsection{Streambanks and Vegetation}

Streambank and vegetation characteristics such as percentage canopy cover, type and amount of streamside vegetation, bank angle, percentage stable banks, and percentage and depth of undercut banks, are often included in monitoring surveys. The impact of riparian restoration varies with channel characteristics, but a study in Mendocino County, CA found reaches with passively restored riparian vegetation (exclusion fencing) on streams heavily impacted by cattle grazing exhibited increased complexity, and decreased width relative to control reaches with no fencing (Opperman and Merenlender, 2004).

Canopy cover is measured using a spherical densitometer (typically convex). Percentage canopy can influence stream temperature regimes through shading and is 
important for small and large organic debris recruitment (Larsen et al., 2004). Opperman and Merenlender (2004) found that pool temperature in reaches with restored riparian vegetation was $2.2^{\circ} \mathrm{C}$ to $4.5^{\circ} \mathrm{C}$ cooler than control (unrestored) reaches. Kaufmann and others (1999) found mid-stream canopy measurements with a spherical densitometer to have 15 times more variance due to difference in stream than to variance from repeat visits, putting them in the most robust group of metrics.

Bank stability, slope, and percentage and depth of undercuts were significant indicators of difference between reference and managed sites in the Columbia River basin. Streambanks in reference sites were 5.9\% more stable, bank angles were $8.2^{\circ}$ less steep, and undercut banks were $6.5 \%$ more common and $0.03 \mathrm{~m}$ deeper than on managed steams (Kershner et al., 2004).

Measurements and metrics collected by stream surveys and used for monitoring depend on both the nature of the stream channel and the purpose of the survey. Habitat unit measurements (including pool frequency) may not be the most robust measurements but they characterize the condition of the stream channel, and when examined over sufficient spatio-temporal scales may detect change. More quantitative measurements, such as pool depth, width-to-depth ratio, and bank and vegetation measurements, contain less observer error, and thus are likely more accurate indicators of stream heterogeneity and available fish habitat. 


\section{CHAPTER 4. METHODS}

\subsection{Study Design}

This study examines all freshwater habitats available to anadromous salmonids in mainstem Scotts Creek across two years, 1997 and 2013, for the purpose of evaluating change between the two sample periods. To accomplish this, an original habitat typing survey conducted in 1997 by the CDFW was used as a baseline, and a subsequent survey was conducted in 2013 using equivalent protocols. Additional baseline data, primarily for LWD, came from existing research by Dodson, (2005); Leicester, (2005); and Monteverde and others (2011). Both the 1997 and 2013 surveys began approximately 1.0 river km upstream from the Highway 1 bridge near the mouth of Scotts Creek and ended at a natural barrier to anadromy (waterfall) approximately 12 river $\mathrm{km}$ above the start point (Figure 4.1.1). Both surveys were conducted during summer base flow conditions. 


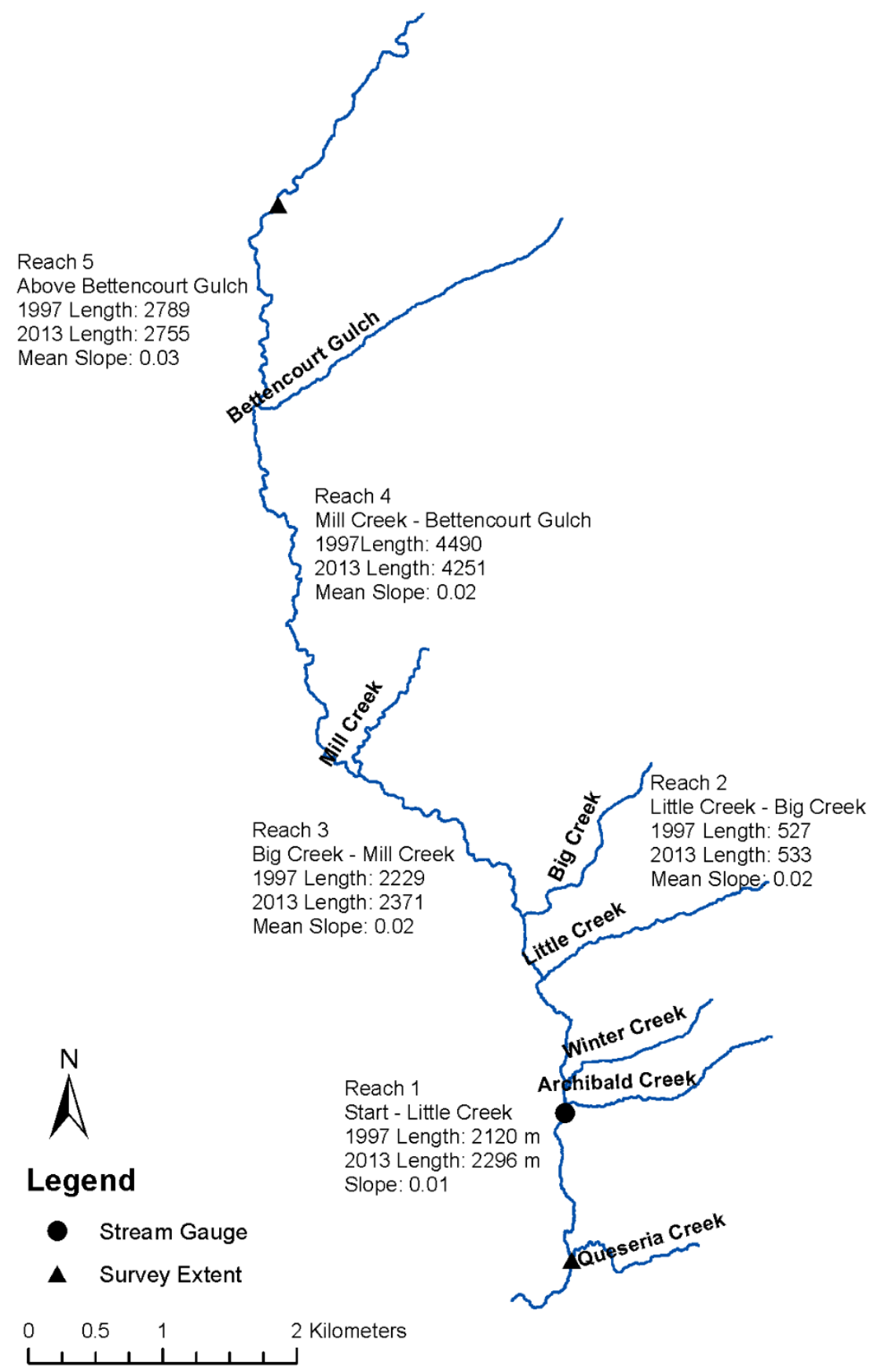

Figure 4.1.1: Scotts Creek survey extent and reaches, delineated by tributary confluences (minus Archibald Creek and Winter Creek, which are often dry at the confluence in the summer), including the location of the streamgage below Archibald Creek. 


\subsubsection{Parameters and Survey}

The survey procedures and data sheet are based on those found in Section III, pages III-30 - III-47 of the California Salmonid Stream Habitat Restoration Manual (Flosi et al., 2004) $)^{2}$ and are provided in Appendix A. Measurements collected and methods are outlined in Table 4.1.1. All measurements were taken in feet and tenths of feet where applicable.

Prior to beginning the 2013 survey, minor changes were made to the survey form and procedures found in Flosi and others (2004). Specifically, all data was recorded at every individual habitat unit, instead of the recommended every $10^{\text {th }}$ unit. The "percent exposed substrate" category was omitted, substrate composition was changed to include percentages of all substrate types (i.e., silt/clay, sand $[<0.2 \mathrm{~cm}]$, gravel $[0.2-6.4 \mathrm{~cm}]$, small cobble [6.4-12.7 cm] large cobble [12.7-25.4 cm], boulder [>25.4 cm] and bedrock), rather than "dominant and co -dominant." Global Positioning System (GPS) waypoints were collected at the downstream edge of every habitat unit, and bankfull width and depth were measured once per day. Examples of both survey forms are provided as Appendix B.

The large woody debris survey form (LWD form) was adopted from Leicester (2005), which was itself modified from the form presented in Flosi and others (2004). Minor modifications were made to simplify the Leicester (2005) form to increase the speed of data collection in the field. Wood was recorded if it was within one of three length classes; $1.8-3.7 \mathrm{~m}, 3.7-6.1 \mathrm{~m}$, and $>6.1 \mathrm{~m}\left(6-12^{\prime}, 12^{\prime}-20^{\prime}\right.$, and $>20^{\prime}$, respectively), and within one of four diameter classes; $0.2-0.6 \mathrm{~m}, 0.6-0.9 \mathrm{~m}, 0.9-1.2 \mathrm{~m}$, and $>1.2 \mathrm{~m}\left(0.5^{\prime}\right.$ $-2^{\prime}, 2^{\prime}-3^{\prime}, 3^{\prime}-4^{\prime}$, and $>4^{\prime}$, respectively). When discernable, the species of tree was recorded. The "function" of the wood was assigned to one of four categories: 1) lowflow

\footnotetext{
${ }^{2}$ available online at http://www.dfg.ca.gov/fish/resources/habitatmanual.asp.
} 
pool - within the wetted channel and causing localized scour, but not limited to within a pool habitat unit; 2) lowflow extra - in the wetted channel, but not causing scour; 3) bankfull backwater - above the lowflow channel, but within the bankfull channel and creating or enhancing a backwater pool; and 4) bankfull extra - within the bankfull channel but not creating or enhancing a backwater. Wood was recorded as either a single piece, as part of an aggregate (2-4 pieces), or as part of a jam ( $>4$ pieces). I also noted the presence of a root wad, whether the wood was dead or alive, and classified the approximate size of the jam as small, medium, or large. An example of the LWD survey form is provided as Appendix C.

Table 4.1.1: Stream habitat measurements taken by each surveyor (A and B) during the 2013 habitat survey of Scotts Creek (Santa Cruz County, CA).

\begin{tabular}{llc}
\hline Measurement & Method/Instrument & Surveyor \\
\hline Habitat Type & Based on level IV descriptions & $\mathrm{A}$ \\
GPS Coordinates & Garmin Montana 650 & $\mathrm{B}$ \\
Temperature (air and water) & Thermometer & $\mathrm{A}$ \\
Length of Habitat Unit & Cloth Tape, measured along & $\mathrm{A}$ \\
& thalwag & \\
Mean Wetted Width & Average of three measurements, & $\mathrm{A} / \mathrm{B}$ \\
& Cloth Tape/Philadelphia Rod & \\
Depth, maximum, pool tail crest, & Philadelphia Rod & $\mathrm{B}$ \\
and mean & & \\
Pool Tail Embeddedness & Visual Estimate & $\mathrm{A}$ \\
Pool Tail Substrate & Visual Estimate & $\mathrm{A}$ \\
Shelter Value & Visual Estimate & $\mathrm{A}$ \\
Percent Instream Cover & Visual Estimate & $\mathrm{A}$ \\
Substrate Composition & Visual Estimate & $\mathrm{A}$ \\
Percent Canopy & Convex Spherical Densiometer & $\mathrm{A}$ \\
Length of Disturbed Bank & Visual Estimate & $\mathrm{A}$ \\
Percent Bank Vegetated & Visual Estimate & $\mathrm{A}$ \\
Dominant Vegetation & Visual Estimate & $\mathrm{A}$ \\
LWD Form & & $\mathrm{B}$ \\
\hline
\end{tabular}

Surveying began on 28 June 2013 and ended on 03 September 2013; a total of 30 days were spent conducting the survey. The starting point for the survey was determined 
by walking the stream and noting the point at which individual pool, riffle, or run habitats could be identified, as opposed to primarily estuarine dominated habitat. Four people conducted the survey, two people at a time. Surveyor A (primary author) remained constant for the entire survey, and surveyor B rotated between three people.

\subsection{Error Study}

A confidence study determined the amount of variance in residual pool measurements among different surveyors. Seven participants surveyed 20 marked pools, measuring pool tail crest depth, maximum depth, and length. Prior to the survey a short classroom training was held for all participants to define residual pools and pool tail crest. The surveyors had different degrees of education and experience, including two NOAA scientists, four hydrology or fisheries ecology graduate students, and one hydrology field assistant. All had previous experience taking a variety of stream measurements.

The results of the confidence survey distinguished actual morphological change from observer error. The standard deviation of residual pool depth for each of the 20 surveyed pools were averaged to generate a "grand deviation" across all pools. Although certain pools were too deep to wade completely (thereby making the maximum depth measurement more difficult) the standard deviations of these pools during the confidence study were not greater than the rest of the pools, in which wading did not hinder maximum depth measurements. The "grand deviation" was determined to be $\pm 8.0 \mathrm{~cm}$, thus any change greater than this was interpreted as strong evidence in support of morphological change, as opposed to difference due to measurement error. 


\subsection{Reach Delineation and Additional Metrics}

For analysis, I divided the $12 \mathrm{~km}$ segment of mainstem Scotts Creek that supports anadromous salmonids into 5 different reaches, using tributary confluences to define reach boundaries. Reaches were numbered consecutively from downstream to upstream and reach lengths, as calculated from survey length measurements, varied from $527 \mathrm{~m}$ (reach 2, between Little Creek and Big Creek; 1997) to 4,490 m (reach 4, between Mill Creek and Bettencourt Gulch; 1997). The reaches had a minimum distance between survey years of $6 \mathrm{~m}$ (reach 2), and a maximum distance between survey years of $239 \mathrm{~m}$ (reach 4). Stream gradient ranged between 0.01 (reach 1) and 0.03 (reach 5; Figure 4.1.1). Using tributaries as reach boundaries had the dual benefits of using marked reference points on both surveys, ensuring the reach boundaries were in the same locations for both surveys, and allowing for comparison of reaches within a year directly below and above a tributary. Reach boundaries are shown in Figure 4.1.1.

In addition to the measurements listed in section 4.1.1, metrics used in this study to determine habitat change included residual pool depth, percentage primary pools, pool abundance, and pool depth diversity. Following Lisle (1987) residual pool depth was defined as the difference in depth or bed elevation between a pool and the downstream riffle crest. For this study, "riffle crest" was replaced with "pool tail crest." Residual pool measurements allowed for pool depth measurements independent of flow. Primary pools are defined in the Final Recovery Plan for Central California Coast Coho Salmon Evolutionarily Significant Unit as pool habitats having a maximum depth $\geq 91 \mathrm{~cm}(3 \mathrm{ft})$, and the plan sets a basin-wide goal of $>49 \%$ of pools being primary pools (NMFS, 2012). Pool abundance was characterized in three ways: 1) number of pools per reach; 2) total length of stream in pool habitat per reach; and 3) percentage of reach length in pool habitat 
per reach. Pool depth diversity was measured as the standard deviation of all residual pool depths per reach and a higher standard deviation indicated greater diversity in pool depth (Mossop and Bradford, 2005; Madej, 1999).

The total number of riffle habitat units was slightly confounded by the use of the "step-run" habitat type which comprises a run-riffle sequence and effectively hides the presence of the individual riffles within the step-run. For analysis, one riffle unit was added for every step-run. Although adding just one riffle for every step-run likely underestimates the number of individual riffle units, it is nonetheless a closer approximation of the actual number.

\subsection{Analysis}

Change was determined by a comparison of means by reach for every metric. I used Student's $t$-tests to determine statistically significant change between the two years, using a significance value of $P=0.05$. To maintain an experiment-wise Type I error rate $(\alpha)$ of 0.05, Bonferroni adjusted P-values were used to assess significance when tests involved multiple comparisons.

\subsection{LWD Terminology}

Large woody debris was defined as both "enhancing" pools, and "in association" with pools, and the difference is somewhat important. The system of habitat typing used in this survey fails to capture the importance of wood in pools larger than $60 \%$ of the wetted channel (see conclusion section 7.2.1), however, I recognized this early in the survey and attempted to make comments noting the importance of LWD in particular habitat units. LWD was considered as enhancing a pool if it was the primary piece in a pool unit 5.2 or 5.3 (Lateral Scour Pool-Log Enhanced or Lateral Scour Pool-Root Wad Enhanced; see 
Table 3.1) or backwater unit 6.3 or 6.4 (Backwater Pool - Rootwad Formed, or Backwater Pool - Log Formed; Table 3.1), or if it was noted to be LWD enhanced in the comments of either the primary survey form or the LWD survey form. Large woody debris that was "associated" with a pool was LWD noted on the LWD form occurring in a pool unit and with the function of either lowflow pool or bankfull backwater. These pieces may have been, but were not necessarily instrumental to the pool's formation and/or maintenance. 


\section{CHAPTER 5. RESULTS}

\subsection{Whole-Stream Results}

The 1997 stream survey of the anadromous portion of mainstem Scotts Creek reported 654 different habitat units distributed over a total stream length of $12.12 \mathrm{~km}$, (as measured using a cloth tape). In contrast, the 2013 survey measured $12.21 \mathrm{~km}(+0.09 \mathrm{~km})$ in length (also measured with a cloth tape) and identified 529 distinct habitat units $(-125$ habitat units). The total number of pool habitat units increased by six pools from 1997 ( $N$ $=284)$ to $2013(N=290)$, and the total length of mainstem Scotts Creek classified as pool habitat increased by $61 \mathrm{~m}$ (Table 5.1.1). There was no change in the total percentage pool habitat by length (40\%) across both surveys. The total length of mainstem Scotts Creek classified as riffle habitat increased by $753.47 \mathrm{~m}$ (not including the length of riffles included in step-run habitat units). Pool-riffle-flatwater ratio increased in percentage riffles and decreased in percentage flatwater (Table 5.1.1). Change in channel dimensions included a decrease in total mean residual pool depth of $5.0 \mathrm{~cm}$, with the number of primary pools $(\geq 91 \mathrm{~cm}$ ) decreasing by 14 and mean depth of primary pools increasing by $4.0 \mathrm{~cm}$. Pool depth diversity, measured as the standard deviation of residual pool depths, increased by $0.7 \mathrm{~cm}$ between surveys (Table 5.1.2).

Table 5.1.1: Whole stream habitat related values for 1997 and 2013.

\begin{tabular}{cccccccccc}
\hline $\begin{array}{c}\text { Number of } \\
\text { Pools }\end{array}$ & \multicolumn{2}{c}{$\begin{array}{c}\text { Length in Pool } \\
(\mathbf{m})\end{array}$} & \multicolumn{2}{c}{$\begin{array}{c}\text { Percentage } \\
\text { Pool Habitat }\end{array}$} & \multicolumn{2}{c}{$\begin{array}{c}\text { Length in Riffle } \\
(\mathbf{m})\end{array}$} & \multicolumn{2}{c}{$\begin{array}{c}\text { Pool-Riffle- } \\
\text { Flatwater Ratio (\%) }\end{array}$} \\
\hline 1997 & 2013 & 1997 & 2013 & 1997 & 2013 & 1997 & 2013 & 1997 & 2013 \\
\hline 284 & 290 & 4877.7 & 4938.7 & 40 & 40 & 1106.4 & 1859.9 & $40: 9: 51$ & $40: 15: 44$ \\
\hline
\end{tabular}


Table 5.1.2: Whole stream channel dimension values for 1997 and 2013. Standard deviation values are in parenthesis.

\begin{tabular}{llllllll}
\hline \multicolumn{2}{c}{$\begin{array}{c}\text { Mean Residual Pool } \\
\text { Depth }\end{array}$} & \multicolumn{2}{c}{$\begin{array}{c}\text { Number of } \\
\text { Primary Pools }\end{array}$} & \multicolumn{2}{c}{$\begin{array}{c}\text { Mean Primary Pool } \\
\text { Depth }\end{array}$} & \multicolumn{2}{c}{$\begin{array}{c}\text { Pool Depth } \\
\text { Diversity }\end{array}$} \\
\hline 1997 & 2013 & 1997 & 2013 & 1997 & 2013 & 1997 & 2013 \\
\hline $75.2(29.9)$ & $69.8(30.6)$ & 74 & 60 & $113(30)$ & $117(21)$ & 29.92 & 30.57 \\
\hline
\end{tabular}

Mean canopy cover, bank vegetation, and percentage instream cover increased between 1997 and 2013 by 4.9\%, 30.4\%, and 1.1\% respectively (Table 5.1.3). Discharge at the start of the 1997 survey (09 July 1997) measured $0.13 \mathrm{~m}^{3} / \mathrm{s}$ and declined to $0.08 \mathrm{~m}^{3} / \mathrm{s}$ by the completion of the survey (07 August 1997). In contrast, 2013 start of survey discharge measured $0.07 \mathrm{~m}^{3} / \mathrm{s}$ (25 June 2013) and dropped to $0.02 \mathrm{~m}^{3} / \mathrm{s}$ by survey's end (03 September 2013).

Table 5.1.3: Mean canopy, vegetation, and instream cover values for the entire survey length for 1997 and 2013. Standard deviation values are in parenthesis.

\begin{tabular}{llllll}
\hline \multicolumn{2}{c}{ Canopy Cover (\%) } & \multicolumn{2}{c}{ Bank Vegetation (\%) } & \multicolumn{2}{c}{ Instream Cover (\%) } \\
\hline 1997 & 2013 & 1997 & 2013 & 1997 & 2013 \\
\hline $87.8(19.2)$ & $92.7(10.7)$ & $48.2(31.3)$ & $78.6(25.3)$ & $18.4(17.4)$ & $19.5(21.3)$ \\
\hline
\end{tabular}

\subsection{Habitat Units by Reach}

The total number of habitat units quantified in 2013 increased relative to 1997 survey results in reach 3 , and decreased in all other reaches (Table 5.2.1). The most notable decrease in the total number of distinct units was in reach 4, with a decrease of 120 habitat units. Additionally, the total number of pool habitat units increased from 1997 to 2013 in reaches 1,3 , and 5 , and decreased in reaches 2 and 4 . Both the total length of habitat classified as pool and the percentage pool habitat by length increased in reaches 1 and 5, and decreased in reaches 2, 3, and 4. Mean pool length increased in reaches 4 and 5, decreased in reaches 1 and 3, and remained unchanged in reach 2 . However, only reach 3 
exhibited a statistically significant change in mean pool length from 1997 to 2013

(Student's $t$-test, $t_{(107)}=4.19, \mathrm{P}<0.001$; Table 5.2.1).

Table 5.2.1: Habitat units and pool units for each reach. Values in bold text indicate significant change between survey years using a Bonferroni adjusted alpha $(\alpha)$ level of $0.01(0.05 / 5)$. Standard deviation values are in parentheses.

\begin{tabular}{lllllllllllllll}
\hline & $\begin{array}{c}\text { Number of } \\
\text { Habitat } \\
\text { Units }\end{array}$ & \multicolumn{2}{c}{$\begin{array}{c}\text { Number of } \\
\text { Pool Units }\end{array}$} & \multicolumn{2}{c}{$\begin{array}{l}\text { Total length } \\
\text { in Pool (m) }\end{array}$} & \multicolumn{2}{c}{$\begin{array}{c}\text { Percentage } \\
\text { Pool by } \\
\text { Length }\end{array}$} & & \multicolumn{2}{c}{ Mean Pool Length (m) } \\
\hline Reach & 1997 & 2013 & 1997 & 2013 & 1997 & 2013 & 1997 & 2013 & 1997 & 2013 & df & $t$ value & P-value \\
\hline 1 & 84 & 80 & 27 & 36 & 617 & 722 & 29 & 31 & $23(9.1)$ & $20(9.3)$ & 56 & 1.20 & 0.236 \\
2 & 23 & 21 & 11 & 8 & 208 & 150 & 39 & 28 & $19(10.0)$ & $19(5.8)$ & 16 & 0.03 & 0.975 \\
3 & 146 & 150 & 60 & 73 & 1077 & 964 & 48 & 41 & $18(7.3)$ & $13(5.4)$ & 107 & $\mathbf{4 . 1 9}$ & $<\mathbf{0 . 0 0 1}$ \\
4 & 370 & 250 & 132 & 111 & 2031 & 1872 & 45 & 44 & $15(8.4)$ & $17(8.3)$ & 235 & -1.38 & 0.170 \\
5 & 178 & 135 & 54 & 62 & 945 & 1230 & 34 & 45 & $18(6.4)$ & $20(9.2)$ & 109 & -1.62 & 0.108 \\
\hline
\end{tabular}

The total number of riffles decreased in all reaches except reach 2 . However, the percentage of riffle units increased in reaches 2,4 , and 5, and decreased in reaches 1 and 3. Mean step run length increased in every reach in 2013 , creating the possibility that the number of riffles in each step run were greater in 2013 than in 1997. The total length in riffle, without any correction for riffles contained within step-runs, increased in reaches 1 , 4, and 5, and decreased in reaches 2 and 3 (Table 5.2.2). The pool-riffle-flatwater ratio improved (decreased in flatwater) in reaches 1, 4, and 5, and declined in reaches 2 and 3 (Table 5.2.3). This measurement does not account for riffles contained within step-runs, which would increase the riffle percentage and decrease the flatwater percentage.

Table 5.2.2: Riffle units for each reach.

\begin{tabular}{lllllll}
\hline & \multicolumn{2}{c}{$\begin{array}{c}\text { Number of } \\
\text { Riffles }\end{array}$} & \multicolumn{2}{c}{$\begin{array}{c}\text { \% Riffle } \\
\text { Units }\end{array}$} & \multicolumn{2}{c}{$\begin{array}{c}\text { Length in Riffle } \\
(\mathbf{m})\end{array}$} \\
\hline Reach & 1997 & 2013 & 1997 & 2013 & 1997 & 2013 \\
\hline 1 & 25 & 20 & 30 & 25 & 217 & 240 \\
2 & 5 & 6 & 22 & 29 & 55 & 53 \\
3 & 40 & 33 & 27 & 22 & 189 & 104 \\
4 & 112 & 80 & 30 & 32 & 396 & 584 \\
5 & 64 & 50 & 36 & 37 & 249 & 880 \\
\hline
\end{tabular}


Table 5.2.3: Pool-Riffle-Flatwater ratio for each reach. The federal CCC Coho Salmon Recovery Plan deems $>30 \%$ pools and $>20 \%$ riffle suitable for Coho Salmon.

\begin{tabular}{ccccccc}
\hline & \multicolumn{1}{c}{$\mathbf{1 9 9 7}$} & \multicolumn{3}{c}{$\mathbf{2 0 1 3}$} \\
\hline Reach & $\begin{array}{c}\mathbf{\%} \\
\text { Pool }\end{array}$ & $\begin{array}{c}\text { \% } \\
\text { Riffle }\end{array}$ & $\begin{array}{c}\text { \% } \\
\text { Flatwater }\end{array}$ & $\begin{array}{c}\text { \% } \\
\text { Pool }\end{array}$ & $\begin{array}{c}\text { \% } \\
\text { Riffle }\end{array}$ & $\begin{array}{c}\text { \% } \\
\text { Flatwater }\end{array}$ \\
\hline 1 & 29 & 10 & 61 & 31 & 10 & 58 \\
2 & 39 & 11 & 50 & 28 & 10 & 62 \\
3 & 48 & 8 & 43 & 41 & 4 & 55 \\
4 & 45 & 9 & 46 & 44 & 14 & 42 \\
5 & 34 & 9 & 57 & 45 & 32 & 23 \\
\hline
\end{tabular}

\subsection{Channel Dimensions}

Mean residual pool depth decreased substantially ( $\geq 10 \mathrm{~cm}$ in all cases) from 1997 to 2013 in all stream reaches except reach 4, where it increased by $7 \mathrm{~cm}$ (Table 5.3.1). The increase in reach 4 , however, fell within the bounds of expected measurement error $( \pm 8.0$ $\mathrm{cm}$; see Methods, Section 4.4). Hence, any change greater than this is indicative of morphological change, as opposed to difference due to measurement error. Significant change in mean residual pool depth occurred in reaches $1\left(-28 \mathrm{~cm}\right.$; Student's $t$-test, $t_{(59)}=$ $3.29, \mathrm{P}=0.002)$ and $3\left(-12 \mathrm{~cm}\right.$; Student's $t$-test, $t_{(129)}=2.84, \mathrm{P}=0.005$; Figure 5.3.6). Pool depths and distributions between the two survey years are compared in figures 5.3.1 - 5.3.6.

The number of primary pools (>91 cm; Methods section 4.3) increased in reach 4, and decreased in reaches $1,2,3$, and 5 (Table 5.3.2). The percentage primary pools and the mean depth of primary pools also increased only in reach 4. However, the observed changes in reaches 1 and 2 fell within the bounds of expected measurement error as determined by the confidence study.

Changes in pool depth diversity follow the same pattern as mean depth of deep pools, as diversity increased in reaches 3 and 4, and decreased in reaches 1, 2, and 5. Both 
the lowest and highest pool depth diversity values were derived from the 1997 survey at

\section{$21.21 \mathrm{~cm}($ reach 4) and $42.42 \mathrm{~cm}$ (reach 5), respectively (Table 5.3.1).}

Table 5.3.1: Channel dimension values for each reach. Values in bold text indicate significant change between survey years using a Bonferroni adjusted alpha $(\alpha)$ level of $0.01(0.05 / 5)$. Standard deviation values are in parentheses. An asterisk $(*)$ indicates change in pool depth greater than that expected by surveyor error $( \pm 8.0 \mathrm{~cm})$.

\begin{tabular}{llllllllll}
\hline & \multicolumn{4}{c}{ Mean Residual Pool Depth (cm) } & \multicolumn{3}{c}{ Pool Depth Diversity } & \multicolumn{2}{c}{ n } \\
\hline Reach & 1997 & 2013 & df & $t$-value & P-value & 1997 & 2013 & 1997 & 2013 \\
\hline 1 & $92(32)$ & $\mathbf{6 4}(31)^{*}$ & 59 & 3.29 & 0.002 & 31.56 & 30.53 & 27 & 36 \\
2 & $93(39)$ & $67(24) *$ & 17 & 1.67 & 0.114 & 39.37 & 24.22 & 11 & 8 \\
3 & $74(23)$ & $\mathbf{6 2}(27) *$ & 129 & 2.84 & 0.005 & 23.23 & 27.40 & 60 & 73 \\
4 & $67(21)$ & $74(32)$ & 239 & -2.26 & 0.025 & 21.21 & 31.86 & 132 & 111 \\
5 & $85(42)$ & $75(31) *$ & 111 & 1.45 & 0.150 & 42.42 & 30.84 & 54 & 62 \\
\hline
\end{tabular}

Table 5.3.2: Primary pool ( $\geq 91 \mathrm{~cm}$ deep) values for each reach. Percentage primary pools is relative to the total number of pools in each reach, standard deviation values are in parentheses.

\begin{tabular}{lllllll}
\hline & \multicolumn{2}{c}{$\begin{array}{c}\text { Number of } \\
\text { Primary Pools }\end{array}$} & \multicolumn{2}{c}{$\begin{array}{c}\text { \% Primary } \\
\text { Pools }\end{array}$} & \multicolumn{2}{c}{$\begin{array}{c}\text { Mean Depth of Primary } \\
\text { Pool (cm) }\end{array}$} \\
\hline Reach & 1997 & 2013 & 1997 & 2013 & 1997 & 2013 \\
\hline 1 & 13 & 7 & 48 & 19 & $119(22)$ & $112(24)$ \\
2 & 7 & 1 & 64 & 13 & $118(24)$ & $113(0)$ \\
3 & 16 & 8 & 27 & 11 & $106(11)$ & $115(16)$ \\
4 & 23 & 30 & 17 & 27 & $101(13)$ & $116(25)$ \\
5 & 15 & 14 & 28 & 23 & $132(55)$ & $122(17)$ \\
\hline
\end{tabular}




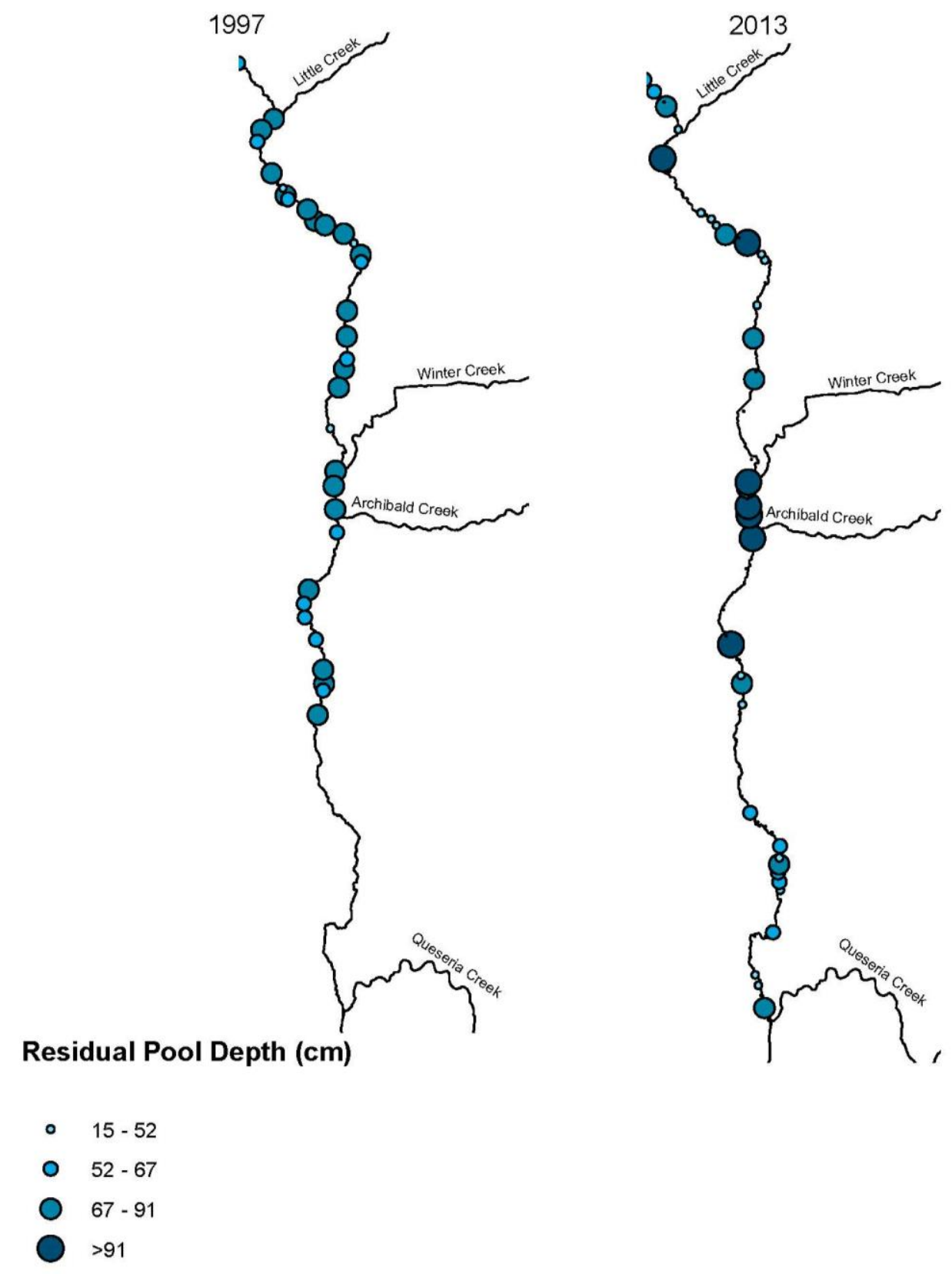

Figure 5.3.1: Residual pool depths and distribution for Reach 1, below Little Creek. 

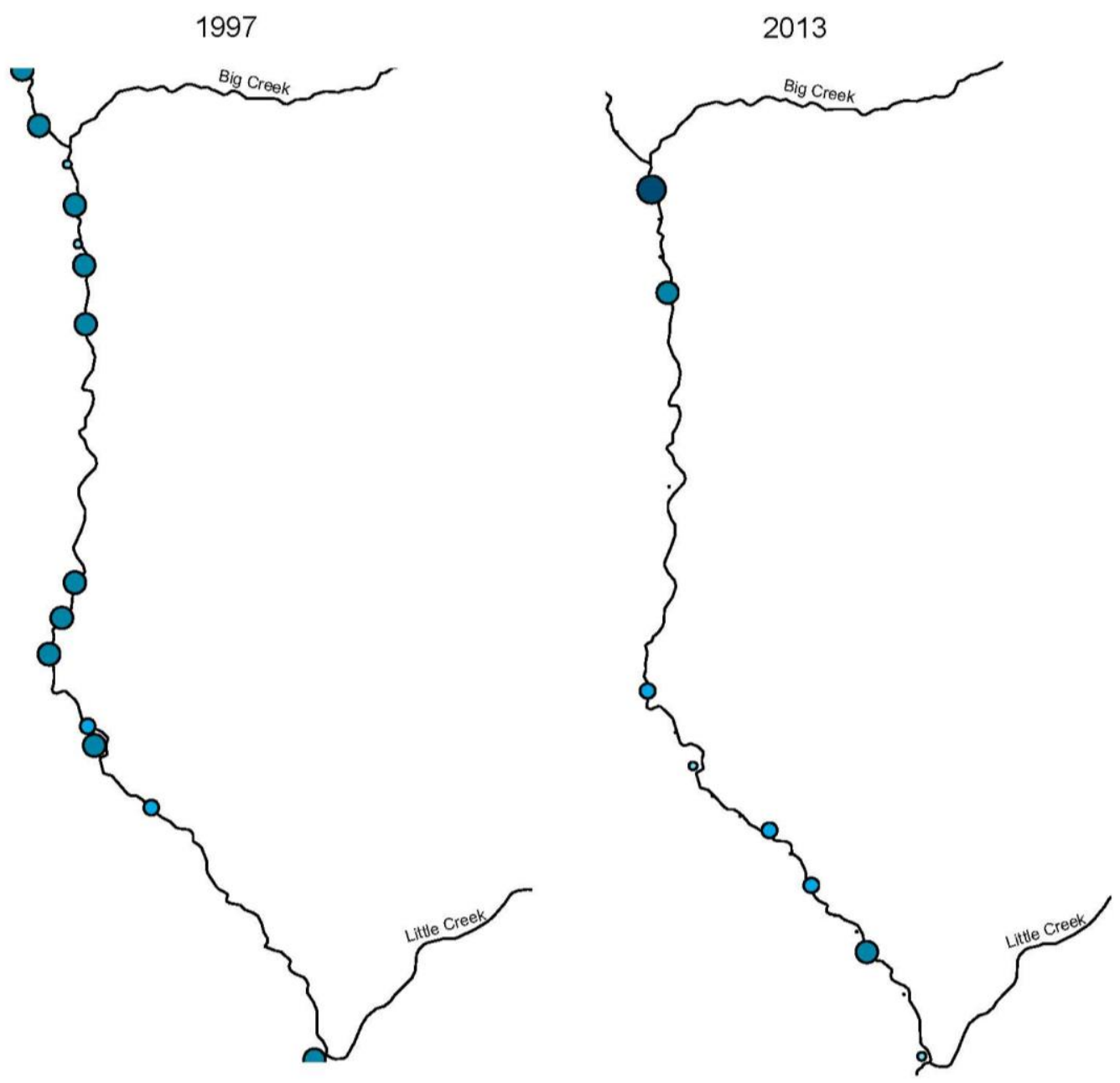

Residual Pool Depth (cm)
- $15-52$
○ $52-67$
O $67-91$
$>>91$

Figure 5.3.2: Residual pool depths and distribution for Reach 2, Little Creek to Big Creek. 


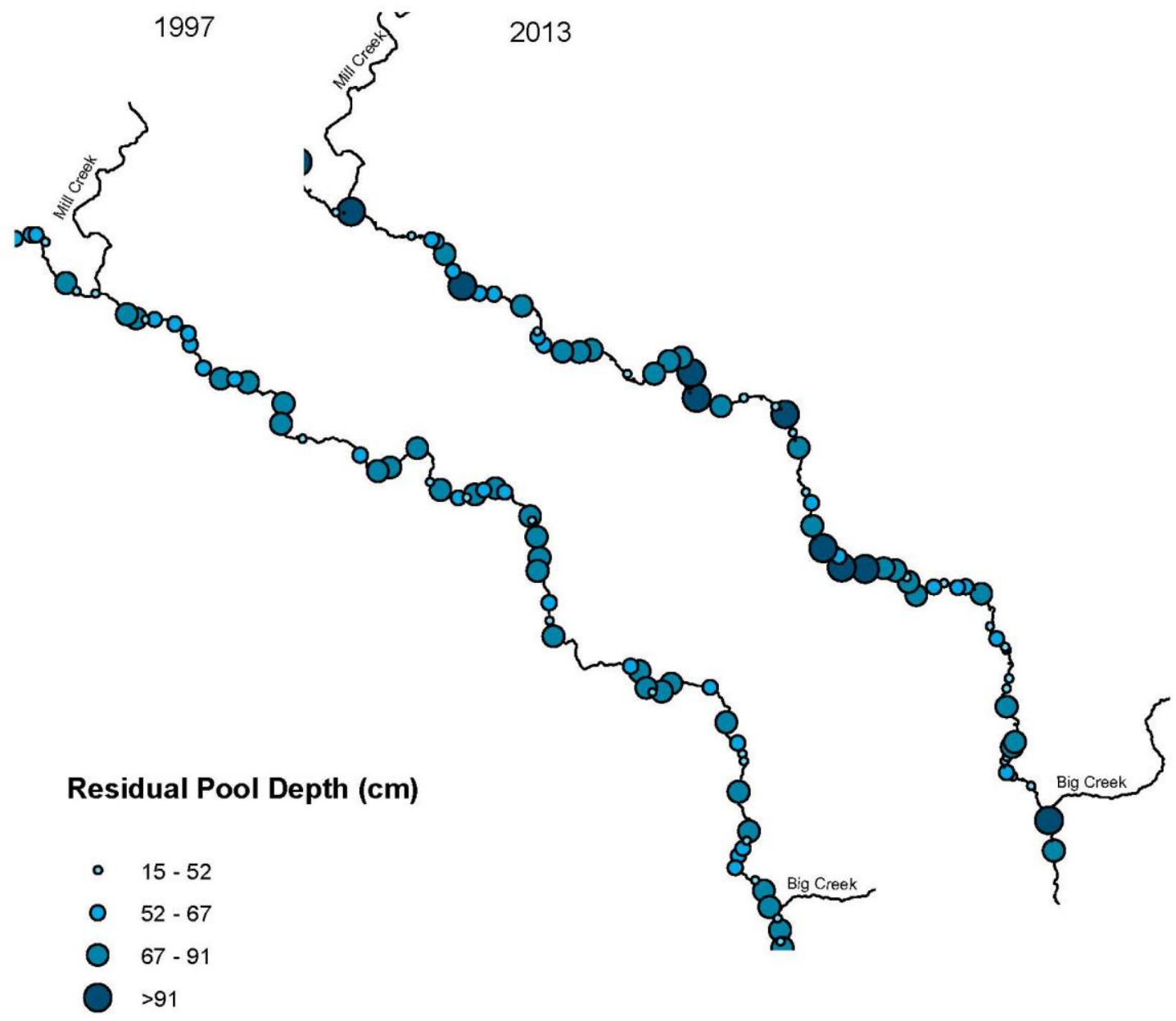

Figure 5.3.3: Residual pool depths and distribution for Reach 3, Big Creek to Mill creek 

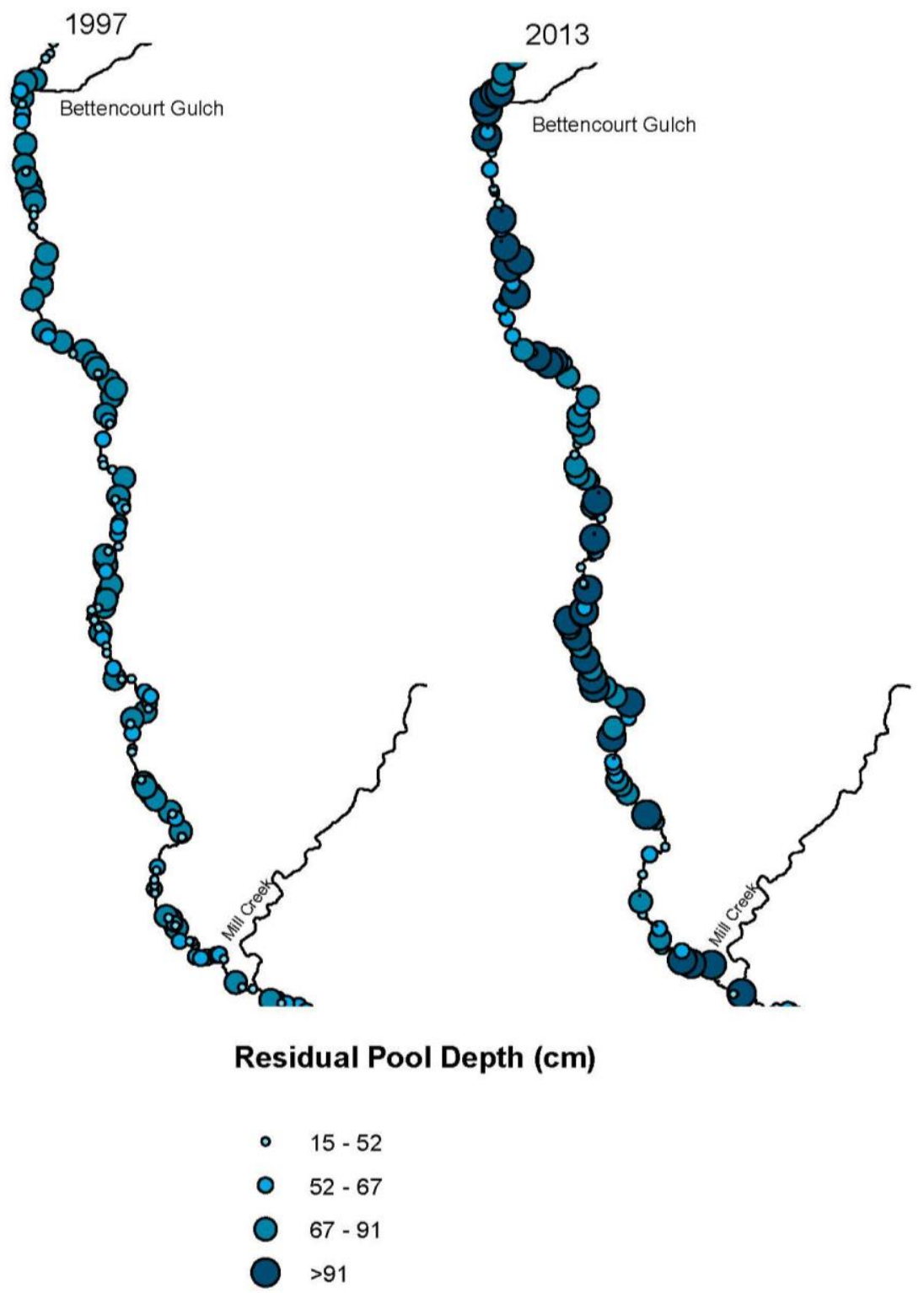

Figure 5.3.4: Residual pool depths and distribution for Reach 4, Mill Creek to Bettencourt Gulch. 


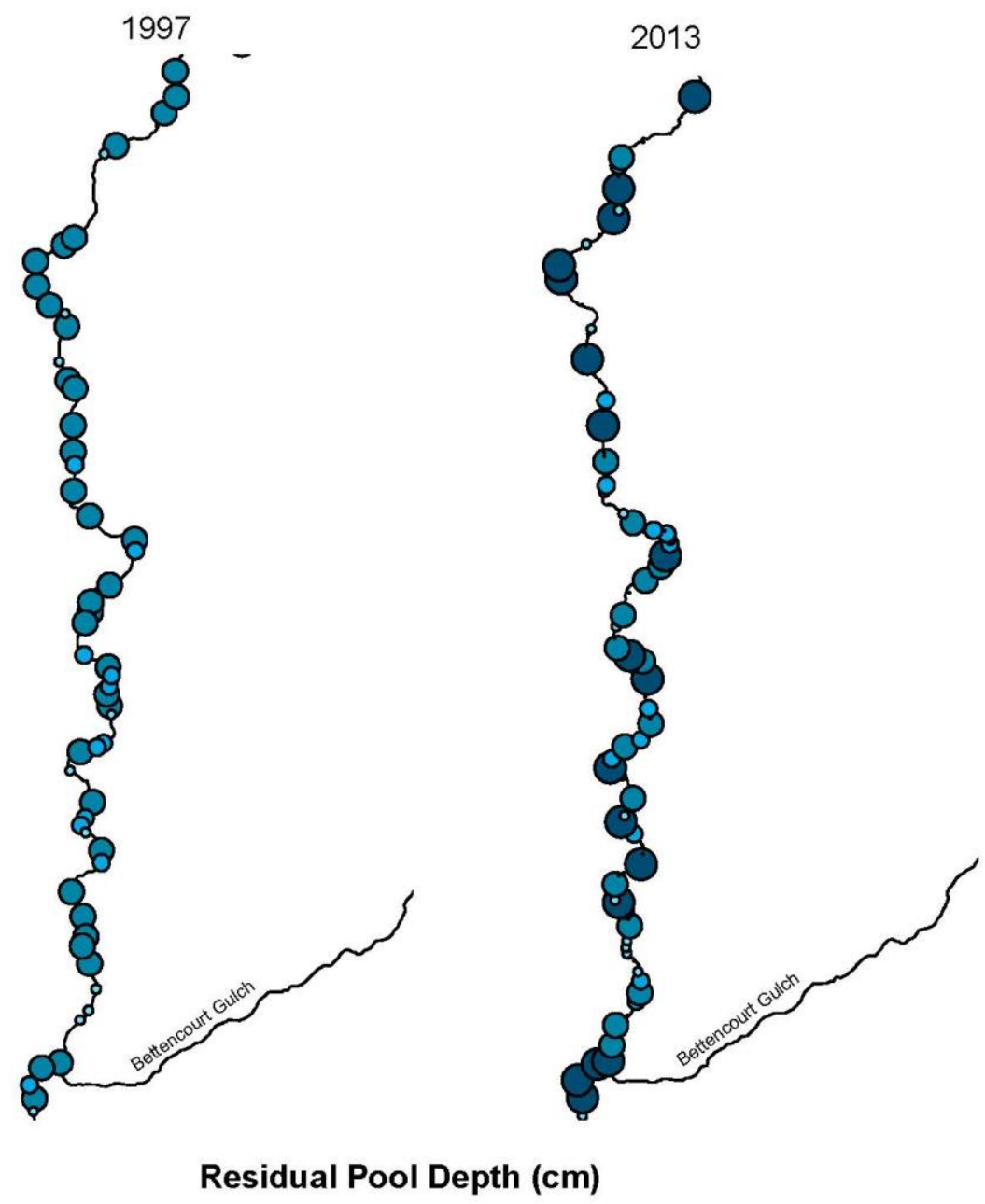

- $15-52$

- $52-67$

- $67-91$

$>>91$

Figure 5.3.5: Residual pool depths and distribution for Reach 5, above Bettencourt Gulch 

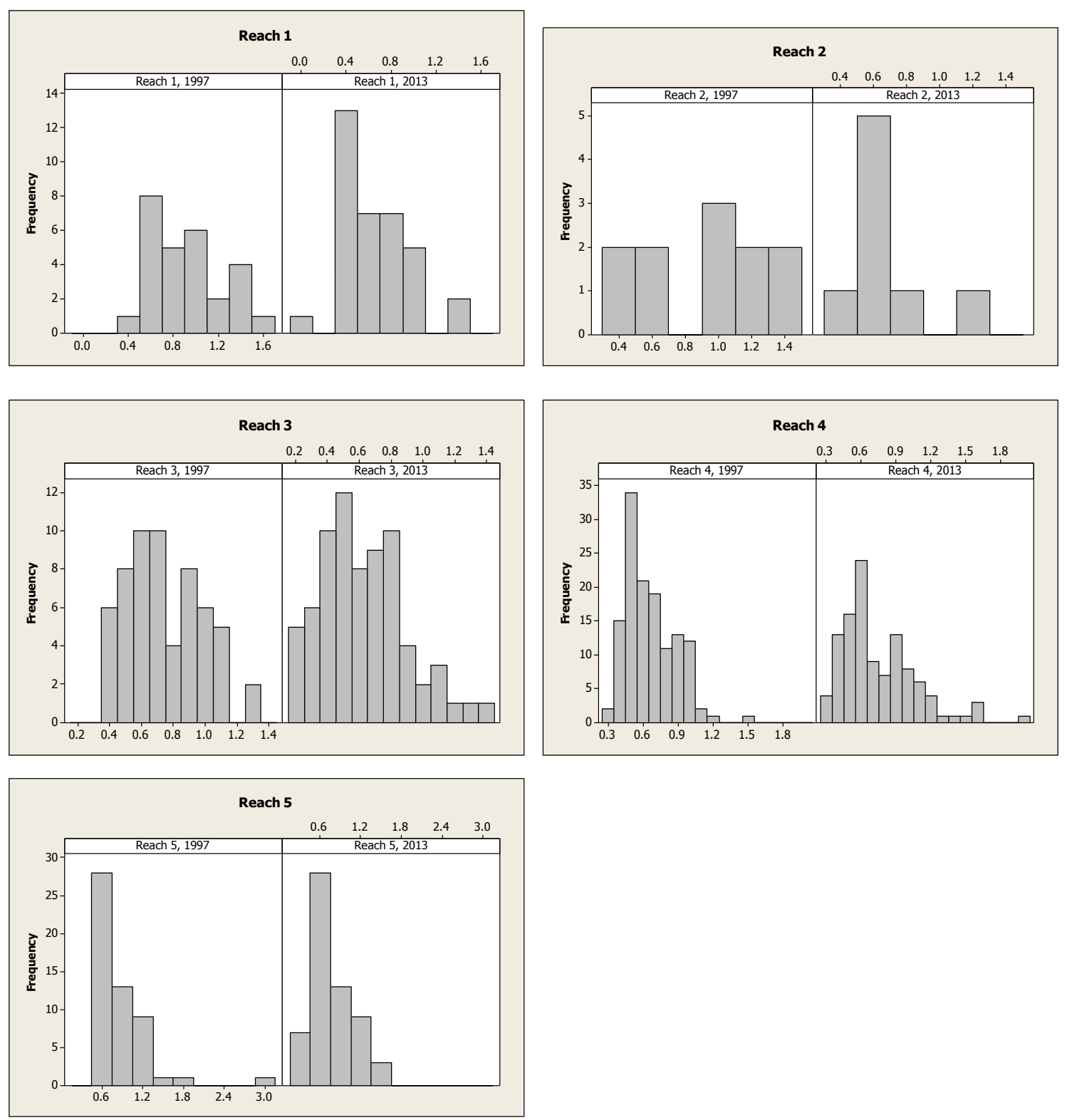

Figure 5.3.6: Histograms showing the frequency distribution of residual pool depths in 1997 and 2013 for each reach

\subsection{Vegetation}

Canopy cover increased in all reaches except reach 5 where it remained unchanged.

The difference in canopy cover between the two survey years increased from upstream to downstream, with a maximum increase of $13 \%$ in reach 1 . The increase in canopy was significant in reach 3 (Student's $t$-test, $t_{(66)}=-2.67, \mathrm{P}=0.010$; Table 5.4.1). The percentage 
of canopy cover composed of coniferous trees increased in reach 3 , decreased in reaches 2, and 5, and remained unchanged in reaches 1 and 4 (Table 5.4.1). However, these values are in relation to the total amount of canopy cover. The actual amount of coniferous canopy increased in reaches 3 and 4, and decreased in reaches 2 and 5. Actual hardwood canopy increased in every reach except reach 4 , in which it remained unchanged (Table 5.4.1). While canopy cover increased in every reach except 5 , coniferous canopy increased at a faster rate than hardwood canopy in reach 3.

Bank vegetation increased dramatically in every reach, with a maximum right and left bank combined increase of $35 \%$ in reach 3 (Table 5.4.2). The increase in vegetation of combined right and left banks was significant in every reach (Student's $t$-test. Reach $1, t_{(66)}$ $=-4.2, \mathrm{P}<0.001 ;$ Reach $2, t_{(27)}=-3.88, \mathrm{P} 0.001 ;$ Reach $3, t_{(71)}=-8.12, \mathrm{P}<0.001 ;$ Reach 4, $t_{(182)}=-10.51, \mathrm{P}<0.001 ;$ Reach 5, $t_{(96)}=-7.77, \mathrm{P}<0.001 ;$ Table 5.4.2).

Table 5.4.1: Mean percentage canopy by reach. Values in bold text indicate significant change between survey years using a Bonferroni adjusted alpha $(\alpha)$ level of $0.01(0.05 / 5)$. Standard deviation values are in parentheses.

\begin{tabular}{|c|c|c|c|c|c|c|c|c|c|c|c|}
\hline \multirow[b]{2}{*}{ Reach } & \multicolumn{5}{|c|}{ \% Canopy Cover } & \multicolumn{2}{|c|}{$\begin{array}{c}\text { \% Conifer } \\
\text { Canopy }\end{array}$} & \multicolumn{2}{|c|}{$\begin{array}{c}\text { \% Hardwood } \\
\text { Canopy }\end{array}$} & \multicolumn{2}{|c|}{$\mathbf{n}$} \\
\hline & 1997 & 2013 & df & $t$-value & P-value & 1997 & 2013 & 1997 & 2013 & 1997 & 2013 \\
\hline 1 & $80(26.5)$ & $92(12.4)$ & 40 & -2.53 & 0.015 & 0 & 0 & 80 & 90 & 33 & 57 \\
\hline 2 & $85(21.8)$ & $96(5.8)$ & 12 & -1.68 & 0.199 & 2 & 0 & 83 & 96 & 12 & 17 \\
\hline 3 & $83(22.5)$ & $92(11.2)$ & 66 & -2.67 & 0.010 & 3 & 4 & 81 & 84 & 54 & 109 \\
\hline 4 & $89(17.2)$ & $92(12.3)$ & 204 & -1.63 & 0.105 & 11 & 12 & 78 & 78 & 124 & 191 \\
\hline 5 & $95(10.3)$ & $94(5.2)$ & 70 & 0.15 & 0.882 & 64 & 50 & 30 & 42 & 57 & 113 \\
\hline
\end{tabular}


Table 5.4.2: Mean percentage bank vegetation for each reach, right and left banks combined. Values in bold text indicate significant change between survey years using a Bonferroni adjusted alpha $(\alpha)$ level of $0.01(0.05 / 5)$. Standard deviation values are in parentheses.

\begin{tabular}{llllllll}
\hline & \multicolumn{3}{c}{ n } & \multicolumn{2}{c}{$\begin{array}{c}\text { \% Bank Vegetation } \\
\text { (both banks) }\end{array}$} & & \% Bank Vegetation \\
\hline Reach & 1997 & 2013 & 1997 & 2013 & df & $t$-value & P-value \\
\hline 1 & 42 & 126 & $58(30.0)$ & $\mathbf{8 0}(27.9)$ & 66 & -4.20 & $<0.001$ \\
2 & 18 & 34 & $48(31.1)$ & $\mathbf{8 0}(23.8)$ & 27 & -3.88 & 0.001 \\
3 & 61 & 218 & $52(32.5)$ & $\mathbf{8 7}(18.6)$ & 71 & -8.12 & $<0.001$ \\
4 & 134 & 383 & $48(33.2)$ & $\mathbf{8 0}(23.6)$ & 182 & -10.51 & $<0.001$ \\
5 & 56 & 230 & $38(23.4)$ & $\mathbf{6 7}(27.9)$ & 96 & -7.77 & $<0.001$ \\
\hline
\end{tabular}

\subsection{Instream Cover and Substrate}

The amount of instream cover increased in reaches 2 and 3, decreased in reaches 4 and 5, and did not change in reach 1 (Table 5.5.1). However, none of the observed changes were statistically significant (Figure 5.5.1). Dominant substrate increased in size from sand to gravel in reaches 2 and 3, remained sand in reach 1, and remained gravel in reaches 4 and 5 (Table 5.5.2).

Table 5.5.1: Mean percentage of instream cover, measured as the percentage of each unit covered, per reach. No significant change was detected between survey years using a Bonferroni adjusted alpha $(\alpha)$ level of $0.01(0.05 / 5)$. Standard deviation values are in parentheses.

\begin{tabular}{llllllll}
\hline & \multicolumn{3}{c}{ Mean \% Instream Cover } & \multicolumn{3}{c}{ n } \\
\hline Reach & 1997 & 2013 & df & $t$-value & P-value & 1997 & 2013 \\
\hline 1 & $21(15.04)$ & $15(18.43)$ & 39 & 1.26 & 0.217 & 20 & 62 \\
2 & $14(14.46)$ & $9(5.72)$ & 9 & 1.01 & 0.337 & 9 & 13 \\
3 & $17(18.13)$ & $23(24.73)$ & 63 & -1.47 & 0.147 & 30 & 102 \\
4 & $20(18.54)$ & $22(22.45)$ & 141 & -0.71 & 0.480 & 66 & 171 \\
5 & $16(16.49)$ & $16(17.32)$ & 42 & 0.01 & 0.988 & 27 & 104 \\
\hline
\end{tabular}

Table 5.2.2: The dominant substrate by reach, sand $<2.03 \mathrm{~mm}$, gravel $2.03-63.5 \mathrm{~mm}$.

\begin{tabular}{ccc}
\hline Reach & $\mathbf{1 9 9 7}$ & $\mathbf{2 0 1 3}$ \\
\hline 1 & Sand & Sand \\
2 & Sand & Gravel \\
3 & Sand & Gravel \\
4 & Gravel & Gravel \\
5 & Gravel & Gravel \\
\hline
\end{tabular}




\subsection{Large Woody Debris}

\subsubsection{Temporal Trends in LWD}

The 1997 habitat survey of Scotts Creek did not include an LWD survey, thus direct comparisons between the two survey years were not possible. However, subsequent LWD surveys were conducted in 2001-02 (Leicester, 2005), 2004-05 (Dodson, 2005), and 201011 (Monteverde et al., 2011), allowing for limited comparisons (Table 5.6.1). Due to different study objectives, the aforementioned LWD surveys followed different protocols from each other and from the 2013 survey, namely surveying two $61 \mathrm{~m}$ reaches every 305 $m$ and extrapolating this across the entire stream, including a portion of Big Creek in the survey (Leicester, 2005). Additionally, previous surveys recorded only pool-forming LWD, used accumulations of LWD as the primary recording unit (i.e., a jam of 1-5 pieces was recorded as 1; Dodson, 2005), and did not assign LWD functionality (Monteverde et al., 2011).

Table 5.6.1: Temporal trends in LWD using results from surveys in 2001-2002 (Leicester, 2005), 20042005 (Dodson, 2005), 2010-2011 (Monteverde et al., 2011) and 2013.

\begin{tabular}{lcccc}
\hline Survey Year & $\mathbf{2 0 0 1}-\mathbf{2 0 0 2}$ & $\mathbf{2 0 0 4}-\mathbf{2 0 0 5}$ & $\mathbf{2 0 1 0 - 2 0 1 1}$ & $\mathbf{2 0 1 3}$ \\
\hline Streams Included & $\begin{array}{c}\text { Scotts Creek and } \\
\text { Big Creek }\end{array}$ & Scotts Creek & Scotts Creek & Scotts Creek \\
\hline Distance Surveyed & $12.1 \mathrm{~km}$ & $8.8 \mathrm{~km}$ & $7.4 \mathrm{~km}$ & $12.1 \mathrm{~km}$ \\
\hline $\begin{array}{l}\text { Total Pieces per } \\
100 \mathrm{~m} .\end{array}$ & 5.4 & $\dagger$ & 163 & 9.8 \\
\hline $\begin{array}{l}\text { Pool Forming } \\
\text { Pieces per 100 } \mathrm{m}\end{array}$ & 62.2 & $\dagger$ & N/A & 6.3 \\
\hline $\begin{array}{l}\text { Conifer Derived } \\
\text { LWD per 100 m }\end{array}$ & 2.1 & N/A & $2.5^{\ddagger}$ & 1.4 \\
\hline $\begin{array}{l}\text { Pool Forming } \\
\text { Conifers per 100 m }\end{array}$ & 1 & N/A & N/A & 0.89 \\
\hline $\begin{array}{l}\text { Number of Large } \\
\text { Log Jams }\end{array}$ & 0 & 13 & N/A & $24 / 19^{\S}$ \\
\hline
\end{tabular}

$\dagger$ This information is unavailable due to the primary recording unit of LWD accumulations, not individual pieces (i.e., an accumulation of 1-5 pieces is recorded as " 1 ").

$\$$ This is an approximate number calculated from approximate percentages.

$\S$ The number of log jams in the whole survey/the number of log jams in the extent of the '04-'05 survey. 
A comparison of the 2013 survey with the three previous LWD surveys showed an increase in LWD in Scotts Creek. This result was primarily due to an increase in hardwood derived LWD, as conifer-derived LWD decreased from 2.1 pieces per $100 \mathrm{~m}$ in $2001-02$ to 1.4 pieces per $100 \mathrm{~m}$ in 2013 . It is important to note that the $2001-02$ survey included the Big Creek drainage, which may have more streamside redwood trees, and the whole survey seems to have a much higher rate of species identification than the 2013 survey, either of which may partially explain this decrease. The amount of structure-forming LWD (pieces causing scour forming a pool or backwater) per $100 \mathrm{~m}$ also increased, but the amount of structure-forming conifer-derived LWD did not change (Table 5.6.1). The most notable difference came from the increase in large log jams between 2001-02 and 2013. No large log jams were observed in Scotts Creek during the 2001-02 survey, whereas 24 large log jams were documented in 2013. The 2004-05 survey categorized log jams by number of pieces, and found 13 jams comprised of $>30$ wood pieces, which would presumably fall into the "large" category. It is unclear whether the presence of at least 13 large jams only three years after no large jams were recorded indicates survey error or is due to log jam destruction and formation due to flow regime.

\subsubsection{Longitudinal Trends in LWD}

In the 2013 survey, the volume and number of structure forming LWD per $\mathrm{km}$ increased from the downstream reaches (1 and 2) to the upstream reaches $(3,4$, and 5), with the most dramatic change occurring between reaches 2 and 3 (Table 5.6.2). The maximum volume and number of pieces occurred in reach 3. However, the maximum number of conifer LWD per km was observed in reach 5 and was more than twice the number of conifer LWD in the second most abundant reach (Reach 3). As with canopy 
cover, reach 5 was the only reach in which conifer-derived LWD was greater than hardwood-derived LWD (Table 5.6.2).

Table 5.6.2: Longitudinal trends in LWD for 2013. Values are for lowflow/pool and bankfull/backwater pieces only.

\begin{tabular}{cccc}
\hline Reach & $\begin{array}{c}\text { Volume }\left(\mathbf{m}^{\mathbf{3}}\right) \text { per } \\
\mathbf{k m}\end{array}$ & Pieces per $\mathbf{~ k m}$ & $\begin{array}{c}\text { Conifer-Derived } \\
\text { Pieces per } \mathbf{~ k m}\end{array}$ \\
\hline 1 & 36.64 & 45.29 & 2.6 \\
2 & 39.73 & 24.27 & 3.8 \\
3 & 109.88 & 97.85 & 14.8 \\
4 & 104.18 & 87.28 & 10.6 \\
5 & 64.95 & 56.62 & 38.5 \\
\hline
\end{tabular}

\subsubsection{LWD and Pools}

Early in the 2013 survey it was recognized that the habitat naming system failed to consistently capture the presence or importance of LWD in pools. Consequently, I amended survey protocol to explicitly note when LWD was contributing to pool formation and/or maintenance and used survey comments to determine the number of LWD enhanced pools (post hoc) in units where this information was not directly recorded.

Although the upper stream reaches contained a higher number of redwood LWD, a higher percentage of primary pools were LWD enhanced in the lower reaches. The percentage of total primary pools that were enhanced by LWD decreased from downstream to upstream, with LWD enhancing six out of seven primary pools in reach $1(86 \%)$, but only three out of 14 in reach $5(21 \%)$. Log jams and single pieces enhanced approximately the same number of primary pools (15 and 14, respectively), and logs and root wads also enhanced an equal number of primary pools (7 each). Over the entire stream, LWD did not appear to have an effect on primary pool depth compared to primary pools without LWD. However, in reach 4 , the reach with the largest number of primary pools and with an approximately 
even number with and without LWD, the mean depth of primary pools enhanced by LWD was substantially deeper (17 $\mathrm{cm}$ difference) than those without LWD. Other reaches display different results, but are skewed due to the dearth of either LWD enhanced or nonenhanced pools. Of the LWD enhancing primary pools that can be identified to species, nine were redwood (seven with a root wad), and four were hardwood (two pieces with a root wad).

I examined the larger size categories of LWD (diameter $>91 \mathrm{~cm}$, length $>6.1 \mathrm{~m}$ ) to determine their association with pools. Over the whole stream, a total of 31 pieces of conifer LWD with a diameter greater than $0.91 \mathrm{~m}$ (excluding lowflow/extra and bankfull/extra pieces) were observed. These pieces were associated with 23 different pool habitat units (if more than one piece was in the same habitat unit, it was counted as only one) (74\%), including eight pieces in log jams (26\%). The survey recorded 28 pieces of hardwood LWD with a diameter greater than $0.91 \mathrm{~m}$, which were associated with 18 pools (67\%). Fifteen pieces were found in log jams. The mean depth of pools associated with large diameter conifer LWD was $15 \mathrm{~cm}$ greater than the mean of pools associated with large diameter hardwood LWD.

A total of 54 conifer pieces and 304 hardwood pieces were in the longest length category (i.e., $>6.1 \mathrm{~m})$. Of the 54 conifer pieces, $43(\sim 80 \%)$ were associated with pool habitat, 25 of which $(\sim 46 \%)$ were associated with log jams. Hardwood LWD > $6.1 \mathrm{~m}$ in length was associated with $212(\sim 70 \%)$ pool habitats, and $113(\sim 53 \%)$ were found in $\log$ jams. 


\section{CHAPTER 6. DISCUSSION}

\subsection{Constraints and Drivers of Change from 1997-2013}

Although streamflow data for Scotts Creek is only available since 2010, discharge patterns can be inferred from data collected at nearby gaging stations. By examining peak discharge information for 1997-2013 from the San Lorenzo River at Big Trees (USGS gage 11160500; http://waterdata.usgs.gov), it is evident that for several years leading up to 1997 through 2013 flows were largely below the long-term average of $224 \mathrm{~m}^{3} / \mathrm{s}$ (Table 6.1 .1 ). The winter of 1997-98 (immediately following the initial habitat survey of Scotts Creek), exhibited the highest magnitude discharge event with a return period of 15 years. In contrast, the winter preceding the 2013 survey was a very low flow year, with one large storm creating a peak flow of approximately $56.6 \mathrm{~m}^{3} / \mathrm{s}$, with an estimated return period of 1.4 years in mainstem Scotts Creek.

In a 1997 study conducted in Washington state, percentage pool area was correlated with LWD volume $/ \mathrm{m}^{2}$ in low (.002-0.02) and moderate (0.02-0.05) slope channels, with the latter showing more sensitivity to LWD volume $/ \mathrm{m}^{2}$ (Beechie and Sibley, 1997). It follows that an increase in LWD in Scotts Creek should lead to an increase in percentage pools; however, our results indicate that while LWD has increased over the entire stream length percentage pools has held steady. Despite a lack of overall change in percentage pools, the system is dynamic and pool frequency is increasing in some reaches and decreasing in others. However, since the previous LWD surveys did not collect location information, I could not determine whether the increases and decreases in percentage pools were linked to a change in LWD loading. 
Table 6.1.1: Peak flows and return periods for the San Lorenzo River at Big Trees (USGS gauge 11160500 ), long-term average flow is $224 \mathrm{~m}^{3} / \mathrm{s}$. The three years preceding the first survey were included due to the impact they could have had on instream habitat.

$\begin{array}{ccc}\text { Water Year } & \text { Peak Flow }\left(\mathbf{m}^{\mathbf{3}} / \mathbf{s}\right) & \text { Return Period (years) } \\ 1995 & 402.1 & 8.8 \\ 1996 & 164.0 & 1.8 \\ 1997 & 322.8 & 3.6 \\ 1998 & 549.3 & 15.8 \\ 1999 & 90.6 & 1.5 \\ 2000 & 213.8 & 2.1 \\ 2001 & 53.8 & 1.2 \\ 2002 & 223.1 & 2.3 \\ 2003 & 373.8 & 5.3 \\ 2004 & 317.1 & 3.3 \\ 2005 & 130.8 & 1.7 \\ 2006 & 376.6 & 5.6 \\ 2007 & 34.3 & 1.1 \\ 2008 & 214.4 & 2.2 \\ 2009 & 108.2 & 1.6 \\ 2010 & 351.1 & 4.4 \\ 2011 & 303.0 & 3.2 \\ 2012 & 75.3 & 1.4\end{array}$

The increase in LWD was primarily driven by an increase in hardwood species, dominantly red alder (Alnus rubra [Bong.]). In line with this, hardwood LWD poolforming pieces increased by 4 pieces per $100 \mathrm{~m}$ from the 2001-02 survey. Little change in conifer LWD was detected. Local hardwood species are smaller in diameter, have smaller root wads, and break down more quickly than local conifer species. Although large diameter pieces $(>0.91 \mathrm{~m})$ of both species were likely to be associated with pool habitat, conifers were slightly more likely than hardwood. Additionally, the pools associated with large diameter conifer LWD were on average deeper than pools associated with large diameter hardwood LWD. Large diameter conifers were twice as likely to have a root wad, which is an important factor in stabilizing instream LWD and influencing persistence, which, along with added roughness, could lead to increased pool depth. 
Although the number of pool-forming hardwood LWD appears to be increasing, these pieces tend to be associated with shallower pools and have a shorter instream life span, likely due to faster decay and greater mobility, than conifer LWD. However, hardwood LWD can play a key role in trapping other debris and accumulating log jams. Large log jams are 3.3 times more abundant per kilometer in the lower reaches, which have a higher hardwood-to-conifer ratio than the upper reaches. A study of hardwood-dominated salmonid streams in California found $\log$ jams to be the primary form in which hardwood LWD impacted stream morphology (Opperman, 2005). Thus, while the size and longevity of instream hardwood may not be enough for individual pieces to create morphological features (e.g., large pools), the length and frequency are sufficient to create large accumulations that can lead to pool formation and provide instream cover. As the lower riparian environment is less hospitable to redwoods than the upper, and as hardwoods are currently the dominant trees along the streambanks, the importance of hardwood LWD should not be overlooked, particularly lower in the system. Additionally, a debris flow in 1955 originating in the Little Creek basin scoured a large area of the channel around and downstream of the confluence. Red alders rapidly colonized the disturbed banks and this stand is currently reaching senescence. Consequently, a large input of alder LWD in these areas is likely in the next few years, as these trees die and fall into the channel.

The Scotts Creek watershed is subject to periodic forest fires. Most recently, the Lockheed Fire in 2009 burned 3,163 ha of the watershed including riparian areas along all of the tributaries. Although the riparian corridor along upper mainstem Scotts Creek was mostly unburned, adjacent forested areas to the south and east did burn. Whereas forest fires have been reported to increase the amount of fine sediment in streams (Potyondy and 
Hardy, 1994), an increase in fine sediment delivery to Scotts Creek could be expected, specifically in the lower depositional portion of the stream. Nonetheless, a 2013 study in the Little Creek basin found the fire had no significant effect on suspended sediment export from the Little Creek watershed the first year following the fire (Loganbill, 2013). It is important to note, however, that streamflows during the first winter after the fire did not reach bankfull, and an increase in fine sediment may have been delivered to the channel and not distributed throughout the system. Additionally, the study concentrated on Little Creek exclusively, and may not be applicable to the greater Scotts Creek watershed. A separate study found sediment size in Scotts Creek increased overall, and amount of sand decreased between 2010 and 2012 in Scotts Creek (Boerman, 2012). Other observations in Mill Creek suggest a large debris/mudflow event supplied tremendous amounts of sediment to the main Mill Creek channel. Grab samples from Mill Creek at Swanton Road from a storm in January 2010, yielded turbidity estimates as high as 40,000 NTUs. This event may have supplied substantial fine sediment to Scotts Creek below Mill Creek, and could have been a source of pool filling in the lower stream reaches (personal communication, Dietterick, 2014).

The study findings show that the dominant substrate in Scotts Creek became coarser from 1997 to 2013. An overall coarsening of substrate does not exclude an increase in fines, especially if they are concentrated in calm pool areas. Additionally, if fine sediment was added to the stream post-fire, subsequent high discharge events may have transported the material out of the system in all but the lower reach. However, these surveys lack the precision to evaluate change in fines accurately for several reasons. First, the 1997 survey only recorded dominant and co-dominant substrate, so the percentage of the total substrate 
composed of fine particles is unknown. Second, fines are typically defined as substrate smaller than $6 \mathrm{~mm}$ (Archer et al, 2004; Roper et al, 2002), which in this survey included silt/clay, sand $(<2.0 \mathrm{~mm})$, and some small sized gravel (i.e., particles $2.0-6.0 \mathrm{~mm})$ which has a broad size class range $(2.0 \mathrm{~mm}-63.5 \mathrm{~mm})$. Thus, an increase in gravel could still lead to an increase in fines, depending on the size of the gravel. Finally, if silt/clay and sand are combined, these finer particles would be classified as the dominant substrate in 2013 in many reaches.

Measurement error must also be considered when assessing and interpreting change. Although measurement error of residual pool depth was relatively small and was controlled for in this study, differences in defining habitat units can also affect pool depth measurements. Several pools in the 2013 survey with glides or pools as the adjacent downstream unit had pool tail crest depths greater than $30.5 \mathrm{~cm}(1 \mathrm{ft})$. The identification of these pool tail crests should possibly be further downstream at a shallower point in the stream. This potentially leads to spuriously shallow residual pool depth measurements, increased pool depth diversity, and, in the case of two adjacent pools, increased pool frequency. An analysis of mean residual pool depth per reach after removing all pools with a tail crest depth of $30.5 \mathrm{~cm}$ or greater did not yield results notably different from the original analysis. With this change, mean residual pool depth in 2013 increased from the original results in reaches 2,4 , and 5 , but not enough to move the amount of change to within the margin of error.

\subsection{Management Implications}

Historically, Scotts Creek ran through old growth redwood forest before emerging to a sinuous, lower depositional zone with a wide floodplain and then into a large, naturally 
functioning estuary. Harmon and others (1986) found streams running through 500-year old redwood stands to have LWD volumes ranging between $240-4500 \mathrm{~m}^{3} / \mathrm{ha}$, the highest amounts of the 11 surveyed temperate forest types. It is likely this was the general condition in Scotts Creek (with the exception of the lower reaches), the downed trees creating many deep pools with complex cover and ample off-channel habitat to provide flow refugia to salmonids during winter high flows. Lower in the system the stream became a meandering or braided channel with large bends and floodplain connectivity, allowing for pool formation, nutrient exchange, and off channel refuge during high flows. Natural disturbances are frequent in the Scotts Creek watershed, including fires, flooding, and mass wasting. The stream is frequently in recovery from one type of event or another, however, extensive landscape alteration that began in late 1800s and early 1900s of the kind not seen in the watershed before, namely clearcutting, agricultural practices and levees, affected the natural stream equilibrium and ecosystem functionality of Scotts Creek to the detriment of aquatic organisms including Coho Salmon.

Scotts Creek is currently still a stream in recovery from anthropogenic activities. Legacy effects from early clear cutting are evident in the near absence of old growth redwoods along the stream corridor and the scarcity of large redwood woody debris in the mid and upper reaches of the stream. If larger trees lead to larger and deeper pools (Bilby and Ward, 1989 as cited in Bilby and Bisson, 1998), Scotts Creek is closing on a century of very little LWD recruitment of the size necessary to form the deepest pools. This lack of recruitment is eased only slightly by the addition of large root wads of previously cut trees which form several of the upper channel's deepest pools. 
Large woody debris is often more important to pool formation in steeper and narrower stream reaches (Bilby and Ward, 1989; Montgomery and Buffington, 1993), so while the upper channel reaches may have relied heavily on LWD to force pool formation, the lower channel, with a lower gradient and less hospitable environment for redwoods, may have exhibited more of a pool-bar morphology. If this was the case, channel mobility and floodplain access were important to pool formation and off channel, high-velocity refuge habitat. The construction of the levees along the lower channel to confine flow towards the Highway 1 bridge opening and protect agricultural fields, constrained channel mobility and prevented access to the larger floodplain area. Currently, a project is underway to restore floodplain connectivity and breach the levees in strategic locations. Post-project monitoring will be essential to evaluate effectiveness and inform future restoration projects.

Presently, based on information in the federal CCC Coho Salmon Recovery Plan, Scotts Creek has sufficient canopy and percentage pools, but is lacking in key pieces of LWD per $100 \mathrm{~m}$, percentage riffles, instream cover, and percentage primary pools (pools deeper than $91 \mathrm{~cm}$ in third order streams and above; Table 6.2.1). While cover complexity does not reach the benchmark established in the recovery plan, the amount of instream cover appears to be increasing. This is likely due to the increase in $\log$ jams and canopy, which can provide small woody debris, large woody debris, and stabilize banks to create undercuts. Though the recorded percentage riffles is below the desired level, the length of riffles is artificially low due to their inclusion in step-run habitat units (which are considered flatwater). However, as riffles are a flow-dependent measurement the low flows 
during the 2013 survey may have resulted in a higher number of reported riffles than during an average or high flow year.

Table 6.2.1: Instream habitat parameter goals as set by the Final Recovery Plan for Central California Coast Coho Salmon Evolutionarily Significant Unit (NMFS, 2012), and actual values as determined by the 2013 Scotts Creek survey.

\begin{tabular}{|c|c|c|c|c|c|c|}
\hline Parameter & Goal & $\begin{array}{l}\text { Total for } \\
\text { Entire } \\
\text { Survev }\end{array}$ & Rating & \multicolumn{3}{|c|}{ Totals by Reach } \\
\hline \multirow{5}{*}{$\begin{array}{c}\text { Yyy7Key } \\
\text { Pieces } \\
\text { LWD/100 } \\
\text { meters }^{1}\end{array}$} & \multirow{5}{*}{$6-11$} & \multirow{5}{*}{3.7} & \multirow{5}{*}{ Fair } & 1 & 0.6 & Poor \\
\hline & & & & 2 & 2.2 & Poor \\
\hline & & & & 3 & 6.1 & Good \\
\hline & & & & 4 & 3.3 & Poor \\
\hline & & & & 5 & 4.9 & Fair \\
\hline \multirow{5}{*}{$\begin{array}{c}\text { Primary } \\
\text { Pools }^{2} \\
(\% \text { of total } \\
\text { pools })\end{array}$} & \multirow{5}{*}{49} & \multirow{5}{*}{21} & \multirow{5}{*}{ Poor } & 1 & 19 & poor \\
\hline & & & & 2 & 13 & poor \\
\hline & & & & 3 & 11 & poor \\
\hline & & & & 4 & 27 & fair \\
\hline & & & & 5 & 23 & poor \\
\hline \multirow{5}{*}{$\begin{array}{l}\text { Shelter } \\
\text { Rating } \\
\text { (Pools) }\end{array}$} & \multirow{5}{*}{$>80$} & \multirow{5}{*}{75} & \multirow{5}{*}{ Fair } & 1 & 70 & fair \\
\hline & & & & 2 & 25 & poor \\
\hline & & & & 3 & 90 & good \\
\hline & & & & 4 & 85 & good \\
\hline & & & & 5 & 47 & poor \\
\hline \multirow{5}{*}{$\begin{array}{l}\text { Canopy } \\
(\%)\end{array}$} & \multirow{5}{*}{$>85$} & \multirow{5}{*}{93} & \multirow{5}{*}{ Very Good } & 1 & 92 & Very Good \\
\hline & & & & 2 & 96 & Very Good \\
\hline & & & & 3 & 92 & Very Good \\
\hline & & & & 4 & 92 & Very Good \\
\hline & & & & 5 & 94 & Very Good \\
\hline \multirow{5}{*}{$\begin{array}{l}\text { Pool:Riffle: } \\
\text { Flatwater } \\
\text { (\% length) }\end{array}$} & \multirow{5}{*}{$\begin{array}{c}>30 \\
\text { Pools } \\
>20 \\
\text { Riffles }\end{array}$} & \multirow{5}{*}{$\begin{array}{l}\text { 40: } \\
\text { 15: } \\
44\end{array}$} & \multirow{5}{*}{$\begin{array}{c}\text { Very } \\
\text { Good/Fair }\end{array}$} & 1 & $31: 10: 58$ & Good/Fair \\
\hline & & & & 2 & $28: 10: 62$ & Fair \\
\hline & & & & 3 & $41: 4: 55$ & V. Good/Poor \\
\hline & & & & 4 & $44: 14: 42$ & V. Good/Fair \\
\hline & & & & 5 & $45: 32: 23$ & Very Good \\
\hline
\end{tabular}

${ }^{1}$ Key pieces of LWD are defined as logs or root wads that 1) are independently stable within the bankfull width and not functionally held by another factor, and 2) can retain other pieces of organic debris, and meet the size criteria outlined on pages 18-19 of Appendix B of the Final CCC Coho Salmon ESU Recovery Plan (NMFS 2012). .

${ }^{2}$ Primary pools are defined as greater than $91 \mathrm{~cm}$ deep in third order streams and above (NMFS 2012). 
The deficit in percentage primary pools is the largest inadequacy in Scotts Creek with an abundance below the standard goal of $49 \%$ (of total pools) identified in the federal CCC Coho Salmon Recovery Plan. Moreover, the percentage of primary pools in the basin appears to be decreasing. The average depth of primary pools across all reaches in 1997 was $113 \mathrm{~cm}$, but dropped as low as $101 \mathrm{~cm}$ in reach 4, indicating that a slight amount of pool filling could have been enough to markedly decrease the number of primary pools. Reach 4 was the only reach with an increase in primary pools, and with a rating above "poor" based on the 2013 survey results. The number of primary pools decreased by 1 pool in reach 5 , less of a decrease than in reaches $1-3$, potentially supporting the idea of pool filling occurring due to the 2010 debris flow from Mill Creek. Additionally, Scotts Creek is lacking in number of key pieces of LWD, meeting the Recovery Plan goal in reach 3 only. An increase in key pieces of LWD could result an increase in the other two lacking parameters, primary pools and instream cover.

The federal CCC Coho Salmon Recovery Plan, as it relates to habitat conditions in Scotts Creek, was written using data from the 1997 habitat survey, and though the updated information from this study shows that there have been changes to instream habitat in the 15 intervening years, the changes have not been significant enough to warrant change to the current conditions described in the plan. Some items, such as instream cover and LWD appear to be improving without direct management action and may only need time to recover to desirable levels. Other items, namely percentage primary pools, appear to be less prevalent and warrant ongoing monitoring to determine if management intervention and improvement is necessary. Monitoring of specific primary pools would be beneficial in confirming the actors affecting their depth and stability, and a more pinpointed study of 
the interaction between LWD and primary pools could further illuminate that relationship. Two points in time are not enough to detect a long-term trend, and it is possible that many of the detected changes between the two survey years are due to events that occurred shortly before the survey (i.e., high flows, debris flows). An ongoing monitoring program in Scotts Creek will help determine long-term trends and the factors and events affecting pool formation and maintenance, to ensure that positive trends are not reversed, negative trends can be managed appropriately, and restoration actions can be monitored for effectiveness. 


\section{CHAPTER 7. CONCLUSION}

In the years between the 1997 survey and the 2013 survey, no major land use change or anthropogenic impact contributing to change in channel morphology and physical habitat conditions occurred in the Scotts Creek watershed. Natural disturbance events between the surveys include an extreme discharge event in 1998 and the Lockheed Fire in 2009; these events may have contributed to observed changes in stream morphology. The pool filling in the lower reaches may be due to an influx of fine sediment from the fire and subsequent debris flow. The high water events could have caused additional scour around features, such as large woody debris, leading to an increase in depth of the pools already formed around these features. It should also be noted that the peak flow in the winter preceding the 2013 survey reached bankfull level, and that the summer of 2013 saw one of the lowest flows in recent time. Low flows may not produce changes to stream morphology, but could impact survey results such as habitat unit delineation (e.g., riffles instead of runs) and the wetted width-to-depth ratio.

Using parameters from the 1997 habitat typing survey I was able to detect change to instream habitat in Scotts Creek between 1997 and 2013. Specifically, the residual pool error study demonstrated that the amount of observer error in this measurement is small, indicating this is a robust measurement for assessing change. The implications of this are that the CDFW habitat typing surveys, though not intended to act as a baseline for trend detection, contain data that could do just that. These surveys are widely conducted on California salmonid streams and may provide important and valuable information beyond their initial assessment. 
The changes documented in Scotts Creek indicate that vegetative parameters are generally improving, and while the overall number of pools has remained steady, pool quality has generally decreased. With only two years of data, it is not possible to determine whether these changes indicate the direction of a long term trend. One objective of this study was to provide a foundation for a long-term habitat study in Scotts Creek, and with one more year of data, trend detection will become possible.

This study served to update the instream habitat knowledge of Scotts Creek, and provides novel information to inform future salmonid studies and restoration actions. Current management practices in the Scotts Creek watershed recognize the sensitivity of the stream to disturbances, and the destructive practices of the past have been abandoned in favor of more responsible land management beginning in the 1940s and gradually improving over the latter half of the $20^{\text {th }}$ century and into the first decade of the $21^{\text {st }}$ century. However, it is evident from Table 6.2.1 that aspects of the stream are still lacking. Based on the 2013 habitat survey, primary steps to restore Scotts Creek to its historical condition include adding large, stable, redwood LWD suitable for deep pool formation to the upper section, and, in the lower sections, breaching the levees and improving floodplain and offchannel habitat connectivity.

Instream habitat is important for its ability to support fish, and a study linking fish production to specific habitat elements in Scotts Creek would support and inform recovery efforts. As Coho Salmon numbers have been increasing in the past few years, now would be an excellent time to begin a rigorous habitat use study. To suggest and inform future restoration action, it will be important to understand how Coho Salmon utilize the habitat in Scotts Creek across various spatiotemporal scales, at various population densities, and 
to identify underperforming areas of the stream. Population data for both Coho Salmon and Steelhead Trout from NOAA Fisheries index electrofishing reaches are available, and could be used as a starting point for future fish occupancy studies. Additional electrofishing reaches near the current ones with different habitat types could allow for comparisons of habitat use. A more detailed description of the habitat within electrofishing reaches, and a more accurate record of where the fish are caught within the reaches could lead to a greater understanding of how salmonids are utilizing the existing habitat in Scotts Creek and how use changes as a function of population size. This study could be focused to pinpoint different types of pools, and/or pools with differing depths and amounts of cover.

\subsection{Study Design}

As one of the objectives of this study was to lay a foundation for a long-term monitoring program on Scotts Creek, it is necessary to think about the most appropriate study design to quantify future status and change in physical salmonid habitat in Scotts Creek. It is neither necessary nor economical to repeat the full habitat survey every summer. Periodicity of the survey should take into account several factors including stream events (i.e., disturbances), available personnel, and funding. Overall, two notable events the 1998 flood and the 2009 Lockheed Fire - occurred in the 15 years between the two habitat surveys presented here, and although differences between the surveys were visually apparent, they were not always easy to quantify. Surveying must be conducted frequently enough to serve as a baseline in the case of a potential stream-altering event. Given the historic frequency of large events, no more than ten, and no fewer than five years between surveys would likely suffice, with additional surveys conducted directly before and after a major event. 
Monitoring studies commonly do not survey the entire stream (i.e., all available anadromous habitat), but instead survey small segments at various locations. While this method would not achieve the desired results of this study, it is much less resource intensive, and could be conducted during the intervening years between full surveys. The 2013 survey averaged $421 \mathrm{~m}$ per day, including days with late starts or early ends, so it is reasonable to expect that a section $0.5 \mathrm{~km}$ could be completed in one full day. If this is the case, four different stream reaches of $0.5 \mathrm{~km}$ in length could be completed in one week. The reaches should be spread throughout the stream, as the stream often behaves or responds differently in the upper reaches than in the lower reaches. Two sections each above and below the Swanton Road Bridge, for example, may be sufficient to extrapolate any detected change to the entire stream. Logical locations for the reaches would encompass NOAA Fisheries' long-term electrofishing index reaches, as they are distributed throughout the watershed and would enable an ongoing occupancy study. This has the drawbacks of introducing new surveyors every year, and possibly each survey day. However, with a thorough training manual and a modified survey form to exclude estimates (for example, since the time commitment is shorter, it may be possible for three people to conduct the survey, one of whom could conduct pebble counts in place of the substrate size estimates), observer error could be kept to a minimum.

\subsection{Survey Design}

There is room in the survey design for improvement; however, any changes to the survey design must not render future surveys incomparable with past work. A modification of the habitat unit classification system may help to more accurately describe individual habitat units. For example, the habitat type, "mid-channel pool," is defined as occupying 
greater than $60 \%$ of the channel width, while, "lateral scour pool - root wad formed," is defined as occupying less than $60 \%$ of the channel width. In the upper reaches of the stream, where the stream is narrow and large root wads are present, many large, root wadformed pools were classified as mid-channel pools. While this does provide information about the pool size relative to the channel, it omits information about how the pool formed (i.e. that the pool is present due to scour around a root wad). Another example of this shortcoming is a pool formed by a channel spanning log jam. This habitat unit also falls under the classification of "mid-channel pool," while it is clearly a distinct type of midchannel pool. A system that allows for multiple variables to be added to the habitat type description independently or one with a separate category for "forming factor" (e.g., USDA Forest Service) may be more descriptive.

With some modifications, the basic structure of the CDFW habitat unit definitions could be maintained, while adding descriptors to make the system more accurate. The proposed system would create more flexibility in describing a habitat unit, and would allow surveyors to add as many descriptors to a unit as necessary. The primary habitat units for everything except pools would remain largely the same: Low Gradient Riffle, High Gradient Riffle, Cascade, Bedrock Sheet, Pocket Water, Glide, Run, Step Run, Edgewater, Dry, Culvert, Not Surveyed, Not Surveyed due to Marsh. The change would come in the pool descriptions. The categories of Scour Pool, and Backwater Pool, would remain, and the Main-Channel Pool category would change to Mid-Channel Pool. The more detailed descriptions of "root-wad enhanced, channel confluence, log enhanced," etc. would be optional add-on's, and as many as necessary could be added. The system would look something like this: 
Primary Units:

- Trench Pool (4.1)

- Step Pool (4.2)

- Mid Channel Pool (5)

- Scour Pool (6)

- Backwater Pool (if no additional descriptor, equivalent to "secondary channel pool) (7)

Descriptive Units:

- $\quad$ Log enhanced (0.1)

- Root wad enhanced (0.2)

- Bedrock formed (0.3)

- Boulder formed (0.4)

- Corner (0.5)

- Confluence (0.6)

- Plunge (0.7)

- Log jam/ Large debris accumulation (for units with channel-spanning log jams) (0.8)

- $\quad$ Dammed (0.9)

In practice, the proposed survey system would call the pool in Figure 7.1, formed by a large log jam across the channel a Mid-Channel Pool, log jam formed (5.7). This is in contrast to the previous (CDFW) system which contained no notation for a log jam and would have simply classified this pool as a Mid-Channel Pool (4.2).

The pool shown in Figure 7.2, because it occupies greater than $60 \%$ of the channel, was also classified as a Mid-Channel Pool (4.2) under the previous system, which leaves out any mention of the functional and important root wad. The proposed system would classify it as a 5.2, a Mid-Channel Pool (occupying greater than $60 \%$ of the wetted channel) enhanced by a root-wad. The adoption of this revised habitat naming system, because it keeps the same basic principles as the original system, would still allow comparisons with 
past surveys (1997 and 2013), but would add description and accuracy to the habitat unit types moving forward.

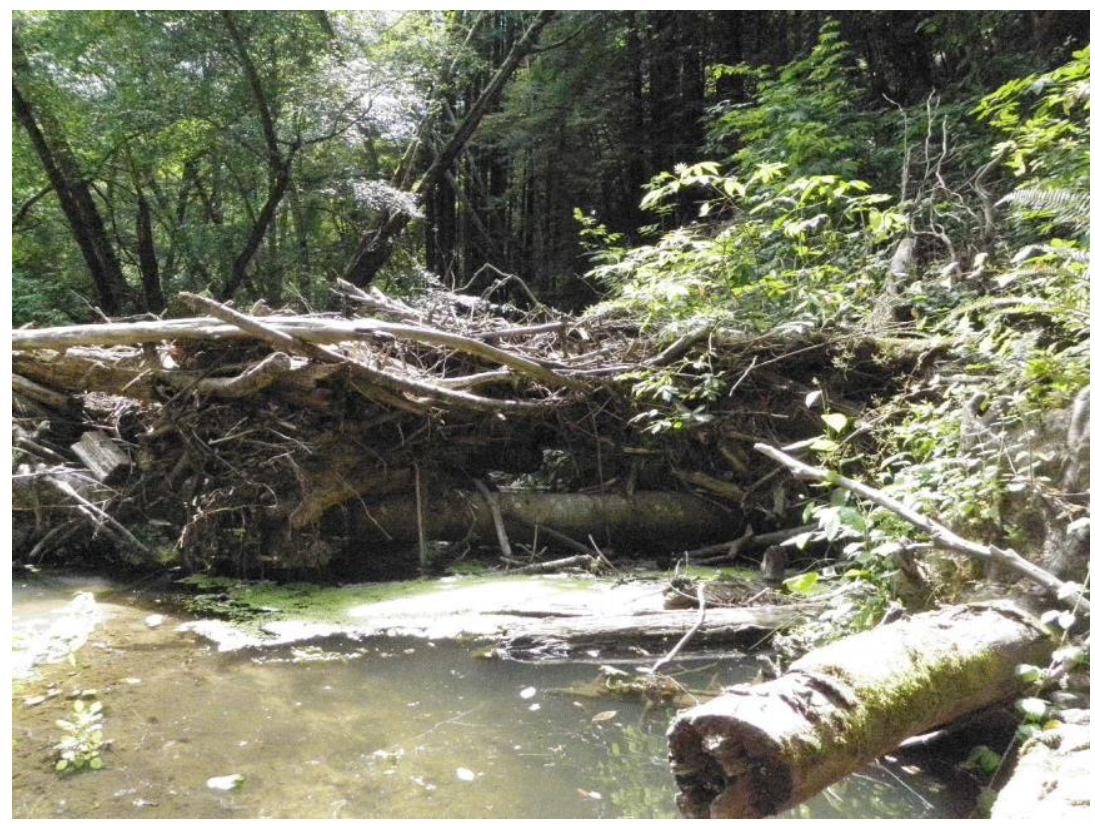

Figure 7.1: A photograph of a logjam in the lower section of Scotts Creek forming a mid-channel pool.

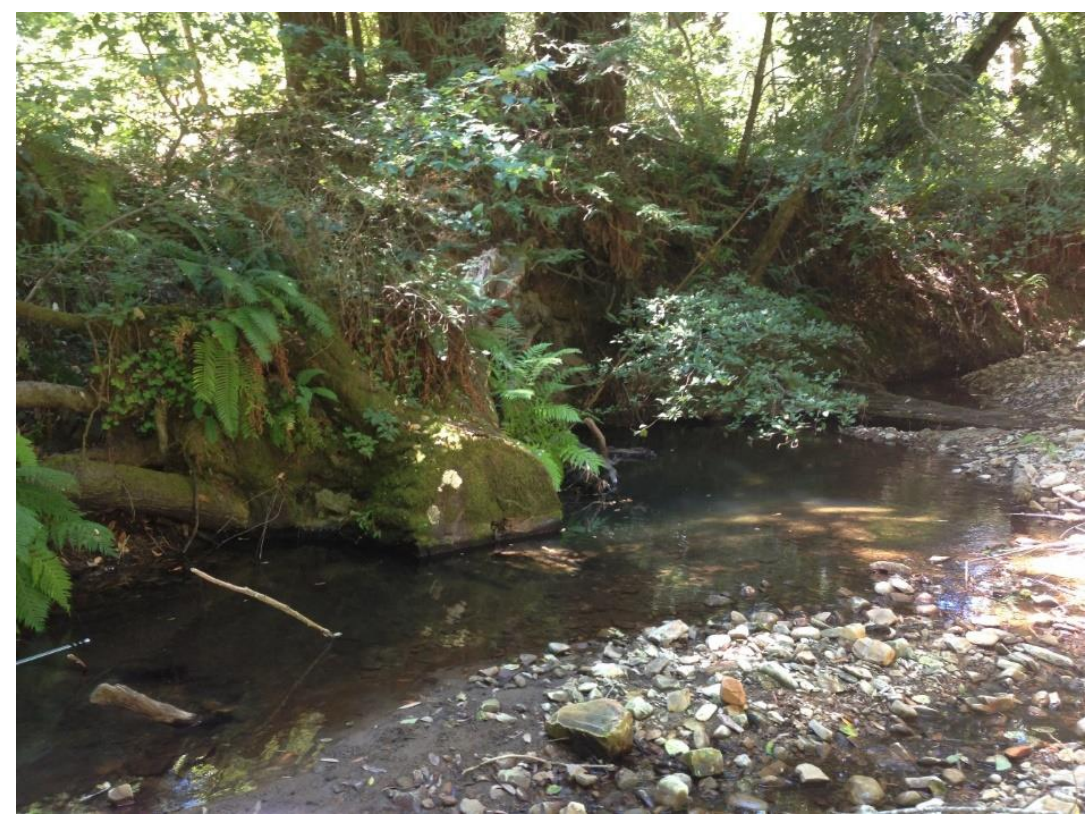

Figure 7.2: A difficult pool to classify under the current habitat unit classification system because it occupies greater than $60 \%$ of the channel, but is obviously enhanced by a root wad 


\section{References}

Analauf, K.J., W. Gaueman, and K.K. Jones, 2011. Detection of Regional Trends in Salmonid Habitat in Coastal Streams, Oregon, Transactions of the American Fisheries Society, 140:1, 52-66

Archer, E.K., B.B. Roper, R.C. Henderson, N. Bouwes, S.C. Mellison, and J.L. Kershner, 2004. Testing common stream sampling methods for broad-scale, long-term monitoring. United States Department of Agriculture, Rocky Mountain Research Station. General Technical Report RMRS-GTR-122.

Armour, C.L., K.P. Burnham, and W.S. Platts, 1983. Field methods and statistical analyses for monitoring small salmonid streams. U.S. Fish and Wildlife Service FWS/OBS$83 / 33$ pp 200

Bain, M.B., T.C. Huges and K.K. Arend. (1999) Trends in methods for assessing freshwater habitats. Fisheries. 24:4, 16-21.

Bain, M.B., and N.J. Stevenson, editors, 1999. Aquatic habitat assessment: common methods. American Fisheries Society, Bethesda, Maryland.

Bauer, Stephen B. and S.C. Ralph, 2001. Strengthening the use of aquatic habitat indicators in Clean Water Act programs. Fisheries. 26(6) 14-25.

Beechie, Timothy J., and T.H. Sibley, 1997. Relationships between channel characteristics, woody debris, and fish habitat in northwestern Washington streams. Transactions of the American Fisheries Society. 126(2) 217-229

Bilby, R.E., and P.A. Bisson, 1998. Function and Distribution of Large Woody Debris. In: River Ecology and Management. Robert J. Naiman, Robert E. Bilby, and Sylvia Kantor (Editors). Springer-Verlag New York, Inc. pp 324-338.

Bisson, Peter A., J.L. Nielsen, R.A. Palmason, and L.E. Grove, 1982. A system of naming habitat types in small streams, with examples of habitat utilization by salmonids during low streamflow. Acquisition and Utilization of Aquatic Habitat inventory Information: Proceedings of a symposium held 28-30 October, 1981, Hilton Hotel, Portland, Oregon.

Boerman, D., 2012. Comparison of Streambed Particle Size Distributions within the Scotts Creek Watershed: Spring 2012 Mid-term Response to the Lockheed Fire. Senior Project, California Polytechnic State University, San Luis Obispo, California.

Brabb, E.E., 1989. Geologic Map of Santa Cruz County, California. Reston, Va.: The Survey.

Brown, Larry R., and P.B. Moyle, 1991. Status of Coho Salmon in California. Report to the National Marine Fisheries Service. Department of Wildlife and Fisheries Biology, University of California Davis, Ca. 
Brown, L.R., P.B. Moyle, and R.M. Yoshiyama, 1994. Historical decline and current status of Coho salmon in California. North American Journal of Fisheries Management. 14, 237-261.

Dodson, T.S., 2005. Large woody debris inventory of Scotts Creek Watershed. Senior Project, California Polytechnic State University, San Luis Obispo, California.

Fitzpatrick, F.A., I.R. Waite, P.J. D’Arconte, M.R. Meador, M.A. Maupin, and M.E. Gurtz, 1998. Revised Methods for Characterizing Stream Habitat in the National WaterQuality Assessment Program. U.S. Geological Survey, Water-Resources Investigations Reports 98-4052.

Flosi, G., S. Downie, J. Hopelain, M. Bird, R. Coey, and B. Collins, 2004. California Salmonid Stream Habitat Restoration Manual, Fourth Edition. State of California, The Resources Agency, California Department of Fish and Game, Wildlife and fisheries Division.

Foster, S.C., C.H. Stein, and K.K. Jones, 2001. A guide to interpreting stream survey reports. Edited by P.A. Bowers. Information Reports 2001-06. Oregon Department of Fish and Wildlife, Portland.

Harmon, M.E., J.F. Franklin, F.J. Swanson, P. Sollins, S.V. Gregory, J.D. Lattin, N.H. Anderson, S.P. Cline, N.G. Aumen, J.R. Sedell, G.W. Lienkaemper, K. Cromack, JR., K.W. Cumming, 1986. Ecology of Coarse Woody Debris in Temperate Ecosystems. In: Advances in Ecological Research Volume 15.

Kaufmann, P.R, P. Levine, E.G. Robison, C. Seeliger, and D.V. Peck, 1999. Quantifying Physical Habitat in Wadeable Streams. EPA/620/R-99/003. U.S. Environmental Protection Agency, Washington, D.C

Keim, R.F., and A.E. Skaugset, 2002. Physical aquatic habitat I. errors associated with measurements and estimation of residual pool volumes. North American Journal of Fisheries Management. 22(1) 145-150

Kershner, J.L., B.B. Roper, N. Bouwes, R. Henderson, and E. Archer, 2004. An analysis of stream habitat conditions in reference and managed watersheds on some federal lands within the Columbia River basin. North American Journal of Fisheries Management. 24(4) 1363-1375.

Knighton, A.D., 1998. Fluvial forms and processes: a new perspective. New York: Oxford University Press.

Larsen, D.P., P.R. Kaufmann, T.M. Kincaid, and N.S. Urquhart, 2004. Detecting persistent change in the habitat of salmon-bearing streams in the Pacific Northwest. Canadian Journal of Fisheries and Aquatic Sciences. 61:283-291 
Leicester, M.A., 2005. Recruitment and function of large woody debris in four California coastal streams. Master's Thesis. Paper 2772. San Jose State University, San Jose, California.

Lisle, T.E., 1987. Using "residual depths" to monitor pool depths independently of discharge. Res. Note PSW-394. Berkeley, CA: Pacific Southwest Forest and Range Experiment Station, Forest Service, U.S. Department of Agriculture.

Loganbill, A.W., 2013. Post-fire response of Little Creek watershed: evaluation of change in sediment production and suspended sediment transport. Master's Thesis. California Polytechnic State University, San Luis Obispo, California.

Madej, M.A.1999. Temporal and spatial variability in thalweg profiles of a gravel-bed river. Earth Surface Processes and Landforms. 24:1153-1169.

McIntosh, B.A., J.R. Sedell, R.F. Thurow, S.E. Clarke, and G.L. Chandler, 2000. Historical changes in pool habitats in the Columbia River basin. Ecological Applications. 10(5) 1478-1496

Meyers, T., and S. Swanson, 1996. Stream morphologic impact of and recovery from major flooding in north-central Nevada. Physical Geography. 17(5) 431-445

Monteverde, D.J., D.R. Theobald, and J.G.W. Smith, 2011. Post fire large woody debris survey of the Scotts Creek Watershed. Senior Project, California Polytechnic State University, San Luis Obispo, California.

Montgomery, D.R., and J.M. Buffington, 1998. Channel processes, classification, and response. River ecology and Management. pp. 13-42.

Moore, K., K. Jones, J. Dambacher, 2006. Methods for stream habitat surveys aquatic Inventories project. Oregon Department of Fish and Wildlife, Aquatic Inventories Project, Conservation and Recovery Program. Corvallis, OR.

Mossop, B., and M.J. Bradford, 2005. Using thalweg profiling to assess and monitor juvenile salmon (Oncorhynchus spp.) habitat in small streams. Canadian Journal of Fisheries and Aquatic Sciences 63:1515-1525

Moyle, P.B., 2002. Inland fishes of California. University of California Press, Berkeley and Los Angeles, CA.

National Marine Fisheries Service, 2012. Final Recovery Plan for Central California Coast Coho Salmon Evolutionarily Significant Unit. National Marine Fisheries Service, Southwest Region, Santa Rosa, California.

Nehlsen, W., J.E. Williams, and J.A. Lichatowich, 1991. Pacific salmon at the crossroads: stocks at risk from California, Oregon, Idaho, and Washington. Fisheries. 16(2) 4-21. 
Newman, S., and S. Swanson, 2008. Assessment of changes in stream and riparian conditions of the Marys River basin, Nevada. Journal of the American Water resources Association. 44(1) 1-13

Nickelson, T.T., J.D. Rodgers, S.L. Johnson, M.F. Solazzi, 1992. Seasonal changes in habitat use by juvenile coho salmon (Oncorhynchus kisutch) in Oregon coastal streams. Canadian Journal of Fisheries and Aquatic Sciences. 49: 783-789.

Opperman, J.J., and A.M. Merenlender, 2004. The Effectiveness of riparian restoration for improving instream fish habitat in four hardwood-dominated California streams. North American Journal of Fisheries Management. 24(3) 822-834

Poole, G.C., C.A. Fiessell, and S.C. Ralph, 1997. In-stream habitat unit classification: inadequacies for monitoring and some consequences for management. Journal of the American Water Resources Association. 33(4) 879-896

Potyondy, J.P., and T. Hardy, 1994. Use of pebble counts to evaluate fine sediment increase in stream channels. Water Resources Bulletin. 30(3) 509-520

Reeves, G.H., F.H. Everest, and J.R. Sedell, 1993. Diversity of juvenile anadromous salmonid assemblages in coastal Oregon basins with different levels of timber harvest. Transactions of the American Fisheries Society. 122(3) 309-317

Roper, B.B., J.L. Kershner, E Archer, R. Henderson, and N. Bouwes, 2002. An evaluation of physical stream habitat attributes used to monitor streams. Journal of the American Water Resources Association. 38(6) 1637-1646.

Rosenfeld, J.S., and L. Huato, 2003. Relationship between large woody debris characteristics and pool formation in small coastal British Columbia streams. North American Journal of Fisheries Management. 23:928-938.

Scotts Creek Watershed Council, 2005. Scotts Creek Watershed Assessment

United States Forest Service, 2010. Stream Inventory Handbook

Wolman, M.G., 1954. A method of sampling coarse river-bed material. Transactions, American Geophysical Union. 35(6) 951-956. 


\section{APPENDICES}

Appendix A: Survey Methods from the California Salmonid Stream Habitat Restoration Handbook, Part III, Habitat Inventory Methods.

\section{LEVEL III and LEVEL IV HABITAT TYPES}

Low Gradient Riffle

High Gradient Riffle

CASCADE

Cascade

Bedrock Sheet

FLATWATER

Pocket Water

Glide

Run

Step Run

Edgewater

MAIN CHANNEL POOL

Trench Pool

Mid-Channel Pool

Channel Confluence Pool

Step Pool

SCOUR POOL

Corner Pool

L. Scour Pool - Log Enhanced

L. Scour Pool - Root Wad Enhanced

L. Scour Pool - Bedrock Formed

L. Scour Pool - Boulder Formed

Plunge Pool

BACKWATER POOLS

Secondary Channel Pool

Backwater Pool - Boulder Formed

Backwater Pool - Root Wad Formed

Backwater Pool - Log Formed

Dammed Pool

ADDITIONAL UNIT DESIGNATIONS

Dry

Culvert

Not Surveyed

Not Surveyed due to a marsh
(LGR)

(HGR)

(CAS)

(BRS)

(POW)

(GLD)

(RUN)

(SRN)

(EDW)

(TRP)

(MCP)

(CCP)

(STP)

(CRP)

(LSL)

(LSR)

(LSBk)

(LSBo)

(PLP)

(SCP)

(BPB)

(BPR)

(BPL)

(DPL)

(DRY)

(CUL)

(NS)

(MAR)
$\{1\}$

$\{2\}$

\{3\}

$\{24\}$

$\{21\}$ 


\section{Level IV Habitat Type Descriptions:}

The following habitat type descriptions are taken from the Pacific Southwest Region Habitat Typing Field Guide, USDA-USFS.

\section{LOW-GRADIENT RIFFLE (LGR) [1.1] $\{1\}$}

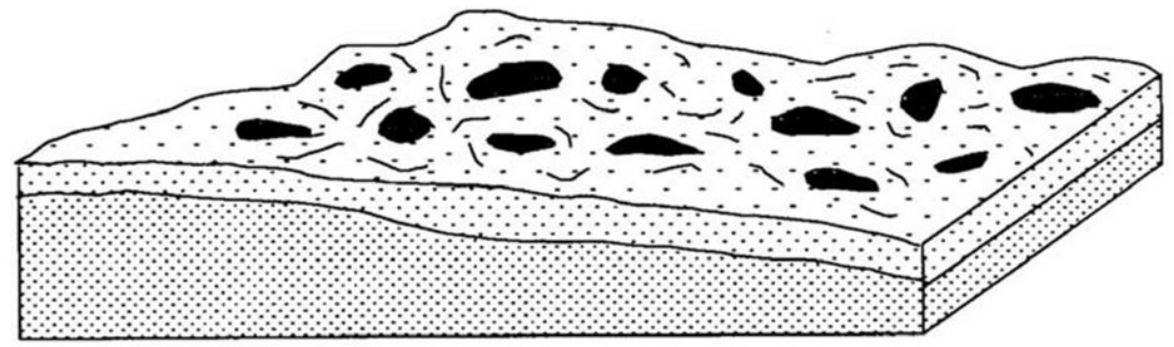

Shallow reaches with swiftly flowing, turbulent water with some partially exposed substrate. Gradient $<4 \%$, substrate is usually cobble dominated.

\section{HIGH-GRADIENT RIFFLE (HGR) [1.2] \{2\}}

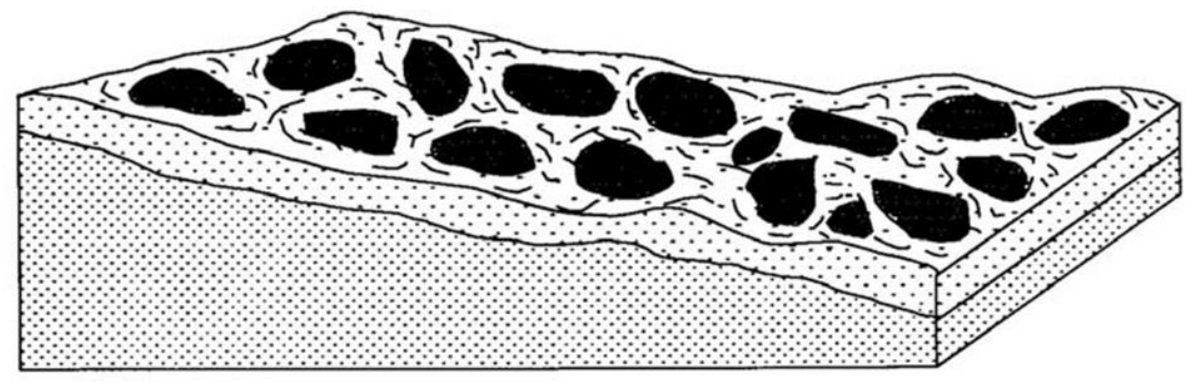

Steep reaches of moderately deep, swift, and very turbulent water. Amount of exposed substrate is relatively high. Gradient is $>4 \%$, and substrate is boulder dominated. 


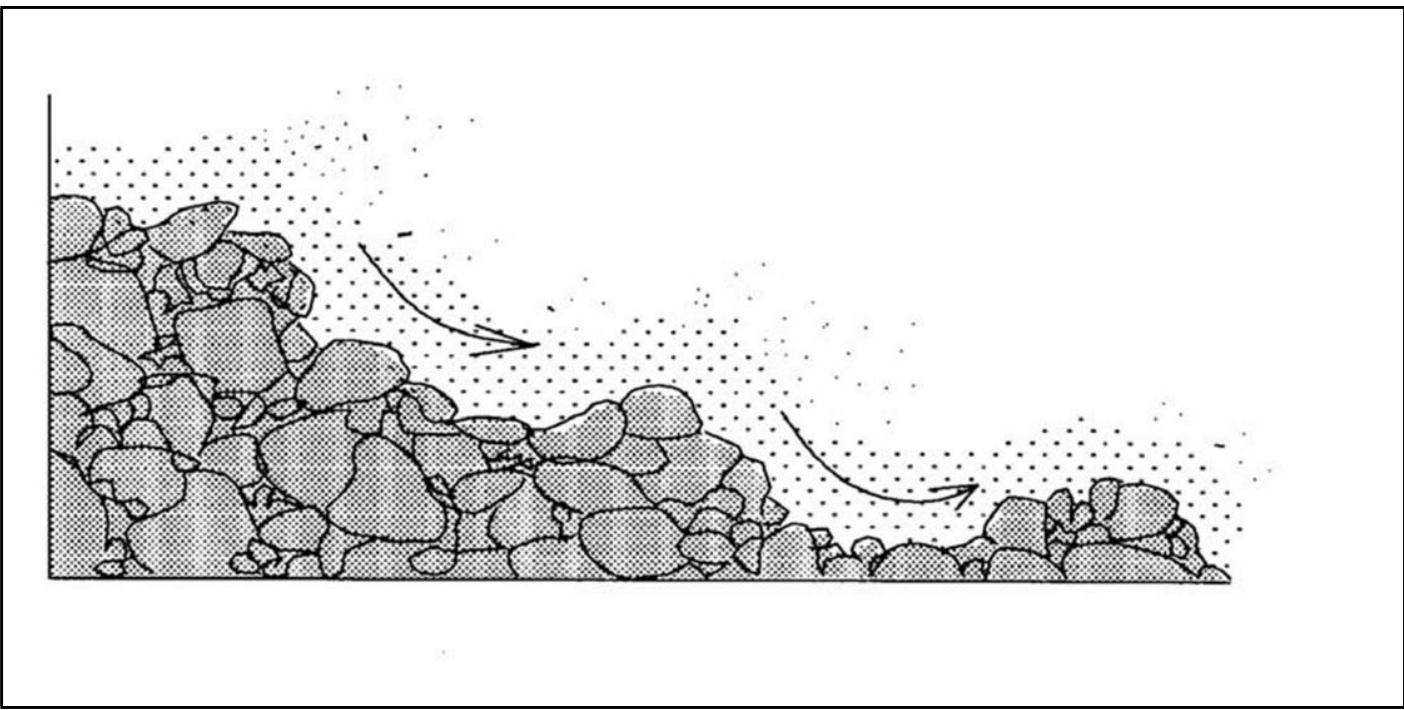

The steepest riffle habitat, consisting of alternating small waterfalls and shallow pools. Substrate is usually bedrock and boulders.

BEDROCK SHEET (BRS) [2.2] \{24\}

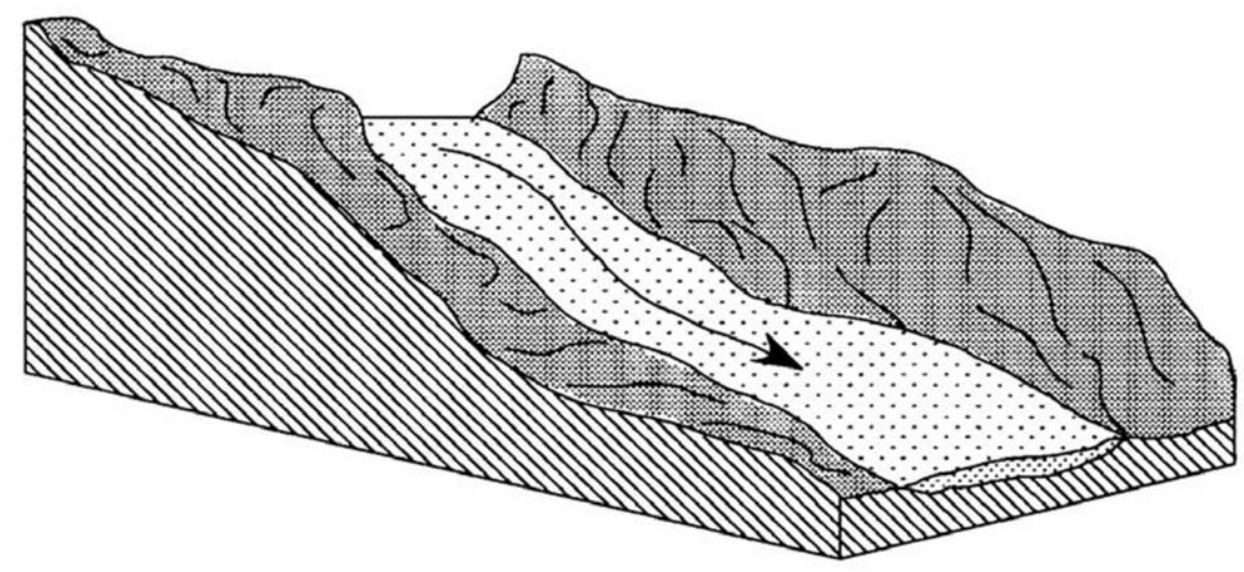

A thin sheet of water flowing over a smooth bedrock surface. Gradients are highly variable. 
POCKET WATER (POW) [3.1] \{21\}

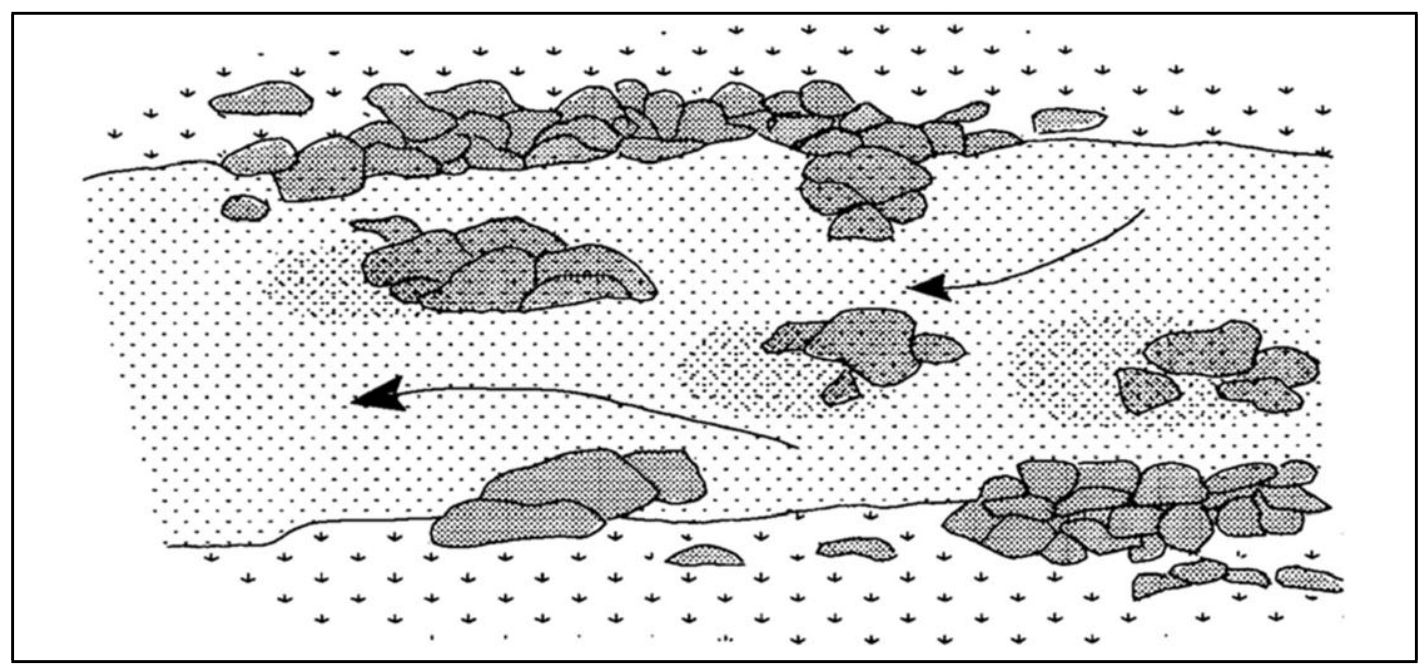

A section of swift-flowing stream containing numerous boulders or other large obstructions which create eddies or scour holes (pockets) behind the obstructions.

GLIDE (GLD) [3.2] \{14\}

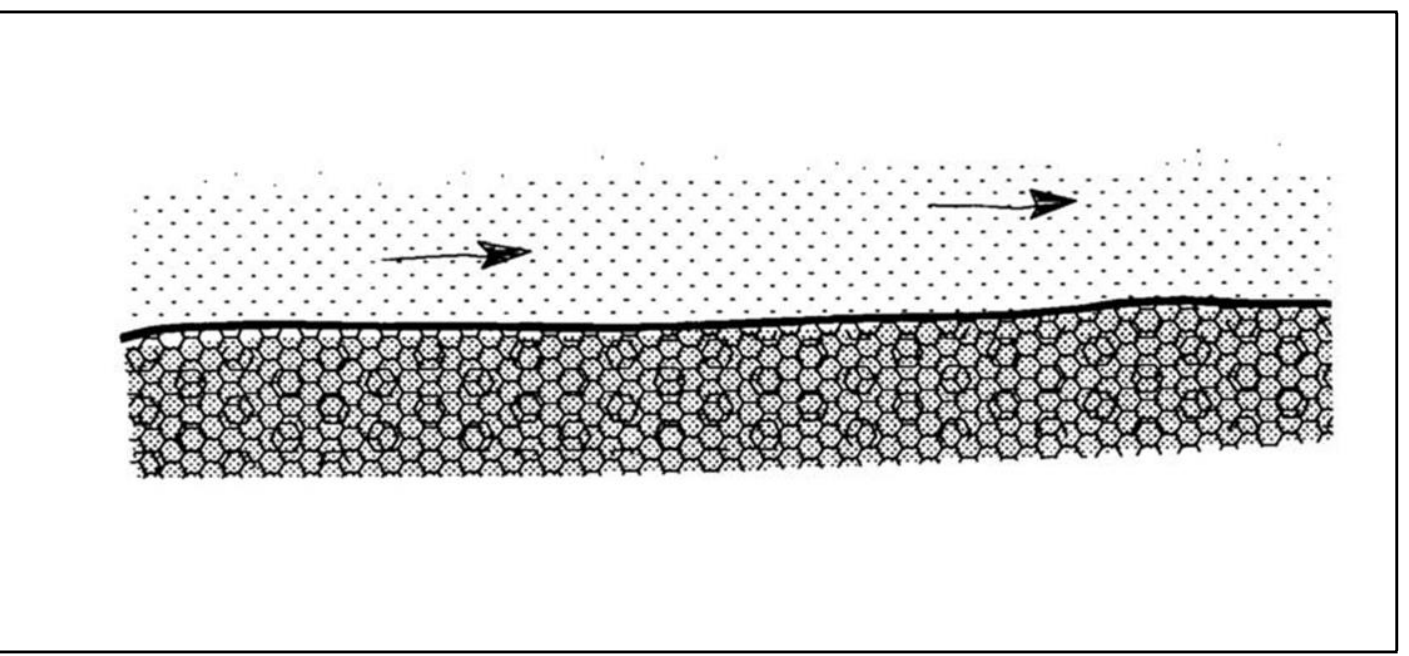

A wide, uniform channel bottom. Flow with low to moderate velocities, lacking pronounced turbulence. Substrate usually consists of cobble, gravel, and sand. 


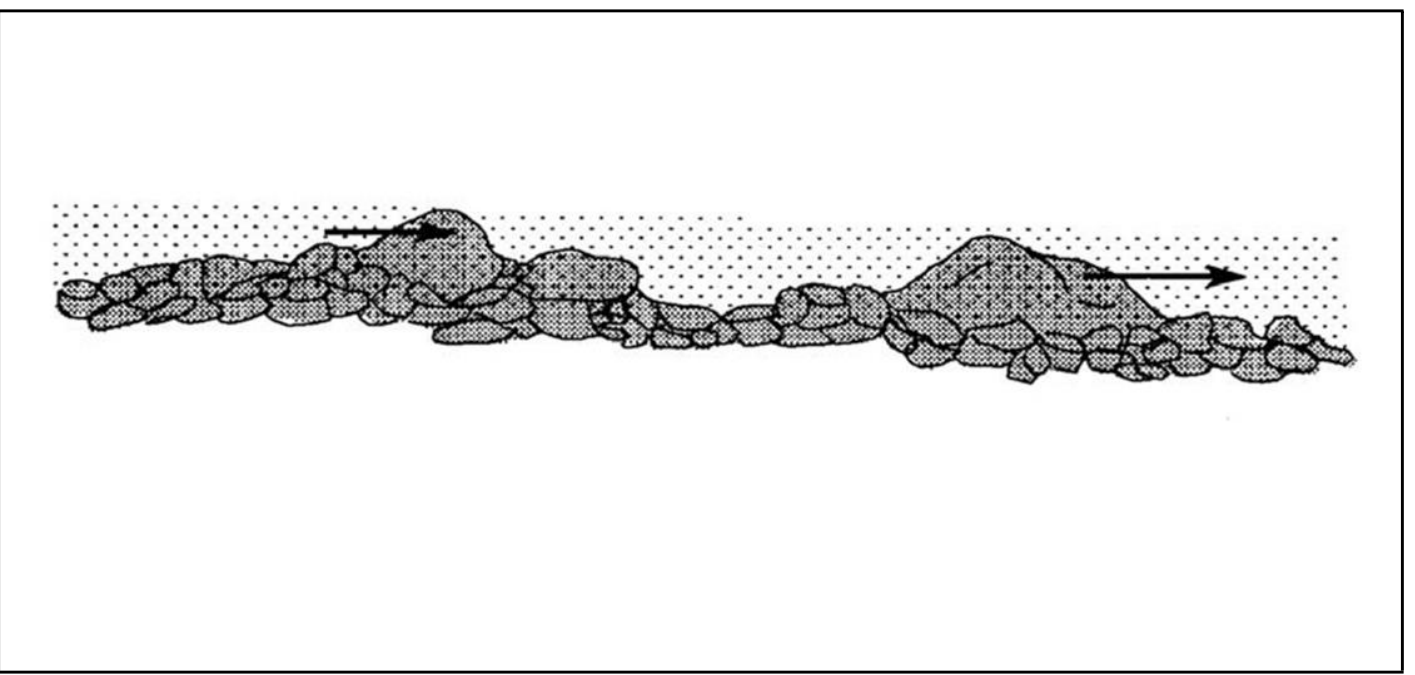

Swiftly flowing reaches with little surface agitation and no major flow obstructions. Often appears as flooded riffles. Typical substrate consists of gravel, cobble, and boulders.

STEP RUN (SRN) [3.4] $\{16\}$

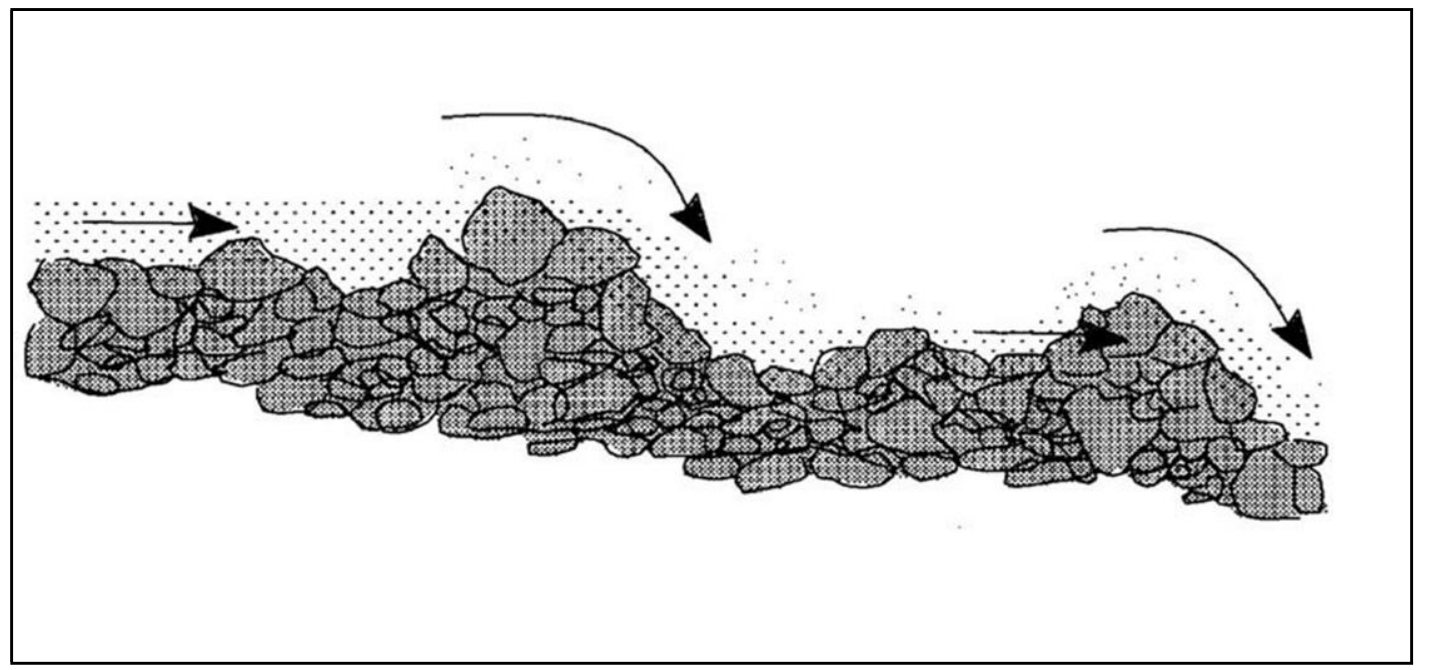

A sequence of runs separated by short riffle steps. Substrate is usually cobble and boulder dominated. 


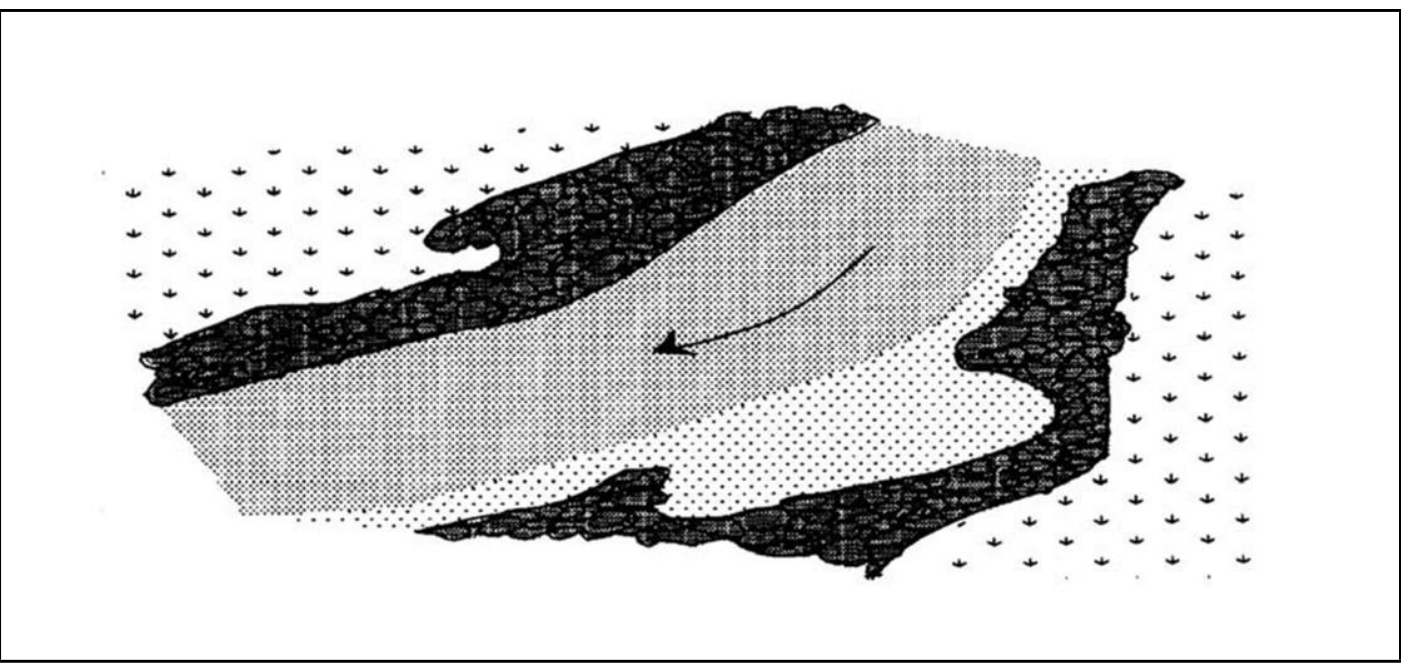

Quiet, shallow area found along the margins of the stream, typically associated with riffles. Water velocity is low and sometimes lacking. Substrate varies from cobbles to boulders.

TRENCH POOLS (TRP) [4.1] $\{8\}$

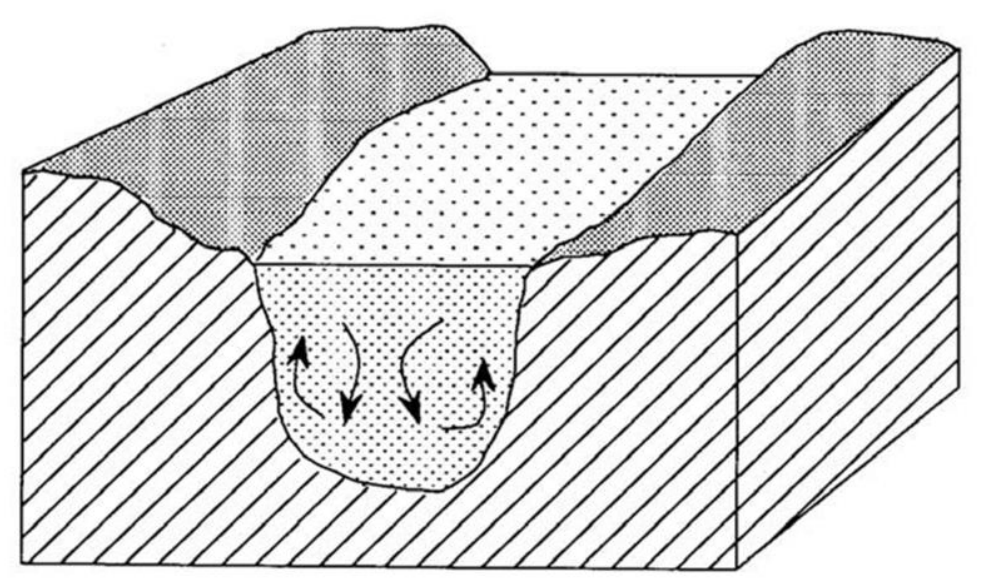

Channel cross sections typically U-shaped with bedrock or coarse grained bottom flanked by bedrock walls. Current velocities are swift and the direction of flow is uniform. 


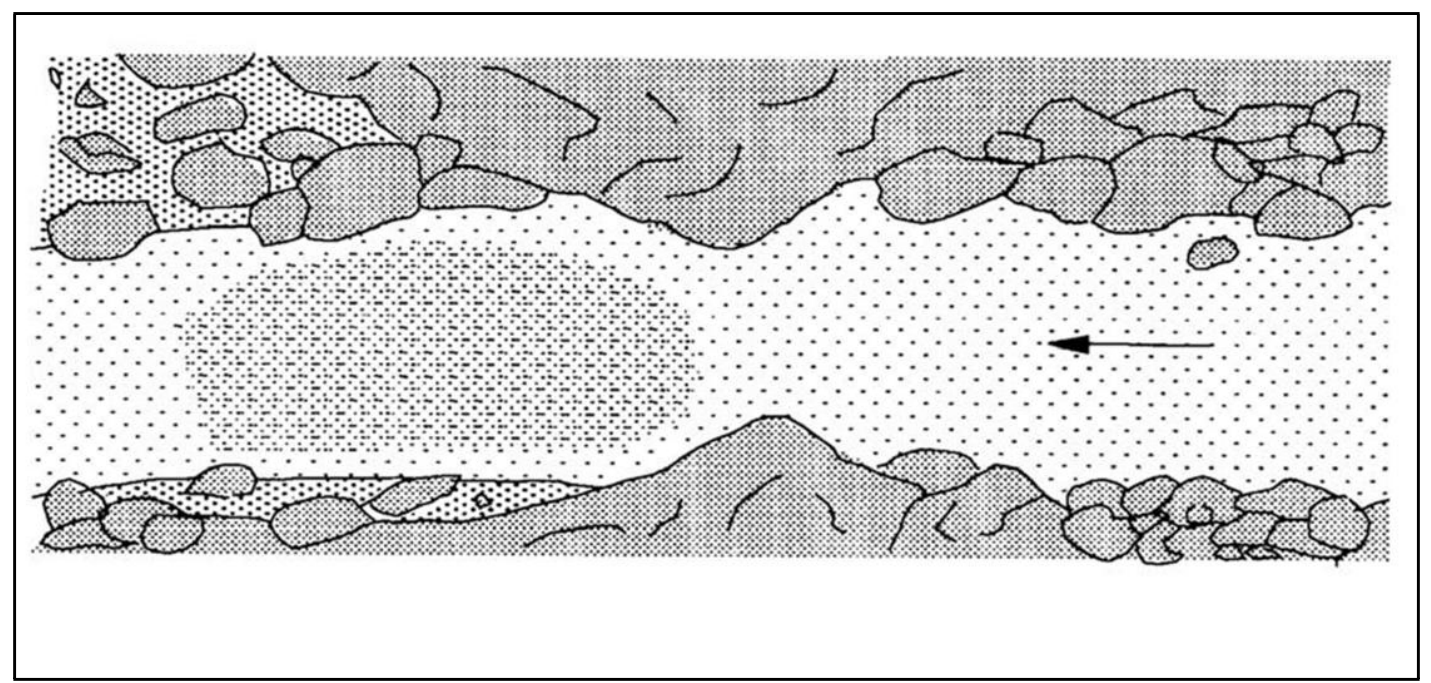

Large pools formed by mid-channel scour. The scour hole encompasses more than $60 \%$ of the wetted channel. Water velocity is slow, and the substrate is highly variable.

\section{CHANNEL CONFLUENCE POOL (CCP) [4.3] \{19\}}

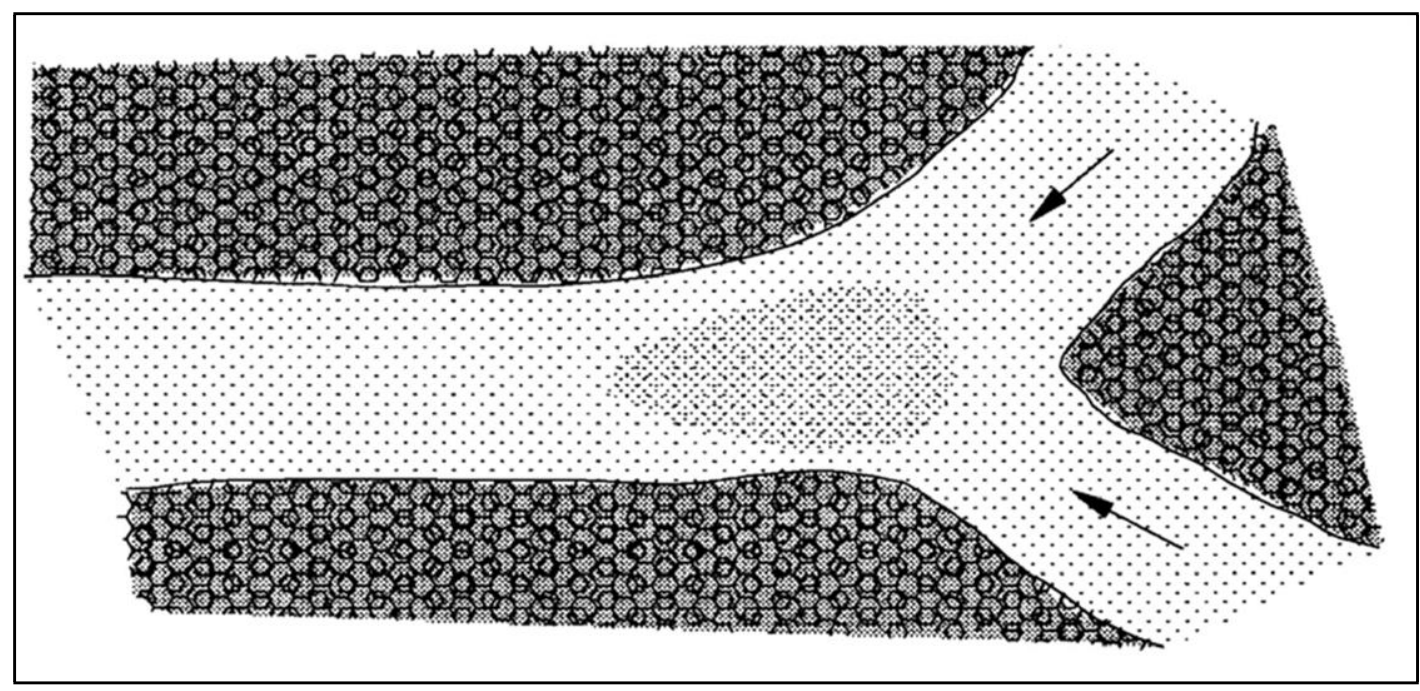

Large pools formed at the confluence of two or more channels. Scour can be due to plunges, lateral obstructions or scour at the channel intersections. Velocity and turbulence are usually greater than those in other pool types. 


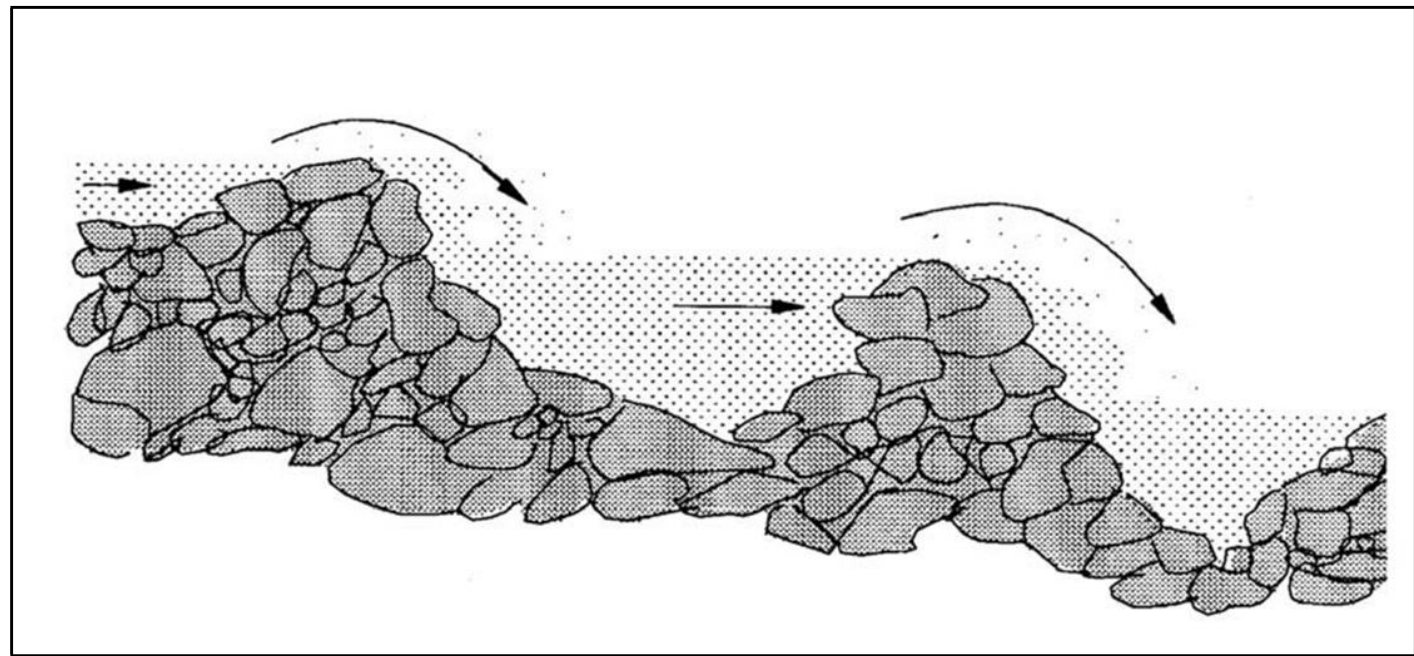

A series of pools separated by short riffles or cascades. Generally found in high- gradient, confined mountain streams dominated by boulder substrate.

CORNER POOL (CRP) [5.1] \{22\}

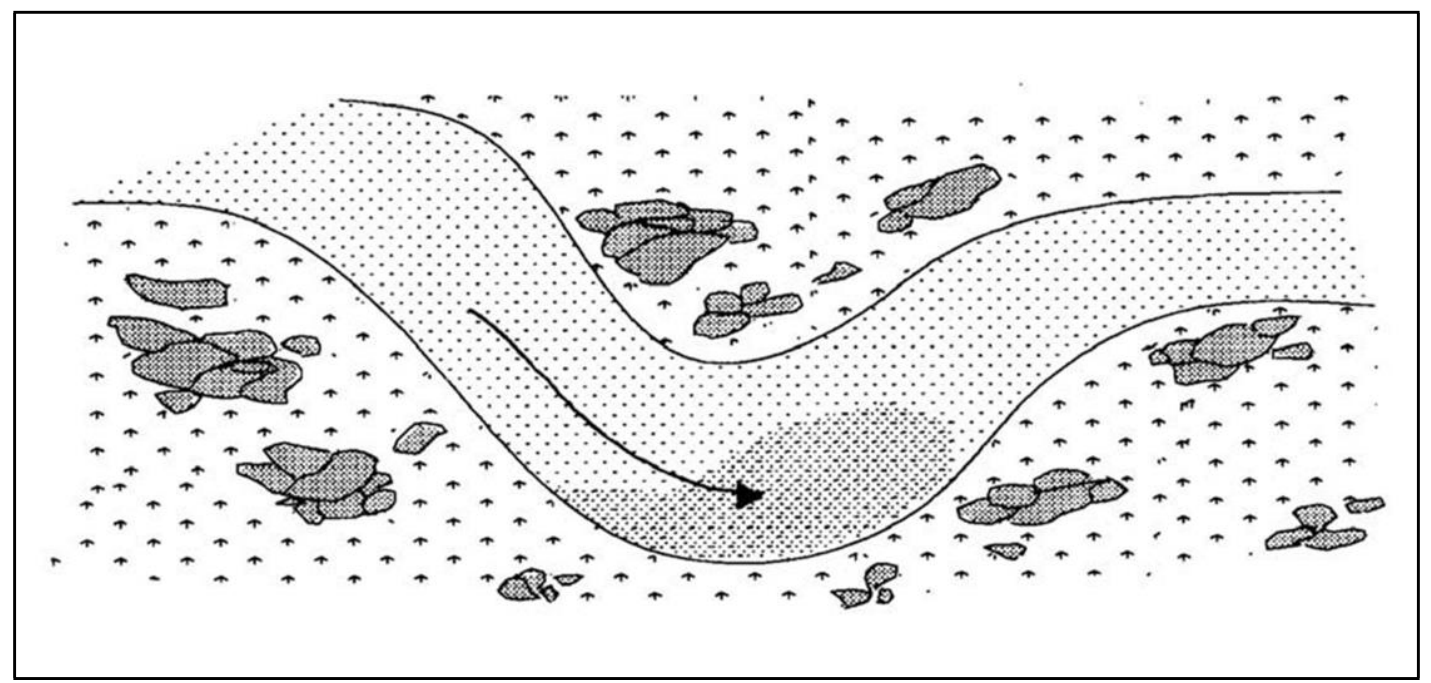

Lateral scour pools formed at a bend in the channel. These pools are common in lowland valley bottoms where stream banks consist of alluvium and lack hard obstructions. 


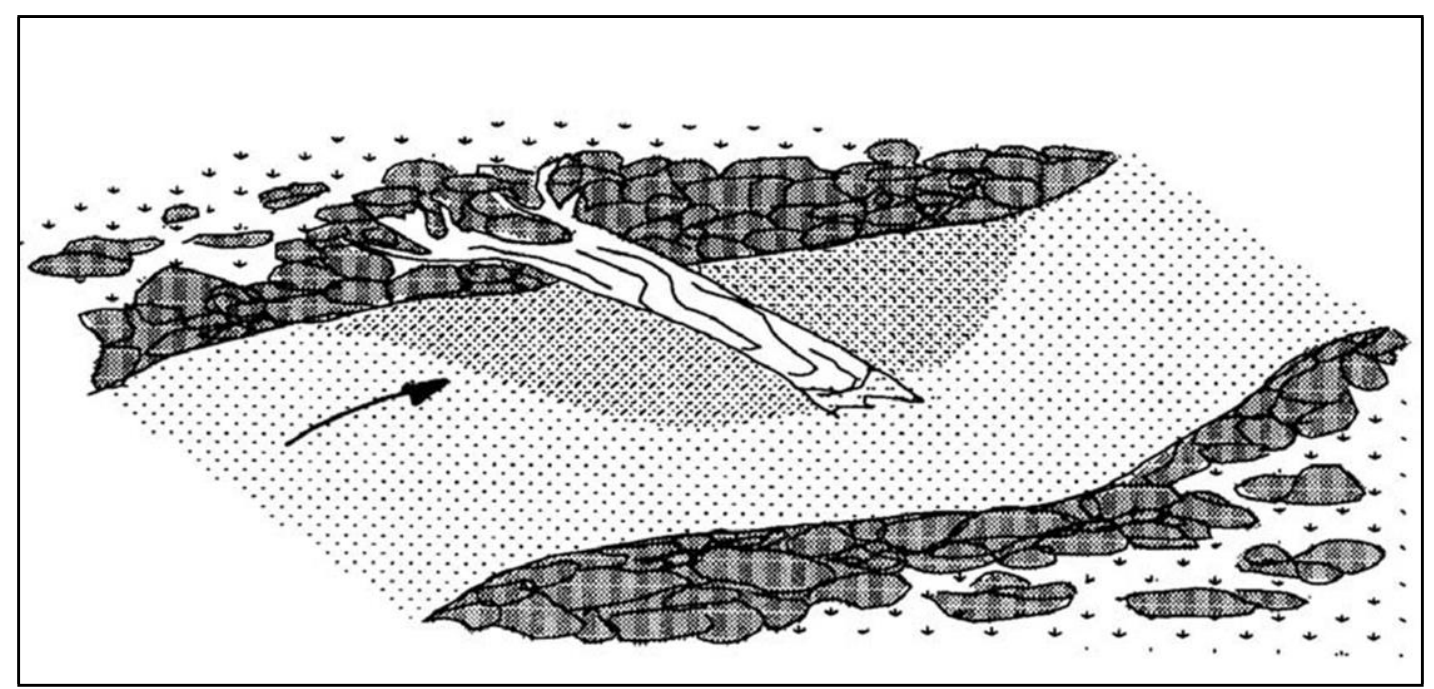

Formed by flow impinging against a partial channel obstruction consisting of large woody debris. The associated scour is generally confined to < $60 \%$ of the wetted channel width.

\section{LATERAL SCOUR POOL - ROOT WAD ENHANCED (LSR) [5.3] \{11\}}

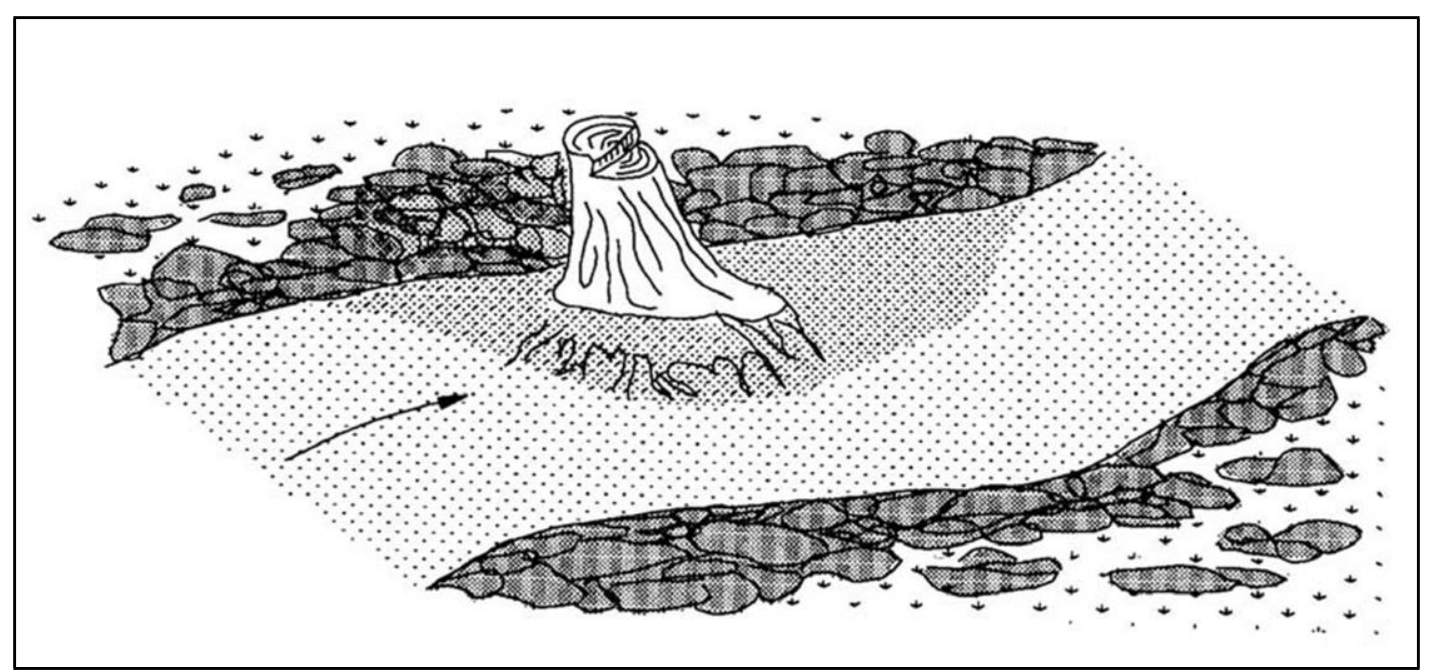

Formed by flow impinging against a partial channel obstruction consisting of a root wad. The associated scour is generally confined to $<60 \%$ of the wetted channel width. 


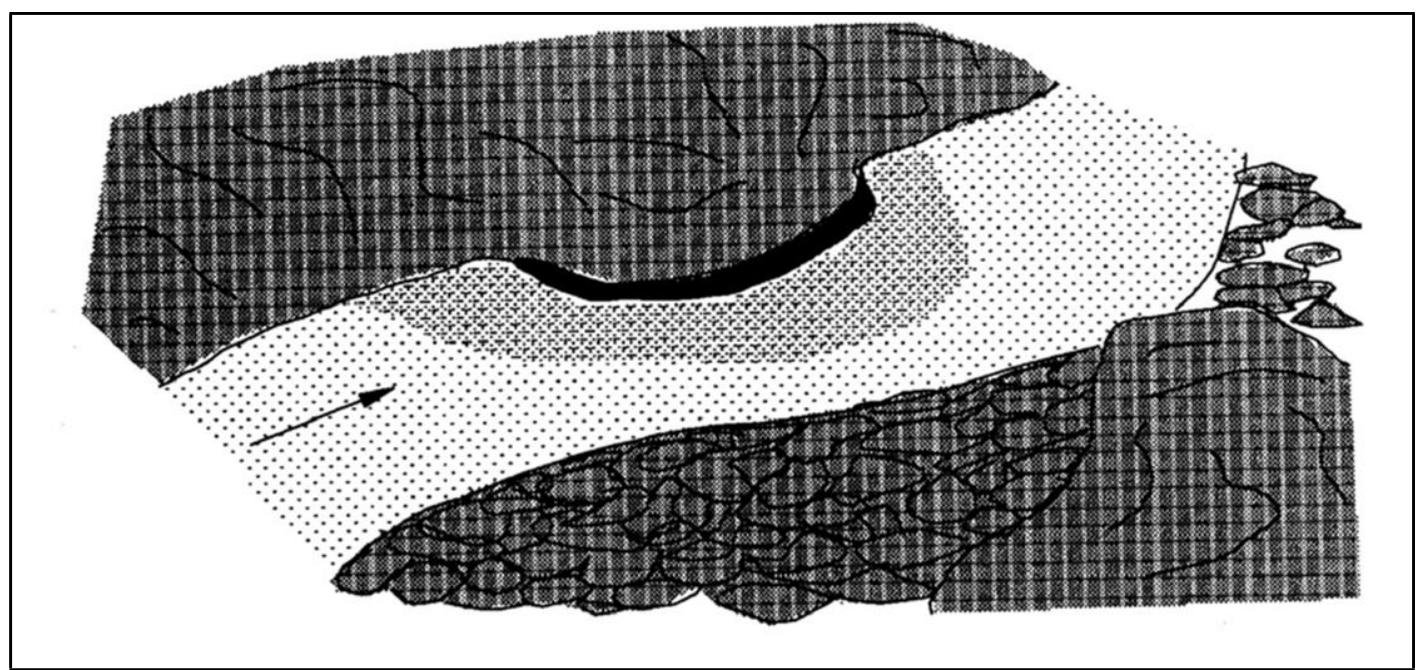

Formed by flow impinging against a bedrock stream bank. The associated scour is generally confined to $<60 \%$ of the wetted channel width.

\section{LATERAL SCOUR POOL - BOULDER FORMED (LSBo) [5.5] \{20\}}

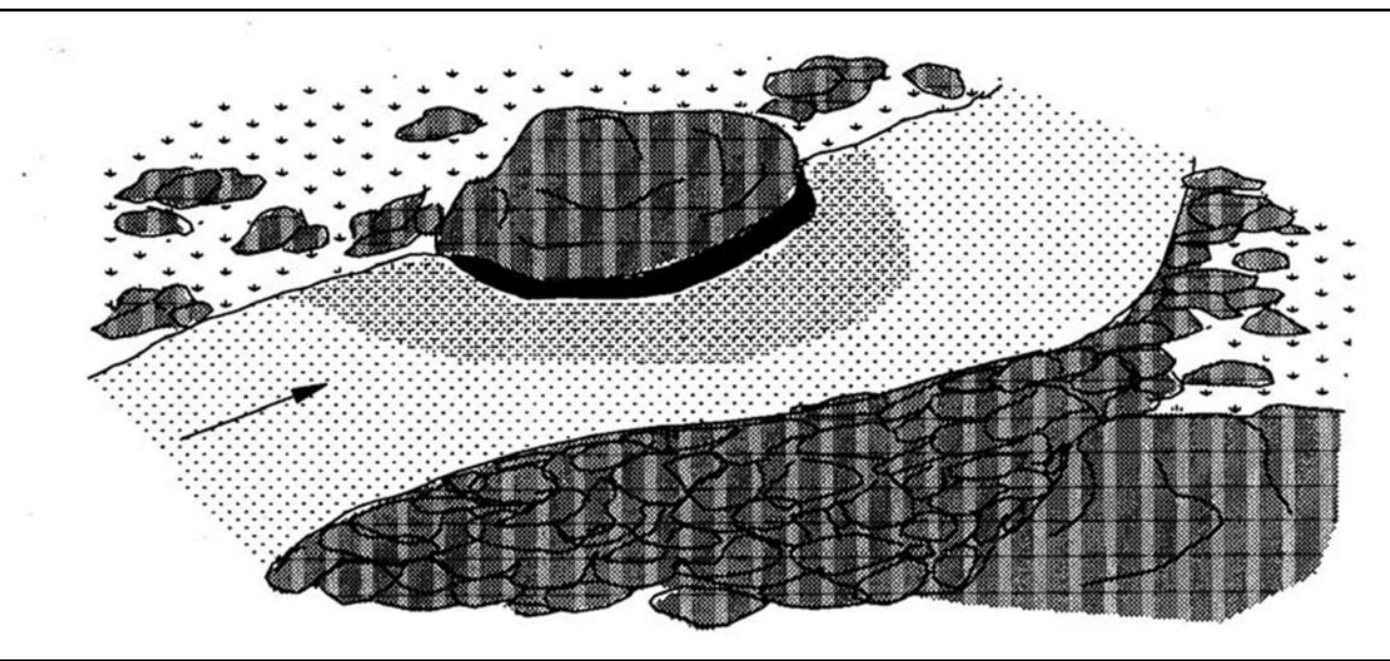

Formed by flow impinging against a partial channel obstruction consisting of a boulder. The associated scour is generally confined to < $60 \%$ of the wetted channel width. 


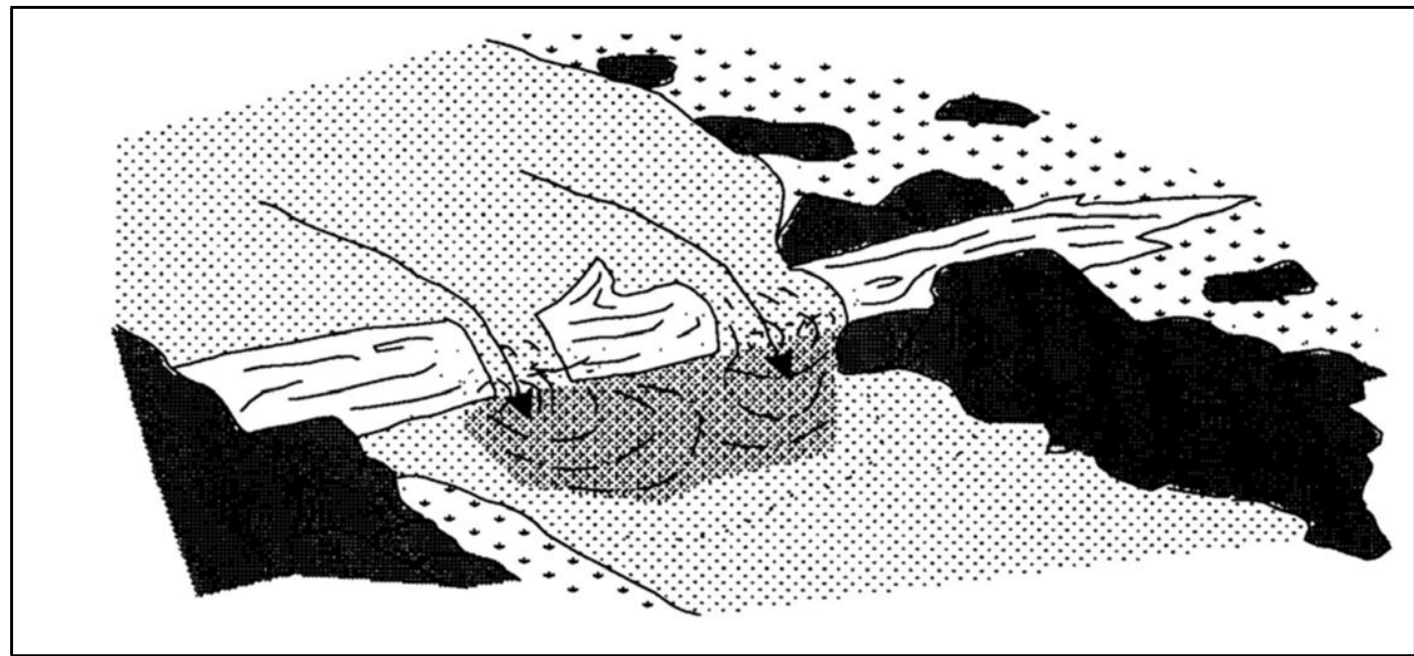

Found where the stream passes over a complete or nearly complete channel obstruction and drops steeply into the streambed below, scouring out a depression; often large and deep. Substrate size is highly variable.

\section{SECONDARY CHANNEL POOL (SCP) [6.1] $\{4\}$}

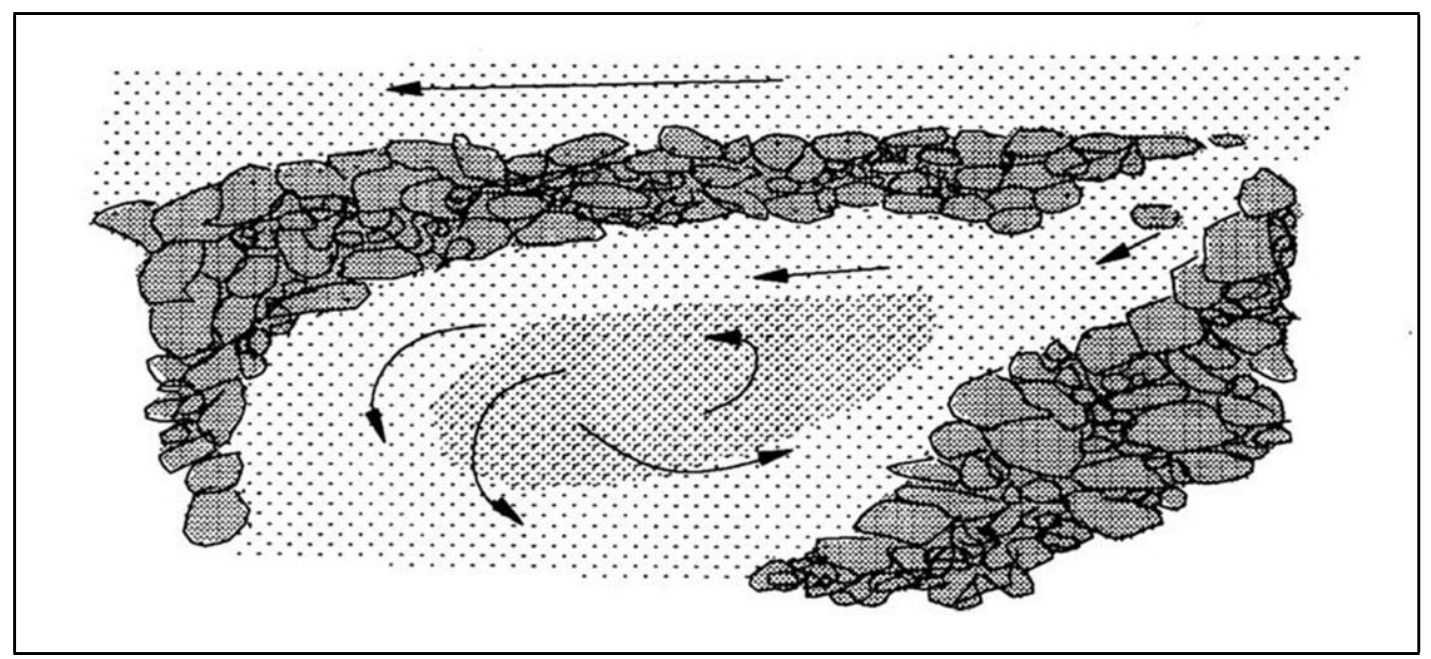

Pools formed outside of the average wetted channel width. During summer, these pools will dry up or have very little flow. Mainly associated with gravel bars and may contain sand and silt substrate. 


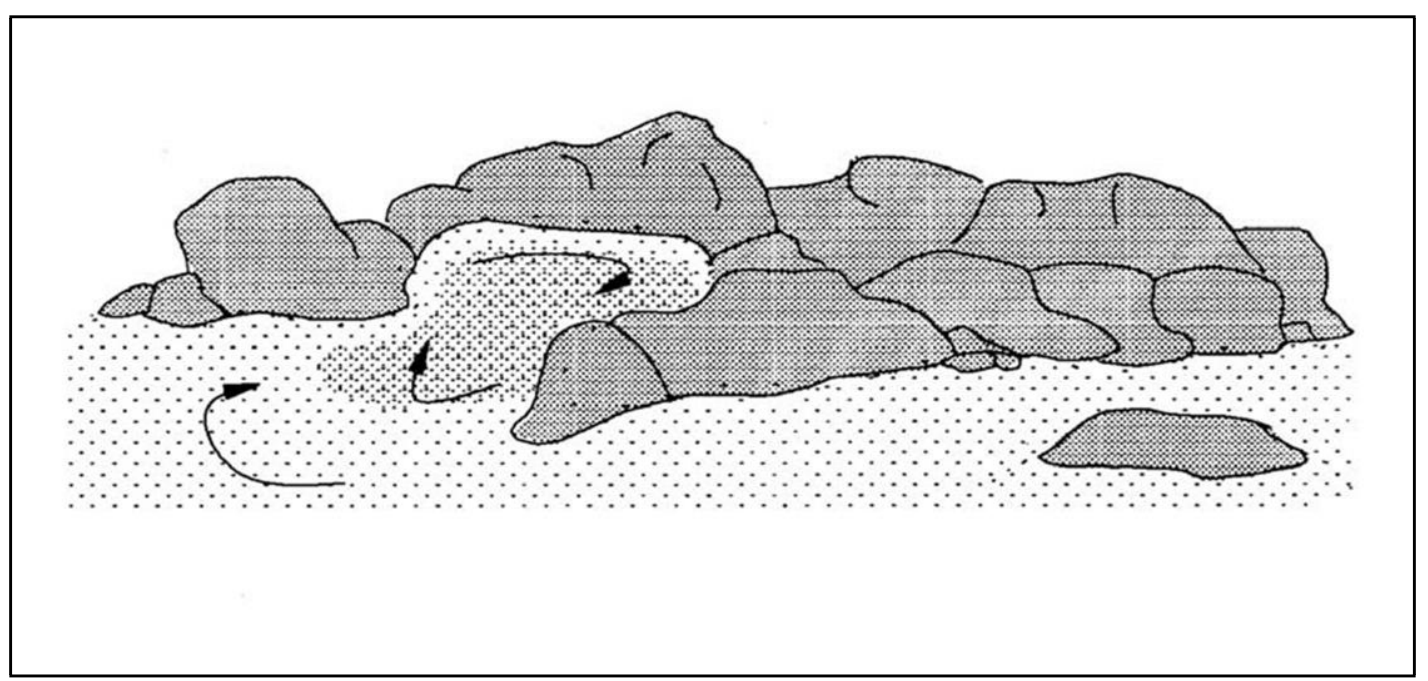

Found along channel margins and caused by eddies around a boulder obstruction. These pools are usually shallow and are dominated by finegrain substrate. Current velocities are quite low.

\section{BACKWATER POOL - ROOT WAD FORMED (BPR) [6.3] \{6\}}

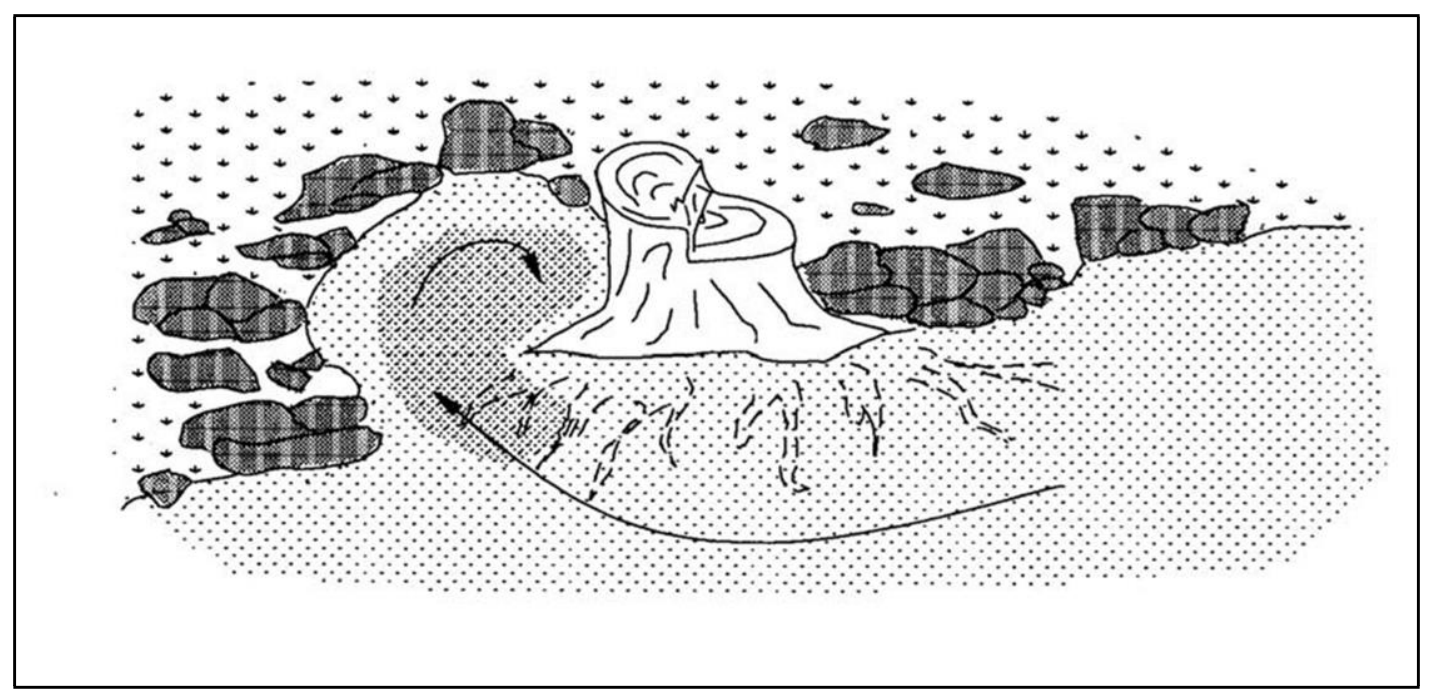

Found along channel margins and caused by eddies around a root wad obstruction. These pools are usually shallow and are dominated by finegrained substrate. Current velocities are quite low. 


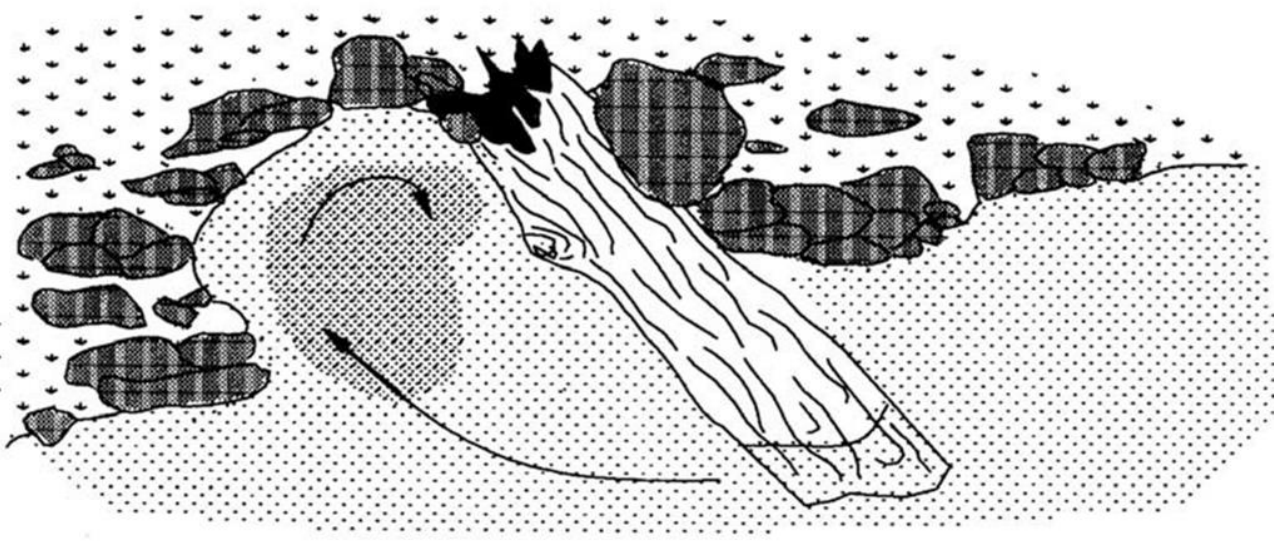

Found along channel margins and caused by eddies around a large woody debris obstruction. These pools are usually shallow and are dominated by fine-grained substrate. Current velocities are quite low.

DAMMED POOLS (DPL) [6.5] \{13\}

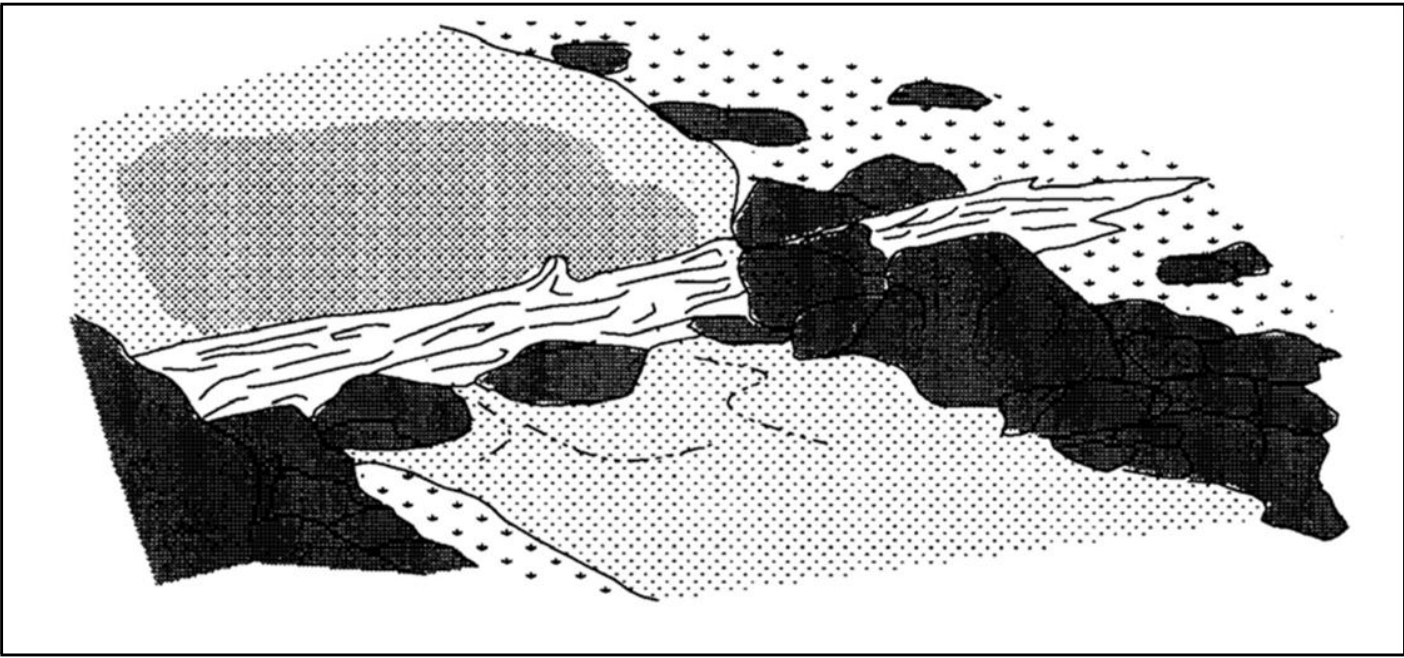

Water impounded from a complete or nearly complete channel blockage (log debris jams, rock landslides or beaver dams). Substrate tends to be dominated by smaller gravel and sand. 


\section{Instream Shelter}

Instream shelter within each habitat unit can be rated according to a standard system. This rating system is a field procedure for habitat inventories which utilizes objective field measurements. It is intended to rate, for each habitat unit, complexity of shelter that serves as instream habitat or that creates areas of diverse velocities which are focal points for salmonids. In this rating system, instream shelter is composed of those elements within a stream channel that provide protection from predation for salmonids, areas of reduced water velocities in which fish can rest and conserve energy, and separation between territorial units to reduce density related competition. This rating does not consider factors related to changes in discharge, such as water depth.

Instream Shelter Complexity. A value rating can be assigned to instream shelter complexity. This rating is a relative measure of the quantity and composition of the instream shelter.

\section{Value Instream Shelter Complexity Value Examples:}

0

1

2

3

- No shelter.

- One to five boulders.

- Bare undercut bank or bedrock ledge.

- Single piece of large wood (>12" diameter and 6' long) defined as large woody debris (LWD).

- One or two pieces of LWD associated with any amount of small wood $(<12 "$ diameter) defined as small woody debris (SWD).

- Six or more boulders per 50 feet.

- Stable undercut bank with root mass, and less than 12" undercut.

- A single root wad lacking complexity.

- Branches in or near the water.

- Limited submersed vegetative fish cover.

- Bubble curtain.

- Combinations of (must have at least two cover types):

LWD/boulders/root wads.

- Three or more pieces of LWD combined with SWD.

- Three or more boulders combined with LWD/SWD.

- Bubble curtain combined with LWD or boulders.

- Stable undercut bank with greater than 12" undercut, associated with root mass or LWD.

- Extensive submersed vegetative fish cover.

Instream Shelter Percent Covered. Instream shelter percent covered is a measure of the area of a habitat unit occupied by instream shelter. The area is estimated from an overhead view. 


\section{Instructions for Completing the Habitat Inventory Data Form}

1) Form No. - Print in the form number. Number the forms sequentially beginning with "01" on the first page and "02" on the second and so on.

2) Date - Enter the day's date: $\mathrm{mm} / \mathrm{dd} / \mathrm{yy}$.

3) Stream Name -Enter the stream name identified on the 7.5 minute USGS quadrangle.

Unnamed streams should be entered as unnamed tributary to name of receiving stream.

4) Legal - Enter the township, range and section of the stream confluence or from where the survey started identified on the 7.5 minute USGS quadrangle.

5) Surveyors - Enter the names of the surveyors with the note taker listed first.

6) Lat - Enter the latitude taken from the 7.5-minute USGS quadrangle at the confluence of the stream (Part II- Instructions for Completing Watershed Overview Work Sheet).

7) Long - Enter the longitude taken from the 7.5-minute USGS quadrangle at the confluence of the stream (Part II- Instructions for Completing Watershed Overview Work Sheet).

8) Quad - Enter the name of the 7.5-minute USGS quadrangle on which the confluence of the stream appears.

9) Channel Type - Record the channel type determined from completing the Stream Channel Type Work Sheet (Part III). Record in the comments the habitat unit number in which the channel type change occurs in.

10) Reach - Enter the reach number beginning with 1 for the lowermost channel type in the basin. Each stream channel type change proceeding upstream will be designated by a new stream reach number.

11) BFW - Measure and enter the stream width at bankfull discharge elevation in the first appropriate velocity crossover on each new data sheet. Use the methods described in the Stream Channel Type Worksheet (Part III).

12)@HU\# - Record the habitat unit number at which the bankfull width was measured.

13) Time - At the beginning of each page enter the time in military time (24-hour clock). 
14) Water Temperature - At the beginning of each page record the water temperature to the nearest degree Fahrenheit. Take water temperatures in the middle of the habitat unit, in flowing water.

15) Air Temperature - At the beginning of each page record the air temperature to the nearest degree Fahrenheit. Take air temperatures in the middle of the habitat unit, within one foot of the water surface.

16) Flow Measurement - Record the flow at the beginning and the end of the survey, at the same location. Record in cubic feet/second.

17) Page Length - Sum the mean lengths for the page.

18) Total Length - Sum all the page lengths through the current page.

19) Habitat Unit Number - Enter the habitat unit number. Record these numbers in sequential order, beginning with "0001" at the survey start. When numbering side channels begin with the number of the unit where the split or divide begins; use a new column and entirely fill it out for each subsequent side channel unit, and number the units sequentially adding a ".1", ".2", etc. as appropriate to describe the exact position of the side channel units. Example of a side channel with two habitat units:

\begin{tabular}{|l|c|c|c|c|c|}
\hline Habitat Unit Number & 0005 & 0006 & 0006.1 & 0006.2 & 0007 \\
\hline Habitat Unit Type & 5.3 & 1.1 & & & 4.2 \\
\hline Side Channel Type & & & 1.1 & 3.2 & \\
\hline
\end{tabular}

20) Habitat Unit Type - Determine the type of habitat unit and enter the appropriate habitat type number code. If the unit is dry, use 7.0 for the habitat unit type. If a stream length is contained within a culvert, use 8.0 for the habitat unit type. If the length of stream was not surveyed due to lack of access, use 9.0 for the habitat type. If the length of stream was not surveyed due to a marsh, use 9.1 for the habitat unit type. Record all pertinent information in the comments.

21) Side Channel Type - Determine the type of habitat unit and enter the appropriate habitat type number code.

22) Mean Length - Enter the thalweg length of the habitat unit, in feet.

23) Mean Width - Measure two or more wetted channel widths within the habitat unit.

Calculate and enter the mean width for the habitat unit, in feet.

24) Mean Depth - Take several random depth measurements across the unit with a stadia rod. Calculate and enter the mean depth, in feet. 
25) Maximum Depth - Enter the measured maximum depth for each habitat unit, in feet.

26) Depth Pool Tail Crest - Measure the maximum thalweg depth at the pool tail crest, in feet. This measurement is taken only in pool habitat units and is used to determine the pool's residual volume.

27) Pool Tail Embeddedness - Percent cobble embeddedness is determined at pool tail-outs where spawning is likely to occur. Sample at least five small cobbles (2.5" to 5.0") in diameter and estimate the amount of the stone buried in the sediment. This is done by

removing the cobble from the streambed and observing the line between the "shiny" buried portion and the duller exposed portion. Estimate the percent of the lower shiny portion using the corresponding number for the $25 \%$ ranges. Average the samples for a mean cobble embeddedness rating. Additionally, a value of 5 is assigned to tail-outs deemed unsuited for spawning due to inappropriate substrate particle size, having a bedrock tailout, or other considerations:

$$
\begin{aligned}
& 1=0 \text { to } 25 \% \\
& 2=26 \text { to } 50 \% \\
& 3=51 \text { to } 75 \% \\
& 4=76 \text { to } 100 \% \\
& 5=\text { unsuitable for spawning }
\end{aligned}
$$

28) Pool Tail Substrate - Enter the letter code (A through G) for the dominant substrate composition of the tail-out for all pools.

29) Large Woody Debris Count Diameter $>1$ ' and Length from 6'to 20' Record the number of pieces of large woody debris that have a diameter greater than one foot and a length between six and twenty feet, and are wholly or partially within the bankfull discharge elevation of that habitat unit.

30) Large Woody Debris Count Diameter $>$ 1' and Length $>$ 20' - Record the number of pieces of large woody debris that have a diameter greater than one foot and a length greater than twenty feet, and are located wholly or partially within the bankfull discharge elevation of that habitat unit.

31) Shelter Value - Enter the number code (0 to 3 ) that corresponds to the dominant structural shelter type that exists in the unit (Part III- Instream Shelter Complexity). 
32) Percent Unit Covered - Enter the percentage of the unit occupied by the structural shelter. Classify 100 percent of the shelter by the types indicated on the form. Note: bubble curtain includes white water.

33) Substrate Composition - Enter a "1" for the dominant substrate and a "2" for the co- dominant substrate. Note: changes in the dominant and codominant substrate may indicate that the channel type has changed.

34) Percent Exposed Substrate - Enter the estimated percentage of the bottom substrate of the unit that is exposed above the water surface.

35) Percent Total Canopy - Enter the percentage of the stream area that is influenced by the tree canopy. The canopy is measured using a spherical densiometer at the upstream end of each habitat unit in the center of the wetted channel. (Appendix M).

36) Percent Hardwood Trees - Estimate the percent of the total canopy consisting of hardwood, or broadleafed, trees. For watershed where the entire canopy consists of hardwood trees, use this field to distinguish deciduous trees, or trees that provide partial year shade and leaf-drop.

37) Percent Coniferous Trees - Estimate the percent of the total canopy consisting of coniferous, or needle leafed, trees. For watersheds where the entire canopy consists of hardwood trees, use this field to distinguish evergreen trees, or trees that provide year- round shade.

38) Right Bank Composition - Observed from the base of the stream bank to the bankfull discharge level. Enter the number (1 through 4) for the right bank composition type corresponding to the list located on the lower left hand side of the form. Enter one number only. The right bank is the right side of the stream when facing downstream.

39) Right Bank Dominant Vegetation - Enter the number (5 through 9) for the right bank dominant vegetation type, from bankfull to 20 feet upslope, corresponding to the list located on the lower left hand side of the form. Enter one number only.

40) Percent Right Bank Vegetated - Estimate the total percentage of the right bank covered with vegetation from bankfull discharge level to 20 feet upslope.

41) Left Bank Composition - Observed from the lower bank to the bankfull discharge level.

Enter the number (1 through 4) for the left bank composition type corresponding to the list located on the lower left hand side of the form. 
Enter one number only. The left bank is the left side of the stream when facing downstream.

42) Left Bank Dominant Vegetation - Enter the number (5 through 9) for the left bank dominant composition type, from bankfull to 20 feet upslope, corresponding to the list located on the lower left hand side of the form. Enter one number only.

43) Percent Left Bank Vegetated - Estimate the total percentage of the left bank covered with vegetation from bankfull discharge level to 20 feet upslope.

44) Comments - Add comments which characterize important habitat unit observations.

There are ten comment categories. Comments are begun with an initial letter code which identifies its assigned category.

a. "S" for an instream habitat structure or bank stabilization project

b. "C" for a channel type change

c. " $\mathrm{D}$ " to document a water diversion

d. "T" to record a tributary

e. "E" for bank erosion or a landslide observation

f. "B" to document a biological observation or sampling site

g. "P" for a fish passage problem

h. " $A$ " to record a stream access point

i. "G" for a location recorded with a GPS receiver

j. " $O$ " for all other comments that do not fall into an above category.

45) The back of the data sheet has an additional area for comments that will not fit on the front, and an area for diagrams to describe a comment. 


\section{Appendix B: Example Data Sheets}

\section{Data Sheet}

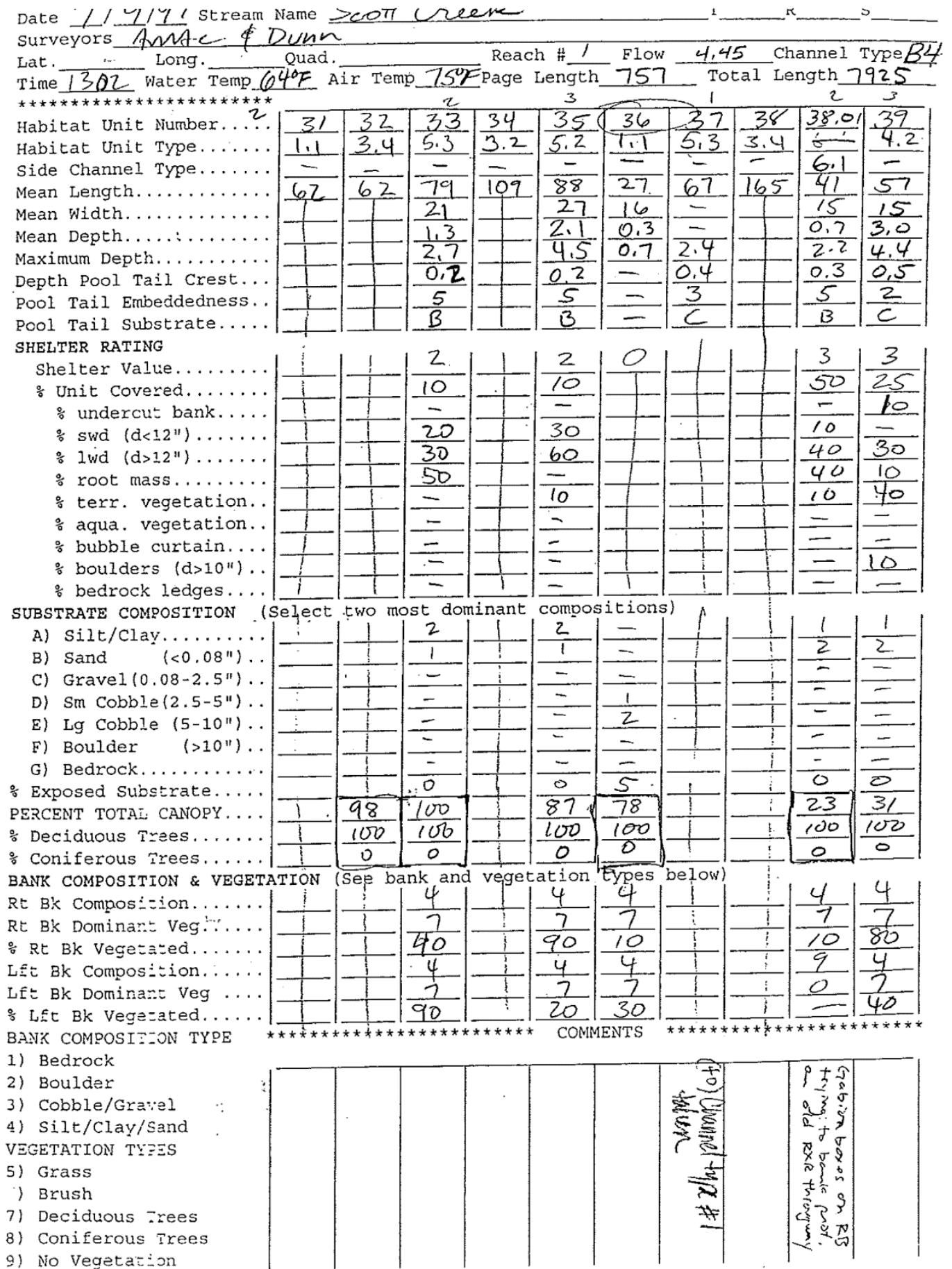

2013 Data Sheet 


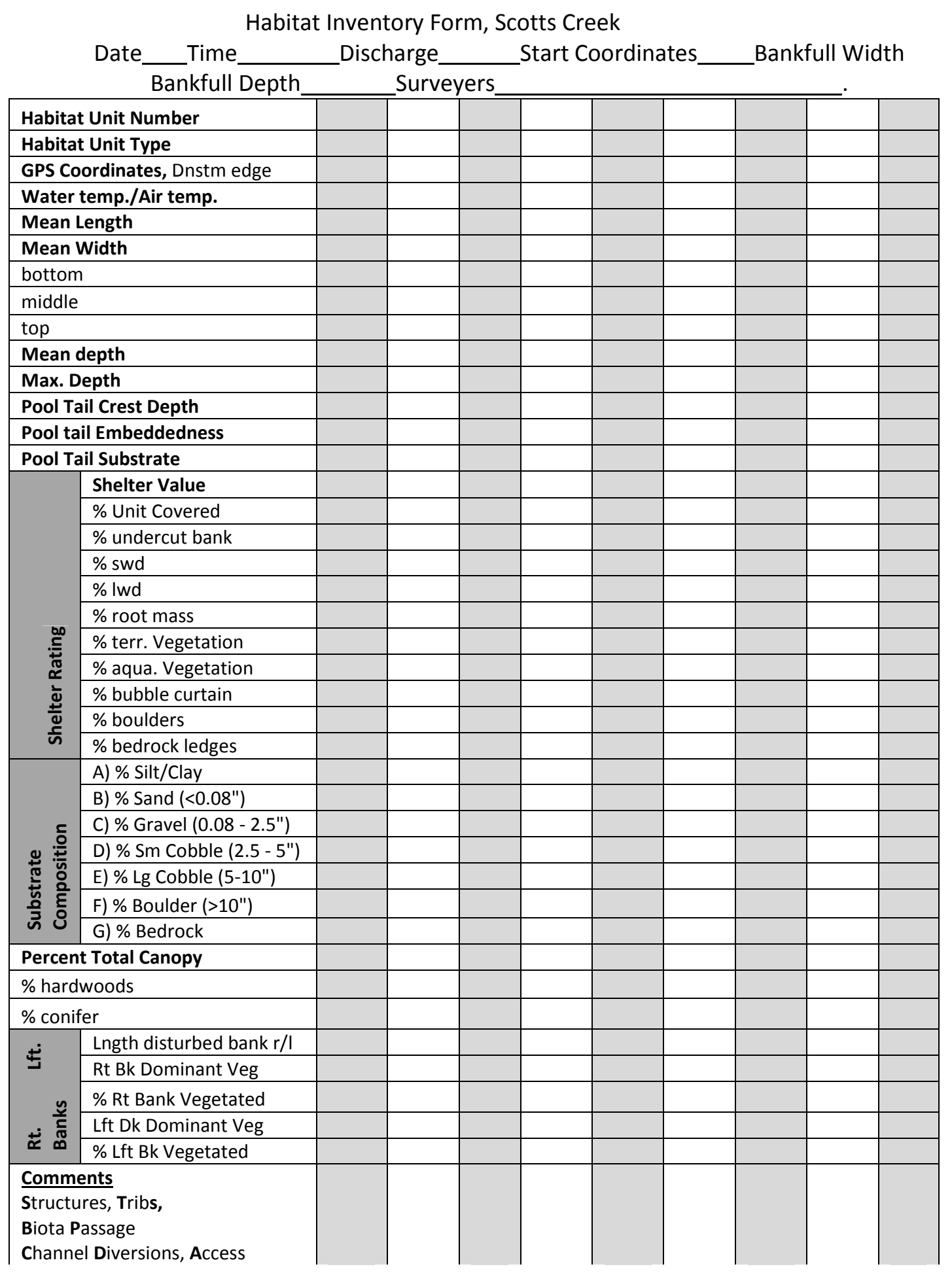




\section{Appendix C: Example LWD Forms}

LWD form used in Lieciester, 2005, for survey years 2000-2001

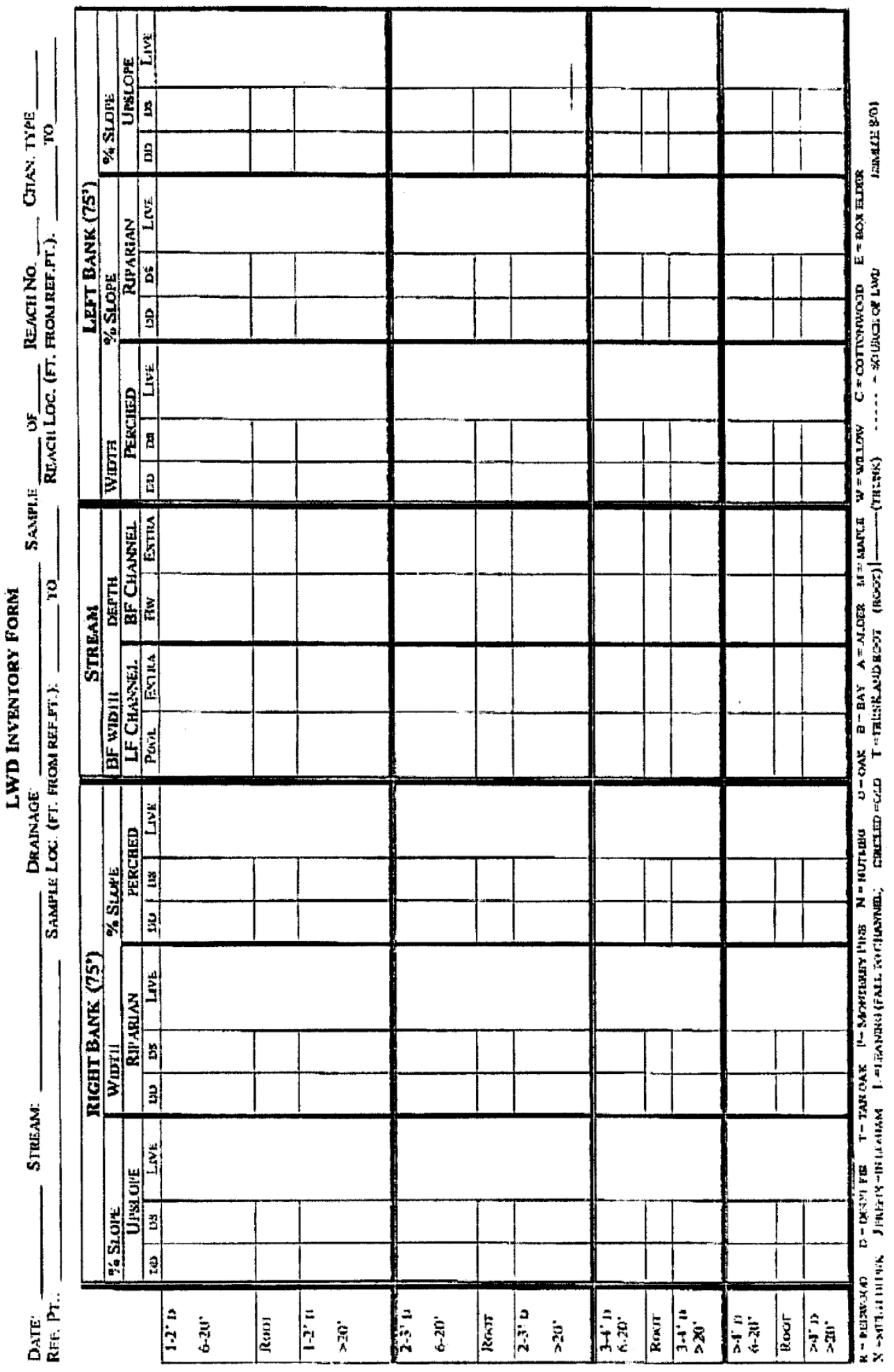




\section{LWD Form}

\section{Scotts Creek Large Woody Debris}

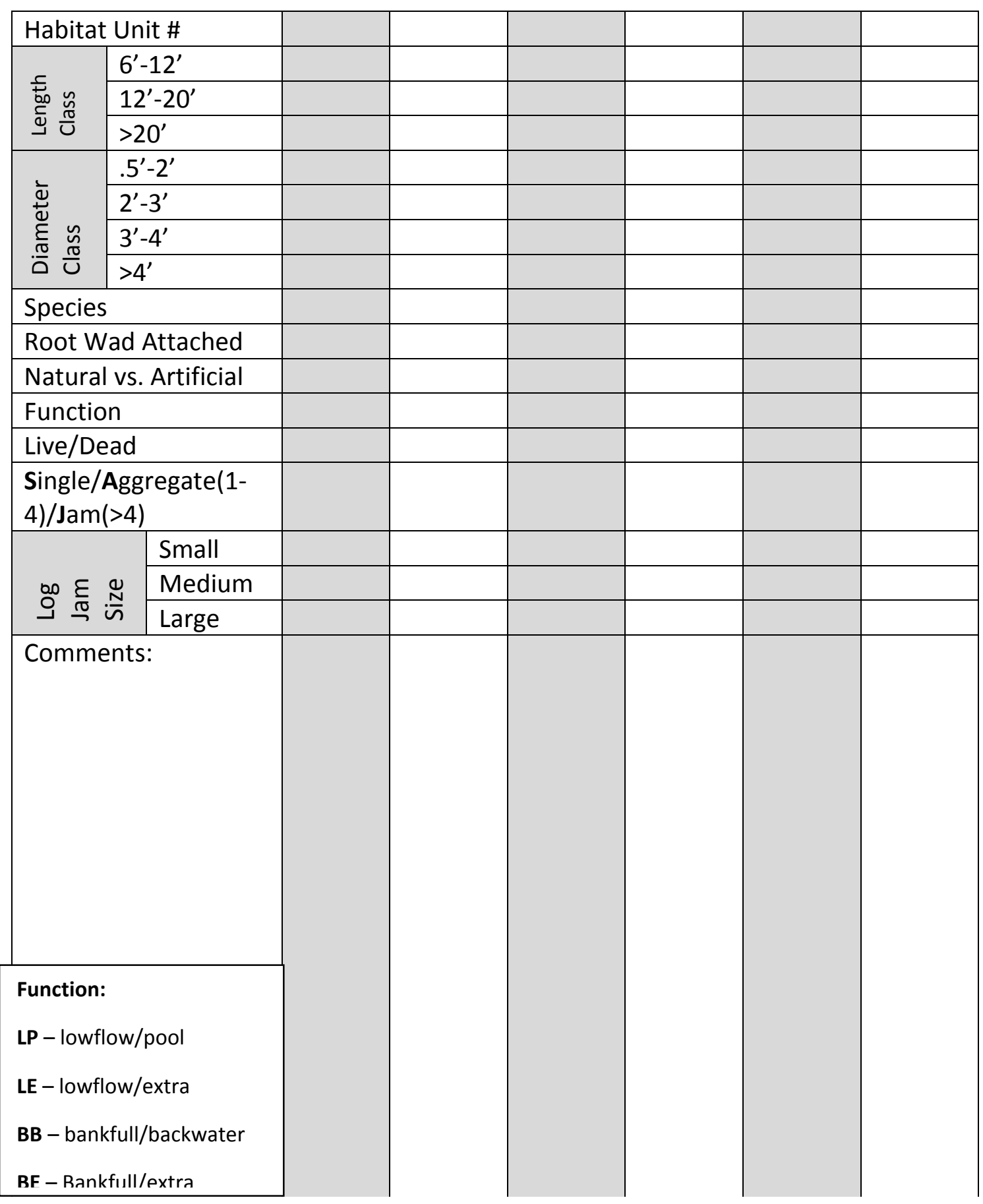




\section{Appendix D: Survey Data}

\begin{tabular}{|c|c|c|c|c|c|c|c|c|c|c|c|c|c|c|c|c|c|c|c|c|c|c|c|c|c|c|c|c|c|c|c|c|c|c|c|}
\hline 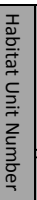 & 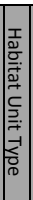 & 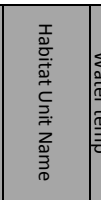 & 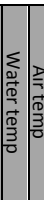 & 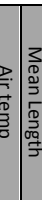 & 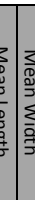 & 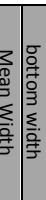 & 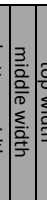 & 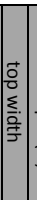 & 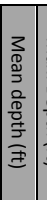 & 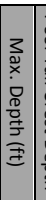 & 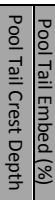 & 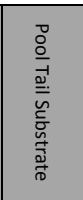 & 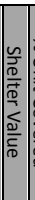 & 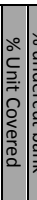 & 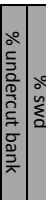 & $\frac{2}{\grave{\Sigma}}$ & 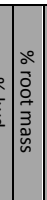 & 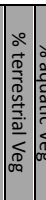 & 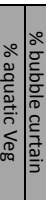 & $\begin{array}{l}2 \\
\frac{0}{0} \\
0 \\
\frac{c}{2} \\
\frac{0}{0} \\
\frac{1}{3}\end{array}$ & 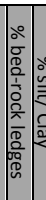 & 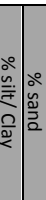 & 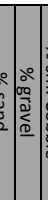 & 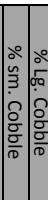 & & 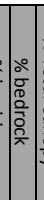 & 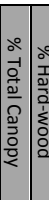 & 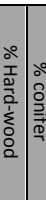 & 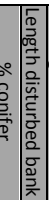 & 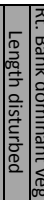 & 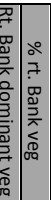 & 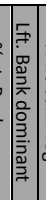 & 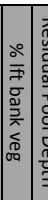 & 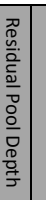 & 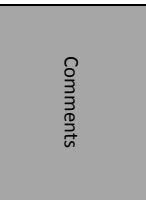 \\
\hline 1 & $\begin{array}{l}3 . \\
2 \\
\end{array}$ & Glide & & 50 & 018 & 1822 & $2|17| 1$ & 15 & & 2 & & & 2 & 10 & 50 & & 25 & 25 & & & & & 050 & $15 \mid 10$ & 100 & 0 & & & 0 & 0 & \begin{tabular}{l|l|}
9 & 0 \\
\end{tabular} & 6 & \begin{tabular}{|c|}
10 \\
0 \\
\end{tabular} & & \\
\hline 2 & $\begin{array}{c}5 . \\
1 \\
\end{array}$ & $\begin{array}{c}\text { Corner } \\
\text { Pool }\end{array}$ & & $\begin{array}{c}12 \\
9 \\
\end{array}$ & \begin{tabular}{l|l}
2 & 17 \\
\end{tabular} & \begin{tabular}{l|l}
17 & 15 \\
\end{tabular} & 5191 & 16 & & \begin{tabular}{|c|c|}
3.2 & 0 \\
5 & \\
\end{tabular} & 0.825 & Gravel & 3 & 10 & $0 \mid 2 C$ & \begin{tabular}{l|l}
0 & 5
\end{tabular} & 10 & 65 & \begin{tabular}{l|l}
0 & 0
\end{tabular} & 0 & 02 & 2025 & 530 & 205 & 50 & 0 & & & 0 & $0 \mid 5$ & \begin{tabular}{l|l|}
5 & 10
\end{tabular} & 6 & \begin{tabular}{|c|c|}
10 & 2 \\
0 & 5 \\
\end{tabular} & \begin{tabular}{c|c}
2.4 & 5 \\
5 & \\
\end{tabular} & $\begin{array}{l}\text { Debris. Old fish } \\
\text { trap? }\end{array}$ \\
\hline 3 & $\begin{array}{l}1 . \\
1\end{array}$ & \begin{tabular}{|} 
Low \\
gradient \\
Riffle
\end{tabular} & & 60 & 016 & 1615 & $5 \mid 102$ & 22 & $0.5^{1}$ & $\begin{array}{c}1.2 \\
5\end{array}$ & & & 1 & 5 & \begin{tabular}{|l|l}
0 & 0
\end{tabular} & 0 & 15 & 85 & \begin{tabular}{l|l}
0 & 0
\end{tabular} & 0 & 0 & \begin{tabular}{l|l}
0 & 15
\end{tabular} & 525 & 3020 & 205 & 0 & & & 0 & $0 \mid 5$ & \begin{tabular}{|l|l|}
5 & 5 \\
\end{tabular} & 6 & 95 & & \\
\hline 4 & $\begin{array}{c}5 . \\
1 \\
\end{array}$ & $\begin{array}{c}\text { Corner } \\
\text { Pool }\end{array}$ & & 85 & 52 & 2422 & 2292 & 20 & & 1.70 & 0.3 & & 1 & 53 & 330 & 0 & 34 & 33 & \begin{tabular}{l|l}
0 & 0 \\
\end{tabular} & 0 & $\begin{array}{lll}0 & 1 \\
\end{array}$ & 1055 & 510 & 1010 & 105 & 0 & & & 0 & 06 & $\begin{array}{ll}6 & 95 \\
\end{array}$ & 6 & \begin{tabular}{|l|l}
95 & 1.
\end{tabular} & 1.4 & \\
\hline 5 & $\begin{array}{l}4 . \\
2\end{array}$ & \begin{tabular}{|c|} 
Mid- \\
Channel \\
Pool \\
\end{tabular} & & 66 & 6 & & & & & $1.8 \mid 0$ & 0.5 & & & & & & & & & & & & & & & & & & & & & & & 1.3 & \\
\hline 6 & $\begin{array}{c}1 . \\
1\end{array}$ & \begin{tabular}{|c|} 
Low \\
Gradient \\
Riffle \\
\end{tabular} & & $\begin{array}{c}21 \\
0\end{array}$ & 10 & 2520 & $0 \mid 292$ & 27 & \begin{tabular}{|l|l|l|}
0.5 & 1
\end{tabular} & 1.5 & & & 1 & 3 & \begin{tabular}{|l|l}
0 & 0
\end{tabular} & 0 & 0 & $\left|\begin{array}{c}10 \\
0\end{array}\right|$ & \begin{tabular}{l|l}
0 & 0
\end{tabular} & 0 & \begin{tabular}{l|l}
0 & 1
\end{tabular} & 1020 & 020 & 2020 & 2010 & 0 & & & 0 & 0 & \begin{tabular}{l|l}
6 & 95
\end{tabular} & 56 & 95 & & \\
\hline 7 & $\begin{array}{l}3 . \\
2 \\
\end{array}$ & Glide & & $\begin{array}{c}12 \\
0 \\
\end{array}$ & 2 & 2627 & $7|27| 2$ & 248 & \begin{tabular}{|l|l|}
8 & 8.7 \\
\end{tabular} & 1.5 & & & 1 & 5 & \begin{tabular}{|l|l}
0 & 0
\end{tabular} & 20 & \begin{tabular}{ll|l}
0 & 0
\end{tabular} & 80 & \begin{tabular}{l|l}
0 & 0
\end{tabular} & 0 & 03 & 3035 & 520 & 50 & \begin{tabular}{l|l|}
5 & 5 \\
\end{tabular} & 0 & & & 0 & 0 & \begin{tabular}{l|l}
6 & 95
\end{tabular} & 6 & 95 & & \\
\hline 8 & $\begin{array}{l}1 . \\
1\end{array}$ & \begin{tabular}{|c|} 
Low \\
gradient \\
Riffle \\
\end{tabular} & & 56 & 623 & 2324 & $4 \mid 18$ & 27 & $\begin{array}{c}0.5 \\
6\end{array}$ & 1.4 & & & 1 & 1 & & & & $\mid \begin{array}{c}10 \\
0\end{array}$ & & & & \begin{tabular}{|l|l}
0 & 5
\end{tabular} & 55 & 2010 & 1060 & 0.0 & $90 \mid \begin{array}{cc}10 \\
0\end{array}$ & \begin{tabular}{c|c}
10 & 0 \\
0 & 0
\end{tabular} & \begin{tabular}{l|l|}
0 & 0
\end{tabular} & 0 & \begin{tabular}{l|l|l}
7 & 20
\end{tabular} & $\begin{array}{lll}0 & 6\end{array}$ & 85 & & \\
\hline 9 & $\begin{array}{c}5 . \\
1 \\
\end{array}$ & $\begin{array}{c}\text { Corner } \\
\text { Pool }\end{array}$ & & 80 & 03. & 3127 & $7 \mid 21$ & 45 & 1.43 & \begin{tabular}{|l|l|}
3.4 & 1 \\
\end{tabular} & 1.220 & Sand & 2 & 10 & \begin{tabular}{l|l}
0 & 75
\end{tabular} & 50 & 25 & 0 & \begin{tabular}{l|l}
0 & 0
\end{tabular} & 0 & 02 & 2055 & 55 & $\begin{array}{ll}5 & 5\end{array}$ & \begin{tabular}{l|l|}
5 & 10 \\
\end{tabular} & 00 & \begin{tabular}{|l|l|}
88 & 10 \\
& 0 \\
\end{tabular} & \begin{tabular}{c|c}
10 & 0 \\
0 & 0 \\
\end{tabular} & \begin{tabular}{ll|}
0 & 0 \\
\end{tabular} & 0 & \begin{tabular}{l|l|}
7 & 10
\end{tabular} & 7 & \begin{tabular}{l|l}
85 & 2
\end{tabular} & 2.2 & \\
\hline 10 & $\begin{array}{l}3 . \\
3 \\
\end{array}$ & Run & & $\begin{array}{c}22 \\
9 \\
\end{array}$ & \begin{tabular}{l|l}
2 & 30 \\
\end{tabular} & \begin{tabular}{l|l}
30 & 45 \\
\end{tabular} & 526 & 20 & \begin{tabular}{|c|c}
0.5 & 1 \\
5 & \\
\end{tabular} & 1.8 & & & & 5 & \begin{tabular}{l|l}
0 & 5
\end{tabular} & 590 & \begin{tabular}{ll|l}
0 & 5
\end{tabular} & 0 & \begin{tabular}{l|l}
0 & 0
\end{tabular} & 0 & 0 & \begin{tabular}{l|l}
0 & 25 \\
\end{tabular} & 510 & 1525 & 2525 & 50 & \begin{tabular}{|c|c|}
96 & 10 \\
& 0 \\
\end{tabular} & \begin{tabular}{c|c}
10 & 0 \\
0 & 0 \\
\end{tabular} & \begin{tabular}{l|l|}
0 & 0 \\
\end{tabular} & 0 & \begin{tabular}{l|l}
7 & 80
\end{tabular} & 7 & 95 & & \\
\hline 11 & $\begin{array}{c}1 . \\
1\end{array}$ & \begin{tabular}{|c|} 
Low \\
gradient \\
Riffle \\
\end{tabular} & & 31 & 118 & 1820 & 019 & 15 & $\begin{array}{c}0.3 \\
6\end{array} \mid$ & 0.8 & & & 1 & 5 & \begin{tabular}{l|l}
0 & 0
\end{tabular} & $\mid \begin{array}{c}10 \\
0\end{array}$ & 0 & 0 & \begin{tabular}{l|l}
0 & 0
\end{tabular} & 0 & 0 & \begin{tabular}{l|l}
0 & 0
\end{tabular} & $\begin{array}{lll}0 & 0 & 2\end{array}$ & 2050 & 5030 & 00 & $95 \mid \begin{array}{cc}10 \\
0\end{array}$ & \begin{tabular}{c|c}
10 & 0 \\
0 & 0
\end{tabular} & \begin{tabular}{l|l|}
0 & 0
\end{tabular} & 0 & \begin{tabular}{l|l|}
6 & 90
\end{tabular} & 6 & $\begin{array}{c}10 \\
0\end{array}$ & & \\
\hline 12 & 2. & $\mid \begin{array}{c}\text { Lateral } \\
\text { Scour } \\
\text { Pool - Log } \\
\text { Enhanced }\end{array}$ & & 20 & 014 & 1415 & $5|15|$ & 13 & 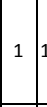 & $|1.7| 0$ & \begin{tabular}{l|l}
0.3 & 50
\end{tabular} & $\begin{array}{c}\text { Sm } \\
\text { Cobble }\end{array}$ & & 25 & \begin{tabular}{|l|l|l|}
0 & 15
\end{tabular} & $15 \mid 85$ & $\begin{array}{ll}5 & 0\end{array}$ & 0 & \begin{tabular}{l|l}
0 & 0
\end{tabular} & 0 & 03 & 3555 & 55 & \begin{tabular}{|l|l}
5 & 0
\end{tabular} & \begin{tabular}{l|l}
0 & 0
\end{tabular} & 0 & $95 \mid$\begin{tabular}{c|c}
1 \\
0
\end{tabular} & \begin{tabular}{c|c}
10 & 0 \\
0 & 0
\end{tabular} & \begin{tabular}{l|l|}
0 & 0
\end{tabular} & 0 & \begin{tabular}{l|l}
6 & 95
\end{tabular} & 7 & \begin{tabular}{|c|c}
10 & 1 \\
0 & 1
\end{tabular} & 1.4 & \\
\hline 13 & $\begin{array}{c}5 . \\
1\end{array}$ & $\begin{array}{l}\text { Corner } \\
\text { Pool }\end{array}$ & & $\begin{array}{l}10 \\
0\end{array}$ & 03 & \begin{tabular}{l|l}
31 & 13
\end{tabular} & 338 & 43 & \begin{tabular}{|l|l} 
& 1 \\
\end{tabular} & $2.4 \mid 0$ & 0.2 & & & 5 & \begin{tabular}{|l|l}
0 & 0
\end{tabular} & \begin{tabular}{l|l}
0 & 0
\end{tabular} & 5 & 95 & \begin{tabular}{l|l}
0 & 0
\end{tabular} & 0 & 02 & 2050 & 5020 & \begin{tabular}{l|l}
10 & 0
\end{tabular} & \begin{tabular}{l|l|}
0 & 0
\end{tabular} & 0 & $95 \mid$\begin{tabular}{c|c}
1 \\
0
\end{tabular} & \begin{tabular}{c|c}
10 & 0 \\
0 & 0
\end{tabular} & \begin{tabular}{l|l|}
0 & 0
\end{tabular} & 0 & \begin{tabular}{l|l}
6 & 95
\end{tabular} & 56 & \begin{tabular}{|c|c|}
10 & 2 \\
0 & 2 \\
\end{tabular} & $2.2 \mathrm{n}$ & $\begin{array}{l}\text { Overlaps with } \\
\text { next unit approx. } \\
15 \mathrm{ft} \text {. On RB }\end{array}$ \\
\hline 14 & $\begin{array}{l}5 . \\
3\end{array}$ & \begin{tabular}{|c|} 
Lateral \\
Scour \\
Pool - \\
Root Wad \\
Enhanced
\end{tabular} & & 42 & 12 & & & & & $\begin{array}{c}2.8 \\
5\end{array}$ & 0.8 & & & & & & & & & & & & & & & & & & & & & & & \begin{tabular}{c|c}
2.0 \\
5
\end{tabular} & $\begin{array}{l}\text { Overlaps with } \\
\text { previous unit } \\
\text { approx. } 15 \mathrm{ft} \text {. On } \\
\text { LB }\end{array}$ \\
\hline 15 & $\begin{array}{l}4 . \\
2\end{array} \mid$ & \begin{tabular}{|c|} 
Mid- \\
Channel \\
Pool \\
\end{tabular} & & 68 & 88 & 3943 & 3413 & 33 & $1.6{ }^{3}$ & \begin{tabular}{c|c}
3.2 & 0 \\
5 &
\end{tabular} & \begin{tabular}{|c|c}
0.8 & 75 \\
5 & 75
\end{tabular} & Gravel & & 5 & 3020 & 200 & 0 & 50 & \begin{tabular}{l|l}
0 & 0
\end{tabular} & 0 & 05 & \begin{tabular}{|l|l}
5 & 45
\end{tabular} & 4535 & 55 & \begin{tabular}{l|l|}
5 & 5
\end{tabular} & 0 & 84 \begin{tabular}{l|l}
1 \\
0
\end{tabular} & \begin{tabular}{c|c}
10 & 0 \\
0 & 0
\end{tabular} & \begin{tabular}{l|l|}
0 & 0
\end{tabular} & 0 & \begin{tabular}{l|c|}
7 & 10 \\
7
\end{tabular} & 6 & \begin{tabular}{c|c}
10 & 2 \\
0 & 2
\end{tabular} & 2.4 & \\
\hline 16 & $\begin{array}{l}5 . \\
3\end{array}$ & $\begin{array}{c}\text { Lateral } \\
\text { Scour } \\
\text { Pool - } \\
\text { Root Wad } \\
\text { Enhanced }\end{array}$ & & 27 & 272 & 2833 & 325 & $25:$ & 1.82 & \begin{tabular}{|l|l|}
2.7 & 1 \\
\end{tabular} & 1.495 & Gravel & & 30 & \begin{tabular}{|l|l}
0 & 10
\end{tabular} & \begin{tabular}{l|l}
10 & 10
\end{tabular} & 015 & 65 & \begin{tabular}{l|l}
0 & 0
\end{tabular} & 0 & 05 & \begin{tabular}{l|l}
5 & 20
\end{tabular} & 2020 & $202 C$ & 2015 & 50 & \begin{tabular}{|l|l}
23 & 1 \\
& 0
\end{tabular} & \begin{tabular}{c|c}
10 & 0 \\
0 & 0
\end{tabular} & \begin{tabular}{l|l|}
0 & 0
\end{tabular} & 0 & \begin{tabular}{l|l}
6 & 90
\end{tabular} & 0 & \begin{tabular}{|l|l}
50 & 1
\end{tabular} & 1.3 & RB pipes \\
\hline 17 & $\begin{array}{c}4 . \\
2\end{array}$ & \begin{tabular}{|c|} 
Mid- \\
Channel \\
Pool
\end{tabular} & & $\begin{array}{c}15 \\
8\end{array}$ & $\begin{array}{l}5 \\
8\end{array}$ & & & & & $2.4 \mid 0$ & 0.6 & & & & & & & & & & & & & & & & & & & & & & & 1.8 & \\
\hline 18 & $\begin{array}{l}3 . \\
3 \\
\end{array}$ & Run & & 82 & 32 & 2225 & 518 & 23 & \begin{tabular}{c|c}
1.2 & \\
5 & 2 \\
\end{tabular} & 2.3 & & & & 5 & 050 & 5010 & 025 & 15 & \begin{tabular}{l|l}
0 & 0
\end{tabular} & 0 & 01 & 1060 & 5010 & 105 & \begin{tabular}{l|l|}
5 & 5
\end{tabular} & 0 & \begin{tabular}{|c|c|}
90 & $\begin{array}{c}1 \\
0\end{array}$ \\
\end{tabular} & \begin{tabular}{c|c}
10 & 0 \\
\end{tabular} & \begin{tabular}{l|l|}
0 & 20 \\
\end{tabular} & 0 & 790 & 7 & 90 & & $\begin{array}{l}\text { RB train turn- } \\
\text { around }\end{array}$ \\
\hline 19 & $\begin{array}{l}1 . \\
1\end{array}$ & \begin{tabular}{|c|} 
Low \\
gradient \\
Riffle \\
\end{tabular} & & 40 & 10 & & & & & & & & & & & & & & & & & & & & & & & & & & & & & & \\
\hline 20 & \begin{tabular}{|c|}
3. \\
2 \\
\end{tabular} & Glide 6 & 637 & 7090 & 02 & 2723 & 329 & 28 & \begin{tabular}{c|c}
0.9 & 2 \\
2 & 2 \\
\end{tabular} & 2.1 & & & 3 & 25 & 2050 & 5015 & 515 & 0 & \begin{tabular}{l|l}
0 & 0
\end{tabular} & 0 & 07 & 7520 & 205 & 00 & \begin{tabular}{l|l|}
0 & 0
\end{tabular} & 0 & \begin{tabular}{l|l}
94 & 11 \\
\end{tabular} & \begin{tabular}{c|c}
10 & 0 \\
0 & 0 \\
\end{tabular} & \begin{tabular}{l|l|}
0 & 0
\end{tabular} & 0 & \begin{tabular}{l|l}
7 & 95
\end{tabular} & 55 & 90 & & \\
\hline 21 & $\begin{array}{l}4 . \\
2\end{array} \mid$ & $\begin{array}{c}\text { Mid- } \\
\text { Channel } \\
\text { Pool }\end{array}$ & 637 & 7080 & 302 & 2628 & 829 & $222^{2}$ & \begin{tabular}{c|c}
2.5 & 3 \\
3 &
\end{tabular} & \begin{tabular}{c|c}
3.5 & 1 \\
6 &
\end{tabular} & $\begin{array}{c}1.4 \\
4\end{array}$ & B & 3 & 80 & $0 \mid 4 c$ & 1020 & 0 & 103 & 300 & 0 & 04 & 4545 & 4510 & 00 & \begin{tabular}{|l|l|}
0 & 0
\end{tabular} & 0 & 74 \begin{tabular}{l|l}
1 \\
0
\end{tabular} & \begin{tabular}{c|c}
10 & 0 \\
0 & 0
\end{tabular} & \begin{tabular}{l|l|}
0 & 0
\end{tabular} & 0 & \begin{tabular}{l|l}
7 & 85
\end{tabular} & 5 & $94^{2}$ & $\begin{array}{c}2.1 \\
2\end{array}$ & $\begin{array}{l}\text { Log jam across } \\
\text { entire chanel. } \\
\text { Few large logs } \\
\text { but tons of SWD }\end{array}$ \\
\hline 22 & $\begin{array}{l}3 . \\
4 \\
\end{array}$ & Step Run 6 & 657 & $70 \begin{array}{c}19 \\
0\end{array}$ & \begin{tabular}{l|l}
9 & 2 \\
\end{tabular} & 2022 & 220 & $17{ }^{C}$ & \begin{tabular}{c|c}
0.7 & 1 \\
9 & \\
\end{tabular} & $\begin{array}{c}1.7 \\
5 \\
\end{array}$ & & & 3 & 10 & 050 & 5030 & 010 & 10 & \begin{tabular}{l|l}
0 & 0
\end{tabular} & 0 & 00 & 030 & 3015 & $252 C$ & 2010 & 00 & \begin{tabular}{|c|c|}
98 & 1 \\
0
\end{tabular} & \begin{tabular}{c|c}
10 & 0 \\
0 & \\
\end{tabular} & \begin{tabular}{l|l|l}
0 & 0 \\
\end{tabular} & 0 & \begin{tabular}{l|l}
7 & 95
\end{tabular} & 57 & 95 & & \\
\hline 23 & $\begin{array}{l}3 . \\
2\end{array}$ & Glide 6 & 647 & 7076 & 63 & 3117 & 737 & 38 & 0.72 & 2.1 & & & 1 & 3 & \begin{tabular}{l|l}
0 & 0
\end{tabular} & $0 \quad 50$ & 045 & 5 & \begin{tabular}{l|l}
0 & 0
\end{tabular} & 0 & 02 & 2525 & 2525 & $151 c$ & 100 & 0 & 87 \begin{tabular}{l|l}
1 \\
0
\end{tabular} & \begin{tabular}{c|c}
10 & 0 \\
0 & 0 \\
\end{tabular} & \begin{tabular}{l|l|}
0 & 0
\end{tabular} & 0 & \begin{tabular}{l|l}
7 & 95
\end{tabular} & 57 & 95 & & $\begin{array}{l}\text { Fish trap upper } \\
\text { bound of unit }\end{array}$ \\
\hline 24 & $\begin{array}{c}3 . \\
4 \\
\end{array}$ & Step Run 6 & 657 & \begin{tabular}{l|c}
10 \\
0
\end{tabular} & \begin{tabular}{l|l}
0 & 2 \\
0 & 2 \\
\end{tabular} & 2938 & $818=$ & 31 & \begin{tabular}{c|c}
0.5 & 1 \\
5 & \\
\end{tabular} & \begin{tabular}{|c|}
1.5 \\
5 \\
\end{tabular} & & & 1 & 2 & 050 & 50 & 50 & 0 & \begin{tabular}{l|l}
0 & 0
\end{tabular} & 0 & 05 & 525 & 2515 & 2030 & \begin{tabular}{|l|l|}
30 & 5 \\
\end{tabular} & 0 & \begin{tabular}{|c|c|}
98 & 11 \\
\end{tabular} & \begin{tabular}{c|c}
10 & 0 \\
0 & 0 \\
\end{tabular} & \begin{tabular}{l|l|}
0 & 0 \\
\end{tabular} & 0 & \begin{tabular}{l|l}
6 & 90
\end{tabular} & 7 & 90 & & \\
\hline 25 & \begin{tabular}{|l|}
3. \\
2 \\
\end{tabular} & \begin{tabular}{l|l} 
Glide & 6 \\
\end{tabular} & 657 & \begin{tabular}{l|r}
77 & 27 \\
9
\end{tabular} & \begin{tabular}{|l|l|}
9 & 2 \\
\end{tabular} & 2731 & 135 & 15 & \begin{tabular}{|l|l|} 
& 1 \\
\end{tabular} & \begin{tabular}{|c|}
1.4 \\
8 \\
\end{tabular} & & & 2 & 5 & \begin{tabular}{l|l}
0 & 0
\end{tabular} & $0 \quad 50$ & 00 & 0 & \begin{tabular}{l|l}
0 & 0
\end{tabular} & 0 & 02 & 2525 & 2525 & 1510 & \begin{tabular}{|l|l|}
10 & 0 \\
\end{tabular} & 0 & \begin{tabular}{|l|l}
94 & 1 \\
\end{tabular} & \begin{tabular}{c|c}
10 & 0 \\
0 & 0 \\
\end{tabular} & \begin{tabular}{l|l|l}
0 & 0
\end{tabular} & 0 & \begin{tabular}{l|l}
7 & 95
\end{tabular} & 7 & 95 & & \\
\hline 26 & $\begin{array}{l}3 . \\
4\end{array}$ & Step Run & 65 & 7690 & 0 & \begin{tabular}{l|l}
22 & 15 \\
\end{tabular} & 529 & 21 & $\begin{array}{c}0.6 \\
2\end{array}$ & $\begin{array}{c}1.5 \\
1\end{array}$ & & & 1 & 5 & \begin{tabular}{l|l}
0 & 80 \\
\end{tabular} & \begin{tabular}{l|l}
0 & 10
\end{tabular} & 0 & 10 & \begin{tabular}{l|l}
0 & 0
\end{tabular} & 0 & 00 & \begin{tabular}{l|l}
0 & 0
\end{tabular} & 05 & 1075 & 7510 & 00 & 98 & \begin{tabular}{c|c}
10 & 0 \\
\end{tabular} & \begin{tabular}{l|l|}
0 & 0
\end{tabular} & 0 & \begin{tabular}{l|l}
7 & 95
\end{tabular} & 7 & 95 & & $\begin{array}{c}\text { Deversion(?) pipe } \\
\text { and structure at } \\
\text { top of unit. S }\end{array}$ \\
\hline
\end{tabular}




\begin{tabular}{|c|c|c|c|c|c|c|c|c|c|c|c|c|c|c|c|c|c|c|c|c|c|c|c|c|c|c|c|c|c|c|c|c|c|c|}
\hline 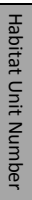 & 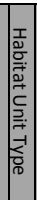 & 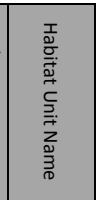 & 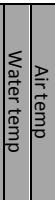 & 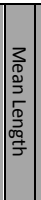 & 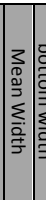 & 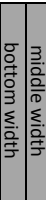 & 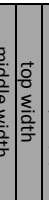 & 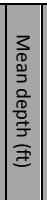 & 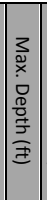 & 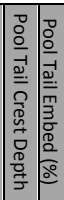 & 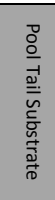 & 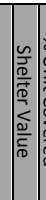 & 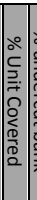 & 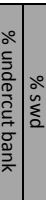 & $\frac{\circ}{\Sigma}$ & 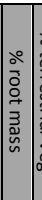 & 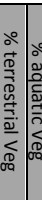 & 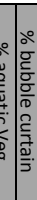 & 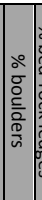 & & & 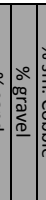 & 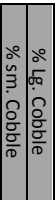 & 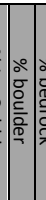 & 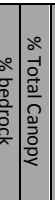 & 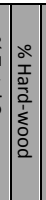 & 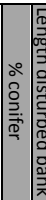 & 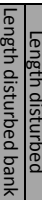 & 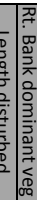 & 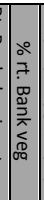 & & & 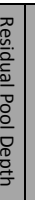 & 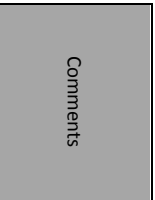 \\
\hline 27 & $\begin{array}{l}5 . \\
2\end{array}$ & $\begin{array}{c}\text { Lateral } \\
\text { Scour } \\
\text { Pool - Log } \\
\text { Enhanced }\end{array}$ & 6679 & 60 & 202 & 2020 & 021 & $\begin{array}{c}1.2 \\
4\end{array}$ & $\begin{array}{c}2.8 \\
9\end{array}$ & 1.260 & B & 3 & 10 & \begin{tabular}{l|l}
0 & 40
\end{tabular} & 40 & 10 & 100 & 0 & 0 & 00 & 0 & 51 & 1075 & 510 & 0.99 & $\mid \begin{array}{c}10 \\
0\end{array}$ & 00 & 00 & $\begin{array}{lll}0 & 6\end{array}$ & 95 & 69 & 951 & 1.6 & \\
\hline 28 & $\begin{array}{l}3 . \\
2\end{array}$ & Glide & 6681 & 90 & 182 & 2112 & 221 & $\begin{array}{c}0.6 \\
7 \\
\end{array}$ & $\begin{array}{c}0.9 \\
2\end{array}$ & & & 1 & 3 & \begin{tabular}{l|l}
0 & 0
\end{tabular} & 0 & 75 & 250 & 0 & 0 & $0: 5$ & 530 & 0202 & 2015 & 510 & \begin{tabular}{|c|c|}
0 & 10 \\
0
\end{tabular} & \begin{tabular}{|c|}
10 \\
0 \\
\end{tabular} & $0 \mid 0$ & 0 & 107 & 90 & 79 & 90 & & $\begin{array}{c}.+1, \text { Ford } \\
\text { crossing. A }\end{array}$ \\
\hline 29 & $\begin{array}{l}5 . \\
3 \\
.\end{array}$ & \begin{tabular}{|c|} 
Lateral \\
Scour \\
Pool - \\
Root Wad \\
Enhanced
\end{tabular} & 6680 & 69 & 262 & $21 \mid 29$ & 927 & $\left|\begin{array}{c}1.1 \\
7\end{array}\right|$ & $\begin{array}{c}3.0 \\
2\end{array}$ & $\begin{array}{c}0.5 \\
8\end{array}$ & c & 31 & 15 & 2025 & 20 & 201 & 150 & 0 & 0 & 02 & 2020 & 040 & \begin{tabular}{|l|l|}
15 & 5 \\
\end{tabular} & 0 & 0.89 & $\mid \begin{array}{c}10 \\
0\end{array}$ & 00 & \begin{tabular}{l|l}
0 & 0
\end{tabular} & 0 & 95 & 79 & $902^{2}$ & $\begin{array}{l}2.4 \\
4\end{array}$ & \\
\hline 30 & $\begin{array}{l}5 . \\
2\end{array}$ & $\begin{array}{c}\text { Lateral } \\
\text { Scour } \\
\text { Pool - Log } \\
\text { Enhanced }\end{array}$ & 5960 & 42 & 252 & $27 \mid 26$ & 623 & 1.4 & $\begin{array}{c}1.6 \\
6\end{array}$ & \begin{tabular}{c|c}
0.5 & 40 \\
3 & 0
\end{tabular} & C & 3 & 202 & 2010 & 40 & $\left.10\right|^{2}$ & $20 \mid 0$ & 0 & 0 & 02 & & 015 & \begin{tabular}{|l|l|}
5 & 10
\end{tabular} & 0 & \begin{tabular}{l|l|l|}
0 & 94
\end{tabular} & 4 & $0 \mid 0$ & \begin{tabular}{|l|l}
0 & 0
\end{tabular} & 0 & 90 & $7 \mid 7$ & $75^{1}$ & $\begin{array}{l}1.1 \\
3\end{array}$ & Start of day, $7 / 15$ \\
\hline 31 & $\begin{array}{l}3 . \\
4 \\
\end{array}$ & Step Run & \begin{tabular}{l|l}
60 & 60 \\
\end{tabular} & $\begin{array}{c}19 \\
1\end{array}$ & 152 & \begin{tabular}{|l|l|}
23 & 11 \\
\end{tabular} & 111 & $\begin{array}{c}0.8 \\
1\end{array}$ & $\begin{array}{c}1.6 \\
2\end{array}$ & & & & & 1510 & 10 & 60 & 50 & 0 & 0 & 00 & 010 & 0203 & 3030 & 10 & \begin{tabular}{|l|l|}
0 & 99 \\
\end{tabular} & $\begin{array}{c}10 \\
0\end{array}$ & 00 & 0 & 0 & 60 & 69 & 90 & & \\
\hline 32 & $\begin{array}{c}4 . \\
2\end{array}$ & \begin{tabular}{|c} 
Mid- \\
Channel \\
Pool
\end{tabular} & 6066 & 44 & 181 & $11 \mid 24$ & 419 & $\begin{array}{c}1.9 \\
4\end{array} \mid$ & $\begin{array}{c}3.6 \\
9\end{array}$ & \begin{tabular}{c|c}
0.4 & 40 \\
7 & 40
\end{tabular} & D & 36 & 60 & $\begin{array}{lll}0 & 30\end{array}$ & 30 & 0 & 1030 & 0 & 0 & 02 & 2530 & 0201 & $\begin{array}{lll}10 & 10 \\
\end{array}$ & 5 & $\begin{array}{lll}0 & 62\end{array}$ & $\begin{array}{c}10 \\
0\end{array}$ & 00 & 0 & 06 & $\left|\begin{array}{c}10 \\
0\end{array}\right|$ & 79 & 953 & $\begin{array}{l}3.2 \\
2\end{array}$ & $\begin{array}{c}\text { Log Jam-formed } \\
\text { pool }\end{array}$ \\
\hline 33 & $\begin{array}{c}4 . \\
2\end{array}$ & \begin{tabular}{|c|} 
Mid- \\
Channel \\
Pool
\end{tabular} & 6162 & 97 & 241 & 1927 & 725 & $\left|\begin{array}{c}1.3 \\
8\end{array}\right|$ & $\begin{array}{c}2.5 \\
4\end{array}$ & 50 & C & 34 & 40 & 030 & 15 & 5 & 500 & \begin{tabular}{l|l}
0 & 0
\end{tabular} & 0 & 03 & & 0301 & \begin{tabular}{|l|l|}
10 & 10 \\
\end{tabular} & 0 & \begin{tabular}{l|l|}
0 & 75 \\
\end{tabular} & $\mid \begin{array}{c}10 \\
0\end{array}$ & 0 & \begin{tabular}{l|l}
0 & 0
\end{tabular} & $\begin{array}{ll}0 & 7\end{array}$ & $\mid \begin{array}{c}10 \\
0\end{array}$ & $7{ }^{1}$ & $\begin{array}{c}10 \\
0\end{array}$ & & \\
\hline 34 & $\begin{array}{l}3 . \\
4 .\end{array}$ & Step Run & 6163 & $\begin{array}{c}31 \\
5\end{array}$ & 282 & \begin{tabular}{|l|l|l|}
25 & 12 \\
\end{tabular} & 248 & $\begin{array}{c}0.5 \\
8\end{array}$ & 1.3 & & & 32 & 25 & 1530 & 35 & 20 & \begin{tabular}{l|l}
0 & 0
\end{tabular} & 0 & 0 & 0.0 & 05 & 102 & 2060 & 5 & 0.89 & $\begin{array}{c}10 \\
0\end{array}$ & 00 & $\begin{array}{ll}0 & 0\end{array}$ & 06 & $\begin{array}{c}10 \\
0\end{array}$ & 71 & $\begin{array}{c}10 \\
0\end{array}$ & & \\
\hline 35 & $\begin{array}{l}3 . \\
2 \\
\end{array}$ & Glide & \begin{tabular}{|l|l|}
61 & 62 \\
\end{tabular} & $\begin{array}{c}40 \\
0\end{array}$ & 34 & \begin{tabular}{|l|l}
48 & 26 \\
\end{tabular} & 2628 & $\begin{array}{c}0.8 \\
6\end{array}$ & $\begin{array}{c}1.7 \\
5\end{array}$ & & & 3 & 53 & 3020 & 30 & 10 & 100 & \begin{tabular}{l|l}
0 & 0
\end{tabular} & 0 & 02 & & $\begin{array}{llll}0 & 30 & 1\end{array}$ & \begin{tabular}{|l|l|}
10 & 10 \\
\end{tabular} & 0 & \begin{tabular}{l|l|}
0 & 97 \\
\end{tabular} & $\begin{array}{c}10 \\
0\end{array}$ & \begin{tabular}{l|l}
0 & 1
\end{tabular} & $15 \mid 0$ & $\begin{array}{ll}0 & 7\end{array}$ & $\begin{array}{c}10 \\
0\end{array}$ & $7{ }^{1}$ & $\begin{array}{c}10 \\
0\end{array}$ & &.+132, staff gage \\
\hline 36 & $\begin{array}{l}5 . \\
3 \\
.\end{array}$ & $\mid \begin{array}{c}\text { Lateral } \\
\text { Scour } \\
\text { Pool - } \\
\text { Root Wad } \\
\text { Enhanced }\end{array}$ & & 68 & 252 & 2828 & 818 & 3.8 & 4.5 & \begin{tabular}{|c|c|}
1.3 & 60 \\
2 & 60
\end{tabular} & C & 3 & 10 & 1020 & 20 & 45 & 50 & \begin{tabular}{l|l}
0 & 0
\end{tabular} & 0 & 03 & & 0301 & \begin{tabular}{|l|l|}
110 & 0
\end{tabular} & 0 & \begin{tabular}{l|l|l|}
0 & 82
\end{tabular} & $\mid \begin{array}{c}10 \\
0\end{array}$ & 0 & $\begin{array}{ll}0 & 0\end{array}$ & \begin{tabular}{l|l}
0 & 6
\end{tabular} & 80 & 7 & \begin{tabular}{c|c}
10 & 3 \\
0 &
\end{tabular} & $\begin{array}{l}3.1 \\
8\end{array}$ & \\
\hline 37 & $\begin{array}{c}1 . \\
1\end{array}$ & $\begin{array}{c}\text { Low } \\
\text { gradient } \\
\text { Riffle }\end{array}$ & 6165 & $\begin{array}{c}10 \\
5\end{array}$ & 221 & 1810 & 1039 & $\mid \begin{array}{c}0.8 \\
6\end{array}$ & 1.5 & & & 2 & 104 & \begin{tabular}{l|l}
40 & 10
\end{tabular} & 0 & 40 & 0 & \begin{tabular}{l|l}
0 & 0
\end{tabular} & 0 & 00 & 0 & & 2050 & 20 & \begin{tabular}{l|l|l}
0 & 99
\end{tabular} & $\mid \begin{array}{c}10 \\
0\end{array}$ & 0 & \begin{tabular}{l|l}
0 & 0
\end{tabular} & \begin{tabular}{l|l}
0 & 7
\end{tabular} & 80 & $6{ }^{1}$ & $\begin{array}{c}10 \\
0\end{array}$ & & \\
\hline 38 & $\begin{array}{l}5 . \\
2\end{array}$ & $\begin{array}{c}\text { Lateral } \\
\text { Scour } \\
\text { Pool - Log } \\
\text { Enhanced }\end{array}$ & 6165 & 53 & 36 & 3936 & 3633 & $\left|\begin{array}{c}1.8 \\
1\end{array}\right|$ & $\begin{array}{c}3.9 \\
5\end{array}$ & $\begin{array}{c}0.3 \\
9\end{array}$ & B & 3 & 30 & \begin{tabular}{l|l}
0 & 20
\end{tabular} & 80 & 0 & $0 \mid 0$ & 0 & 0 & 03 & & $\begin{array}{llll}0 & 20 & 1\end{array}$ & $\begin{array}{lll}110 & 0\end{array}$ & 0 & 0.91 & 10 & $0 \mid 0$ & \begin{tabular}{|l|l}
0 & 0
\end{tabular} & \begin{tabular}{l|l}
0 & 7
\end{tabular} & 70 & 69 & $953^{3}$ & $\begin{array}{l}3.5 \\
6\end{array}$ & \\
\hline 39 & $\begin{array}{l}5 . \\
2\end{array}$ & $\begin{array}{c}\text { Lateral } \\
\text { Scour } \\
\text { Pool - Log } \\
\text { Enhanced }\end{array}$ & \begin{tabular}{|l|l}
6165 & 65 \\
\end{tabular} & 72 & 253 & 3323 & 2320 & 1.9 & 4.8 & 1.7 & C & 32 & 25 & \begin{tabular}{l|l}
0 & 50
\end{tabular} & 50 & 0 & $0 \mid 0$ & \begin{tabular}{l|l}
0 & 0
\end{tabular} & 0 & \begin{tabular}{l|l}
0 & 1
\end{tabular} & & 020 & $\begin{array}{ll}0 & 0\end{array}$ & 5 & \begin{tabular}{l|l|l|}
0 & 99
\end{tabular} & $\mid \begin{array}{c}10 \\
0\end{array}$ & 00 & $\begin{array}{ll}0 & 0\end{array}$ & \begin{tabular}{l|l}
0 & 7
\end{tabular} & 90 & 79 & \begin{tabular}{l|l}
90 & 3
\end{tabular} & 3.1 & \\
\hline 40 & $\begin{array}{c}1 . \\
1\end{array}$ & \begin{tabular}{|c|} 
Low \\
gradient \\
Riffle
\end{tabular} & 6270 & 38 & 272 & 2028 & 2834 & $\begin{array}{c}0.7 \\
5\end{array}$ & 1.2 & & & 1 & 5 & \begin{tabular}{l|l}
0 & 0
\end{tabular} & 0 & 0 & \begin{tabular}{c|c}
10 & 0 \\
0 & 0
\end{tabular} & $\begin{array}{lll}0 & 0\end{array}$ & 0 & 00 & \begin{tabular}{l|l}
0 & 5
\end{tabular} & 02 & 2075 & 55 & \begin{tabular}{l|l|}
0 & 78 \\
\end{tabular} & 3 & 00 & \begin{tabular}{l|l}
0 & 0
\end{tabular} & \begin{tabular}{l|l}
0 & 6
\end{tabular} & 95 & $6 \mid 8$ & 80 & & \\
\hline 41 & $\begin{array}{l}3 . \\
2\end{array}$ & Glide & 6266 & 44 & 333 & 3432 & 3234 & 1.3 & 2.4 & & & 34 & 402 & 2010 & 20 & 30 & 200 & 0 & 0 & 03 & & $\begin{array}{lll}0 & 25 \\
\end{array}$ & \begin{tabular}{ll|}
0 & 0
\end{tabular} & 0 & \begin{tabular}{|l|l|}
0 & 82 \\
\end{tabular} & $\mid \begin{array}{c}10 \\
0\end{array}$ & 0 & \begin{tabular}{l|l}
0 & 0
\end{tabular} & \begin{tabular}{l|l}
0 & 7
\end{tabular} & 90 & 79 & 95 & & \\
\hline 42 & $\begin{array}{c}4 . \\
2\end{array}$ & \begin{tabular}{|c|} 
Mid- \\
Channel \\
Pool
\end{tabular} & \begin{tabular}{|l|l|}
6366 \\
\end{tabular} & 31 & 383 & 3443 & 4336 & 2.8 & 4.4 & 1.9 & C & 3 & 30 & $10 \mid 0$ & 50 & 30 & 100 & $\begin{array}{lll}0 & 0\end{array}$ & 0 & 03 & & $\begin{array}{llll}0 & 20 & 1\end{array}$ & \begin{tabular}{|l|l|}
110 & 0
\end{tabular} & 0 & \begin{tabular}{l|l|l|}
0 & 81
\end{tabular} & $\left|\begin{array}{c}10 \\
0\end{array}\right|$ & $0 \mid 0$ & \begin{tabular}{l|l}
0 & 0
\end{tabular} & \begin{tabular}{l|l}
0 & 6
\end{tabular} & $\begin{array}{c}10 \\
0\end{array}$ & 79 & 902 & 2.5 & Start $7 / 16$ \\
\hline 43 & $\begin{array}{l}4 . \\
2\end{array}$ & \begin{tabular}{|c|} 
Mid- \\
Channel \\
Pool
\end{tabular} & 6265 & 50 & 393 & $\begin{array}{lll}36 & 50\end{array}$ & 5032 & $\begin{array}{c}3.6 \\
4\end{array}$ & 6 & $\begin{array}{c}1.3 \\
5\end{array}$ & C & 3 & 75 & \begin{tabular}{l|l}
0 & 50
\end{tabular} & 50 & 0 & \begin{tabular}{l|l}
0 & 0 \\
2
\end{tabular} & $\begin{array}{ll}0 & 0\end{array}$ & 0 & 03 & & $\begin{array}{llll}0 & 20 & 1\end{array}$ & $\begin{array}{lll}110 & 0\end{array}$ & 0 & \begin{tabular}{l|c|}
0 & 10 \\
0
\end{tabular} & $\mid \begin{array}{c}10 \\
0\end{array}$ & \begin{tabular}{l|l}
0 & 0
\end{tabular} & \begin{tabular}{l|l}
0 & 0
\end{tabular} & \begin{tabular}{l|l}
0 & 6
\end{tabular} & $\begin{array}{c}10 \\
0\end{array}$ & $77^{1}$ & $\begin{array}{c}10 \\
0\end{array}$ & $\begin{array}{l}4.6 \\
5\end{array}$ & $\begin{array}{c}\text { Channel spanning } \\
\text { log jam }\end{array}$ \\
\hline 44 & $\begin{array}{l}3 . \\
2\end{array}$ & Glide & 6164 & $\begin{array}{c}33 \\
0\end{array}$ & 23 & \begin{tabular}{|l|l|}
32 & 22 \\
\end{tabular} & 2214 & $\begin{array}{c}1.0 \\
1\end{array}$ & 2.1 & & & 3 & 15 & 1015 & 10 & $30 \mid 2$ & $20 \mid 0$ & 0 & 0 & 02 & & $0 \mid$\begin{tabular}{l|l}
0 & 1 \\
\end{tabular} & \begin{tabular}{|l|l|}
10 & 15 \\
\end{tabular} & 510 & $0 \mid$\begin{tabular}{c|c}
10 \\
0
\end{tabular} & $\begin{array}{c}10 \\
0\end{array}$ & 0 & \begin{tabular}{|l|l}
0 & 0 \\
0
\end{tabular} & \begin{tabular}{l|l}
0 & 7
\end{tabular} & 20 & 77 & 75 & & $\begin{array}{l}\text { RB train tracks, } \\
\text { crib wall }\end{array}$ \\
\hline 45 & $\begin{array}{c}3 . \\
4 \\
\end{array}$ & Step Run & 6362 & \begin{tabular}{|c|}
43 \\
7
\end{tabular} & 20 & 1417 & 1729 & $\begin{array}{c}0.9 \\
4\end{array}$ & 1.6 & & & 32 & 20 & 1010 & 35 & 201 & 100 & 0 & 5 & \begin{tabular}{l|l}
0 & 1
\end{tabular} & & 0202 & 2020 & 10 & \begin{tabular}{l|l|}
0 & 91 \\
\end{tabular} & $\begin{array}{c}10 \\
0\end{array}$ & 00 & 00 & $\begin{array}{ll}0 & 7\end{array}$ & $\begin{array}{c}10 \\
0\end{array}$ & 71 & $\begin{array}{c}10 \\
0\end{array}$ & & $\begin{array}{l}\text { RB single tall } \\
\text { redwood }\end{array}$ \\
\hline 46 & $\begin{array}{c}4 . \\
2\end{array}$ & \begin{tabular}{|c|} 
Mid- \\
Channel \\
Pool \\
\end{tabular} & 6361 & 58 & 292 & 2930 & 3028 & 2.1 & 3 & 0.650 & D & 2 & 5 & \begin{tabular}{l|l}
0 & 10
\end{tabular} & 90 & 0 & $0 \mid 0$ & 0 & 0 & $\begin{array}{lll}0 & 5 \\
r\end{array}$ & & 0202 & $25 \mid 25$ & 5 & $0\left|\begin{array}{c}10 \\
0\end{array}\right|$ & 10 & 00 & 0.0 & 07 & $\left|\begin{array}{c}10 \\
0\end{array}\right|$ & 79 & 902 & 2.4 & \\
\hline 47 & $\begin{array}{c}1 . \\
1\end{array}$ & \begin{tabular}{|c|} 
Low \\
gradient \\
Riffle
\end{tabular} & 6366 & 59 & 32 & 2834 & 3433 & $\mid \begin{array}{c}0.9 \\
3\end{array}$ & 1.9 & & & 2 & $0 \mid$ & \begin{tabular}{l|l}
0 & 40
\end{tabular} & 50 & 5 & $0 \mid 0$ & 0 & 5 & 0 & 0 & 202 & 2040 & 20 & \begin{tabular}{l|l|l}
0 & 95
\end{tabular} & $\mid \begin{array}{c}10 \\
0\end{array}$ & 00 & 00 & $\begin{array}{ll}0 & 7\end{array}$ & 90 & 79 & 95 & & \\
\hline 48 & $\begin{array}{l}3 . \\
2\end{array}$ & Glide & \begin{tabular}{|l|l|}
63 & 64
\end{tabular} & $\begin{array}{c}20 \\
0\end{array}$ & 30 & \begin{tabular}{|l|l|}
33 & 28 \\
\end{tabular} & 2829 & $\begin{array}{c}0.7 \\
5\end{array}$ & 1.8 & & & 2 & 5 & \begin{tabular}{l|l}
0 & 20
\end{tabular} & 50 & 20 & $0 \mid 0$ & 0 & 10 & \begin{tabular}{l|l}
0 & 1
\end{tabular} & & 0352 & 2020 & 5 & \begin{tabular}{l|c|}
0 & 10 \\
0
\end{tabular} & $\begin{array}{c}10 \\
0\end{array}$ & $0 \mid 0$ & 00 & 07 & $\begin{array}{c}10 \\
0\end{array}$ & 71 & $\begin{array}{c}10 \\
0\end{array}$ & & \\
\hline 49 & $\begin{array}{l}5 . \\
3 \\
\end{array}$ & \begin{tabular}{|c|} 
Lateral \\
Scour \\
Pool - \\
Root Wad \\
Enhanced
\end{tabular} & 6366 & 33 & 272 & 2929 & 2922 & 1.2 & 2.9 & \begin{tabular}{|c|c}
0.4 & 60 \\
5 &
\end{tabular} & D & 3 & 40 & \begin{tabular}{l|l}
0 & 10
\end{tabular} & 30 & 50 & $0 \mid 0$ & 0 & 10 & 0 & & 0 & $\begin{array}{lll}15 & 15 \\
\end{array}$ & 510 & \begin{tabular}{l|c|}
10 \\
0
\end{tabular} & $\begin{array}{c}10 \\
0\end{array}$ & $0 \mid 0$ & 00 & 07 & 95 & 79 & 95 & 5 & \\
\hline 50 & $\begin{array}{c}1 . \\
1\end{array}$ & \begin{tabular}{|c|} 
Low \\
gradient \\
Riffle \\
\end{tabular} & 6264 & $\begin{array}{c}13 \\
2\end{array}$ & 24 & 2225 & 2526 & $\begin{array}{c}0.6 \\
7\end{array}$ & 0.9 & & & 2 & 5 & \begin{tabular}{l|l}
0 & 20
\end{tabular} & 0 & 40 & $0 \mid 0$ & 0 & 40 & $0: 5$ & 5 & 152 & 2025 & 530 & \begin{tabular}{l|l|l}
0 & 98 \\
\end{tabular} & $\mid \begin{array}{c}10 \\
0\end{array}$ & 00 & \begin{tabular}{l|l}
0 & 0
\end{tabular} & \begin{tabular}{l|l}
0 & 7
\end{tabular} & 95 & 79 & 95 & & $\begin{array}{c}+132 \mathrm{RB} \text { trail } \\
\text { leading to RR xing } \\
\text { sign and tracks }\end{array}$ \\
\hline
\end{tabular}




\begin{tabular}{|c|c|c|c|c|c|c|c|c|c|c|c|c|c|c|c|c|c|c|c|c|c|c|c|c|c|c|c|c|c|c|c|c|c|c|c|c|}
\hline 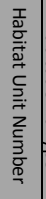 & & 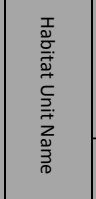 & 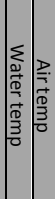 & 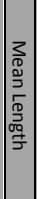 & 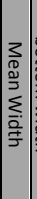 & 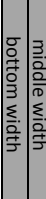 & 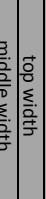 & 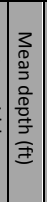 & 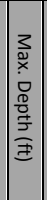 & 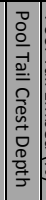 & 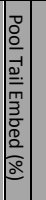 & 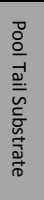 & 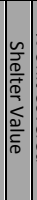 & 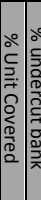 & 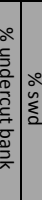 & $\frac{o 0}{\sum}$ & 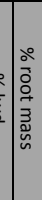 & 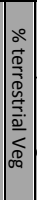 & & & 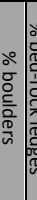 & & \begin{tabular}{l} 
\\
\hdashline \\
\hdashline \\
0 \\
0 \\
2 \\
2
\end{tabular} & 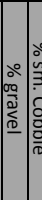 & 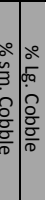 & 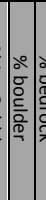 & & 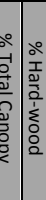 & 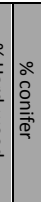 & 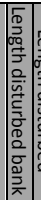 & 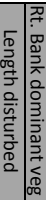 & 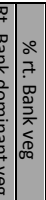 & 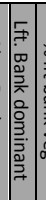 & 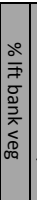 & 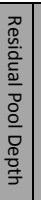 & 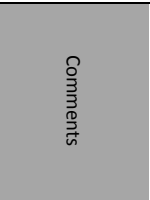 \\
\hline 51 & $\begin{array}{l}. \\
3 \\
\end{array}$ & Run & 6366 & 65 & 22 & 2622 & 2219 & \begin{tabular}{c|}
1.1 \\
3 \\
\end{tabular} & 2.1 & & & & 2 & 50 & \begin{tabular}{l|l}
0 & 20 \\
\end{tabular} & 0.0 & 40 & 0 & 0 & 03 & 30 & \begin{tabular}{l|l}
0 & 5
\end{tabular} & 10 & 102 & 2530 & 20 & 008 & \begin{tabular}{c|c}
98 & 10 \\
0
\end{tabular} & 0 & 0 & \begin{tabular}{l|l}
0 & 7 \\
\end{tabular} & 790 & 72 & 25 & & LB cliff \\
\hline 52 & $\begin{array}{l}4 . \\
2\end{array}$ & $\begin{array}{c}\text { Mid- } \\
\text { Channel } \\
\text { Pool } \\
\end{array}$ & 6266 & 53 & 21 & 1917 & 1727 & $\begin{array}{c}1.6 \\
8\end{array}$ & 2.3 & 1.1 & & C & 3 & 05 & $50 \mid 0$ & 0 & 30 & 0 & 0 & \begin{tabular}{l|l}
0 & 2
\end{tabular} & 20 & 020 & 30 & $10 \mid 1$ & 1015 & 515 & 095 & $99 \begin{array}{c}10 \\
0\end{array}$ & 0 & 0 & \begin{tabular}{l|l}
5 & 7
\end{tabular} & $7 \begin{array}{c}10 \\
0\end{array}$ & 73 & 30 & 1.2 & \\
\hline 53 & $\begin{array}{l}3 . \\
4 \\
\end{array}$ & Step Run & 6366 & $\begin{array}{c}29 \\
8\end{array}$ & 19 & $27 \mid 17$ & 1714 & $\begin{array}{c}1.0 \\
3 \\
\end{array}$ & 1.8 & & & & 3 & & 1020 & 020 & 030 & 5 & 0 & \begin{tabular}{l|l}
0 & 1
\end{tabular} & 15 & \begin{tabular}{l|l}
0 & 5
\end{tabular} & 5 & 102 & 2535 & 520 & \begin{tabular}{l|l}
0 & 95
\end{tabular} & \begin{tabular}{l|c}
10 \\
99
\end{tabular} & 0 & 02 & \begin{tabular}{l|l}
25 & 7
\end{tabular} & \begin{tabular}{|c|c|} 
& 10 \\
0
\end{tabular} & 75 & 50 & & End of day, $7 / 16$ \\
\hline 54 & $\begin{array}{l}4 . \\
2\end{array} \mid$ & \begin{tabular}{c|} 
Mid- \\
Channel \\
Pool \\
\end{tabular} & 6163 & 47 & 18 & $14 \mid 19$ & 1921 & 1.1 & 2 & 0.7 & & $\mathrm{D}$ & & 300 & $0 \quad 50$ & $\begin{array}{l}0 \\
0\end{array}$ & 50 & 0 & 0 & \begin{tabular}{l|l}
0 & $c$ \\
\end{tabular} & 0 & 030 & 40 & 205 & 55 & 0 & \begin{tabular}{l|l}
0 & 96
\end{tabular} & \begin{tabular}{l|l}
96 & 60
\end{tabular} & 0 & 0 & \begin{tabular}{l|l}
0 & 7
\end{tabular} & 720 & 73 & 30 & 1.3 & $\begin{array}{c}\text { Bridge provides } \\
\text { "canopy cover" } \\
\text { Start } 7 / 17\end{array}$ \\
\hline 55 & $\begin{array}{c}5 . \\
4\end{array}$ & \begin{tabular}{|c|} 
Lateral \\
Scour \\
Pool - \\
Bedrock \\
Formed \\
\end{tabular} & 6063 & 39 & 18 & 2119 & 1915 & $\mid \begin{array}{c}1.2 \\
3\end{array}$ & 2.5 & 1 & 50 & C & 1 & $5 \mid 0$ & \begin{tabular}{l|l}
0 & 0
\end{tabular} & 0 & 0 & 5 & 0 & \begin{tabular}{l|l}
0 & 9
\end{tabular} & 95 & 030 & 30 & 205 & 55 & 10 & 0 \begin{tabular}{l|l}
10 \\
0
\end{tabular} & \begin{tabular}{l|l}
10 & 44 \\
0 & 4
\end{tabular} & 40 & 0 & \begin{tabular}{l|l}
0 & 7
\end{tabular} & 715 & \begin{tabular}{|l|l|l|l|}
5 & 7 & 1 &
\end{tabular} & 10 & 1.5 & $\begin{array}{c}\text { "Bedrock } \\
\text { formed" = bridge } \\
\text { abutment. } 66 \% \\
\text { "canopy cover" is } \\
\text { from bridge }\end{array}$ \\
\hline 56 & $\begin{array}{l}3 . \\
4 \\
\end{array}$ & Step Run & 6065 & 85 & 14 & 1514 & 1414 & $\begin{array}{c}0.6 \\
5\end{array}$ & 0.8 & & & & 2 & & \begin{tabular}{l|l}
0 & 0
\end{tabular} & 0 & 10 & 80 & 0 & \begin{tabular}{l|l}
0 & 1
\end{tabular} & $10 \mathrm{C}$ & \begin{tabular}{l|l}
0 & 0
\end{tabular} & 10 & $10 \mid 1$ & 1040 & 30 & $0 \mid 95$ & $95 \begin{array}{c}10 \\
0\end{array}$ & 0 & 0 & \begin{tabular}{|l|l}
0 & 6
\end{tabular} & $6 \quad 95$ & \begin{tabular}{|l|l|l|l}
5 & 7 & 1 &
\end{tabular} & 15 & & \\
\hline 57 & $\begin{array}{c}4 . \\
2\end{array}$ & \begin{tabular}{|c|} 
Mid- \\
Channel \\
Pool
\end{tabular} & 6165 & 73 & 20 & 1430 & 3016 & 5 & 3.8 & 0.7 & & c & 3 & \begin{tabular}{|l|ll}
0 & 1 &
\end{tabular} & $10 \mid 0$ & 40 & 0 & 0 & 0 & 0 & 0 & $0 \mid 30$ & 30 & $30 \mid 1$ & 100 & $0 \mid \mathrm{c}$ & \begin{tabular}{|l|l|}
0 & 85
\end{tabular} & $85 \mid \begin{array}{c}10 \\
0\end{array}$ & 0 & 0 & \begin{tabular}{l|l}
0 & 6
\end{tabular} & \begin{tabular}{ll|}
6 & 10
\end{tabular} & 7 & $20 \mid$ & 3.1 & LWD enhanced \\
\hline 58 & $\begin{array}{l}3 . \\
3 \\
\end{array}$ & Run & 6167 & 73 & 17 & 1615 & 1916 & \begin{tabular}{|c|}
0.4 \\
5 \\
\end{tabular} & $\begin{array}{c}0.7 \\
5 \\
\end{array}$ & & & & & $5 \mid 4$ & 4010 & 30 & 020 & 0 & 0 & $\begin{array}{lll}0 & 0 \\
0\end{array}$ & 0 & \begin{tabular}{l|l}
0 & 0 \\
\end{tabular} & 10 & 305 & 5010 & 0 & \begin{tabular}{|c|c|}
0 & 10 \\
\end{tabular} & \begin{tabular}{l|c}
10 & 10 \\
0 & 0 \\
\end{tabular} & 0 & 02 & \begin{tabular}{l|l}
20 & 7 \\
\end{tabular} & 790 & 7 & 95 & & \\
\hline 59 & $\begin{array}{c}5 . \\
1 \\
\end{array}$ & $\begin{array}{c}\text { Corner } \\
\text { Pool }\end{array}$ & 6264 & $\begin{array}{c}11 \\
8\end{array}$ & 21 & 1625 & 2522 & $\begin{array}{c}1.1 \\
8\end{array}$ & 3 & $\begin{array}{c}0.6 \\
5\end{array}$ & 75 & c & 2 & & 1530 & 010 & 040 & 5 & 0 & \begin{tabular}{l|l}
0 & $\mathrm{C}$ \\
\end{tabular} & 0 & 020 & 20 & 302 & 2010 & 0 & 0 \begin{tabular}{c|c}
10 \\
0
\end{tabular} & \begin{tabular}{l|c}
10 & 10 \\
0 & 0
\end{tabular} & 0 & 0 & \begin{tabular}{l|l}
0 & 7 \\
\end{tabular} & $\begin{array}{c}10 \\
0\end{array}$ & 75 & 50 & $\begin{array}{c}2.3 \\
5\end{array}$ & Photo \\
\hline 60 & $\begin{array}{l}4 . \\
2\end{array}$ & \begin{tabular}{|c|} 
Mid- \\
Channel \\
Pool \\
\end{tabular} & 6265 & 75 & 21 & 2220 & 2022 & 2.4 & 2.4 & $\begin{array}{c}1.1 \\
5\end{array}$ & & D & & 103 & 3030 & 0 & 30 & 10 & 0 & 0 & 0 & 030 & 30 & 305 & 55 & 0 & 0 \begin{tabular}{c|c}
10 \\
0
\end{tabular} & \begin{tabular}{l|c}
10 & 10 \\
0 & 0
\end{tabular} & 0 & 0 & \begin{tabular}{l|l}
0 & 7
\end{tabular} & $\begin{array}{l}7 \\
7\end{array}$ & 579 & 90 & $\begin{array}{c}1.2 \\
5\end{array}$ & \\
\hline 61 & $\begin{array}{l}5 . \\
3\end{array}$ & $\begin{array}{c}\text { Lateral } \\
\text { Scour } \\
\text { Pool - } \\
\text { Root Wad } \\
\text { Enhanced }\end{array}$ & 6366 & 37 & 19 & $22 \mid 20$ & 2015 & 5 & 2.5 & 1 & 50 & $\mathrm{D}$ & 3 & 53 & 3015 & 5 & $\begin{array}{l}530 \\
5\end{array}$ & 0 & 0 & 0 & 0 & \begin{tabular}{l|l}
0 & 20
\end{tabular} & 30 & 305 & 55 & 10 & \begin{tabular}{|l|l|l}
0 & 99
\end{tabular} & $99 \mid \begin{array}{c}10 \\
0\end{array}$ & 0 & 15 & \begin{tabular}{l|l}
0 & 7
\end{tabular} & $\begin{array}{l}7 \\
7\end{array}$ & 579 & 90 & 1.5 & photo \\
\hline 62 & $\begin{array}{c}1 . \\
1\end{array}$ & \begin{tabular}{|c|} 
Low \\
gradient \\
Riffle
\end{tabular} & \begin{tabular}{|l|l}
6267 \\
\end{tabular} & 56 & 20 & 152 & 2124 & $4\left|\begin{array}{c}0.5 \\
4\end{array}\right|$ & 0.8 & & & & 1 & & \begin{tabular}{l|l}
0 & 50
\end{tabular} & 050 & 0 & 0 & 0 & 0 & \begin{tabular}{l|l}
0 & $c$
\end{tabular} & \begin{tabular}{l|l}
0 & 0
\end{tabular} & 10 & 103 & 3030 & 20 & & & & & & & & & & \\
\hline 62.1 & $\begin{array}{c}6 . \\
1\end{array}$ & $\left|\begin{array}{c}\text { Secondar } \\
\text { y Channel } \\
\text { Pool }\end{array}\right|$ & 6267 & 55 & 13 & $11 \mid 14$ & 1414 & \begin{tabular}{c|c}
1.2 \\
6
\end{tabular} & $\begin{array}{c}1.8 \\
5\end{array}$ & $\begin{array}{c}0.3 \\
5\end{array}$ & & & & \begin{tabular}{l|l}
10 & 0
\end{tabular} & \begin{tabular}{l|l}
0 & 50
\end{tabular} & 050 & $\begin{array}{lll}0 & 0\end{array}$ & 0 & 0 & \begin{tabular}{l|l}
0 & 0
\end{tabular} & 0 & & 050 & 00 & \begin{tabular}{l|l}
0 & 0
\end{tabular} & 0 & 0 & & & & & & & & 1.5 & $\begin{array}{c}\text { Dry channel } \\
\text { upstream, } \\
\text { connects to main } \\
\text { channel at } \\
\text { downstream end }\end{array}$ \\
\hline 63 & $\begin{array}{l}5 . \\
2\end{array}$ & \begin{tabular}{|c|} 
Lateral \\
Scour \\
Pool - Log \\
Enhanced \\
\end{tabular} & 6269 & 27 & 20 & 24 & 15 & 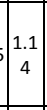 & $\begin{array}{c}2.2 \\
5\end{array}$ & 0.8 & & D & 3 & 750 & \begin{tabular}{l|l}
0 & 30
\end{tabular} & 030 & 030 & 5 & 0 & 0 & 5 & \begin{tabular}{l|l}
0 & 0
\end{tabular} & & $15 \mid 2$ & 2525 & 525 & 0 \begin{tabular}{c|c}
10 & 0 \\
0
\end{tabular} & \begin{tabular}{l|l}
10 & 10 \\
0 & 0
\end{tabular} & 0 & 0 & \begin{tabular}{l|l}
0 & 7
\end{tabular} & $\begin{array}{l}7 \\
7\end{array}$ & 75 & 50 & $\begin{array}{c}1.4 \\
5\end{array}$ & $\begin{array}{c}\text { Log jam } \\
\text { enhancec. Photo }\end{array}$ \\
\hline 64 & $\begin{array}{l}3 . \\
4 \\
\end{array}$ & Step Run & $\begin{array}{ll}63 & 68 \\
\end{array}$ & $\begin{array}{c}37 \\
8 \\
\end{array}$ & 23 & 1525 & 2926 & \begin{tabular}{|c|}
0.6 \\
8 \\
\end{tabular} & 1.5 & & & & & 53 & $\begin{array}{lll}30 & 10\end{array}$ & 0 & $\begin{array}{lll}0 & 10\end{array}$ & 10 & 0 & \begin{tabular}{l|l}
0 & 3
\end{tabular} & 30 & \begin{tabular}{l|l}
0 & 0
\end{tabular} & 0 & $10 \mid 2$ & 2040 & 30 & \begin{tabular}{|l|c|}
0 & 10 \\
\end{tabular} & \begin{tabular}{l|c}
10 & 10 \\
0 & 0 \\
\end{tabular} & 0 & 0 & \begin{tabular}{l|l}
0 & 7
\end{tabular} & 790 & 79 & 95 & & \\
\hline 65 & \begin{tabular}{|c|}
5. \\
1 \\
\end{tabular} & $\begin{array}{c}\text { Corner } \\
\text { Pool }\end{array}$ & 6369 & 69 & 28 & 2625 & 2534 & $\begin{array}{c}1.2 \\
8 \\
\end{array}$ & 2.2 & $\begin{array}{c}0.9 \\
5\end{array}$ & & C & & 56 & 600 & 0 & 30 & 10 & 0 & 0 & 0 & 030 & & 400 & \begin{tabular}{l|l}
0 & 0
\end{tabular} & 0 & \begin{tabular}{|l|l|l}
0 & 90 \\
\end{tabular} & \begin{tabular}{c|c}
90 & 10 \\
& 0 \\
\end{tabular} & 0 & 0 & \begin{tabular}{l|l}
0 & 6
\end{tabular} & $6 \quad 85$ & 57 & \begin{tabular}{|c|}
10 \\
0 \\
\end{tabular} & \begin{tabular}{c|}
1.2 \\
5 \\
\end{tabular} & \\
\hline 66 & $\begin{array}{c}5 . \\
1\end{array}$ & $\begin{array}{c}\text { Corner } \\
\text { Pool }\end{array}$ & 6368 & $\begin{array}{c}10 \\
5\end{array}$ & 34 & 3433 & 33 & 1.8 & 5.4 & 0.5 & & C & 1 & $<50$ & \begin{tabular}{l|l}
0 & 0
\end{tabular} & $\begin{array}{c}10 \\
0\end{array}$ & 0 & 0 & 0 & \begin{tabular}{l|l}
0 & $\mathrm{c}-2$
\end{tabular} & 0 & \begin{tabular}{l|l}
0 & 40
\end{tabular} & $0 \mid 30$ & $30 \mid 0$ & \begin{tabular}{l|l}
0 & 0
\end{tabular} & 0 & $\mid$\begin{tabular}{l|l}
0 & 86 \\
\end{tabular} & \begin{tabular}{l|l}
36 & 90
\end{tabular} & 0 & 0 & \begin{tabular}{l|l}
0 & 6
\end{tabular} & $\begin{array}{ll}6 & 50\end{array}$ & \begin{tabular}{|l|l|l}
7 & 8 & 2
\end{tabular} & 80 & 4.9 & $\begin{array}{c}\text { Pool occupies } \\
>60 \% \text { of channel, } \\
\text { so } 4.2 \text { ? }\end{array}$ \\
\hline 66.1 & $\begin{array}{c}6 . \\
1\end{array}$ & $\left|\begin{array}{c}\text { Secondar } \\
\text { y Channel } \\
\text { Pool }\end{array}\right|$ & 6570 & 46 & 13 & 2012 & 128 & $\begin{array}{c}1.2 \\
5\end{array}$ & 1.4 & 1.4 & & C & & \begin{tabular}{|l|l}
5 & 0
\end{tabular} & \begin{tabular}{l|l}
0 & 10
\end{tabular} & 030 & 030 & 10 & 20 & 0 & 0 & $\begin{array}{lll}0 & 40\end{array}$ & 060 & & & & & & & & & & & & 0 & $\begin{array}{c}\text { Dry channel } \\
\text { upstream, } \\
\text { connects to main } \\
\text { channel at } \\
\text { downstream end }\end{array}$ \\
\hline 67 & $\begin{array}{r}3 . \\
4 \\
\end{array}$ & Step Run & 6368 & $\begin{array}{c}16 \\
5 \\
\end{array}$ & 24 & & 2423 & \begin{tabular}{|c|}
1.0 \\
1 \\
\end{tabular} & 2.2 & & & & & \begin{tabular}{|l|l}
5 & 0 \\
\end{tabular} & \begin{tabular}{l|l}
0 & 30
\end{tabular} & 20 & 030 & 0 & 0 & \begin{tabular}{l|l}
0 & 2
\end{tabular} & 20 & \begin{tabular}{l|l}
0 & 0
\end{tabular} & 10 & 203 & $\begin{array}{l}30 \\
30\end{array}$ & 10 & \begin{tabular}{|l|l|l}
0 & 10 \\
0 & 0 \\
\end{tabular} & \begin{tabular}{l|c}
10 & 10 \\
0 & 0 \\
\end{tabular} & 0 & 0 & \begin{tabular}{l|l}
0 & 7
\end{tabular} & 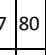 & $7 \mid 7$ & 70 & & \\
\hline 68 & $\begin{array}{c}5 . \\
1 \\
\end{array}$ & $\begin{array}{c}\text { Corner } \\
\text { Pool }\end{array}$ & & 93 & 21 & $23 \mid 24$ & $\begin{array}{l}24 \\
\end{array}$ & $5 \mid 1.3$ & 2.2 & 0.5 & 60 & C & 3 & 102 & 2030 & 10 & 020 & 0 & 0 & \begin{tabular}{l|l}
0 & 2
\end{tabular} & 20 & 020 & & 301 & 1510 & 5 & \begin{tabular}{|l|l|}
0 & 10 \\
\end{tabular} & \begin{tabular}{l|c}
10 & 10 \\
0 & 0 \\
\end{tabular} & 0 & 0 & \begin{tabular}{l|l}
0 & 7
\end{tabular} & $\begin{array}{l}720 \\
\end{array}$ & 6 & 20 & 1.7 & $\begin{array}{c}+34, \text { LB Little } \\
\text { Creek confluence }\end{array}$ \\
\hline 69 & $\begin{array}{l}3 . \\
4 \\
\end{array}$ & Step Run & 6369 & $\begin{array}{c}14 \\
0 \\
\end{array}$ & 21 & 1615 & 1533 & $\begin{array}{c}0.6 \\
1 \\
\end{array}$ & 1 & & & & & & 3020 & 020 & $\begin{array}{lll}0 & 10\end{array}$ & 0 & 0 & \begin{tabular}{l|l}
0 & 2
\end{tabular} & 20 & \begin{tabular}{l|l}
0 & 0
\end{tabular} & 0 & 303 & 3020 & 20 & \begin{tabular}{|l|l}
0 & 95
\end{tabular} & \begin{tabular}{l|c}
10 \\
99
\end{tabular} & 0 & 0 & \begin{tabular}{l|l}
0 & 5
\end{tabular} & 595 & 56 & 90 & & \\
\hline 70 & $\begin{array}{l}5 . \\
3\end{array}$ & \begin{tabular}{|c|} 
Lateral \\
Scour \\
Pool - \\
Root Wad \\
Enhanced \\
\end{tabular} & 6369 & 52 & 29 & 3330 & 3025 & 5 & $\begin{array}{c}3.1 \\
5\end{array}$ & 0.3 & 30 & C & 3 & \begin{tabular}{|l|l}
20 & 0
\end{tabular} & \begin{tabular}{l|l}
0 & 10
\end{tabular} & 0 & 060 & 0 & 0 & \begin{tabular}{l|l}
0 & 1
\end{tabular} & 10 & 020 & & $30 \mid 1$ & 1010 & 5 & \begin{tabular}{l|l}
0 & 95
\end{tabular} & $99 \mid \begin{array}{c}10 \\
0\end{array}$ & 0 & 25 & \begin{tabular}{l|l}
0 & 6
\end{tabular} & $\begin{array}{ll}6 & 80\end{array}$ & 6 & $\begin{array}{c}10 \\
0\end{array} \mid$ & $\begin{array}{c}2.8 \\
5\end{array}$ & photo \\
\hline 71 & $\begin{array}{c}1 . \\
1\end{array}$ & \begin{tabular}{|c|} 
Low \\
gradient \\
Riffle
\end{tabular} & & 25 & 26 & 25 & 27 & $7 \begin{array}{c}0.3 \\
7\end{array}$ & 0.5 & & & & & \begin{tabular}{|l|l}
5 & 0
\end{tabular} & \begin{tabular}{l|l}
0 & 50
\end{tabular} & \begin{tabular}{l|l}
0 & 0
\end{tabular} & 0 & 35 & 0 & \begin{tabular}{l|l}
0 & 1
\end{tabular} & 15 & \begin{tabular}{l|l}
0 & 0
\end{tabular} & 0 & 204 & 4030 & 10 & 0 \begin{tabular}{c|c}
10 \\
0
\end{tabular} & \begin{tabular}{c|c}
10 & 10 \\
0 & 0
\end{tabular} & 0 & 0 & \begin{tabular}{l|l}
0 & 6
\end{tabular} & $6 \begin{array}{c}10 \\
0\end{array}$ & 6 & $\begin{array}{c}10 \\
0\end{array}$ & & \\
\hline 72 & $\begin{array}{l}3 . \\
2 \\
\end{array}$ & Glide & 6367 & 73 & 27 & 27 & 26 & 50.7 & 1.2 & & & & & \begin{tabular}{|l|l}
5 & 4
\end{tabular} & $40 \mid 40$ & \begin{tabular}{l|l}
0 & 0
\end{tabular} & 10 & 10 & 0 & 0 & 0 & 020 & & 405 & 55 & 0 & $\begin{array}{ll}0 & 96 \\
\end{array}$ & \begin{tabular}{c|c}
96 & 10 \\
0
\end{tabular} & 0 & 0 & \begin{tabular}{l|l}
0 & 6 \\
\end{tabular} & \begin{tabular}{|c|c|}
6 & 10 \\
0
\end{tabular} & 6 & \begin{tabular}{|c|}
10 \\
0 \\
\end{tabular} & & End of day, $7 / 17$ \\
\hline 73 & $\begin{array}{c}5 . \\
3\end{array}$ & \begin{tabular}{|c|} 
Lateral \\
Scour \\
Pool - \\
Root Wad \\
Enhanced
\end{tabular} & 6164 & 61 & & $26 \mid 21$ & 2120 & $\mid \begin{array}{c}1.1 \\
9\end{array}$ & 2.9 & 1.1 & & C & & \begin{tabular}{|l|l|l|} 
& 11
\end{tabular} & $10 \mid 0$ & 0 & 90 & 0 & 0 & 0 & 0 & 0 & & $40 \mid 1$ & 1515 & 55 & 0 \begin{tabular}{l|l}
10 \\
0
\end{tabular} & \begin{tabular}{l|l}
10 & 10 \\
0 & 0
\end{tabular} & 0 & 0 & \begin{tabular}{l|l}
0 & 6
\end{tabular} & 695 & 56 & 90 & 1.8 & $\begin{array}{c}\text { Start } 7 / 18 \text {. Root } \\
\text { wad still } \\
\text { embedded in } \\
\text { bank }\end{array}$ \\
\hline
\end{tabular}




\begin{tabular}{|c|c|c|c|c|c|c|c|c|c|c|c|c|c|c|c|c|c|c|c|c|c|c|c|c|c|c|c|c|c|c|c|c|}
\hline 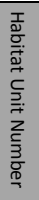 & 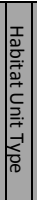 & 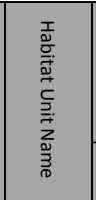 & 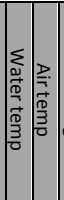 & $\left|\begin{array}{c}3 \\
0 \\
0 \\
3 \\
5 \\
0 \\
3 \\
00 \\
\frac{1}{5} \\
5\end{array}\right|$ & 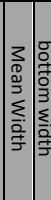 & 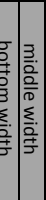 & 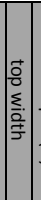 & 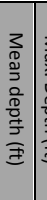 & 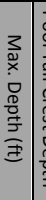 & 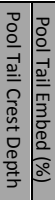 & 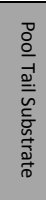 & 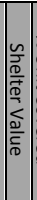 & 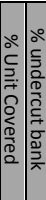 & 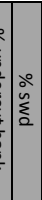 & 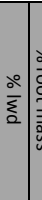 & & 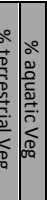 & 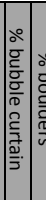 & 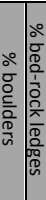 & 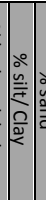 & 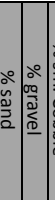 & 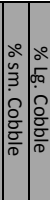 & 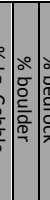 & & 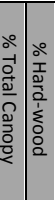 & 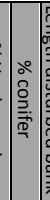 & 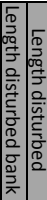 & 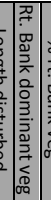 & 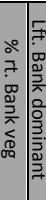 & 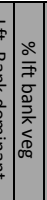 & 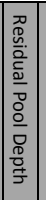 & 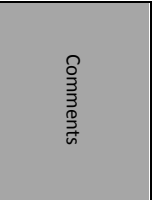 \\
\hline 74 & \begin{tabular}{|c|}
3. \\
4 \\
\end{tabular} & Step Run & 6064 & 69 & 2520 & 2031 & 25 & \begin{tabular}{|c|c|}
0.6 & 1 \\
8 & \\
\end{tabular} & 1.4 & & & & 1020 & 020 & \begin{tabular}{l|l}
0 & 2
\end{tabular} & 203 & \begin{tabular}{l|l|l}
30 & 0
\end{tabular} & \begin{tabular}{l|l}
0 & 1
\end{tabular} & $10 \mid 0$ & 0 & $\begin{array}{lll}0 & 20 \\
\end{array}$ & 3040 & 010 & 09 & \begin{tabular}{c|c}
95 & 10 \\
& 0 \\
\end{tabular} & 0 & \begin{tabular}{l|l}
0 & 0
\end{tabular} & \begin{tabular}{l|l}
0 & 7
\end{tabular} & \begin{tabular}{l|l}
95 & 6
\end{tabular} & 695 & & \\
\hline 75 & $\begin{array}{c}5 . \\
1\end{array}$ & $\begin{array}{c}\text { Corner } \\
\text { Pool }\end{array}$ & 5962 & 54 & 2125 & 2518 & 19 & \begin{tabular}{|c|c|}
1.2 & 2 \\
3 & \\
\end{tabular} & 2.80 & $\begin{array}{lll}0.7 & 40 \\
\end{array}$ & c & & 1050 & 00 & 05 & \begin{tabular}{l|l}
50 & 0
\end{tabular} & \begin{tabular}{l|l|}
0 & 0 \\
\end{tabular} & 00 & \begin{tabular}{l|l}
0 & 0 \\
\end{tabular} & 101 & 1030 & 1010 & 030 & 09 & \begin{tabular}{|c|c|}
99 & 10 \\
0
\end{tabular} & 0 & \begin{tabular}{l|l}
0 & 0
\end{tabular} & 069 & \begin{tabular}{l|l}
90 & 6
\end{tabular} & 695 & 2.1 & \\
\hline 76 & $\begin{array}{l}1 . \\
1\end{array}$ & \begin{tabular}{|c|} 
Low \\
gradient \\
Riffle
\end{tabular} & 6062 & 57 & 1815 & 1915 & 20 & $\begin{array}{c}0.6 \\
3\end{array}$ & 0.9 & & & & 200 & 20 & 30 & \begin{tabular}{l|l}
0 & 4
\end{tabular} & 450 & 05 & $5 \mid 0$ & 0 & \begin{tabular}{l|l|}
0 & 40
\end{tabular} & 2525 & 510 & 09 & \begin{tabular}{l|c|}
92 & 10 \\
0
\end{tabular} & 0 & \begin{tabular}{l|l}
0 & 0
\end{tabular} & 0.69 & \begin{tabular}{l|l}
90 & 7
\end{tabular} & 790 & & \\
\hline 77 & \begin{tabular}{|c|}
3. \\
3 \\
\end{tabular} & Run & 6062 & 59 & \begin{tabular}{|l|l|}
22 & $2 C$ \\
\end{tabular} & 2033 & 14 & \begin{tabular}{|c|c|}
0.9 & 1 \\
6 & 1 \\
\end{tabular} & 1.3 & & & 3 & $15 \mid 0$ & 30 & 402 & $20 \mid 0$ & \begin{tabular}{l|l|}
0 & 0
\end{tabular} & \begin{tabular}{l|l}
0 & 1
\end{tabular} & 100 & 0 & \begin{tabular}{l|l|}
0 & 40 \\
\end{tabular} & & 510 & \begin{tabular}{l|l} 
& 1 \\
\end{tabular} & \begin{tabular}{|c|c|}
10 & 10 \\
0 & 0 \\
\end{tabular} & 0 & \begin{tabular}{l|l}
0 & 0
\end{tabular} & $\begin{array}{lll}0 & 6 & 5\end{array}$ & \begin{tabular}{l|l}
50 & 6
\end{tabular} & 685 & & \\
\hline 78 & $\begin{array}{l}4 . \\
2\end{array}$ & $\begin{array}{c}\text { Mid- } \\
\text { Channel } \\
\text { Pool }\end{array}$ & 6162 & 64 & \begin{tabular}{|l|l|l}
22 & 14
\end{tabular} & 1431 & 21 & \begin{tabular}{c|c}
1.2 & \\
9 &
\end{tabular} & 2.7 & 175 & $\mathrm{E}$ & & 1010 & 0 & 90 & $0 \mid 0$ & \begin{tabular}{l|l|}
0 & 0
\end{tabular} & 0 & \begin{tabular}{l|l}
0 & 0
\end{tabular} & 252 & $25 \quad 15$ & 1025 & 510 & $00^{1}$ & \begin{tabular}{c|c|}
10 & 10 \\
0 & 0
\end{tabular} & 0 & \begin{tabular}{|l|l|l|l}
0 & 0
\end{tabular} & $\begin{array}{lll}0 & 6 & 5\end{array}$ & \begin{tabular}{l|l}
50 & 6
\end{tabular} & 685 & $1.7 \mathrm{f}$ & $\begin{array}{c}\text { Log Jam formed, } \\
\text { cement blocks in } \\
\text { stream large log } \\
\text { jam on RB } \\
\text { forming pool and } \\
\text { backwater. Jam } \\
\text { includes large } \\
\text { (>6x10ft) metal } \\
\text { "bucket" }\end{array}$ \\
\hline 78.1 & 6. & \begin{tabular}{|c|} 
Backwate \\
r Pool - \\
log \\
formed
\end{tabular} & 6062 & 59 & \begin{tabular}{|l|l|}
10 & 11 \\
\end{tabular} & 119 & & \begin{tabular}{c|c}
0.6 & 1 \\
4 &
\end{tabular} & $\begin{array}{c}1.0 \\
5\end{array}$ & & & & & & & & & & & & & & & & & & & & & & $\left|\begin{array}{c}1.0 \\
5\end{array}\right|$ & $\begin{array}{l}\text { Jam formed, } \\
\text { connects } \\
\text { upstream end }\end{array}$ \\
\hline 79 & $\begin{array}{l}3 . \\
2\end{array}$ & Glide & 6162 & 66 & 1921 & 2115 & 22 & \begin{tabular}{c|c}
1.0 & \\
5 & 2
\end{tabular} & 2.7 & & & 1 & \begin{tabular}{l|l}
$<5$ & 0
\end{tabular} & 0 & $\left.0\right|^{1}$ & \begin{tabular}{c|c}
10 & 0 \\
0 & 0
\end{tabular} & \begin{tabular}{l|l|}
0 & 0
\end{tabular} & 0 & \begin{tabular}{l|l}
0 & 0
\end{tabular} & 101 & 1555 & \begin{tabular}{l|l}
15 & 5
\end{tabular} & 50 & 0 & \begin{tabular}{c|c}
10 & 10 \\
0 & 0
\end{tabular} & 03 & $\begin{array}{lll}30 & 0\end{array}$ & $\begin{array}{lll}0 & 6 & 7\end{array}$ & \begin{tabular}{l|l}
75 & 6
\end{tabular} & 575 & & $\begin{array}{c}\text { Redwood stand } \\
\text { on top of cliff, RB } \\
\text {. LB, cement } \\
\text { blocks stabilizing } \\
\text { bank }\end{array}$ \\
\hline 80 & \begin{tabular}{|c|}
5. \\
1 \\
\end{tabular} & $\begin{array}{c}\text { Corner } \\
\text { Pool }\end{array}$ & \begin{tabular}{|l|l|}
63 & 64 \\
\end{tabular} & \begin{tabular}{|c|}
10 \\
5 \\
\end{tabular} & \begin{tabular}{|l|l|}
20 & 22 \\
\end{tabular} & 2220 & 18 & \begin{tabular}{|c|c|}
1.5 & 2 \\
3 & 2 \\
\end{tabular} & \begin{tabular}{|l|l|}
2.6 & 0 \\
\end{tabular} & \begin{tabular}{c|c}
0.4 & 50 \\
5 & \\
\end{tabular} & d & 3 & \begin{tabular}{|l|l}
5 & 10
\end{tabular} & 00 & 05 & \begin{tabular}{l|l}
50 & 1
\end{tabular} & \begin{tabular}{l|l|l}
10 & 10
\end{tabular} & 02 & 20 | 0 & 202 & $25 \mid 30$ & $10 \mid 10$ & .05 & 07 & \begin{tabular}{|c|c|}
79 & 10 \\
& 0 \\
\end{tabular} & \begin{tabular}{|l|l|}
0 & 1 \\
\end{tabular} & \begin{tabular}{l|l}
10 & 0
\end{tabular} & 06 & 95 & 585 & \begin{tabular}{|c|}
2.1 \\
5 \\
\end{tabular} & \\
\hline 81 & $\begin{array}{l}3 . \\
4\end{array}$ & Step Run & 6264 & $\begin{array}{c}62 \\
9\end{array}$ & 2718 & 1820 & 43 & \begin{tabular}{|c|c}
0.7 & 1 \\
6 & 1
\end{tabular} & 1.8 & & & 3 & \begin{tabular}{|l|l}
5 & 20
\end{tabular} & 015 & $15 \mid 1$ & \begin{tabular}{l|l}
15 & 1
\end{tabular} & 1010 & 01 & $15 \mid 0$ & 01 & 1025 & 2025 & $\begin{array}{ll}520 & 20\end{array}$ & \begin{tabular}{l|l}
0 & 9
\end{tabular} & \begin{tabular}{c|c|}
98 & 10 \\
0
\end{tabular} & 0 & \begin{tabular}{l|l}
0 & 0
\end{tabular} & \begin{tabular}{l|l|l}
0 & 7 & 9
\end{tabular} & \begin{tabular}{l|l}
95 & 6
\end{tabular} & $\begin{array}{ll}6 & 90\end{array}$ & & $\begin{array}{c}.+629 \text { Big Creek } \\
\text { House (Sinclair's } \\
\text { House) }+1, \text { LB } \\
\text { access through LC } \\
\text { hayfield (gate) }\end{array}$ \\
\hline 82 & $\begin{array}{c}5 . \\
6 \\
\end{array}$ & $\begin{array}{c}\text { Plunge } \\
\text { Pool }\end{array}$ & & 41 & 46 & 4344 & 50 & 1.7 & \begin{tabular}{|l|l|}
3 & 0 \\
\end{tabular} & \begin{tabular}{|c|}
0.7 \\
5 \\
\end{tabular} & c & 1 & \begin{tabular}{l|l}
$<5$ & 0
\end{tabular} & 75 & \begin{tabular}{|l|l}
0 & 1
\end{tabular} & 105 & 50 & \begin{tabular}{l|l}
0 & 1
\end{tabular} & 100 & 102 & 2030 & 255 & $5 \mid 10$ & 0.8 & \begin{tabular}{|c|c|}
88 & 10 \\
& 0 \\
\end{tabular} & 0 & \begin{tabular}{l|l}
0 & 0
\end{tabular} & \begin{tabular}{l|l}
0 & 7
\end{tabular} & \begin{tabular}{l|l}
90 & 5
\end{tabular} & 510 & $\begin{array}{c}2.2 \\
5\end{array}$ & .41, old fish trap \\
\hline 83 & $\begin{array}{c}1 . \\
1\end{array}$ & \begin{tabular}{|c|} 
Low \\
gradient \\
Riffle
\end{tabular} & 6368 & 91 & 4250 & 5038 & 38 & \begin{tabular}{c|c}
0.6 & 1 \\
3 &
\end{tabular} & $\begin{array}{c}1.4 \\
5\end{array}$ & & & & $5 \quad 20$ & 030 & 03 & 301 & 105 & 0 & \begin{tabular}{l|l}
0 & 0
\end{tabular} & 0 & $\begin{array}{lll}0 & 15 & 2\end{array}$ & 2035 & 530 & 09 & \begin{tabular}{l|c|}
92 & 10 \\
0
\end{tabular} & 0 & $\begin{array}{lll}0 & 0\end{array}$ & 069 & $95 \quad 7$ & 730 & & \\
\hline 84 & $\begin{array}{l}3 . \\
2 \\
\end{array}$ & Glide & 6270 & 48 & $36 \quad 38$ & \begin{tabular}{|l|l}
38 & 36
\end{tabular} & 33 & \begin{tabular}{|c|c|}
0.8 & 1 \\
1 & \\
\end{tabular} & 1.4 & & & & $<580$ & 00 & \begin{tabular}{l|l}
0 & 1
\end{tabular} & 101 & 100 & 00 & \begin{tabular}{l|l}
0 & 0
\end{tabular} & 252 & 2530 & 200 & 00 & 09 & \begin{tabular}{|c|c|}
92 & 10 \\
& 0 \\
\end{tabular} & 0 & $\begin{array}{lll}0 & 0\end{array}$ & $\begin{array}{lll}0 & 6 & 8\end{array}$ & 857 & 740 & & \\
\hline 85 & $\begin{array}{l}4 . \\
3\end{array}$ & $\begin{array}{c}\text { Channel } \\
\text { Confluenc } \\
\text { e Pool }\end{array}$ & 6270 & 57 & 3233 & 3331 & 31 & \begin{tabular}{|c|c}
2.3 & 4 \\
4 & 4
\end{tabular} & 4.60 & 0.9 & c & 2 & 560 & 010 & \begin{tabular}{|l|l|l|}
0 & 3
\end{tabular} & $\begin{array}{lll}30 & 0\end{array}$ & \begin{tabular}{l|l|l}
0 & 0
\end{tabular} & 00 & 0 & 202 & 2050 & 100 & 00 & $\left.0\right|^{1}$ & \begin{tabular}{c|c}
10 & 10 \\
0 & 0
\end{tabular} & 0 & \begin{tabular}{|l|l|l|} 
& 0
\end{tabular} & \begin{tabular}{l|l|l}
0 & 7 & 4
\end{tabular} & \begin{tabular}{l|l}
40 & 7
\end{tabular} & \begin{tabular}{l|l|}
7 & 40
\end{tabular} & 3.7 & $\begin{array}{l}\text { Redwoods on RB } \\
\text { but a bay just in } \\
\text { fromnt of the. } \\
+57, \mathrm{LB}, \mathrm{Big} \\
\text { Creek confluence }\end{array}$ \\
\hline 86 & \begin{tabular}{|l|}
3. \\
3 \\
\end{tabular} & Run & \begin{tabular}{|l|l|}
62 & 67 \\
\end{tabular} & \begin{tabular}{|c|}
19 \\
5 \\
\end{tabular} & \begin{tabular}{|l|l}
11 & 9 \\
\end{tabular} & \begin{tabular}{|l|l|}
9 & 10 \\
\end{tabular} & 15 & \begin{tabular}{|c|c|}
0.6 & 1 \\
3 & 1 \\
\end{tabular} & 1.3 & & & 3 & 0 & 40 & 30 & $0 \mid \begin{array}{ll}0 & 11\end{array}$ & 1020 & 0 & \begin{tabular}{l|l}
0 & 0
\end{tabular} & 0 & $0 \quad 20$ & 3040 & 010 & 06 & 6298 & 2 & 020 & \begin{tabular}{l|l|l}
0 & 7 & 1 \\
\end{tabular} & \begin{tabular}{c|c}
10 & 7 \\
0 & 7 \\
\end{tabular} & \begin{tabular}{|c|} 
\\
\\
0
\end{tabular} & & \\
\hline 86.1 & 6. & $\left|\begin{array}{c}\text { Secondar } \\
\text { y Channel } \\
\text { Pool }\end{array}\right|$ & 16265 & 78 & 85 & \begin{tabular}{l|l|l}
5 & 11
\end{tabular} & & \begin{tabular}{c|c}
0.6 & \\
9 & 2
\end{tabular} & 2.1 & 0 & $\mathrm{E}$ & 3 & 0 & 40 & 0 & \begin{tabular}{l|l}
0 & 1
\end{tabular} & 1040 & 01 & 100 & 0 & $\begin{array}{lll}0 & 50 & 2\end{array}$ & 2525 & & & & & & & & & 2.1 & $\begin{array}{l}\text { Water exchange } \\
\text { at downstream } \\
\text { end but no fish } \\
\text { passage. Lots of } \\
\text { fish } \\
\end{array}$ \\
\hline 86.2 & $\begin{array}{l}6 . \\
3\end{array}$ & $\begin{array}{c}\text { Backwate } \\
\text { r Pool - } \\
\text { rootwad } \\
\text { formed }\end{array} \mid$ & 6665 & 40 & 1317 & 179 & & \begin{tabular}{c|c}
0.7 & 1 \\
5 & 1
\end{tabular} & 1.2 & 0 & c & & & & & 50 & 50 & & & 303 & 3040 & & & & & & & & & & 1.2 & $\begin{array}{c}\text { Water exchange } \\
\text { in middle of pool, } \\
\text { but no fish } \\
\text { passage. Lots of } \\
\text { fish. Root wad } \\
\text { still rooted in } \\
\text { bank. } \\
\end{array}$ \\
\hline 87 & $\begin{array}{l}4 . \\
2\end{array}$ & \begin{tabular}{|c|} 
Mid- \\
Channel \\
Pool
\end{tabular} & 6266 & 53 & 1415 & 1513 & 14 & \begin{tabular}{c|c}
1.1 & 1 \\
6 & 1
\end{tabular} & 1.8 & 1 & c & & \begin{tabular}{l|l}
5 & 0
\end{tabular} & 20 & 305 & 500 & $0 \mid 0$ & 0 & 0 & 202 & 2030 & $15 \mid 10$ & 105 & $\left.0\right|^{1}$ & \begin{tabular}{c|c}
10 & 10 \\
0 & 0
\end{tabular} & 0 & 0 & 069 & 956 & 595 & 0.8 & $+50 \mathrm{ft}, \mathrm{RB}$ trail. \\
\hline 88 & $\begin{array}{c}3 . \\
4 \\
\end{array}$ & Step Run & 6264 & 86 & 1514 & 148 & 24 & \begin{tabular}{|c|c|}
0.5 & 0 \\
9 & \\
\end{tabular} & 0.9 & & & 2 & $5 \quad 40$ & 020 & \begin{tabular}{|l|l}
0 & 4 \\
\end{tabular} & 400 & \begin{tabular}{l|l|l}
0 & 0
\end{tabular} & 0 & 0 & 0 & $530=$ & 3030 & 305 & \begin{tabular}{|l|l}
0 & 1 \\
\end{tabular} & \begin{tabular}{c|c}
10 & 10 \\
0 & 0 \\
\end{tabular} & 0 & $\begin{array}{lll}0 & 0\end{array}$ & 6 & \begin{tabular}{c|c}
10 & 6 \\
0 & 6 \\
\end{tabular} & $5 \mid \begin{array}{c}10 \\
0\end{array}$ & & \\
\hline 89 & $\begin{array}{c}5 . \\
3 \\
\end{array}$ & \begin{tabular}{|c|} 
Lateral \\
Scour \\
Pool - \\
Root Wad \\
Enhanced
\end{tabular} & 6365 & 46 & $17 \quad 24$ & 2413 & 13 & \begin{tabular}{c|c}
0.7 & \\
4 & 1
\end{tabular} & $1.30^{0}$ & $\begin{array}{c}0.4 \\
5\end{array}$ & C & & \begin{tabular}{l|l}
5 & 0
\end{tabular} & 0 & 0 & \begin{tabular}{c|c}
10 & 0 \\
0 & 0
\end{tabular} & \begin{tabular}{l|l|l}
0 & 0
\end{tabular} & 0 & \begin{tabular}{l|l}
0 & 0
\end{tabular} & 202 & 2040 & 200 & 0.0 & 0 & \begin{tabular}{c|c|}
10 & 10 \\
0 & 0
\end{tabular} & 03 & 305 & 56 & \begin{tabular}{l|l}
75 & 6
\end{tabular} & 680 & $\left|\begin{array}{c}0.8 \\
5\end{array}\right|$ & $\begin{array}{c}.+10, \text { LB trail } \\
\text { access through } \\
\text { hay field above } \\
\text { big creek }\end{array}$ \\
\hline 90 & $\begin{array}{c}5 . \\
4\end{array}$ & \begin{tabular}{|c|} 
Lateral \\
Scour \\
Pool - \\
Bedrock \\
Formed \\
\end{tabular} & 6168 & 50 & 1513 & 1319 & 13 & \begin{tabular}{c|c}
1.4 & 2 \\
3 &
\end{tabular} & \begin{tabular}{c|c}
2.8 & \\
5 & 0
\end{tabular} & 0.730 & c & 1 & $<5 \mid 0$ & 0 & 80 & \begin{tabular}{l|l}
0 & 0
\end{tabular} & \begin{tabular}{l|l|l}
0 & 0
\end{tabular} & 02 & 200 & 305 & 5010 & $\begin{array}{ll}5 & 0\end{array}$ & 0.0 & 0 & \begin{tabular}{c|c|}
10 & 10 \\
0 & 0
\end{tabular} & 01 & 130 & 066 & \begin{tabular}{l|l}
60 & 6
\end{tabular} & 580 & $\begin{array}{c}2.1 \\
5\end{array}$ & Start $7 / 22$ \\
\hline 91 & $\begin{array}{l}3 . \\
2\end{array}$ & Glide & & 32 & \begin{tabular}{|l|l}
14 & 13 \\
\end{tabular} & \begin{tabular}{|l|l}
13 & 16 \\
\end{tabular} & 14 & \begin{tabular}{|c|c}
0.4 & 0 \\
4 &
\end{tabular} & \begin{tabular}{|c|}
0.9 \\
5
\end{tabular} & & & 2 & 550 & 00 & 0 & 500 & \begin{tabular}{l|l|l}
0 & 0
\end{tabular} & 00 & 0 & 152 & $2030=$ & 305 & 50 & ${ }^{0}$ & \begin{tabular}{c|c|}
10 & 10 \\
0 & 0
\end{tabular} & 0 & \begin{tabular}{l|l}
0 & 0
\end{tabular} & $\begin{array}{llll}0 & 6 & 3\end{array}$ & 306 & $5 \mid$\begin{tabular}{c|c}
10 \\
0
\end{tabular} & & \\
\hline
\end{tabular}




\begin{tabular}{|c|c|c|c|c|c|c|c|c|c|c|c|c|c|c|c|c|c|c|c|c|c|c|c|c|c|c|c|c|c|c|c|c|c|c|}
\hline 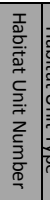 & 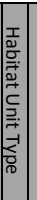 & 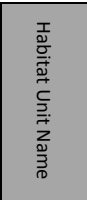 & 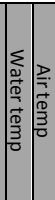 & 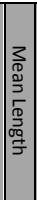 & 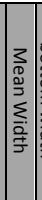 & 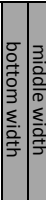 & 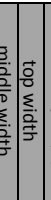 & 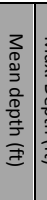 & 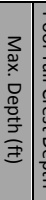 & 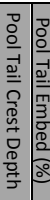 & 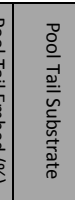 & 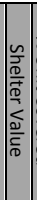 & 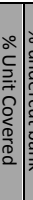 & 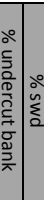 & $\frac{\circ}{\sum}$ & 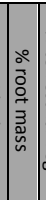 & 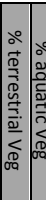 & 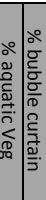 & 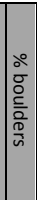 & 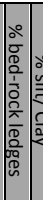 & & 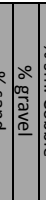 & 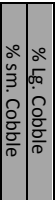 & 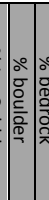 & & 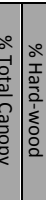 & 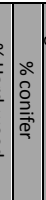 & 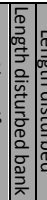 & 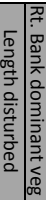 & 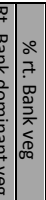 & 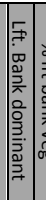 & 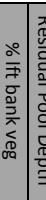 & 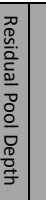 & 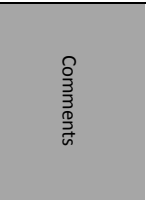 \\
\hline 92 & $\begin{array}{ll}5 . \\
3 \\
R\end{array}$ & \begin{tabular}{|c|} 
Lateral \\
Scour \\
Pool - \\
Root Wad \\
Enhanced \\
\end{tabular} & \begin{tabular}{|l|l}
6165 & 65 \\
&
\end{tabular} & 40 & 21 & 1414 & 1434 & \begin{tabular}{c|c}
1.0 & 2 \\
1 &
\end{tabular} & \begin{tabular}{c|c}
2.3 & 0 \\
5 &
\end{tabular} & $\begin{array}{ccc}0.8 & 30 \\
5 & 30\end{array}$ & 0 & 3 & $40 \sqrt{1}$ & \begin{tabular}{l|l}
10 & 0
\end{tabular} & 0 & 85 & \begin{tabular}{|l|l}
0 & 5
\end{tabular} & $5 \mid 0$ & 0 & 03 & & 030 & \begin{tabular}{|l|l|}
5 & 5 \\
\end{tabular} & 00 & \begin{tabular}{l|l}
0 & 98
\end{tabular} & \begin{tabular}{l|c}
98 & 10 \\
0
\end{tabular} & $\begin{array}{l}0 \\
0\end{array}$ & 0 & \begin{tabular}{l|l}
0 & 6
\end{tabular} & 695 & 69 & 95 1. & 1.5 & \\
\hline 93 & $\begin{array}{ll}5 . \\
3 \\
R\end{array}$ & \begin{tabular}{|c|} 
Lateral \\
Scour \\
Pool - \\
Root Wad \\
Enhanced \\
\end{tabular} & \begin{tabular}{|l|l}
6164 & 64 \\
\end{tabular} & 28 & 22 & $34 \mid 18$ & 1815 & 1.22 & 2.10 & 0.520 & d & & 10 & \begin{tabular}{l|l|l}
0 & 20
\end{tabular} & \begin{tabular}{l|l}
0 & 0
\end{tabular} & 70 & $0 \mid 1 c$ & 100 & 0 & 02 & 2525 & 525 & $15 \mid 10$ & 0 & $0 \mid 9$ & 94 \begin{tabular}{c|c}
10 \\
0
\end{tabular} & 0 & 0 & \begin{tabular}{l|l}
0 & 6
\end{tabular} & 690 & 77 & \begin{tabular}{l|l}
70 & 1.
\end{tabular} & 1.6 & \\
\hline 94 & $\begin{array}{l}4 . \\
2\end{array}$ & \begin{tabular}{|c|} 
Mid- \\
Channel \\
Pool \\
\end{tabular} & 6165 & 30 & 13 & $15 \mid 15$ & 158 & \begin{tabular}{|c|c}
1.2 & 2 \\
8 & 2
\end{tabular} & \begin{tabular}{l|l}
2.3 & 1
\end{tabular} & 1.420 & c & 3 & 40 & $0 \mid 5$ & 50 & 45 & \begin{tabular}{|l|l|l|l|l|l}
0 & 0
\end{tabular} & \begin{tabular}{l|l}
0 & 0
\end{tabular} & 0 & 02 & 2025 & 540 & \begin{tabular}{|l|l|}
10 & 5 \\
\end{tabular} & 00 & \begin{tabular}{l|l}
0 & 9
\end{tabular} & \begin{tabular}{l|c}
91 & 10 \\
0
\end{tabular} & 0 & 0 & \begin{tabular}{l|l}
0 & 6
\end{tabular} & 690 & 69 & 900. & 0.9 & Log enhanced \\
\hline 94.1 & $\begin{array}{l}6 . \\
4\end{array}$ & $\begin{array}{l}\text { Backwate } \\
\text { r Pool - } \\
\text { log } \\
\text { formed }\end{array}$ & 6065 & 31 & 9 & $8 \mid 13$ & 137 & \begin{tabular}{c|c}
0.5 & 0 \\
8 &
\end{tabular} & $\begin{array}{c}0.9 \\
5\end{array}$ & & & & 101 & 1030 & 020 & 30 & 100 & \begin{tabular}{l|l}
0 & 0
\end{tabular} & 0 & 0 & & & & & & & & & & & & & $\begin{array}{c}0.9 \\
5\end{array}$ & \\
\hline 95 & 2. & \begin{tabular}{|c} 
Lateral \\
Scour \\
Pool - Log \\
Enhanced
\end{tabular} & \begin{tabular}{|l|l}
61 & 65 \\
\end{tabular} & 49 & 13 & 723 & 2310 & \begin{tabular}{|c|c}
1.3 & 2 \\
9 & 2
\end{tabular} & 2.90 & 0.330 & 0 & 3 & 10 & \begin{tabular}{l|l}
0 & 0
\end{tabular} & 45 & 45 & \begin{tabular}{|l|l|l|l|l|l}
0 & 0
\end{tabular} & \begin{tabular}{l|l}
0 & 0
\end{tabular} & 0 & 02 & & 530 & \begin{tabular}{l|l|}
15 & 5
\end{tabular} & 00 & 0 \begin{tabular}{l|l}
1 & 1 \\
0 & 0
\end{tabular} & \begin{tabular}{l|c}
10 & 10 \\
0 & 0
\end{tabular} & \begin{tabular}{ll|l}
0 & 0 \\
0
\end{tabular} & 0 & \begin{tabular}{l|l}
0 & 6
\end{tabular} & 695 & 69 & \begin{tabular}{|l|l|l|}
90 & 2
\end{tabular} & 2.6 & \\
\hline 96 & $\begin{array}{ll}5 . \\
3 \\
R\end{array}$ & \begin{tabular}{|c|} 
Lateral \\
Scour \\
Pool - \\
Root Wad \\
Enhanced \\
\end{tabular} & 6264 & 31 & 19 & 1026 & 2620 & \begin{tabular}{|c|c}
1.1 & 2 \\
3 &
\end{tabular} & $\left.2.9\right|^{0}$ & $\begin{array}{c}0.2 \\
5\end{array}$ & c & 3 & 40 & \begin{tabular}{l|l}
0 & 10
\end{tabular} & 0 & 80 & \begin{tabular}{l|l}
0 & 0
\end{tabular} & \begin{tabular}{l|l}
0 & 0
\end{tabular} & 0 & 103 & 3030 & 030 & 100 & 00 & 0 \begin{tabular}{l|l}
1 & 1 \\
0
\end{tabular} & \begin{tabular}{c|c}
10 & 10 \\
0 & 0
\end{tabular} & \begin{tabular}{ll|l}
0 & 0 \\
0
\end{tabular} & 0 & \begin{tabular}{l|l}
0 & 6
\end{tabular} & 695 & 68 & 802 & $\begin{array}{c}2.6 \\
5\end{array}$ & \\
\hline 97 & \begin{tabular}{|l|}
3. \\
4 \\
\end{tabular} & Step Run & 6264 & $\begin{array}{c}20 \\
6 \\
\end{array}$ & 19 & 2015 & 1521 & \begin{tabular}{|c|c|}
0.6 & 1 \\
2 & \\
\end{tabular} & 1.2 & & & 3 & & 030 & 020 & 0 & 50 & \begin{tabular}{l|l}
0 & 0
\end{tabular} & 0 & 05 & \begin{tabular}{l|l}
5 & 5
\end{tabular} & 30 & $\begin{array}{lll}45 & 15 \\
\end{array}$ & 500 & 09 & \begin{tabular}{l|c}
10 \\
09
\end{tabular} & \begin{tabular}{|l|l|}
0 & 0 \\
\end{tabular} & 0 & \begin{tabular}{l|l}
0 & 6
\end{tabular} & 695 & 69 & 95 & & $\begin{array}{l}\text { one riffle } 20 \mathrm{ft} \\
\text { long } \\
\end{array}$ \\
\hline 98 & \begin{tabular}{|l|}
5. \\
1
\end{tabular} & $\begin{array}{l}\text { Corner } \\
\text { Pool }\end{array}$ & 6165 & 48 & 17 & 2112 & 1217 & \begin{tabular}{|c|c}
1.7 & 2 \\
5 &
\end{tabular} & 2.90 & 0.3 & c & 3 & & \begin{tabular}{l|l}
45 & 0
\end{tabular} & 0 & 50 & \begin{tabular}{|l|l}
5 & 0
\end{tabular} & \begin{tabular}{l|l}
0 & 0
\end{tabular} & 0 & 02 & 2030 & 030 & \begin{tabular}{|l|l|}
15 & 5 \\
\end{tabular} & 00 & $0 \begin{array}{lll}1 & 1 \\
0\end{array}$ & \begin{tabular}{c|c}
10 & 10 \\
0 & 0
\end{tabular} & \begin{tabular}{ll|l}
0 & 0 \\
\end{tabular} & $\begin{array}{ll}0 \\
0\end{array}$ & \begin{tabular}{l|l}
0 & 6
\end{tabular} & 695 & 6 & \begin{tabular}{c|c}
10 & 2. \\
0 & 2.
\end{tabular} & 2.6 & $\begin{array}{c}\text { Huge undercut } \\
\text { bank, } 3.3 \text { feet } \\
\text { undercut. }\end{array}$ \\
\hline 99 & \begin{tabular}{|l}
3. \\
3 \\
\end{tabular} & run & \begin{tabular}{|l|l|}
62 & 68 \\
\end{tabular} & 93 & 16 & 1714 & 1417 & \begin{tabular}{|c|}
0.6 \\
4 \\
\end{tabular} & 1 & & & 3 & 10 & 525 & 525 & 0 & \begin{tabular}{|l|l}
45 & 0
\end{tabular} & \begin{tabular}{l|l}
0 & 0 \\
\end{tabular} & 0 & 0 & \begin{tabular}{l|l}
0 & 5
\end{tabular} & 535 & 3020 & 10 & $\begin{array}{lll}0 & 9\end{array}$ & \begin{tabular}{c|c}
92 & 10 \\
0
\end{tabular} & \begin{tabular}{|l|l|}
0 \\
\end{tabular} & 0 & \begin{tabular}{l|l}
0 & 7
\end{tabular} & 795 & 69 & 95 & & \\
\hline 100 & \begin{tabular}{|l|}
5. \\
1 \\
\end{tabular} & $\begin{array}{c}\text { Corner } \\
\text { Pool }\end{array}$ & 6267 & 23 & 10 & \begin{tabular}{|l|l}
17 & 7
\end{tabular} & \begin{tabular}{|l|l|}
7 & 5 \\
\end{tabular} & \begin{tabular}{|c|c|}
0.8 & 1 \\
5 & \\
\end{tabular} & 1.50 & \begin{tabular}{l|l}
0.3 & 15
\end{tabular} & $d$ & 3 & 401 & 1530 & 020 & 25 & \begin{tabular}{|l|l}
10 & 0
\end{tabular} & \begin{tabular}{l|l}
0 & 0
\end{tabular} & 0 & 0 & \begin{tabular}{l|l}
5 & 10
\end{tabular} & 035 & \begin{tabular}{|l|l|}
40 & 10 \\
\end{tabular} & $\begin{array}{llll}0 & 0 & 0\end{array}$ & 0 & & & & & 695 & 69 & \begin{tabular}{l|l}
95 & 1.
\end{tabular} & 1.2 & \\
\hline 101 & \begin{tabular}{|l|}
3. \\
3 \\
\end{tabular} & run & & 35 & 11 & 5 & 16 & \begin{tabular}{|c|c|}
0.7 & 1 \\
7 & 1 \\
\end{tabular} & 1.3 & & & 3 & 102 & 2025 & 525 & 30 & \begin{tabular}{|l|l}
0 & 0
\end{tabular} & \begin{tabular}{l|l}
0 & 0
\end{tabular} & 0 & 0 & \begin{tabular}{l|l}
5 & 10 \\
\end{tabular} & 035 & \begin{tabular}{l|l}
40 & 10 \\
\end{tabular} & $\begin{array}{llll}0 & 0 & \\
\end{array}$ & \begin{tabular}{|l|l|}
0 & 10 \\
\end{tabular} & \begin{tabular}{l|c}
10 & 10 \\
0 & 0 \\
\end{tabular} & \begin{tabular}{|l|l|}
0 & 0 \\
\end{tabular} & 0 & \begin{tabular}{l|l}
0 & 6 \\
\end{tabular} & 690 & 78 & 80 & & \\
\hline 102 & \begin{tabular}{|l|}
5. \\
1 \\
\end{tabular} & $\begin{array}{c}\text { Corner } \\
\text { Pool } \\
\end{array}$ & 6266 & 41 & 16 & $16 \quad 13$ & 1319 & \begin{tabular}{|c|c|}
1.3 & 2 \\
5 & 2 \\
\end{tabular} & \begin{tabular}{|l|l|}
2.4 & 0 \\
\end{tabular} & \begin{tabular}{c|c}
0.8 & 40 \\
5 & \\
\end{tabular} & 0 & 3 & 103 & 350 & 25 & 40 & \begin{tabular}{|l|l}
0 & 0
\end{tabular} & \begin{tabular}{l|l}
0 & 0
\end{tabular} & 0 & \begin{tabular}{l|l}
0 & 1
\end{tabular} & 1525 & 530 & \begin{tabular}{ll|}
30 & 0 \\
\end{tabular} & 0 & \begin{tabular}{l|l}
0 & 98 \\
\end{tabular} & \begin{tabular}{|c|c}
98 & 10 \\
0
\end{tabular} & \begin{tabular}{|l|l|}
0 \\
\end{tabular} & 0 & \begin{tabular}{l|l}
0 & 6
\end{tabular} & 6 90 & 68 & \begin{tabular}{|l|l}
80 & 1 \\
\end{tabular} & $\begin{array}{c}1.5 \\
5 \\
\end{array}$ & \\
\hline 103 & \begin{tabular}{c|}
3 \\
4
\end{tabular} & Step Run & 6265 & $\begin{array}{c}14 \\
6 \\
\end{array}$ & 17 & 1917 & 1715 & \begin{tabular}{l|l|}
0.7 & 1
\end{tabular} & 1.9 & & & 3 & 10 & \begin{tabular}{l|l}
0 & 25
\end{tabular} & 525 & 25 & $25 \mid 0$ & \begin{tabular}{l|l}
0 & 0
\end{tabular} & 0 & 0 & \begin{tabular}{l|l}
0 & 5
\end{tabular} & 525 & \begin{tabular}{|l|l|}
50 & 15 \\
\end{tabular} & 55 & \begin{tabular}{l|l}
0 & 9 \\
\end{tabular} & \begin{tabular}{l|l}
92 & 90
\end{tabular} & \begin{tabular}{ll|}
0 & 10 \\
\end{tabular} & 0 & $15 \mid 6$ & 690 & 69 & 90 & & $\begin{array}{c}\text { one riffle, } 6 \mathrm{ft} \\
\text { long }\end{array}$ \\
\hline 104 & \begin{tabular}{|l|l}
5. & \\
2 & $P$ \\
& $P$ \\
\end{tabular} & \begin{tabular}{|c|} 
Lateral \\
Scour \\
Pool - Log \\
Enhanced \\
\end{tabular} & 6367 & 29 & 14 & $15 \mid 10$ & 1018 & \begin{tabular}{|c|c|}
1.0 & 1 \\
4 &
\end{tabular} & \begin{tabular}{c|c}
1.6 & 0 \\
5 & 0
\end{tabular} & 0.315 & d & 3 & 30 & \begin{tabular}{l|l}
0 & 30
\end{tabular} & 0.70 & 0 & \begin{tabular}{|l|l}
0 & 0
\end{tabular} & \begin{tabular}{l|l}
0 & 0
\end{tabular} & 0 & \begin{tabular}{l|l}
0 & 1
\end{tabular} & & $030=$ & \begin{tabular}{|l|l|}
30 & 10 \\
\end{tabular} & 000 & \begin{tabular}{l|l}
0 & 9
\end{tabular} & \begin{tabular}{l|l}
93 & 95
\end{tabular} & 5 & 0 & \begin{tabular}{l|l}
0 & 6
\end{tabular} & 690 & 6 & \begin{tabular}{c|c}
10 & 1 \\
0 & 5
\end{tabular} & $\begin{array}{c}1.3 \\
5\end{array}$ & \\
\hline 105 & $\begin{array}{ll}5 . \\
3 \\
R\end{array}$ & \begin{tabular}{|c|} 
Lateral \\
Scour \\
Pool - \\
Root Wad \\
Enhanced \\
\end{tabular} & \begin{tabular}{|l|l}
63 & 67 \\
\end{tabular} & 18 & 18 & 1825 & 2510 & \begin{tabular}{c|c}
0.8 & 1 \\
9 &
\end{tabular} & $1.40^{0}$ & $\begin{array}{c}0.5 \\
5\end{array}$ & & 3 & 50 & \begin{tabular}{l|l}
0 & $2 C$
\end{tabular} & \begin{tabular}{l|l}
0 & 10
\end{tabular} & 60 & \begin{tabular}{|l|l}
10 & 0
\end{tabular} & \begin{tabular}{l|l}
0 & 0
\end{tabular} & 0 & \begin{tabular}{l|l}
0 & 1
\end{tabular} & 1520 & 025 & \begin{tabular}{|l|l|}
25 & 10 \\
\end{tabular} & 05 & $\begin{array}{ll}0 & 7\end{array}$ & \begin{tabular}{l|c}
70 \\
71
\end{tabular} & 0 & 0 & \begin{tabular}{l|l}
0 & 6
\end{tabular} & 680 & 69 & 950 & $\begin{array}{c}0.8 \\
5\end{array}$ & \\
\hline 106 & \begin{tabular}{|l|}
3. \\
3 \\
\end{tabular} & run & 6367 & 50 & 10 & $10 \mid 10$ & 1011 & \begin{tabular}{|l|l|l|}
0.6 & 0
\end{tabular} & 0.9 & & & 2 & 5 & \begin{tabular}{l|l}
0 & 0
\end{tabular} & 30 & 0 & \begin{tabular}{l|l}
70 & 0
\end{tabular} & \begin{tabular}{l|l}
0 & 0
\end{tabular} & 0 & 0 & \begin{tabular}{l|l}
5 & 10
\end{tabular} & 025 & \begin{tabular}{|l|l|}
25 & 30 \\
\end{tabular} & 05 & \begin{tabular}{|l|l}
0 & 7
\end{tabular} & \begin{tabular}{c|c}
73 & 10 \\
0
\end{tabular} & \begin{tabular}{l|l|}
0 \\
\end{tabular} & 0 & $\begin{array}{ll}0 & 7\end{array}$ & 790 & 6 & $\begin{array}{c}10 \\
0 \\
\end{array}$ & & \\
\hline 107 & \begin{tabular}{|l|}
5. \\
1 \\
\end{tabular} & $\begin{array}{c}\text { Corner } \\
\text { Pool }\end{array}$ & 6368 & 43 & 10 & $11 \mid 11$ & 118 & \begin{tabular}{|l|l|}
1.3 & 2
\end{tabular} & \begin{tabular}{l|l}
2.2 & 0
\end{tabular} & 0.2 & c & & 201 & 100 & 0 & 90 & \begin{tabular}{l|l}
0 & 0
\end{tabular} & \begin{tabular}{l|l}
0 & 0
\end{tabular} & 0 & 03 & 3030 & 025 & \begin{tabular}{|l|l|}
10 & 5 \\
\end{tabular} & 0 & \begin{tabular}{|l|l}
0 & 1 \\
0 & 0 \\
\end{tabular} & \begin{tabular}{l|c}
10 & 10 \\
0 & 0 \\
\end{tabular} & \begin{tabular}{|l|l|} 
& 0 \\
\end{tabular} & 15 & \begin{tabular}{l|l}
0 & 7
\end{tabular} & 795 & 6 & \begin{tabular}{|c|c|}
10 & 2 \\
0 & 2 \\
\end{tabular} & 2 & \\
\hline \begin{tabular}{c|c}
107 \\
1
\end{tabular} & \begin{tabular}{|l|}
3. \\
3 \\
\end{tabular} & run & 6368 & 42 & 6 & \begin{tabular}{|l|l}
5 & 7 \\
\end{tabular} & \begin{tabular}{|l|l|}
7 & 5 \\
\end{tabular} & \begin{tabular}{|c|c|}
0.5 & 0 \\
7 & 0 \\
\end{tabular} & 0.8 & & & & & & & & & & & & & & & & & & & & & & & & & \\
\hline 108 & $\begin{array}{l}3 . \\
3\end{array}$ & run & 6269 & 64 & 10 & 8 & 11 & \begin{tabular}{c|c}
0.5 \\
3
\end{tabular} & 1.5 & & & 1 & 5 & \begin{tabular}{l|l}
0 & 0
\end{tabular} & $\begin{array}{c}10 \\
0\end{array}$ & 0 & \begin{tabular}{l|l}
0 & 0
\end{tabular} & \begin{tabular}{l|l}
0 & 0
\end{tabular} & 0 & 0 & \begin{tabular}{l|l}
0 & 5
\end{tabular} & & \begin{tabular}{|l|l|}
35 & 30 \\
\end{tabular} & 010 & 09 & $99 \begin{array}{c}10 \\
0\end{array}$ & 0 & 0 & \begin{tabular}{l|l}
0 & 6
\end{tabular} & 695 & 68 & 80 & & Split channel, RB \\
\hline $\begin{array}{c}108 . \\
1\end{array}$ & $\begin{array}{l}4 . \\
2\end{array}$ & \begin{tabular}{|c|} 
Mid- \\
Channel \\
Pool \\
\end{tabular} & & 31 & 9 & \begin{tabular}{|l|l|l}
5 & 13
\end{tabular} & 13 & \begin{tabular}{c|c}
1.5 & 2 \\
1 &
\end{tabular} & \begin{tabular}{c|c}
2.6 & 0 \\
5 &
\end{tabular} & $\begin{array}{c}0.4 \\
5\end{array}$ & c & 3 & & 1010 & 040 & 40 & \begin{tabular}{l|l}
0 & 0
\end{tabular} & \begin{tabular}{l|l}
0 & 0
\end{tabular} & 0 & 04 & 4545 & 510 & \begin{tabular}{ll|}
0 & 0 \\
\end{tabular} & 0 & 09 & 99 & & & & & & & $2.2 \mathrm{~S}$ & Split Channel, LB \\
\hline 109 & $\begin{array}{ll}5 . \\
3 \\
R\end{array}$ & \begin{tabular}{|c|} 
Lateral \\
Scour \\
Pool - \\
Root Wad \\
Enhanced \\
\end{tabular} & 6269 & 39 & 14 & $11 \mid 16$ & 1614 & \begin{tabular}{|c|c}
0.6 & 1 \\
7 &
\end{tabular} & 1.50 & \begin{tabular}{l|l}
0.3 & 1
\end{tabular} & d & 3 & 20 & \begin{tabular}{l|l}
5 & 20
\end{tabular} & 030 & 45 & \begin{tabular}{l|l}
0 & 0 \\
\end{tabular} & 0.0 & 0 & 0 & $5 \quad 10$ & 025 & 2525 & 510 & 098 & \begin{tabular}{l|c}
98 \\
98
\end{tabular} & 0 & 0 & \begin{tabular}{l|l}
0 & 7
\end{tabular} & 795 & 68 & \begin{tabular}{|l|l}
80 & 1
\end{tabular} & 1.2 & \\
\hline 110 & \begin{tabular}{|c|}
3 \\
4
\end{tabular} & Step Run & \begin{tabular}{|l|l}
63 & 68
\end{tabular} & 20 & 12 & $14 \mid 16$ & 167 & \begin{tabular}{|c|c}
0.5 & 1 \\
8 & \\
\end{tabular} & $\begin{array}{c}1.6 \\
5\end{array}$ & & & 3 & 5 & \begin{tabular}{l|l|l|l|l}
0 & 30
\end{tabular} & \begin{tabular}{l|l}
0 & 30
\end{tabular} & 10 & 300 & \begin{tabular}{l|l}
0 & 0
\end{tabular} & 0 & 0 & \begin{tabular}{l|l}
0 & 10
\end{tabular} & $020=$ & 3530 & 05 & 07 & \begin{tabular}{c|c}
79 & 10 \\
0
\end{tabular} & 0 & 0 & \begin{tabular}{l|l}
0 & 6
\end{tabular} & 695 & $\begin{array}{lll}6 & 6\end{array}$ & 80 & & \\
\hline 111 & $\begin{array}{l}4 . \\
2\end{array}$ & \begin{tabular}{|c|} 
Mid- \\
Channel \\
Pool \\
\end{tabular} & 6268 & 25 & 45 & 44 & 46 & $\begin{array}{c}1.1 \\
4\end{array}$ & 30 & 0.2 & c & 3 & & \begin{tabular}{l|l}
0 & 35
\end{tabular} & $\begin{array}{l}535 \\
\end{array}$ & 30 & \begin{tabular}{|l|l|l|l|l|l}
0 & 0
\end{tabular} & \begin{tabular}{l|l|l}
0 & 0
\end{tabular} & 0 & 02 & 2020 & 050 & \begin{tabular}{|l|l|}
5 & 5 \\
\end{tabular} & 0 & 0 & $99 \begin{array}{c}10 \\
0\end{array}$ & 0 & 02 & $25 \mid 6$ & 690 & 6 & \begin{tabular}{|l|l|l|}
5 & 2
\end{tabular} & $2.8 \mathrm{~L}$ & $\begin{array}{c}\text { Log jam formed } \\
\text { pool }\end{array}$ \\
\hline 112 & $\begin{array}{l}3 . \\
2\end{array}$ & Glide & 6166 & 81 & 26 & $46 \mid 14$ & 1418 & \begin{tabular}{|c|c}
0.6 & 1 \\
1 & 1 \\
\end{tabular} & 1.1 & & & 3 & & 3030 & 0 & 30 & 10 & \begin{tabular}{l|l}
0 & 0
\end{tabular} & 0 & 0 & \begin{tabular}{l|l}
5 & 5
\end{tabular} & 550 & $\begin{array}{lll}30 & 10 \\
\end{array}$ & 000 & \begin{tabular}{|l|l|}
0 & 1 \\
0 & 0
\end{tabular} & \begin{tabular}{l|c}
10 & 10 \\
0 & 0
\end{tabular} & \begin{tabular}{|l|l|}
0 & 0 \\
\end{tabular} & 0 & \begin{tabular}{l|l}
0 & 6
\end{tabular} & 680 & 73 & 30 & & End of day, $7 / 22$ \\
\hline
\end{tabular}




\begin{tabular}{|c|c|c|c|c|c|c|c|c|c|c|c|c|c|c|c|c|c|c|c|c|c|c|c|c|c|c|c|c|c|c|c|c|c|c|}
\hline 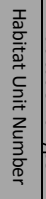 & & 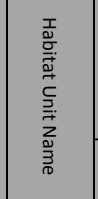 & 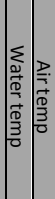 & 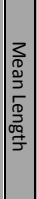 & 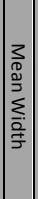 & 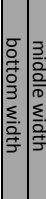 & 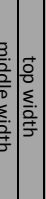 & 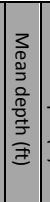 & 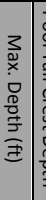 & 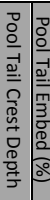 & 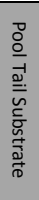 & 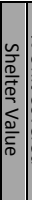 & 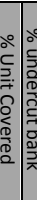 & 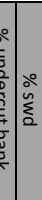 & $\frac{\circ}{\grave{\Sigma}}$ & 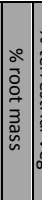 & 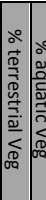 & 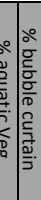 & 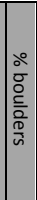 & 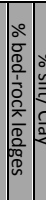 & 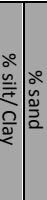 & 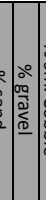 & 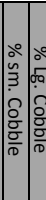 & 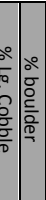 & 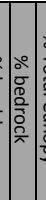 & 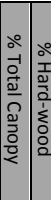 & 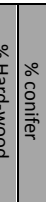 & 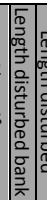 & 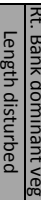 & 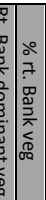 & 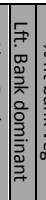 & 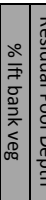 & 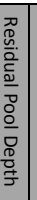 & $\begin{array}{l}\mathfrak{0} \\
3 \\
3 \\
\frac{0}{3} \\
\vec{\omega}\end{array}$ \\
\hline 113 & $\begin{array}{l}4 . \\
2\end{array}$ & \begin{tabular}{|c|} 
Mid- \\
Channel \\
Pool
\end{tabular} & 6167 & 39 & 15 & $18 \mid 18$ & 189 & $\begin{array}{c}1.2 \\
9\end{array}$ & $2.1]^{0}$ & \begin{tabular}{c|c}
0.3 & 20 \\
5 & $2 C$
\end{tabular} & c & 3 & 200 & 030 & 40 & 0 & $30 \mid 0$ & 0.0 & 0 & 01 & 1520 & $0 \mid 50$ & 105 & 50 & 0 & \begin{tabular}{|c|c|}
10 & 10 \\
0 & 0
\end{tabular} & \begin{tabular}{l|l}
0 & 0 \\
0 & 0
\end{tabular} & 0 & \begin{tabular}{l|l}
0 & 6
\end{tabular} & 690 & 73 & $300^{1}$ & $\begin{array}{c}1.7 \\
5\end{array}$ & Start, $7 / 23$ \\
\hline 114 & $\begin{array}{l}1 . \\
1\end{array}$ & \begin{tabular}{|c|} 
Low \\
gradient \\
Riffle
\end{tabular} & & 10 & 11 & 9 & 12 & & & & & & & & & & & & & & & & & & & & & & & & & & & \\
\hline 115 & $\begin{array}{l}4 . \\
2\end{array}$ & \begin{tabular}{|c|} 
Mid- \\
Channel \\
Pool
\end{tabular} & 6265 & 39 & 15 & 1216 & 1616 & $\mid \begin{array}{c}1.3 \\
2\end{array}$ & \begin{tabular}{c|c}
2.1 & 0 \\
5 &
\end{tabular} & \begin{tabular}{c|c}
0.3 & 40 \\
5 &
\end{tabular} & $d$ & 3 & $5 \mid 1$ & 060 & 10 & 15 & \begin{tabular}{l|l}
0 & 0
\end{tabular} & \begin{tabular}{l|l}
0 & 0
\end{tabular} & 0 & 02 & 2020 & 040 & 155 & 50 & 0 & \begin{tabular}{|c|c|}
10 & 10 \\
0 & 0
\end{tabular} & \begin{tabular}{l|l}
0 & 0 \\
0 &
\end{tabular} & 01 & 106 & 680 & 68 & \begin{tabular}{l|l}
80 & 1
\end{tabular} & 1.8 & \\
\hline 116 & \begin{tabular}{|l|}
3. \\
4 \\
\end{tabular} & Step Run & 6266 & 65 & 12 & 1612 & 127 & \begin{tabular}{c|c}
0.6 \\
3
\end{tabular} & $\begin{array}{c}0.9 \\
5 \\
\end{array}$ & & & & $5 \mid 5$ & 550 & 0 & 45 & \begin{tabular}{l|l}
0 & 0
\end{tabular} & $\begin{array}{ll}0 & 0\end{array}$ & 0 & 01 & 1010 & $020=$ & 3025 & 255 & 01 & \begin{tabular}{|c|c|}
10 & 10 \\
0 & 0 \\
\end{tabular} & \begin{tabular}{l|l|}
0 & 0 \\
\end{tabular} & $0 \mid$ & \begin{tabular}{l|l}
0 & 6
\end{tabular} & 6 & 68 & 80 & & \\
\hline $\begin{array}{c}116 . \\
1\end{array}$ & $\begin{array}{l}6 . \\
4\end{array}$ & \begin{tabular}{|c|} 
Backwate \\
r Pool - \\
log \\
formed
\end{tabular} & 6264 & 21 & 14 & $10 \mid 18$ & 18 & $\begin{array}{c}2.7 \\
2\end{array}$ & $\begin{array}{c}4.6 \\
5\end{array}$ & & & & 100 & $0 \mid 10$ & 40 & $|40| 1$ & $10 \mid 0$ & $\begin{array}{l}0 \\
0\end{array}$ & 0 & 02 & 2525 & 550 & & & & & & & & & & & $\begin{array}{c}4.6 \\
5\end{array}$ & $\begin{array}{l}\text { Log jam formed, } \\
\text { not connected to } \\
\text { main channel }\end{array}$ \\
\hline 117 & $\begin{array}{l}5 . \\
3\end{array}$ & \begin{tabular}{|c|} 
Lateral \\
Scour \\
Pool - \\
Root Wad \\
Enhanced \\
\end{tabular} & 6264 & 29 & 9 & 716 & 165 & $\mid \begin{array}{c}0.9 \\
6\end{array}$ & \begin{tabular}{c|c}
1.5 & 0 \\
5 &
\end{tabular} & \begin{tabular}{c|c}
0.5 & 40 \\
5 &
\end{tabular} & d & 3 & 400 & $\begin{array}{l}0 \\
0\end{array}$ & 50 & 40 & $0 \mid 0$ & \begin{tabular}{l|l}
0 & 0
\end{tabular} & 0 & 02 & 2020 & 030 & $15 \mid 1 C$ & 105 & 0 & 94 \begin{tabular}{c|c}
10 \\
0
\end{tabular} & \begin{tabular}{l|l}
0 & 0 \\
0 & 0
\end{tabular} & 0 & \begin{tabular}{l|l}
0 & 6
\end{tabular} & 680 & 6 & 90 & 1 & \\
\hline 118 & $\begin{array}{l}1 . \\
1\end{array}$ & $\begin{array}{c}\text { Low } \\
\text { gradient } \\
\text { Riffle }\end{array}$ & & 18 & 10 & 5 & 14 & $\begin{array}{c}0.1 \\
6\end{array}$ & $\begin{array}{c}0.2 \\
5\end{array}$ & & & & & & & & & & & & & & & & & & & & & & & & & \\
\hline 119 & $\begin{array}{l}4 . \\
2\end{array}$ & \begin{tabular}{|c|} 
Mid- \\
Channel \\
Pool \\
\end{tabular} & 6267 & 74 & 13 & $14 \mid 14$ & 1412 & $\begin{array}{c}1.3 \\
5\end{array}$ & $\left.2.1\right|^{0}$ & \begin{tabular}{c|c}
0.3 & 20 \\
5 &
\end{tabular} & d & 3 & 103 & 50 & 0 & $|10|$ & \begin{tabular}{|l|l|l|l}
0 & 5
\end{tabular} & 5 & 50 & 02 & 2020 & 030 & 55 & 520 & 007 & 7075 & 525 & 50 & \begin{tabular}{l|l}
0 & 6
\end{tabular} & 670 & 69 & $955^{1}$ & $\begin{array}{c}1.7 \\
5\end{array}$ & \\
\hline 120 & $\begin{array}{l}3 . \\
3 \\
\end{array}$ & Run & 6370 & 65 & 25 & 12 & 38 & \begin{tabular}{|c|}
0.5 \\
8
\end{tabular} & $\begin{array}{c}1.2 \\
5 \\
\end{array}$ & & & 3 & 600 & $\begin{array}{l}0 \\
0\end{array}$ & 15 & 0 & 3030 & 300 & 0 & 01 & & 030 & 2525 & 250 & 05 & 5994 & 46 & 0 & \begin{tabular}{|l|l}
0 & 7 \\
\end{tabular} & \begin{tabular}{|c|c|}
70 \\
0
\end{tabular} & 6 & \begin{tabular}{|c|}
10 \\
0 \\
\end{tabular} & & \\
\hline 121 & $\begin{array}{l}4 . \\
2\end{array}$ & $\begin{array}{c}\text { Mid- } \\
\text { Channel } \\
\text { Pool }\end{array}$ & 6270 & 65 & 22 & 3816 & 1613 & $\mid \begin{array}{c}1.5 \\
6\end{array}$ & \begin{tabular}{c|c}
3.2 & 0 \\
5 &
\end{tabular} & 0.4 & & 3 & 505 & 520 & 40 & $5 \mid 1$ & 1515 & 150 & 0 & 03 & 3030 & 020 & 1010 & 100 & 0 & \begin{tabular}{|l|l}
90 & 99
\end{tabular} & \begin{tabular}{l|l|l}
39 & 1
\end{tabular} & $0 \mid$ & \begin{tabular}{l|l}
0 & 7
\end{tabular} & 790 & 6 & \begin{tabular}{c|c}
10 & 2. \\
0 & 5
\end{tabular} & $\begin{array}{c}2.8 \\
5\end{array}$ & $\begin{array}{c}\text { Log jam, seems } \\
\text { like two } \\
\text { seperately } \\
\text { formed pools, } \\
\text { top one corner } \\
\text { pool, bottom by } \\
\text { log jam, } \\
\text { connected by a } \\
\text { thalwag } 1.6 \text { feet } \\
\text { deep } \\
\end{array}$ \\
\hline 122 & \begin{tabular}{|l|}
3. \\
3
\end{tabular} & Run & & 59 & 15 & 1310 & 1021 & \begin{tabular}{c|c}
0.5 \\
3
\end{tabular} & $\begin{array}{c}0.8 \\
5 \\
\end{array}$ & & & 3 & 305 & 540 & 0 & 30 & 250 & $\begin{array}{ll}0 & 0\end{array}$ & 0 & 01 & 1010 & 0252 & 2525 & 255 & 0 & \begin{tabular}{|c|c|}
93 & 10 \\
& 0 \\
\end{tabular} & \begin{tabular}{l|l|}
10 & 0 \\
\end{tabular} & 0 & \begin{tabular}{l|l}
0 & 6 \\
\end{tabular} & 699 & 6 & \begin{tabular}{|c|}
10 \\
0 \\
\end{tabular} & & \\
\hline 123 & $\begin{array}{l}5 . \\
3\end{array}$ & \begin{tabular}{|c|} 
Lateral \\
Scour \\
Pool - \\
Root Wad \\
Enhanced
\end{tabular} & & 43 & 17 & $21 \mid 17$ & 1712 & $\begin{array}{c}1.3 \\
1\end{array}$ & 2.80 & 0.3 & c & 3 & 1 & \begin{tabular}{l|l}
0 & 0 \\
2
\end{tabular} & 0 & $90 \mid$ & \begin{tabular}{|l|l|l|l}
0 & 0
\end{tabular} & 0 & 0 & 03 & & 030 & $\begin{array}{ll}10 & 0\end{array}$ & \begin{tabular}{l|l|}
0 & 0
\end{tabular} & 08 & 82 \begin{tabular}{c|c}
10 \\
0
\end{tabular} & \begin{tabular}{l|l|}
10 & 0 \\
0
\end{tabular} & 0 & \begin{tabular}{l|l}
0 & 6
\end{tabular} & 6 (60 & 6 & \begin{tabular}{c|c}
10 & 2 \\
0 & 2
\end{tabular} & 2.5 & \\
\hline 124 & \begin{tabular}{|l|}
5. \\
1 \\
\end{tabular} & $\begin{array}{c}\text { Corner } \\
\text { Pool }\end{array}$ & & 25 & 11 & 12 & 9 & \begin{tabular}{|c|}
1.3 \\
3
\end{tabular} & \begin{tabular}{l|l}
1.9 & 1
\end{tabular} & 1.4 & c & 3 & 104 & 1030 & 0 & 201 & \begin{tabular}{|l|l}
10 & 0
\end{tabular} & \begin{tabular}{l|l}
0 & 0
\end{tabular} & 0 & 03 & 3030 & 030 & 100 & \begin{tabular}{ll|}
0 & 0 \\
\end{tabular} & 0 & \begin{tabular}{|c|c|}
10 & 10 \\
0 & 0 \\
\end{tabular} & \begin{tabular}{l|l|}
10 & 0 \\
\end{tabular} & 0 & \begin{tabular}{l|l}
0 & 6
\end{tabular} & $\begin{array}{ll}6 & 50\end{array}$ & 79 & \begin{tabular}{l|l}
95 & 0.
\end{tabular} & 0.5 & \\
\hline 125 & \begin{tabular}{|l|}
3. \\
4 \\
\end{tabular} & Step Run & 6270 & 57 & 16 & 916 & 1623 & \begin{tabular}{|c|c|}
0.3 & \\
8 & 0 \\
\end{tabular} & 0.7 & \begin{tabular}{l|l}
0 & 0
\end{tabular} & 0 & 2 & 100 & 0 & 0 & 0 & 50 & \begin{tabular}{l|l}
0 & 0
\end{tabular} & 0 & 05 & \begin{tabular}{l|l}
5 & 5 \\
\end{tabular} & 50 & 2015 & 155 & 06 & \begin{tabular}{|l|c|}
69 & 10 \\
& 0 \\
\end{tabular} & \begin{tabular}{|l|l|}
10 & 0 \\
\end{tabular} & 0 & \begin{tabular}{l|l}
0 & 6 \\
\end{tabular} & 695 & 56 & $\begin{array}{c}10 \\
0 \\
\end{array}$ & & $\begin{array}{c}\text { one riffle } 29 \mathrm{ft} \text {., q } \\
\text { run } 28 \mathrm{ft} \text {. }\end{array}$ \\
\hline 126 & $\begin{array}{l}4 . \\
2\end{array}$ & \begin{tabular}{|c|} 
Mid- \\
Channel \\
Pool \\
\end{tabular} & 6270 & 40 & 28 & 2350 & 5011 & 1.9 & $3.40^{0}$ & $\begin{array}{c}0.8 \\
5\end{array}$ & c & 3 & 600 & \begin{tabular}{l|l}
0 & 40
\end{tabular} & 40 & $20 \mid$ & \begin{tabular}{l|l}
0 & 0
\end{tabular} & \begin{tabular}{l|l}
0 & 0
\end{tabular} & 0 & 03 & 3535 & 520 & 55 & \begin{tabular}{l|l}
5 & 0
\end{tabular} & 07 & \begin{tabular}{|c|c}
10 & 10 \\
0
\end{tabular} & \begin{tabular}{l|l}
10 & 0 \\
0
\end{tabular} & $0 \mid$ & \begin{tabular}{l|l}
0 & 6
\end{tabular} & $6 \begin{array}{c}10 \\
0\end{array}$ & 6 & \begin{tabular}{c|c}
10 & 2 \\
0 & 5
\end{tabular} & $\begin{array}{c}2.5 \\
5\end{array}$ & $\begin{array}{c}\text { log jam. LB trail } \\
\text { access }\end{array}$ \\
\hline 127 & $\begin{array}{l}1 . \\
1\end{array}$ & \begin{tabular}{|c|} 
Low \\
gradient \\
Riffle
\end{tabular} & & 36 & 9 & 11 & 6 & $\begin{array}{c}0.3 \\
5\end{array}$ & $\begin{array}{c}0.5 \\
5\end{array}$ & \begin{tabular}{l|l}
0 & 0
\end{tabular} & 0 & & 100 & 030 & 0 & 0 & 403 & 300 & 0 & 0 & \begin{tabular}{l|l}
0 & 0
\end{tabular} & 30 & 5010 & 100 & 08 & $85 \mid$\begin{tabular}{c|c}
10 \\
0
\end{tabular} & \begin{tabular}{l|l}
10 & 0 \\
0
\end{tabular} & 0 & \begin{tabular}{l|l}
0 & 6
\end{tabular} & $6 \begin{array}{c}10 \\
0\end{array}$ & 6 & $\begin{array}{c}10 \\
0\end{array}$ & & \\
\hline 128 & $\begin{array}{l}4 . \\
2\end{array}$ & $\begin{array}{c}\text { Mid- } \\
\text { Channel } \\
\text { Pool } \\
\end{array}$ & 6374 & 37 & 15 & 627 & 2713 & $\begin{array}{c}1.3 \\
4\end{array}$ & $3.22^{0}$ & $\begin{array}{c}0.5 \\
5\end{array}$ & c & 3 & 900 & $\begin{array}{lll}0 & 40\end{array}$ & 40 & 0 & 02 & 200 & 0 & \begin{tabular}{l|l}
0 & 4
\end{tabular} & 4040 & 020 & 0 & \begin{tabular}{l|l|}
0 & 0
\end{tabular} & 0 & 64 \begin{tabular}{c|c}
10 \\
0
\end{tabular} & \begin{tabular}{l|l|}
10 & 0 \\
0
\end{tabular} & 0 & \begin{tabular}{l|l}
0 & 6
\end{tabular} & 675 & 6 & \begin{tabular}{c|c}
10 & 2 \\
0 & 5
\end{tabular} & $\begin{array}{c}2.6 \\
5\end{array}$ & Log jam \\
\hline 129 & $\begin{array}{l}3 . \\
4 \\
\end{array}$ & Step Run & 6373 & 70 & 16 & 1312 & 1223 & $\begin{array}{c}0.4 \\
6\end{array}$ & 0.8 & & & 3 & 100 & 035 & 20 & $25 \mid 1$ & $10 \mid 0$ & \begin{tabular}{l|l}
0 & 0
\end{tabular} & 0 & 05 & \begin{tabular}{l|l}
5 & 5 \\
\end{tabular} & 25 & $402 C$ & 205 & 0 & 9974 & 4426 & 50 & \begin{tabular}{l|l}
0 & 6
\end{tabular} & 695 & 6 & \begin{tabular}{|c|}
10 \\
0 \\
\end{tabular} & & 2 riffles, 2 runs \\
\hline 130 & $\begin{array}{l}5 . \\
3\end{array}$ & $\mid \begin{array}{c}\text { Lateral } \\
\text { Scour } \\
\text { Pool - } \\
\text { Root Wad } \\
\text { Enhanced }\end{array}$ & 6272 & 56 & 28 & 2338 & 3824 & $\begin{array}{c}2.1 \\
9\end{array}$ & \begin{tabular}{c|l}
4.0 & 0 \\
5 &
\end{tabular} & 0.9 & & 3 & 305 & 535 & 30 & 30 & \begin{tabular}{l|l}
0 & 0
\end{tabular} & \begin{tabular}{l|l}
0 & 0
\end{tabular} & 0 & 03 & 3535 & 520 & 55 & \begin{tabular}{l|l}
5 & 0
\end{tabular} & 09 & \begin{tabular}{|l|l}
95 & 80
\end{tabular} & 3020 & 0 & \begin{tabular}{l|l}
0 & 6
\end{tabular} & $6 \quad 60$ & 69 & $90^{3}$ & $\begin{array}{c}3.1 \\
5\end{array}$ & \\
\hline 131 & $\begin{array}{l}3 . \\
4 \\
\end{array}$ & Step Run & & \begin{tabular}{c|}
12 \\
2 \\
\end{tabular} & 18 & \begin{tabular}{|l|l|}
24 & 14 \\
\end{tabular} & 1416 & $\begin{array}{c}0.6 \\
6\end{array}$ & 1.4 & & & 3 & 51 & .020 & 0 & 40 & 300 & $\begin{array}{ll}0 & 0\end{array}$ & 0 & 0 & \begin{tabular}{l|l}
5 & 5
\end{tabular} & $520=$ & 353 & $\begin{array}{lll}35 & 0 \\
\end{array}$ & 06 & \begin{tabular}{|c|c|}
67 & 10 \\
& 0 \\
\end{tabular} & \begin{tabular}{|l|l|}
10 & 0 \\
\end{tabular} & 0 & \begin{tabular}{l|l}
0 & 6 \\
\end{tabular} & \begin{tabular}{|c|c|}
6 & 10 \\
0
\end{tabular} & 6 & $\begin{array}{c}10 \\
0 \\
\end{array}$ & & 1 riffle $10 \mathrm{ft}$. \\
\hline 132 & \begin{tabular}{|l|}
5. \\
1 \\
\end{tabular} & \begin{tabular}{c|} 
Corner \\
Pool
\end{tabular} & 6274 & 57 & 21 & 16 & 26 & \begin{tabular}{c|}
2.3 \\
6
\end{tabular} & 3.90 & 0.2 & c & 3 & 305 & $5 \quad 25$ & 40 & 30 & \begin{tabular}{l|l}
0 & 0
\end{tabular} & \begin{tabular}{l|l}
0 & 0
\end{tabular} & 0 & 03 & 3535 & 525 & 50 & \begin{tabular}{l|l|}
0 & 0
\end{tabular} & 01 & \begin{tabular}{|c|c|}
10 & 10 \\
0 & 0 \\
\end{tabular} & \begin{tabular}{|l|l|}
10 & 0 \\
\end{tabular} & 0 & \begin{tabular}{l|l}
0 & 7
\end{tabular} & \begin{tabular}{ll|l|}
7 & 80
\end{tabular} & 6 & \begin{tabular}{|l|l|l|}
80 & 3
\end{tabular} & 3.7 & $\begin{array}{l}\text { log enhanced at } \\
\text { downstream end }\end{array}$ \\
\hline 133 & $\begin{array}{l}3 . \\
4 \\
\end{array}$ & Step Run & & 27 & 8 & 5 & 10 & & & & & & & & & & & & & & & & & & & & & & & & & & & \\
\hline 134 & $\begin{array}{l}4 . \\
2\end{array}$ & \begin{tabular}{|c|} 
Mid- \\
Channel \\
Pool \\
\end{tabular} & & 37 & 10 & 10 & & $\begin{array}{c}1.7 \\
4\end{array}$ & \begin{tabular}{c|c}
2.3 & 0 \\
5 &
\end{tabular} & 0.525 & c & 3 & 800 & 030 & 40 & 30 & $0 \mid 0$ & 0.0 & 0 & 03 & 3535 & 525 & 50 & 0.0 & 09 & \begin{tabular}{|c|c}
97 & 10 \\
& 0
\end{tabular} & \begin{tabular}{l|l}
10 & 0 \\
0
\end{tabular} & 0 & \begin{tabular}{l|l}
0 & 6
\end{tabular} & 690 & 78 & $\left.80\right|^{1}$ & $\begin{array}{c}1.8 \\
5\end{array}$ & \\
\hline $\begin{array}{c}134 . \\
1\end{array}$ & $\begin{array}{l}6 . \\
4\end{array}$ & \begin{tabular}{|c|} 
Backwate \\
r Pool - \\
log \\
formed
\end{tabular} & & 27 & & 5 & & & 2.3 & 0 & & & & & & & & & & & & & & & & & & & & & & & & $\begin{array}{c}\text { Not connected to } \\
\text { main channel }\end{array}$ \\
\hline
\end{tabular}




\begin{tabular}{|c|c|c|c|c|c|c|c|c|c|c|c|c|c|c|c|c|c|c|c|c|c|c|c|c|c|c|c|c|c|c|c|c|c|c|}
\hline 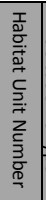 & & 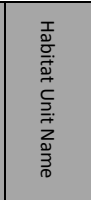 & 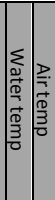 & 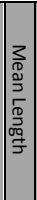 & 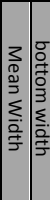 & 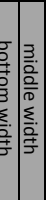 & $=\mid \begin{array}{l}\frac{0}{0} \\
\frac{0}{0} \\
\frac{\leq}{2} \\
\frac{2}{5}\end{array}$ & 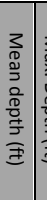 & 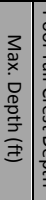 & 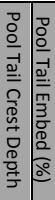 & 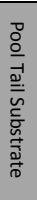 & 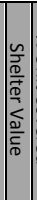 & 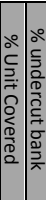 & 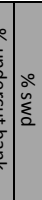 & $\frac{.0}{\grave{2}}$ & 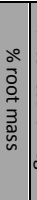 & 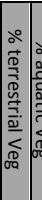 & 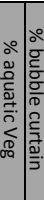 & 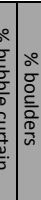 & 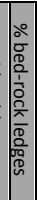 & & 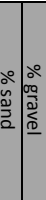 & 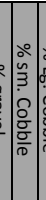 & 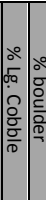 & & 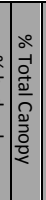 & 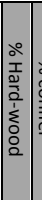 & 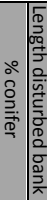 & 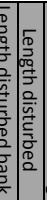 & 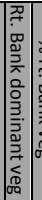 & 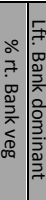 & 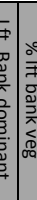 & 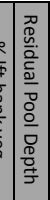 & 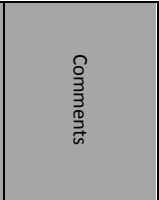 \\
\hline 135 & \begin{tabular}{|l|}
3. \\
4 \\
\end{tabular} & Step Run & & $\begin{array}{c}11 \\
7 \\
\end{array}$ & 1311 & 117 & 21 & \begin{tabular}{|c|c|}
0.4 & 1 \\
4 & \\
\end{tabular} & \begin{tabular}{|c|}
1.0 \\
5 \\
\end{tabular} & & & & $10 \mid 5$ & 50 & 40 & 20 & 03 & 350 & 0 & 0 & 0 & 535 & 550 & $10 \mid 0$ & 0.0 & 53 & \begin{tabular}{|c|}
10 \\
0 \\
\end{tabular} & $0 \quad 20$ & 200 & 79 & 907 & 7 9c & & $\begin{array}{l}\text { two runs, two } \\
\text { riffles }\end{array}$ \\
\hline $\begin{array}{c}135 . \\
1\end{array}$ & $\begin{array}{l}6 . \\
4\end{array}$ & $\begin{array}{c}\text { Backwate } \\
\text { r Pool - } \\
\text { log } \\
\text { formed }\end{array}$ & & 43 & 5 & & & & & & & & & & & & & & & & & & & & & & & & & & & & & Log jam formed \\
\hline 136 & \begin{tabular}{|l|}
5. \\
1 \\
\end{tabular} & $\begin{array}{c}\text { Corner } \\
\text { Pool }\end{array}$ & 74 & 42 & \begin{tabular}{|l|l|}
18 & 21 \\
\end{tabular} & \begin{tabular}{l|l}
21 & 15 \\
\end{tabular} & 519 & \begin{tabular}{|c|}
1.9 \\
5
\end{tabular} & $4 \mid 0$ & 0.1 & c & & 1025 & $5 \mid 5$ & 0 & 70 & 0 & 0 & 0 & 0 & 202 & 2035 & 5151 & $10 \mid 0$ & 0.0 & $\begin{array}{c}10 \\
0\end{array}$ & \begin{tabular}{c|}
10 \\
0 \\
\end{tabular} & $0 \mid 0$ & 0.0 & 76 & \begin{tabular}{l|l}
60 & 6 \\
\end{tabular} & $6 \mid$\begin{tabular}{c|c}
10 \\
0
\end{tabular} & \begin{tabular}{|l|l|}
0 & 3.9 \\
\end{tabular} & \\
\hline 137 & $\begin{array}{l}3 . \\
3 \\
\end{array}$ & Run & 6373 & $\begin{array}{c}13 \\
4 \\
\end{array}$ & 1419 & 1912 & 210 & \begin{tabular}{|c|c|}
0.7 & 1 \\
8 & \\
\end{tabular} & 1.5 & & & 3 & \begin{tabular}{|l|l}
5 & 5
\end{tabular} & 545 & 0 & 50 & 0 & \begin{tabular}{l|l}
0 & 0
\end{tabular} & 0 & 0 & 5 & \begin{tabular}{l|l}
5 & 35
\end{tabular} & 5351 & 155 & 50 & $\mid \begin{array}{c}10 \\
0\end{array}$ & \begin{tabular}{|c|c|}
10 \\
0
\end{tabular} & \begin{tabular}{l|l}
0 & 0 \\
\end{tabular} & 00 & $6{ }^{1}$ & \begin{tabular}{c|c}
10 & 6 \\
0 & 6 \\
\end{tabular} & 675 & & End $7 / 23$ \\
\hline 138 & $\begin{array}{l}5 . \\
2\end{array}$ & \begin{tabular}{|c|} 
Lateral \\
Scour \\
Pool - Log \\
Enhanced
\end{tabular} & 6166 & 31 & 1210 & & 611 & \begin{tabular}{c|c}
1.6 & 2 \\
1 &
\end{tabular} & $2.70^{0}$ & \begin{tabular}{c|c}
0.4 & 20 \\
5 &
\end{tabular} & $d$ & 2 & \begin{tabular}{|l|l}
5 & 0
\end{tabular} & 20 & 60 & 0 & $20 \mid c$ & \begin{tabular}{l|l}
0 & 0
\end{tabular} & $\begin{array}{ll}0 & 0\end{array}$ & 0 & 152 & 2050 & 010 & \begin{tabular}{l|l}
5 & 0
\end{tabular} & 0.0 & $\left|\begin{array}{c}10 \\
0\end{array}\right|$ & $\begin{array}{c}10 \\
0\end{array}$ & \begin{tabular}{l|l}
0 & 0
\end{tabular} & \begin{tabular}{l|l|l}
0 & 0
\end{tabular} & 69 & \begin{tabular}{l|l}
95 & 7
\end{tabular} & $7 \mid 80$ & $\begin{array}{l}0.2 \\
5\end{array}$ & $\begin{array}{l}\text { Alder Trail access. } \\
\text { Start } 7 / 24\end{array}$ \\
\hline $\begin{array}{c}138 . \\
1\end{array}$ & $\begin{array}{l}6 . \\
3\end{array}$ & $\begin{array}{c}\text { Backwate } \\
\text { r Pool - } \\
\text { rootwad } \\
\text { formed }\end{array}$ & & 15 & 7 & & & \begin{tabular}{c|c}
0.6 & 1 \\
9 &
\end{tabular} & \begin{tabular}{c|c}
1.3 & 0 \\
5 &
\end{tabular} & $\begin{array}{c}0.4 \\
5\end{array}$ & c & 1 & & & & & & & & & & & & & & & & & & & & & 0.9 & \\
\hline 139 & $\begin{array}{l}3 . \\
4\end{array}$ & Step Run & 6366 & $\begin{array}{c}10 \\
0\end{array}$ & 1211 & 118 & 18 & \begin{tabular}{c|c}
0.5 & 0 \\
9 &
\end{tabular} & 0.9 & & & 3 & \begin{tabular}{|l|l}
20 & 5
\end{tabular} & 520 & 10 & 0 & 40 & \begin{tabular}{l|l}
0 & 0
\end{tabular} & $0 \quad 5$ & 0 & 5 & 520 & 0402 & 2010 & $10 \mid 0$ & 79 & $\mid \begin{array}{c}10 \\
0\end{array}$ & $0 \mid 0$ & 020 & 7 & \begin{tabular}{c|c}
10 & 6 \\
0 &
\end{tabular} & 680 & & \begin{tabular}{|c|} 
Two riffles (10 ft., \\
$20 \mathrm{ft}$.$) , two runs.$ \\
Road \\
stabilization, \\
"dead man's \\
curve." \\
\end{tabular} \\
\hline 140 & $\begin{array}{l}5 . \\
2\end{array}$ & $\begin{array}{c}\text { Lateral } \\
\text { Scour } \\
\text { Pool - Log } \\
\text { Enhanced }\end{array}$ & 6272 & 80 & 1718 & 1817 & 717 & \begin{tabular}{c|c}
1.4 & 2 \\
2 &
\end{tabular} & \begin{tabular}{c|c}
2.6 & 0 \\
5 & 0
\end{tabular} & 0.5 & & 3 & $15 \mid 0$ & 5 & 35 & 0 & $60 \mid$ & \begin{tabular}{l|l}
0 & 0
\end{tabular} & $\begin{array}{ll}0 & 0\end{array}$ & 0 & $15 \mid 1$ & 1540 & 020 & \begin{tabular}{|l|l}
5 & 5
\end{tabular} & $5 \mid$\begin{tabular}{l|l}
5 & 0
\end{tabular} & 71 & $\mid \begin{array}{c}10 \\
0\end{array}$ & \begin{tabular}{l|l}
0 & 0
\end{tabular} & 0.0 & 6 & \begin{tabular}{c|c}
10 & 6 \\
0 & 6
\end{tabular} & $\left.6\right|_{0} ^{10}$ & \begin{tabular}{l|l|}
0 & 2.1 \\
0 & 5
\end{tabular} & \\
\hline 141 & $\begin{array}{l}5 . \\
3\end{array}$ & \begin{tabular}{|c|} 
Lateral \\
Scour \\
Pool - \\
Root Wad \\
Enhanced \\
\end{tabular} & & 77 & 1917 & 1717 & 23 & \begin{tabular}{c|c}
1.2 & \\
1 &
\end{tabular} & $2.5^{1}$ & \begin{tabular}{c|c}
1.7 & 70 \\
5 &
\end{tabular} & c & 3 & $15 \mid 0$ & 0 & 0 & $\begin{array}{c}10 \\
0\end{array}$ & 0 & \begin{tabular}{l|l}
0 & 0
\end{tabular} & $\begin{array}{ll}0 & 0\end{array}$ & 0 & 252 & 2525 & 520 & \begin{tabular}{l|l}
5 & 0
\end{tabular} & 0.0 & 92 & 10 & $0 \mid 0$ & 0.0 & $\left.6\right|^{1}$ & \begin{tabular}{c|c}
10 & 6 \\
0 & 6
\end{tabular} & $6 \mid \begin{array}{c}10 \\
0\end{array}$ & \begin{tabular}{l|l}
0 & 0.7 \\
0 & 5
\end{tabular} & \\
\hline 142 & \begin{tabular}{|c|}
3 \\
4 \\
\end{tabular} & Step Run & 6370 & $\begin{array}{c}24 \\
0\end{array}$ & \begin{tabular}{|l|l|}
13 & 23 \\
\end{tabular} & 239 & 8 & \begin{tabular}{|c|c}
0.7 & 1 \\
6 & \\
\end{tabular} & \begin{tabular}{|c|}
1.4 \\
5 \\
\end{tabular} & & & 3 & $10 \mid 0$ & 45 & 10 & 0 & 45 & \begin{tabular}{l|l}
0 & 0
\end{tabular} & 0 & 0 & 5 & 520 & 0353 & $\begin{array}{lll}30 & 5\end{array}$ & $\begin{array}{lll}5 & 0\end{array}$ & 98 & \begin{tabular}{|c|c}
10 \\
0 \\
\end{tabular} & \begin{tabular}{l|l}
9 & 9 \\
\end{tabular} & 9 & 79 & \begin{tabular}{l|l}
95 & 6 \\
\end{tabular} & \begin{tabular}{l|l}
6 & 10 \\
0
\end{tabular} & & $\begin{array}{l}\text { Two riffles (10, } \\
30 \mathrm{ft} .) \text { two runs } \\
\end{array}$ \\
\hline 143 & $\begin{array}{l}4 . \\
2\end{array}$ & $\begin{array}{c}\text { Mid- } \\
\text { Channel } \\
\text { Pool }\end{array}$ & 6164 & 40 & \begin{tabular}{|l|l}
15 & 8 \\
\end{tabular} & 822 & 215 & \begin{tabular}{c|c}
1.6 & \\
8 & 2
\end{tabular} & $2.70^{0}$ & \begin{tabular}{c|c}
0.2 & 60 \\
5 &
\end{tabular} & c & 3 & $15 \mid 0$ & 25 & 25 & 50 & 0 & \begin{tabular}{l|l}
0 & 0
\end{tabular} & $\begin{array}{ll}0 & 0\end{array}$ & 0 & 202 & $\begin{array}{lll}25 & 30\end{array}$ & 010 & \begin{tabular}{l|l|l}
10 & 5
\end{tabular} & \begin{tabular}{l|l}
5 & 0
\end{tabular} & $\left|\begin{array}{c}10 \\
0\end{array}\right|$ & $\begin{array}{c}10 \\
0\end{array}$ & \begin{tabular}{l|l}
0 & 0
\end{tabular} & \begin{tabular}{ll|l}
0 & 25
\end{tabular} & 69 & \begin{tabular}{l|l}
95 & 6
\end{tabular} & $6 \quad 60$ & $0 \begin{array}{c}2.4 \\
5\end{array}$ & \\
\hline $\begin{array}{c}143 . \\
1\end{array}$ & 6. & \begin{tabular}{|c|}
$\begin{array}{c}\text { Secondar } \\
\text { y Channel } \\
\text { Pool }\end{array}$ \\
\end{tabular} & & 37 & $\# \#$ & & & \begin{tabular}{c|c}
1.0 & \\
5 & 1
\end{tabular} & 1.40 & 0.1 & & & & & & & & & & & & & & & & & & & & & & & 1.3 & $\begin{array}{l}\text { Connects at } \\
\text { bottom }\end{array}$ \\
\hline 144 & $\begin{array}{l}3 . \\
3 \\
\end{array}$ & Run & & 68 & \begin{tabular}{|l|l|l}
14 & 15
\end{tabular} & 15 & 13 & \begin{tabular}{|c|c|}
0.5 & 0 \\
5 & 0 \\
\end{tabular} & 0.9 & & & & & \begin{tabular}{|c|}
10 \\
0
\end{tabular} & 0 & 0 & 0 & \begin{tabular}{l|l}
0 & 0
\end{tabular} & \begin{tabular}{l|l}
0 & 0
\end{tabular} & 0 & 0 & \begin{tabular}{l|l}
5 & 15
\end{tabular} & 535 & 3510 & 100 & $\begin{array}{c}10 \\
0\end{array}$ & \begin{tabular}{c|}
10 \\
0
\end{tabular} & \begin{tabular}{l|l}
0 & 0 \\
\end{tabular} & 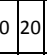 & 69 & \begin{tabular}{l|l}
95 & 6
\end{tabular} & \begin{tabular}{l|l}
6 & 70
\end{tabular} & & \\
\hline 145 & $\begin{array}{c}5 . \\
2\end{array}$ & $\begin{array}{c}\text { Lateral } \\
\text { Scour } \\
\text { Pool - Log } \\
\text { Enhanced }\end{array}$ & 6265 & 34 & 1213 & 1310 & 13 & $\begin{array}{c}1.3 \\
2\end{array}$ & \begin{tabular}{l|l}
2 & 0
\end{tabular} & 0.8 & & 3 & 2010 & 030 & 30 & 5 & 5 & \begin{tabular}{l|l}
0 & 0
\end{tabular} & $\begin{array}{ll}0 & 0\end{array}$ & 0 & 202 & 2030 & & \begin{tabular}{l|l}
10 & 5
\end{tabular} & 50 & $\mid \begin{array}{c}10 \\
0\end{array}$ & $\mid \begin{array}{c}10 \\
0\end{array}$ & \begin{tabular}{l|l}
0 & 0
\end{tabular} & \begin{tabular}{l|l|l}
0 & 0
\end{tabular} & 6 & \begin{tabular}{c|c}
10 & 6 \\
0 & 6
\end{tabular} & 670 & \begin{tabular}{l|l}
0 & 1.2
\end{tabular} & \\
\hline 146 & $\begin{array}{l}3 . \\
3\end{array}$ & Run & 6266 & 88 & 1313 & \begin{tabular}{|l|l|}
13 & 8 \\
\end{tabular} & 18 & \begin{tabular}{|c|c}
0.6 & 1 \\
5 & \\
\end{tabular} & $\begin{array}{c}1.3 \\
5\end{array}$ & & & & \begin{tabular}{l|l}
5 & 10
\end{tabular} & 080 & 0 & 10 & 0 & \begin{tabular}{l|l}
0 & 0
\end{tabular} & \begin{tabular}{l|l}
0 & 0
\end{tabular} & 0 & 101 & 1040 & 030 & 100 & 0.0 & 97 & \begin{tabular}{c|}
10 \\
0
\end{tabular} & \begin{tabular}{l|l}
0 & 0
\end{tabular} & 00 & $6{ }^{1}$ & \begin{tabular}{c|c}
10 & 7 \\
0 & 7
\end{tabular} & \begin{tabular}{l|l}
7 & 80
\end{tabular} & & \\
\hline 147 & $\begin{array}{l}5 . \\
3\end{array}$ & \begin{tabular}{|c|} 
Lateral \\
Scour \\
Pool - \\
Root Wad \\
Enhanced
\end{tabular} & 6265 & 48 & 2118 & 1832 & 213 & \begin{tabular}{c|c}
1.5 & 4 \\
5 &
\end{tabular} & \begin{tabular}{c|c}
4.2 & 0 \\
5 & 0
\end{tabular} & 0.7 & c & 3 & \begin{tabular}{l|l}
10 & 5
\end{tabular} & 5 & 20 & 60 & 10 & \begin{tabular}{l|l}
0 & 0
\end{tabular} & \begin{tabular}{l|l}
0 & 0
\end{tabular} & 0 & 303 & 3030 & 05 & \begin{tabular}{l|l}
5 & 0
\end{tabular} & \begin{tabular}{l|l}
0 & 0
\end{tabular} & 98 & $\left|\begin{array}{c}10 \\
0\end{array}\right|$ & \begin{tabular}{l|l}
0 & 0
\end{tabular} & \begin{tabular}{l|l|}
0 & 15
\end{tabular} & 6 & \begin{tabular}{l|l}
90 & 6
\end{tabular} & 650 & $\begin{array}{c}3.5 \\
5\end{array}$ & \\
\hline 148 & $\begin{array}{c}3 . \\
2 \\
\end{array}$ & Glide & 6266 & 47 & \begin{tabular}{|l|l|l}
15 & 13
\end{tabular} & 13 & 16 & \begin{tabular}{|c|c|}
0.8 & 1 \\
4 & 1 \\
\end{tabular} & 1.5 & & & & $<5 \quad 50$ & 050 & 0 & 0 & 0 & \begin{tabular}{l|l}
0 & 0
\end{tabular} & \begin{tabular}{l|l}
0 & 0
\end{tabular} & 0 & 252 & 2535 & 510 & \begin{tabular}{l|l}
5 & 0
\end{tabular} & \begin{tabular}{l|l}
0 & 0
\end{tabular} & 99 & $\begin{array}{c}10 \\
0 \\
\end{array}$ & \begin{tabular}{l|l}
0 & 0
\end{tabular} & $\begin{array}{lll}0 & 0\end{array}$ & $6{ }^{1}$ & \begin{tabular}{c|c}
10 & 6 \\
0 & 6 \\
\end{tabular} & 675 & & \\
\hline 149 & $\begin{array}{l}5 . \\
3\end{array}$ & \begin{tabular}{|c|} 
Lateral \\
Scour \\
Pool - \\
Root Wad \\
Enhanced
\end{tabular} & & 25 & \begin{tabular}{|l|l|l}
14 & 16
\end{tabular} & 16 & 11 & \begin{tabular}{c|c}
1.3 & 1 \\
5 &
\end{tabular} & \begin{tabular}{c|c}
1.9 & 0 \\
5 &
\end{tabular} & $\begin{array}{c}0.2 \\
5\end{array}$ & c & & 2010 & $0 \mid 40$ & 0 & 50 & 0 & \begin{tabular}{l|l}
0 & 0
\end{tabular} & \begin{tabular}{l|l}
0 & 0
\end{tabular} & 0 & 303 & 3040 & 00 & \begin{tabular}{l|l}
0 & 0
\end{tabular} & \begin{tabular}{l|l}
0 & 0
\end{tabular} & $\left|\begin{array}{c}10 \\
0\end{array}\right|$ & $\left|\begin{array}{c}10 \\
0\end{array}\right|$ & \begin{tabular}{l|l}
0 & 0
\end{tabular} & \begin{tabular}{ll|}
0 & 0
\end{tabular} & 69 & \begin{tabular}{l|l}
95 & 6
\end{tabular} & \begin{tabular}{l|l}
6 & 75 \\
6
\end{tabular} & \begin{tabular}{l|l|l|}
5 & 1.7 \\
\end{tabular} & \\
\hline 150 & \begin{tabular}{|c|} 
\\
3 \\
\end{tabular} & Run & 6266 & $\begin{array}{c}18 \\
0\end{array}$ & 1111 & 11 & & \begin{tabular}{|c|c|}
0.4 & 0 \\
6 & 0 \\
\end{tabular} & 0.9 & & & 2 & & 50 & 0 & 50 & 0 & \begin{tabular}{l|l}
0 & 0
\end{tabular} & \begin{tabular}{l|l}
0 & 0
\end{tabular} & 0 & 101 & 1035 & 535 & $10 \mid$ & \begin{tabular}{l|l}
0 & 0
\end{tabular} & $\begin{array}{c}10 \\
0 \\
\end{array}$ & \begin{tabular}{|c|}
10 \\
0 \\
\end{tabular} & \begin{tabular}{l|l}
0 & 0
\end{tabular} & $\begin{array}{lll}0 & 0\end{array}$ & 6 & \begin{tabular}{l|l}
90 & 6 \\
\end{tabular} & \begin{tabular}{c|c}
6 & 10 \\
\end{tabular} & & \\
\hline $\begin{array}{c}150 . \\
1\end{array}$ & $\begin{array}{l}6 . \\
3\end{array}$ & $\begin{array}{c}\text { Backwate } \\
\text { r Pool - } \\
\text { rootwad } \\
\text { formed }\end{array}$ & & 32 & 5 & & & \begin{tabular}{c|c}
0.3 \\
6
\end{tabular} & 0.8 & & & & & & & & & & & & & & & & & & & & & & & & 0.8 & $\begin{array}{c}\text { Not connected to } \\
\text { main channel }\end{array}$ \\
\hline $\begin{array}{c}150 . \\
2\end{array}$ & $\begin{array}{l}6 . \\
3\end{array}$ & $\begin{array}{c}\text { Backwate } \\
\text { r Pool - } \\
\text { rootwad } \\
\text { formed }\end{array}$ & & 20 & 9 & & & $\left.0.7\right|^{1}$ & $\begin{array}{c}1.3 \\
5\end{array}$ & & & & & & & & & & & & & & & & & & & & & & & & $\begin{array}{c}1.3 \\
5\end{array}$ & $\begin{array}{c}\text { Not connected to } \\
\text { main channel. } \\
\text { End of day } 7 / 24\end{array}$ \\
\hline 151 & $\begin{array}{c}5 . \\
1 \\
\end{array}$ & $\begin{array}{c}\text { Corner } \\
\text { Pool }\end{array}$ & 6064 & 66 & 1625 & 2512 & 210 & \begin{tabular}{|c|c|}
1.2 & 2 \\
6 & \\
\end{tabular} & \begin{tabular}{|c|l}
2.2 & 0 \\
5 & 0 \\
\end{tabular} & 0.6 & c & 3 & 1020 & 020 & 0 & 60 & 0 & \begin{tabular}{l|l}
0 & 0
\end{tabular} & \begin{tabular}{l|l}
0 & 0
\end{tabular} & 0 & 303 & 3530 & 05 & 50 & \begin{tabular}{l|l}
0 & 0
\end{tabular} & 99 & 693 & 3140 & 400 & $8 \mid 1$ & 106 & $\begin{array}{ll}6 & 95\end{array}$ & \begin{tabular}{|c|c|}
5 & 1.6 \\
5
\end{tabular} & Start $7 / 25$ \\
\hline 152 & $\begin{array}{l}3 . \\
4\end{array}$ & Step Run & 6163 & 92 & \begin{tabular}{|l|l}
15 & 19 \\
\end{tabular} & 1912 & 215 & \begin{tabular}{|c|c|}
0.6 & 1 \\
4 & \\
\end{tabular} & 1.5 & & & 2 & $\begin{array}{lll}5 & 20\end{array}$ & 030 & 0 & 50 & 0 & \begin{tabular}{l|l}
0 & 0
\end{tabular} & \begin{tabular}{l|l}
0 & 0
\end{tabular} & 0 & 101 & 1025 & 5302 & \begin{tabular}{l|l}
20 & 5
\end{tabular} & 50 & 97 & 831 & 1730 & 300 & 6 & \begin{tabular}{l|l}
70 & 6
\end{tabular} & $\begin{array}{lll}6 & 80\end{array}$ & & $\begin{array}{c}\text { two riffles }(10,20 \\
\text { ft.) one run }\end{array}$ \\
\hline
\end{tabular}




\begin{tabular}{|c|c|c|c|c|c|c|c|c|c|c|c|c|c|c|c|c|c|c|c|c|c|c|c|c|c|c|c|c|c|c|c|c|c|c|}
\hline 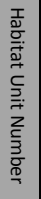 & & 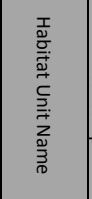 & 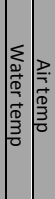 & 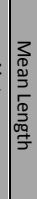 & $\mid \begin{array}{c}3 \\
0 \\
0 \\
\frac{2}{3} \\
\sum \\
\frac{2}{2} \\
\frac{0}{5}\end{array}$ & 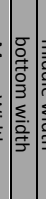 & 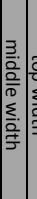 & 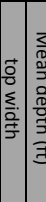 & 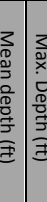 & 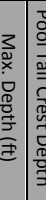 & 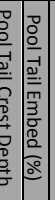 & 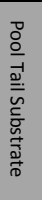 & 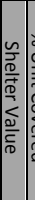 & 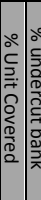 & 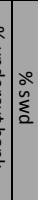 & $\frac{\circ}{\grave{\alpha}}$ & 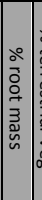 & 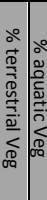 & 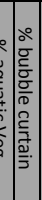 & 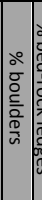 & & $\begin{array}{lll}2 \\
2 \\
2\end{array}$ & 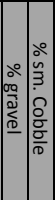 & 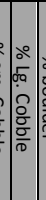 & 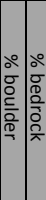 & 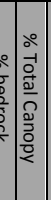 & 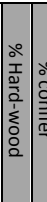 & 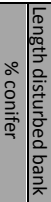 & 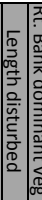 & 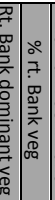 & 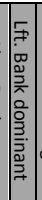 & 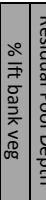 & 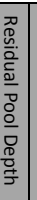 & 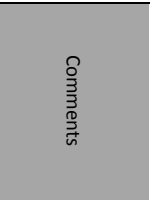 \\
\hline 153 & $\begin{array}{ll}5 . \\
3 \\
R\end{array}$ & \begin{tabular}{|c|} 
Lateral \\
Scour \\
Pool - \\
Root Wad \\
Enhanced \\
\end{tabular} & 6 & 360 & 18 & 3152 & & $15 \frac{1 .}{2}$ & \begin{tabular}{l|l}
1.6 & 3. \\
2 & 3.
\end{tabular} & $3.2 \quad \begin{array}{r}0 . \\
5\end{array}$ & 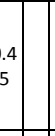 & & 37 & 750 & 20 & 30 & 30 & 1010 & 00 & 0 & 030 & 0303 & 3010 & 00 & \begin{tabular}{l|l}
0 & 0
\end{tabular} & 0.85 & \begin{tabular}{l|l}
89 & 1
\end{tabular} & 1110 & $0 \mid 6$ & \begin{tabular}{l|l|}
6 & 90
\end{tabular} & 6 & $955^{2}$ & $\begin{array}{c}2.7 \\
5\end{array}$ & Log jam \\
\hline 154 & $\begin{array}{l}3 . \\
4\end{array}$ & Step Run & 6064 & \begin{tabular}{|l|l}
12 \\
3
\end{tabular} & 18 & 3151 & 132 & \begin{tabular}{|l|r|}
26 & 0. \\
& 2 \\
\end{tabular} & \begin{tabular}{l|c}
0.4 & 0. \\
2 & 5 \\
\end{tabular} & $\begin{array}{l}0.7 \\
5 \\
\end{array}$ & & & \begin{tabular}{|l|l}
3 & 1
\end{tabular} & 101 & 030 & 0 & $\left.30\right|^{2}$ & $20 \mid 0$ & 0 & 0 & \begin{tabular}{l|l}
0 & 5
\end{tabular} & 52 & 2045 & 520 & \begin{tabular}{l|l}
5 & 0
\end{tabular} & \begin{tabular}{l|l|}
099 \\
\end{tabular} & \begin{tabular}{c|c}
10 & 0 \\
0 & 0 \\
\end{tabular} & \begin{tabular}{l|l}
0 & 0
\end{tabular} & 06 & \begin{tabular}{|c|c|}
6 & 10 \\
& 0 \\
\end{tabular} & 6 & \begin{tabular}{|c|}
10 \\
0 \\
\end{tabular} & & $\begin{array}{c}\text { one riffle (28 ft) } \\
\text { one run }\end{array}$ \\
\hline 155 & $\begin{array}{ll}5 . \\
3 \\
R\end{array}$ & \begin{tabular}{|c|} 
Lateral \\
Scour \\
Pool - \\
Root Wad \\
Enhanced \\
\end{tabular} & 6 & $4 \quad 54$ & 21 & 1262 & & 152 & \begin{tabular}{l|l}
2.0 & 4. \\
2 & 4.
\end{tabular} & $4.2 \quad \begin{array}{l}0 . \\
5\end{array}$ & .4 & c & 3 & $1 \mathrm{c}$ & 020 & 30 & $40 \mid$ & \begin{tabular}{l|l}
0 & 0
\end{tabular} & 0 & 0 & \begin{tabular}{l|l}
0 & 30
\end{tabular} & 0303 & \begin{tabular}{l|l|}
30 & 5 \\
\end{tabular} & 5 & \begin{tabular}{l|l}
0 & 0
\end{tabular} & 098 & $\begin{array}{c}10 \\
0\end{array}$ & \begin{tabular}{l|l}
0 & 0
\end{tabular} & 106 & 6 \begin{tabular}{c|c|}
10 \\
0
\end{tabular} & 6 & $60^{3}$ & $\begin{array}{c}3.7 \\
5\end{array}$ & \\
\hline 156 & \begin{tabular}{|l|}
3. \\
2 \\
\end{tabular} & Glide & 6166 & 5 & 16 & $5 \mid 15$ & & 190. & \begin{tabular}{r|r}
.8 & 1. \\
& 5 \\
\end{tabular} & $\begin{array}{c}1.1 \\
5\end{array}$ & & & 2 & 0 & 50 & 0 & 02 & \begin{tabular}{l|l}
25 & 0 \\
\end{tabular} & 0 & 25 & \begin{tabular}{l|l}
0 & 15 \\
\end{tabular} & 515 & 2530 & 010 & \begin{tabular}{|l|l}
5 & 0 \\
\end{tabular} & \begin{tabular}{|c|c|}
0 & 10 \\
0
\end{tabular} & \begin{tabular}{|c|c}
10 & 0 \\
0 & 0 \\
\end{tabular} & \begin{tabular}{l|l}
0 & 0 \\
\end{tabular} & 206 & \begin{tabular}{l|c|}
6 & 10 \\
0
\end{tabular} & 6 & 50 & & \\
\hline 157 & $\begin{array}{ll}5 . \\
2\end{array}$ & $\begin{array}{c}\text { Lateral } \\
\text { Scour } \\
\text { Pool - Log } \\
\text { Enhanced }\end{array}$ & 6164 & 442 & 21 & 19 & & $22 \frac{1 .}{7}$ & \begin{tabular}{c|c}
1.2 & 1. \\
7 &
\end{tabular} & \begin{tabular}{l|l}
1.6 & 1
\end{tabular} & 1 & c & & 400 & 20 & 80 & 0 & \begin{tabular}{l|l}
0 & 0
\end{tabular} & 0 & 0 & \begin{tabular}{l|l|l}
0 & 25
\end{tabular} & 5253 & 3020 & 00 & \begin{tabular}{l|l}
0 & 0
\end{tabular} & $0 \begin{array}{c}10 \\
0\end{array}$ & $\left|\begin{array}{c}10 \\
0\end{array}\right|$ & \begin{tabular}{l|l}
0 & 0
\end{tabular} & $17 \mid 6$ & $6\left|\begin{array}{c}10 \\
0\end{array}\right|$ & 6 & \begin{tabular}{l|l|l}
70 & 0
\end{tabular} & 0.6 & \\
\hline 158 & \begin{tabular}{|l|}
3. \\
2 \\
\end{tabular} & Glide & 6164 & $4 \mid 42$ & 22 & 22 & & \begin{tabular}{|l|r|r}
22 & 0. \\
\end{tabular} & \begin{tabular}{l|l}
.4 & 0. \\
2 & 0. \\
\end{tabular} & .6 & & & $1 \mid$ & $<5 \mid 1$ & 060 & 0 & $20 \mid$ & \begin{tabular}{l|l}
0 & 0
\end{tabular} & 0 & 10 & 030 & 0303 & $30 \mid 0$ & 01 & 100 & 099 & \begin{tabular}{|c|c}
10 & 0 \\
0 & 0 \\
\end{tabular} & \begin{tabular}{l|l}
0 & 0 \\
\end{tabular} & 56 & \begin{tabular}{|c|c|}
6 & 10 \\
\end{tabular} & 6 & 60 & & \\
\hline 159 & $\begin{array}{l}4 . \\
2\end{array}$ & \begin{tabular}{|c|} 
Mid- \\
Channel \\
Pool \\
\end{tabular} & 6164 & $4 \mid 38$ & 16 & $5: 221$ & 17 & $8{ }^{1 .}$ & \begin{tabular}{l|c}
1.7 \\
4 & 5.
\end{tabular} & \begin{tabular}{l|l}
3.4 & 0. \\
5 &
\end{tabular} & .2 & c & 32 & 200 & 30 & 20 & 40 & $10 \mid 0$ & 0 & 0 & \begin{tabular}{l|l}
0 & 30
\end{tabular} & 030 & 2010 & 05 & \begin{tabular}{|l|l}
5 & 0
\end{tabular} & 093 & $\mid \begin{array}{c}10 \\
0\end{array}$ & \begin{tabular}{l|l}
0 & 0
\end{tabular} & 206 & $6 \mid$\begin{tabular}{c|c|}
10 \\
0
\end{tabular} & 7 & $40^{3}$ & $\begin{array}{c}3.2 \\
5\end{array}$ & \\
\hline 160 & \begin{tabular}{|l|}
3 \\
4
\end{tabular} & Step Run & 6162 & 282 & 13 & \begin{tabular}{|l|l|}
3 & 8 \\
\end{tabular} & 131 & \begin{tabular}{|l|r|}
18 & 0. \\
& 1 \\
\end{tabular} & \begin{tabular}{l|l}
.7 & 1. \\
1 & 1. \\
\end{tabular} & 1.9 & & & & 102 & 010 & 0 & 35 & 035 & 50 & 0 & \begin{tabular}{l|l}
0 & 10
\end{tabular} & $0 \mid 15$ & 4020 & 010 & \begin{tabular}{|l|l}
5 & 0 \\
\end{tabular} & \begin{tabular}{|c|c|}
0 & 10 \\
0
\end{tabular} & \begin{tabular}{|c|c}
10 & 0 \\
0 & 0 \\
\end{tabular} & \begin{tabular}{l|l}
0 & 20 \\
\end{tabular} & $0 \mid 6$ & \begin{tabular}{l|l|}
6 & 90 \\
\end{tabular} & 6 & $\begin{array}{c}10 \\
0 \\
\end{array}$ & & $\begin{array}{c}\text { one riffle ( } 22 \mathrm{ft} .), \\
\text { one run }\end{array}$ \\
\hline 161 & $\begin{array}{l}4 . \\
2\end{array}$ & \begin{tabular}{|c|} 
Mid- \\
Channel \\
Pool \\
\end{tabular} & & 43 & 16 & $5|18| 1$ & 151 & $16{ }_{6}^{1 .}$ & \begin{tabular}{l|l}
1.7 & 2. \\
6 & 5
\end{tabular} & \begin{tabular}{l|l}
. .8 & 0. \\
5 & 5
\end{tabular} & .4 & c & & 200 & 20 & 20 & 40 & \begin{tabular}{l|l}
0 & 0
\end{tabular} & 0 & 0 & \begin{tabular}{l|l}
0 & 25
\end{tabular} & 5303 & 305 & 5 & $\begin{array}{lll}5 & 0\end{array}$ & $0 \begin{array}{c}10 \\
0\end{array}$ & $\begin{array}{c}10 \\
0\end{array}$ & \begin{tabular}{l|l}
0 & 20
\end{tabular} & 00 & 650 & 6 & \begin{tabular}{l|l}
95 & 2.
\end{tabular} & 2.4 & \\
\hline 162 & \begin{tabular}{|l|}
3. \\
2 \\
\end{tabular} & Glide & 6265 & 539 & 15 & 516 & 131 & 16 \begin{tabular}{r|r}
0. \\
1
\end{tabular} & \begin{tabular}{l|l}
0.7 & 1. \\
1 & \\
\end{tabular} & .1 & & & 33 & 302 & 020 & 0 & $30 \mid 3$ & $30 \mid 0$ & 0 & 0 & 030 & 0304 & 400 & 0 & \begin{tabular}{l|l}
0 & 0
\end{tabular} & \begin{tabular}{l|l|}
0 & 95 \\
\end{tabular} & \begin{tabular}{|c|c}
10 & 0 \\
0 & 0 \\
\end{tabular} & \begin{tabular}{l|l}
0 & 0
\end{tabular} & $0 \mid 6$ & \begin{tabular}{l|l|}
6 & 60
\end{tabular} & 7 & 80 & & \\
\hline 163 & $\begin{array}{l}4 . \\
2\end{array}$ & \begin{tabular}{|c|} 
Mid- \\
Channel \\
Pool \\
\end{tabular} & 6165 & 550 & 15 & $5|16| 1$ & $19 \mid 1$ & 102. & $\begin{array}{lll}2.1 & 3 . & \\
.\end{array}$ & \begin{tabular}{l|l}
3.7 & 0.8
\end{tabular} & .8 & b & 37 & $75 \mid 2$ & 010 & 50 & 20 & \begin{tabular}{l|l}
0 & 0
\end{tabular} & 0 & 0 & \begin{tabular}{l|l}
0 & 40
\end{tabular} & $0|40| 2$ & 200 & 0 & \begin{tabular}{l|l}
0 & 0
\end{tabular} & 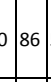 & \begin{tabular}{|l|l|}
50 & 5
\end{tabular} & \begin{tabular}{l|l}
50 & 20
\end{tabular} & $0 \mid 8$ & \begin{tabular}{l|l|}
8 & 60
\end{tabular} & 7 & \begin{tabular}{l|l|l}
75 & 2
\end{tabular} & 2.9 & Log Jam \\
\hline 164 & \begin{tabular}{c|c}
3 \\
4
\end{tabular} & Step Run & 6264 & 460 & 11 & 10 & & \begin{tabular}{|l|l|}
17 & 0. \\
& 6 \\
\end{tabular} & \begin{tabular}{l|l}
.5 & 0. \\
6 & \\
\end{tabular} & .9 & & & 2 & \begin{tabular}{|l|l|l}
5 & 0 \\
\end{tabular} & 50 & 20 & 03 & $30 \mid 0$ & 0 & 0 & \begin{tabular}{l|l}
0 & 5 \\
\end{tabular} & 510 & 2530 & 0201 & 100 & 088 & \begin{tabular}{l|l}
85 & 1 \\
\end{tabular} & $15 \mid 0$ & 06 & \begin{tabular}{l|l|}
6 & 85 \\
\end{tabular} & 5 & 95 & & $\begin{array}{c}\text { two riffles }(18,5 \\
\text { ft.), one run }\end{array}$ \\
\hline 165 & $\begin{array}{l}4 . \\
2\end{array}$ & \begin{tabular}{|c|} 
Mid- \\
Channel \\
Pool \\
\end{tabular} & 6365 & 583 & 20 & 17 & & \begin{tabular}{l|l}
22 & 1.
\end{tabular} & \begin{tabular}{l|l}
1.1 & 2.
\end{tabular} & $2.9 \begin{array}{c}0.2 \\
5\end{array}$ & $\begin{array}{l}.2 \\
5\end{array}$ & b & 39 & 950 & 40 & 30 & 20 & \begin{tabular}{l|l}
0 & 10
\end{tabular} & 00 & 0 & 030 & 0403 & $30 \mid 0$ & 0 & \begin{tabular}{l|l}
0 & 0
\end{tabular} & 085 & \begin{tabular}{|l|l|}
97 & 3
\end{tabular} & \begin{tabular}{l|l}
3 & 0
\end{tabular} & 07 & 770 & 7 & \begin{tabular}{c|c}
10 & 2. \\
0 & 5
\end{tabular} & $\begin{array}{c}2.6 \\
5\end{array}$ & $\begin{array}{l}\text { Log jam } 67 \text { feet } \\
\text { long }\end{array}$ \\
\hline 166 & $\begin{array}{l}3 . \\
2\end{array}$ & Glide & 6265 & 589 & 13 & \begin{tabular}{l|l|l}
3 & 22 & 1
\end{tabular} & 11 & \begin{tabular}{|l|l|l}
5 & 0. \\
8
\end{tabular} & \begin{tabular}{l|l}
.8 & 1. \\
\end{tabular} & 1.6 & & & 2 & \begin{tabular}{|l|l}
5 & 0 \\
\end{tabular} & 50 & 0 & 50 & \begin{tabular}{l|l}
0 & 0
\end{tabular} & 0 & 0 & 030 & 0303 & 3010 & 00 & \begin{tabular}{l|l}
0 & 0 \\
\end{tabular} & \begin{tabular}{|c|c|}
0 & 10 \\
0
\end{tabular} & \begin{tabular}{|c|c|}
10 & 0 \\
0 & 0 \\
\end{tabular} & \begin{tabular}{l|l}
0 & 0 \\
\end{tabular} & 706 & 695 & 5 & 25 & & \\
\hline 167 & $\begin{array}{l}3 . \\
4\end{array}$ & Step Run & 6265 & 573 & 5 & 5 & 4 & \begin{tabular}{|l|r|}
6 & 0. \\
\end{tabular} & \begin{tabular}{l|c}
.4 & 1. \\
5 & 5 \\
\end{tabular} & 5 & & & 31 & 102 & 525 & 0 & 25 & $25 \mid 0$ & 0 & 0 & \begin{tabular}{l|l}
0 & 0
\end{tabular} & 03 & $\begin{array}{lll}35 & 40\end{array}$ & 025 & \begin{tabular}{l|l}
0 & 0
\end{tabular} & \begin{tabular}{|l|l|}
049 \\
\end{tabular} & \begin{tabular}{|c|c}
10 & 0 \\
0 & 0 \\
\end{tabular} & \begin{tabular}{l|l}
0 & 0 \\
\end{tabular} & 106 & \begin{tabular}{|c|c|}
6 & 10 \\
\end{tabular} & 6 & 80 & & $\begin{array}{l}\text { two riffle, two } \\
\text { run }\end{array}$ \\
\hline 168 & 2. & \begin{tabular}{|c|} 
Lateral \\
Scour \\
Pool - Log \\
Enhanced
\end{tabular} & 6265 & 535 & 12 & 262 & 22 & 8 \begin{tabular}{l|l}
1. \\
5
\end{tabular} & \begin{tabular}{c|c}
1.1 & 1. \\
5 & 5
\end{tabular} & \begin{tabular}{l|l}
. .6 & 0. \\
5 & 0.
\end{tabular} & .435 & c & 32 & 201 & 025 & 25 & 40 & \begin{tabular}{l|l}
0 & 0
\end{tabular} & 0 & 0 & $\begin{array}{lll}0 & 30\end{array}$ & 0303 & 305 & 5 & \begin{tabular}{l|l}
0 & 0
\end{tabular} & 094 & $\begin{array}{c}10 \\
0\end{array}$ & \begin{tabular}{l|l}
0 & 0
\end{tabular} & 06 & \begin{tabular}{l|l|}
6 & 10 \\
0
\end{tabular} & 6 & 90 & $\begin{array}{c}1.2 \\
5\end{array}$ & \\
\hline 169 & \begin{tabular}{c|}
3 \\
4
\end{tabular} & Step Run & 6263 & $3 \begin{array}{c}24 \\
7\end{array}$ & 15 & 58 & 231 & \begin{tabular}{l|l}
13 & 0. \\
1
\end{tabular} & \begin{tabular}{l|l}
0.8 & 1.
\end{tabular} & 1.6 & & & 3 & \begin{tabular}{|l|l}
5 & 1 \\
\end{tabular} & 020 & 30 & 202 & 200 & 0 & 0 & \begin{tabular}{l|l}
0 & 15
\end{tabular} & 5152 & 2020 & 030 & \begin{tabular}{l|l}
0 & 0
\end{tabular} & 099 & \begin{tabular}{|c|c}
10 & 0 \\
0 & 0 \\
\end{tabular} & \begin{tabular}{l|l}
0 & 0 \\
\end{tabular} & 06 & 6 \begin{tabular}{c|c|}
6 \\
0
\end{tabular} & 6 & \begin{tabular}{|c|}
10 \\
0 \\
\end{tabular} & & $\begin{array}{c}\text { one riffle, } 36 \mathrm{ft} . \\
\text { End } 7 / 25\end{array}$ \\
\hline 170 & $\begin{array}{ll}5 . \\
3 \\
R\end{array}$ & $\begin{array}{c}\text { Lateral } \\
\text { Scour } \\
\text { Pool - } \\
\text { Root Wad } \\
\text { Enhanced }\end{array}$ & 6063 & 349 & 13 & $\begin{array}{lll}3 & 13 & 1\end{array}$ & 18 & $9 \begin{array}{ll}1 . \\
7\end{array}$ & \begin{tabular}{l|l}
1.1 & 2. \\
7 & 9
\end{tabular} & \begin{tabular}{l|c}
.3 & 0. \\
9 & 6
\end{tabular} & $\begin{array}{l}.1 \\
6\end{array}$ & c & 33 & 30 & 10 & 0 & 80 & \begin{tabular}{l|l}
0 & 0
\end{tabular} & 0 & 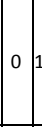 & & 0203 & 3010 & 00 & $0 \mid$\begin{tabular}{l|l}
0 & 20
\end{tabular} & 099 & 257 & 7525 & 008 & 860 & 6 & 902 & $\begin{array}{c}2.2 \\
3\end{array}$ & Access \\
\hline 171 & \begin{tabular}{l|}
1. \\
1
\end{tabular} & \begin{tabular}{|c|} 
Low \\
gradient \\
Riffle
\end{tabular} & & 18 & 9 & 9 & & $8 \begin{array}{l}0 . \\
2\end{array}$ & \begin{tabular}{l|l}
0.2 & 0. \\
2 & 6
\end{tabular} & $\begin{array}{l}0.2 \\
6\end{array}$ & & & 0 & & & & & & & & & & 595 & & & & & & & & & & & \\
\hline 172 & $\begin{array}{ll}5 . \\
3 \\
R\end{array}$ & \begin{tabular}{|c|} 
Lateral \\
Scour \\
Pool - \\
Root Wad \\
Enhanced \\
Enal
\end{tabular} & 6062 & 270 & 13 & 38 & 23 & $9 \begin{array}{r}1 . \\
1\end{array}$ & \begin{tabular}{c|c}
1.8 & 3. \\
1 & 3.
\end{tabular} & $1 \mid \begin{array}{c}0.1 \\
3\end{array}$ & \begin{tabular}{l|l|}
3 & 40 \\
3 & 40
\end{tabular} & c & 3 & 201 & 015 & 10 & $60 \mid$ & \begin{tabular}{l|l}
5 & 0
\end{tabular} & 0 & 0 & 025 & 5303 & 3010 & 05 & \begin{tabular}{l|l}
0 & 0
\end{tabular} & 0 & 109 & 9040 & 008 & $8 \quad 20$ & 6 & $955^{2}$ & $\begin{array}{c}2.9 \\
7\end{array}$ & Access \\
\hline 173 & $\begin{array}{l}1 . \\
1\end{array}$ & \begin{tabular}{|c|} 
Low \\
gradient \\
Riffle
\end{tabular} & 6062 & 231 & 19 & 9 & & \begin{tabular}{l|l}
29 & 0.
\end{tabular} & \begin{tabular}{l|l}
0.3 & 0.
\end{tabular} & .4 & & & 1 & $<50$ & 80 & 0 & 0 & $20 \mid 0$ & 0 & 0 & 0.0 & 01 & 1050 & 045 & \begin{tabular}{l|l}
0 & 0
\end{tabular} & 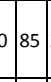 & \begin{tabular}{l|l}
25 & 7
\end{tabular} & \begin{tabular}{l|l}
75 & 0
\end{tabular} & 0 & \begin{tabular}{l|c}
6 & 10 \\
0
\end{tabular} & 8 & 95 & & \\
\hline 174 & $\begin{array}{ll}5 . \\
3 \\
R \\
R\end{array}$ & \begin{tabular}{|c|} 
Lateral \\
Scour \\
Pool - \\
Root Wad \\
Enhanced \\
\end{tabular} & 6062 & 293 & 21 & 1291 & 171 & $17 \begin{array}{r}1 . \\
9\end{array}$ & \begin{tabular}{l|l}
1.1 & 2. \\
9 & 8
\end{tabular} & \begin{tabular}{c|c}
99 \\
8 & 0.2
\end{tabular} & 3 & c & 3 & & $\begin{array}{lll}0 & 10\end{array}$ & 10 & 40 & \begin{tabular}{|l|l}
20 & 0
\end{tabular} & 0 & 0 & \begin{tabular}{l|l}
0 & 25
\end{tabular} & 5253 & 3015 & $\begin{array}{lll}5 & 5\end{array}$ & \begin{tabular}{l|l}
0 & 0
\end{tabular} & $\begin{array}{lll}0 & 87\end{array}$ & \begin{tabular}{|l|l}
50 & 5
\end{tabular} & \begin{tabular}{l|l}
50 & 0
\end{tabular} & 56 & $6 \mid \begin{array}{c}10 \\
0\end{array}$ & 8 & $90^{2}$ & $\begin{array}{c}2.7 \\
5\end{array}$ & \\
\hline 175 & \begin{tabular}{|l|}
3. \\
2 \\
\end{tabular} & Glide & 6163 & 59 & 17 & 717 & 92 & \begin{tabular}{|l|r}
25 & 0. \\
& 7 \\
\end{tabular} & \begin{tabular}{l|c}
.6 & 1. \\
7 & 6 \\
\end{tabular} & $\begin{array}{l}.6 \\
6 \\
\end{array}$ & & & 3 & \begin{tabular}{|l|l}
5 & 0
\end{tabular} & 55 & 0 & 5 & \begin{tabular}{l|l}
40 & 0
\end{tabular} & 0 & 0 & \begin{tabular}{l|l}
0 & 10 \\
\end{tabular} & 0105 & 5020 & $\begin{array}{lll}0 & 10\end{array}$ & \begin{tabular}{l|l}
0 & 0
\end{tabular} & \begin{tabular}{|l|l|}
0 & 94 \\
\end{tabular} & \begin{tabular}{c|c}
10 & 0 \\
0 & \\
\end{tabular} & \begin{tabular}{l|l}
0 & 0 \\
\end{tabular} & 06 & \begin{tabular}{|c|c|}
6 & 10 \\
& 0 \\
\end{tabular} & 7 & 80 & & \\
\hline 176 & $\begin{array}{l}4 . \\
2\end{array}$ & \begin{tabular}{|c|} 
Mid- \\
Channel \\
Pool \\
\end{tabular} & & 51 & 17 & 7251 & 161 & $10{ }^{1}$. & \begin{tabular}{l|l}
1.5 & 2. \\
3 & 3
\end{tabular} & \begin{tabular}{l|l}
3 & 0.4 \\
3 &
\end{tabular} & .4 & c & 3 & 80 & 45 & 40 & 5 & \begin{tabular}{l|l}
0 & 10
\end{tabular} & 00 & 0 & \begin{tabular}{l|l}
0 & 25
\end{tabular} & 525 & 2520 & \begin{tabular}{ll|}
0 & 5 \\
\end{tabular} & \begin{tabular}{l|l}
0 & 0
\end{tabular} & \begin{tabular}{ll|l}
0 & 80
\end{tabular} & \begin{tabular}{c|c}
10 & 0 \\
0 & 0
\end{tabular} & \begin{tabular}{l|l}
0 & 0
\end{tabular} & 00 & $6 \mid$\begin{tabular}{c|c|}
10 \\
0
\end{tabular} & 6 & \begin{tabular}{c|c}
10 & 1 \\
0 & 3
\end{tabular} & $\begin{array}{c}1.7 \\
3\end{array}$ & Log Jam \\
\hline 177 & $\begin{array}{l}3 . \\
2 \\
\end{array}$ & Glide & 6272 & \begin{tabular}{l|l}
2 & 22 \\
\end{tabular} & 16 & 510 & \begin{tabular}{|l|l}
8 & 3 \\
\end{tabular} & \begin{tabular}{|l|r|}
30 & 0. \\
\end{tabular} & \begin{tabular}{|c|c}
0.7 & 1. \\
1 & 6 \\
\end{tabular} & $\begin{array}{l}.2 \\
6 \\
\end{array}$ & & & 3 & \begin{tabular}{|l|l|}
10 & 11 \\
\end{tabular} & 055 & 15 & 0 & $\begin{array}{ll}20 & 0\end{array}$ & 0 & 0 & \begin{tabular}{l|l}
0 & 15
\end{tabular} & 515 & 3030 & \begin{tabular}{l|l|l|l|l|l|l|l} 
& 10
\end{tabular} & $\begin{array}{lll}0 & 0\end{array}$ & \begin{tabular}{ll|}
0 & 78 \\
\end{tabular} & \begin{tabular}{c|c}
10 & 0 \\
0 & \\
\end{tabular} & \begin{tabular}{l|l}
0 & 0 \\
\end{tabular} & 06 & \begin{tabular}{|c|c|}
6 & 10 \\
\end{tabular} & 6 & \begin{tabular}{|c|}
10 \\
0
\end{tabular} & & \\
\hline
\end{tabular}




\begin{tabular}{|c|c|c|c|c|c|c|c|c|c|c|c|c|c|c|c|c|c|c|c|c|c|c|c|c|c|c|c|c|c|c|c|c|c|c|}
\hline 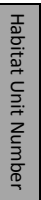 & & 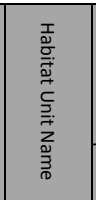 & 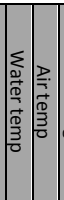 & 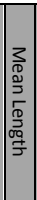 & 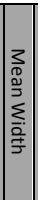 & 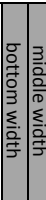 & 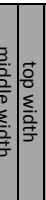 & 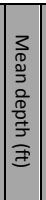 & 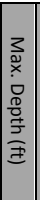 & 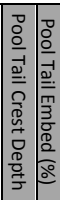 & 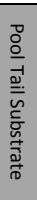 & 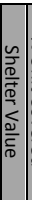 & 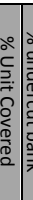 & & 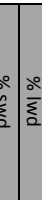 & 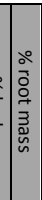 & 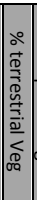 & & 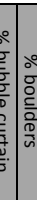 & 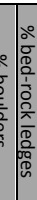 & 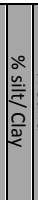 & 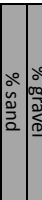 & 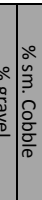 & 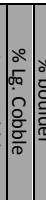 & & 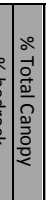 & 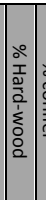 & 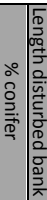 & 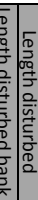 & 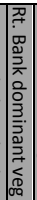 & 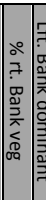 & & 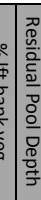 & 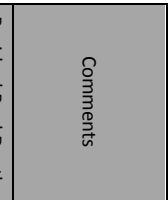 \\
\hline 178 & \begin{tabular}{|l|}
5. \\
1 \\
\end{tabular} & $\begin{array}{l}\text { Corner } \\
\text { Pool }\end{array}$ & & 36 & 23 & 3023 & 2317 & 1.7 & \begin{tabular}{|c|}
2.4 \\
2 \\
\end{tabular} & 0.5 & c & 3 & & 202 & 2010 & 50 & 0 & 00 & 0 & 0 & 40 & 402 & 200 & 0 & 0.0 & 65 & \begin{tabular}{c|}
10 \\
0 \\
\end{tabular} & \begin{tabular}{l|l}
0 & 0
\end{tabular} & \begin{tabular}{l|l}
0 & 0
\end{tabular} & 6 & \begin{tabular}{|c|c|}
10 & \\
0 & 6 \\
\end{tabular} & \begin{tabular}{c|c}
10 \\
6 \\
\end{tabular} & \begin{tabular}{c|c}
0 & 1.9 \\
0 & 2 \\
\end{tabular} & \begin{tabular}{|c|}
9.1 turns into log \\
formed 4.2
\end{tabular} \\
\hline 179 & $\begin{array}{l}5 . \\
2\end{array}$ & \begin{tabular}{|c|} 
Lateral \\
Scour \\
Pool - Log \\
Enhanced
\end{tabular} & & 32 & 13 & $17 \mid 12$ & 1210 & $\begin{array}{c}1.5 \\
2\end{array}$ & $\begin{array}{c}2.2 \\
5\end{array}$ & $\begin{array}{c}1.0 \\
4\end{array}$ & $\mathrm{~b}$ & 3 & 95 & $0 \mid 1 c$ & 1020 & 0 & 60 & 00 & 0 & 0 & 50 & 500 & 0.0 & 0 & 0.0 & $\begin{array}{c}10 \\
0\end{array}$ & $\begin{array}{c}10 \\
0\end{array}$ & \begin{tabular}{l|l}
0 & 0
\end{tabular} & \begin{tabular}{l|l}
0 & 0
\end{tabular} & 6 & 80 & 6 \begin{tabular}{c|c}
10 \\
0
\end{tabular} & \begin{tabular}{l|l}
0 & 1.2 \\
0 & 1
\end{tabular} & \\
\hline 180 & $\begin{array}{l}3 . \\
4\end{array}$ & Step Run & 6267 & $\begin{array}{c}20 \\
2\end{array}$ & 12 & 1015 & 1512 & $\begin{array}{c}0.5 \\
4\end{array}$ & $\begin{array}{c}1.0 \\
9\end{array}$ & & & 3 & 5 & $5 \mid 2$ & 2510 & 30 & 30 & 00 & \begin{tabular}{l|l}
0 & 0
\end{tabular} & 0 & 10 & 102 & 2040 & 20 & \begin{tabular}{l|l}
0 & 0
\end{tabular} & $\begin{array}{c}10 \\
0\end{array}$ & $\begin{array}{c}10 \\
0\end{array}$ & \begin{tabular}{l|l}
0 & 0
\end{tabular} & \begin{tabular}{l|l}
0 & 0
\end{tabular} & 6 & $\begin{array}{c}10 \\
0\end{array}$ & 690 & & \begin{tabular}{|c|}
+120 LB dry trib \\
heavily \\
vegetated. Two \\
riffles (37, $27 \mathrm{ft})$, \\
three runs. \\
\end{tabular} \\
\hline 181 & $\begin{array}{l}4 . \\
2\end{array}$ & $\begin{array}{c}\text { Mid- } \\
\text { Channel } \\
\text { Pool } \\
\end{array}$ & 6166 & 19 & 12 & 1215 & 1510 & $\begin{array}{c}1.2 \\
1\end{array}$ & $\begin{array}{c}1.7 \\
5\end{array}$ & $\begin{array}{c}0.3 \\
5\end{array}$ & b & 1 & 5 & \begin{tabular}{l|l}
0 & 8
\end{tabular} & \begin{tabular}{l|l}
30 & 0
\end{tabular} & 0 & 20 & 00 & \begin{tabular}{l|l}
0 & 0
\end{tabular} & 0 & 35 & $35 \mid 2$ & $\begin{array}{ll}20 & 5\end{array}$ & 5 & \begin{tabular}{l|l}
0 & 0
\end{tabular} & $\begin{array}{c}10 \\
0\end{array}$ & $\begin{array}{c}10 \\
0\end{array}$ & \begin{tabular}{l|l}
0 & 0
\end{tabular} & $\begin{array}{lll}0 & 0\end{array}$ & 6 & $\begin{array}{c}10 \\
0\end{array} \mid$ & 6 \begin{tabular}{c|c}
10 \\
0
\end{tabular} & \begin{tabular}{l|l}
0 & 1.4 \\
0 & 1.4
\end{tabular} & \\
\hline 182 & $\begin{array}{l}1 . \\
1\end{array}$ & \begin{tabular}{|c|} 
Low \\
gradient \\
Riffle
\end{tabular} & & 15 & 12 & 10 & 13 & $\begin{array}{c}0.3 \\
1\end{array} \mid$ & $\left|\begin{array}{c}0.4 \\
5\end{array}\right|$ & & & & & & & & & & & & & & & & & & & & & & & & & \\
\hline 183 & $\begin{array}{l}4 . \\
2\end{array}$ & \begin{tabular}{|c|} 
Mid- \\
Channel \\
Pool \\
\end{tabular} & 6267 & 32 & 23 & 1333 & 3322 & $\begin{array}{c}1.3 \\
9\end{array}$ & $\begin{array}{c}2.6 \\
2\end{array} \mid$ & $\begin{array}{c}0.3 \\
5\end{array}$ & c & & 52 & $20 \mid 7$ & 0 & 0 & 10 & 00 & 0 & 0 & 15 & 155 & 5015 & 5 & \begin{tabular}{l|l}
0 & 0
\end{tabular} & 82 & $\begin{array}{c}10 \\
0\end{array}$ & $0 \mid 0$ & \begin{tabular}{l|l}
0 & 0
\end{tabular} & 6 & $\left|\begin{array}{c}10 \\
0\end{array}\right|$ & 6 \begin{tabular}{c|c}
10 \\
0
\end{tabular} & \begin{tabular}{l|l}
0 & 2.2 \\
0 & 7
\end{tabular} & 2 Log enhanced \\
\hline 184 & $\begin{array}{l}3 . \\
4 \\
\end{array}$ & Step Run & 6167 & $\begin{array}{c}16 \\
1\end{array}$ & 13 & 2211 & 117 & 0.5 & 1.2 & & & 3 & 5 & \begin{tabular}{l|l}
5 & 11
\end{tabular} & \begin{tabular}{l|l}
15 & 0
\end{tabular} & 20 & 50 & 00 & \begin{tabular}{l|l}
0 & 0
\end{tabular} & 0 & 20 & 203 & 3020 & 10 & 00 & 80 & $\begin{array}{c}10 \\
0\end{array}$ & \begin{tabular}{l|l}
0 & 0 \\
\end{tabular} & \begin{tabular}{l|l}
0 & 0
\end{tabular} & 6 & 956 & \begin{tabular}{l|l}
6 & 95
\end{tabular} & & \\
\hline 185 & $\begin{array}{l}4 . \\
2\end{array}$ & $\begin{array}{c}\text { Mid- } \\
\text { Channel } \\
\text { Pool } \\
\end{array}$ & 6166 & 43 & 10 & 712 & 1211 & $\begin{array}{c}1.2 \\
2\end{array}$ & $\begin{array}{c}2.1 \\
4\end{array} \mid$ & $\begin{array}{c}0.2 \\
9\end{array}$ & c & 3 & \begin{tabular}{l|l}
30 & 1
\end{tabular} & 100 & \begin{tabular}{l|l}
0 & 0
\end{tabular} & 40 & 50 & 00 & \begin{tabular}{l|l}
0 & 0
\end{tabular} & $\begin{array}{ll}0 & 0\end{array}$ & 20 & 203 & $30 \mid 20$ & 10 & 0.0 & 69 & $\begin{array}{c}10 \\
0\end{array}$ & \begin{tabular}{l|l}
0 & 0
\end{tabular} & $\begin{array}{lll}0 & 0\end{array}$ & 7 & 956 & 6 \begin{tabular}{c|c}
10 \\
0
\end{tabular} & \begin{tabular}{l|l}
0 & 1.8 \\
0 & 5
\end{tabular} & \\
\hline 186 & $\begin{array}{l}1 . \\
1\end{array}$ & \begin{tabular}{|c|} 
Low \\
gradient \\
Riffle
\end{tabular} & 6266 & 50 & 10 & 1117 & 711 & $\begin{array}{c}0.3 \\
4\end{array}$ & $\left|\begin{array}{c}0.4 \\
5\end{array}\right|$ & & & 1 & 60 & $0 \mid 2$ & \begin{tabular}{l|l}
0 & 0
\end{tabular} & 20 & 60 & 00 & 0 & $\begin{array}{lll}0 & 0\end{array}$ & 0 & 03 & 3040 & 30 & \begin{tabular}{l|l}
0 & 0
\end{tabular} & 87 & $\begin{array}{c}10 \\
0\end{array}$ & $0 \mid 0$ & $\begin{array}{lll}0 & 0\end{array}$ & 7 & 80 & 6 \begin{tabular}{c|c}
10 \\
0
\end{tabular} & & \\
\hline 187 & $\begin{array}{l}5 . \\
2\end{array}$ & \begin{tabular}{|c|} 
Lateral \\
Scour \\
Pool - Log \\
Enhanced
\end{tabular} & 6264 & 51 & 16 & $14 \mid 22$ & 2211 & $\left|\begin{array}{c}1.0 \\
2\end{array}\right|$ & $\begin{array}{c}2.0 \\
1\end{array}$ & $\begin{array}{c}0.2 \\
3\end{array}$ & d & 3 & 10 & 03 & 3550 & 10 & 5 & 00 & 0 & $\begin{array}{ll}0 & 0\end{array}$ & 20 & 204 & 1015 & 5 & 0.0 & $\begin{array}{c}10 \\
0\end{array}$ & $\begin{array}{c}10 \\
0\end{array}$ & $0 \mid 0$ & \begin{tabular}{l|l}
0 & 0
\end{tabular} & 7 & 156 & $6 \mid$\begin{tabular}{c|c}
10 \\
0
\end{tabular} & \begin{tabular}{l|l}
0 & 1.7 \\
0 & 8
\end{tabular} & end $7 / 29$ \\
\hline 188 & $\begin{array}{l}3 . \\
4\end{array}$ & Step Run & 6064 & 54 & 12 & 1118 & 186 & $\begin{array}{c}0.8 \\
4\end{array}$ & $\begin{array}{c}1.3 \\
6\end{array}$ & & & 2 & 51 & 103 & 3050 & 10 & 0 & 00 & \begin{tabular}{l|l}
0 & 0
\end{tabular} & $\begin{array}{ll}0 & 0\end{array}$ & 0 & 52 & 2040 & 305 & \begin{tabular}{l|l}
5 & 0
\end{tabular} & $\begin{array}{c}10 \\
0\end{array}$ & $\begin{array}{c}10 \\
0\end{array}$ & \begin{tabular}{l|l}
0 & 0
\end{tabular} & \begin{tabular}{l|l}
0 & 0
\end{tabular} & 7 & 206 & 690 & & $\begin{array}{c}\text { Start } 7 / 30 . \text { two } \\
\text { riffles }(5,12 \mathrm{ft}) \\
\text { one run }\end{array}$ \\
\hline 189 & $\begin{array}{l}4 . \\
2\end{array}$ & $\begin{array}{c}\text { Mid- } \\
\text { Channel } \\
\text { Pool }\end{array}$ & 6063 & 84 & 15 & 2617 & 173 & $\begin{array}{c}1.9 \\
7\end{array}$ & $\begin{array}{c}4.4 \\
7\end{array} \mid$ & 0.310 & c & 3 & 20 & \begin{tabular}{l|l}
5 & 4
\end{tabular} & \begin{tabular}{l|l}
10 & 45
\end{tabular} & 510 & 0 & $0 \mid 0$ & \begin{tabular}{l|l}
0 & 0
\end{tabular} & \begin{tabular}{l|l}
0 & 0
\end{tabular} & 25 & & 305 & 5 & \begin{tabular}{l|l}
0 & 0
\end{tabular} & $\begin{array}{c}10 \\
0\end{array}$ & $\begin{array}{c}10 \\
0\end{array}$ & 0.0 & $0 \begin{array}{ll}0 & 10\end{array}$ & 7 & 75 & 7780 & $0 \begin{array}{l}4.1 \\
7\end{array}$ & $1 \begin{array}{c}\text { two pools } \\
\text { connected by } \\
\text { deep water, } \\
\text { second is wood } \\
\text { formed. Log jam }\end{array}$ \\
\hline 190 & $\begin{array}{l}1 . \\
1\end{array}$ & \begin{tabular}{|c|} 
Low \\
gradient \\
Riffle
\end{tabular} & 6164 & 55 & 10 & 710 & 1013 & $\begin{array}{c}0.4 \\
5\end{array}$ & $\begin{array}{c}0.9 \\
8\end{array} \mid$ & & & 1 & 5 & 59 & \begin{tabular}{l|l}
35 & 0
\end{tabular} & 0 & 0 & $0 \mid 0$ & \begin{tabular}{l|l}
0 & 0
\end{tabular} & \begin{tabular}{l|l}
0 & 0
\end{tabular} & 5 & 103 & 3040 & 15 & 0 & 98 & $\begin{array}{c}10 \\
0\end{array}$ & 0 & \begin{tabular}{l|l}
0 & 0
\end{tabular} & 7 & 80 & \begin{tabular}{l|l}
6 & 95
\end{tabular} & & \\
\hline 191 & $\begin{array}{l}4 . \\
2\end{array}$ & \begin{tabular}{|c|} 
Mid- \\
Channel \\
Pool \\
\end{tabular} & 6064 & 37 & 14 & 1318 & 1810 & $\begin{array}{c}1.4 \\
1\end{array}$ & $\begin{array}{c}2.3 \\
2\end{array}$ & 0.320 & d & & 52 & 203 & 3030 & 20 & 0 & 00 & 00 & $\begin{array}{ll}0 & 0\end{array}$ & 30 & 303 & 305 & 50 & \begin{tabular}{l|l}
0 & 0
\end{tabular} & $\begin{array}{c}10 \\
0\end{array}$ & $\begin{array}{c}10 \\
0\end{array}$ & 0.0 & $\begin{array}{lll}0 & 0\end{array}$ & 7 & 956 & 695 & $5 \frac{2.0}{2}$ & \\
\hline 192 & $\begin{array}{c}1 . \\
1\end{array}$ & \begin{tabular}{|c|} 
Low \\
gradient \\
Riffle
\end{tabular} & 6064 & 63 & 9 & $10 \mid 6$ & 610 & $\left|\begin{array}{c}0.6 \\
3\end{array}\right|$ & $\begin{array}{c}1.1 \\
5\end{array}$ & & & 3 & 601 & 101 & 1010 & 60 & 0 & 00 & 0.0 & 05 & 5 & 52 & 2535 & 255 & 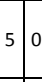 & 86 & $\begin{array}{c}10 \\
0\end{array}$ & \begin{tabular}{l|l}
0 & 0
\end{tabular} & 0.0 & 7 & $\left|\begin{array}{c}10 \\
0\end{array}\right|$ & 6 \begin{tabular}{c|c}
10 \\
0
\end{tabular} & & \\
\hline 193 & $\begin{array}{l}4 . \\
2\end{array}$ & \begin{tabular}{|c|} 
Mid- \\
Channel \\
Pool \\
\end{tabular} & 6064 & 86 & 13 & 10 & 15 & $\begin{array}{c}1.8 \\
6\end{array} \mid$ & $\begin{array}{c}3.3 \\
3\end{array}$ & $\begin{array}{c}0.5 \\
9\end{array}$ & c & 75 & 05 & 505 & 00 & 0 & 0 & 00 & 0.0 & $\begin{array}{ll}0 & 0\end{array}$ & 40 & 401 & 105 & 5 & 00 & 96 & $\begin{array}{c}10 \\
0\end{array}$ & 0.0 & $\begin{array}{lll}0 & 0\end{array}$ & 7 & $\left|\begin{array}{c}10 \\
0\end{array}\right|$ & 695 & 5 & 7 Log jam, nutmeg \\
\hline 194 & $\begin{array}{l}1 . \\
1\end{array}$ & $\begin{array}{c}\text { Low } \\
\text { gradient } \\
\text { Riffle }\end{array}$ & 6064 & 45 & 17 & 1522 & 2215 & $\begin{array}{c}0.4 \\
4\end{array}$ & $\begin{array}{c}0.8 \\
1\end{array}$ & & & 1 & 5 & 02 & 2080 & 0 & 0 & 00 & 00 & $\begin{array}{ll}0 & 0\end{array}$ & 0 & 03 & 3545 & 20 & 0 & 97 & $\begin{array}{c}10 \\
0\end{array}$ & 0 & 0.0 & 6 & $\left|\begin{array}{c}10 \\
0\end{array}\right|$ & 6 \begin{tabular}{c|c}
10 \\
0
\end{tabular} & & \\
\hline $\begin{array}{c}194 . \\
1\end{array}$ & $\begin{array}{l}6 . \\
4 \\
\end{array}$ & & 6064 & 36 & 11 & & & $\begin{array}{c}1.2 \\
5\end{array}$ & 1.7 & $\begin{array}{c}0.0 \\
5\end{array}$ & & 3 & 75 & 0.4 & 1050 & 0 & 0 & 100 & 00 & $\begin{array}{ll}0 & 0\end{array}$ & 30 & 303 & 3010 & & & & & & & & & & $\begin{array}{c}1.6 \\
5\end{array}$ & \\
\hline 195 & $\begin{array}{l}4 . \\
2\end{array}$ & $\begin{array}{c}\text { Mid- } \\
\text { Channel } \\
\text { Pool } \\
\end{array}$ & 6064 & 30 & 15 & 1516 & 1615 & $\begin{array}{c}1.1 \\
9\end{array}$ & $\begin{array}{c}2.2 \\
6\end{array}$ & \begin{tabular}{c|c}
0.2 & 20 \\
1 & 20
\end{tabular} & d & 3 & 5 & $10 \mid 4 c$ & 100 & 50 & 0 & 00 & 00 & $\begin{array}{ll}0 & 0\end{array}$ & 25 & 303 & 3015 & 0 & 0 & $\mid \begin{array}{c}10 \\
0\end{array}$ & $\begin{array}{c}10 \\
0\end{array}$ & 0.0 & 0.0 & 6 & 956 & 695 & 5 & 0 root wad formed \\
\hline 196 & $\begin{array}{l}4 . \\
2\end{array}$ & $\begin{array}{c}\text { Mid- } \\
\text { Channel } \\
\text { Pool }\end{array}$ & 6165 & 36 & 24 & 1532 & 3224 & 1.6 & $\begin{array}{c}2.9 \\
5\end{array}$ & $\begin{array}{c}0.9 \\
4\end{array}$ & c & 1 & $<5$ & \begin{tabular}{l|l}
0 & 11 \\
0
\end{tabular} & 1010 & 0 & 5 & 00 & 075 & $\begin{array}{ll}5 & 0\end{array}$ & 20 & 252 & 2510 & 101 & 100 & 98 & $\begin{array}{c}10 \\
0\end{array}$ & \begin{tabular}{l|l}
0 & 0
\end{tabular} & $\begin{array}{lll}0 & 0\end{array}$ & 6 & 906 & $\left.6\right|_{0} ^{10}$ & \begin{tabular}{l|l}
0 & $2 . C_{1}$ \\
0 & 1
\end{tabular} & $0 \begin{array}{c}\text { Boulders }= \\
\text { concrete blocks. } \\
\text { Concrete } \\
\text { blocks } / \text { slabs on } \\
\text { RB }\end{array}$ \\
\hline 197 & $\begin{array}{l}3 . \\
4\end{array}$ & Step Run & & \begin{tabular}{c|}
10 \\
0
\end{tabular} & 16 & 2415 & \begin{tabular}{|l|l|}
15 & 10
\end{tabular} & $\begin{array}{c}0.7 \\
5\end{array}$ & $\begin{array}{c}2.2 \\
2\end{array}$ & & & & 5 & $15 \mid 2$ & $\begin{array}{ll}5 & 30\end{array}$ & 0 & 0 & 03 & 300 & $\begin{array}{ll}0 & 0\end{array}$ & 0 & 204 & 1030 & 10 & \begin{tabular}{l|l}
0 & 96
\end{tabular} & $6 \begin{array}{c}10 \\
0\end{array}$ & 0 & 0.0 & 0 & 6 & 80 & \begin{tabular}{l|l}
6 & 10 \\
0
\end{tabular} & & $\begin{array}{c}\text { two riffles }(10,25 \\
\mathrm{ft}) \text { one run }\end{array}$ \\
\hline 198 & $\begin{array}{l}4 . \\
2\end{array}$ & \begin{tabular}{|c|} 
Mid- \\
Channel \\
Pool \\
\end{tabular} & 6165 & 20 & 18 & 1035 & 395 & 1.1 & 1.8 & \begin{tabular}{c|c}
0.2 & 15 \\
6 & 15
\end{tabular} & c & 3 & 80 & \begin{tabular}{l|l}
0 & 2
\end{tabular} & 2540 & 35 & 0 & 0 & 0.0 & $\begin{array}{ll}0 & 0\end{array}$ & 30 & 303 & 305 & 5 & 0.0 & 93 & $\begin{array}{c}10 \\
0\end{array}$ & 0.0 & 0.0 & 6 & 956 & 6 \begin{tabular}{c|c}
10 \\
0
\end{tabular} & \begin{tabular}{l|l}
0 & 1.5 \\
0 & 4
\end{tabular} & Log Jam \\
\hline 199 & $\begin{array}{l}3 . \\
4\end{array}$ & Step Run & & \begin{tabular}{c|}
10 \\
0
\end{tabular} & 15 & \begin{tabular}{|l|l}
5 & 15 \\
\end{tabular} & 1525 & 0.4 & \begin{tabular}{|c|}
0.7 \\
8
\end{tabular} & & & & 5 & \begin{tabular}{l|l}
0 & 5
\end{tabular} & 5050 & 0 & 0 & 0 & 00 & $\begin{array}{ll}0 & 0\end{array}$ & 10 & 103 & 3030 & 15 & $5 \mid 0$ & $\begin{array}{c}10 \\
0\end{array}$ & $\begin{array}{c}10 \\
0\end{array}$ & \begin{tabular}{l|l}
0 & 0
\end{tabular} & $\begin{array}{lll}0 & 0\end{array}$ & 6 & 956 & 69 & 5 & $\begin{array}{c}\text { two riffles, }(15, \\
15 \mathrm{ft})\end{array}$ \\
\hline 200 & $\begin{array}{l}3 . \\
4\end{array}$ & Step Run & 6167 & $\begin{array}{c}32 \\
5\end{array}$ & 17 & 2512 & 1214 & $\begin{array}{c}0.8 \\
3\end{array}$ & $\left|\begin{array}{c}1.7 \\
2\end{array}\right|$ & & & 3 & 15 & $5 \mid 2$ & \begin{tabular}{l|l}
25 & 30
\end{tabular} & 30 & 15 & 00 & \begin{tabular}{l|l}
0 & 0
\end{tabular} & $\begin{array}{lll}0 & 0\end{array}$ & 25 & 252 & & 10 & \begin{tabular}{l|l}
5 & 0
\end{tabular} & 93 & $\begin{array}{c}10 \\
0\end{array} \mid$ & \begin{tabular}{l|l}
0 & 0
\end{tabular} & \begin{tabular}{l|l}
0 & 0
\end{tabular} & 6 & 956 & 69 & & $\begin{array}{c}\text { + } 113 \text { LB Mill } \\
\text { creek confluence. } \\
\text { More of a step } \\
\text { glide }\end{array}$ \\
\hline
\end{tabular}




\begin{tabular}{|c|c|c|c|c|c|c|c|c|c|c|c|c|c|c|c|c|c|c|c|c|c|c|c|c|c|c|c|c|c|c|c|c|c|c|}
\hline 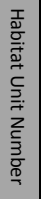 & & 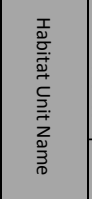 & 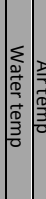 & 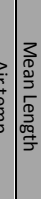 & 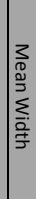 & 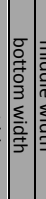 & 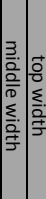 & 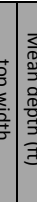 & 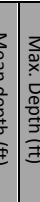 & 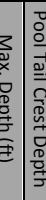 & 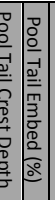 & 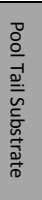 & 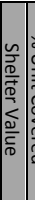 & 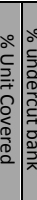 & 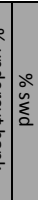 & $\frac{\circ}{\grave{c}}$ & 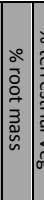 & 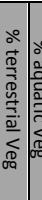 & 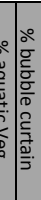 & 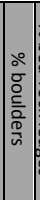 & & 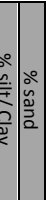 & 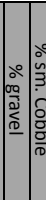 & 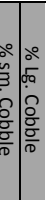 & 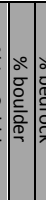 & & 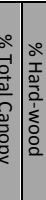 & 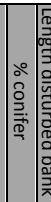 & 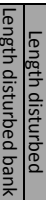 & 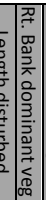 & 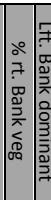 & 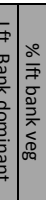 & 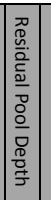 & $\begin{array}{l}\mathfrak{0} \\
3 \\
3 \\
\frac{0}{3} \\
\vec{\omega}\end{array}$ \\
\hline 201 & $\begin{array}{l}4 . \\
2\end{array}$ & \begin{tabular}{|c|} 
Mid- \\
Channel \\
Pool \\
\end{tabular} & 636 & 8834 & 18 & 143 & 3010 & 10 & $\begin{array}{l}0.03 . \\
3\end{array}$ & \begin{tabular}{l|l}
3.8 & 0. \\
6 & 9
\end{tabular} & 9 & c & 36 & 600 & 50 & 45 & 5 & $0 \mid c$ & 0 & 0 & $03 c$ & 3035 & 305 & 50 & 0 & 096 & $96 \begin{array}{c}10 \\
0\end{array}$ & 0 & \begin{tabular}{l|l}
0 & 0
\end{tabular} & 06 & \begin{tabular}{c|c}
10 & 6 \\
0 & 6
\end{tabular} & $6 \mid \begin{array}{c}10 \\
0\end{array}$ & $\begin{array}{c}3.5 \\
7\end{array} \mid$ & Log Jam \\
\hline 202 & \begin{tabular}{|c|}
3. \\
4 \\
\end{tabular} & Step Run & 637 & \begin{tabular}{l|l}
8 & 42
\end{tabular} & 9 & 101 & 117 & $7 \begin{array}{r}0 . \\
3\end{array}$ & \begin{tabular}{l|c}
5 & 1. \\
3 & 5 \\
\end{tabular} & \begin{tabular}{l|}
.1 \\
5 \\
\end{tabular} & & & 31 & 101 & 010 & 25 & $\left.20\right|^{3}$ & $35 \mid c$ & $\begin{array}{l}0 \\
0\end{array}$ & 0 & 0 & 1010 & 403 & 3010 & 0 & \begin{tabular}{l|l}
0 & 83 \\
\end{tabular} & \begin{tabular}{c|c}
33 & 10 \\
0
\end{tabular} & 00 & \begin{tabular}{l|l}
0 & 0 \\
\end{tabular} & 06 & \begin{tabular}{|c|c|}
10 & 6 \\
0 & 6 \\
\end{tabular} & \begin{tabular}{|c|c|}
6 & 10 \\
\end{tabular} & & $\begin{array}{c}\text { one riffle (10 ft), } \\
\text { one run }\end{array}$ \\
\hline 203 & $\begin{array}{l}4 . \\
2\end{array}$ & \begin{tabular}{|c|} 
Mid- \\
Channel \\
Pool \\
\end{tabular} & 658 & 450 & 16 & 73 & 3012 & $12 \frac{1 .}{3}$ & $\begin{array}{ll}.0 & 2 . \\
3 & 9\end{array}$ & \begin{tabular}{l|l}
2.0 & 0. \\
9 & 2
\end{tabular} & $\begin{array}{l}2.4 \\
2\end{array}$ & c & 39 & 950 & 50 & 50 & 0 & $0 \mid c$ & $\begin{array}{lll}0 & 0\end{array}$ & 0 & 030 & 3030 & 3016 & 100 & 0 & 065 & $65 \mid \begin{array}{c}10 \\
0\end{array}$ & 0 & \begin{tabular}{l|l}
0 & 0
\end{tabular} & 06 & \begin{tabular}{c|c}
10 & 6 \\
0 & 6
\end{tabular} & $6 \mid \begin{array}{c}10 \\
0\end{array}$ & $\begin{array}{c}1.6 \\
7\end{array}$ & \\
\hline 204 & $\begin{array}{l}3 . \\
4\end{array}$ & Step Run & & $\begin{array}{c}33 \\
0\end{array}$ & 14 & 121 & 1120 & 200. & \begin{tabular}{l|l}
7 & 1. \\
4 & 8
\end{tabular} & $\begin{array}{l}.7 \\
8\end{array}$ & & & 32 & & 020 & 20 & $\left.20\right|^{2}$ & 200 & \begin{tabular}{l|l}
0 & 0
\end{tabular} & 0 & 01 & 1515 & 253 & 3010 & 5 & $0 \mid 76$ & \begin{tabular}{c|c}
76 & 10 \\
0
\end{tabular} & 0 & \begin{tabular}{l|l}
0 & 0
\end{tabular} & 0.6 & \begin{tabular}{|l|l|}
90 & 6
\end{tabular} & \begin{tabular}{l|l}
6 & 90
\end{tabular} & & $\begin{array}{c}\text { four riffles (25, } \\
25,10,25 \mathrm{ft})\end{array}$ \\
\hline $\begin{array}{c}204 . \\
1\end{array}$ & $\begin{array}{l}6 . \\
4 \\
\end{array}$ & \begin{tabular}{|c} 
Backwate \\
r Pool - \\
log \\
formed \\
\end{tabular} & & 14 & 8 & & 8 & 0. & 81. & .5 & & & & & & & & & & & & & & & & & & & & & & & $1.5^{n}$ & $\begin{array}{l}\text { not connected to } \\
\text { main channel }\end{array}$ \\
\hline $\begin{array}{c}204 . \\
2\end{array}$ & $\begin{array}{l}6 . \\
4 \\
\end{array}$ & \begin{tabular}{|c|} 
Backwate \\
r Pool - \\
log \\
formed
\end{tabular} & & 12 & 24 & & 24 & 1. & $\begin{array}{l}3.2 \\
4 \\
4\end{array}$ & \begin{tabular}{l|l}
.3 & 0. \\
7
\end{tabular} & .4 & & & & & & & & & & & & & & & & & & & & & & $\begin{array}{c}1.9 \\
7\end{array}$ & \\
\hline 205 & $\begin{array}{l}4 . \\
2\end{array}$ & \begin{tabular}{|c|} 
Mid- \\
Channel \\
Pool \\
\end{tabular} & 627 & 240 & 21 & 20 & 21 & 21. & \begin{tabular}{l|l}
.9 & 4 \\
8 & 4
\end{tabular} & 4 . & .3 & c & 3 & 950 & 35 & 40 & 25 & $0 \mid c$ & \begin{tabular}{l|l}
0 & 0
\end{tabular} & 0 & 030 & 3030 & $30 \mid 1$ & 10 & 0 & \begin{tabular}{l|l}
0 & 85
\end{tabular} & \begin{tabular}{l|c}
85 \\
0
\end{tabular} & 0 & \begin{tabular}{l|l}
0 & 0
\end{tabular} & $0 \mid 7$ & \begin{tabular}{l|l}
80 & 6
\end{tabular} & $6 \mid \begin{array}{c}10 \\
0\end{array}$ & 3.7 & Log Jam \\
\hline 206 & $\begin{array}{l}3 . \\
4 \\
\end{array}$ & Step Run & 627 & $\begin{array}{ll}19 \\
1 \\
\end{array}$ & 14 & 10 & \begin{tabular}{|l|l|}
8 & 23 \\
\end{tabular} & $\begin{array}{r}23 . \\
8 \\
\end{array}$ & \begin{tabular}{c|c}
7 & 1. \\
3 & 1 \\
\end{tabular} & $\begin{array}{l}1.5 \\
1 \\
\end{array}$ & & & 3 & 105 & 30 & 10 & 55 & $0 \mid 0$ & \begin{tabular}{l|l}
0 & 0
\end{tabular} & 0 & 015 & 1515 & 5010 & 105 & 0 & \begin{tabular}{|l|c|}
5 & 10 \\
\end{tabular} & \begin{tabular}{l|l}
10 & 80 \\
0 & 80 \\
\end{tabular} & 20 & \begin{tabular}{l|l}
0 & 0
\end{tabular} & \begin{tabular}{l|l|l}
0 & 8
\end{tabular} & 95 & $7 \mid 90$ & & 1 riffle, $43 \mathrm{ft}$. \\
\hline $\begin{array}{c}206 . \\
1\end{array}$ & $\begin{array}{l}6 . \\
4 \\
\end{array}$ & \begin{tabular}{|c|} 
Backwate \\
r Pool - \\
log \\
formed
\end{tabular} & & 20 & 12 & & & 0. & $\begin{array}{l}9 \\
9 \\
9 \\
9\end{array}$ & \begin{tabular}{l|l}
.4 & 0. \\
1 & 8
\end{tabular} & 8 & & & & & & & & & & & & & & & & & & & & & & $\begin{array}{c}1.2 \\
3\end{array}$ & \\
\hline 207 & $\begin{array}{l}4 . \\
2\end{array}$ & \begin{tabular}{|c|} 
Mid- \\
Channel \\
Pool \\
\end{tabular} & 616 & 9933 & 25 & 233 & 3814 & $142_{2}^{2}$ & \begin{tabular}{l|l}
3 & 4. \\
2 & 5
\end{tabular} & \begin{tabular}{l|l}
1.2 & 0. \\
5 & 5
\end{tabular} & 5 & b & 36 & 600 & 35 & 40 & 25 & $0 \mid c$ & $\begin{array}{lll}0 & 0\end{array}$ & 0 & 02 & 2540 & $25 \mid 1 C$ & 100 & 0 & 0 \begin{tabular}{c|c}
10 \\
0
\end{tabular} & \begin{tabular}{l|c}
10 & 10 \\
0 & 0
\end{tabular} & 0 & \begin{tabular}{l|l}
0 & 0
\end{tabular} & 0.6 & 606 & $6 \quad 80$ & 4.1 & log Jam \\
\hline 208 & $\begin{array}{l}1 . \\
1\end{array}$ & \begin{tabular}{|c|} 
Low \\
gradient \\
Riffle \\
\end{tabular} & 626 & 7760 & 13 & 14 & 5 & $\left.19\right|_{8} ^{0 .}$ & $\begin{array}{cc}2 & 0 . \\
8 & 9\end{array}$ & $\begin{array}{l}0.6 \\
9\end{array}$ & & & 1 & $5 \mid 0$ & 80 & 0 & 20 & $0 \mid c$ & $\begin{array}{lll}0 & 0\end{array}$ & 0 & $0 \mid 0$ & 00 & 207 & 7010 & 0 & 0 \begin{tabular}{c|c}
10 \\
0
\end{tabular} & \begin{tabular}{l|l}
10 & 10 \\
0 & 0
\end{tabular} & 0 & \begin{tabular}{l|l}
0 & 0
\end{tabular} & 0.7 & \begin{tabular}{l|l}
80 & 6
\end{tabular} & \begin{tabular}{l|l}
6 & 75
\end{tabular} & & \\
\hline 209 & $\begin{array}{l}5 . \\
1\end{array}$ & $\begin{array}{c}\text { Corner } \\
\text { Pool }\end{array}$ & 626 & 496 & 17 & 192 & 2013 & 13.4 & 8. & $.5 \mid \begin{array}{c}0.2 \\
6\end{array}$ & \begin{tabular}{l|l}
.2 & 20 \\
6 & 20
\end{tabular} & $d$ & 31 & $10 \mid 1$ & 020 & 5 & 65 & $0 \mid c$ & $\begin{array}{lll}0 & 0\end{array}$ & 0 & 030 & 3030 & 3010 & 100 & 0 & 095 & $99 \begin{array}{c}10 \\
0\end{array}$ & 0 & \begin{tabular}{l|l}
0 & 5
\end{tabular} & 5 & \begin{tabular}{l|l}
80 & 6
\end{tabular} & 690 & \begin{tabular}{|c|c|}
3.2 & $\mathrm{~T}$ \\
4 & \\
\end{tabular} & $\begin{array}{c}\text { Trees on LB roots } \\
\text { add complexity. } \\
\text { End } 7 / 30 \\
\end{array}$ \\
\hline 210 & $\begin{array}{l}1 . \\
1 \\
\end{array}$ & $\begin{array}{c}\text { Low } \\
\text { gradient } \\
\text { Riffle }\end{array}$ & & 11 & 9 & 8 & 10 & $\left.10\right|_{8} ^{0 .}$ & $\begin{array}{ll}1 & 0 . \\
8 & 9\end{array}$ & $\begin{array}{l}0.1 \\
9\end{array}$ & & & & & & & & & & & & 0.0 & 306 & 6010 & & & & & & & & & & start 8/1 \\
\hline 211 & $\begin{array}{l}4 . \\
2\end{array}$ & \begin{tabular}{|c|} 
Mid- \\
Channel \\
Pool \\
\end{tabular} & 606 & 662 & 18 & 101 & 1629 & 29. & $\begin{array}{cc}3 & 2 . \\
7 & 1\end{array}$ & \begin{tabular}{l|l}
.2 & 0. \\
1 & 4
\end{tabular} & \begin{tabular}{l|l|} 
& 15 \\
\end{tabular} & c & 32 & $20 \mid 1$ & 020 & 35 & 35 & $0 \mid c$ & \begin{tabular}{l|l}
0 & 0
\end{tabular} & 0 & 02 & 2020 & 401 & 155 & 0 & 0 \begin{tabular}{c|c}
10 \\
0
\end{tabular} & \begin{tabular}{l|c}
10 & 10 \\
0 & 0
\end{tabular} & 0 & \begin{tabular}{l|l}
0 & 0
\end{tabular} & \begin{tabular}{l|l|l}
0 & 7
\end{tabular} & \begin{tabular}{|l|l|l}
85 & 6
\end{tabular} & 695 & $\begin{array}{c}2.0 \\
7\end{array} \mid$ & $\begin{array}{c}\text { connected } 4.2 \\
\text { and } 6.3\end{array}$ \\
\hline 212 & $\begin{array}{l}3 . \\
4\end{array}$ & Step Run & 606 & 55 & 10 & 6 & 14 & $\begin{array}{c}0 . \\
8\end{array}$ & \begin{tabular}{l|c}
3 & 1. \\
8 & 3 \\
\end{tabular} & $\begin{array}{l}.0 \\
3 \\
\end{array}$ & & & 2 & $<50$ & 90 & 10 & 0 & $0 \mid c$ & $\begin{array}{lll}0 & 0\end{array}$ & 0 & & 55 & 2535 & 3520 & 10 & 0 & & & & & & & & $\begin{array}{l}\text { two riffles }(47,66 \\
\text { ft), one run }\end{array}$ \\
\hline $\begin{array}{c}212 . \\
1\end{array}$ & \begin{tabular}{c|}
6. \\
1
\end{tabular} & \begin{tabular}{|c|} 
Secondar \\
y Channel \\
Pool \\
\end{tabular} & & 33 & 6 & & 6 & $\begin{array}{r}1 . \\
1\end{array}$ & $\begin{array}{l}.0 \\
1.9 \\
1 .\end{array}$ & \begin{tabular}{l|l}
.6 & 0.2 \\
9 & 6
\end{tabular} & 6 & & & & & & & & & & & & & & & & & & & & & & $\begin{array}{c}1.4 \\
3\end{array}$ & $\begin{array}{c}\text { Dry at top, } \\
\text { connects at } \\
\text { bottom }\end{array}$ \\
\hline 213 & $\begin{array}{l}4 . \\
2\end{array}$ & \begin{tabular}{|c|} 
Mid- \\
Channel \\
Pool \\
\end{tabular} & 7 & 047 & 10 & 14 & \begin{tabular}{l|l}
9 & 7
\end{tabular} & $7 \begin{array}{l}1 . \\
7\end{array}$ & $\begin{array}{l}5 \\
7 \\
4\end{array}$ & \begin{tabular}{l|l}
2.0 \\
4 & 0.
\end{tabular} & .2 & & 3 & 300 & 55 & 15 & 30 & $0 \mid c$ & \begin{tabular}{l|l}
0 & 0
\end{tabular} & 0 & 02 & 2525 & 252 & 205 & 0 & \begin{tabular}{|l|l}
0 & 91 \\
\end{tabular} & \begin{tabular}{l|c}
91 & 10 \\
0
\end{tabular} & 0 & \begin{tabular}{l|l}
0 & 0
\end{tabular} & 06 & \begin{tabular}{c|c}
10 & 7 \\
0 & 7
\end{tabular} & 785 & $\begin{array}{c}1.8 \\
4\end{array}$ & \\
\hline 214 & \begin{tabular}{l|}
1. \\
1 \\
\end{tabular} & \begin{tabular}{|c|} 
Low \\
gradient \\
Riffle
\end{tabular} & & 9 & 8 & 7 & 8 & $8{ }_{8} \quad \begin{array}{l}0 . \\
6\end{array}$ & $\begin{array}{c}2 \\
6 \\
6\end{array}$ & 9.2 & & & & & & & & & & & & & & & & & & & & & & & & \\
\hline 215 & 2 & \begin{tabular}{|c|} 
Lateral \\
Scour \\
Pool - Log \\
Enhanced \\
\end{tabular} & 606 & 7721 & 8 & 8 & \begin{tabular}{|l|l}
9 & 7
\end{tabular} & $7 \begin{array}{l}0 . \\
3\end{array}$ & $\begin{array}{l}9 \\
3 \\
3\end{array}$ & \begin{tabular}{l|c}
.4 & 0. \\
5 & 8
\end{tabular} & 8 & & 3 & 200 & 90 & 0 & 10 & 0 & 0.0 & 0 & 02 & 2530 & 3010 & 105 & 0 & $0 \mid 95$ & $95 \mid \begin{array}{c}10 \\
0\end{array}$ & 0 & \begin{tabular}{l|l}
0 & 0
\end{tabular} & 0.6 & 907 & \begin{tabular}{l|l}
7 & 70
\end{tabular} & $\begin{array}{c}1.1 \\
7\end{array}$ & SDA, RB \\
\hline 216 & 2 & \begin{tabular}{|c|} 
Lateral \\
Scour \\
Pool - Log \\
Enhanced
\end{tabular} & 606 & 7720 & 9 & 7 & 10 & $10 \frac{1 .}{1}$ & $\begin{array}{l}1 \\
1 \\
1\end{array}$ & \begin{tabular}{l|l}
.5 & 0. \\
7 & 0.5
\end{tabular} & .5 & c & 3 & 100 & 50 & 50 & 0 & $0 \mid c$ & $\begin{array}{lll}0 & 0\end{array}$ & 0 & 030 & & 400 & \begin{tabular}{l|l}
0 & 0
\end{tabular} & 0 & 0 \begin{tabular}{c|c}
10 \\
0
\end{tabular} & \begin{tabular}{c|c}
10 & 10 \\
0 & 0
\end{tabular} & 0 & \begin{tabular}{l|l}
0 & 0
\end{tabular} & 0.6 & 957 & \begin{tabular}{l|l}
7 & 40
\end{tabular} & $\mid \begin{array}{c}1.0 \\
7\end{array}$ & \\
\hline 217 & $\begin{array}{l}4 . \\
2\end{array}$ & \begin{tabular}{|c|} 
Mid- \\
Channel \\
Pool \\
\end{tabular} & 616 & 6751 & 9 & \begin{tabular}{|l|l}
10 & 1 \\
$\mathbf{n}$
\end{tabular} & \begin{tabular}{l|l}
11 & 7
\end{tabular} & $7 \begin{array}{c}1 . \\
7\end{array}$ & 63. & \begin{tabular}{l|l}
3.3 & 0. \\
9 & 6
\end{tabular} & $\begin{array}{l}0.6 \\
6\end{array}$ & b & 3 & 750 & 30 & 70 & 0 & 00 & 0.0 & 0 & 03 & 3535 & $25 \mid 5$ & \begin{tabular}{l|l}
5 & 0
\end{tabular} & 0 & 095 & \begin{tabular}{l|c}
95 & 10 \\
0
\end{tabular} & 0 & \begin{tabular}{l|l}
0 & 0
\end{tabular} & \begin{tabular}{l|l|l}
0 & 6
\end{tabular} & \begin{tabular}{|c|c}
10 & 6 \\
0 & 6
\end{tabular} & $6 \mid \begin{array}{c}10 \\
0\end{array}$ & $\begin{array}{c}2.7 \\
3\end{array}$ & $\begin{array}{l}\text { Log Jam along } \\
\text { entire LB }\end{array}$ \\
\hline 218 & \begin{tabular}{|l|}
3. \\
2 \\
\end{tabular} & Glide & 616 & 6765 & 7 & $7 \mid 7$ & 78 & $8 \begin{array}{r}0 . \\
3\end{array}$ & \begin{tabular}{c|c}
4 & 0. \\
3 & 8 \\
\end{tabular} & $\begin{array}{l}8.8 \\
8\end{array}$ & & & 7 & 50 & 80 & 10 & 10 & 0 & 0.0 & 0 & 01 & 1515 & 4030 & 300 & 0 & 0 & \begin{tabular}{l|c}
97 & 10 \\
9
\end{tabular} & 0 & \begin{tabular}{l|l}
0 & 0
\end{tabular} & \begin{tabular}{|l|l|}
0 & 6
\end{tabular} & 806 & \begin{tabular}{|l|l|}
6 & 10 \\
\end{tabular} & & \\
\hline 219 & 1. & \begin{tabular}{|c|} 
Low \\
gradient \\
Riffle
\end{tabular} & & 15 & 8 & 8 & 7 & $7 \begin{array}{r}0 . \\
2\end{array}$ & $\begin{array}{l}1 \\
2 \\
2\end{array}$ & 3 & & & & & & & & & & & & & & & & & & & & & & & & \\
\hline 220 & \begin{tabular}{|c|}
5. \\
1 \\
\end{tabular} & $\begin{array}{c}\begin{array}{c}\text { Corner } \\
\text { Pool }\end{array} \\
\end{array}$ & 617 & 034 & 12 & \begin{tabular}{|l|l|l|l} 
& 1 \\
\end{tabular} & 1514 & $14 \begin{array}{r}1 . \\
8\end{array}$ & \begin{tabular}{c|c|}
0 & 1. \\
8 & 6 \\
\end{tabular} & \begin{tabular}{c|c}
.9 & 0. \\
6 & 5 \\
\end{tabular} & $\begin{array}{l}1 \\
5 \\
\end{array}$ & c & 3 & 101 & 010 & 35 & 35 & $0 \mid c$ & $\begin{array}{lll}0 & 0\end{array}$ & 0 & 030 & 3030 & 355 & 50 & 0 & \begin{tabular}{l|l|}
0 & 10 \\
& 0 \\
\end{tabular} & \begin{tabular}{l|c}
10 & 10 \\
0 & 0 \\
\end{tabular} & 0 & \begin{tabular}{l|l}
0 & 0 \\
\end{tabular} & \begin{tabular}{|l|l|}
0 & 6 \\
\end{tabular} & \begin{tabular}{|c|c|}
10 & 6 \\
0 & 6 \\
\end{tabular} & $\begin{array}{ll}6 & 90 \\
\end{array}$ & \begin{tabular}{|c|}
1.8 \\
1
\end{tabular} & \\
\hline 221 & $\begin{array}{l}1 . \\
1\end{array}$ & \begin{tabular}{|c|} 
Low \\
gradient \\
Riffle
\end{tabular} & 616 & 7744 & 12 & 14 & 9 & $9 \mid \begin{array}{c}0 . \\
3\end{array}$ & \begin{tabular}{c|c}
3 & 0 \\
3 & 8
\end{tabular} & $\begin{array}{l}.7 \\
8\end{array}$ & & & 1 & 105 & 95 & 0 & 0 & \begin{tabular}{l|l}
0 & 0 \\
0
\end{tabular} & $\begin{array}{lll}0 & 0\end{array}$ & 0 & \begin{tabular}{l|l}
0 & 0
\end{tabular} & 0.0 & 4050 & 5010 & 0 & \begin{tabular}{l|l}
0 & 97
\end{tabular} & \begin{tabular}{c|c}
97 & 10 \\
0
\end{tabular} & 0 & \begin{tabular}{l|l}
0 & 0
\end{tabular} & 0 & \begin{tabular}{l|l}
90 & 6
\end{tabular} & $6 \mid \begin{array}{c}10 \\
0\end{array}$ & & \\
\hline 222 & \begin{tabular}{|l|}
3. \\
3
\end{tabular} & Run & 616 & \begin{tabular}{l|l}
99 & 75 \\
9
\end{tabular} & 13 & \begin{tabular}{|l|l|} 
& 9 \\
\end{tabular} & 1414 & $17 \begin{array}{r}0 . \\
8\end{array}$ & \begin{tabular}{c|c}
5 & 1. \\
8 & 8 \\
\end{tabular} & $\begin{array}{l}.4 \\
8\end{array}$ & & & 1 & $<5$ & 1 & 0 & 50 & 0 & \begin{tabular}{l|l}
0 & 0 \\
\end{tabular} & 0 & \begin{tabular}{|l|l|}
0 & 1 \\
\end{tabular} & 1010 & $35 \mid 4 c$ & $\begin{array}{lll}40 & 5 \\
\end{array}$ & 0 & \begin{tabular}{|l|c|}
0 & 10 \\
& 0 \\
\end{tabular} & \begin{tabular}{c|c}
10 & 10 \\
0 & 0 \\
\end{tabular} & \begin{tabular}{|l|l|} 
& 0 \\
\end{tabular} & \begin{tabular}{l|l}
0 & 0 \\
\end{tabular} & \begin{tabular}{|l|l|}
0 & 6 \\
\end{tabular} & \begin{tabular}{|c|c|}
10 & 6 \\
0 & 6 \\
\end{tabular} & \begin{tabular}{|c|c|}
6 & 10 \\
\end{tabular} & & \\
\hline
\end{tabular}




\begin{tabular}{|c|c|c|c|c|c|c|c|c|c|c|c|c|c|c|c|c|c|c|c|c|c|c|c|c|c|c|c|c|c|c|c|c|c|c|}
\hline 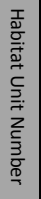 & & 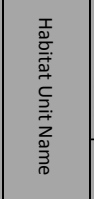 & 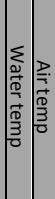 & 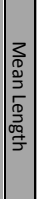 & 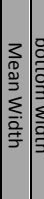 & 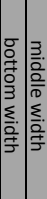 & 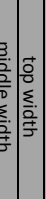 & 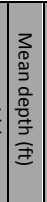 & 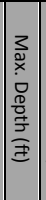 & 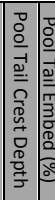 & 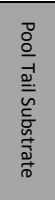 & 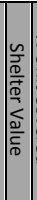 & 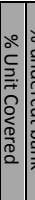 & & $\frac{\partial}{\sum}$ & $=$ & 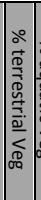 & 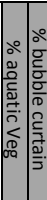 & 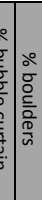 & 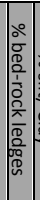 & 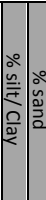 & 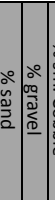 & 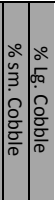 & 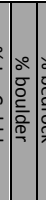 & & 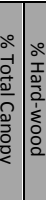 & 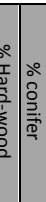 & 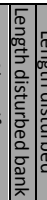 & 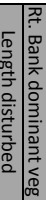 & 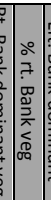 & 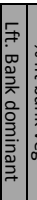 & 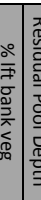 & $\begin{array}{l}\frac{0}{2} \\
\frac{0}{2} \\
0 \\
\frac{0}{0} \\
0 \\
0 \\
\frac{0}{5} \\
\end{array}$ & 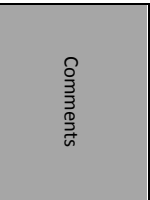 \\
\hline 223 & $\begin{array}{l}3 . \\
2\end{array}$ & Glide & 6269 & 26 & $18 \mid 1$ & 17 & 19 & 0.6 & $\begin{array}{c}1.1 \\
1\end{array}$ & & & 0 & 1 & $0 \mid 5 c$ & 5050 & 0 & 0 & \begin{tabular}{|l|l|l|l|l}
0 & 0
\end{tabular} & \begin{tabular}{l|l}
0 & 0
\end{tabular} & 0 & 3030 & 3040 & 00 & 0 & 0 & \begin{tabular}{c|c}
10 & 10 \\
0
\end{tabular} & \begin{tabular}{l|l}
10 & 0 \\
0 & 0
\end{tabular} & 0 & \begin{tabular}{l|l}
0 & 6
\end{tabular} & $5 \mid$\begin{tabular}{c|c}
10 \\
0
\end{tabular} & 6 & $\begin{array}{c}10 \\
0\end{array}$ & & $\begin{array}{c}\text { Access. Main Line } \\
\text { Trail crosses } \\
\text { creek at apple } \\
\text { orchard. }\end{array}$ \\
\hline 224 & \begin{tabular}{|c|}
5. \\
1 \\
\end{tabular} & \begin{tabular}{c|} 
Corner \\
Pool
\end{tabular} & 6169 & $\begin{array}{c}10 \\
0\end{array}$ & 141 & \begin{tabular}{|l|l}
19 & 11 \\
\end{tabular} & 1112 & 1.1 & $\begin{array}{c}1.9 \\
6\end{array}$ & $\begin{array}{c}0.4 \\
7\end{array}$ & b & 2 & 15 & \begin{tabular}{l|l}
0 & 5 \\
\end{tabular} & $5 \mid 0$ & 90 & 5 & \begin{tabular}{l|l}
0 & 0 \\
\end{tabular} & 0 & 0 & 3035 & 3530 & 50 & 0 & $0: 1$ & \begin{tabular}{c|c}
10 & 10 \\
0 & 0 \\
\end{tabular} & \begin{tabular}{l|l}
10 & 0 \\
\end{tabular} & 0 & \begin{tabular}{l|l}
0 & 6 \\
\end{tabular} & 590 & 77 & $75 \mid \begin{array}{c}1 . \\
9\end{array}$ & $\begin{array}{ll}.4 \\
9\end{array}$ & $\begin{array}{c}\text { Access. Main } \\
\text { Line Trail crosses }\end{array}$ \\
\hline 225 & \begin{tabular}{|l|}
3. \\
2 \\
\end{tabular} & Glide & 6172 & 46 & \begin{tabular}{|l|l}
15 & 1
\end{tabular} & \begin{tabular}{|l|l|}
12 & 10 \\
\end{tabular} & 1022 & $\begin{array}{c}0.3 \\
6 \\
\end{array}$ & $\begin{array}{c}0.7 \\
8\end{array}$ & & & 2 & 53 & 303 & $30 \mid 0$ & 40 & 0 & $\begin{array}{ll}0 & 0\end{array}$ & 0 & 0 & 1010 & 1070 & $\begin{array}{lll}10 & 0 \\
\end{array}$ & 0 & \begin{tabular}{|l|l|l|}
0 & 1 \\
\end{tabular} & \begin{tabular}{l|c}
10 & 10 \\
0 & 0 \\
\end{tabular} & \begin{tabular}{l|l}
0 & 0 \\
\end{tabular} & $0 \mid 1$ & $\begin{array}{ll}10 & 7 \\
\end{array}$ & 75 & 69 & 90 & & \\
\hline 226 & $\begin{array}{l}4 . \\
2\end{array}$ & \begin{tabular}{|c|} 
Mid- \\
Channel \\
Pool \\
\end{tabular} & 6372 & 70 & 232 & 2229 & 2917 & $\mid \begin{array}{c}1.7 \\
5\end{array}$ & $\left|\begin{array}{c}3.0 \\
5\end{array}\right|$ & $\left|\begin{array}{c}0.1 \\
3\end{array}\right|$ & c & 3 & 80 & 05 & 5050 & 0 & 0 & \begin{tabular}{l|l}
0 & 0
\end{tabular} & 0 & 0 & $15 \mid 25$ & $25|40|$ & 100 & 0 & 0 & \begin{tabular}{c|c}
10 & 10 \\
0 & 0
\end{tabular} & \begin{tabular}{l|l}
10 & 0 \\
0 & 0
\end{tabular} & 0 & \begin{tabular}{l|l}
0 & 7
\end{tabular} & 780 & 69 & $\left.95\right|_{2} ^{2}$ & 2.9 & Log Jam \\
\hline 227 & $\begin{array}{l}1 . \\
1\end{array}$ & \begin{tabular}{|c|} 
Low \\
gradient \\
Riffle
\end{tabular} & & 49 & 141 & 1715 & 1510 & $\left|\begin{array}{c}0.1 \\
9\end{array}\right|$ & 0.3 & & & 2 & 5 & \begin{tabular}{l|l}
0 & $6 c$
\end{tabular} & \begin{tabular}{l|l}
0 & 0
\end{tabular} & 40 & 0 & \begin{tabular}{l|l}
0 & 0
\end{tabular} & 0 & 0 & \begin{tabular}{l|l}
0 & 0
\end{tabular} & 030 & 4030 & 00 & $00^{1}$ & \begin{tabular}{c|c}
10 & 10 \\
0 & 0
\end{tabular} & \begin{tabular}{l|l|l}
10 & 0 \\
0 & 0
\end{tabular} & 0 & \begin{tabular}{l|l}
0 & 7
\end{tabular} & 790 & \begin{tabular}{|l|l|l|}
7 & 8
\end{tabular} & 80 & & \\
\hline 228 & \begin{tabular}{|c|}
3 \\
4 \\
\end{tabular} & Step Run & \begin{tabular}{|l|l|}
63 & 74
\end{tabular} & 58 & $11 \mid 1$ & 108 & $\begin{array}{lll}8 & 14 \\
\end{array}$ & \begin{tabular}{|c|}
0.3 \\
9 \\
\end{tabular} & \begin{tabular}{|c|}
0.7 \\
5 \\
\end{tabular} & & & 1 & 5 & \begin{tabular}{|l|l|}
0 & 10 \\
\end{tabular} & \begin{tabular}{l|l}
0 & 0 \\
0 & 0 \\
\end{tabular} & 0 & 0 & \begin{tabular}{l|l}
0 & 0 \\
\end{tabular} & 0 & 0 & $5 \quad 5$ & $\begin{array}{lll}5 & 30 \\
\end{array}$ & 4020 & 0.0 & $0{ }^{1}$ & \begin{tabular}{c|c}
10 & 10 \\
0 & 0 \\
\end{tabular} & \begin{tabular}{l|l}
10 & 0 \\
\end{tabular} & 0 & \begin{tabular}{l|l}
0 & 6 \\
\end{tabular} & 595 & \begin{tabular}{|l|l|l|}
7 & 8
\end{tabular} & 80 & & $\begin{array}{c}1 \text { riffle (14 ft.), } 1 \\
\text { run }\end{array}$ \\
\hline 229 & \begin{tabular}{|l|}
3. \\
2 \\
\end{tabular} & Glide & 6273 & $\begin{array}{c}10 \\
0 \\
\end{array}$ & 161 & \begin{tabular}{|l|l|l|}
14 & 13 \\
\end{tabular} & 1320 & \begin{tabular}{|c|}
0.8 \\
4 \\
\end{tabular} & $\begin{array}{c}1.4 \\
9\end{array}$ & & & 2 & 53 & \begin{tabular}{l|l}
35 & 0
\end{tabular} & \begin{tabular}{l|l}
0 & 0
\end{tabular} & 20 & 50 & \begin{tabular}{|l|l|l|l|l}
0 & 0
\end{tabular} & 0 & 0 & 1515 & 1560 & 100 & 0 & 0 & & \begin{tabular}{l|l}
10 & 0 \\
0 & 0 \\
\end{tabular} & 5 & \begin{tabular}{l|l}
0 & 6 \\
\end{tabular} & 595 & \begin{tabular}{|l|l}
6 & 1 \\
\end{tabular} & \begin{tabular}{c|}
10 \\
0 \\
\end{tabular} & & \\
\hline 230 & \begin{tabular}{|l|}
5. \\
1 \\
\end{tabular} & $\begin{array}{c}\text { Corner } \\
\text { Pool }\end{array}$ & 6271 & 40 & 162 & 20 & 11 & \begin{tabular}{|c|}
1.1 \\
6 \\
\end{tabular} & $\begin{array}{c}1.7 \\
6 \\
\end{array}$ & $\begin{array}{c}0.8 \\
9 \\
\end{array}$ & $b / c$ & & 52 & $25 \mid 2$ & \begin{tabular}{l|l}
55 & 0
\end{tabular} & 25 & 25 & \begin{tabular}{l|l}
0 & 0
\end{tabular} & 0 & 0 & 2030 & 3040 & 100 & 0 & \begin{tabular}{l|l} 
& 1 \\
\end{tabular} & \begin{tabular}{c|c}
10 & 10 \\
0 & 0 \\
\end{tabular} & \begin{tabular}{l|l}
10 & 0 \\
0 & 0 \\
\end{tabular} & 0 & \begin{tabular}{l|l}
0 & 6
\end{tabular} & 595 & \begin{tabular}{|l|l}
6 & 9
\end{tabular} & 950 & 7 & \\
\hline 231 & $\begin{array}{l}3 . \\
2\end{array}$ & Glide & 6372 & $\begin{array}{c}19 \\
3\end{array}$ & 141 & \begin{tabular}{l|l}
11 & 11 \\
\end{tabular} & 1121 & $\left.1 \mid \begin{array}{c}0.7 \\
4\end{array}\right]$ & $\begin{array}{c}1.6 \\
4\end{array}$ & & & & 10 & 030 & 3030 & 20 & 0 & 200 & 0 & 0 & $15 \mid 15$ & $15|40|=$ & 300 & 0 & $\left.0\right|^{1}$ & & \begin{tabular}{l|l}
10 & 0 \\
0 & 0
\end{tabular} & 402 & $20 \mid 6$ & 580 & \begin{tabular}{|l|l|}
6 & 8
\end{tabular} & 85 & & $\begin{array}{l}\text { (+50) Swanton } \\
\text { Rd. Bridge. } \\
(+150) \text { Some kind } \\
\text { of structure, } \\
\text { looks like a fish } \\
\text { trap. RB } \\
\text { stabilized with } \\
\text { small logs, crib } \\
\text { wall } \\
\end{array}$ \\
\hline 232 & $\begin{array}{l}5 . \\
1\end{array}$ & $\begin{array}{l}\text { Corner } \\
\text { Pool }\end{array}$ & & 65 & $162^{2}$ & \begin{tabular}{|l|l}
21 & 14
\end{tabular} & 1413 & $\begin{array}{c}1.7 \\
7\end{array}$ & $\begin{array}{c}2.3 \\
9\end{array}$ & $\begin{array}{c}0.5 \\
1\end{array}$ & c & 3 & 20 & \begin{tabular}{l|l}
0 & 0
\end{tabular} & \begin{tabular}{l|l}
0 & 0
\end{tabular} & 0 & 50 & \begin{tabular}{l|l}
0 & 0
\end{tabular} & 050 & 0 & $15 \mid 15$ & 1530 & 250 & 15 & 0 & 90 \begin{tabular}{c|c}
10 \\
0
\end{tabular} & \begin{tabular}{l|l}
10 & 0 \\
0 & 0
\end{tabular} & 0 & $0 \quad 5$ & $5 \mid \begin{array}{c}10 \\
0\end{array}$ & 7 & \begin{tabular}{c|c}
10 & 1 \\
0 & 8
\end{tabular} & $\begin{array}{l}1.8 \\
8\end{array}$ & $\begin{array}{c}\text { Boulders from } \\
\text { retaining } \\
\text { wall/bank } \\
\text { stabilization } \\
\end{array}$ \\
\hline 233 & $\begin{array}{l}3 . \\
4\end{array}$ & Step Run & 6270 & $\begin{array}{c}13 \\
7\end{array}$ & 101 & 1311 & 116 & $\begin{array}{c}0.7 \\
3\end{array}$ & 2 & & & 3 & & 030 & 3040 & 15 & 15 & \begin{tabular}{l|l}
0 & 0
\end{tabular} & 0 & 0 & 1010 & 1055 & 205 & 0 & 0 & \begin{tabular}{l|c}
10 & 10 \\
0
\end{tabular} & \begin{tabular}{l|l}
10 \\
0
\end{tabular} & 0 & \begin{tabular}{l|l}
0 & 5
\end{tabular} & $5 \mid \begin{array}{c}10 \\
0\end{array}$ & 6 & $\begin{array}{c}10 \\
0\end{array}$ & & $\begin{array}{l}\text { "step Glide" } 1 \\
\text { riffle (30 ft.) } 2 \\
\text { glides }\end{array}$ \\
\hline 234 & \begin{tabular}{|c|}
5. \\
1 \\
\end{tabular} & $\begin{array}{c}\text { Corner } \\
\text { Pool }\end{array}$ & 69 & 76 & 8 & \begin{tabular}{|l|l}
6 & 8 \\
\end{tabular} & $\begin{array}{lll}8 & 10 \\
\end{array}$ & $\begin{array}{c}1.1 \\
4\end{array}$ & $\begin{array}{c}2.1 \\
5\end{array}$ & $\begin{array}{c}0.5 \\
6\end{array}$ & c & 3 & & 2515 & \begin{tabular}{l|l}
5 & 0
\end{tabular} & 50 & 10 & 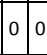 & 0 & 0 & 2020 & 2050 & 100 & 0 & $0{ }^{1}$ & \begin{tabular}{c|c}
10 & 10 \\
0 & 0 \\
\end{tabular} & \begin{tabular}{l|l}
10 & 0 \\
\end{tabular} & 0 & \begin{tabular}{l|l}
0 & 7
\end{tabular} & 795 & 69 & \begin{tabular}{l|l}
9 & 1 \\
\end{tabular} & $\begin{array}{l}1.5 \\
9 \\
\end{array}$ & \\
\hline 235 & \begin{tabular}{|l|}
3. \\
2 \\
\end{tabular} & Glide & 6369 & $\begin{array}{c}14 \\
5 \\
\end{array}$ & 111 & 10 & 11 & \begin{tabular}{|c|}
0.7 \\
5 \\
\end{tabular} & $\begin{array}{c}1.4 \\
3\end{array}$ & & & 3 & 15 & 030 & 3030 & 40 & 0 & $\begin{array}{ll}0 & 0\end{array}$ & 0 & 0 & 2020 & 2045 & $\begin{array}{lll}10 & 5 \\
\end{array}$ & 0 & \begin{tabular}{|l|l|l} 
& 1 \\
\end{tabular} & \begin{tabular}{c|c}
10 & 10 \\
0 & 0 \\
\end{tabular} & \begin{tabular}{l|l}
10 & 0 \\
\end{tabular} & 0 & \begin{tabular}{l|l}
0 & 6
\end{tabular} & 595 & \begin{tabular}{|l|l}
6 & 9
\end{tabular} & 95 & & \\
\hline 236 & $\begin{array}{l}3 . \\
4\end{array}$ & Step Run & 6369 & $\mid \begin{array}{c}10 \\
0\end{array}$ & 171 & 11 & 22 & 20.3 & $\begin{array}{c}0.8 \\
5\end{array}$ & & & 3 & 53 & 304 & \begin{tabular}{l|l}
10 & 0
\end{tabular} & 30 & 0 & $\begin{array}{ll}0 & 0\end{array}$ & 0 & 0 & $5 \quad 5$ & $550=$ & 3010 & 00 & $\left.0\right|^{1}$ & \begin{tabular}{c|c}
10 & 10 \\
0 & 0
\end{tabular} & \begin{tabular}{l|l}
10 & 0 \\
0 & 0
\end{tabular} & 0 & \begin{tabular}{l|l}
0 & 7
\end{tabular} & 795 & \begin{tabular}{|l|l}
6 & 9
\end{tabular} & 95 & & $\begin{array}{c}2 \text { riffles }(10,15 \\
\text { ft.), } 1 \text { run. } \\
\text { Bankfull } \\
\text { measurements } \\
\text { take. End of Day } \\
8 / 1 \\
\end{array}$ \\
\hline 237 & 2 & \begin{tabular}{|c|} 
Lateral \\
Scour \\
Pool - Log \\
Enhanced \\
\end{tabular} & 6062 & 52 & 152 & 2213 & 1311 & $1 \begin{array}{c}1.2 \\
6\end{array}$ & $\begin{array}{c}2.3 \\
2\end{array}$ & $\begin{array}{c}0.2 \\
6\end{array} \mid 15$ & c & 3 & 153 & & 525 & 540 & 0 & $\begin{array}{ll}0 & 0\end{array}$ & 0 & 0 & 2020 & 2050 & 100 & 0 & $0{ }^{1}$ & \begin{tabular}{c|c}
10 & 10 \\
0 & 0
\end{tabular} & \begin{tabular}{l|l}
10 & 0 \\
0 & 0
\end{tabular} & 0 & \begin{tabular}{l|l}
0 & 6
\end{tabular} & 590 & 69 & $955^{2}$ & 6 & Start $8 / 5$ \\
\hline $\begin{array}{c}237 . \\
1\end{array}$ & \begin{tabular}{c|}
6. \\
1
\end{tabular} & \begin{tabular}{|c|}
$\begin{array}{c}\text { Secondar } \\
\text { y Channel } \\
\text { Pool }\end{array}$ \\
\end{tabular} & & 26 & 16 & & & $\begin{array}{c}1.0 \\
7\end{array}$ & $\begin{array}{c}1.4 \\
4\end{array}$ & $\begin{array}{c}0.1 \\
8\end{array}$ & b & 3 & & 403 & \begin{tabular}{l|l}
30 & 30
\end{tabular} & $\begin{array}{lll}0 & 0\end{array}$ & 0 & $\begin{array}{ll}0 & 0\end{array}$ & \begin{tabular}{l|l}
0 & 0
\end{tabular} & 0 & 5050 & 500 & \begin{tabular}{l|l}
0 & 0
\end{tabular} & 0 & 0 & & & & & & & & $\begin{array}{l}1.2 \\
6\end{array}$ & \\
\hline 238 & \begin{tabular}{|c|}
1. \\
1
\end{tabular} & \begin{tabular}{|c|} 
Low \\
gradient \\
Riffle
\end{tabular} & 6063 & 40 & 13 & \begin{tabular}{l|l}
11 & 9
\end{tabular} & \begin{tabular}{l|l|l}
9 & 19 \\
\end{tabular} & $\begin{array}{c}0.3 \\
4\end{array}$ & $\begin{array}{c}0.6 \\
8\end{array}$ & & & 1 & $<5$ & $0 \mid 0$ & $0 \quad 50$ & 0 & 50 & 0 & 0 & 0 & 1010 & $1035=$ & 3510 & 00 & $\left.0\right|^{1}$ & \begin{tabular}{c|c}
10 & 10 \\
0 & 0
\end{tabular} & \begin{tabular}{l|l}
10 \\
0
\end{tabular} & 0 & \begin{tabular}{l|l}
0 & 6
\end{tabular} & 595 & \begin{tabular}{|l|l}
6 & 9
\end{tabular} & 95 & & \\
\hline 239 & $\begin{array}{l}5 . \\
3 \\
\end{array}$ & \begin{tabular}{|c|} 
Lateral \\
Scour \\
Pool - \\
Root Wad \\
Enhanced
\end{tabular} & 6063 & 35 & 221 & 1923 & 2325 & 51.3 & $\begin{array}{c}2.8 \\
1\end{array}$ & $\begin{array}{c}0.2 \\
1\end{array} \mid 1 C$ & c & 3 & & & 2020 & 30 & 0 & 00 & 0 & 0 & 3030 & 3020 & \begin{tabular}{|l|l|}
15 & 5
\end{tabular} & 0 & 09 & 9795 & \begin{tabular}{l|l}
95 & 5
\end{tabular} & 0 & \begin{tabular}{l|l}
0 & 6
\end{tabular} & 585 & 69 & 952 & 2.6 & \\
\hline 240 & $\begin{array}{l}4 . \\
2\end{array}$ & \begin{tabular}{|c|} 
Mid- \\
Channel \\
Pool \\
\end{tabular} & 6165 & 63 & 282 & 2539 & 3921 & $\begin{array}{c}2.6 \\
8\end{array}$ & $\begin{array}{c}5.0 \\
8\end{array}$ & $\begin{array}{c}0.2 \\
6\end{array} \mid$ & c & 3 & & \begin{tabular}{l|l}
5 & 0
\end{tabular} & \begin{tabular}{l|l|l}
0 & 85
\end{tabular} & 510 & 0 & $\begin{array}{ll}0 & 0\end{array}$ & 0 & 0 & 3030 & 3020 & \begin{tabular}{|l|l|}
5 & 5
\end{tabular} & 0 & $\left.0\right|^{1}$ & \begin{tabular}{c|c}
10 & 95 \\
0 &
\end{tabular} & \begin{tabular}{l|l}
95 & 5
\end{tabular} & 0 & \begin{tabular}{l|l}
0 & 6
\end{tabular} & 525 & 68 & $\left.85\right|^{4}$ & $\begin{array}{l}1.8 \\
2\end{array}$ & $\begin{array}{c}\text { Log enhanced, } \\
\text { two connected } \\
\text { pools } \\
\end{array}$ \\
\hline 241 & $\begin{array}{c}3 . \\
4\end{array}$ & Step Run & 6265 & $\begin{array}{c}25 \\
6\end{array}$ & 12 & 6111 & 1120 & $\left|\begin{array}{c}0.3 \\
7\end{array}\right|$ & $\begin{array}{c}1.3 \\
8\end{array} \mid$ & & & 3 & 52 & 2030 & 3010 & 20 & 20 & $\begin{array}{ll}0 & 0\end{array}$ & \begin{tabular}{l|l}
0 & 0
\end{tabular} & 0 & $10 \mid 10$ & $1030=$ & 3020 & 00 & 09 & 9394 & \begin{tabular}{l|l}
94 & 6
\end{tabular} & 02 & \begin{tabular}{l|l}
20 & 6
\end{tabular} & 595 & 89 & 95 & & $\begin{array}{c}3 \text { riffles ( } 38,63, \\
25 \mathrm{ft} .), 2 \text { runs. } \\
(+236) \text { LB trib } \\
\text { with water }\end{array}$ \\
\hline 242 & $\begin{array}{l}4 . \\
2\end{array}$ & \begin{tabular}{|c|} 
Mid- \\
Channel \\
Pool \\
\end{tabular} & 6265 & 47 & 172 & 2020 & 2011 & 10.8 & $\begin{array}{c}2.3 \\
6\end{array}$ & $\begin{array}{c}0.1 \\
4\end{array} \mid 1 C$ & c & & 5 & 105 & 580 & 5 & 0 & $\begin{array}{ll}0 & 0\end{array}$ & 0 & 0 & 3030 & 3025 & \begin{tabular}{l|l}
10 & 5
\end{tabular} & 0 & 09 & 9898 & \begin{tabular}{l|l}
88 & 2
\end{tabular} & 0 & \begin{tabular}{l|l}
0 & 6
\end{tabular} & $5 \mid \begin{array}{c}10 \\
0\end{array}$ & 6 & 95 & 2.2 & \\
\hline 243 & \begin{tabular}{|l|}
3. \\
4 \\
\end{tabular} & Step Run & & 71 & 111 & \begin{tabular}{|l|l|}
11 & 11 \\
\end{tabular} & \begin{tabular}{|l|l|}
11 & 12 \\
\end{tabular} & \begin{tabular}{|c|}
0.2 \\
9 \\
\end{tabular} & $\begin{array}{c}0.5 \\
8 \\
\end{array}$ & & & 3 & 5 & 5 & $15 \mid 5$ & 0 & 45 & 0 & 0 & 0 & $\begin{array}{lll}5 & 5 \\
\end{array}$ & $\begin{array}{lll}5 & 40 \\
\end{array}$ & 3515 & 50 & 09 & \begin{tabular}{l|c}
97 & 10 \\
& 0 \\
\end{tabular} & \begin{tabular}{l|l}
0 & 0 \\
0 & \\
\end{tabular} & 0 & 0.6 & 595 & 69 & 95 & & $\begin{array}{c}2 \text { riffles }(31,14 \\
\text { ft. }), 1 \text { run } \\
\end{array}$ \\
\hline 244 & $\begin{array}{l}4 . \\
2\end{array}$ & \begin{tabular}{|c|} 
Mid- \\
Channel \\
Pool \\
\end{tabular} & & 45 & 121 & 1214 & 1411 & 10.8 & $\begin{array}{c}2.5 \\
7\end{array}$ & $\begin{array}{c}0.2 \\
4\end{array} \mid 3$ & c & 3 & & \begin{tabular}{l|l|l}
20 & 0
\end{tabular} & $0 \quad 60$ & 20 & 0 & $\begin{array}{ll}0 & 0\end{array}$ & 0 & 0 & 3030 & 3025 & 150 & 0 & 0 & \begin{tabular}{c|c}
10 & 10 \\
0 & 0
\end{tabular} & \begin{tabular}{l|l}
10 & 0 \\
0 & 0
\end{tabular} & 0 & \begin{tabular}{l|l}
0 & 6
\end{tabular} & 595 & 6 & $\begin{array}{c}10 \\
0\end{array}$ & 3 & \\
\hline 245 & \begin{tabular}{|l|}
3. \\
3 \\
\end{tabular} & Run & & 34 & 81 & \begin{tabular}{l|l}
11 & 7 \\
\end{tabular} & \begin{tabular}{l|l}
7 & 7 \\
\end{tabular} & $\begin{array}{c}0.6 \\
6 \\
\end{array}$ & \begin{tabular}{|c|}
0.9 \\
4
\end{tabular} & & & 3 & & \begin{tabular}{l|l}
0 & $3 \mathrm{C}$ \\
\end{tabular} & \begin{tabular}{l|l}
30 & 70 \\
\end{tabular} & 0 & 0 & $\begin{array}{lll}0 & 0 \\
\end{array}$ & 0 & 0 & 1010 & 1060 & 200 & 0 & 0 & 90 \begin{tabular}{r|r}
10 \\
0
\end{tabular} & \begin{tabular}{l|l}
10 & 0 \\
\end{tabular} & $0 \mid$ & \begin{tabular}{l|l}
0 & 6 \\
\end{tabular} & \begin{tabular}{|c|}
50 \\
0
\end{tabular} & 6 & \begin{tabular}{|c|}
10 \\
0
\end{tabular} & & \\
\hline
\end{tabular}




\begin{tabular}{|c|c|c|c|c|c|c|c|c|c|c|c|c|c|c|c|c|c|c|c|c|c|c|c|c|c|c|c|c|c|c|c|c|c|c|c|c|}
\hline 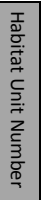 & & 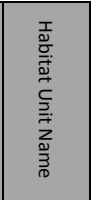 & 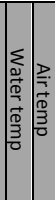 & 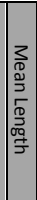 & 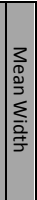 & 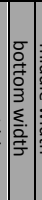 & & 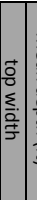 & & 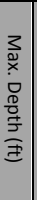 & 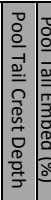 & & 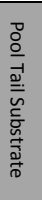 & 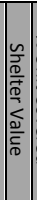 & 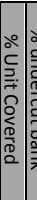 & 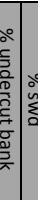 & $\frac{\circ}{\sum}$ & 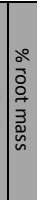 & 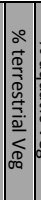 & & 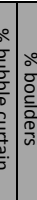 & 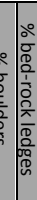 & 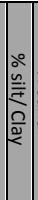 & 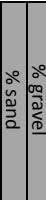 & 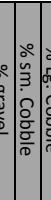 & 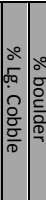 & 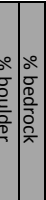 & 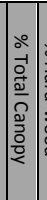 & 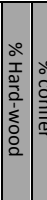 & 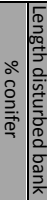 & 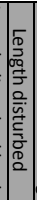 & 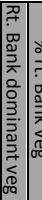 & 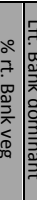 & 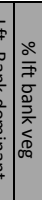 & 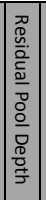 & 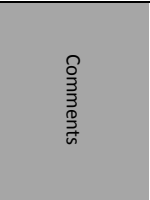 \\
\hline 246 & $\begin{array}{l}4 . \\
2\end{array}$ & \begin{tabular}{|c|} 
Mid- \\
Channel \\
Pool \\
\end{tabular} & 6369 & 35 & 11 & 7 & $12 \mid 1$ & \begin{tabular}{|l|l}
13 & 1 \\
1
\end{tabular} & $1.72^{2}$ & $\begin{array}{c}2.5 \\
8\end{array}$ & $\begin{array}{c}0.2 \\
5\end{array}$ & & & 3 & & 400 & 0 & 40 & 20 & 00 & \begin{tabular}{l|l}
0 & 0
\end{tabular} & 0 & 40 & 4020 & 00 & \begin{tabular}{|l|l|} 
& 0 \\
\end{tabular} & 00 & 84 & $\begin{array}{c}10 \\
0\end{array}$ & \begin{tabular}{l|l}
0 & 0
\end{tabular} & 0 & \begin{tabular}{|l|l}
6 & 5
\end{tabular} & 50 & 695 & $\begin{array}{c}2.3 \\
3\end{array}$ & End $8 / 5$ \\
\hline 247 & $\begin{array}{l}1 . \\
1\end{array}$ & \begin{tabular}{|c|} 
Low \\
gradient \\
Riffle \\
\end{tabular} & 6167 & 19 & 12 & 13 & & $10^{0}$ & \begin{tabular}{c|c}
0.2 & 0 \\
9 &
\end{tabular} & $\begin{array}{c}0.5 \\
6\end{array}$ & & & & 3 & 25 & \begin{tabular}{l|l}
0 & $2 C$
\end{tabular} & \begin{tabular}{l|l}
0 & 5
\end{tabular} & 0 & 75 & 00 & \begin{tabular}{l|l}
0 & 0
\end{tabular} & 0 & 0 & 050 & 050 & \begin{tabular}{l|l}
0 & 0
\end{tabular} & 0.0 & $95^{1}$ & $\begin{array}{c}10 \\
0\end{array}$ & $0 \mid 0$ & 0 & \begin{tabular}{|l|l}
6 & 1
\end{tabular} & 106 & 6 60 & & Start 8/6 \\
\hline 248 & $\begin{array}{l}4 . \\
2\end{array}$ & \begin{tabular}{|c|} 
Mid- \\
Channel \\
Pool \\
\end{tabular} & 6064 & 32 & 11 & 10 & 11 & $122^{1}$ & \begin{tabular}{c|c}
1.0 \\
7
\end{tabular} & $\begin{array}{c}2.0 \\
1\end{array}$ & \begin{tabular}{c|c}
0.2 & 4 \\
5 &
\end{tabular} & 40 & $d$ & 3 & 201 & $10 \mid 5$ & 0 & 55 & 30 & $0 \mid 0$ & \begin{tabular}{l|l}
0 & 0
\end{tabular} & 0 & 20 & 2040 & 015 & $5 \mid 0$ & \begin{tabular}{l|l|l}
0 & 0
\end{tabular} & 97 & $\begin{array}{c}10 \\
0\end{array}$ & $0 \mid 0$ & 0 & 71 & 106 & 6 & $\begin{array}{c}1.7 \\
6\end{array} \mid$ & \\
\hline 249 & $\begin{array}{l}1 . \\
1\end{array}$ & \begin{tabular}{|c|} 
Low \\
gradient \\
Riffle \\
\end{tabular} & 6064 & 57 & 11 & 12 & 901 & $110^{0}$ & \begin{tabular}{c|c}
0.4 & 0 \\
1 &
\end{tabular} & $\begin{array}{c}0.7 \\
4\end{array}$ & & & & 3 & $20 \mid \mathrm{C}$ & $0 \mid 3$ & \begin{tabular}{l|l}
0 & 0
\end{tabular} & 0 & 70 & 00 & \begin{tabular}{l|l}
0 & 0
\end{tabular} & 0 & 0 & $\begin{array}{lll}0 & 50\end{array}$ & \begin{tabular}{l|l|l|l|}
0 & 50
\end{tabular} & \begin{tabular}{|l|l}
0 & 0
\end{tabular} & \begin{tabular}{l|l|l}
0 & 0
\end{tabular} & 83 & $\begin{array}{c}10 \\
0\end{array}$ & \begin{tabular}{l|l}
0 & 0
\end{tabular} & 0 & \begin{tabular}{|l|l|}
7 & 2
\end{tabular} & 20 & $\begin{array}{l}6 \\
5\end{array}$ & & \\
\hline 250 & $\begin{array}{c}5 . \\
1\end{array}$ & $\begin{array}{c}\text { Corner } \\
\text { Pool }\end{array}$ & 6064 & 45 & 14 & 11 & 121 & $\left.18\right|^{1}$ & $\begin{array}{c}1.0 \\
5\end{array}$ & $\begin{array}{c}2.1 \\
2\end{array}$ & $\begin{array}{c}0.2 \\
4\end{array}$ & & & 3 & 25 & 54 & 040 & 5 & 10 & 00 & \begin{tabular}{l|l}
0 & 0
\end{tabular} & 0 & 25 & 2530 & 3020 & \begin{tabular}{l|l}
0 & 0
\end{tabular} & 00 & $90^{1}$ & \begin{tabular}{c|c}
10 & 0 \\
0 & 0
\end{tabular} & $0 \mid 0$ & 0 & \begin{tabular}{l|l}
7 & 4
\end{tabular} & 406 & 695 & $\begin{array}{c}1.8 \\
8\end{array}$ & $\begin{array}{c}\text { Log jam on right, } \\
\text { doesn't span } \\
\text { channel }\end{array}$ \\
\hline 251 & $\begin{array}{l}3 . \\
3\end{array}$ & Run & 6063 & 27 & 13 & 18 & & 7 & \begin{tabular}{c|c}
0.7 & 1 \\
3 &
\end{tabular} & $\begin{array}{c}1.2 \\
2\end{array}$ & & & & 3 & 65 & 02 & \begin{tabular}{l|l}
0 & 80
\end{tabular} & 0 & 0 & 00 & \begin{tabular}{l|l}
0 & 0
\end{tabular} & 0 & 10 & 1035 & 3535 & $10 \mid 0$ & 00 & 99 & $0 \mid 0$ & $0 \mid 5$ & 0 & $\begin{array}{ll}6 & 6\end{array}$ & 60 & 660 & & $\begin{array}{c}\text { Log Jam on right, } \\
\text { doesn't span } \\
\text { channel. } \\
\text { Together with } \\
245 \text { log jam } \\
\text { creates shallow } \\
\text { off } \\
\text { channel/backwat } \\
\text { er. Not good } \\
\text { summer habitat, } \\
\text { but possibly good } \\
\text { winter } \\
\text { backwater. }\end{array}$ \\
\hline 252 & $\begin{array}{l}4 . \\
2\end{array}$ & \begin{tabular}{|c|} 
Mid- \\
Channel \\
Pool \\
\end{tabular} & 6164 & 21 & 11 & 7 & 151 & $10^{1}$ & \begin{tabular}{c|c}
1.4 & 2 \\
2 &
\end{tabular} & $\begin{array}{c}2.4 \\
2\end{array}$ & $\begin{array}{c}0.4 \\
5\end{array}$ & 30 & $d$ & 3 & 10 & \begin{tabular}{l|l}
0 & 5
\end{tabular} & 5 & 90 & 0 & $0 \mid 0$ & \begin{tabular}{l|l}
0 & 0
\end{tabular} & 0 & 30 & 3020 & 2015 & \begin{tabular}{l|l}
5 & 0
\end{tabular} & 0.0 & $95^{1}$ & \begin{tabular}{c|c}
10 & 0 \\
0 & 0
\end{tabular} & 00 & 0 & $6 \mid 5$ & 56 & 6 90 & $\begin{array}{c}1.9 \\
7\end{array}$ & \\
\hline 253 & $\begin{array}{l}3 . \\
4 \\
\end{array}$ & Step Run & 6165 & 68 & 13 & 10 & 72 & $21{ }^{0}$ & \begin{tabular}{c|c}
0.2 & 0 \\
6 &
\end{tabular} & $\begin{array}{c}0.6 \\
4 \\
\end{array}$ & & & & 3 & 10 & 02 & \begin{tabular}{l|l}
0 & 45
\end{tabular} & 30 & 5 & 00 & \begin{tabular}{l|l}
0 & 0
\end{tabular} & \begin{tabular}{l|l}
0 & 0
\end{tabular} & 0 & $\begin{array}{lll}0 & 40 \\
\end{array}$ & 4055 & $\begin{array}{lll}5 & 0 \\
\end{array}$ & $\begin{array}{lll}0 & 0 \\
\end{array}$ & \begin{tabular}{c|c}
10 & 1 \\
0 & \\
\end{tabular} & \begin{tabular}{|c|c}
10 & 0 \\
0 & 0 \\
\end{tabular} & \begin{tabular}{l|l}
0 & 15 \\
\end{tabular} & 50 & 76 & 656 & 595 & & $\begin{array}{c}2 \text { riffles (16, } 30 \\
\mathrm{ft}), 1 \text { run }\end{array}$ \\
\hline 254 & $\begin{array}{l}3 . \\
2\end{array}$ & Glide & 6165 & $\begin{array}{c}10 \\
0 \\
\end{array}$ & 16 & 21 & 18 & $9^{0}$ & \begin{tabular}{c|c}
0.7 & 1 \\
6 & \\
\end{tabular} & \begin{tabular}{|c|}
1.3 \\
5 \\
\end{tabular} & & & & 3 & 101 & 102 & 535 & 30 & 0 & 00 & \begin{tabular}{l|l}
0 & 0
\end{tabular} & $\begin{array}{ll}0 & 0\end{array}$ & 15 & 1555 & 5510 & \begin{tabular}{l|l}
5 & 0
\end{tabular} & 00 & $85^{1}$ & \begin{tabular}{|c|c}
10 & 0 \\
0 & \\
\end{tabular} & \begin{tabular}{l|l}
0 & 0
\end{tabular} & 0 & 69 & 956 & 695 & & \\
\hline 255 & $\begin{array}{l}3 . \\
4\end{array}$ & Step Run & 6164 & 72 & 10 & 9 & $5 \mid 1$ & $\left.17\right|^{0}$ & \begin{tabular}{c|l}
0.0 & 0 \\
4 & 0
\end{tabular} & 0.1 & & & & 3 & $\begin{array}{lll}5 & 1\end{array}$ & $10 \mid 2$ & 030 & 10 & 30 & 00 & \begin{tabular}{l|l}
0 & 0
\end{tabular} & $\begin{array}{ll}0 & 0\end{array}$ & 0 & 030 & 30403 & $30 \mid 0$ & 00 & $\left|\begin{array}{c}10 \\
0\end{array}\right|$ & \begin{tabular}{c|c}
10 & 0 \\
0 & 0
\end{tabular} & \begin{tabular}{l|l}
0 & 5
\end{tabular} & 5 & 69 & 956 & 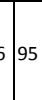 & & $\begin{array}{l}\text { Access, LB trail to } \\
\text { Purdy Ranch Rd } \\
\text { just below } \\
\text { Squirrel Flats }\end{array}$ \\
\hline 256 & $\begin{array}{l}4 . \\
2\end{array}$ & \begin{tabular}{|c|} 
Mid- \\
Channel \\
Pool \\
\end{tabular} & 6063 & 80 & 15 & 17 & 151 & \begin{tabular}{|l|l}
13 & 1 \\
13
\end{tabular} & $1.6]^{3}$ & $\begin{array}{c}3.4 \\
9\end{array}$ & \begin{tabular}{c|c}
0.1 & 1 \\
8 & 1
\end{tabular} & 10 & d & & 30 & \begin{tabular}{l|l}
0 & $5 \mathrm{C}$
\end{tabular} & \begin{tabular}{l|l}
0 & 0
\end{tabular} & 0 & 0 & 500 & \begin{tabular}{l|l}
0 & 0
\end{tabular} & \begin{tabular}{l|l}
0 & 0
\end{tabular} & 5 & 54 & $4035 \mid 1$ & \begin{tabular}{l|l}
10 & 5
\end{tabular} & 50 & $\begin{array}{c}10 \\
0\end{array}$ & \begin{tabular}{c|c}
10 & 0 \\
0 & 0
\end{tabular} & \begin{tabular}{l|l}
0 & 0
\end{tabular} & 5 & 69 & 956 & 695 & $\begin{array}{c}3.3 \\
1\end{array}$ & \\
\hline 257 & $\begin{array}{l}5 . \\
1 \\
\end{array}$ & $\begin{array}{c}\text { Corner } \\
\text { Pool }\end{array}$ & 6064 & $\begin{array}{c}15 \\
5\end{array}$ & 18 & 13 & & $20^{1}$ & \begin{tabular}{c|c}
1.7 & 2 \\
2 & \\
\end{tabular} & \begin{tabular}{c|}
2.6 \\
9
\end{tabular} & \begin{tabular}{|c|c|}
0.3 & 4 \\
3 & \\
\end{tabular} & 40 & c & 3 & 10 & \begin{tabular}{l|l}
0 & 1 \\
0
\end{tabular} & \begin{tabular}{l|l}
0 & 40
\end{tabular} & 30 & 0 & 00 & \begin{tabular}{l|l}
0 & 0
\end{tabular} & $\begin{array}{lll}0 & 10\end{array}$ & 15 & 1530 & 3015 & $5 \quad 5$ & \begin{tabular}{|l|l|}
5 & 15 \\
\end{tabular} & \begin{tabular}{c|}
10 \\
0
\end{tabular} & \begin{tabular}{c|c}
10 & 0 \\
0 & \\
\end{tabular} & \begin{tabular}{l|l}
0 & 15
\end{tabular} & 50 & \begin{tabular}{|l|l|}
6 & 8
\end{tabular} & 80 & 6 & $\begin{array}{c}2.3 \\
6\end{array}$ & $\begin{array}{c}\text { Combo } 5.1 \text { and } \\
5.2\end{array}$ \\
\hline 258 & \begin{tabular}{|l|}
3. \\
2 \\
\end{tabular} & Glide & 6064 & 71 & 17 & 20 & 131 & $19^{0}$ & \begin{tabular}{l|l}
0.8 & 1 \\
4 & \\
\end{tabular} & \begin{tabular}{|c|}
1.5 \\
2 \\
\end{tabular} & & & & 3 & 53 & $35 \mid 2$ & \begin{tabular}{l|l}
0 & 0
\end{tabular} & 35 & 10 & 00 & \begin{tabular}{l|l}
0 & 0
\end{tabular} & $\begin{array}{ll}0 & 0\end{array}$ & 20 & 2040 & 1020 & 00 & 00 & 95 & \begin{tabular}{c|c}
10 & 0 \\
0 & 0 \\
\end{tabular} & \begin{tabular}{l|l}
0 & 0 \\
\end{tabular} & 0 & \begin{tabular}{l|l}
6 & 1 \\
\end{tabular} & $\begin{array}{l}10 \\
0 \\
\end{array}$ & 695 & & \\
\hline 259 & $\begin{array}{l}5 . \\
2\end{array}$ & \begin{tabular}{|c|} 
Lateral \\
Scour \\
Pool - Log \\
Enhanced
\end{tabular} & 6163 & $\mid \begin{array}{c}10 \\
6\end{array}$ & 13 & 19 & 12 & 9 & \begin{tabular}{c|c}
1.9 & 3 \\
1 &
\end{tabular} & $\begin{array}{c}3.2 \\
6\end{array}$ & $\left|\begin{array}{c|c}1.4 \\
3\end{array}\right|$ & 40 & c & 3 & $50 \mid 1$ & $10 \mid 2 C$ & \begin{tabular}{l|l}
0 & 35
\end{tabular} & 30 & 5 & 00 & \begin{tabular}{l|l}
0 & 0
\end{tabular} & $\begin{array}{ll}0 & 0\end{array}$ & 20 & 2035 & 3520 & \begin{tabular}{|l|l}
5 & 0
\end{tabular} & 00 & 93 & \begin{tabular}{c|c}
10 & 0 \\
0 & 0
\end{tabular} & \begin{tabular}{l|l}
0 & 0
\end{tabular} & 10 & $\begin{array}{ll}6 & 9\end{array}$ & 95 & 590 & $\begin{array}{c}1.8 \\
3\end{array}$ & $\begin{array}{l}\text { log jam with root } \\
\text { wad enhanced. } \\
\text { SDA LB }\end{array}$ \\
\hline 260 & $\begin{array}{l}3 . \\
2\end{array}$ & Glide & & 91 & 14 & 9 & & \begin{tabular}{|l|l|}
18 & 0 \\
\end{tabular} & \begin{tabular}{c|c}
0.7 & 1 \\
8 &
\end{tabular} & \begin{tabular}{|c|}
1.6 \\
8
\end{tabular} & & & & 3 & \begin{tabular}{|l|l}
5 & 1
\end{tabular} & $10 \mid 2$ & $\begin{array}{lll}0 & 30\end{array}$ & 35 & 5 & 00 & \begin{tabular}{l|l}
0 & 0
\end{tabular} & $\begin{array}{ll}0 & 0\end{array}$ & 20 & 2050 & 5010 & \begin{tabular}{l|l}
0 & 0
\end{tabular} & 00 & \begin{tabular}{c|c}
10 \\
0
\end{tabular} & \begin{tabular}{|c|c}
10 & 0 \\
0 & 0 \\
\end{tabular} & \begin{tabular}{l|l}
0 & 0
\end{tabular} & 0 & $6{ }^{6} 1$ & $\begin{array}{c}10 \\
0\end{array}$ & 595 & & \\
\hline 261 & $\begin{array}{l}4 . \\
2\end{array}$ & \begin{tabular}{|c|} 
Mid- \\
Channel \\
Pool \\
\end{tabular} & 6169 & 76 & 14 & 18 & 16 & 7 & \begin{tabular}{c|c}
1.7 & 3 \\
1 &
\end{tabular} & $\begin{array}{c}3.9 \\
5\end{array}$ & $\begin{array}{c}0.4 \\
2\end{array}$ & & c & 3 & 5 & 02 & 045 & 30 & 0 & 00 & \begin{tabular}{l|l}
0 & 0
\end{tabular} & $\begin{array}{ll}0 & 0\end{array}$ & 10 & 1040 & 4030 & $10 \mid 0$ & 00 & $\begin{array}{c}10 \\
0\end{array}$ & \begin{tabular}{c|c}
10 & 0 \\
0 & 0
\end{tabular} & \begin{tabular}{l|l}
0 & 0
\end{tabular} & 15 & 69 & 90 & 550 & $\begin{array}{c}3.5 \\
3\end{array}$ & $\begin{array}{l}\text { Log formed but } \\
\text { no channel } \\
\text { spanning jam }\end{array}$ \\
\hline 262 & $\begin{array}{l}3 . \\
4\end{array}$ & Step Run & 62 & $\begin{array}{c}11 \\
8\end{array}$ & 10 & 7 & 15 & 8 & \begin{tabular}{c|c}
0.7 & 1 \\
1 &
\end{tabular} & $\begin{array}{c}1.8 \\
4\end{array}$ & & & & 3 & 10 & 52 & 035 & 30 & 10 & 00 & \begin{tabular}{l|l}
0 & 0
\end{tabular} & $\begin{array}{ll}0 & 0\end{array}$ & 10 & 1050 & 5020 & $10 \mid 0$ & 00 & $85^{1}$ & \begin{tabular}{c|c}
10 & 0 \\
0 & 0
\end{tabular} & 0.0 & 0 & 69 & 956 & 6 & & $\begin{array}{l}1 \text { riffle }(25 \mathrm{ft}), 2 \\
\text { glide. "step } \\
\text { glide." }\end{array}$ \\
\hline 263 & $\begin{array}{l}4 . \\
2\end{array}$ & \begin{tabular}{|c|} 
Mid- \\
Channel \\
Pool
\end{tabular} & 6367 & 73 & 12 & 8 & 20 & 9 & \begin{tabular}{c|c}
1.5 & 2 \\
1 &
\end{tabular} & $\begin{array}{c}2.7 \\
5\end{array}$ & $\begin{array}{c}0.1 \\
3\end{array}$ & & c & 3 & 80 & 05 & $\begin{array}{lll}0 & 0\end{array}$ & 50 & 0 & 00 & \begin{tabular}{l|l}
0 & 0
\end{tabular} & $\begin{array}{ll}0 & 0\end{array}$ & 15 & 1560 & 6010 & 00 & 00 & $90^{1}$ & \begin{tabular}{c|c}
10 & 0 \\
0 & 0
\end{tabular} & 030 & 0 & 69 & 907 & 790 & $\begin{array}{c}2.6 \\
2\end{array}$ & \\
\hline 264 & $\begin{array}{l}1 . \\
1\end{array}$ & \begin{tabular}{|c|} 
Low \\
gradient \\
Riffle \\
\end{tabular} & & $\begin{array}{c}12 \\
4\end{array}$ & 10 & 9 & \begin{tabular}{l|l}
3 & 1
\end{tabular} & 18 & \begin{tabular}{c|c}
0.2 & 0 \\
8 &
\end{tabular} & $\begin{array}{c}0.6 \\
4\end{array}$ & & & & 3 & & $0 \mid 9 c$ & \begin{tabular}{l|l}
0 & 0
\end{tabular} & 0 & 10 & \begin{tabular}{l|l}
0 & 0 \\
0
\end{tabular} & \begin{tabular}{l|l}
0 & 0
\end{tabular} & $\begin{array}{ll}0 & 0\end{array}$ & 0 & 040 & 4040 & 200 & 00 & $\mid \begin{array}{c}10 \\
0\end{array}$ & \begin{tabular}{c|c}
10 & 0 \\
0 & 0
\end{tabular} & \begin{tabular}{l|l}
0 & 0
\end{tabular} & 0 & 69 & 957 & $\begin{array}{l}7 \\
7\end{array}$ & & \\
\hline 265 & $\begin{array}{l}4 . \\
2\end{array}$ & \begin{tabular}{|c} 
Mid- \\
Channel \\
Pool
\end{tabular} & 6368 & 73 & 13 & 18 & 11 & 11 & $\begin{array}{c}1.4 \\
5\end{array}$ & $\begin{array}{c}2.4 \\
1\end{array}$ & \begin{tabular}{c|c}
0.1 \\
9
\end{tabular} \mid 3 & 30 & $d$ & 3 & 52 & 203 & \begin{tabular}{l|l}
0 & 10
\end{tabular} & 40 & 0 & \begin{tabular}{l|l}
0 & 0
\end{tabular} & \begin{tabular}{l|l}
0 & 0
\end{tabular} & $\begin{array}{ll}0 & 0\end{array}$ & 15 & 1550 & 5020 & 00 & 00 & \begin{tabular}{c|c}
10 & $s$ \\
0 &
\end{tabular} & 955 & \begin{tabular}{l|l}
5 & 0
\end{tabular} & 20 & 65 & 50 & 650 & $\begin{array}{c}2.2 \\
2\end{array} \mid$ & \\
\hline 266 & $\begin{array}{l}3 . \\
4\end{array}$ & Step Run & 6473 & 59 & 16 & 11 & 73 & 30 & \begin{tabular}{c|}
0.6 \\
6
\end{tabular} & 1.6 & & & & 3 & & $25 \mid 2$ & $5 \quad 0$ & 25 & 25 & \begin{tabular}{l|l}
0 & 0 \\
0
\end{tabular} & \begin{tabular}{l|l}
0 & 0
\end{tabular} & \begin{tabular}{l|l}
0 & 0
\end{tabular} & 5 & $\begin{array}{lll}5 & 40\end{array}$ & 4040 & 100 & 00 & 62 & \begin{tabular}{c|c}
10 & 0 \\
0 & 0
\end{tabular} & \begin{tabular}{l|l}
0 & 0
\end{tabular} & 0 & $6^{6}$ & $\begin{array}{c}10 \\
0\end{array}$ & 695 & & $\begin{array}{l}1 \text { riffle }(25 \mathrm{ft}), 1 \\
\text { run. Bankfull } \\
\text { measurements } \\
\text { taken }\end{array}$ \\
\hline 267 & $\begin{array}{l}4 . \\
2\end{array}$ & \begin{tabular}{|c|} 
Mid- \\
Channel \\
Pool
\end{tabular} & 6370 & 48 & 25 & 30 & 301 & \begin{tabular}{|l|l}
14 & 2 \\
\end{tabular} & 2.2 & $\begin{array}{c}3.7 \\
5\end{array}$ & $\left|\begin{array}{c|c}0.3 \\
5\end{array}\right| 4$ & 40 & c & 3 & & 05 & 050 & 0 & 0 & 0 & \begin{tabular}{l|l}
0 & 0
\end{tabular} & $\begin{array}{lll}0 & 0\end{array}$ & 15 & 1550 & & \begin{tabular}{l|l}
0 & 0
\end{tabular} & 00 & 549 & 964 & 40 & 0 & $6^{1}$ & $\begin{array}{c}10 \\
0\end{array}$ & 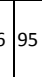 & 3.4 & $\begin{array}{l}\text { Log jam, channel } \\
\text { spanning. } 15^{\prime} L x \\
30^{\prime} \text { W. End } 8 / 6\end{array}$ \\
\hline
\end{tabular}




\begin{tabular}{|c|c|c|c|c|c|c|c|c|c|c|c|c|c|c|c|c|c|c|c|c|c|c|c|c|c|c|c|c|c|c|c|c|c|c|c|}
\hline 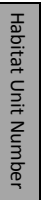 & & 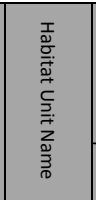 & 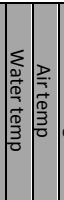 & 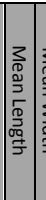 & 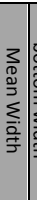 & 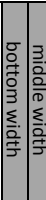 & 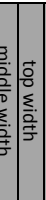 & 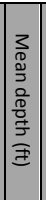 & 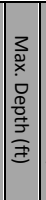 & 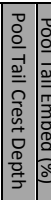 & 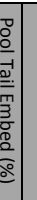 & 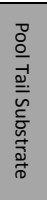 & 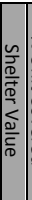 & 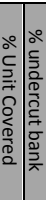 & 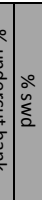 & $\frac{\curvearrowright}{\grave{2}}$ & 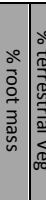 & 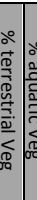 & 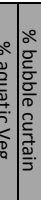 & 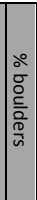 & 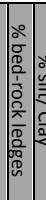 & 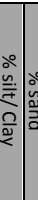 & 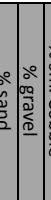 & 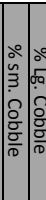 & 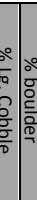 & 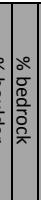 & 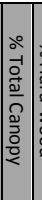 & 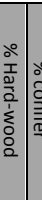 & 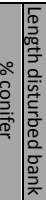 & 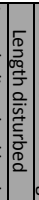 & 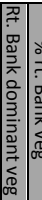 & 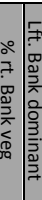 & 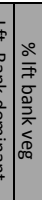 & 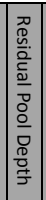 & 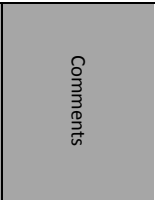 \\
\hline 268 & $\begin{array}{l}5 . \\
3\end{array}$ & \begin{tabular}{|c|} 
Lateral \\
Scour \\
Pool - \\
Root Wad \\
Enhanced
\end{tabular} & 6270 & 37 & 13 & $14 \mid 20$ & 205 & $\begin{array}{c}1.1 \\
8\end{array}$ & $\begin{array}{c}2.2 \\
4\end{array}$ & $\begin{array}{c}0.6 \\
9\end{array}$ & & & 3 & 4010 & $\begin{array}{ll}0 & 10\end{array}$ & 40 & \begin{tabular}{l|l}
40 & 0
\end{tabular} & 0 o & \begin{tabular}{l|l}
0 & 0
\end{tabular} & 0 & 02 & & 2050 & $\begin{array}{ll}10 & 0\end{array}$ & \begin{tabular}{l|l}
0 & 0
\end{tabular} & 0 & 96 & \begin{tabular}{l|l}
98 & 2
\end{tabular} & \begin{tabular}{l|l}
2 & 5
\end{tabular} & 0 & $\begin{array}{lll}6 & 8\end{array}$ & $80 \mid 6$ & 675 & $\begin{array}{c}1.5 \\
5\end{array}$ & End $8 / 6$ \\
\hline 269 & $\begin{array}{l}4 . \\
2\end{array}$ & \begin{tabular}{|c|} 
Mid- \\
Channel \\
Pool \\
\end{tabular} & 6165 & \begin{tabular}{|l|l|l|}
7 & 1
\end{tabular} & 14 & $5 \mid 27$ & $27 \mid 9$ & 1.9 & 3.6 & $\begin{array}{c}0.4 \\
3\end{array} \mid 2$ & 20 & d & 3 & 2020 & 020 & 30 & $30 \mid 0$ & \begin{tabular}{l|l}
0 & 0
\end{tabular} & $\begin{array}{l}0 \\
0\end{array}$ & 0 & 03 & 354 & 4020 & 50 & \begin{tabular}{l|l}
0 & 0
\end{tabular} & 0 & $\left|\begin{array}{c}10 \\
0\end{array}\right|$ & \begin{tabular}{c|c}
10 & 0 \\
0 & 0
\end{tabular} & \begin{tabular}{l|l}
0 & 0
\end{tabular} & 0 & 69 & $90 \quad 6$ & 695 & $\begin{array}{c}3.1 \\
7\end{array} \mid$ & $\begin{array}{c}\text { Start } 8 / 7 . \text { Log } \\
\text { jam across } \\
\text { channel }\end{array}$ \\
\hline 270 & $\begin{array}{l}1 . \\
1\end{array}$ & $\begin{array}{c}\text { Low } \\
\text { gradient } \\
\text { Riffle } \\
\end{array}$ & 6164 & \begin{tabular}{|l|l}
46 & 1
\end{tabular} & 14 & $\begin{array}{ll}9 & 5\end{array}$ & 529 & $\left|\begin{array}{c}0.4 \\
2\end{array}\right|$ & $\begin{array}{c}1.1 \\
4\end{array}$ & & & & 3 & \begin{tabular}{l|l}
25 & 0
\end{tabular} & 20 & 40 & \begin{tabular}{l|l}
0 & 4
\end{tabular} & 40 & \begin{tabular}{l|l}
0 & 0
\end{tabular} & 0 & 0 & 5 & $5 \quad 50$ & 400 & \begin{tabular}{l|l}
0 & 0
\end{tabular} & 0 & $\mid \begin{array}{c}10 \\
0\end{array}$ & $\begin{array}{c}10 \\
0\end{array} \mid$ & \begin{tabular}{l|l|l}
0 & 15
\end{tabular} & 50 & \begin{tabular}{|l|l|}
6 & 7
\end{tabular} & \begin{tabular}{l|l}
70 & 6
\end{tabular} & 6 6 95 & & \\
\hline 271 & $\begin{array}{l}4 . \\
2\end{array}$ & $\begin{array}{c}\text { Mid- } \\
\text { Channel } \\
\text { Pool } \\
\end{array}$ & \begin{tabular}{|l|l|}
61 & 63 \\
\end{tabular} & 52 & 132 & $20 \mid 14$ & 144 & $\begin{array}{c}1.6 \\
3\end{array}$ & $\begin{array}{c}3.1 \\
5\end{array}$ & $\begin{array}{c}0.3 \\
2\end{array}$ & & c & 3 & \begin{tabular}{l|l}
80 & 5
\end{tabular} & 515 & 65 & $10 \mid 0$ & 05 & $\begin{array}{l}5 \\
5\end{array}$ & 0 & 03 & 303 & 3035 & 50 & \begin{tabular}{l|l}
0 & 0
\end{tabular} & 0 & 91 & \begin{tabular}{c|c}
10 & 0 \\
0 & 0
\end{tabular} & \begin{tabular}{l|l}
0 & 0
\end{tabular} & 15 & $\begin{array}{ll}6 & 9\end{array}$ & \begin{tabular}{l|l}
95 & 6
\end{tabular} & 585 & $\begin{array}{c}2.8 \\
3\end{array}$ & \begin{tabular}{|c|} 
Access, trail to \\
gate near top of \\
squirrel flats \\
\end{tabular} \\
\hline 272 & $\begin{array}{c}1 . \\
1\end{array}$ & $\begin{array}{c}\text { Low } \\
\text { gradient } \\
\text { Riffle } \\
\end{array}$ & 62 & 81 & 5 & \begin{tabular}{|l|l}
4 & 6
\end{tabular} & \begin{tabular}{l|l|}
6 & 6
\end{tabular} & $\begin{array}{c}0.3 \\
6\end{array}$ & $\left|\begin{array}{c}0.7 \\
8\end{array}\right|$ & & & & 3 & \begin{tabular}{|l|l}
10 & 0
\end{tabular} & 50 & 0 & \begin{tabular}{|l|l}
50 & 0
\end{tabular} & 0 & \begin{tabular}{l|l}
0 & 0
\end{tabular} & 0 & 0 & \begin{tabular}{l|l}
5 & 5
\end{tabular} & 570 & 200 & \begin{tabular}{l|l}
0 & 0
\end{tabular} & 0 & $87 \mid 9$ & \begin{tabular}{l|l}
90 & 1
\end{tabular} & $10 \mid 0$ & 0 & \begin{tabular}{|l|l|}
6 & 9
\end{tabular} & \begin{tabular}{l|l}
95 & 6
\end{tabular} & 6 6 95 & & \\
\hline 273 & $\begin{array}{c}4 . \\
2\end{array}$ & $\begin{array}{c}\text { Mid- } \\
\text { Channel } \\
\text { Pool }\end{array}$ & 6266 & 572 & 202 & 2925 & \begin{tabular}{l|l}
25 & 6
\end{tabular} & 4 & $\begin{array}{c}5.4 \\
1\end{array}$ & $\begin{array}{c}0.2 \\
7\end{array}$ & & c & 3 & $\begin{array}{lll}30 & 10\end{array}$ & $\begin{array}{ll}0 & 10\end{array}$ & 35 & \begin{tabular}{l|l}
30 & 1 \\
\end{tabular} & 150 & \begin{tabular}{l|l}
0 & 0
\end{tabular} & 0 & 03 & 303 & 3040 & $\begin{array}{ll}0 & 0\end{array}$ & \begin{tabular}{l|l}
0 & 0
\end{tabular} & 0 & 63 & \begin{tabular}{l|l}
5 & 9
\end{tabular} & \begin{tabular}{l|l}
95 & 0
\end{tabular} & 0 & $\begin{array}{ll}8 & 6\end{array}$ & \begin{tabular}{l|l}
60 & 6
\end{tabular} & 6 & $\left|\begin{array}{c}5.1 \\
4\end{array}\right|$ & $\begin{array}{c}\text { Appears a barrier } \\
\text { to fish passage at } \\
\text { current flows. } \\
\text { Log jam }\end{array}$ \\
\hline 274 & $\begin{array}{c}1 . \\
1\end{array}$ & $\begin{array}{c}\text { Low } \\
\text { gradient } \\
\text { Riffle }\end{array}$ & 6266 & 50 & 9 & \begin{tabular}{l|l}
6 & 10
\end{tabular} & 1012 & $\begin{array}{c}0.2 \\
6\end{array}$ & 0.4 & & & & 3 & $\begin{array}{lll}10 & 0\end{array}$ & 30 & 0 & \begin{tabular}{l|l}
30 & 4
\end{tabular} & 40 & \begin{tabular}{l|l}
0 & 0
\end{tabular} & 0 & 0 & \begin{tabular}{l|l}
0 & 0
\end{tabular} & \begin{tabular}{l|l|l|l|}
0 & 45
\end{tabular} & 4510 & $10 \mid 0$ & 0 & 83 & \begin{tabular}{l|l}
50 & 5
\end{tabular} & \begin{tabular}{l|l}
50 & 0
\end{tabular} & 0 & $\begin{array}{ll}7 & 7\end{array}$ & \begin{tabular}{l|l}
70 & 6
\end{tabular} & 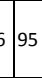 & & \\
\hline 275 & $\begin{array}{c}5 . \\
1 \\
\end{array}$ & \begin{tabular}{c|} 
Corner \\
Pool
\end{tabular} & 6267 & 65 & 14 & 1214 & 1416 & $\begin{array}{c}1.4 \\
9\end{array}$ & $\begin{array}{c}2.4 \\
5\end{array}$ & \begin{tabular}{|c|c|}
0.3 & 4 \\
& \\
\end{tabular} & 40 & d & 3 & 2015 & 530 & 25 & \begin{tabular}{|l|l|}
20 & 11
\end{tabular} & 100 & \begin{tabular}{l|l}
0 & 0
\end{tabular} & 0 & 01 & 151 & 1560 & \begin{tabular}{|l|l}
10 & 0
\end{tabular} & \begin{tabular}{l|l}
0 & 0
\end{tabular} & 0 & \begin{tabular}{|c|c|}
10 \\
0
\end{tabular} & \begin{tabular}{c|c}
10 & 0 \\
0 & 0 \\
\end{tabular} & \begin{tabular}{l|l}
0 & 0
\end{tabular} & 0 & 72 & $20 \mid 6$ & 695 & $\begin{array}{c}2.1 \\
1 \\
\end{array}$ & \\
\hline 276 & $\begin{array}{l}4 . \\
2\end{array}$ & \begin{tabular}{c|} 
Mid- \\
Channel \\
Pool \\
\end{tabular} & 6264 & 381 & 15 & \begin{tabular}{l|l|l}
16 & 14
\end{tabular} & 1414 & $\begin{array}{c}1.6 \\
1\end{array}$ & $\begin{array}{c}2.9 \\
5\end{array}$ & \begin{tabular}{c|c}
0.4 & 2 \\
1 &
\end{tabular} & 20 & c & 2 & \begin{tabular}{l|l}
10 & 0
\end{tabular} & 10 & 0 & 306 & 60 & $\begin{array}{l}0 \\
0\end{array}$ & 0 & 01 & 151 & 1565 & 50 & \begin{tabular}{l|l}
0 & 0
\end{tabular} & 0 & $\mid \begin{array}{c}10 \\
0\end{array}$ & \begin{tabular}{c|c}
10 & 0 \\
0 & 0
\end{tabular} & \begin{tabular}{l|l}
0 & 0
\end{tabular} & 0 & 69 & $90 \quad 6$ & 6 & $\begin{array}{c}2.5 \\
4\end{array}$ & \\
\hline 277 & $\begin{array}{l}5 . \\
2\end{array}$ & \begin{tabular}{|c|} 
Lateral \\
Scour \\
Pool - Log \\
Enhanced
\end{tabular} & 6265 & 28 & 10 & \begin{tabular}{|l|l|l|}
14 & 10
\end{tabular} & 107 & $\begin{array}{c}1.3 \\
3\end{array}$ & 2 & $\mid \begin{array}{c}0.8 \\
2\end{array}$ & 20 & c & 3 & \begin{tabular}{l|l}
40 & 5
\end{tabular} & 525 & 30 & \begin{tabular}{l|l|l}
30 & 0
\end{tabular} & 0 o & $\begin{array}{l}0 \\
0\end{array}$ & 0 & 02 & 202 & 2055 & 50 & \begin{tabular}{l|l}
0 & 0
\end{tabular} & 0 & $\left|\begin{array}{c}10 \\
0\end{array}\right|$ & \begin{tabular}{c|c}
10 & 0 \\
0 & 0
\end{tabular} & \begin{tabular}{l|l}
0 & 0
\end{tabular} & 0 & 69 & $90 \quad 6$ & 590 & $\left|\begin{array}{c}1.1 \\
8\end{array}\right|$ & \\
\hline 278 & \begin{tabular}{c|}
1. \\
1
\end{tabular} & \begin{tabular}{|c|} 
Low \\
gradient \\
Riffle
\end{tabular} & \begin{tabular}{ll|l|}
62 & 64 \\
\end{tabular} & 32 & 7 & \begin{tabular}{l|l}
7 & 7
\end{tabular} & \begin{tabular}{l|l|}
7 & 8
\end{tabular} & $\begin{array}{c}0.2 \\
8\end{array}$ & 0.5 & & & & 1 & \begin{tabular}{l|l}
10 & 0
\end{tabular} & 50 & 0 & \begin{tabular}{|l|l}
50 & 0
\end{tabular} & \begin{tabular}{l|l}
0 & 0
\end{tabular} & \begin{tabular}{l|l}
0 & 0
\end{tabular} & 0 & 0 & 0 & \begin{tabular}{l|l|l}
0 & 50 & 5
\end{tabular} & 500 & \begin{tabular}{l|l}
0 & 0
\end{tabular} & 0 & $\mid \begin{array}{c}10 \\
0\end{array}$ & \begin{tabular}{c|c}
10 & 0 \\
0 & 0
\end{tabular} & \begin{tabular}{l|l}
0 & 0
\end{tabular} & 0 & $\begin{array}{ll}6 & 6\end{array}$ & 60 & $\begin{array}{l}5 \\
5\end{array}$ & & \\
\hline 279 & $\begin{array}{l}4 . \\
2\end{array} \mid$ & $\begin{array}{c}\text { Mid- } \\
\text { Channel } \\
\text { Pool } \\
\end{array}$ & 6166 & \begin{tabular}{|l|l|l|}
62 & 1
\end{tabular} & 15 & 826 & \begin{tabular}{l|l|}
26 & 12
\end{tabular} & $\begin{array}{c}2.3 \\
6\end{array}$ & $\left|\begin{array}{c}4.7 \\
1\end{array}\right|$ & $\begin{array}{c}0.1 \\
3\end{array} \mid 1$ & 10 & c & 3 & $40 \quad 0$ & 10 & 20 & \begin{tabular}{l|l}
40 & 2
\end{tabular} & $25 \mid c$ & \begin{tabular}{l|l}
0 & 0
\end{tabular} & 0 & 53 & 304 & $40 \mid 40$ & $\begin{array}{lll}10 & 0\end{array}$ & \begin{tabular}{l|l}
0 & 0
\end{tabular} & 0 & 94 & \begin{tabular}{c|c}
10 & 0 \\
0 & 0
\end{tabular} & \begin{tabular}{l|l}
0 & 15
\end{tabular} & 50 & $\begin{array}{lll}6 & 7\end{array}$ & \begin{tabular}{l|l}
75 & 7
\end{tabular} & $\begin{array}{l}7 \\
7\end{array}$ & $\mid \begin{array}{c}4.5 \\
8\end{array}$ & \\
\hline 280 & $\begin{array}{l}3 . \\
4 \\
\end{array}$ & Step Run & 6369 & 601 & 16 & 129 & 927 & $\begin{array}{c}0.4 \\
2\end{array}$ & $\begin{array}{c}0.7 \\
1\end{array}$ & & & & 2 & $<5 \mid 10$ & & 0 & \begin{tabular}{l|l}
90 & 0
\end{tabular} & \begin{tabular}{l|l}
0 & 0
\end{tabular} & $\begin{array}{ll}0 & 0\end{array}$ & 0 & 01 & 101 & 1050 & 300 & \begin{tabular}{l|l}
0 & 0
\end{tabular} & 0 & 98 & \begin{tabular}{|c|c}
10 & 0 \\
0 & 0 \\
\end{tabular} & \begin{tabular}{l|l}
0 & 0
\end{tabular} & 0 & 69 & \begin{tabular}{l|l}
90 & 6
\end{tabular} & 6 & & $\begin{array}{c}1 \text { riffle }(20 \mathrm{ft}) 2 \\
\text { run }\end{array}$ \\
\hline 281 & $\begin{array}{l}5 . \\
2\end{array}$ & \begin{tabular}{|c} 
Lateral \\
Scour \\
Pool - Log \\
Enhanced
\end{tabular} & 69 & 631 & 172 & \begin{tabular}{|l|l|l|}
27 & 15
\end{tabular} & 1510 & $\begin{array}{c}1.5 \\
3\end{array} \mid$ & $\begin{array}{c}2.7 \\
9\end{array} \mid$ & $\begin{array}{c}0.1 \\
9\end{array}$ & & & 3 & $80 \mid 10$ & $\begin{array}{ll}0 & 10\end{array}$ & 40 & 103 & $30 \mathrm{C}$ & $\begin{array}{ll}0 & 0\end{array}$ & 0 & 02 & 252 & 2535 & 150 & \begin{tabular}{l|l}
0 & 0
\end{tabular} & 0 & $\left|\begin{array}{c}10 \\
0\end{array}\right|$ & \begin{tabular}{c|c}
10 & 0 \\
0 & 0
\end{tabular} & \begin{tabular}{l|l}
0 & 0
\end{tabular} & 15 & 75 & \begin{tabular}{l|l}
50 & 6
\end{tabular} & $\begin{array}{l}5 \\
5\end{array}$ & 2.6 & SDA \\
\hline 282 & $\begin{array}{l}3 . \\
4 \\
\end{array}$ & Step Run & & 36 & 8 & 108 & 87 & $\begin{array}{c}0.4 \\
1\end{array}$ & $\begin{array}{c}0.5 \\
7\end{array}$ & & & & 1 & $\begin{array}{lll}5 & 30\end{array}$ & 030 & 0 & \begin{tabular}{|l|l}
40 & 0
\end{tabular} & \begin{tabular}{l|l}
0 & 0
\end{tabular} & \begin{tabular}{l|l}
0 & 0
\end{tabular} & 0 & 01 & 101 & 10602 & 200 & \begin{tabular}{l|l}
0 & 0
\end{tabular} & 0 & \begin{tabular}{|c|c|}
10 & 1 \\
0 & \\
\end{tabular} & \begin{tabular}{|c|c}
10 & 0 \\
0 & 0 \\
\end{tabular} & \begin{tabular}{l|l}
0 & 0 \\
\end{tabular} & 5 & $\begin{array}{ll}6 & 9\end{array}$ & $95 \mid 6$ & $\begin{array}{l}6 \\
5\end{array}$ & & \\
\hline 283 & $\begin{array}{l}4 . \\
2\end{array}$ & \begin{tabular}{|c|} 
Mid- \\
Channel \\
Pool \\
\end{tabular} & 6369 & 75 & 17 & $13 \mid 20$ & & $\mid \begin{array}{c}3.8 \\
8\end{array}$ & $\begin{array}{c}5.3 \\
4\end{array}$ & \begin{tabular}{c|c}
0.2 & 4 \\
1 & 4
\end{tabular} & 40 & d & 3 & \begin{tabular}{l|l}
5 & 25
\end{tabular} & 50 & 25 & \begin{tabular}{l|l|l}
30 & 1
\end{tabular} & $10 \mathrm{C}$ & \begin{tabular}{l|l}
0 & 0
\end{tabular} & 0 & 102 & 252 & 2535 & 50 & \begin{tabular}{l|l}
0 & 0
\end{tabular} & 10 & 89 & \begin{tabular}{c|c}
10 & 0 \\
0 & 0
\end{tabular} & \begin{tabular}{l|l}
0 & 0
\end{tabular} & 0 & 66 & \begin{tabular}{l|l}
60 & 6
\end{tabular} & 595 & $\begin{array}{c}5.1 \\
3\end{array}$ & \\
\hline 284 & $\begin{array}{c}1 . \\
1\end{array}$ & \begin{tabular}{|c|} 
Low \\
gradient \\
Riffle
\end{tabular} & & $37 \mid$ & 7 & \begin{tabular}{|l|l|}
3 & 8
\end{tabular} & 89 & $\begin{array}{c}0.2 \\
2\end{array}$ & 0.4 & & & & 1 & \begin{tabular}{|l|l}
5 & 0
\end{tabular} & 50 & 0 & $0 \mid 5$ & $50 \mathrm{c}$ & $\begin{array}{ll}0 & 0\end{array}$ & 0 & 0 & \begin{tabular}{l|l}
0 & 0
\end{tabular} & \begin{tabular}{ll|l}
0 & 55
\end{tabular} & 405 & 50 & 0 & $\left|\begin{array}{c}10 \\
0\end{array}\right|$ & $\begin{array}{c}10 \\
0\end{array}$ & \begin{tabular}{l|l}
0 & 0
\end{tabular} & 0 & 68 & \begin{tabular}{l|l}
85 & 6
\end{tabular} & 6 6 95 & & \\
\hline 285 & \begin{tabular}{|l|}
3. \\
2 \\
\end{tabular} & Glide & \begin{tabular}{|l|l|}
62 & 67 \\
\end{tabular} & \begin{tabular}{|c|c|}
10 & 1 \\
0 & 1 \\
\end{tabular} & 13 & 9218 & 1813 & \begin{tabular}{|c|}
0.5 \\
2 \\
\end{tabular} & 1.3 & & & & 3 & 20 & $\begin{array}{ll}0 & 10\end{array}$ & 20 & $25 \mid 2$ & $25 \mathrm{C}$ & \begin{tabular}{l|l}
0 & 0
\end{tabular} & 0 & 02 & 202 & 2045 & 105 & 50 & 0 & 96 & \begin{tabular}{|c|c}
10 & 0 \\
0 & 0 \\
\end{tabular} & $\begin{array}{lll}0 & 30 \\
\end{array}$ & 0 & 69 & $90 \quad 6$ & 695 & & \\
\hline 286 & $\begin{array}{l}4 . \\
2\end{array}$ & $\begin{array}{c}\text { Mid- } \\
\text { Channel } \\
\text { Pool } \\
\end{array}$ & \begin{tabular}{|l|l|}
62 & 70 \\
\end{tabular} & 431 & 11 & \begin{tabular}{l|l|l|}
13 & 12
\end{tabular} & 129 & $\begin{array}{c}1.8 \\
1\end{array}$ & 2.9 & 0.5 & & c & 3 & 5020 & 00 & 10 & \begin{tabular}{|l|l}
60 & 1
\end{tabular} & 10 c & $\begin{array}{ll}0 & 0\end{array}$ & 0 & 02 & 202 & 2050 & \begin{tabular}{|l|l}
10 & 0
\end{tabular} & \begin{tabular}{l|l}
0 & 0
\end{tabular} & 0 & $\left|\begin{array}{c|c}10 \\
0\end{array}\right|$ & $\begin{array}{c}10 \\
0\end{array}$ & \begin{tabular}{l|l}
0 & 0
\end{tabular} & 0 & 66 & \begin{tabular}{l|l}
60 & 8
\end{tabular} & \begin{tabular}{l|l}
8 & 90
\end{tabular} & 2.4 & End $8 / 7$ \\
\hline 287 & $\begin{array}{c}5 . \\
1\end{array}$ & $\begin{array}{c}\text { Corner } \\
\text { Pool }\end{array}$ & 6267 & 51 & 12 & \begin{tabular}{l|l}
9 & 17
\end{tabular} & $17 \mid 10$ & $\left|\begin{array}{c}2.0 \\
6\end{array}\right|$ & $\begin{array}{c}3.4 \\
2\end{array}$ & \begin{tabular}{c|c}
0.3 \\
1
\end{tabular} & 30 & d & 3 & 5040 & 0 & 10 & \begin{tabular}{|l|l}
40 & 0
\end{tabular} & 0 & \begin{tabular}{l|l}
0 & 0
\end{tabular} & 0 & 02 & 202 & 2050 & \begin{tabular}{|l|l}
10 & 0
\end{tabular} & \begin{tabular}{l|l}
0 & 0
\end{tabular} & 0 & 97 & 406 & 60 & 5 & 90 & \begin{tabular}{l|l}
0 & 8
\end{tabular} & 880 & $\left|\begin{array}{c}3.1 \\
1\end{array}\right|$ & \begin{tabular}{|c|} 
Big redwood on \\
LB, roots create \\
undercut, $5.5 \mathrm{ft}$. \\
Undercut. Start \\
8.8 \\
\end{tabular} \\
\hline 288 & $\begin{array}{l}4 . \\
2\end{array}$ & $\begin{array}{c}\text { Mid- } \\
\text { Channel } \\
\text { Pool } \\
\end{array}$ & 6366 & 26 & 9 & \begin{tabular}{l|l|l}
7 & 12
\end{tabular} & 128 & $\begin{array}{c}1.2 \\
4\end{array}$ & $\begin{array}{c}2.0 \\
1\end{array}$ & \begin{tabular}{c|c}
0.2 & 3 \\
2 & \\
\end{tabular} & 30 & d & 1 & \begin{tabular}{|l|l}
5 & 0
\end{tabular} & 0 & 0 & \begin{tabular}{c|c}
10 & 0 \\
0 & 0
\end{tabular} & \begin{tabular}{l|l}
0 & 0
\end{tabular} & \begin{tabular}{l|l}
0 & 0
\end{tabular} & 0 & 02 & 202 & 2040 & 20 & \begin{tabular}{l|l}
0 & 0
\end{tabular} & 0 & 965 & 505 & \begin{tabular}{l|l}
50 & 0
\end{tabular} & 0 & 90 & \begin{tabular}{l|l}
0 & 6
\end{tabular} & 550 & $\begin{array}{c}1.7 \\
9\end{array}$ & \\
\hline 289 & $\begin{array}{c}1 . \\
1\end{array}$ & \begin{tabular}{c|} 
Low \\
gradient \\
Riffle
\end{tabular} & 6265 & 921 & 11 & \begin{tabular}{l|l}
8 & 11
\end{tabular} & 1115 & $\mid \begin{array}{c}0.2 \\
4\end{array}$ & $\left|\begin{array}{c}0.3 \\
6\end{array}\right|$ & & & & 1 & \begin{tabular}{|l|l}
5 & 0
\end{tabular} & 85 & 5 & \begin{tabular}{l|l}
10 & 0
\end{tabular} & 0 & $\begin{array}{ll}0 & 0\end{array}$ & 0 & 0 & \begin{tabular}{l|l}
0 & 0
\end{tabular} & $\begin{array}{lll}0 & 35\end{array}$ & 3530 & $\begin{array}{lll}30 & 0\end{array}$ & 0 & $\mid \begin{array}{c}10 \\
0\end{array}$ & 982 & \begin{tabular}{l|l}
2 & 0
\end{tabular} & 0 & 66 & 606 & 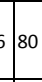 & & \\
\hline 290 & \begin{tabular}{|c|}
5. \\
1 \\
\end{tabular} & $\begin{array}{c}\text { Corner } \\
\text { Pool }\end{array}$ & 6265 & 491 & 161 & 1511 & 1121 & $\begin{array}{c}0.9 \\
1\end{array}$ & \begin{tabular}{|c|}
1.6 \\
2
\end{tabular} & \begin{tabular}{l|l}
0.2 & 4 \\
\end{tabular} & 40 & d & 2 & 540 & 010 & 0 & \begin{tabular}{l|l}
40 & 0
\end{tabular} & 0 o & \begin{tabular}{l|l}
0 & 0
\end{tabular} & 0 & 03 & 303 & 3530 & 50 & \begin{tabular}{l|l}
0 & 0
\end{tabular} & 0 & 98 & \begin{tabular}{l|l}
99 & 1
\end{tabular} & \begin{tabular}{l|l|l}
1 & 10
\end{tabular} & 0 & 69 & 90 & 695 & $\begin{array}{c}1.4 \\
2 \\
\end{array}$ & \\
\hline 291 & $\begin{array}{c}1 . \\
1\end{array}$ & \begin{tabular}{|c|} 
Low \\
gradient \\
Riffle
\end{tabular} & & $|10| 1$ & $18 \mid 2$ & 21 & 14 & $\begin{array}{c}0.1 \\
9\end{array}$ & $\left|\begin{array}{c}0.2 \\
1\end{array}\right|$ & & & & & & & & & & & & & & & & & & & & & & & & & & \\
\hline 292 & $\begin{array}{l}6 . \\
4\end{array}$ & $\begin{array}{c}\text { Backwate } \\
\text { r Pool - }\end{array}$ & 6366 & $\mid \begin{array}{c}10 \\
0\end{array}$ & 13 & 1214 & 1413 & $\begin{array}{c}1.6 \\
2\end{array}$ & $\begin{array}{c}4.4 \\
2\end{array}$ & \begin{tabular}{c|c}
0.2 & 4 \\
1 & 4
\end{tabular} & 4 & d & 3 & 3010 & \begin{tabular}{l|l}
0 & 5
\end{tabular} & 50 & \begin{tabular}{l|l}
30 & 5
\end{tabular} & 5 & \begin{tabular}{l|l}
0 & 0
\end{tabular} & 0 & 03 & & 3030 & $\begin{array}{ll}10 & 0\end{array}$ & \begin{tabular}{l|l}
0 & 0
\end{tabular} & 0 & 93 & $\begin{array}{c}10 \\
0\end{array}$ & \begin{tabular}{l|l}
0 & 0
\end{tabular} & 0 & 69 & & 695 & $\mid \begin{array}{c}4.2 \\
1\end{array}$ & $\begin{array}{l}\text { Run gradually } \\
\text { turns into } 5.2 \\
\text { turns in to } 6.4\end{array}$ \\
\hline
\end{tabular}




\begin{tabular}{|c|c|c|c|c|c|c|c|c|c|c|c|c|c|c|c|c|c|c|c|c|c|c|c|c|c|c|c|c|c|c|c|c|c|c|c|}
\hline 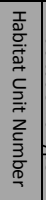 & & 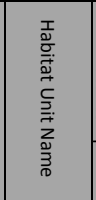 & 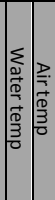 & 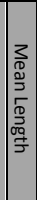 & $\mid \begin{array}{c}3 \\
0 \\
0 \\
\frac{2}{3} \\
\sum \\
\frac{2}{2} \\
\frac{0}{5}\end{array}$ & 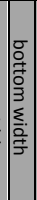 & 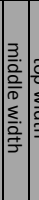 & 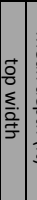 & 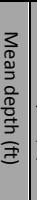 & 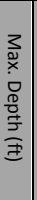 & 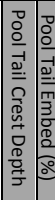 & 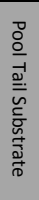 & 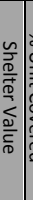 & 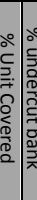 & 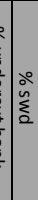 & $\frac{o}{\grave{\Sigma}}$ & 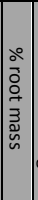 & 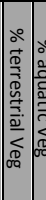 & 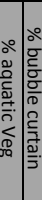 & 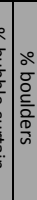 & 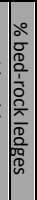 & & 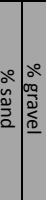 & $\mid$\begin{tabular}{lll}
0 \\
\hdashline
\end{tabular} & & 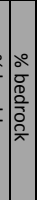 & 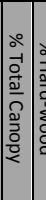 & 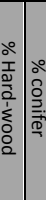 & 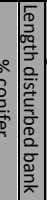 & 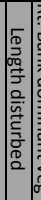 & 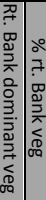 & 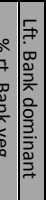 & 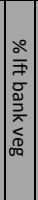 & 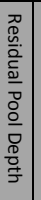 & 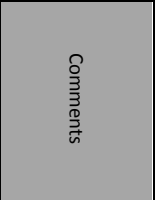 \\
\hline & & \begin{tabular}{c|}
$\log$ \\
formed
\end{tabular} & & & & & & & & & & & & & & & & & & & & & & & & & & & & & & & & & \\
\hline 293 & $\begin{array}{c}1 . \\
1\end{array}$ & \begin{tabular}{|c|} 
Low \\
gradient \\
Riffle
\end{tabular} & & 6 & 13 & & 13 & & $\begin{array}{c}0.1 \\
3\end{array}$ & $\begin{array}{c}0.1 \\
8\end{array}$ & & & & & & & & & & & & & & & & & & & & & & & & & \\
\hline 294 & $\begin{array}{l}4 . \\
2\end{array}$ & $\begin{array}{c}\text { Mid- } \\
\text { Channel } \\
\text { Pool } \\
\end{array}$ & 6269 & 21 & 16 & 14 & 151 & 19 & $\begin{array}{c}1.1 \\
9\end{array}$ & $\begin{array}{c}1.6 \\
8\end{array}$ & $\begin{array}{c}0.1 \\
5\end{array} \mid 20$ & $d$ & & $10 \mid 0$ & 15 & 80 & 0 & 50 & \begin{tabular}{l|l}
0 & 0
\end{tabular} & 0 & 0 & $15 \mid 1$ & 1540 & 0205 & 50 & 0 & $97^{1}$ & \begin{tabular}{c|c}
10 & 0 \\
0 & 0
\end{tabular} & \begin{tabular}{l|l|l}
0 & 0
\end{tabular} & 0 & \begin{tabular}{l|l}
6 & 95
\end{tabular} & 55 & 9 & $\begin{array}{c}1.5 \\
3\end{array}$ & \\
\hline 295 & $\begin{array}{l}5 . \\
2\end{array}$ & $\begin{array}{c}\text { Lateral } \\
\text { Scour } \\
\text { Pool - Log } \\
\text { Enhanced }\end{array}$ & 6367 & 24 & 19 & 10 & 252 & $21{ }^{0}$ & $\begin{array}{c}0.8 \\
9\end{array}$ & $\begin{array}{c}1.5 \\
9\end{array}$ & 0.230 & c & 35 & $50 \mid 0$ & 30 & 70 & 0 & \begin{tabular}{l|l}
0 & 0
\end{tabular} & \begin{tabular}{l|l}
0 & 0
\end{tabular} & 0 & 0 & & 2535 & $5 \mid 150$ & \begin{tabular}{l|l}
0 & 0
\end{tabular} & 0 & $98^{1}$ & \begin{tabular}{c|c}
10 & 0 \\
0 &
\end{tabular} & \begin{tabular}{l|l|l}
0 & 0
\end{tabular} & 0 & 6 \begin{tabular}{c|c}
10 \\
0
\end{tabular} & \begin{tabular}{l|l}
0 & 6 \\
0 & 6
\end{tabular} & 90 & \begin{tabular}{c|}
1.3 \\
9
\end{tabular} & SDA river left \\
\hline 296 & \begin{tabular}{|l|}
3. \\
4 \\
\end{tabular} & Step Run & 6365 & $\begin{array}{c}14 \\
6 \\
\end{array}$ & 13 & 21 & 10 & 80 & 0.4 & 0.9 & & & 2 & $5 \mid 4$ & 0 & 0 & 20 & 40 & \begin{tabular}{l|l}
0 & 0
\end{tabular} & 0 & 0 & 5 & \begin{tabular}{l|l}
5 & 40 \\
\end{tabular} & 040 & 100 & 0 & $86{ }^{1}$ & \begin{tabular}{c|c}
10 & 0 \\
0 & 0 \\
\end{tabular} & 035 & 0 & \begin{tabular}{l|l}
6 & 90 \\
\end{tabular} & 0 & 95 & & $\begin{array}{c}1 \text { riffle }(48 \mathrm{ft}) 2 \\
\text { run }\end{array}$ \\
\hline 297 & $\begin{array}{l}4 . \\
2\end{array}$ & $\begin{array}{c}\text { Mid- } \\
\text { Channel } \\
\text { Pool } \\
\end{array}$ & $\begin{array}{ll}6267 \\
\end{array}$ & 50 & 15 & 8 & 261 & 10 & $\begin{array}{c}0.9 \\
8\end{array}$ & $\begin{array}{c}1.6 \\
8\end{array}$ & $\begin{array}{c}0.1 \\
9\end{array} \mid 15$ & d & 3 & $10 \mid 0$ & 20 & 80 & 0 & & \begin{tabular}{l|l}
0 & 0
\end{tabular} & 0 & 0 & 202 & 2030 & $0: 20 \mid 1 C$ & 100 & 0 & 95 & \begin{tabular}{c|c}
10 & 0 \\
0 & 0
\end{tabular} & \begin{tabular}{l|l|l}
0 & 0
\end{tabular} & 0 & \begin{tabular}{l|l}
6 & 50
\end{tabular} & \begin{tabular}{l|l}
0 & 6 \\
\end{tabular} & 80 & $\begin{array}{c}1.4 \\
9\end{array}$ & \\
\hline 298 & \begin{tabular}{|l|}
3. \\
3 \\
\end{tabular} & Run & 6475 & $\begin{array}{c}13 \\
6 \\
\end{array}$ & 11 & 10 & \begin{tabular}{l|l}
9 & 1 \\
\end{tabular} & $14^{0}$ & $\begin{array}{c}0.4 \\
5 \\
\end{array}$ & \begin{tabular}{c|}
1.2 \\
6 \\
\end{tabular} & & & 3 & 950 & 30 & 40 & 30 & \begin{tabular}{l|l}
0 & 0 \\
0
\end{tabular} & \begin{tabular}{l|l}
0 & 0
\end{tabular} & 0 & 0 & \begin{tabular}{|l|l}
10 & 1 \\
\end{tabular} & \begin{tabular}{l|l}
10 & 70 \\
\end{tabular} & \begin{tabular}{l|l|l|l|l|l} 
& 10 & 0 \\
\end{tabular} & \begin{tabular}{l|l}
0 & 0 \\
\end{tabular} & 0 & 972 & 2080 & 300 & 0 & \begin{tabular}{c|c}
6 & 10 \\
& 0 \\
\end{tabular} & \begin{tabular}{l|l|}
0 & 6 \\
0
\end{tabular} & 95 & & \\
\hline $\begin{array}{c}298 . \\
1 \\
\end{array}$ & \begin{tabular}{|l|}
3. \\
4 \\
\end{tabular} & Step Run & 6372 & 58 & 7 & 10 & 6 & $4^{0}$ & $\begin{array}{c}0.3 \\
9\end{array}$ & \begin{tabular}{c|}
1.0 \\
6 \\
\end{tabular} & & & 2 & $<5 \quad 0$ & 10 & 20 & 70 & 00 & \begin{tabular}{l|l}
0 & 0
\end{tabular} & 0 & 0 & 5 & \begin{tabular}{l|l}
5 & 40 \\
\end{tabular} & $0 \mid 4016$ & 100 & 0 & \begin{tabular}{|l|l|}
89 & 6 \\
\end{tabular} & \begin{tabular}{l|l}
60 & 40 \\
\end{tabular} & 100 & 0 & \begin{tabular}{c|c}
6 & 10 \\
& 0 \\
\end{tabular} & \begin{tabular}{|l|l|}
0 & 6 \\
\end{tabular} & 95 & & $\begin{array}{c}2 \text { riffles }(13,28 \mathrm{ft}) \\
2 \text { runs. } \\
\end{array}$ \\
\hline $\begin{array}{c}298 . \\
2 \\
\end{array}$ & 7 & Dry & & & $\begin{array}{c}\# \# \\
\# \\
\end{array}$ & & & & & & & & & & & & & & & & & & & & & & & & & & & & & & \\
\hline 299 & $\begin{array}{l}4 . \\
2\end{array}$ & $\begin{array}{c}\text { Mid- } \\
\text { Channel } \\
\text { Pool }\end{array}$ & 6369 & 60 & 17 & & 221 & 12 & $\begin{array}{c}1.1 \\
7\end{array}$ & 2.2 & $\begin{array}{c}0.5 \\
7\end{array} \mid$ & c & 3 & 300 & 45 & 10 & 45 & $0 \mid 0$ & \begin{tabular}{l|l}
0 & 0
\end{tabular} & 0 & 0 & $10 \mid 1$ & 1070 & $0: 10 \quad 0$ & \begin{tabular}{l|l}
0 & 0
\end{tabular} & 0 & $91^{1}$ & \begin{tabular}{c|c}
10 & 0 \\
0 & 0
\end{tabular} & $0 \mid 10$ & 0 & 7880 & \begin{tabular}{l|l|l}
30 & 6
\end{tabular} & 95 & $\begin{array}{c}1.6 \\
3\end{array}$ & $\begin{array}{c}\text { wide area with } 3 \\
\text { pools all } \\
\text { connected by } \\
\text { deep channels. } \\
\text { Huge red alder } \\
\text { rootwad, }>10 \mathrm{ft} \\
\text { tall. } \\
\end{array}$ \\
\hline 300 & $\begin{array}{l}1 . \\
1\end{array}$ & $\begin{array}{c}\text { Low } \\
\text { gradient } \\
\text { Riffle }\end{array}$ & & 22 & 4 & 4 & & 40 & 0.2 & $\begin{array}{c}0.2 \\
5\end{array}$ & & & 1 & $<50$ & 50 & 0 & 0 & 50 & \begin{tabular}{l|l}
0 & 0
\end{tabular} & \begin{tabular}{l|l}
0 & 0
\end{tabular} & 0 & 0 & \begin{tabular}{l|l}
0 & 95
\end{tabular} & $5: 5 \mid 0$ & 0.0 & 0 & & & & & & & & & $\begin{array}{c}\text { A secondary } \\
\text { channel } \\
\text { seperated by } \\
\text { very low (about } \\
.5 \mathrm{ft} \text { ) cobble bar. } \\
\text { Didn't record. } \\
\end{array}$ \\
\hline 301 & $\begin{array}{l}5 . \\
2\end{array}$ & $\begin{array}{c}\text { Lateral } \\
\text { Scour } \\
\text { Pool - Log } \\
\text { Enhanced }\end{array}$ & 6367 & 45 & 14 & 4 & 211 & $17^{1}$ & $\begin{array}{c}1.3 \\
2\end{array}$ & $\begin{array}{c}2.1 \\
4\end{array}$ & $\mid \begin{array}{c}0.1 \\
9\end{array}$ & c & 3 & 300 & 20 & 80 & 0 & $0 \mid c$ & \begin{tabular}{l|l}
0 & 0
\end{tabular} & 0 & 0 & 202 & 2050 & $0: 10 \quad 0$ & 0.0 & 0 & \begin{tabular}{|l|l|}
88 & 9
\end{tabular} & \begin{tabular}{l|l}
96 & 4
\end{tabular} & 40 & 0 & $6{ }^{6} \begin{array}{c}10 \\
0\end{array}$ & \begin{tabular}{l|l}
0 \\
0
\end{tabular} & 80 & \begin{tabular}{c|}
1.9 \\
5
\end{tabular} & \\
\hline 302 & $\begin{array}{l}4 . \\
2\end{array}$ & $\begin{array}{c}\text { Mid- } \\
\text { Channel } \\
\text { Pool } \\
\end{array}$ & 6366 & 72 & 22 & 17 & 381 & 12 & $\begin{array}{c}1.5 \\
2\end{array}$ & $\begin{array}{c}2.4 \\
8\end{array}$ & 0.4 & c & 3 & 402 & 020 & 30 & 30 & $0 \mid 0$ & \begin{tabular}{l|l}
0 & 0
\end{tabular} & \begin{tabular}{l|l}
0 & 0
\end{tabular} & 0 & 353 & & $0: 100$ & \begin{tabular}{l|l}
0 & 0
\end{tabular} & 0 & 98 9 & \begin{tabular}{l|l}
90 & 10
\end{tabular} & 00 & 10 & \begin{tabular}{l|l}
6 & 95
\end{tabular} & \begin{tabular}{l|l|l}
5 & 6
\end{tabular} & 95 & \begin{tabular}{c|c}
2.0 \\
8
\end{tabular} & End $8 / 8$ \\
\hline 303 & $\begin{array}{l}3 . \\
4\end{array}$ & Step Run & 6473 & 34 & 13 & 12 & 151 & 13 & $\begin{array}{c}0.5 \\
8\end{array}$ & $\begin{array}{c}1.2 \\
2\end{array}$ & & & 3 & 300 & 25 & 75 & 0 & $0 \mid c$ & \begin{tabular}{l|l}
0 & 0
\end{tabular} & 0 & 0 & 5 & 535 & $545 \mid 1 C$ & 100 & 0 & \begin{tabular}{|c|c|}
10 & 1 \\
0 &
\end{tabular} & \begin{tabular}{c|c}
10 & 0 \\
0 & 0
\end{tabular} & \begin{tabular}{l|l|}
0 & 0
\end{tabular} & 0 & \begin{tabular}{l|l}
765 \\
6
\end{tabular} & 556 & \begin{tabular}{|c|}
10 \\
0
\end{tabular} & & \begin{tabular}{|c|} 
Start $8 / 12.1$ \\
riffle $(10 \mathrm{ft}) 1$ run. \\
Adrian's first day. \\
\end{tabular} \\
\hline 304 & $\begin{array}{l}4 . \\
2\end{array}$ & $\begin{array}{c}\text { Mid- } \\
\text { Channel } \\
\text { Pool } \\
\end{array}$ & 6470 & 43 & 15 & 13 & 181 & $143^{2}$ & $\begin{array}{c}2.6 \\
8\end{array}$ & 3.4 & $\left|\begin{array}{c}0.1 \\
2\end{array}\right| 10$ & d & 3 & 150 & 20 & 25 & 40 & 0 & 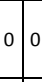 & 0 & 0 & 202 & 2045 & $5 \quad 105$ & 50 & 0 & \begin{tabular}{|c|c|c}
10 & 1 \\
0 &
\end{tabular} & \begin{tabular}{c|c}
10 & 0 \\
0 & 0
\end{tabular} & \begin{tabular}{l|l|}
0 & 0
\end{tabular} & 0 & \begin{tabular}{l|l}
6 & 75
\end{tabular} & \begin{tabular}{l|l|}
5 & 6
\end{tabular} & $\begin{array}{c}10 \\
0\end{array}$ & $\begin{array}{c}3.2 \\
8\end{array}$ & \\
\hline 305 & $\begin{array}{l}3 . \\
4\end{array}$ & Step Run & 6469 & 59 & 13 & 14 & 121 & $\left.14\right|^{0}$ & $\begin{array}{c}0.6 \\
5\end{array}$ & $\begin{array}{c}1.2 \\
8\end{array}$ & & & & $5 \mid 1$ & $0 \mid 30$ & 20 & 35 & $0 \mid c$ & \begin{tabular}{l|l}
0 & 0
\end{tabular} & \begin{tabular}{l|l}
0 & 0
\end{tabular} & 0 & 5 & 535 & 545 & 100 & 0 & $92^{1}$ & \begin{tabular}{c|c}
10 & 0 \\
0 & 0
\end{tabular} & \begin{tabular}{l|l|l}
0 & 0
\end{tabular} & 10 & 690 & \begin{tabular}{l|l|l}
0 & 6 \\
\end{tabular} & 90 & & $\begin{array}{c}2 \text { riffles (10ft, } 10 \\
\mathrm{ft}, \text { ) } 2 \text { runs. Trail } \\
\text { crosses. RB dry } \\
\text { trib. }\end{array}$ \\
\hline $\begin{array}{c}305 . \\
1 \\
\end{array}$ & $\begin{array}{l}6 . \\
3 \\
\end{array}$ & & & 15 & 11 & & 11 & & 1.3 & $\begin{array}{c}1.6 \\
4 \\
\end{array}$ & 0.5 & & 3 & 502 & 030 & 0 & 50 & 00 & 0.0 & 0 & 0 & 353 & 3520 & 010 & & & & & & & & & & $\begin{array}{c}1.1 \\
4 \\
\end{array}$ & \\
\hline 306 & \begin{tabular}{|l|}
3. \\
2 \\
\end{tabular} & Glide & 6471 & $\begin{array}{c}13 \\
8\end{array}$ & 11 & 14 & \begin{tabular}{l|l}
6 & 1
\end{tabular} & $133^{0}$ & $\begin{array}{c}0.6 \\
4 \\
\end{array}$ & \begin{tabular}{|c|}
1.1 \\
2
\end{tabular} & & & 1 & $<5 \mid 0$ & $\begin{array}{c}10 \\
0\end{array}$ & 0 & 0 & 00 & \begin{tabular}{l|l}
0 & 0
\end{tabular} & \begin{tabular}{l|l}
0 & 0
\end{tabular} & 0 & 5 & 530 & $0501 c$ & 100 & 0 & \begin{tabular}{|c|c|}
10 & 1 \\
0 & \\
\end{tabular} & \begin{tabular}{c|c}
10 & 0 \\
0 & 0 \\
\end{tabular} & 065 & 0 & 670 & \begin{tabular}{l|l|l}
0 & 6 \\
\end{tabular} & 90 & & $\begin{array}{c}\text { (+86) water } \\
\text { intake (?) box. }\end{array}$ \\
\hline 307 & $\begin{array}{l}4 . \\
2\end{array}$ & $\begin{array}{c}\text { Mid- } \\
\text { Channel } \\
\text { Pool }\end{array}$ & \begin{tabular}{|l|l|}
64 & 71 \\
\end{tabular} & 42 & 13 & 13 & 141 & 13 & $\begin{array}{c}1.0 \\
9\end{array}$ & 1.8 & $\begin{array}{c}0.4 \\
2\end{array}$ & c & 2 & 100 & 5 & 5 & 85 & $5 \mid c$ & \begin{tabular}{l|l}
0 & 0
\end{tabular} & \begin{tabular}{l|l}
0 & 0
\end{tabular} & 0 & 252 & 2525 & 5205 & 50 & 0 & \begin{tabular}{|l|l|l}
86 & 9
\end{tabular} & \begin{tabular}{l|l}
98 & 2
\end{tabular} & 235 & 0 & \begin{tabular}{l|l}
6 & 5
\end{tabular} & \begin{tabular}{l|l|l}
5 & 6 \\
\end{tabular} & 75 & $\begin{array}{c}1.3 \\
8\end{array}$ & \\
\hline 308 & \begin{tabular}{|l|}
3. \\
3 \\
\end{tabular} & Run & 6471 & 63 & 10 & 13 & 8 & 8 & $\begin{array}{c}0.3 \\
7\end{array}$ & $\begin{array}{c}0.6 \\
6 \\
\end{array}$ & & & 2 & $<5 \quad 0$ & 70 & 0 & 30 & $0 \mid c$ & 0.0 & \begin{tabular}{l|l}
0 & 0
\end{tabular} & 0 & 5 & \begin{tabular}{l|l}
5 & 40 \\
\end{tabular} & 04010 & 100 & 0 & 999 & $98 \quad 2$ & \begin{tabular}{l|l|}
2 & 0
\end{tabular} & 0 & \begin{tabular}{l|l}
6 & 90
\end{tabular} & \begin{tabular}{l|l|l}
0 & 6 \\
\end{tabular} & 80 & & LB seeping water \\
\hline 309 & \begin{tabular}{|l|}
5. \\
1 \\
\end{tabular} & \begin{tabular}{c|} 
Corner \\
Pool
\end{tabular} & 6574 & 72 & 9 & 8 & 10 & \begin{tabular}{|l|l}
8 & 1
\end{tabular} & 1.2 & \begin{tabular}{|c|}
2.0 \\
5 \\
\end{tabular} & 0.540 & d & 3 & 300 & 10 & 45 & 0 & 45 & 0 & $\begin{array}{l}0 \\
0\end{array}$ & 0 & 303 & 3030 & $\begin{array}{lllll}0 & 10 & 0\end{array}$ & \begin{tabular}{l|l}
0 & 0
\end{tabular} & 0 & $96{ }^{1}$ & \begin{tabular}{c|c}
10 & 0 \\
0 & 0 \\
\end{tabular} & \begin{tabular}{ll|l}
0 & 0 \\
\end{tabular} & 30 & \begin{tabular}{c|c}
6 & 10 \\
& 0 \\
\end{tabular} & \begin{tabular}{|l|l|}
0 & 6 \\
\end{tabular} & 60 & \begin{tabular}{c|}
1.5 \\
5 \\
\end{tabular} & \\
\hline 310 & $\begin{array}{l}3 . \\
4 \\
\end{array}$ & Step Run & 6573 & 64 & 12 & 7 & $13{ }_{1}$ & $15^{0}$ & $\begin{array}{c}0.4 \\
6 \\
\end{array}$ & $\begin{array}{c}0.7 \\
6 \\
\end{array}$ & & & 1 & $<5 \quad 0$ & $\begin{array}{c}10 \\
0\end{array}$ & 0 & 0 & 00 & $0 \quad 0$ & $\begin{array}{l}0 \\
0\end{array}$ & 0 & 5 & \begin{tabular}{l|l}
5 & 40
\end{tabular} & 03020 & 200 & 0 & \begin{tabular}{|c|c|}
10 & 1 \\
0 & \\
\end{tabular} & \begin{tabular}{c|c}
10 & 0 \\
0 & 0 \\
\end{tabular} & \begin{tabular}{l|l|}
0 & 0
\end{tabular} & 0 & \begin{tabular}{l|l}
6 & 90
\end{tabular} & \begin{tabular}{l|l|l|l|l|l|l|}
0 & 6
\end{tabular} & 90 & & $\begin{array}{c}1 \text { riffle }(20 \mathrm{ft}) 1 \\
\text { run }\end{array}$ \\
\hline 311 & $\begin{array}{l}4 . \\
2\end{array}$ & $\begin{array}{c}\text { Mid- } \\
\text { Channel } \\
\text { Pool }\end{array}$ & 6575 & 55 & 13 & 15 & 17 & 8 & $\begin{array}{c}2.0 \\
8\end{array}$ & $\begin{array}{c}3.1 \\
2\end{array}$ & $\begin{array}{c}0.5 \\
6\end{array} \mid 20$ & d & 3 & $50 \mid 1$ & 010 & 35 & 35 & 50 & \begin{tabular}{l|l}
0 & 0
\end{tabular} & $\begin{array}{l}0 \\
0\end{array}$ & 5 & $15 \mid 1$ & 1550 & \begin{tabular}{l|l|l}
0 & 15 & 5
\end{tabular} & \begin{tabular}{l|l}
5 & 0
\end{tabular} & 0 & $85^{1}$ & \begin{tabular}{c|c}
10 & 0 \\
0 & 0
\end{tabular} & \begin{tabular}{l|l|}
0 & 0
\end{tabular} & 0 & \begin{tabular}{l|l}
6 & 40
\end{tabular} & 106 & 50 & $\begin{array}{c}2.5 \\
6\end{array}$ & Ed 8/12 \\
\hline 312 & $\begin{array}{c}1 . \\
1\end{array}$ & $\begin{array}{c}\text { Low } \\
\text { gradient } \\
\text { Riffle }\end{array}$ & & 12 & 9 & 6 & & 12 & $\begin{array}{c}0.2 \\
1\end{array}$ & $\begin{array}{c}0.3 \\
1\end{array}$ & & & 2 & 100 & 50 & 50 & 0 & 00 & \begin{tabular}{l|l}
0 & 0
\end{tabular} & $\begin{array}{l}0 \\
0\end{array}$ & 0 & 0 & \begin{tabular}{l|l}
0 & 20
\end{tabular} & $0 \quad 5030$ & \begin{tabular}{ll|l}
30 & 0
\end{tabular} & 0 & 71 & \begin{tabular}{c|c}
10 & 0 \\
0 & 0
\end{tabular} & \begin{tabular}{l|l|}
0 & 10
\end{tabular} & 0 & \begin{tabular}{l|l}
6 & 95
\end{tabular} & \begin{tabular}{l|l|l}
55 & 6
\end{tabular} & 95 & & Start $8 / 13$ \\
\hline 313 & $\begin{array}{l}5 . \\
2\end{array}$ & $\begin{array}{c}\text { Lateral } \\
\text { Scour } \\
\text { Pool - Log } \\
\text { Enhanced }\end{array}$ & 6263 & 91 & 16 & 12 & & 10 & $\begin{array}{c}3.1 \\
1\end{array}$ & 5.3 & $0.2 \mid 20$ & d & 3 & 500 & 10 & 35 & 55 & 00 & \begin{tabular}{l|l}
0 & 0
\end{tabular} & $\begin{array}{l}0 \\
0\end{array}$ & 0 & 252 & & $5 \quad 105$ & \begin{tabular}{l|l}
5 & 0
\end{tabular} & 0 & 96 & \begin{tabular}{c|c}
10 & 0 \\
0 & 0
\end{tabular} & \begin{tabular}{l|l|}
0 & 10
\end{tabular} & 0 & \begin{tabular}{l|l}
6 & 90
\end{tabular} & \begin{tabular}{l|l|l}
0 & 6 \\
\end{tabular} & 95 & 5.1 & \\
\hline
\end{tabular}




\begin{tabular}{|c|c|c|c|c|c|c|c|c|c|c|c|c|c|c|c|c|c|c|c|c|c|c|c|c|c|c|c|c|c|c|c|c|c|c|}
\hline 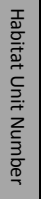 & 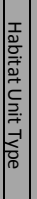 & 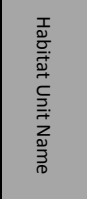 & 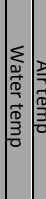 & 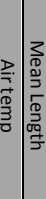 & 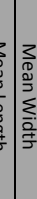 & 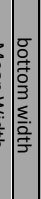 & 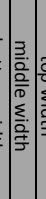 & $\begin{array}{l}0 \\
\frac{0}{0} \\
\frac{0}{0} \\
\frac{j}{3} \\
\frac{2}{3} \\
\end{array}$ & 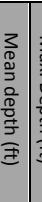 & 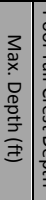 & 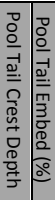 & 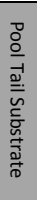 & 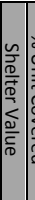 & 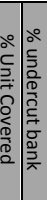 & $\mid$ & $\frac{\circ}{\sum}$ & 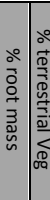 & 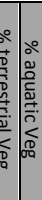 & 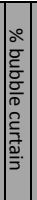 & 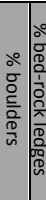 & 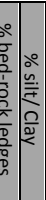 & 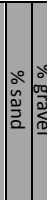 & 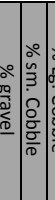 & 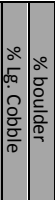 & 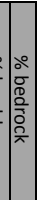 & 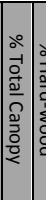 & 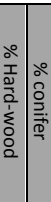 & 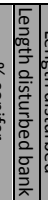 & 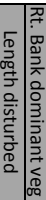 & 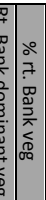 & 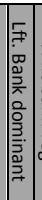 & 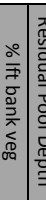 & 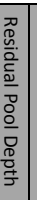 & 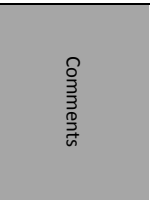 \\
\hline 314 & \begin{tabular}{|l|}
3. \\
2 \\
\end{tabular} & Glide & 616 & \begin{tabular}{l|l}
62 & 85
\end{tabular} & 510 & 010 & 0101 & 11 & \begin{tabular}{c|c}
0.9 & 1 \\
2 & \\
\end{tabular} & \begin{tabular}{c|}
1.6 \\
2 \\
\end{tabular} & & & 32 & 2030 & 0 & 0 & \begin{tabular}{l|l}
70 & 0
\end{tabular} & \begin{tabular}{l|l}
0 & 0
\end{tabular} & 0 & \begin{tabular}{l|l}
0 & 0
\end{tabular} & \begin{tabular}{l|l|}
0 & 25
\end{tabular} & 254 & $40 \mid 10$ & \begin{tabular}{l|l|l|l|l|l|l}
0 & 0
\end{tabular} & 0 & 909 & \begin{tabular}{l|l}
98 & 2
\end{tabular} & & \begin{tabular}{l|l}
0 & 6
\end{tabular} & 695 & 6 & 95 & & $\begin{array}{c}\text { Almost a pool(?) } \\
\text { BF taken }\end{array}$ \\
\hline 315 & $\begin{array}{l}3 . \\
4\end{array}$ & Step Run & 616 & \begin{tabular}{l|l}
62 & 93
\end{tabular} & 311 & 111 & $1 \mid 10$ & $130^{0}$ & \begin{tabular}{c|c}
0.7 & 1 \\
1 &
\end{tabular} & $\begin{array}{c}1.6 \\
2\end{array}$ & & & 1 & $<540$ & 40 & 0 & \begin{tabular}{l|l}
0 & 0
\end{tabular} & \begin{tabular}{l|l}
0 & 0
\end{tabular} & 0 & \begin{tabular}{l|l}
0 & $2 C$
\end{tabular} & 2025 & 252 & $25 \mid 10$ & \begin{tabular}{l|l|}
5 & 0 \\
\end{tabular} & 10 & \begin{tabular}{l|l}
99 & 6
\end{tabular} & \begin{tabular}{l|l}
60 & 40
\end{tabular} & \begin{tabular}{ll|l}
0 & 0
\end{tabular} & \begin{tabular}{l|l}
0 & 7
\end{tabular} & 720 & 8 & 70 & & $\begin{array}{c}2 \text { riffles }(20,5 \mathrm{ft}), \\
1 \text { run. "Step } \\
\text { Glide" }\end{array}$ \\
\hline 316 & $\begin{array}{l}4 . \\
2\end{array}$ & \begin{tabular}{|c|} 
Mid- \\
Channel \\
Pool \\
\end{tabular} & 626 & $63 \quad 57$ & \begin{tabular}{l|l}
7 & 12
\end{tabular} & 213 & 314 & 8 & \begin{tabular}{c|c}
1.0 & 2 \\
1 &
\end{tabular} & \begin{tabular}{c|c}
2.2 & 0 \\
8 &
\end{tabular} & \begin{tabular}{c|c}
0.1 & 20 \\
8 &
\end{tabular} & d & 2 & 5 40 & 10 & 5 & \begin{tabular}{l|l}
35 & 0
\end{tabular} & \begin{tabular}{l|l}
0 & 0
\end{tabular} & 0 & \begin{tabular}{l|l}
0 & 10
\end{tabular} & 1015 & 154 & 4025 & \begin{tabular}{l|l}
5 & 0
\end{tabular} & 10 & \begin{tabular}{|c|c}
10 & 1 \\
0 & $C$
\end{tabular} & \begin{tabular}{c|c}
10 & 0 \\
0 & 0
\end{tabular} & 10 & \begin{tabular}{|l|l}
0 & 7
\end{tabular} & $\begin{array}{l}7 \\
20\end{array}$ & 6 & \begin{tabular}{l|l|l}
80 & 2.
\end{tabular} & 2.1 & \\
\hline 317 & $\begin{array}{l}1 . \\
1\end{array}$ & \begin{tabular}{|c|} 
Low \\
gradient \\
Riffle \\
\end{tabular} & & 14 & $4 \quad 13$ & \begin{tabular}{ll|}
3 & 8 \\
\end{tabular} & & $\left.17\right|^{0}$ & \begin{tabular}{c|c}
0.2 & 0 \\
2 & 0
\end{tabular} & 0.6 & & & 1 & 0 & $\begin{array}{c}10 \\
0\end{array}$ & 0 & \begin{tabular}{l|l}
0 & 0
\end{tabular} & $0 \mid 0$ & 0 & \begin{tabular}{l|l}
0 & 0
\end{tabular} & \begin{tabular}{l|l|}
0 & 5
\end{tabular} & 54 & 4040 & \begin{tabular}{l|l|}
10 & 0
\end{tabular} & 0 & \begin{tabular}{c|c}
10 & 1 \\
0 &
\end{tabular} & \begin{tabular}{c|c}
10 & 0 \\
0 & 0
\end{tabular} & 0 & 0 & & & & & \\
\hline 318 & $\begin{array}{l}5 . \\
1\end{array}$ & $\begin{array}{l}\text { Corner } \\
\text { Pool }\end{array}$ & 626 & 6568 & 816 & 617 & 7151 & $15^{0}$ & \begin{tabular}{c|c}
0.9 & 3 \\
7 &
\end{tabular} & \begin{tabular}{c|c}
3.0 & 0 \\
1 &
\end{tabular} & \begin{tabular}{c|c}
0.2 & 20 \\
2 & 20
\end{tabular} & d & 2 & 5 40 & 0 & 0 & \begin{tabular}{l|l}
35 & 15
\end{tabular} & 150 & 0 & \begin{tabular}{l|l}
0 & $1 C$ \\
\end{tabular} & 1025 & 253 & 350 & \begin{tabular}{l|l|}
0 & 0 \\
\end{tabular} & 15 & $988^{1}$ & \begin{tabular}{c|c}
10 & 0 \\
0 & 0
\end{tabular} & 15 & \begin{tabular}{l|l}
0 & 6
\end{tabular} & $\begin{array}{ll}6 & 60\end{array}$ & 6 & 852. & $\begin{array}{c}2.7 \\
9\end{array}$ & \\
\hline 319 & $\begin{array}{l}1 . \\
1\end{array}$ & \begin{tabular}{|c} 
Low \\
gradient \\
Riffle
\end{tabular} & & 22 & $\begin{array}{ll}2 & 15\end{array}$ & 515 & & 140 & $0.33^{0}$ & $\begin{array}{c}0.4 \\
6\end{array}$ & & & 1 & & $\left|\begin{array}{c}10 \\
0\end{array}\right|$ & 0 & \begin{tabular}{l|l}
0 & 0
\end{tabular} & \begin{tabular}{l|l}
0 & 0
\end{tabular} & 0 & \begin{tabular}{l|l}
0 & 0
\end{tabular} & $0 \mid 5$ & 54 & 4040 & \begin{tabular}{l|l|}
10 & 0 \\
\end{tabular} & 0 & \begin{tabular}{l|l}
96 & 9
\end{tabular} & $95 \mid 5$ & 50 & \begin{tabular}{l|l}
0 & 8
\end{tabular} & \begin{tabular}{ll|}
8 & 10
\end{tabular} & 6 & 95 & & $\begin{array}{c}\text { GPS pts off } \\
\text { somewhere } \\
\text { between } 308 \text { and } \\
315, \text { back on } \\
\text { track at } 315 .\end{array}$ \\
\hline 320 & $\begin{array}{l}4 . \\
2\end{array}$ & \begin{tabular}{|c|} 
Mid- \\
Channel \\
Pool \\
\end{tabular} & 626 & $65 \mid 82$ & $\begin{array}{ll}2 & 14\end{array}$ & & $4|15| 1$ & 12 & \begin{tabular}{c|c}
1.0 & 2 \\
9 &
\end{tabular} & $\left.2.7\right|^{0}$ & \begin{tabular}{c|c}
0.3 & 20 \\
8 & 20
\end{tabular} & c & 21 & 1050 & 10 & 0 & \begin{tabular}{l|l}
40 & 0
\end{tabular} & \begin{tabular}{l|l}
0 & 0
\end{tabular} & 0 & \begin{tabular}{l|l}
0 & 0
\end{tabular} & 0.25 & 253 & $35 \mid 10$ & \begin{tabular}{l|l|}
5 & 0 \\
\end{tabular} & 0 & \begin{tabular}{|l|l|}
99 & 9
\end{tabular} & \begin{tabular}{l|l}
98 & 2
\end{tabular} & 0 & \begin{tabular}{l|l}
0 & 6
\end{tabular} & $\begin{array}{ll}6 & 50\end{array}$ & 7 & $102^{2}$ & $\begin{array}{c}2.3 \\
2\end{array}$ & \\
\hline 321 & 1. & \begin{tabular}{|c|} 
Low \\
gradient \\
Riffle \\
\end{tabular} & & 11 & 130 & & 30 & & \begin{tabular}{c|c}
0.2 & 0 \\
7 &
\end{tabular} & $\begin{array}{c}0.3 \\
3\end{array}$ & & & & & & & & & & & & & & & & & & & & & & & & \\
\hline 322 & \begin{tabular}{|c|}
5. \\
1 \\
\end{tabular} & \begin{tabular}{|c|} 
Corner \\
Pool
\end{tabular} & 636 & 6596 & 620 & & 0171 & $14^{1}$ & \begin{tabular}{c|c}
1.2 & 2 \\
3 & \\
\end{tabular} & \begin{tabular}{c|c}
2.5 & 0 \\
5 & 0 \\
\end{tabular} & \begin{tabular}{l|l}
0.2 & 30 \\
\end{tabular} & c & 31 & 1040 & 0 & 0 & \begin{tabular}{l|l}
50 & 0 \\
\end{tabular} & \begin{tabular}{l|l}
0 & 0 \\
\end{tabular} & 0 & \begin{tabular}{l|l}
0 & $1 C$ \\
\end{tabular} & 1020 & 203 & 3015 & \begin{tabular}{|l|l|}
5 & 0 \\
\end{tabular} & 10 & \begin{tabular}{|l|l}
76 & 7 \\
\end{tabular} & $\begin{array}{ll}75 & 25 \\
\end{array}$ & 525 & \begin{tabular}{l|l}
0 & 6 \\
\end{tabular} & $\begin{array}{ll}6 & 15 \\
\end{array}$ & 7 & 202 & \begin{tabular}{c|}
2.3 \\
5
\end{tabular} & \\
\hline 323 & \begin{tabular}{l|}
3. \\
4 \\
\end{tabular} & Step Run & 637 & 7065 & 516 & 613 & $315 \mid 2$ & $211^{0}$ & \begin{tabular}{c|c}
0.4 & 1 \\
8 & \\
\end{tabular} & 1.4 & & & 31 & 105 & 20 & 50 & \begin{tabular}{l|l}
25 & 0
\end{tabular} & 0 | 0 & 0 & \begin{tabular}{l|l}
0 & 0
\end{tabular} & \begin{tabular}{l|l|l|}
0 & 10
\end{tabular} & 103 & $35 \mid 301$ & 150 & 0 & $966^{1}$ & \begin{tabular}{c|c}
10 & 0 \\
0 &
\end{tabular} & 0 & \begin{tabular}{l|l}
0 & 7
\end{tabular} & \begin{tabular}{ll|l|}
7 & 80
\end{tabular} & 6 & 40 & & $\begin{array}{l}1 \text { riffle, }(13 \mathrm{ft}) 1 \\
\text { run. "Step Glide" }\end{array}$ \\
\hline 324 & $\begin{array}{l}4 . \\
2\end{array}$ & \begin{tabular}{|c|} 
Mid- \\
Channel \\
Pool \\
\end{tabular} & 657 & 7322 & 225 & 521 & 1252 & 28 & \begin{tabular}{c|c}
1.0 & 1 \\
6 & 1
\end{tabular} & $1.90^{0}$ & $\begin{array}{c}0.3 \\
6\end{array}$ & c & 38 & 800 & 20 & 80 & \begin{tabular}{|l|l}
0 & 0
\end{tabular} & \begin{tabular}{l|l}
0 & 0
\end{tabular} & 0 & \begin{tabular}{l|l}
0 & 0
\end{tabular} & \begin{tabular}{l|l|}
0 & 25
\end{tabular} & 253 & 3015 & \begin{tabular}{|l|l|}
5 & 0 \\
\end{tabular} & 0 & $71^{1}$ & \begin{tabular}{c|c}
10 & 0 \\
0 &
\end{tabular} & 0 & $10 \mid 6$ & 695 & 6 & $755^{1}$ & $\begin{array}{c}1.5 \\
4\end{array}$ & \\
\hline 325 & $\begin{array}{l}3 . \\
4 \\
\end{array}$ & Step Run & 668 & 8089 & $\begin{array}{l}9 \\
\end{array}$ & 7 & 81 & $10^{0}$ & \begin{tabular}{c|c}
0.6 & 1 \\
5 & \\
\end{tabular} & 1.9 & & & 3 & 2520 & 15 & 20 & 1035 & 350 & 0 & \begin{tabular}{l|l}
0 & 0
\end{tabular} & \begin{tabular}{l|l|}
0 & 15 \\
\end{tabular} & 154 & 4025 & \begin{tabular}{l|l|}
5 & 0 \\
\end{tabular} & 0 & \begin{tabular}{|l|l}
37 & 1 \\
\end{tabular} & \begin{tabular}{c|c}
10 & 0 \\
0 & 0 \\
\end{tabular} & 0 & \begin{tabular}{|l|l|}
0 & 6 \\
\end{tabular} & \begin{tabular}{|c|c|}
6 & 10 \\
0
\end{tabular} & 6 & \begin{tabular}{|c|}
10 \\
0 \\
\end{tabular} & & $\begin{array}{l}1 \text { riffle }(32 \mathrm{ft}) 1 \\
\text { run. Step Glide }\end{array}$ \\
\hline 326 & $\begin{array}{l}4 . \\
2\end{array}$ & \begin{tabular}{|c|} 
Mid- \\
Channel \\
Pool \\
\end{tabular} & 657 & 7534 & \begin{tabular}{l|l}
4 & 9
\end{tabular} & 10 & 09 & 9 & $1.22^{2}$ & \begin{tabular}{c|c}
2.1 & 0 \\
5 &
\end{tabular} & $\begin{array}{c}0.4 \\
9\end{array}$ & c & 3 & 1010 & 0 & 10 & \begin{tabular}{l|l|l}
70 & 10
\end{tabular} & $10 \mid 0$ & 0 & \begin{tabular}{l|l}
0 & 0
\end{tabular} & \begin{tabular}{l|l|}
0 & 25
\end{tabular} & 254 & 455 & \begin{tabular}{l|l|l|l|l|l|l}
0 & 0
\end{tabular} & 0 & \begin{tabular}{|c|c|}
10 & 1 \\
0 & $c$
\end{tabular} & \begin{tabular}{c|c}
10 & 0 \\
0 & 0
\end{tabular} & 0 & \begin{tabular}{l|l}
0 & 6
\end{tabular} & 695 & 6 & $60{ }^{1}$ & $\begin{array}{c}1.6 \\
6\end{array}$ & \\
\hline 327 & \begin{tabular}{|c|}
3. \\
4 \\
\end{tabular} & Step Run & 668 & 82 \begin{tabular}{c|c}
11 \\
4
\end{tabular} & \begin{tabular}{l|l}
1 & 13 \\
\end{tabular} & $\begin{array}{ll}3 & 9 \\
\end{array}$ & 92 & $20^{0}$ & \begin{tabular}{c|c}
0.4 & 0 \\
6 & \\
\end{tabular} & $\begin{array}{c}0.7 \\
5 \\
\end{array}$ & & & 3 & 1015 & 20 & 10 & 2035 & \begin{tabular}{l|l}
35 & 0
\end{tabular} & 0 & \begin{tabular}{l|l}
0 & 0
\end{tabular} & \begin{tabular}{l|l|}
0 & 5
\end{tabular} & 54 & 4035 & \begin{tabular}{|l|l|}
10 & 0 \\
\end{tabular} & 0 & \begin{tabular}{|l|l}
53 & 9
\end{tabular} & 98 2 & 0 & \begin{tabular}{l|l}
0 & 6 \\
\end{tabular} & \begin{tabular}{|c|c|}
6 & 10 \\
0
\end{tabular} & 6 & 95 & & $\begin{array}{c}1 \text { riffle }(63 \mathrm{ft}) 2 \\
\text { runs }\end{array}$ \\
\hline 328 & $\begin{array}{l}4 . \\
2\end{array}$ & \begin{tabular}{|c|} 
Mid- \\
Channel \\
Pool \\
\end{tabular} & 658 & $83 \quad 37$ & \begin{tabular}{l|l}
7 & 18
\end{tabular} & 820 & $0|18| 1$ & 16 & \begin{tabular}{c|c}
1.7 & 3 \\
7 &
\end{tabular} & \begin{tabular}{c|c}
3.2 & 0 \\
5 &
\end{tabular} & $\begin{array}{c}0.2 \\
5\end{array}$ & c & 3 & 1040 & 0 & 20 & \begin{tabular}{l|l}
30 & 10
\end{tabular} & 100 & 0 & \begin{tabular}{l|l}
0 & 0
\end{tabular} & \begin{tabular}{l|l|}
0 & 25
\end{tabular} & 254 & 455 & \begin{tabular}{l|l|}
0 & 0 \\
\end{tabular} & 0 & \begin{tabular}{|l|l}
58 & 9
\end{tabular} & \begin{tabular}{l|l}
96 & 4
\end{tabular} & 0 & 106 & 6 \begin{tabular}{c|c}
10 \\
0
\end{tabular} & 7 & 85 & 3 & \\
\hline 329 & \begin{tabular}{|c|}
3. \\
4 \\
\end{tabular} & Step Run & 657 & 7754 & $\begin{array}{lll}4 & 11\end{array}$ & 116 & \begin{tabular}{l|l|}
6 & 8 \\
\end{tabular} & 80 & $0.44^{0}$ & $\begin{array}{c}0.9 \\
4 \\
\end{array}$ & & & & $<5 \mid 0$ & 50 & 0 & \begin{tabular}{l|l}
0 & 50
\end{tabular} & 500 & 0 & \begin{tabular}{l|l}
0 & 0
\end{tabular} & \begin{tabular}{l|l|}
0 & 5
\end{tabular} & 54 & 4040 & \begin{tabular}{l|l|}
10 & 0 \\
\end{tabular} & 0 & 96 & \begin{tabular}{l|l}
99 & 1
\end{tabular} & 0 & \begin{tabular}{l|l}
0 & 6
\end{tabular} & 665 & 7 & 65 & & $\begin{array}{c}1 \text { riffle }(20 \mathrm{ft}), 1 \\
\text { run }\end{array}$ \\
\hline 330 & $\begin{array}{l}4 . \\
2\end{array}$ & \begin{tabular}{|c|} 
Mid- \\
Channel \\
Pool \\
\end{tabular} & 667 & 7578 & \begin{tabular}{l|l}
8 & 13
\end{tabular} & 38 & 201 & 10 & \begin{tabular}{c|c}
1.7 & 3 \\
9 &
\end{tabular} & \begin{tabular}{c|c}
3.1 & 0 \\
8 &
\end{tabular} & $\begin{array}{c}0.3 \\
1\end{array}$ & & 3 & 1030 & 10 & 10 & \begin{tabular}{l|l}
50 & 0
\end{tabular} & \begin{tabular}{l|l}
0 & 0
\end{tabular} & 0 & \begin{tabular}{l|l}
0 & 0
\end{tabular} & \begin{tabular}{l|l}
0 & 20
\end{tabular} & 205 & 5010 & \begin{tabular}{l|l|}
0 & 0 \\
\end{tabular} & 0 & \begin{tabular}{|c|c}
10 & 1 \\
0 &
\end{tabular} & \begin{tabular}{c|c}
10 & 0 \\
0 & 0
\end{tabular} & 0 & \begin{tabular}{l|l}
0 & 5
\end{tabular} & 590 & 6 & $80^{2}$ & $\begin{array}{c}2.8 \\
7\end{array}$ & \\
\hline 331 & \begin{tabular}{|c|}
3 \\
4 \\
\end{tabular} & Step Run & 667 & \begin{tabular}{l|l}
72 & 48
\end{tabular} & $\begin{array}{lll}8 & 11\end{array}$ & 110 & \begin{tabular}{|l|l|l|}
0 & 7 & 1 \\
\end{tabular} & $16^{0}$ & \begin{tabular}{l|l}
0.3 & 0 \\
3 & \\
\end{tabular} & $\begin{array}{c}0.5 \\
1 \\
\end{array}$ & & & 3 & 540 & 0 & 0 & \begin{tabular}{l|l}
40 & 20
\end{tabular} & 200 & 0 & \begin{tabular}{l|l}
0 & 0
\end{tabular} & \begin{tabular}{l|l|l}
0 & 10
\end{tabular} & 107 & 7010 & \begin{tabular}{l|l|}
0 & 0 \\
\end{tabular} & 0 & $999^{1}$ & \begin{tabular}{c|c}
10 & 0 \\
0 & 0 \\
\end{tabular} & 0 & \begin{tabular}{l|l}
5 & 5
\end{tabular} & $5 \quad 40$ & 6 & 95 & & $\begin{array}{c}1 \text { riffle }(20 \mathrm{ft}), 1 \\
\text { run }\end{array}$ \\
\hline 332 & $\begin{array}{l}4 . \\
2\end{array}$ & \begin{tabular}{|c|} 
Mid- \\
Channel \\
Pool \\
\end{tabular} & 657 & 7531 & 112 & 216 & 614 & 7 & \begin{tabular}{c|c}
1.7 & 3 \\
3 & 3
\end{tabular} & $3.22^{0}$ & \begin{tabular}{c|c}
0.2 & 30 \\
5 &
\end{tabular} & c & 3 & 1535 & 10 & 0 & $35 \mid 20$ & 200 & 0 & \begin{tabular}{l|l}
0 & 0
\end{tabular} & \begin{tabular}{l|l|l}
0 & 10 \\
\end{tabular} & 106 & 6015 & \begin{tabular}{l|l|}
5 & 0
\end{tabular} & 0 & \begin{tabular}{l|l}
79 & 7
\end{tabular} & 7525 & 50 & \begin{tabular}{l|l}
0 & 6
\end{tabular} & 690 & 6 & 802. & $\begin{array}{c}2.9 \\
5\end{array}$ & \\
\hline 333 & $\begin{array}{l}1 . \\
1\end{array}$ & \begin{tabular}{|c|} 
Low \\
gradient \\
Riffle \\
\end{tabular} & 657 & \begin{tabular}{l|l}
72 & 50
\end{tabular} & \begin{tabular}{l|l}
0 & 9
\end{tabular} & 7 & 8 & 12 & \begin{tabular}{c|c}
0.3 & 0 \\
8 &
\end{tabular} & $\begin{array}{c}0.7 \\
2\end{array}$ & & & 2 & \begin{tabular}{l|l}
5 & 0
\end{tabular} & 65 & 0 & \begin{tabular}{l|l}
0 & 35
\end{tabular} & 350 & 0 & \begin{tabular}{l|l}
0 & 0
\end{tabular} & \begin{tabular}{l|l|}
0 & 0
\end{tabular} & 04 & 4050 & \begin{tabular}{|l|l|}
10 & 0 \\
\end{tabular} & 0 & \begin{tabular}{l|l}
98 & 9
\end{tabular} & \begin{tabular}{l|l}
95 & 5
\end{tabular} & 0 & \begin{tabular}{l|l}
0 & 6
\end{tabular} & 695 & 6 & 50 & & \\
\hline 334 & \begin{tabular}{|c|}
5. \\
1 \\
\end{tabular} & $\begin{array}{c}\text { Corner } \\
\text { Pool }\end{array}$ & 657 & 7160 & \begin{tabular}{l|l}
0 & 14
\end{tabular} & 412 & \begin{tabular}{ll|l}
2 & 9 & 2
\end{tabular} & 21 & \begin{tabular}{c|c}
1.0 & 2 \\
4 & \\
\end{tabular} & \begin{tabular}{|c|c}
2.0 & 0 \\
5 & \\
\end{tabular} & \begin{tabular}{l|l}
0.2 & 20 \\
2 & \\
\end{tabular} & c & 3 & $\begin{array}{ll}5 & 20\end{array}$ & 10 & 10 & \begin{tabular}{l|l}
60 & 0
\end{tabular} & \begin{tabular}{l|l}
0 & 0
\end{tabular} & 0 & \begin{tabular}{l|l}
0 & 0
\end{tabular} & $\begin{array}{lll}0 & 30\end{array}$ & 303 & 355 & \begin{tabular}{l|l|l|}
0 & 0
\end{tabular} & 0 & \begin{tabular}{|l|l}
98 & 4
\end{tabular} & 4060 & 0 & \begin{tabular}{l|l}
0 & 8
\end{tabular} & 850 & 6 & 601 & \begin{tabular}{|l|}
1.8 \\
3
\end{tabular} & End $8 / 13$ \\
\hline 335 & $\begin{array}{l}. \\
1 \\
\end{array}$ & \begin{tabular}{|c|} 
Low \\
gradient \\
Riffle
\end{tabular} & & 27 & \begin{tabular}{l|l}
7 & 14
\end{tabular} & 421 & & 7 & \begin{tabular}{c|c}
0.2 & 0 \\
7 &
\end{tabular} & $\begin{array}{c}0.9 \\
1\end{array}$ & & & 2 & \begin{tabular}{l|l}
5 & 0
\end{tabular} & 0 & 0 & \begin{tabular}{c|c}
10 & 0 \\
0 & 0
\end{tabular} & \begin{tabular}{l|l}
0 & 0
\end{tabular} & 0 & \begin{tabular}{l|l}
0 & 0
\end{tabular} & 0.5 & 54 & 4040 & \begin{tabular}{|l|l|}
10 & 0 \\
\end{tabular} & 0 & \begin{tabular}{l|l}
99 & 5
\end{tabular} & \begin{tabular}{l|l}
50 & 50
\end{tabular} & 0 & \begin{tabular}{l|l}
0 & 8
\end{tabular} & 860 & 6 & 50 & & Start $8 / 14$ \\
\hline 336 & $\begin{array}{l}4 . \\
2\end{array}$ & \begin{tabular}{|c|} 
Mid- \\
Channel \\
Pool \\
\end{tabular} & 627 & 7453 & $\begin{array}{lll}3 & 10\end{array}$ & 07 & 18 & 6 & \begin{tabular}{c|c}
0.9 & 1 \\
2 &
\end{tabular} & \begin{tabular}{c|c}
1.3 & 0 \\
6 &
\end{tabular} & \begin{tabular}{c|c}
0.1 & 20 \\
8 &
\end{tabular} & $d$ & 3 & 700 & 30 & 40 & \begin{tabular}{l|l}
30 & 0
\end{tabular} & $0 \quad 0$ & 0 & \begin{tabular}{l|l}
0 & 0
\end{tabular} & 025 & 253 & 3510 & \begin{tabular}{|l|l|}
5 & 0 \\
\end{tabular} & 0 & $93 \quad 6$ & \begin{tabular}{l|l}
60 & 40
\end{tabular} & 0 & \begin{tabular}{l|l}
0 & 8
\end{tabular} & 870 & 6 & 85 & $\begin{array}{c}1.1 \\
8\end{array}$ & $\begin{array}{l}\text { Log Jam, m/l, } \\
\text { spans channel }\end{array}$ \\
\hline 337 & $\begin{array}{c}1 . \\
1\end{array}$ & \begin{tabular}{|c|} 
Low \\
gradient \\
Riffle \\
\end{tabular} & & 12 & 210 & 06 & & 14 & \begin{tabular}{c|c}
0.2 & 0 \\
6 &
\end{tabular} & $\begin{array}{c}0.3 \\
4\end{array}$ & & & & & & & & & & & & & & & & & & & & & & & & \\
\hline 338 & $\begin{array}{l}4 . \\
2\end{array}$ & \begin{tabular}{|c|} 
Mid- \\
Channel \\
Pool
\end{tabular} & 627 & 7443 & $\begin{array}{lll}3 & 13\end{array}$ & $\begin{array}{lll}314 \\
\end{array}$ & $4 \mid 16$ & 101 & $1.44^{2}$ & \begin{tabular}{c|c}
2.8 & 0 \\
5 &
\end{tabular} & $\begin{array}{c}0.2 \\
4\end{array}$ & c & 3 & 250 & 5 & 0 & \begin{tabular}{l|l}
90 & 0
\end{tabular} & 0 o & 0 & \begin{tabular}{l|l}
0 & 5
\end{tabular} & 530 & 303 & 3010 & \begin{tabular}{l|l|}
0 & 0 \\
\end{tabular} & 0 & 929 & \begin{tabular}{l|l}
98 & 2
\end{tabular} & 202 & 206 & 695 & 6 & 80 & $\begin{array}{c}2.6 \\
1\end{array}$ & $\begin{array}{l}\text { Combo } 4.2 \text { and } \\
\text { 6.3. Almost a } \\
5.3 \text {, but big } \\
\text { enough for a } 4.2\end{array}$ \\
\hline 339 & $\begin{array}{c}3 . \\
4\end{array}$ & Step Run & 647 & $72 \begin{array}{c}31 \\
0\end{array}$ & 1 & 6 & 121 & 10 & \begin{tabular}{c|c}
0.7 & 1 \\
1 &
\end{tabular} & $\begin{array}{c}1.5 \\
5\end{array}$ & & & 3 & 5 & 20 & 25 & \begin{tabular}{|l|l|l}
25 & 10 \\
\end{tabular} & $10 \mid 0$ & 0 & \begin{tabular}{|l|l}
0 & 0
\end{tabular} & \begin{tabular}{l|l|l}
0 & 5
\end{tabular} & 54 & 4040 & $10 \mid 0$ & 0 & 839 & \begin{tabular}{l|l}
90 & 10
\end{tabular} & 0 & \begin{tabular}{l|l}
0 & 6
\end{tabular} & 690 & 6 & 95 & & $\begin{array}{c}3 \text { riffles }(20,16, \\
11 \mathrm{ft}) 3 \text { runs. Last } \\
110 \text { feet = glide. }\end{array}$ \\
\hline
\end{tabular}




\begin{tabular}{|c|c|c|c|c|c|c|c|c|c|c|c|c|c|c|c|c|c|c|c|c|c|c|c|c|c|c|c|c|c|c|c|c|c|c|c|c|c|}
\hline 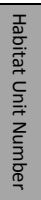 & 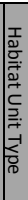 & 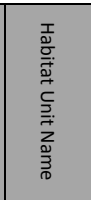 & 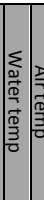 & 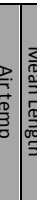 & & 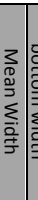 & 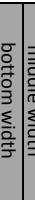 & & 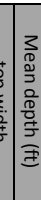 & 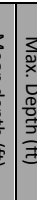 & 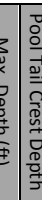 & 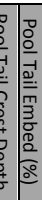 & 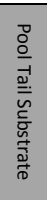 & 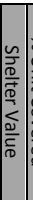 & 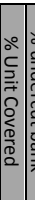 & 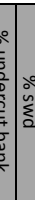 & $\frac{2}{\grave{c}}$ & 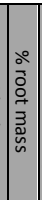 & 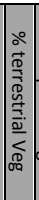 & 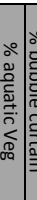 & 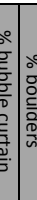 & 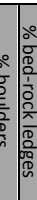 & 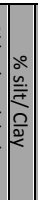 & 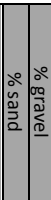 & 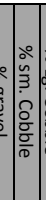 & 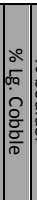 & & 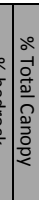 & 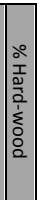 & 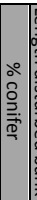 & 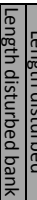 & 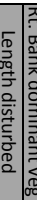 & 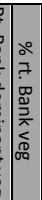 & & 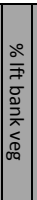 & 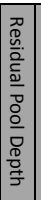 & 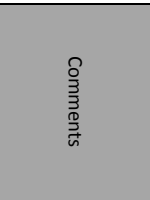 \\
\hline 340 & $\begin{array}{l}4 . \\
2\end{array}$ & \begin{tabular}{|c|} 
Mid- \\
Channel \\
Pool \\
\end{tabular} & 647 & $76 \mid 6$ & & 121 & 101 & $17 \mid 8$ & $\begin{array}{c}1.1 \\
2\end{array}$ & 13. & \begin{tabular}{c|c}
.2 & 0. \\
2 & 9
\end{tabular} & \begin{tabular}{l|l}
.3 & 40 \\
9 & 40
\end{tabular} & c & 3 & 90 & $\begin{array}{lll}5 & 10\end{array}$ & 55 & 30 & 0 & 00 & $0 \mid 0$ & $\begin{array}{ll}0 & 0\end{array}$ & 25 & 2530 & 020 & 0 & \begin{tabular}{l|l}
0 & 0
\end{tabular} & 94 & $\begin{array}{c}10 \\
0\end{array}$ & 0 & 0 & 0 & 90 & 6 & 60 & $\begin{array}{c}2.8 \\
3\end{array}$ & Log jam \\
\hline 341 & $\begin{array}{l}3 . \\
4 .\end{array}$ & Step Run & 637 & 799 & & 98 & \begin{tabular}{l|l}
8 & 1
\end{tabular} & 108 & $\begin{array}{c}0.4 \\
7\end{array}$ & $\begin{array}{rl}4 & 1 . \\
2\end{array}$ & & & & 3 & 5 & 405 & 5 & 50 & 0 & 0 & 0.0 & 0 & 5 & 535 & 535 & 20 & \begin{tabular}{l|l}
0 & 0
\end{tabular} & $\begin{array}{c}10 \\
0\end{array}$ & $\begin{array}{c}10 \\
0\end{array}$ & 0 & 0 & 06 & 75 & 7 & 40 & & $\begin{array}{c}2 \text { riffle }(33,34 \mathrm{ft}) \\
1 \text { run }\end{array}$ \\
\hline 342 & $\begin{array}{l}4 . \\
2\end{array}$ & \begin{tabular}{|c|} 
Mid- \\
Channel \\
Pool \\
\end{tabular} & 637 & $73 \mid 6$ & & $11 \mid \varepsilon$ & \begin{tabular}{l|l}
8 & 1
\end{tabular} & 1410 & $\begin{array}{cc}0 & 1.3 \\
8\end{array}$ & \begin{tabular}{c|c|c}
3 & 2. \\
3 & 9
\end{tabular} & \begin{tabular}{c|c}
.3 & 0. \\
9 & 1
\end{tabular} & \begin{tabular}{l|l}
.5 & 35 \\
1
\end{tabular} & c & 32 & 20 & \begin{tabular}{l|l}
0 & 5
\end{tabular} & 10 & 85 & 0 & 0 & \begin{tabular}{l|l}
0 & 0
\end{tabular} & \begin{tabular}{l|l}
0 & 0
\end{tabular} & 25 & 2530 & 015 & 5 & \begin{tabular}{l|l}
0 & 0
\end{tabular} & $\begin{array}{c}10 \\
0\end{array}$ & $\begin{array}{c}10 \\
0\end{array}$ & 0 & \begin{tabular}{|l|l}
0 & 1 \\
\end{tabular} & 106 & 95 & 6 & 85 & $\begin{array}{c}1.8 \\
8\end{array}$ & \\
\hline 343 & 3. & Step Run & $63 \mid 7$ & $73 \mid 9$ & & 111 & 101 & 149 & $9 \begin{array}{c}0.3 \\
9\end{array}$ & \begin{tabular}{l|l}
3 & 1 \\
9
\end{tabular} & & & & 2 & & \begin{tabular}{l|l}
0 & 10
\end{tabular} & 0 & 90 & 0 & 0 & $0 \mid 0$ & \begin{tabular}{l|l}
0 & 0
\end{tabular} & 10 & 1040 & 035 & 5 & \begin{tabular}{l|l}
0 & 0
\end{tabular} & $\begin{array}{c}10 \\
0\end{array}$ & $\begin{array}{c}10 \\
0\end{array}$ & 0 & 0 & 06 & 95 & 6 & 90 & & \begin{tabular}{|l} 
temp probe. 2 \\
riffle $(57,10 \mathrm{ft}) 1$ \\
glide. Step glide
\end{tabular} \\
\hline 344 & $\begin{array}{l}4 . \\
2\end{array}$ & \begin{tabular}{|c|} 
Mid- \\
Channel \\
Pool \\
\end{tabular} & 657 & $7 6 \longdiv { 5 }$ & 44 & 8 & 9 & \begin{tabular}{l|l}
9 & 7
\end{tabular} & $7 \begin{array}{c}1.5 \\
3\end{array}$ & \begin{tabular}{r|r}
5 & 3. \\
3 & 2
\end{tabular} & \begin{tabular}{c|c}
.4 \\
2 & 0.
\end{tabular} & \begin{tabular}{l|l}
.3 & 30 \\
4 & 30
\end{tabular} & c & 2 & 10 & 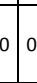 & 0 & 90 & 10 & 00 & \begin{tabular}{l|l}
0 & 0
\end{tabular} & $\begin{array}{ll}0 & 0\end{array}$ & 25 & 2530 & 020 & 0 & \begin{tabular}{l|l}
0 & 0
\end{tabular} & 073 & $\begin{array}{c}10 \\
0\end{array}$ & 0 & 02 & 256 & 95 & 6 & 80 & $\begin{array}{c}3.0 \\
8\end{array}$ & \\
\hline 345 & $\begin{array}{l}4 . \\
2\end{array}$ & \begin{tabular}{|c|} 
Mid- \\
Channel \\
Pool \\
\end{tabular} & 657 & $79 \mid 5$ & 7 & 9 & \begin{tabular}{l|l}
7 & 1
\end{tabular} & 146 & $6 \begin{array}{c}2.4 \\
9\end{array}$ & \begin{tabular}{l|l}
4 & 4
\end{tabular} & $4 \mid \begin{array}{c}0 . \\
9\end{array}$ & \begin{tabular}{l|l}
.5 & 40 \\
9 &
\end{tabular} & c & 2 & 5 & 105 & 5 & 0 & 0 & 0 & $0 \mid 0$ & 080 & 25 & 2520 & 05 & 0 & $0 \mid 25$ & 1589 & 99 & 1 & \begin{tabular}{l|l}
0 & 1
\end{tabular} & 106 & 15 & 6 & 80 & $\begin{array}{c}3.4 \\
1\end{array}$ & \\
\hline 346 & $\begin{array}{l}1 . \\
1\end{array}$ & \begin{tabular}{|c|} 
Low \\
gradient \\
Riffle
\end{tabular} & 668 & $82\left[\begin{array}{l}1 \\
0\end{array}\right.$ & \begin{tabular}{l|l}
1 & 1
\end{tabular} & 151 & 161 & 1612 & $.2 \begin{array}{c}0.3 \\
2\end{array}$ & \begin{tabular}{l|l}
3 & 0. \\
2 & 6
\end{tabular} & & & & 1 & $<5 \mid$ & $0 \mid 50$ & 0 & 0 & 50 & 0 & \begin{tabular}{l|l}
0 & 0
\end{tabular} & \begin{tabular}{l|l}
0 & 0
\end{tabular} & 5 & 535 & 535 & 20 & \begin{tabular}{l|l}
0 & 0
\end{tabular} & 077 & $\begin{array}{c}10 \\
0\end{array}$ & 0 & $15 \mid 2$ & 206 & 80 & 6 & 80 & & $\begin{array}{l}(+80) \text { road } \\
\text { crossing - ford }\end{array}$ \\
\hline 347 & $\begin{array}{l}4 . \\
2\end{array}$ & \begin{tabular}{|c|} 
Mid- \\
Channel \\
Pool \\
\end{tabular} & $65 \mid 8$ & $85 \mid 5$ & 562 & 201 & 122 & $272 C$ & $\begin{array}{c}2.0 \\
5\end{array}$ & \begin{tabular}{c|c}
0 & 4. \\
9 & 9
\end{tabular} & \begin{tabular}{c|c}
.1 & 0. \\
9 & 6
\end{tabular} & \begin{tabular}{l|l}
.3 & 10 \\
6 & 10
\end{tabular} & c.d & 3 & 20 & $5 \mid 2 C$ & 030 & 40 & 5 & 0 & \begin{tabular}{l|l}
0 & 0
\end{tabular} & $\begin{array}{ll}0 & 0\end{array}$ & 25 & 2540 & 010 & 0 & \begin{tabular}{l|l}
0 & 0
\end{tabular} & 060 & $\begin{array}{c}10 \\
0\end{array}$ & 0 & 10 & 06 & 60 & 6 & 10 & $\begin{array}{c}3.8 \\
3\end{array}$ & \\
\hline 348 & $\begin{array}{l}1 . \\
1\end{array}$ & \begin{tabular}{|c|} 
Low \\
gradient \\
Riffle
\end{tabular} & & 2 & & 9 & 6 & 11 & $1 \begin{array}{c}0.2 \\
5\end{array}$ & $\begin{array}{l}2 \\
5\end{array}$ & & & & 1 & $<5$ & $0 \mid$\begin{tabular}{c|c}
10 \\
0
\end{tabular} & 0 & 0 & 0 & 00 & 0.0 & $\begin{array}{ll}0 & 0\end{array}$ & 0 & 040 & 040 & 20 & $0 \mid 0$ & & & & & & & & & & \\
\hline 349 & $\begin{array}{l}5 . \\
3 \\
\end{array}$ & & 668 & $85 \mid 3$ & & 171 & 112 & 2316 & $6 \begin{array}{c}1.0 \\
5\end{array}$ & \begin{tabular}{l|l}
0 & 1. \\
\end{tabular} & \begin{tabular}{l|r}
0. \\
.7 \\
8
\end{tabular} & \begin{tabular}{l|l}
3 & 25 \\
\end{tabular} & d & 3 & 15 & $0 \mid 25$ & 535 & 540 & 0 & 0 & \begin{tabular}{l|l}
0 & 0
\end{tabular} & \begin{tabular}{l|l}
0 & 0
\end{tabular} & 20 & 2530 & 0 & 5 & $0 \mid 0$ & $\begin{array}{c}10 \\
0\end{array}$ & 95 & 5 & 0 & 06 & 85 & 6 & 90 & \begin{tabular}{|c|}
1.3 \\
2 \\
\end{tabular} & \\
\hline 350 & \begin{tabular}{|l|}
3. \\
2
\end{tabular} & Glide & 678 & $83 \mid 7$ & & 111 & $16 \mathrm{~s}$ & 97 & $\begin{array}{c}0.6 \\
3\end{array}$ & \begin{tabular}{l|l}
6 & 1. \\
\end{tabular} & & & & 3 & 53 & 3030 & 0 & 30 & 10 & 00 & $0 \mid 0$ & $\begin{array}{ll}0 & 0\end{array}$ & 25 & 2530 & 020 & 0 & $0 \mid 0$ & 095 & 95 & 5 & 0 & 06 & 90 & 6 & 90 & & \\
\hline 351 & $\begin{array}{l}4 . \\
2\end{array}$ & \begin{tabular}{|c|} 
Mid- \\
Channel \\
Pool \\
\end{tabular} & 668 & 835 & 571 & 172 & 241 & $13 \mid 13$ & $\begin{array}{c}3.5 \\
2\end{array}$ & $\begin{array}{cc}5 & 3 . \\
2 & 2\end{array}$ & \begin{tabular}{l|l}
.2 & 0. \\
2 &
\end{tabular} & 3 & c & 3 & & $40 \mid 10$ & 0 & 40 & 0 & $\begin{array}{lll}0 & 0\end{array}$ & \begin{tabular}{l|l}
0 & 0
\end{tabular} & \begin{tabular}{l|l}
0 & 0
\end{tabular} & 25 & 2540 & 010 & 0 & $\begin{array}{lll}0 & 0\end{array}$ & $\begin{array}{lll}0 & 5\end{array}$ & 50 & 50 & 0 & 08 & 10 & 8 & 80 & $\begin{array}{c}2.9 \\
2\end{array}$ & \\
\hline $\begin{array}{c}351 . \\
1\end{array}$ & $\begin{array}{l}6 . \\
3\end{array}$ & & \begin{tabular}{l|l}
67 & 8
\end{tabular} & $83 \mid 3$ & 39 & \begin{tabular}{l|l}
9 & 1
\end{tabular} & 101 & 115 & $5 \mid \begin{array}{c}0.9 \\
3\end{array}$ & \begin{tabular}{c|c}
9 & 3. \\
3 & 5
\end{tabular} & \begin{tabular}{c|c}
.0 \\
5 & 0.
\end{tabular} & & & 3 & 25 & $50 \mid 0$ & 0 & 40 & 0 & 0 & \begin{tabular}{l|l}
0 & 0
\end{tabular} & \begin{tabular}{l|l}
0 & 0
\end{tabular} & & & & & & & & & & & & & & 2.9 & $\begin{array}{c}\text { Backwater (off } \\
\text { channel) pool, } \\
\text { but no obvious } \\
\text { forming factor. } \\
\text { Top of } 347.1 \\
\text { connects to } \\
\text { bottom of } 347 . \\
\text { Four ft. undercut. }\end{array}$ \\
\hline 352 & 3. & Step Run & 698 & 82 & 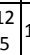 & 111 & 13 & 912 & .20 .8 & \begin{tabular}{r|r}
8 & 1. \\
1 & 1.
\end{tabular} & & & & 3 & 10 & 25225 & 525 & 525 & 0 & 0 & \begin{tabular}{l|l}
0 & 0
\end{tabular} & \begin{tabular}{l|l}
0 & 0
\end{tabular} & 5 & 540 & 040 & 10 & 00 & $\begin{array}{l}0 \\
07\end{array}$ & 60 & 40 & 0 & 07 & 75 & 8 & 50 & & $\begin{array}{c}2 \text { riffles }(5,27 \mathrm{ft}) \\
2 \text { runs. }\end{array}$ \\
\hline 353 & \begin{tabular}{|l|}
5 \\
1 \\
\end{tabular} & $\begin{array}{c}\text { Corner } \\
\text { Pool }\end{array}$ & 688 & 809 & \begin{tabular}{l|l}
2 & 1
\end{tabular} & 111 & \begin{tabular}{|l|l}
12 & 1
\end{tabular} & 128 & $8 \begin{array}{c}1.2 \\
9\end{array}$ & \begin{tabular}{r|r}
2 & 2. \\
& 8 \\
\end{tabular} & \begin{tabular}{l|c}
2 & 0.2 \\
8 & 8 \\
\end{tabular} & \begin{tabular}{l|l}
2 & 20 \\
8 & \\
\end{tabular} & c & 1 & $<5$ & 1010 & 0 & 0 & 0 & 0 & \begin{tabular}{l|l}
0 & 0
\end{tabular} & \begin{tabular}{l|l}
0 & 80
\end{tabular} & 15 & $\begin{array}{lll}15 & 40\end{array}$ & 010 & 0 & 020 & & 40 & 60 & 0 & 0.8 & 70 & 6 & 80 & 2 & \\
\hline 354 & $\begin{array}{l}1 . \\
1\end{array}$ & \begin{tabular}{|c|} 
Low \\
gradient \\
Riffle \\
\end{tabular} & 678 & & $\begin{array}{ll}10 \\
3\end{array}$ & 14 & \begin{tabular}{l|l}
8 & 1
\end{tabular} & 132 & $0 \begin{array}{c}0.2 \\
6\end{array}$ & $\begin{array}{ccc}2 & 0 . \\
\vdots & 1\end{array}$ & & & & 2 & $<5$ & 1080 & 0 & 0 & 10 & 0 & \begin{tabular}{l|l}
0 & 0
\end{tabular} & \begin{tabular}{l|l}
0 & 0
\end{tabular} & 5 & 535 & 535 & 20 & $\begin{array}{lll}0 & 0\end{array}$ & $\begin{array}{ll}0 & 91\end{array}$ & 95 & 5 & 0 & 06 & 90 & 8 & 80 & & $\begin{array}{l}\text { Near top of unit, } \\
\text { shed (?) on LB. } \\
\text { Horse camp? }\end{array}$ \\
\hline 355 & $\begin{array}{l}4 . \\
2\end{array}$ & \begin{tabular}{|c|} 
Mid- \\
Channel \\
Pool \\
\end{tabular} & 658 & $\left.81\right|_{2} ^{1}$ & \begin{tabular}{l|l}
10 & 2 \\
2 &
\end{tabular} & $24 \sqrt{2}$ & 204 & \begin{tabular}{l|l|l}
40 & 1.
\end{tabular} & $\begin{array}{c}1.2 \\
2\end{array}$ & 2.2. & \begin{tabular}{c|c}
.1 & 0.2 \\
5 & 2
\end{tabular} & $\begin{array}{l}.2 \\
2\end{array}$ & & 3 & 75 & $0 \mid 2 C$ & 55 & 525 & 0 & 0 & \begin{tabular}{l|l}
0 & 0
\end{tabular} & \begin{tabular}{l|l}
0 & 0
\end{tabular} & 20 & 2030 & 020 & 10 & 0 & 070 & 60 & 40 & 0 & 06 & 95 & 6 & 90 & $\begin{array}{c}1.9 \\
3\end{array}$ & \\
\hline 356 & $\begin{array}{l}1 . \\
1\end{array}$ & \begin{tabular}{|c|} 
Low \\
gradient \\
Riffle
\end{tabular} & & 1 & $18 \mid 1$ & \begin{tabular}{l|l}
13 & 1
\end{tabular} & 11 & 14 & $\begin{array}{l}4 \\
0.2 \\
3\end{array}$ & \begin{tabular}{c|c}
2 & 0. \\
3
\end{tabular} & & & & 1 & $<5$ & $0 \mid \begin{array}{c}10 \\
0\end{array}$ & 0 & 0 & 0 & 0 & \begin{tabular}{l|l}
0 & 0
\end{tabular} & \begin{tabular}{l|l}
0 & 0
\end{tabular} & 5 & 540 & 0.40 & 10 & $0 \mid 0$ & & & & & & & & & & \\
\hline 357 & $\begin{array}{l}3 . \\
2 .\end{array}$ & Glide & 667 & 776 & 50 & 161 & 11 & 1422 & $2 \begin{array}{c}0.6 \\
7\end{array}$ & \begin{tabular}{l|l}
6 & 1. \\
\end{tabular} & & & & 3 & 10 & 2015 & 540 & 040 & 5 & 0 & \begin{tabular}{l|l}
0 & 0
\end{tabular} & \begin{tabular}{l|l}
0 & 0
\end{tabular} & 10 & 1030 & 0.40 & 10 & 00 & $\begin{array}{ll}0 & 85\end{array}$ & 40 & 60 & 0 & 0.8 & 80 & 8 & 80 & & End 8/14 \\
\hline 358 & $\begin{array}{l}4 . \\
2\end{array}$ & \begin{tabular}{|c|} 
Mid- \\
Channel \\
Pool
\end{tabular} & 646 & $69 \mid 4$ & 181 & 172 & 222 & \begin{tabular}{l|l}
22 & 7
\end{tabular} & $7 \begin{array}{c}1.2 \\
2\end{array}$ & 22. & $.3 \mid \begin{array}{c}0.2 \\
6\end{array}$ & \begin{tabular}{l|l|l}
.2 & 10 \\
6
\end{tabular} & d & 3 & 15 & 3530 & 025 & 540 & 0 & 0 & \begin{tabular}{l|l}
0 & 0
\end{tabular} & \begin{tabular}{l|l}
0 & 0
\end{tabular} & 20 & 2030 & 025 & 5 & 0 & 073 & 50 & 50 & 0 & 06 & 90 & 6 & $900^{2}$ & $\begin{array}{c}2.0 \\
4\end{array}$ & Start $8 / 15$ \\
\hline 359 & $\begin{array}{l}1 . \\
1\end{array}$ & \begin{tabular}{|c|} 
Low \\
gradient \\
Riffle
\end{tabular} & & 2 & $25 \mid$ & 12 & 7 & 16 & \begin{tabular}{c|c}
0.2 \\
4
\end{tabular} & $\begin{array}{cc}2 & 0 . \\
2 & \end{array}$ & & & & 1 & $<5$ & $0 \mid 2$ & 80 & 0 & 0 & 0 & \begin{tabular}{l|l}
0 & 0
\end{tabular} & \begin{tabular}{l|l}
0 & 0
\end{tabular} & 0 & \begin{tabular}{|l|l|}
0 & 40
\end{tabular} & $0 \mid 40$ & 20 & 0 & & & & & & & & & & \\
\hline 360 & $\begin{array}{l}3 . \\
3\end{array}$ & Run & 647 & 705 & 501 & \begin{tabular}{l|l}
12 & 1
\end{tabular} & 161 & 129 & $\begin{array}{c}0.5 \\
4\end{array}$ & \begin{tabular}{l|l}
5 & 1. \\
\end{tabular} & & & & 2 & 5 & 0 & 0 & 30 & 10 & $\begin{array}{lll}0 & 0\end{array}$ & \begin{tabular}{l|l}
0 & 0
\end{tabular} & \begin{tabular}{l|l}
0 & 0
\end{tabular} & 5 & $\begin{array}{lll}5 & 35\end{array}$ & 535 & 20 & \begin{tabular}{l|l}
0 & 0
\end{tabular} & 75 & 70 & 30 & 0 & 06 & 95 & 6 & 5 & & \\
\hline 361 & $\begin{array}{l}5 . \\
3\end{array}$ & \begin{tabular}{|c|} 
Lateral \\
Scour \\
Pool - \\
Root Wad \\
Enhanced
\end{tabular} & 646 & 696 & 51 & 11 & \begin{tabular}{l|l}
9 & 1
\end{tabular} & 1510 & $\begin{array}{c}0.9 \\
0\end{array}$ & \begin{tabular}{r|r}
9 & 1. \\
2 & 5
\end{tabular} & \begin{tabular}{c|c}
.9 & 0. \\
5 & 4
\end{tabular} & \begin{tabular}{l|l}
.2 & 30 \\
4 & 30
\end{tabular} & c & 3 & & $10 \mid 2$ & 0 & 40 & 0 & 0 & \begin{tabular}{l|l}
0 & 0
\end{tabular} & \begin{tabular}{l|l}
0 & 0
\end{tabular} & 20 & & 025 & 5 & $\begin{array}{lll}0 & 0\end{array}$ & 94 & $\begin{array}{c}10 \\
0\end{array}$ & 0 & 0 & 0 & 90 & 7 & 5 & $\begin{array}{c}1.7 \\
1\end{array}$ & $\begin{array}{c}\text { Root wad } \\
\text { enhancing pool is } \\
\text { from living red } \\
\text { alder, still } \\
\text { standing }\end{array}$ \\
\hline 362 & 1. & \begin{tabular}{|c|} 
Low \\
gradient \\
Riffle
\end{tabular} & 657 & & 47 & \begin{tabular}{l|l}
9 & 1
\end{tabular} & 10 & \begin{tabular}{l|l|l}
5 & 11
\end{tabular} & $\begin{array}{c}1 \\
0.2 \\
5\end{array}$ & \begin{tabular}{c|c}
2 & 0. \\
5 & 1
\end{tabular} & & & & 1 & 5 & 09 & 50 & 0 & 5 & 0 & \begin{tabular}{l|l}
0 & 0
\end{tabular} & \begin{tabular}{l|l}
0 & 0
\end{tabular} & 0 & $\begin{array}{lll}0 & 30\end{array}$ & $040=$ & 30 & 0.0 & 88 & $\begin{array}{c}10 \\
0\end{array}$ & 0 & 0 & 0.6 & 95 & 6 & 5 & & \\
\hline 363 & $\begin{array}{l}4 . \\
2\end{array}$ & \begin{tabular}{|c|} 
Mid- \\
Channel \\
Pool
\end{tabular} & 647 & 758 & 33 & $21 \mid 2$ & $22 \mid 1$ & $18 \mid 24$ & $\begin{array}{c}4.6 \\
7\end{array}$ & \begin{tabular}{r|r}
6 & 3. \\
7 & 5
\end{tabular} & \begin{tabular}{l|c}
.4 & 0.2 \\
5 & 6
\end{tabular} & \begin{tabular}{l|l}
.2 & 25 \\
6 & 25
\end{tabular} & d & 2 & 5 & 151 & 0 & 40 & 0 & 0 & \begin{tabular}{l|l}
0 & 0
\end{tabular} & \begin{tabular}{l|l}
0 & 5
\end{tabular} & 25 & 2525 & 55 & 0 & 02 & 099 & 99 & 1 & 0 & 56 & 90 & 6 & 75 & $\begin{array}{c}3.1 \\
9\end{array}$ & $\begin{array}{c}\text { On a bend. } \\
\text { Access, trail to } \\
\text { road }\end{array}$ \\
\hline
\end{tabular}




\begin{tabular}{|c|c|c|c|c|c|c|c|c|c|c|c|c|c|c|c|c|c|c|c|c|c|c|c|c|c|c|c|c|c|c|c|c|c|c|c|}
\hline 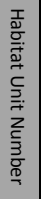 & & 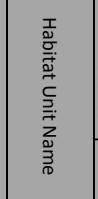 & 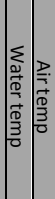 & 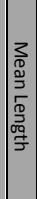 & 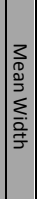 & 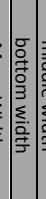 & 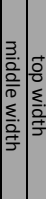 & 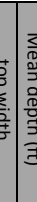 & 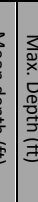 & 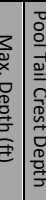 & 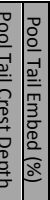 & 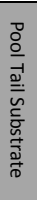 & 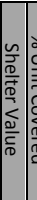 & 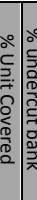 & 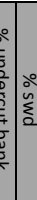 & $\frac{\circ}{\grave{c}}$ & 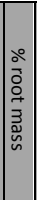 & 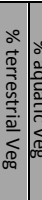 & 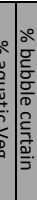 & $\begin{array}{l}\frac{0}{2} \\
\overline{0} \\
\frac{0}{2} \\
\frac{0}{0} \\
\frac{0}{3}\end{array}$ & 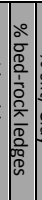 & & 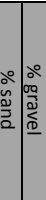 & 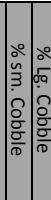 & 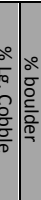 & 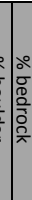 & 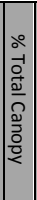 & 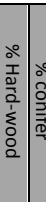 & 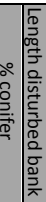 & 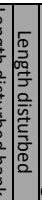 & 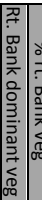 & 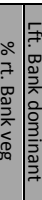 & 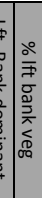 & 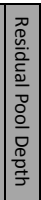 & 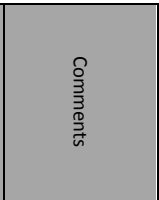 \\
\hline 364 & $\begin{array}{l}5 . \\
2\end{array}$ & \begin{tabular}{|c|} 
Lateral \\
Scour \\
Pool - Log \\
Enhanced
\end{tabular} & 6579 & 33 & 24 & $4 \quad 242$ & 2423 & 23.1. & \begin{tabular}{l|l}
6 & 2. \\
9
\end{tabular} & \begin{tabular}{l|l}
.5 & 1. \\
5 & 1
\end{tabular} & $\begin{array}{l}.0 \\
1\end{array}$ & c & $\begin{array}{lll}3 & 2\end{array}$ & 200 & \begin{tabular}{l|l|l}
0 & 45
\end{tabular} & 5 & 10 & $0 \mid 0$ & \begin{tabular}{l|l}
0 & 0
\end{tabular} & 0 & 0 & 2020 & 2050 & \begin{tabular}{|l|l|l|l|l}
10 & 0
\end{tabular} & \begin{tabular}{l|l}
0 & 0
\end{tabular} & 0 & 97 & \begin{tabular}{l|l}
80 & $2 C$
\end{tabular} & $20 \mid 0$ & 0 & \begin{tabular}{|l|l}
6 & 9
\end{tabular} & $95 \mid 6$ & 685 & $\begin{array}{c}1.5 \\
4\end{array}$ & \\
\hline 365 & $\begin{array}{l}4 . \\
2\end{array}$ & \begin{tabular}{|c|} 
Mid- \\
Channel \\
Pool
\end{tabular} & 6479 & 84 & 22 & 232 & 2220 & $20 \frac{1 .}{5}$ & \begin{tabular}{l|l}
3 & 2. \\
5 & 1
\end{tabular} & \begin{tabular}{l|l}
.8 & 0.8 \\
1 & 1
\end{tabular} & $\begin{array}{l}.8 \\
1\end{array}$ & c & 3 & & 515 & 35 & 30 & $5 \mid 0$ & \begin{tabular}{l|l}
0 & 0
\end{tabular} & 0 & 0 & 3030 & 3035 & 50 & \begin{tabular}{l|l}
0 & 0
\end{tabular} & 0 & 78 & \begin{tabular}{l|l}
95 & 5
\end{tabular} & \begin{tabular}{l|l}
5 & 0
\end{tabular} & 0 & \begin{tabular}{|l|l|}
6 & 9
\end{tabular} & \begin{tabular}{l|l}
95 & 6
\end{tabular} & 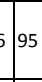 & 2 & \\
\hline 366 & $\begin{array}{c}1 . \\
1\end{array}$ & \begin{tabular}{|c|} 
Low \\
gradient \\
Riffle
\end{tabular} & 6683 & $\mid \begin{array}{c}10 \\
0\end{array}$ & 16 & 5201 & 199 & 90. & $\begin{array}{l}3 \\
4 \\
4\end{array}$ & $\begin{array}{l}0.4 \\
6\end{array}$ & & & $1<$ & $<5 \mid 0$ & 0 & 0 & 0 & 50 & $\begin{array}{l}0 \\
0\end{array}$ & 0 & 0 & \begin{tabular}{l|l}
0 & 0
\end{tabular} & $\begin{array}{lll}0 & 35\end{array}$ & 3530 & 300 & 0 & 93 & \begin{tabular}{l|l}
60 & $4 C$
\end{tabular} & $40 \mid 0$ & 0 & $\begin{array}{ll}6 & 9\end{array}$ & $95 \mid 6$ & $5 \mid \begin{array}{c}10 \\
0\end{array}$ & & \\
\hline 367 & $\begin{array}{l}3 . \\
2\end{array}$ & Glide & 6583 & 36 & 8 & 9 & \begin{tabular}{l|l}
10 & 6
\end{tabular} & $6 \quad \begin{array}{l}0 . \\
8\end{array}$ & $\begin{array}{ll}5 & 0 . \\
3 & 8\end{array}$ & $\begin{array}{l}0.9 \\
8\end{array}$ & & & $1<$ & $<50$ & 0 & 0 & 0 & 50 & \begin{tabular}{l|l}
0 & 0
\end{tabular} & 0 & 0 & 3535 & 3530 & 00 & \begin{tabular}{l|l}
0 & 0
\end{tabular} & 0 & 87 & 4060 & $60 \mid 0$ & 0 & $6^{1}$ & \begin{tabular}{c|c}
10 & 6 \\
0 & 6
\end{tabular} & 695 & & $\begin{array}{c}(+35) \text { RB trib. No } \\
\text { water at } \\
\text { confluence but } \\
\text { yes water about } \\
20 \mathrm{ft} \text {. up the trib. } \\
\begin{array}{c}\text { Very incised } \\
\text { canyon. }\end{array} \\
\end{array}$ \\
\hline 368 & $\begin{array}{l}4 . \\
2\end{array}$ & \begin{tabular}{|c|} 
Mid- \\
Channel \\
Pool \\
\end{tabular} & 6687 & 40 & 37 & 732 & 42 & 2. & $\begin{array}{l}7 \\
7 . \\
5\end{array}$ & \begin{tabular}{l|l}
1.0 & 0. \\
5 & 2
\end{tabular} & 2.4 & & 36 & 600 & \begin{tabular}{l|l}
0 & 40
\end{tabular} & 60 & 0 & $0 \mid 0$ & \begin{tabular}{l|l}
0 & 0
\end{tabular} & 0 & 0 & $30 \mid 3$ & $\begin{array}{ll}30 & 30\end{array}$ & \begin{tabular}{|l|l}
5 \\
\end{tabular} & \begin{tabular}{l|l}
5 & 0
\end{tabular} & 0 & 87 & & 0 & 0 & \begin{tabular}{|l|l|l|}
6 & 2
\end{tabular} & \begin{tabular}{l|l}
20 & 6
\end{tabular} & 670 & $\begin{array}{c}3.6 \\
3\end{array}$ & Log Jam \\
\hline 369 & $\begin{array}{l}1 . \\
1\end{array}$ & \begin{tabular}{|c|} 
Low \\
gradient \\
Riffle
\end{tabular} & 6687 & 53 & 13 & 8 & \begin{tabular}{|l|l|}
6 & 25
\end{tabular} & $25\left(\begin{array}{l}0 . \\
2\end{array}\right.$ & $\begin{array}{ll}2 & 0 . \\
2 & 2\end{array}$ & 2.4 & & & 2 & 53 & 035 & 50 & 35 & $0 \mid c$ & \begin{tabular}{l|l}
0 & 0
\end{tabular} & 0 & 0 & 00 & $\begin{array}{lll}0 & 35\end{array}$ & 5530 & 300 & 0 & 95 & \begin{tabular}{l|l}
90 & $1 C$
\end{tabular} & $10 \mid 0$ & 0 & $\begin{array}{ll}8 & 8\end{array}$ & $80 \mid 6$ & 690 & & \\
\hline 370 & $\begin{array}{l}4 . \\
2\end{array}$ & \begin{tabular}{|c|} 
Mid- \\
Channel \\
Pool
\end{tabular} & 6582 & $\begin{array}{c}16 \\
5\end{array}$ & 23 & 3252 & $24 \mid 19$ & 191. & $6 \begin{array}{c}3 . \\
5\end{array}$ & \begin{tabular}{l|l}
.5 & 0.2 \\
5 & 4
\end{tabular} & \begin{tabular}{l|l}
$4^{2}$ & 20 \\
\end{tabular} & c & 31 & 102 & 020 & 25 & 25 & 10 & \begin{tabular}{l|l}
0 & 0
\end{tabular} & 0 & 10 & $10 \mid$ & $10 \mid 40$ & \begin{tabular}{l|l|l|l} 
& 10
\end{tabular} & $10 \mid 0$ & 10 & 71 & \begin{tabular}{l|l|l}
30 & 7
\end{tabular} & \begin{tabular}{l|l}
70 & 0
\end{tabular} & 10 & \begin{tabular}{|l|l|}
6 & 8
\end{tabular} & 85 & 6 & $\begin{array}{c}3.3 \\
1\end{array}$ & 85 \\
\hline 371 & $\begin{array}{l}3 . \\
4 \\
\end{array}$ & Step Run & $\begin{array}{lll}65 & 83 \\
\end{array}$ & $\begin{array}{c}10 \\
0 \\
\end{array}$ & 15 & 5191 & 1314 & $14 \begin{array}{r}0 . \\
3\end{array}$ & \begin{tabular}{r|r}
5 & 1. \\
3 & 1 \\
\end{tabular} & $\begin{array}{l}1.0 \\
1 \\
\end{array}$ & & & 31 & 102 & 525 & 25 & 25 & $0 \mid 0$ & \begin{tabular}{l|l}
0 & 0
\end{tabular} & 0 & 0 & 252 & 2525 & 5205 & 50 & 0 & $\begin{array}{c}10 \\
0 \\
\end{array}$ & 955 & $5 \mid 0$ & 0 & \begin{tabular}{|l|l|}
6 & 8
\end{tabular} & \begin{tabular}{l|l}
80 & 6
\end{tabular} & 590 & & $\begin{array}{l}2 \text { riffles }(12,10 \mathrm{ft}) \\
2 \text { run. Step Gilde }\end{array}$ \\
\hline 372 & $\begin{array}{l}4 . \\
2\end{array}$ & $\begin{array}{c}\text { Mid- } \\
\text { Channel } \\
\text { Pool }\end{array}$ & 6585 & 95 & 15 & 5142 & 239 & $9 \begin{array}{r}2 . \\
3\end{array}$ & \begin{tabular}{l|l}
1 & 6. \\
3 & 1
\end{tabular} & \begin{tabular}{l|c}
.6 & 0. \\
1 & 8
\end{tabular} & \begin{tabular}{l|l|}
.1 & 30 \\
8 &
\end{tabular} & d & 34 & 400 & \begin{tabular}{ll|l}
0 & 20
\end{tabular} & 20 & 60 & $0 \mid 0$ & \begin{tabular}{l|l}
0 & 0
\end{tabular} & 0 & 0 & $15 \mid 1$ & 1560 & 55 & \begin{tabular}{l|l}
5 & 0
\end{tabular} & 0 & 91 & 455 & \begin{tabular}{l|l}
55 & 0
\end{tabular} & 0 & 85 & \begin{tabular}{l|l}
50 & 6
\end{tabular} & $6 \quad 60$ & $\begin{array}{c}6.4 \\
3\end{array}$ & \begin{tabular}{|c|} 
Huge redwood \\
rootwad with cut \\
stump on its side \\
in stream forming \\
pool, maybe \\
more of a 6.3 . \\
Stump diameter \\
$=4.5^{\prime}$. \\
\end{tabular} \\
\hline 373 & $\begin{array}{l}1 . \\
1\end{array}$ & $\begin{array}{c}\text { Low } \\
\text { gradient } \\
\text { Riffle }\end{array}$ & 6686 & 63 & 12 & 17 & $8 \quad 10$ & $100_{6}^{0 .}$ & 30. & .6 & & & 2 & 50 & 030 & 35 & 30 & 50 & \begin{tabular}{l|l}
0 & 0
\end{tabular} & 0 & 0 & 00 & \begin{tabular}{l|l}
0 & 40
\end{tabular} & $401 C$ & 100 & 0 & $\begin{array}{c}10 \\
0\end{array}$ & \begin{tabular}{c|c}
10 & 0 \\
0 & 0
\end{tabular} & \begin{tabular}{l|l}
0 & 0
\end{tabular} & 20 & 65 & $5 \mid 6$ & $\begin{array}{l}5 \\
5\end{array}$ & & \\
\hline 374 & $\begin{array}{l}3 . \\
2\end{array}$ & Glide & 6683 & 75 & 18 & 8102 & \begin{tabular}{|l|l|}
25 & 18 \\
\end{tabular} & $18 \begin{array}{r}0 . \\
1\end{array}$ & \begin{tabular}{r|r}
5 & 1. \\
1 & 1 \\
\end{tabular} & $\begin{array}{l}.0 \\
1 \\
\end{array}$ & & & & 56 & 00 & 0 & 40 & 0 & \begin{tabular}{l|l}
0 & 0
\end{tabular} & 0 & 0 & 252 & \begin{tabular}{l|l}
25 & 5
\end{tabular} & 50 & $\begin{array}{ll}0 & 0\end{array}$ & 0 & 95 & 2575 & 750 & 5 & 69 & $90 \quad 6$ & $\begin{array}{l}6 \\
6\end{array}$ & & \\
\hline 375 & $\begin{array}{l}4 . \\
2\end{array}$ & \begin{tabular}{|c|} 
Mid- \\
Channel \\
Pool \\
\end{tabular} & 6785 & 69 & 20 & 183 & \begin{tabular}{l|l}
36 & 5
\end{tabular} & 5 & $\begin{array}{ll}0 & 2 . \\
3 & 2\end{array}$ & \begin{tabular}{c|c}
25 & 0. \\
2 & 2
\end{tabular} & 2.3 & c & 31 & 155 & 525 & 50 & 20 & 0 & \begin{tabular}{l|l}
0 & 0
\end{tabular} & 0 & 0 & 252 & 2545 & 55 & \begin{tabular}{l|l}
0 & 0
\end{tabular} & 0 & 91 & 0 \begin{tabular}{c|c}
$1 c$ \\
0
\end{tabular} & \begin{tabular}{l|l}
10 & 15 \\
0 & 15
\end{tabular} & 510 & 82 & $25 \mid 8$ & 850 & 2.2 & Log jam \\
\hline 376 & $\begin{array}{l}1 . \\
1\end{array}$ & \begin{tabular}{|c|} 
Low \\
gradient \\
Riffle
\end{tabular} & & 18 & 9 & 5 & 13 & 133. & $\begin{array}{ll}3 & 0 . \\
3 & 4\end{array}$ & $\begin{array}{l}0.7 \\
4\end{array}$ & & & 0 & $<5 \mid 0$ & $0 \begin{array}{c}10 \\
0\end{array}$ & 0 & 0 & 0 & \begin{tabular}{l|l}
0 & 0
\end{tabular} & 0 & 0 & 00 & \begin{tabular}{l|l}
0 & 60
\end{tabular} & & & & & & & & & & & & \\
\hline 377 & $\begin{array}{l}4 . \\
2\end{array}$ & $\begin{array}{c}\text { Mid- } \\
\text { Channel } \\
\text { Pool } \\
\end{array}$ & 6784 & 38 & 17 & 713 & 2413 & 13.1. & $\begin{array}{l}2 \\
2 \\
2\end{array}$ & \begin{tabular}{l|l}
2.2 & 0. \\
2
\end{tabular} & .330 & c & 32 & 200 & 0 & 60 & 0 & 0 & \begin{tabular}{l|l}
0 & 0
\end{tabular} & 0 & 20 & $\left.15\right|_{1}$ & 1525 & 525 & 150 & 5 & 93 & $0 \mid$\begin{tabular}{c|c}
$1 c$ \\
0
\end{tabular} & \begin{tabular}{l|l}
10 & 0 \\
0 & 0
\end{tabular} & 0 & 69 & \begin{tabular}{l|l}
95 & 8
\end{tabular} & 880 & $\begin{array}{c}1.9 \\
2\end{array}$ & $\begin{array}{c}\text { Bedrock = } \\
\text { cement blocks }\end{array}$ \\
\hline 378 & $\begin{array}{l}4 . \\
2\end{array}$ & $\begin{array}{c}\text { Mid- } \\
\text { Channel } \\
\text { Pool }\end{array}$ & 6779 & 40 & 16 & 5132 & $24 \mid 12$ & $12 \frac{1 .}{2}$ & $\begin{array}{l}9 \\
2 \\
2\end{array}$ & \begin{tabular}{l|c}
3.5 & 0. \\
5 & 8
\end{tabular} & \begin{tabular}{l|l|}
8.4 & 40 \\
\end{tabular} & c & 34 & $45 \mid 1$ & 030 & 30 & 30 & 0 & 0.0 & 0 & 0 & 252 & $25 \mid 30$ & $\begin{array}{l}0 \\
0\end{array}$ & \begin{tabular}{l|l}
0 & 0
\end{tabular} & 0 & 96 & 0 \begin{tabular}{c|c}
$1 c$ \\
0
\end{tabular} & \begin{tabular}{l|l}
10 & 0 \\
0 & 0
\end{tabular} & 10 & 69 & \begin{tabular}{l|l}
90 & 8
\end{tabular} & 880 & $\begin{array}{c}3.0 \\
7\end{array}$ & \begin{tabular}{|c|} 
Large $\left(>4^{\prime}\right.$ \\
diameter) \\
redwood log \\
across stream \\
blocks fish \\
passage at this \\
flow. End of day \\
$8 / 15$
\end{tabular} \\
\hline 379 & $\begin{array}{l}3 . \\
4\end{array}$ & Step Run & 6370 & $\left|\begin{array}{c}13 \\
8\end{array}\right|$ & 18 & 8251 & $15 \mid 14$ & $\left.14\right|_{8} ^{0 .}$ & \begin{tabular}{l|r}
2 & 1. \\
3 & 4
\end{tabular} & 4 & & & 3 & 52 & 025 & 50 & 25 & 0 & \begin{tabular}{l|l|l}
0 & 0
\end{tabular} & 0 & 0 & 5 & $\begin{array}{ll}5 & 35\end{array}$ & 5352 & 200 & 0 & 90 & $0 \mid$\begin{tabular}{c|c}
$1 c$ \\
0
\end{tabular} & \begin{tabular}{c|c}
10 & 0 \\
0 & 0
\end{tabular} & 0 & 69 & \begin{tabular}{l|l}
95 & 6
\end{tabular} & $\begin{array}{l}6 \\
5\end{array}$ & & $\begin{array}{c}3 \text { riffles }\left(19^{\prime}, 3^{\prime}, ?\right) \\
2 \text { runs. }+100 \mathrm{LB} \\
\text { barbed wire } \\
\text { fence. Start } 8 / 19\end{array}$ \\
\hline 380 & $\begin{array}{l}5 . \\
3\end{array}$ & \begin{tabular}{|c|} 
Lateral \\
Scour \\
Pool - \\
Root Wad \\
Enhanced
\end{tabular} & 6369 & 49 & 13 & $\begin{array}{llll}3 & 14\end{array}$ & 1411 & $11{ }_{8}^{0 .}$ & & \begin{tabular}{c|c}
.8 & 0. \\
1 & 9
\end{tabular} & \begin{tabular}{l|l|}
9 & 40 \\
9 & 40
\end{tabular} & $d$ & 3 & 100 & \begin{tabular}{l|l}
0 & 15
\end{tabular} & 30 & 45 & 10 & 0.0 & 0 & 0 & 101 & 1030 & 0302 & 200 & 0 & 91 & 0 \begin{tabular}{c|c}
$1 c$ \\
0
\end{tabular} & \begin{tabular}{l|l}
10 & 0 \\
0 & 0
\end{tabular} & 0 & $\begin{array}{ll}6 & 5\end{array}$ & $55 \mid 6$ & 680 & $\begin{array}{c}1.5 \\
2\end{array}$ & \\
\hline 381 & $\begin{array}{l}3 . \\
4\end{array}$ & Step Run & 6368 & $\left(\begin{array}{c}11 \\
4\end{array}\right.$ & 12 & 211 & 818 & $18{ }_{8}^{0 .} 8$ & 51. & 1.3 & & & 3 & 3011 & 030 & 35 & 20 & 5 & 0.0 & 0 & 0 & 101 & 1025 & 5302 & 250 & 0 & 83 & $\begin{array}{ll}5 & 95\end{array}$ & \begin{tabular}{l|l}
95 & 0
\end{tabular} & 0 & $\begin{array}{ll}6 & 9\end{array}$ & $90 \quad 6$ & 580 & & $\begin{array}{c}3 \text { riffles }\left(1^{\prime}, 8^{\prime}, 5^{\prime}\right) \\
4 \text { glides. Step } \\
\text { glide }\end{array}$ \\
\hline $\begin{array}{c}381 . \\
1\end{array}$ & $\begin{array}{l}6 . \\
4\end{array}$ & \begin{tabular}{|c|} 
Backwate \\
r Pool - \\
log \\
formed
\end{tabular} & & 17 & & & 15 & 8 & $\begin{array}{ll}5 & 2 . \\
3 & 8\end{array}$ & \begin{tabular}{l|l}
2.7 & 0. \\
8 & 2
\end{tabular} & $\begin{array}{l}.7 \\
2\end{array}$ & & 3 & $20 \mid 1$ & .010 & 50 & 10 & 20 & 0.0 & 0 & 0 & 202 & 2020 & & & & & & & & & & & $\begin{array}{c}2.0 \\
6\end{array}$ & \\
\hline 382 & $\left|\begin{array}{l}4 . \\
2\end{array}\right|$ & $\begin{array}{c}\text { Mid- } \\
\text { Channel } \\
\text { Pool } \\
\end{array}$ & \begin{tabular}{|l|l}
63 & 70 \\
\end{tabular} & 11 & 13 & \begin{tabular}{ll|l}
3 & 18 \\
\end{tabular} & 7 & 7 & $\begin{array}{l}7 \\
7 \\
4\end{array}$ & \begin{tabular}{l|l}
. .7 & 0. \\
4 &
\end{tabular} & $\begin{array}{l}0.320 \\
\end{array}$ & c & 3 & 600 & \begin{tabular}{l|l}
0 & 40
\end{tabular} & 60 & 0 & 0 & \begin{tabular}{l|l}
0 & 0
\end{tabular} & 0 & 0 & 101 & 1035 & 5351 & 100 & 0 & & & 0 & 0 & \begin{tabular}{l|l}
6 & 5
\end{tabular} & $50 \mid 6$ & 6 & $\begin{array}{c}1.4 \\
4\end{array}$ & \\
\hline
\end{tabular}




\begin{tabular}{|c|c|c|c|c|c|c|c|c|c|c|c|c|c|c|c|c|c|c|c|c|c|c|c|c|c|c|c|c|c|c|c|c|c|c|}
\hline 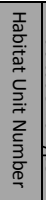 & & 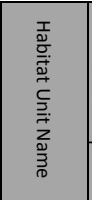 & 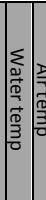 & & 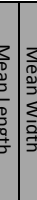 & 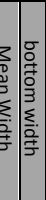 & 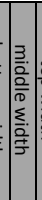 & 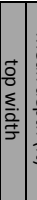 & & 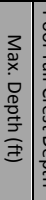 & 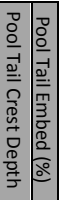 & 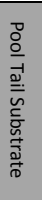 & 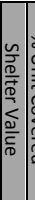 & 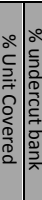 & a & 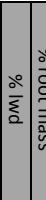 & 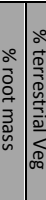 & 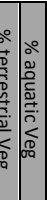 & 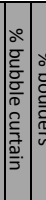 & 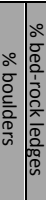 & 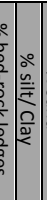 & 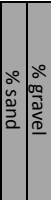 & 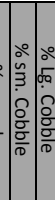 & 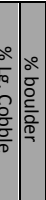 & 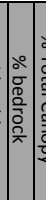 & 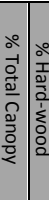 & 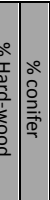 & 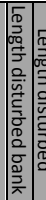 & 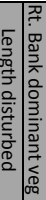 & 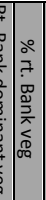 & 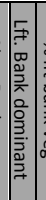 & 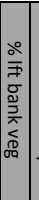 & 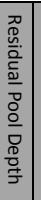 & 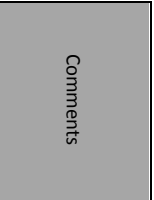 \\
\hline 383 & $\begin{array}{l}4 . \\
2\end{array}$ & \begin{tabular}{|c|} 
Mid- \\
Channel \\
Pool \\
\end{tabular} & 637 & 7155 & $551 c$ & 107 & 12 & 101 & $1.1{ }^{1}$ & \begin{tabular}{c|c}
1.9 & 0 \\
1 &
\end{tabular} & \begin{tabular}{c|c}
0.6 & 40 \\
2 &
\end{tabular} & c & 32 & 2035 & 50 & 303 & $30 \quad 5$ & 50 & 00 & \begin{tabular}{l|l}
0 & 0
\end{tabular} & 025 & 3030 & \begin{tabular}{l|l}
0 & 10 \\
\end{tabular} & 50 & 09 & \begin{tabular}{l|l}
99 & 0
\end{tabular} & $\begin{array}{cc}0 \\
0 \\
0\end{array}$ & 0 & 08 & 360 & 69 & 95 & $\begin{array}{c}1.2 \\
9\end{array}$ & \\
\hline 384 & \begin{tabular}{|l|}
1. \\
2 \\
\end{tabular} & & & 4 & $4 \mid 1 c$ & 10 & 10 & & \begin{tabular}{c|c}
0.1 & 0 \\
4 & \\
\end{tabular} & \begin{tabular}{c|}
0.1 \\
5
\end{tabular} & & & & & & & & & & & & & & & & & & & & & & & & \\
\hline 385 & $\begin{array}{c}1 . \\
1\end{array}$ & \begin{tabular}{|c|} 
Low \\
gradient \\
Riffle \\
\end{tabular} & 646 & 699 & $01 c$ & 1010 & 8 & $\left.12\right|^{0}$ & \begin{tabular}{c|c}
0.3 & 0 \\
7 &
\end{tabular} & $\begin{array}{c}0.6 \\
4\end{array}$ & & & 2 & \begin{tabular}{l|l}
5 & 0
\end{tabular} & 50 & 50 & \begin{tabular}{l|l}
0 & 0
\end{tabular} & \begin{tabular}{l|l|l}
0 & 0
\end{tabular} & 00 & 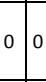 & 0 & $\begin{array}{lll}5 & 30\end{array}$ & 03030 & 300 & 09 & 9760 & 5040 & 0 & \begin{tabular}{l|l}
0 & 6
\end{tabular} & 595 & 69 & 90 & & \\
\hline 386 & \begin{tabular}{|l|}
3. \\
2 \\
\end{tabular} & Glide & 636 & 6845 & \begin{tabular}{l|l}
15 & 13 \\
\end{tabular} & \begin{tabular}{l|l|}
13 & 12 \\
\end{tabular} & 211 & $\left.17\right|^{0}$ & \begin{tabular}{c|c}
0.5 & 1 \\
7 &
\end{tabular} & 1.1 & & & 31 & 1025 & 525 & 252 & \begin{tabular}{l|l}
25 & 0
\end{tabular} & 00 & 0 & 0 & 015 & 1530 & 03010 & 100 & 09 & \begin{tabular}{l|l}
96 & 60
\end{tabular} & 5040 & 02 & 26 & 595 & 69 & 95 & & \\
\hline 387 & $\begin{array}{l}4 . \\
2\end{array}$ & \begin{tabular}{|c|} 
Mid- \\
Channel \\
Pool \\
\end{tabular} & 637 & $73 \mid \begin{array}{c}1 c \\
5\end{array}$ & 5 & 1717 & 719 & $15^{1}$ & \begin{tabular}{c|c}
1.4 & 2 \\
4
\end{tabular} & \begin{tabular}{c|c}
2.6 & 0 \\
2 & 0
\end{tabular} & 0.5 & c & 3 & 15 & 510 & \begin{tabular}{|l|l|}
30 & 4
\end{tabular} & $40 \quad 5$ & 50 & 00 & 0 & 025 & \begin{tabular}{|l|l}
25 & 4
\end{tabular} & \begin{tabular}{l|l|l}
4 & 5
\end{tabular} & \begin{tabular}{l|l|l}
5 & 0
\end{tabular} & 09 & \begin{tabular}{l|c}
97 & 10 \\
9
\end{tabular} & \begin{tabular}{l|l}
10 & 0 \\
0
\end{tabular} & 0 & \begin{tabular}{l|l}
0 & 6
\end{tabular} & 595 & 6 & $\mid$\begin{tabular}{c|c}
10 \\
0
\end{tabular} & $\begin{array}{c}2.1 \\
2\end{array}$ & $\begin{array}{l}\text { long pool, top } \\
\text { part = 5.2/5.3 }\end{array}$ \\
\hline 388 & \begin{tabular}{|l|}
3. \\
2 \\
\end{tabular} & Glide & 657 & 7397 & \begin{tabular}{l|l}
7 & 13 \\
\end{tabular} & 13 & 519 & 5 & \begin{tabular}{c|c}
0.6 & 1 \\
8 & \\
\end{tabular} & 1.5 & & & 3 & 525 & 520 & 252 & $20 \quad 10$ & 100 & 00 & 0 & 020 & 2025 & 52510 & 100 & 08 & 8150 & 5050 & 0 & \begin{tabular}{l|l}
0 & 6
\end{tabular} & 590 & 69 & 90 & & (+73) Bridge \\
\hline 389 & 1. & \begin{tabular}{|c|} 
Low \\
gradient \\
Riffle
\end{tabular} & & 27 & 7711 & 115 & 12 & $15^{0}$ & \begin{tabular}{c|c}
0.2 & 0 \\
5 &
\end{tabular} & $\begin{array}{c}0.3 \\
8\end{array}$ & & & 2 & \begin{tabular}{l|l}
5 & 0
\end{tabular} & 20 & \begin{tabular}{|l|l}
30 & 4
\end{tabular} & $45 \quad 5$ & 50 & 0 & \begin{tabular}{l|l}
0 & 0
\end{tabular} & 0 & \begin{tabular}{|ll}
5 & 35
\end{tabular} & $5352 C$ & 200 & 09 & \begin{tabular}{l|c}
10 & 10 \\
0
\end{tabular} & \begin{tabular}{l|l}
10 & 0 \\
0 & 0
\end{tabular} & 0 & \begin{tabular}{l|l}
0 & 6
\end{tabular} & 595 & 6 & \begin{tabular}{c|}
10 \\
0
\end{tabular} & & \\
\hline 390 & $\begin{array}{c}5 . \\
3 \\
\end{array}$ & \begin{tabular}{|c|} 
Lateral \\
Scour \\
Pool - \\
Root Wad \\
Enhanced \\
\end{tabular} & 65 & $74 \mid 36$ & 3011 & 1115 & 511 & 6 & \begin{tabular}{c|c}
0.9 & 1 \\
9 & 1
\end{tabular} & $1.50^{0}$ & \begin{tabular}{c|c}
0.3 & 10 \\
4 & 10
\end{tabular} & e & 3 & \begin{tabular}{l|l}
5 & 40
\end{tabular} & 0 & \begin{tabular}{|l|l|l|} 
& 6
\end{tabular} & \begin{tabular}{l|l}
60 & 0
\end{tabular} & \begin{tabular}{l|l|l}
0 & 0
\end{tabular} & 00 & \begin{tabular}{l|l}
0 & 0
\end{tabular} & \begin{tabular}{l|l|l|}
0 & 10
\end{tabular} & \begin{tabular}{|l|l|}
10 & 35
\end{tabular} & 53510 & 100 & $0^{1}$ & \begin{tabular}{c|c}
10 & 98 \\
0 & 98
\end{tabular} & \begin{tabular}{l|l}
88 & 2
\end{tabular} & 0 & \begin{tabular}{l|l}
0 & 6
\end{tabular} & 5 & 6 & $90]^{1}$ & $\begin{array}{c}1.1 \\
6\end{array}$ & $\begin{array}{l}\text { Root wads from } \\
\text { living alders in } \\
\text { bank }\end{array}$ \\
\hline 391 & \begin{tabular}{|l|}
1. \\
1 \\
\end{tabular} & \begin{tabular}{|c|} 
Low \\
gradient \\
Riffle \\
\end{tabular} & 667 & 743 & 3310 & 106 & 13 & 12 & \begin{tabular}{c|c}
0.3 & 0 \\
3 &
\end{tabular} & $\begin{array}{c}0.6 \\
9\end{array}$ & & & 1 & \begin{tabular}{l|l}
5 & 0
\end{tabular} & $\begin{array}{c}10 \\
0\end{array}$ & \begin{tabular}{l|l}
0 & 0 \\
0
\end{tabular} & \begin{tabular}{l|l}
0 & 0
\end{tabular} & \begin{tabular}{l|l|}
0 & 0
\end{tabular} & 0 & \begin{tabular}{l|l}
0 & 0
\end{tabular} & 00 & $\begin{array}{lll}0 & 30\end{array}$ & $0353 c$ & 305 & 09 & \begin{tabular}{|c|c}
10 & 10 \\
\end{tabular} & \begin{tabular}{l|l|}
0 & 0 \\
0 & 0
\end{tabular} & 05 & \begin{tabular}{l|l}
5 & 6
\end{tabular} & 595 & 568 & 85 & & \\
\hline 392 & $\begin{array}{l}4 . \\
2\end{array}$ & \begin{tabular}{|c|} 
Mid- \\
Channel \\
Pool \\
\end{tabular} & 657 & $74 \mid 88$ & $88 \mid 13$ & 1312 & 217 & 10 & \begin{tabular}{c|c}
1.4 & 2 \\
4 &
\end{tabular} & \begin{tabular}{c|c}
2.5 & 0 \\
1 &
\end{tabular} & \begin{tabular}{c|c|}
0.4 & 20 \\
8 &
\end{tabular} & e & 32 & 2010 & 10 & \begin{tabular}{|l|l}
25 & 5
\end{tabular} & \begin{tabular}{l|l}
55 & 0
\end{tabular} & \begin{tabular}{l|l|}
0 & 0
\end{tabular} & 0 & \begin{tabular}{l|l}
0 & 0
\end{tabular} & \begin{tabular}{ll|l}
0 & 25
\end{tabular} & \begin{tabular}{|l|l|}
25 & 25
\end{tabular} & $\begin{array}{l}520 \\
20\end{array}$ & \begin{tabular}{l|l|}
10 & 0
\end{tabular} & 09 & \begin{tabular}{l|l}
94 & 50
\end{tabular} & \begin{tabular}{ll|l}
50 & 50
\end{tabular} & 0 & 0 & & 6 & 95 & $\begin{array}{c}2.0 \\
3\end{array}$ & $\begin{array}{l}\text { Root wad } \\
\text { enhanced }\end{array}$ \\
\hline 393 & $\begin{array}{c}1 . \\
1\end{array}$ & \begin{tabular}{|c|} 
Low \\
gradient \\
Riffle
\end{tabular} & 657 & $71 \mid 2$ & 13 & 1310 & 010 & $20^{0}$ & \begin{tabular}{c|c}
0.3 & 0 \\
3 &
\end{tabular} & $\begin{array}{c}0.3 \\
8\end{array}$ & & & 1 & \begin{tabular}{l|l}
$<5$ & 0
\end{tabular} & $\begin{array}{c}10 \\
0\end{array}$ & 0 & \begin{tabular}{l|l}
0 & 0
\end{tabular} & \begin{tabular}{l|l|}
0 & 0
\end{tabular} & 0 & \begin{tabular}{l|l}
0 & 0
\end{tabular} & \begin{tabular}{l|l|}
0 & 0
\end{tabular} & $\begin{array}{lll}0 & 40\end{array}$ & 04020 & 200 & 09 & \begin{tabular}{l|l}
96 & 40
\end{tabular} & $\begin{array}{ll}10 & 60\end{array}$ & 0 & \begin{tabular}{l|l}
0 & 6
\end{tabular} & 5 & 68 & 80 & & \\
\hline 394 & $\begin{array}{l}4 . \\
2\end{array}$ & \begin{tabular}{|c|} 
Mid- \\
Channel \\
Pool \\
\end{tabular} & 677 & 725 & \begin{tabular}{l|l}
77 & 19
\end{tabular} & 1920 & 023 & 14 & \begin{tabular}{c|c}
2.3 & 4 \\
7 &
\end{tabular} & \begin{tabular}{c|c}
4.1 & 0 \\
5 &
\end{tabular} & \begin{tabular}{c|c|}
0.3 & 20 \\
1 &
\end{tabular} & $d$ & 32 & 2010 & 10 & \begin{tabular}{|l|l}
10 & 7
\end{tabular} & $70 \quad 0$ & \begin{tabular}{l|l|}
0 & 0
\end{tabular} & 0 & \begin{tabular}{l|l}
0 & 0
\end{tabular} & \begin{tabular}{ll|}
0 & 30
\end{tabular} & 3030 & \begin{tabular}{l|l|l}
0 & 5
\end{tabular} & \begin{tabular}{l|l}
5 & 0
\end{tabular} & $0^{1}$ & \begin{tabular}{|c|c|}
10 & 80 \\
0 &
\end{tabular} & $\begin{array}{lll}30 & 20\end{array}$ & 01 & 106 & 575 & 56 & 90 & $\begin{array}{c}3.8 \\
4\end{array}$ & $\begin{array}{l}\text { Root wad } \\
\text { enhanced }\end{array}$ \\
\hline 395 & $\begin{array}{l}4 . \\
2\end{array}$ & \begin{tabular}{|c|} 
Mid- \\
Channel \\
Pool \\
\end{tabular} & 677 & 725 & $\begin{array}{lll}55 & 14\end{array}$ & 1414 & \begin{tabular}{ll|l}
4 & 14 \\
\end{tabular} & $14^{1}$ & \begin{tabular}{c|c}
1.7 & 3 \\
2 &
\end{tabular} & $\begin{array}{c}3.0 \\
5\end{array}$ & 1 & c & 34 & 4010 & 30 & 303 & $30 \quad 0$ & \begin{tabular}{l|l|}
0 & 0
\end{tabular} & 0 & \begin{tabular}{l|l}
0 & 0
\end{tabular} & $\begin{array}{lll}0 & 25\end{array}$ & 2535 & 5105 & \begin{tabular}{l|l}
5 & 0
\end{tabular} & 08 & \begin{tabular}{|l|l|}
85 & 95
\end{tabular} & \begin{tabular}{|l|l|}
95 & 5
\end{tabular} & 0 & \begin{tabular}{l|l}
0 & 6
\end{tabular} & $\mid$\begin{tabular}{c|c}
10 \\
0
\end{tabular} & 69 & $955^{2}$ & $\begin{array}{c}2.0 \\
5\end{array}$ & \\
\hline 396 & $\begin{array}{l}1 . \\
1\end{array}$ & \begin{tabular}{|c|} 
Low \\
gradient \\
Riffle \\
\end{tabular} & 668 & 8038 & \begin{tabular}{l|l}
38 & 11
\end{tabular} & \begin{tabular}{l|l|l}
11 & 14
\end{tabular} & 46 & 13 & \begin{tabular}{c|c}
0.1 & 0 \\
9 & 0
\end{tabular} & 0.3 & & & 1 & \begin{tabular}{l|l}
5 & 0
\end{tabular} & 0 & 0 & \begin{tabular}{l|c}
0 & 10
\end{tabular} & \begin{tabular}{l|l}
10 & 0 \\
0 & 0
\end{tabular} & 0 & \begin{tabular}{l|l}
0 & 0
\end{tabular} & \begin{tabular}{l|l|}
0 & 0
\end{tabular} & $\begin{array}{lll}0 & 30\end{array}$ & $\begin{array}{llll}0 & 45 & 20\end{array}$ & 205 & 08 & \begin{tabular}{|l|l|}
82 & 75
\end{tabular} & 5525 & 0 & \begin{tabular}{l|l}
0 & 6
\end{tabular} & 5 & 6 & \begin{tabular}{c|}
10 \\
0
\end{tabular} & & \\
\hline 397 & \begin{tabular}{|l|}
3. \\
2 \\
\end{tabular} & Glide & 657 & 7558 & \begin{tabular}{l|l}
88 & 13 \\
\end{tabular} & \begin{tabular}{|l|l|}
13 & 13 \\
\end{tabular} & 312 & $15^{0}$ & \begin{tabular}{c|c}
0.5 & 1 \\
4 & \\
\end{tabular} & 1.2 & & & 2 & \begin{tabular}{l|l}
5 & 5
\end{tabular} & 20 & 750 & \begin{tabular}{l|l}
0 & 0
\end{tabular} & \begin{tabular}{l|l|}
0 & 0
\end{tabular} & 00 & \begin{tabular}{l|l}
0 & 0
\end{tabular} & $\begin{array}{lll}0 & 15\end{array}$ & \begin{tabular}{|l|l}
15 & 50 \\
\end{tabular} & \begin{tabular}{l|l|l}
0 & 15 & 5
\end{tabular} & \begin{tabular}{l|l}
5 & 0 \\
\end{tabular} & 00 & \begin{tabular}{|c|c|}
10 & $1 c$ \\
0 & 0 \\
\end{tabular} & \begin{tabular}{|l|l|}
10 & 0 \\
\end{tabular} & 0 & \begin{tabular}{l|l}
0 & 6
\end{tabular} & 590 & 69 & 95 & & \\
\hline 398 & $\begin{array}{l}4 . \\
2\end{array}$ & \begin{tabular}{|c|} 
Mid- \\
Channel \\
Pool \\
\end{tabular} & 657 & $71 \mid 28$ & 816 & 1615 & 522 & $10^{0}$ & \begin{tabular}{c|c}
0.8 & 1 \\
7 &
\end{tabular} & \begin{tabular}{c|c}
1.2 & 0 \\
5 &
\end{tabular} & $\begin{array}{c}0.4 \\
1\end{array}$ & c & 35 & 5520 & 0 & \begin{tabular}{|l|l|l|}
70 & 1
\end{tabular} & $10 \quad 0$ & \begin{tabular}{l|l|}
0 & 0
\end{tabular} & 0 & \begin{tabular}{l|l}
0 & 0
\end{tabular} & \begin{tabular}{ll|l}
0 & 25
\end{tabular} & \begin{tabular}{|l|l|}
25 & 30
\end{tabular} & $\begin{array}{lllll}0 & 10 & 10\end{array}$ & 100 & 0 & \begin{tabular}{|c|c}
10 & 10 \\
0 & 0
\end{tabular} & \begin{tabular}{l|l|}
0 & 0 \\
0 & 0 \\
\end{tabular} & 0 & \begin{tabular}{l|l}
0 & 6
\end{tabular} & 590 & 69 & 95 & $\begin{array}{c}0.8 \\
4\end{array}$ & \\
\hline 399 & $\begin{array}{l}1 . \\
1\end{array}$ & \begin{tabular}{|c|} 
Low \\
gradient \\
Riffle
\end{tabular} & 657 & 725 & 508 & 8 | 10 & 8 & 5 & \begin{tabular}{c|c}
0.2 & 0 \\
8 &
\end{tabular} & $\begin{array}{c}0.6 \\
5\end{array}$ & & & 3 & $\begin{array}{lll}5 & 30\end{array}$ & 30 & \begin{tabular}{l|l}
0 & 3
\end{tabular} & \begin{tabular}{l|l}
30 & 10
\end{tabular} & 100 & 0 & \begin{tabular}{l|l}
0 & 0
\end{tabular} & \begin{tabular}{ll|l}
0 & 5
\end{tabular} & \begin{tabular}{|l|l}
5 & 30
\end{tabular} & 03030 & 300 & 09 & 94 \begin{tabular}{c|c}
10 \\
0
\end{tabular} & \begin{tabular}{l|l}
10 & 0 \\
0 & 0 \\
\end{tabular} & 0 & \begin{tabular}{l|l}
0 & 6
\end{tabular} & 575 & 568 & 85 & & \\
\hline 400 & $\begin{array}{l}4 . \\
2\end{array}$ & $\begin{array}{c}\text { Mid- } \\
\text { Channel } \\
\text { Pool }\end{array}$ & 657 & 7073 & 315 & 1518 & 815 & 12 & \begin{tabular}{c|c}
2.3 & 4 \\
7 &
\end{tabular} & \begin{tabular}{c|c}
4.2 & 0 \\
2 & 0
\end{tabular} & 0.3 & & 34 & 4510 & 10 & \begin{tabular}{|l|l|}
10 & 7
\end{tabular} & \begin{tabular}{l|l}
70 & 0
\end{tabular} & \begin{tabular}{l|l|}
0 & 0
\end{tabular} & 0 & \begin{tabular}{l|l}
0 & 0
\end{tabular} & $\begin{array}{lll}0 & 30\end{array}$ & 3030 & 055 & \begin{tabular}{l|l}
5 & 0
\end{tabular} & 09 & \begin{tabular}{|l|l}
93 & 2
\end{tabular} & 2 298 & 5 & 0 & 595 & 58 & $80^{3}$ & $\begin{array}{c}3.9 \\
2\end{array}$ & $\begin{array}{c}\text { Formed by a } \\
\text { huge rootwad, } \\
\text { combo } 4.2 \text { and } \\
6.3, \text { pipe across } \\
\text { stream } \\
\end{array}$ \\
\hline 401 & $\begin{array}{c}1 . \\
1\end{array}$ & \begin{tabular}{|c|} 
Low \\
gradient \\
Riffle \\
\end{tabular} & & $2 C$ & $\begin{array}{lll}20 & 14\end{array}$ & $14 \mid 12$ & & 16 & \begin{tabular}{c|c}
0.2 & 0 \\
6 &
\end{tabular} & $\begin{array}{c}0.4 \\
2\end{array}$ & & & 1 & \begin{tabular}{l|l}
$<5$ & 0
\end{tabular} & $\begin{array}{c}10 \\
0\end{array}$ & 00 & \begin{tabular}{l|l}
0 & 0
\end{tabular} & \begin{tabular}{l|l|}
0 & 0
\end{tabular} & 0 & \begin{tabular}{l|l}
0 & 0
\end{tabular} & $\begin{array}{lll}0 & 0\end{array}$ & $\begin{array}{lll}0 & 25\end{array}$ & $\begin{array}{lll}530 & 32\end{array}$ & 2520 & 0 & & & & & & & & & \\
\hline 402 & $\begin{array}{c}5 . \\
1\end{array}$ & $\begin{array}{c}\text { Corner } \\
\text { Pool }\end{array}$ & 657 & 73.96 & 616 & $16 \mid 16$ & 620 & 13 & \begin{tabular}{c|c}
1.3 & 3 \\
1 &
\end{tabular} & \begin{tabular}{c|c}
3.8 & 0 \\
8 &
\end{tabular} & \begin{tabular}{c|c|}
0.2 & 50 \\
2 &
\end{tabular} & c & 31 & 1020 & 20 & 402 & \begin{tabular}{l|l}
20 & 0
\end{tabular} & \begin{tabular}{l|l|l}
0 & 0
\end{tabular} & 0 & \begin{tabular}{l|l}
0 & 0
\end{tabular} & $\begin{array}{lll}0 & 25\end{array}$ & 2530 & 0105 & \begin{tabular}{l|l}
5 & 5
\end{tabular} & \begin{tabular}{|l|l|l|l|l}
0 & 8
\end{tabular} & \begin{tabular}{|l|l|l}
85 & 20
\end{tabular} & \begin{tabular}{ll|l}
20 & 80
\end{tabular} & 0 & \begin{tabular}{|l|l|l|}
0 & 8
\end{tabular} & \begin{tabular}{l|l|}
3 & 75
\end{tabular} & \begin{tabular}{l|l|l}
5 & 6
\end{tabular} & $955^{3}$ & $\begin{array}{c}3.6 \\
6 \\
\end{array}$ & $\begin{array}{l}\text { Top of pool, two } \\
\text { artificial cement } \\
\text { logs cabled to RB }\end{array}$ \\
\hline 403 & $\begin{array}{l}1 . \\
1\end{array}$ & \begin{tabular}{|c|} 
Low \\
gradient \\
Riffle \\
\end{tabular} & 656 & 6895 & $\begin{array}{ll}30 & 10\end{array}$ & $\begin{array}{lll}10 & 7\end{array}$ & 14 & 9 & \begin{tabular}{c|c}
0.3 & 1 \\
9
\end{tabular} & $\begin{array}{c}1.2 \\
1\end{array}$ & & & 3 & \begin{tabular}{l|l}
5 & 20
\end{tabular} & 60 & \begin{tabular}{|l|l}
0 & 0
\end{tabular} & \begin{tabular}{l|l}
0 & 20
\end{tabular} & \begin{tabular}{l|l|}
20 & 0
\end{tabular} & 0 & \begin{tabular}{l|l}
0 & 0
\end{tabular} & $\begin{array}{lll}0 & 0\end{array}$ & $\begin{array}{lll}0 & 30\end{array}$ & 0303 & \begin{tabular}{l|l|l}
30 & 10
\end{tabular} & 0 & \begin{tabular}{|l|l|}
99 & 98
\end{tabular} & \begin{tabular}{l|l}
88 & 2
\end{tabular} & 0 & 06 & 5 & 69 & 90 & & End of day $8 / 19$ \\
\hline 404 & $\begin{array}{l}4 . \\
2\end{array}$ & $\begin{array}{c}\text { Mid- } \\
\text { Channel } \\
\text { Pool }\end{array}$ & 636 & & 1223 & 2336 & 626 & 6 & \begin{tabular}{c|c}
1.9 & 3 \\
5 &
\end{tabular} & \begin{tabular}{c|c}
3.6 & 0 \\
5 & 0
\end{tabular} & 0.2 & c & 3 & 405 & 30 & \begin{tabular}{|l|l|}
30 & 3
\end{tabular} & \begin{tabular}{l|l}
30 & 5
\end{tabular} & 50 & 0 & 0 & $0 \mid 30$ & 3025 & 55 & \begin{tabular}{l|l|}
5 & 5
\end{tabular} & \begin{tabular}{|l|l|l|l|}
0 & 8
\end{tabular} & \begin{tabular}{|l|l|l}
80 & 60
\end{tabular} & 5040 & 15 & \begin{tabular}{l|l}
0 & 6
\end{tabular} & 560 & 67 & 75 & $\begin{array}{c}3.4 \\
5\end{array}$ & $\begin{array}{c}\text { Log jam, } 36^{\prime} \mathrm{w} x \\
6 \text { 'h } \times 17^{\prime} l . \text { Start } \\
8 / 20 . \text { Start } \\
\text { Raney } \\
\end{array}$ \\
\hline 405 & \begin{tabular}{|l|}
1. \\
1
\end{tabular} & \begin{tabular}{|c|} 
Low \\
gradient \\
Riffle
\end{tabular} & 636 & $67 \mid 28$ & 86 & \begin{tabular}{l|l}
6 & 6
\end{tabular} & 6 & 70 & 0.30 & 0.4 & & & 2 & 100 & 50 & 05 & $50 \quad 0$ & \begin{tabular}{l|l|l}
0 & 0
\end{tabular} & 00 & \begin{tabular}{l|l}
0 & 0
\end{tabular} & 00 & 030 & 0303 & 3010 & 09 & 9798 & \begin{tabular}{l|l|}
98 & 2
\end{tabular} & 0 & \begin{tabular}{l|l}
0 & 6
\end{tabular} & 580 & 62 & 20 & & \\
\hline 406 & $\begin{array}{l}4 . \\
2\end{array}$ & $\begin{array}{c}\text { Mid- } \\
\text { Channel } \\
\text { Pool }\end{array}$ & 636 & 6543 & 1315 & 1512 & 219 & 15 & \begin{tabular}{c|c}
1.2 & 3 \\
7 & 3
\end{tabular} & 3.30 & \begin{tabular}{l|l|l|l|}
0.2 & 10 \\
\end{tabular} & c & 1 & 0 & $\begin{array}{c}10 \\
0\end{array}$ & 0 & \begin{tabular}{l|l}
0 & 0
\end{tabular} & \begin{tabular}{l|l|l}
0 & 0
\end{tabular} & 00 & 0 & $0 \mid 30$ & & 0100 & 0.0 & 09 & \begin{tabular}{l|c}
98 & 10 \\
0
\end{tabular} & \begin{tabular}{l|l}
10 & 0 \\
0 & 0
\end{tabular} & 0 & $0 \mid 6$ & 590 & 6 & 90 & 3.1 & $\begin{array}{c}\text { (+40) LB trib with } \\
\text { water. } \\
\text { Bettencourt } \\
\text { Gulch. } \\
\end{array}$ \\
\hline 407 & 1. & \begin{tabular}{|c|} 
Low \\
gradient \\
Riffle
\end{tabular} & 636 & & \begin{tabular}{l|l}
99 & 13
\end{tabular} & 1315 & 8 & & 0.3 & 0.6 & & & 1 & 0 & 0 & 0 & $\left.0\right|_{0} ^{10}$ & $\begin{array}{lll}10 & 0 \\
0 & & \end{array}$ & 0 & \begin{tabular}{l|l}
0 & 0
\end{tabular} & $\begin{array}{lll}0 & 0 \\
\end{array}$ & 0 & 0353 & 350 & $\mid$\begin{tabular}{l|l}
0 & 8
\end{tabular} & \begin{tabular}{l|l}
80 & 2
\end{tabular} & 298 & 0 & \begin{tabular}{l|l}
0 & 6
\end{tabular} & 590 & 6 & 60 & & \\
\hline
\end{tabular}




\begin{tabular}{|c|c|c|c|c|c|c|c|c|c|c|c|c|c|c|c|c|c|c|c|c|c|c|c|c|c|c|c|c|c|c|c|c|c|}
\hline 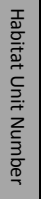 & & 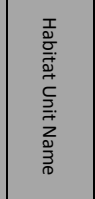 & 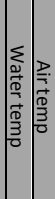 & 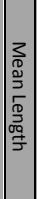 & 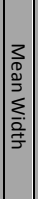 & 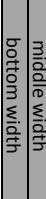 & 要 & 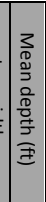 & 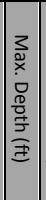 & 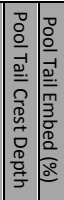 & 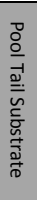 & 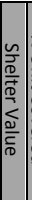 & 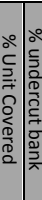 & 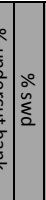 & 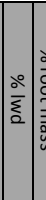 & 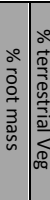 & 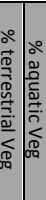 & 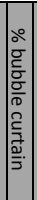 & 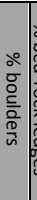 & & 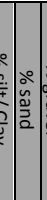 & 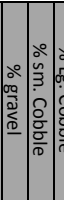 & 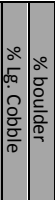 & 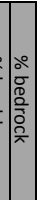 & 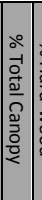 & 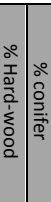 & 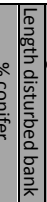 & 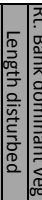 & 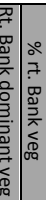 & 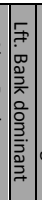 & 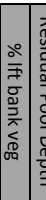 & 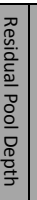 & $\begin{array}{l}\hat{2} \\
3 \\
3 \\
\frac{0}{0} \\
\stackrel{\omega}{\vec{\omega}}\end{array}$ \\
\hline 408 & $\begin{array}{l}4 . \\
2\end{array}$ & $\begin{array}{c}\text { Mid- } \\
\text { Channel } \\
\text { Pool }\end{array}$ & 6364 & 82 & 21 & 1625 & 2521 & $1 \begin{array}{c}1.2 \\
3\end{array}$ & 2.7 & \begin{tabular}{c|c}
0.2 & 10 \\
5 & 10
\end{tabular} & $d$ & 3 & 2515 & 520 & 60 & \begin{tabular}{l|l}
5 & 0
\end{tabular} & \begin{tabular}{l|l}
0 & 0
\end{tabular} & 0 & 0 & 030 & 030 & 3010 & \begin{tabular}{l|l|}
0 & 0 \\
\end{tabular} & 0 & 955 & \begin{tabular}{l|l}
50 & 50
\end{tabular} & 00 & 017 & 735 & 7 & 802 & $\begin{array}{c}2.4 \\
5\end{array}$ & $\begin{array}{c}\text { Long piece of } \\
\text { LWD parallel to } \\
\text { channel, } \\
\text { artificially placed } \\
\text { and cabled into } \\
\text { place to protect } \\
\text { right bank. } \\
\text { Cabins on RB. } \\
\end{array}$ \\
\hline 409 & $\begin{array}{l}3 . \\
2\end{array}$ & Glide & 6267 & $\begin{array}{c}10 \\
3\end{array}$ & 25 & 2125 & 2528 & $\begin{array}{c}8.6 \\
5\end{array}$ & $\begin{array}{c}1.0 \\
5\end{array}$ & & & 3 & $20 \mid 5$ & 515 & 305 & \begin{tabular}{l|l}
50 & 0
\end{tabular} & \begin{tabular}{l|l}
0 & 0
\end{tabular} & 0 & 0 & 030 & 030 & 3010 & \begin{tabular}{l|l|}
0 & 0 \\
\end{tabular} & 0 & 996 & \begin{tabular}{l|l}
60 & 40
\end{tabular} & 100 & 00 & \begin{tabular}{l|l}
6 & 40
\end{tabular} & 6 & 60 & & $\begin{array}{l}\text { Huge redwood } \\
\text { stump on RB, but } \\
\text { not creating a } \\
\text { pool. Another } \\
\text { teathered Doug } \\
\text { Fir. }\end{array}$ \\
\hline 410 & $\begin{array}{l}4 . \\
2\end{array}$ & $\begin{array}{c}\text { Mid- } \\
\text { Channel } \\
\text { Pool } \\
\end{array}$ & 6368 & 40 & 21 & 2822 & 2214 & $4 \mid \begin{array}{c}0.9 \\
3\end{array}$ & 2.4 & 0.120 & c & 3 & 1010 & 030 & $0 \mid 6$ & \begin{tabular}{l|l}
60 & 0
\end{tabular} & \begin{tabular}{l|l}
0 & 0
\end{tabular} & 0 & 0 & 030 & 030 & 305 & \begin{tabular}{l|l|}
5 & 0
\end{tabular} & 0 & 93 & \begin{tabular}{c|c}
10 & 0 \\
0 & 0
\end{tabular} & \begin{tabular}{l|l|}
0 & 0
\end{tabular} & $10 T^{7}$ & 790 & 7 & \begin{tabular}{|l|l|}
10 & 2
\end{tabular} & 2.3 & \\
\hline 411 & $\begin{array}{l}3 . \\
4 \\
\end{array}$ & Step Run & 6367 & $\begin{array}{c}18 \\
7\end{array}$ & 13 & \begin{tabular}{|l|l|}
14 & 10 \\
\end{tabular} & 1015 & \begin{tabular}{|c|}
0.6 \\
7
\end{tabular} & $\begin{array}{c}1.6 \\
5\end{array}$ & & & 3 & 1020 & 020 & 103 & 3020 & 200 & 0 & 0 & \begin{tabular}{l|l}
0 & 10 \\
\end{tabular} & 010 & 25252 & $20 \mid$\begin{tabular}{l|l}
20 \\
\end{tabular} & 00 & 949 & \begin{tabular}{l|l}
95 & 5 \\
\end{tabular} & 50 & 06 & 6 65 & 6 & 95 & & $\begin{array}{l}2 \text { riffles }\left(21^{\prime}, 9^{\prime}\right) 3 \\
\text { glides. Step glide }\end{array}$ \\
\hline 412 & $\begin{array}{c}5 \\
1 \\
\end{array}$ & \begin{tabular}{c|} 
Corner \\
Pool
\end{tabular} & 6468 & 54 & 14 & $15 \mid 13$ & 1313 & \begin{tabular}{|c|}
1.2 \\
5
\end{tabular} & 2.5 & 0.4 & c & 3 & 2030 & 020 & $\left.10\right|^{3}$ & \begin{tabular}{l|l}
35 & 5
\end{tabular} & \begin{tabular}{l|l}
5 & 0 \\
\end{tabular} & 0 & 0 & 025 & 530 & 3010 & \begin{tabular}{|l|l|}
5 & 0 \\
\end{tabular} & 0 & 75 & \begin{tabular}{l|l}
90 & 10
\end{tabular} & 015 & $0 \mid 6$ & \begin{tabular}{l|l}
6 & 40
\end{tabular} & 6 & \begin{tabular}{|l|l|l|}
80 & 2
\end{tabular} & 2.1 & \\
\hline 413 & $\begin{array}{c}1 . \\
1\end{array}$ & \begin{tabular}{|c|} 
Low \\
gradient \\
Riffle
\end{tabular} & & 9 & 8 & 7 & 8 & {$\left[\begin{array}{c}0.2 \\
2\end{array}\right.$} & 0.6 & & & & & & & & & & & & & \begin{tabular}{|l|l|l}
45 & 45 & 1
\end{tabular} & & & & & & & & & & & \\
\hline 414 & $\begin{array}{c}5 . \\
1 \\
\end{array}$ & \begin{tabular}{c|} 
Corner \\
Pool
\end{tabular} & 6372 & 66 & 11 & 811 & 1113 & \begin{tabular}{|c|}
1.3 \\
6
\end{tabular} & $\begin{array}{c}2.7 \\
5\end{array}$ & \begin{tabular}{|l|l|}
0.3 & 25 \\
\end{tabular} & c & 3 & 3010 & 010 & 305 & \begin{tabular}{|l|l}
50 & 0 \\
\end{tabular} & \begin{tabular}{l|l}
0 & 0
\end{tabular} & 0 & 0 & 020 & 020 & \begin{tabular}{l|l|l}
30 & 20 & 1 \\
\end{tabular} & 100 & 0 & \begin{tabular}{|c|c|}
10 \\
0
\end{tabular} & \begin{tabular}{l|l}
98 & 2 \\
\end{tabular} & 20 & 06 & 690 & 6 & 202 & \begin{tabular}{c|}
2.4 \\
5
\end{tabular} & \\
\hline 415 & $\begin{array}{c}1 . \\
1\end{array}$ & \begin{tabular}{|c|} 
Low \\
gradient \\
Riffle
\end{tabular} & 6472 & 4 & 12 & 138 & $8 \mid 16$ & $6 \begin{array}{c}0.2 \\
4\end{array}$ & 0.3 & & & 1 & 0 & 50 & 0 & \begin{tabular}{l|l}
0 & 50
\end{tabular} & 500 & 0 & 0 & \begin{tabular}{l|l}
0 & 0
\end{tabular} & 00 & 30403 & \begin{tabular}{l|l|}
30 & 0 \\
\end{tabular} & 0 & $99 \mid \mathrm{s}$ & \begin{tabular}{l|l}
95 & 5
\end{tabular} & $5 \mid 00$ & $0 \mid 6$ & \begin{tabular}{l|l}
6 & 80
\end{tabular} & 6 & 60 & & \\
\hline 416 & $\begin{array}{l}4 . \\
2\end{array}$ & \begin{tabular}{|c|} 
Mid- \\
Channel \\
Pool \\
\end{tabular} & 6470 & 93 & 14 & $16 \mid 14$ & $14 \mid 11$ & $1 \begin{array}{c}1.2 \\
3\end{array}$ & 2.4 & \begin{tabular}{l|l|l|}
0.2 & 10
\end{tabular} & d & 3 & $10 \mid 0$ & 40 & 06 & \begin{tabular}{l|l}
60 & 0
\end{tabular} & \begin{tabular}{l|l}
0 & 0
\end{tabular} & 0 & 0 & 020 & 020 & \begin{tabular}{|l|l|l|}
30 & 20 & 1
\end{tabular} & 100 & 0 & 99 & \begin{tabular}{l|l}
98 & 2
\end{tabular} & 20 & 56 & \begin{tabular}{l|l}
6 & 50
\end{tabular} & 6 & \begin{tabular}{l|l}
90 & 2.
\end{tabular} & 2.2 & $\begin{array}{c}\text { Combo 4.2 } \\
\text { (rootwad) and } \\
5.1\end{array}$ \\
\hline 417 & $\begin{array}{l}5 . \\
3\end{array}$ & \begin{tabular}{|c|} 
Lateral \\
Scour \\
Pool - \\
Root Wad \\
Enhanced \\
\end{tabular} & 6570 & 54 & 9 & \begin{tabular}{|l|l|l|l|} 
& 11
\end{tabular} & 106 & $\mid \begin{array}{c}0.9 \\
8\end{array}$ & 1.7 & 0.540 & d & 3 & 3030 & 015 & \begin{tabular}{l|l}
0 & 5
\end{tabular} & \begin{tabular}{l|l}
55 & 0
\end{tabular} & \begin{tabular}{l|l}
0 & 0
\end{tabular} & 0 & 0 & \begin{tabular}{l|l}
0 & 25
\end{tabular} & 525 & \begin{tabular}{l|l|l|l|l|l}
30 & 10 & 1
\end{tabular} & 100 & 0 & 99 & \begin{tabular}{l|l}
98 & 2
\end{tabular} & \begin{tabular}{l|l|}
2 & 0
\end{tabular} & 56 & \begin{tabular}{l|l}
6 & 80
\end{tabular} & 6 & \begin{tabular}{l|l}
80 & 1.
\end{tabular} & 1.2 & (4.2?) \\
\hline 418 & $\begin{array}{l}3 . \\
4 \\
\end{array}$ & Step Run & 6568 & \begin{tabular}{c|}
12 \\
2 \\
\end{tabular} & 10 & \begin{tabular}{|l|l|}
6 & 10 \\
\end{tabular} & 1013 & \begin{tabular}{|c|}
0.6 \\
1 \\
\end{tabular} & 1.4 & & & 3 & 520 & $\begin{array}{ll}0 & 15\end{array}$ & 253 & \begin{tabular}{l|l}
35 & 5
\end{tabular} & $5 \mid 0$ & 0 & 0 & \begin{tabular}{l|l}
0 & 5
\end{tabular} & 55 & \begin{tabular}{|l|l|l|}
40 & 30 & 2
\end{tabular} & 200 & 0 & 95 & 2080 & 300 & 06 & \begin{tabular}{l|l|l}
6 & 80
\end{tabular} & 6 & 80 & & $\begin{array}{l}\text { BF taken. } 3 \text { riffles } \\
\left(11^{\prime}, 6^{\prime}, 3^{\prime}\right) 3 \text { runs } \\
\end{array}$ \\
\hline 419 & $\begin{array}{l}4 . \\
2\end{array}$ & \begin{tabular}{|c|} 
Mid- \\
Channel \\
Pool \\
\end{tabular} & 6570 & 34 & 13 & \begin{tabular}{l|l}
13 & 15
\end{tabular} & 1512 & $2 \begin{array}{c}0.8 \\
6\end{array}$ & 1.6 & \begin{tabular}{l|l}
0.1 & 40
\end{tabular} & d & 3 & 300 & 30 & 07 & \begin{tabular}{l|l}
70 & 0
\end{tabular} & \begin{tabular}{l|l}
0 & 0
\end{tabular} & 0 & 0 & \begin{tabular}{l|l|l}
0 & 20
\end{tabular} & 020 & \begin{tabular}{|l|l|}
40 & 20 \\
\end{tabular} & 00 & 0 & 91 & \begin{tabular}{l|l}
95 & 5
\end{tabular} & 50 & 06 & \begin{tabular}{l|l}
6 & 80
\end{tabular} & 6 & \begin{tabular}{|l|l}
90 & 1.
\end{tabular} & 1.5 & \\
\hline 420 & $\begin{array}{l}4 . \\
2\end{array}$ & \begin{tabular}{|c|} 
Mid- \\
Channel \\
Pool
\end{tabular} & 6468 & 55 & 13 & \begin{tabular}{|l|l|}
12 & 17
\end{tabular} & 179 & $\left|\begin{array}{c}0.9 \\
1\end{array}\right|$ & 1.8 & $\begin{array}{lll}0.5 & 15\end{array}$ & c & 3 & \begin{tabular}{l|l}
5 & 25 \\
\end{tabular} & 525 & \begin{tabular}{l|l}
25 & 2
\end{tabular} & \begin{tabular}{l|l}
25 & 0
\end{tabular} & \begin{tabular}{l|l}
0 & 0
\end{tabular} & 0 & 0 & \begin{tabular}{l|l|l}
0 & 20
\end{tabular} & 020 & \begin{tabular}{l|l|l}
30 & 20 & 1
\end{tabular} & 100 & 0 & 959 & \begin{tabular}{l|l}
98 & 2
\end{tabular} & \begin{tabular}{l|l|}
2 & 0
\end{tabular} & 06 & \begin{tabular}{l|l}
6 & 30
\end{tabular} & 6 & \begin{tabular}{|l|l|l}
80 & 1
\end{tabular} & 1.3 & \\
\hline 421 & $\begin{array}{l}4 . \\
2\end{array}$ & \begin{tabular}{|c|} 
Mid- \\
Channel \\
Pool
\end{tabular} & 6566 & 39 & 11 & \begin{tabular}{|l|l}
9 & 11
\end{tabular} & $11 \mid 12$ & $2 \begin{array}{c}1.2 \\
4\end{array}$ & 1.8 & \begin{tabular}{l|l}
0.7 & 0
\end{tabular} & e & 1 & 50 & 50 & 50 & \begin{tabular}{l|l}
0 & 0
\end{tabular} & \begin{tabular}{l|l}
0 & 0
\end{tabular} & 0 & 0 & \begin{tabular}{l|l}
0 & 30
\end{tabular} & 030 & $\begin{array}{lll}30 & 5 \\
\end{array}$ & 50 & 0 & 91 & \begin{tabular}{l|l}
25 & 75
\end{tabular} & \begin{tabular}{ll|l}
5 & 10
\end{tabular} & 08 & \begin{tabular}{l|l|l}
8 & 20
\end{tabular} & 6 & \begin{tabular}{|l|l}
90 & 1
\end{tabular} & 1.1 & \\
\hline 422 & $\begin{array}{c}1 . \\
1\end{array}$ & $\begin{array}{c}\text { Low } \\
\text { gradient } \\
\text { Riffle }\end{array}$ & 6567 & 77 & 11 & 127 & 715 & $5 \begin{array}{c}0.3 \\
4\end{array}$ & 1.2 & & & 3 & 2020 & 020 & \begin{tabular}{|l|l}
0 & 1
\end{tabular} & 1050 & \begin{tabular}{l|l}
50 & 0
\end{tabular} & 0 & 0 & \begin{tabular}{l|l}
0 & 0
\end{tabular} & 0 & 25353 & 355 & 0 & 835 & \begin{tabular}{l|l}
50 & 50
\end{tabular} & \begin{tabular}{l|l}
0 & 0 \\
\end{tabular} & 06 & \begin{tabular}{l|l}
6 & 40
\end{tabular} & 6 & 80 & & End of day $8 / 20$ \\
\hline 423 & $\begin{array}{l}4 . \\
2\end{array}$ & \begin{tabular}{|c|} 
Mid- \\
Channel \\
Pool
\end{tabular} & 6265 & 33 & 14 & $15 \mid 11$ & 1117 & $7 \begin{array}{c}1.3 \\
3\end{array}$ & 2.8 & 0.120 & d & 3 & 1030 & 00 & 203 & $30 \quad 15$ & 150 & 0 & 5 & 520 & 020 & \begin{tabular}{|l|l|l|l|}
30 & 15 & 1
\end{tabular} & 100 & 5 & 915 & 5050 & 00 & $0 \mid 6$ & 695 & 6 & \begin{tabular}{l|l}
95 & 2 \\
\end{tabular} & 2.7 & Start $8 / 21$ \\
\hline 424 & $\begin{array}{l}3 . \\
3 \\
\end{array}$ & Run & 6365 & 69 & 15 & 1712 & 1217 & \begin{tabular}{|c|}
0.6 \\
5 \\
\end{tabular} & 1.2 & & & 3 & 1025 & 525 & 252 & $25 \mid 0$ & \begin{tabular}{l|l}
0 & 0
\end{tabular} & 0 & 0 & 020 & 020 & 20201 & $15 \mid 5$ & 0 & 92 & \begin{tabular}{l|l}
95 & 5
\end{tabular} & 50 & 00 & \begin{tabular}{l|l}
6 & 80
\end{tabular} & 6 & 90 & & Combo $3.3,3.2$ \\
\hline 425 & $\begin{array}{l}4 . \\
2\end{array}$ & \begin{tabular}{|c|} 
Mid- \\
Channel \\
Pool \\
\end{tabular} & 6265 & 55 & 13 & 1716 & $16 \quad 6$ & $\begin{array}{c}1.4 \\
8\end{array}$ & 2.3 & 0.340 & c & 3 & 1015 & 520 & 15 & \begin{tabular}{|l|l}
0 & 0
\end{tabular} & \begin{tabular}{l|l}
0 & 0
\end{tabular} & 0 & 0 & 025 & 525 & 3015 & \begin{tabular}{|l|l|l|}
5 & 0 \\
\end{tabular} & 0 & $99 \mathrm{~s}$ & \begin{tabular}{l|l}
98 & 2
\end{tabular} & \begin{tabular}{l|l|l}
2 & 0
\end{tabular} & $0 \mid 6$ & \begin{tabular}{l|l|l}
6 & 80
\end{tabular} & $7 \mid$ & $10 \mid 2$ & 2 & \\
\hline 426 & $\begin{array}{c}1 . \\
1\end{array}$ & \begin{tabular}{|c|} 
Low \\
gradient \\
Riffle \\
\end{tabular} & & 17 & 5 & \begin{tabular}{|l|l}
6 & 3
\end{tabular} & 3 & $\begin{array}{c}0.2 \\
3\end{array}$ & 0.3 & & & 3 & 7040 & 020 & \begin{tabular}{|l|l}
0 & 4 \\
\end{tabular} & \begin{tabular}{l|l}
40 & 0
\end{tabular} & \begin{tabular}{l|l}
0 & 0
\end{tabular} & 0 & 0 & 0.0 & 00 & 35353 & 300 & 0 & $\mid \begin{array}{c}10 \\
0\end{array}$ & \begin{tabular}{|c|c}
10 & 0 \\
0 & 0
\end{tabular} & \begin{tabular}{l|l|l|}
0 & 0
\end{tabular} & 00 & 650 & 7 & 50 & & \\
\hline 427 & 7 & Dry & 13 & & $\begin{array}{c}\# \# \\
\#\end{array}$ & & & & & & & & & & & & & & & & & & & & & & & & & & & & $\begin{array}{c}\text { Stream } \\
\text { dissappear under } \\
\text { an undercut bank } \\
\text { blocked off by } \\
\text { cobbles and roots }\end{array}$ \\
\hline 428 & $\left|\begin{array}{c}1 . \\
1\end{array}\right|$ & \begin{tabular}{|c|} 
Low \\
gradient \\
Riffle
\end{tabular} & & 9 & 6 & & 47 & $\mid \begin{array}{c}0.1 \\
7\end{array}$ & 0.3 & & & 1 & & 090 & 0 & \begin{tabular}{l|l}
0 & 0
\end{tabular} & \begin{tabular}{l|l}
0 & 0
\end{tabular} & 0 & 0 & \begin{tabular}{l|l}
0 & 0
\end{tabular} & 00 & 35353 & & & & & & & & & & & \\
\hline 429 & $\begin{array}{c}4 . \\
2\end{array}$ & \begin{tabular}{|c|} 
Mid- \\
Channel \\
Pool \\
\end{tabular} & 6268 & 41 & 26 & 2627 & $27 \mid 25$ & $5 \mid \begin{array}{c}2.5 \\
1\end{array}$ & 4.3 & 0.210 & c & 3 & 100 & 10 & \begin{tabular}{|l|l|l}
40 & 4
\end{tabular} & \begin{tabular}{l|l}
40 & 0
\end{tabular} & \begin{tabular}{l|l}
0 & 0
\end{tabular} & 0 & 0 & 020 & 020 & 35151 & 100 & 0 & $\left|\begin{array}{c}10 \\
0\end{array}\right|$ & 955 & 50 & 008 & 860 & 6 & \begin{tabular}{|l|l}
20 & 4
\end{tabular} & 4.1 & \\
\hline 430 & $\begin{array}{l}4 . \\
2\end{array}$ & \begin{tabular}{|c|} 
Mid- \\
Channel \\
Pool \\
\end{tabular} & 6368 & 26 & 20 & 2527 & $27 \mid 9$ & $\begin{array}{c}0.9 \\
4\end{array}$ & 2 & \begin{tabular}{c|c|}
1.1 & 20 \\
1 &
\end{tabular} & $d$ & 3 & 100 & 40 & $50 \mid 1$ & 100 & \begin{tabular}{l|l}
0 & 0
\end{tabular} & 0 & 0 & \begin{tabular}{l|l}
0 & 0
\end{tabular} & 0 & 30303 & \begin{tabular}{l|l|l}
30 & 10
\end{tabular} & 0 & $\left|\begin{array}{c}10 \\
0\end{array}\right|$ & \begin{tabular}{|l|l|}
60 & 40
\end{tabular} & 100 & 06 & \begin{tabular}{l|l}
6 & 75
\end{tabular} & 56 & $200^{0}$ & $\begin{array}{c}0.8 \\
9\end{array}$ & \\
\hline 431 & $\begin{array}{c}3 . \\
4 \\
\end{array}$ & Step Run & 6366 & 44 & 13 & 9213 & 1318 & $8 \begin{array}{c}0.3 \\
1 \\
\end{array}$ & 0.6 & & & 3 & \begin{tabular}{l|l}
5 & 30 \\
\end{tabular} & 040 & \begin{tabular}{|l|l|}
0 & 3 \\
\end{tabular} & \begin{tabular}{l|l}
30 & 0 \\
\end{tabular} & \begin{tabular}{l|l}
0 & 0 \\
\end{tabular} & 0 & 0 & \begin{tabular}{l|l}
0 & 0 \\
\end{tabular} & 0 & \begin{tabular}{|l|l|l|}
40 & 40 & 2 \\
\end{tabular} & 200 & 0 & \begin{tabular}{|l|l|}
98 & 7 \\
\end{tabular} & \begin{tabular}{|l|l|}
75 & 25 \\
\end{tabular} & \begin{tabular}{l|l}
55 & 0 \\
\end{tabular} & \begin{tabular}{|l|l|}
0 & 6 \\
\end{tabular} & \begin{tabular}{l|l}
6 & 75 \\
\end{tabular} & 6 & 75 & & $\begin{array}{c}2 \text { riffles }\left(7^{\prime}, 9^{\prime}\right), 2 \\
\text { runs }\end{array}$ \\
\hline
\end{tabular}




\begin{tabular}{|c|c|c|c|c|c|c|c|c|c|c|c|c|c|c|c|c|c|c|c|c|c|c|c|c|c|c|c|c|c|c|c|c|c|c|c|c|c|}
\hline 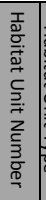 & 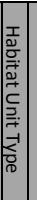 & 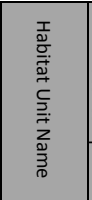 & 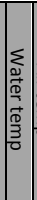 & & 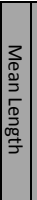 & 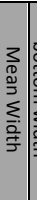 & 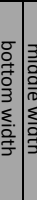 & 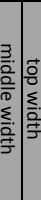 & 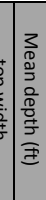 & 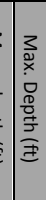 & 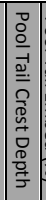 & 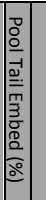 & 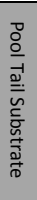 & 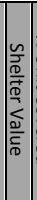 & 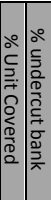 & 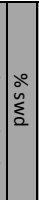 & $\frac{\circ}{\grave{c}}$ & 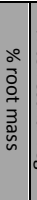 & 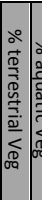 & 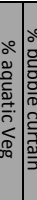 & 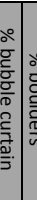 & 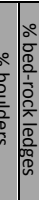 & 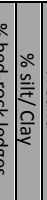 & 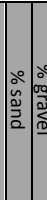 & 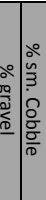 & 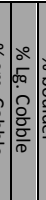 & & 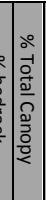 & 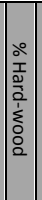 & 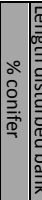 & 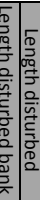 & 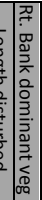 & 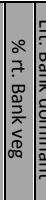 & & 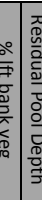 & & 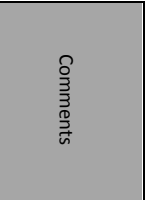 \\
\hline 432 & $\begin{array}{l}4 . \\
2\end{array}$ & \begin{tabular}{|c|} 
Mid- \\
Channel \\
Pool \\
\end{tabular} & 63 & & $\begin{array}{c}12 \\
5\end{array}$ & 19 & & 308 & $8 \begin{array}{c}1.3 \\
4\end{array}$ & $\begin{array}{ll}3 & 2.8 \\
5\end{array}$ & 0.6 & & $d$ & 3 & \begin{tabular}{|l|l|}
50 & 0
\end{tabular} & 20 & 30 & 50 & 0 & 00 & 00 & \begin{tabular}{l|l}
0 & 0
\end{tabular} & 020 & 202 & 2525 & 510 & \begin{tabular}{l|l}
0 & 0
\end{tabular} & 93 & 25 & 75 & \begin{tabular}{l|l}
0 & 0
\end{tabular} & 06 & 70 & 685 & $35 \mid \begin{array}{l}2 . \\
5\end{array}$ & 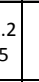 & \\
\hline 433 & $\begin{array}{l}1 . \\
1\end{array}$ & \begin{tabular}{|c|} 
Low \\
gradient \\
Riffle
\end{tabular} & 64 & & 16 & 7 & 877 & $7 \mid 6$ & \begin{tabular}{c|c}
0.2 \\
7
\end{tabular} & $\begin{array}{lll}2 & 0.5\end{array}$ & & & & 3 & 3030 & 0 & $20 \mid 5$ & 50 & 0 & $0 \mid 0$ & 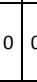 & 0 o 0 & $\begin{array}{lll}0 & 0\end{array}$ & 04 & 4545 & 510 & \begin{tabular}{l|l}
0 & 0
\end{tabular} & 99 & 75 & 25 & \begin{tabular}{l|l}
0 & 0
\end{tabular} & 07 & 40 & \begin{tabular}{|l|l|l}
6 & 80 \\
\end{tabular} & 30 & & \\
\hline 434 & \begin{tabular}{|l|}
3. \\
2
\end{tabular} & Glide & 64 & & 26 & 8 & $6 \mid 1$ & 118 & $\begin{array}{c}0.7 \\
5\end{array}$ & \begin{tabular}{l|c}
7 & 1.1 \\
5 & 5
\end{tabular} & & & & 3 & 1020 & 10 & 0 & 70 & 0 & 00 & 00 & \begin{tabular}{l|l}
0 & 0
\end{tabular} & 030 & 303 & 3010 & 00 & \begin{tabular}{l|l}
0 & 0
\end{tabular} & 99 & 75 & 25 & \begin{tabular}{l|l}
0 & 0
\end{tabular} & 0 & 80 & 67 & 70 & & \\
\hline 435 & $\begin{array}{l}1 . \\
1\end{array}$ & \begin{tabular}{|c|} 
Low \\
gradient \\
Riffle
\end{tabular} & & & 4 & 6 & 5 & 7 & $\begin{array}{c}0.1 \\
8\end{array}$ & 10.2 & & & & 0 & 0 & & & & & & & & 0 & \begin{tabular}{|l|l}
0 & 4
\end{tabular} & $45 \mid 45$ & 510 & & & & & & & & & & & \\
\hline 436 & \begin{tabular}{c|}
3. \\
4
\end{tabular} & Step Run & 63 & & 94 & 9 & 75 & \begin{tabular}{l|l}
5 & 14
\end{tabular} & $\begin{array}{c}0.5 \\
5\end{array}$ & \begin{tabular}{|l|l}
5 & 1.3 \\
\end{tabular} & & & & 3 & 4020 & 20 & 30 & 30 & 0 & 00 & $0 \mid c$ & $\begin{array}{lll}0 & 0\end{array}$ & 010 & 103 & 3535 & 510 & \begin{tabular}{l|l}
0 & 0
\end{tabular} & 99 & 60 & 40 & \begin{tabular}{l|l}
0 & 0
\end{tabular} & 06 & 75 & \begin{tabular}{|l|l|l}
6 & 95
\end{tabular} & 95 & & $\begin{array}{c}3 \text { riffles }\left(2^{\prime}, 8^{\prime},\right. \\
\left.18^{\prime}\right) 3 \text { glides }\end{array}$ \\
\hline 437 & $\begin{array}{l}4 . \\
2\end{array}$ & \begin{tabular}{|c|} 
Mid- \\
Channel \\
Pool \\
\end{tabular} & 64 & & $\begin{array}{c}10 \\
5\end{array}$ & 20 & 141 & 1431 & $1 \begin{array}{c}1.6 \\
8\end{array}$ & $\begin{array}{lll}6 & 3.6\end{array}$ & 0.2 & 10 & $d$ & 3 & 1515 & 15 & 35 & 35 & 0 & 00 & \begin{tabular}{l|l}
0 & 0 \\
\end{tabular} & \begin{tabular}{l|l}
0 & 0
\end{tabular} & 025 & 252 & 2520 & 05 & 0 & 96 & 75 & 25 & \begin{tabular}{l|l}
0 & 0
\end{tabular} & 06 & 75 & 790 & 903. & \begin{tabular}{l|l}
$.4 \mathrm{E}$ & $\mathrm{E}-\mathrm{C}$
\end{tabular} & End of day $8 / 21$ \\
\hline 438 & $\begin{array}{l}4 . \\
2\end{array}$ & \begin{tabular}{|c|} 
Mid- \\
Channel \\
Pool \\
\end{tabular} & 63 & & 56 & 13 & $13 \mid 1$ & $18 \mid 7$ & \begin{tabular}{l|l}
7 & 1
\end{tabular} & 1.9 & 0 & 20 & $d$ & 2 & \begin{tabular}{|l|l}
5 & 40
\end{tabular} & 10 & 0 & 50 & 0 & \begin{tabular}{l|l}
0 & 0 \\
2
\end{tabular} & $0 \mid c$ & 0 & 025 & $25 \mid 3$ & $30 \mid 10$ & 010 & \begin{tabular}{l|l}
0 & 0
\end{tabular} & 96 & 20 & 80 & \begin{tabular}{l|l}
0 & 0
\end{tabular} & 06 & 85 & \begin{tabular}{|l|l}
8 & 75 \\
\end{tabular} & 75 & & \\
\hline 439 & \begin{tabular}{|l|}
3. \\
3 \\
\end{tabular} & Run & 63 & & 84 & 7 & 76 & \begin{tabular}{l|l}
6 & 7
\end{tabular} & $\begin{array}{c}0.5 \\
3\end{array}$ & \begin{tabular}{c|c}
5 & 0.7 \\
5
\end{tabular} & & & & 3 & 1030 & 10 & 0 & 30 & 30 & 0.0 & $0 \mid c$ & \begin{tabular}{l|l}
0 & 0
\end{tabular} & $0 \mid 10$ & 103 & 3030 & 020 & \begin{tabular}{l|l}
0 & 0
\end{tabular} & 94 & 0 & \begin{tabular}{|c|c|}
10 & $c$ \\
0 & \\
\end{tabular} & \begin{tabular}{l|l}
0 & 0
\end{tabular} & 06 & 90 & \begin{tabular}{|l|l}
6 & 95 \\
\end{tabular} & 95 & & \\
\hline 440 & \begin{tabular}{|c|}
1. \\
1
\end{tabular} & \begin{tabular}{|c|} 
Low \\
gradient \\
Riffle
\end{tabular} & 63 & & 22 & 8 & 76 & $6 \mid 10$ & \begin{tabular}{l|c}
0 & 0.1 \\
9
\end{tabular} & 10.3 & & & & 0 & \begin{tabular}{|l|l|}
0 & 0
\end{tabular} & 0 & 0 & 0 & 0 & 00 & 00 & 0 & $\begin{array}{lll}0 & 0\end{array}$ & 02 & 2545 & 530 & \begin{tabular}{l|l}
0 & 0
\end{tabular} & 86 & 20 & 80 & \begin{tabular}{l|l}
0 & 0
\end{tabular} & 08 & 80 & \begin{tabular}{|l|l|l}
6 & 90 \\
\end{tabular} & 90 & & \\
\hline 441 & $\begin{array}{l}4 . \\
2\end{array}$ & \begin{tabular}{|c|} 
Mid- \\
Channel \\
Pool \\
\end{tabular} & 63 & 71 & 88 & 14 & 101 & 1715 & \begin{tabular}{l|l}
5 & 1.0 \\
4
\end{tabular} & \begin{tabular}{l|l}
0.1 \\
0.1
\end{tabular} & 0.3 & 60 & $d$ & 3 & 1010 & 5 & 30 & 40 & 0 & 00 & 00 & $\begin{array}{lll}0 & 15\end{array}$ & 525 & 253 & 3020 & 020 & \begin{tabular}{l|l}
0 & 0
\end{tabular} & 86 & 10 & 901 & 100 & 06 & 80 & \begin{tabular}{|l|l}
6 & 90
\end{tabular} & \begin{tabular}{l|l}
90 & 1.
\end{tabular} & .8 & $\begin{array}{c}\text { Combo } 5.1 \text { and } \\
4.2\end{array}$ \\
\hline 442 & $\begin{array}{l}4 . \\
2\end{array}$ & \begin{tabular}{|c|} 
Mid- \\
Channel \\
Pool \\
\end{tabular} & 63 & 70 & 36 & 13 & 151 & 187 & $\begin{array}{c}0.8 \\
8\end{array}$ & \begin{tabular}{l|l}
8 & 1.7
\end{tabular} & 0.5 & 40 & $d$ & 3 & \begin{tabular}{l|l|}
60 & 5
\end{tabular} & $10 \mid$ & $20 \mid \epsilon$ & 65 & 0 & $0 \mid 0$ & $0 \mid 0$ & 0 & 020 & 203 & 3020 & 010 & \begin{tabular}{l|l}
0 & 0
\end{tabular} & $\mid \begin{array}{c}10 \\
0\end{array}$ & 60 & 40 & \begin{tabular}{l|l}
0 & 0
\end{tabular} & 06 & 70 & \begin{tabular}{|l|l|l}
6 & 90 \\
\end{tabular} & \begin{tabular}{l|l}
90 & 1.
\end{tabular} & .2 & $\begin{array}{l}\text { Root wad } \\
\text { enhanced }\end{array}$ \\
\hline $\begin{array}{c}442 . \\
1\end{array}$ & 6. & $\left|\begin{array}{c}\text { Secondar } \\
\text { y Channel } \\
\text { Pool }\end{array}\right|$ & 63 & 72 & 13 & 9 & & 9 & $\begin{array}{c}1.0 \\
3\end{array}$ & $\begin{array}{lll}0 & 1.6\end{array}$ & 0.1 & & $\mathrm{e}$ & 3 & \begin{tabular}{l|l}
10 & 0 \\
1 &
\end{tabular} & 0 & 50 & 50 & 0 & 00 & 00 & \begin{tabular}{l|l}
0 & 0
\end{tabular} & 015 & 20 & 2520 & 020 & 0 & $\mid \begin{array}{c}10 \\
0\end{array}$ & 20 & 80 & 0.0 & 0 & & & & .5 & $\begin{array}{l}\text { Dry upstream, } \\
\text { connects at } \\
\text { bottom but no } \\
\text { fish passage }\end{array}$ \\
\hline 443 & \begin{tabular}{|c|}
3 \\
4
\end{tabular} & Step Run & 64 & & 30 & 6 & 75 & \begin{tabular}{l|l}
5 & 7
\end{tabular} & $\begin{array}{c}0.4 \\
4\end{array}$ & \begin{tabular}{c|c}
4 & 0.8 \\
5
\end{tabular} & & & & 2 & \begin{tabular}{l|l}
10 & 0
\end{tabular} & 50 & 05 & 50 & 0 & 00 & $0 \mid c$ & $\begin{array}{lll}0 & 0\end{array}$ & \begin{tabular}{lll|}
0 & 10
\end{tabular} & 103 & 3535 & $\begin{array}{lll}5 & 10 \\
\end{array}$ & 00 & $\begin{array}{c}10 \\
0\end{array}$ & 60 & 40 & \begin{tabular}{l|l}
0 & 0
\end{tabular} & 06 & 90 & 690 & 90 & & 1 riffle $\left(6^{\prime}\right) 1$ run \\
\hline 444 & $\begin{array}{l}4 . \\
2\end{array}$ & \begin{tabular}{|c|} 
Mid- \\
Channel \\
Pool \\
\end{tabular} & 64 & 72 & 49 & 14 & 72 & 278 & $\begin{array}{c}1.8 \\
5\end{array}$ & 83.2 & 0.1 & 40 & $d$ & 3 & 5010 & 20 & $40 \mid=$ & 30 & 0 & 00 & 00 & $\begin{array}{lll}0 & 0\end{array}$ & 015 & 153 & 3525 & $5 \mid 10$ & 0 & {$\left[\begin{array}{c}10 \\
0\end{array}\right.$} & 60 & 40 & $0 \mid 0$ & 06 & 95 & \begin{tabular}{|l|l|l}
6 & 90
\end{tabular} & 903. & 11 & $\begin{array}{c}\text { Log jam, } 35^{\prime} \mathrm{w} x \\
8.5^{\prime} \mathrm{h} \times 17^{\prime} \mathrm{l}\end{array}$ \\
\hline 445 & $\begin{array}{l}3 . \\
4\end{array}$ & Step Run & 64 & 78 & 21 & 10 & \begin{tabular}{l|l}
8 & 1
\end{tabular} & $11 \mid 10$ & $\begin{array}{c}0.3 \\
5\end{array}$ & $\begin{array}{ll}3 & 0.6 \\
\end{array}$ & & & & 1 & \begin{tabular}{|l|l}
5 & 0
\end{tabular} & 10 & 90 & 0 & 0 & 00 & 00 & \begin{tabular}{l|l}
0 & 0
\end{tabular} & 05 & 52 & & 530 & \begin{tabular}{l|l}
5 & 0
\end{tabular} & 87 & 50 & 50 & 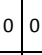 & 06 & 90 & 695 & 95 & & 1 riffle $\left(3^{\prime}\right) 1$ run \\
\hline 446 & $\begin{array}{l}4 . \\
2\end{array}$ & $\begin{array}{c}\text { Mid- } \\
\text { Channel } \\
\text { Pool }\end{array}$ & 64 & & 19 & 9 & 101 & 117 & $\begin{array}{c}1.1 \\
5\end{array}$ & 11.9 & 0.6 & & c & 3 & \begin{tabular}{|l|l}
5 & 40
\end{tabular} & 20 & 40 & 0 & 0 & $0 \mid 0$ & $0 \mid c$ & \begin{tabular}{l|l}
0 & 0
\end{tabular} & \begin{tabular}{lll|}
0 & 15
\end{tabular} & 153 & 3030 & $\begin{array}{lll}0 & 10 \\
\end{array}$ & 0 & {$\left[\begin{array}{c}10 \\
0\end{array}\right.$} & 40 & 60 & \begin{tabular}{l|l}
0 & 0
\end{tabular} & 06 & 90 & \begin{tabular}{|l|l}
6 & 95
\end{tabular} & \begin{tabular}{l|l}
95 & 1.
\end{tabular} & .3 & \\
\hline 447 & \begin{tabular}{l|}
1. \\
1
\end{tabular} & \begin{tabular}{|c|} 
Low \\
gradient \\
Riffle
\end{tabular} & 65 & 74 & 56 & 19 & 72 & 2524 & \begin{tabular}{l|c}
4 & 0.2 \\
4
\end{tabular} & \begin{tabular}{l|l}
2 & 0.4
\end{tabular} & & & & 3 & 1030 & 20 & 10 & 40 & 0 & 0.0 & 00 & \begin{tabular}{l|l}
0 & 0
\end{tabular} & $\begin{array}{lll}0 & 0\end{array}$ & 03 & 3030 & 030 & 100 & 99 & 30 & 70 & 0 & 08 & 80 & \begin{tabular}{|l|l|}
8 & 45
\end{tabular} & 45 & & \\
\hline 448 & \begin{tabular}{|l|}
3. \\
2
\end{tabular} & Glide & 65 & & 39 & 172 & 241 & 1216 & $\begin{array}{c}6 \\
6 \\
5\end{array}$ & \begin{tabular}{|l|l}
5 & 0.9 \\
\end{tabular} & & & & 1 & \begin{tabular}{|l|l}
5 & 25
\end{tabular} & 25 & 0 & 25 & 0 & 00 & $\begin{array}{lll}0 & 2\end{array}$ & \begin{tabular}{l|l}
25 & 0
\end{tabular} & $\begin{array}{lll}0 & 5\end{array}$ & 54 & 4530 & \begin{tabular}{l|l|}
0 & 10 \\
\end{tabular} & \begin{tabular}{l|l}
5 & 0
\end{tabular} & 97 & 70 & 30 & 0 & 0 & 90 & \begin{tabular}{l|l}
8 & 5
\end{tabular} & 5 & & \\
\hline 449 & \begin{tabular}{|l|}
5. \\
1
\end{tabular} & \begin{tabular}{c|} 
Corner \\
Pool
\end{tabular} & 64 & & 83 & 16 & \begin{tabular}{l|l}
16 & 2
\end{tabular} & 2211 & $\begin{array}{c}1.2 \\
5\end{array}$ & \begin{tabular}{|l|l}
2 & 3.3 \\
\end{tabular} & $\begin{array}{c}0.6 \\
5\end{array}$ & & c & 3 & $\begin{array}{lll}10 & 10\end{array}$ & 35 & 35 & 5 & 0 & 00 & 0 & \begin{tabular}{l|l}
0 & 15
\end{tabular} & 510 & 104 & \begin{tabular}{l|l}
40 & 5
\end{tabular} & 51 & 1020 & 092 & 20 & \begin{tabular}{l|l|l}
80 & 1
\end{tabular} & 150 & 06 & 90 & $\begin{array}{lll}6 & 5\end{array}$ & \begin{tabular}{l|r}
2. \\
5
\end{tabular} & $\begin{array}{l}.6 \\
5\end{array}$ & \\
\hline 450 & \begin{tabular}{|l|}
1. \\
1
\end{tabular} & \begin{tabular}{|c|} 
Low \\
gradient \\
Riffle
\end{tabular} & 65 & & $\begin{array}{c}10 \\
5\end{array}$ & 12 & 119 & \begin{tabular}{l|l}
9 & 16
\end{tabular} & $\begin{array}{l}6 \\
0.2 \\
2\end{array}$ & $\begin{array}{lll}2 & 0.4\end{array}$ & & & & 3 & \begin{tabular}{|l|l}
5 & 25
\end{tabular} & 25 & 0 & 25 & 25 & 0.0 & $\begin{array}{lll}0 & 0\end{array}$ & \begin{tabular}{l|l}
0 & 0
\end{tabular} & $\begin{array}{lll}0 & 0\end{array}$ & 03 & 3030 & 030 & 100 & $\mid \begin{array}{c}10 \\
0\end{array}$ & 25 & 75 & \begin{tabular}{l|l}
0 & 0
\end{tabular} & $\begin{array}{lll}0 & 6\end{array}$ & 90 & \begin{tabular}{|l|l}
8 & 20
\end{tabular} & 20 & & \\
\hline 451 & $\begin{array}{l}4 . \\
2\end{array}$ & \begin{tabular}{|c|} 
Mid- \\
Channel \\
Pool
\end{tabular} & 65 & 77 & 98 & 17 & 161 & 17 & $\begin{array}{c}0.8 \\
5\end{array}$ & \begin{tabular}{l|l}
8 & 1.9
\end{tabular} & 0.2 & & c & 3 & 1020 & 30 & 30 & 10 & 0 & \begin{tabular}{l|l}
0 & 0
\end{tabular} & 0 & \begin{tabular}{l|l}
0 & 15
\end{tabular} & 515 & 252 & 2515 & $\begin{array}{ll}5 & 5\end{array}$ & \begin{tabular}{l|l}
0 & 0
\end{tabular} & 99 & 80 & 20 & \begin{tabular}{l|l}
0 & 0
\end{tabular} & 06 & 95 & \begin{tabular}{|l|l|}
6 & 90
\end{tabular} & \begin{tabular}{l|l}
90 & 1.
\end{tabular} & .7 & \\
\hline 452 & $\begin{array}{l}1 . \\
1\end{array}$ & \begin{tabular}{|c|} 
Low \\
gradient \\
Riffle
\end{tabular} & 65 & & 15 & 8 & 5 & \begin{tabular}{l|l}
9 & 10
\end{tabular} & \begin{tabular}{l|c}
0.3 \\
4
\end{tabular} & \begin{tabular}{c|c}
3 & 0.4 \\
5
\end{tabular} & & & & 2 & \begin{tabular}{l|l}
20 & 0
\end{tabular} & 50 & 0 & 0 & 20 & 0.0 & \begin{tabular}{l|l}
0 & 3
\end{tabular} & \begin{tabular}{l|l}
30 & 0
\end{tabular} & \begin{tabular}{l|l|l}
0 & 0
\end{tabular} & \begin{tabular}{|l|l|l|}
0 & 2
\end{tabular} & $20 \mid 40$ & $\begin{array}{lll}0 & 35\end{array}$ & \begin{tabular}{l|l}
5 & 0
\end{tabular} & 94 & 10 & 90 & \begin{tabular}{l|l}
0 & 0
\end{tabular} & $\begin{array}{lll}0 & 6\end{array}$ & 80 & \begin{tabular}{|l|l|l}
6 & 70
\end{tabular} & 70 & & \\
\hline 453 & $\begin{array}{l}4 . \\
2\end{array}$ & \begin{tabular}{|c|} 
Mid- \\
Channel \\
Pool \\
\end{tabular} & 65 & 77 & 78 & 15 & 102 & $23 \mid 12$ & \begin{tabular}{l|l}
1.8 \\
5
\end{tabular} & 85.1 & 0.5 & & & 3 & \begin{tabular}{|l|l|l}
10 & 10
\end{tabular} & 20 & 35 & 30 & 5 & \begin{tabular}{l|l}
0 & 0
\end{tabular} & \begin{tabular}{l|l}
0 & 0
\end{tabular} & \begin{tabular}{l|l}
0 & 0
\end{tabular} & \begin{tabular}{lll|}
0 & 25
\end{tabular} & 252 & 2520 & 0 & 0 & 90 & 10 & 90 & \begin{tabular}{l|l}
0 & 0
\end{tabular} & 06 & 90 & \begin{tabular}{l|l}
6 & 80
\end{tabular} & $\begin{array}{lll}30 & 4 .\end{array}$ & 6 & \\
\hline 454 & \begin{tabular}{|l|}
5. \\
1 \\
\end{tabular} & \begin{tabular}{|c|} 
Corner \\
Pool
\end{tabular} & 65 & 74 & 77 & 10 & 121 & 118 & $\begin{array}{c}1.1 \\
2\end{array}$ & \begin{tabular}{l|l}
1 & 2.3 \\
\end{tabular} & 0.5 & & c & 3 & 2030 & 0 & 20 & 50 & 0 & 00 & $\begin{array}{lll}0 & 0\end{array}$ & $\begin{array}{lll}0 & 0 \\
\end{array}$ & 020 & 202 & 2020 & $\begin{array}{lll}0 & 10 \\
\end{array}$ & \begin{tabular}{l|l}
0 & 10
\end{tabular} & 098 & 20 & 80 & \begin{tabular}{l|l}
0 & 0
\end{tabular} & 0 & 90 & \begin{tabular}{l|l}
6 & 90
\end{tabular} & $\begin{array}{lll}90 & 1 .\end{array}$ & .8 & \\
\hline 455 & $\begin{array}{l}1 . \\
1\end{array}$ & \begin{tabular}{|c|} 
Low \\
gradient \\
Riffle \\
\end{tabular} & 65 & & 33 & 18 & $17 \mid 1$ & $\left.19\right|_{17}$ & \begin{tabular}{l|l}
.7 & 0.3 \\
7
\end{tabular} & $\begin{array}{lll}3 & 0.6\end{array}$ & & & & 1 & $<5 \mid 20$ & 50 & 0 & 20 & 10 & \begin{tabular}{l|l}
0 & 0
\end{tabular} & \begin{tabular}{l|l}
0 & 0
\end{tabular} & \begin{tabular}{l|l}
0 & 0
\end{tabular} & \begin{tabular}{l|l|l}
0 & 0
\end{tabular} & 03 & 3030 & 0 & 100 & 94 & 0 & $\begin{array}{c}10 \\
0\end{array}$ & \begin{tabular}{l|l}
0 & 0
\end{tabular} & 06 & 95 & \begin{tabular}{|l|l|l|}
60 & 90
\end{tabular} & 90 & & $\begin{array}{c}\text { rail crosses. End } \\
\text { of day } 8 / 26\end{array}$ \\
\hline 456 & \begin{tabular}{|c|}
5. \\
1
\end{tabular} & \begin{tabular}{|c|} 
Corner \\
Pool
\end{tabular} & 50 & 60 & \begin{tabular}{|c|}
13 \\
4 \\
\end{tabular} & 14 & 171 & $13 \mid 11$ & $1 \begin{array}{c}1.1 \\
8\end{array}$ & $\begin{array}{cc}1 & 2.7 \\
3\end{array}$ & $\begin{array}{c}0.3 \\
5\end{array}$ & 40 & $d$ & 3 & 1020 & 10 & 20 & 30 & 10 & 0.0 & $0 \mid c$ & \begin{tabular}{l|l}
0 & 10
\end{tabular} & .025 & 252 & \begin{tabular}{l|l}
25 & 0
\end{tabular} & 10 & \begin{tabular}{l|l}
5 & 11
\end{tabular} & 079 & 10 & 90 & \begin{tabular}{l|l}
0 & 0
\end{tabular} & 06 & 90 & \begin{tabular}{l|l}
6 & 95
\end{tabular} & $95 \begin{array}{r}2 . \\
8\end{array}$ & 8 & $\begin{array}{c}\text { Start } 8 / 27 . \text { BF } \\
\text { taken }\end{array}$ \\
\hline 457 & \begin{tabular}{|l|}
3. \\
3 \\
\end{tabular} & Run & 60 & 60 & 15 & 10 & 118 & \begin{tabular}{l|l}
8 & 10 \\
\end{tabular} & $\begin{array}{c}0.2 \\
2\end{array}$ & \begin{tabular}{|c|c}
2 & 0.3 \\
& 8
\end{tabular} & & & & 1 & \begin{tabular}{l|l}
10 & 0
\end{tabular} & 0 & 0 & 0 & \begin{tabular}{|c|c|}
10 & \\
0 & \\
\end{tabular} & 00 & 0 & \begin{tabular}{l|l}
0 & 0
\end{tabular} & \begin{tabular}{ll|l|}
0 & 10 \\
\end{tabular} & 103 & 3030 & $\begin{array}{lll}0 & 20 \\
\end{array}$ & \begin{tabular}{l|l}
0 & 0
\end{tabular} & 89 & 10 & 90 & \begin{tabular}{l|l}
0 & 0
\end{tabular} & 06 & 90 & \begin{tabular}{|l|l}
6 & 95
\end{tabular} & 95 & & \\
\hline 458 & \begin{tabular}{|l|}
5. \\
1
\end{tabular} & \begin{tabular}{|c|} 
Corner \\
Pool
\end{tabular} & 61 & 60 & 90 & 7 & 109 & \begin{tabular}{l|l}
9 & 3 \\
\end{tabular} & $\begin{array}{c}1.1 \\
2\end{array}$ & $\begin{array}{c}1 \\
1 \\
2.0 \\
\end{array}$ & $\begin{array}{c}0.2 \\
9\end{array}$ & 50 & C & 1 & \begin{tabular}{|l|l|l}
5 & 50
\end{tabular} & 0 & 0 & 50 & 0 & 0.0 & 0 & \begin{tabular}{l|l}
0 & 0
\end{tabular} & $\begin{array}{lll}0 & 20\end{array}$ & 202 & 2015 & $\begin{array}{llll}5 & 10 \\
\end{array}$ & \begin{tabular}{l|l|l}
5 & 10
\end{tabular} & 092 & 10 & 90 & \begin{tabular}{l|l}
0 & 0
\end{tabular} & 06 & 80 & 685 & \begin{tabular}{l|r}
35 & 1. \\
6
\end{tabular} & $\begin{array}{l}.7 \\
6 \\
\end{array}$ & \\
\hline
\end{tabular}




\begin{tabular}{|c|c|c|c|c|c|c|c|c|c|c|c|c|c|c|c|c|c|c|c|c|c|c|c|c|c|c|c|c|c|c|c|c|c|c|c|}
\hline 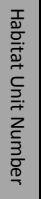 & & 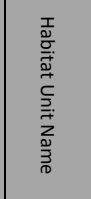 & 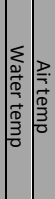 & 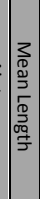 & 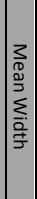 & 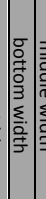 & & 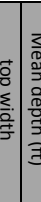 & 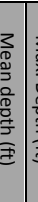 & 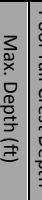 & 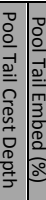 & 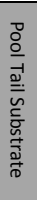 & 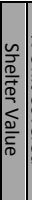 & 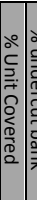 & 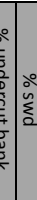 & $\frac{2}{\sum_{0}}$ & 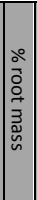 & 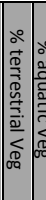 & 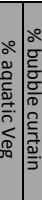 & 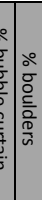 & 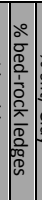 & & 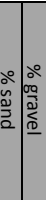 & 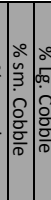 & 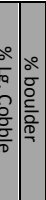 & 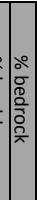 & 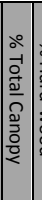 & 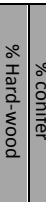 & 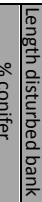 & 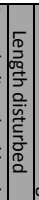 & 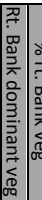 & 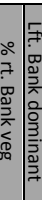 & 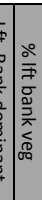 & 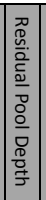 & 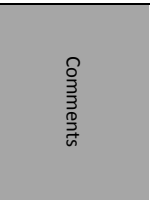 \\
\hline 459 & $\begin{array}{l}1 . \\
1\end{array}$ & $\begin{array}{c}\text { Low } \\
\text { gradient } \\
\text { Riffle } \\
\end{array}$ & 6061 & 122 & 4 & 3 & 4 & $4 \mid \begin{array}{l}0 . \\
1\end{array}$ & \begin{tabular}{l|l}
0.2 & 0 \\
1 &
\end{tabular} & 0.4 & & & 2 & 10 & 0 & 30 & 10 & 60 & $0 \mid 0$ & 0 & 0 & $5 \mid 5$ & 525 & 5530 & 300 & 0 & 93 & 208 & 300 & 0 & $\begin{array}{lll}6 & 5\end{array}$ & $50 \mid 6$ & 670 & & \\
\hline 460 & $\begin{array}{l}4 . \\
2\end{array}$ & $\begin{array}{c}\text { Mid- } \\
\text { Channel } \\
\text { Pool } \\
\end{array}$ & 6163 & 43 & 10 & 4 & $17 \mid$ & $8{ }^{1 .}$ & \begin{tabular}{l|l}
1.0 & 1 \\
6 &
\end{tabular} & \begin{tabular}{c|c}
1.9 & 0 \\
4 &
\end{tabular} & \begin{tabular}{c|c}
0.2 & 40 \\
7 &
\end{tabular} & $d$ & 3 & $\begin{array}{lll}5 & 3 \\
\end{array}$ & 010 & 0 & 30 & 30 & \begin{tabular}{l|l}
0 & 0
\end{tabular} & 0 & 0 & 252 & 2530 & $15 \mid 5$ & 50 & 0 & 935 & 505 & 500 & 0 & \begin{tabular}{|l|l}
6 & 6
\end{tabular} & $65 \mid 6$ & 660 & $\begin{array}{c}1.6 \\
7\end{array} \mid$ & \\
\hline 461 & $\begin{array}{l}1 . \\
1\end{array}$ & \begin{tabular}{|c|} 
Low \\
gradient \\
Riffle \\
\end{tabular} & 6166 & $6 \quad 21$ & 9 & 8 & \begin{tabular}{|l|l}
5 & 1
\end{tabular} & 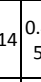 & \begin{tabular}{c|c}
0.4 & 0 \\
5 & 0
\end{tabular} & 0.7 & & & 3 & 5 & 020 & 10 & 30 & 40 & \begin{tabular}{l|l}
0 & 0
\end{tabular} & 0 & 0 & $5 \mid 5$ & 530 & 3030 & 300 & 0 & 895 & \begin{tabular}{l|l}
50 & $5 C$
\end{tabular} & 500 & 0 & $\begin{array}{lll}6 & 5\end{array}$ & $50 \mid 6$ & 590 & & \\
\hline 462 & $\begin{array}{l}4 . \\
2\end{array}$ & $\begin{array}{c}\text { Mid- } \\
\text { Channel } \\
\text { Pool } \\
\end{array}$ & 6369 & 943 & 10 & 141 & 10 & 52. & \begin{tabular}{l|l}
2.1 & 3 \\
4 &
\end{tabular} & \begin{tabular}{c|c}
3.0 & 0 \\
8 &
\end{tabular} & $\begin{array}{c}0.1 \\
6\end{array}$ & & 3 & 601 & 020 & 40 & 30 & $0 \mid 0$ & \begin{tabular}{l|l}
0 & 0
\end{tabular} & 0 & 0 & 252 & 2525 & $15 \mid 1 C$ & 100 & 0 & 88 & 505 & 500 & 0 & $\begin{array}{lll}6 & 8\end{array}$ & $80 \mid 6$ & 675 & $\begin{array}{c}2.9 \\
2\end{array} \mid$ & SDA left bank \\
\hline 463 & $\begin{array}{l}1 . \\
1\end{array}$ & \begin{tabular}{|c|} 
Low \\
gradient \\
Riffle
\end{tabular} & 6369 & 932 & 12 & \begin{tabular}{l|l}
5 & 1
\end{tabular} & 102 & $200_{2}^{0}$ & \begin{tabular}{l|l}
0.2 & 0 \\
2 &
\end{tabular} & $\begin{array}{c}0.4 \\
4\end{array}$ & & & 1 & 5 & $5 \quad 10$ & 0 & 0 & 85 & $0 \mid 0$ & 0 & 0 & $5 \mid 5$ & 525 & 3030 & 305 & 0 & 906 & \begin{tabular}{l|l}
60 & $4 C$
\end{tabular} & 400 & 0 & $\begin{array}{lll}6 & 7\end{array}$ & $75 \mid 6$ & 660 & & \\
\hline 464 & \begin{tabular}{|l|}
3. \\
3
\end{tabular} & Run & 6268 & 825 & 14 & 4201 & 121 & $11 \mid \begin{array}{c}0 . \\
6\end{array}$ & \begin{tabular}{l|l}
.6 & 1 \\
6 & 1 \\
\end{tabular} & 1.3 & & & 2 & \begin{tabular}{|l|l}
5 & 3 \\
\end{tabular} & 50 & 0 & 35 & 30 & \begin{tabular}{l|l}
0 & 0
\end{tabular} & 0 & 0 & 202 & 2025 & $5251 C$ & 100 & 0 & 96 & $406 \mathrm{C}$ & $50 \mid 0$ & 0 & $\begin{array}{ll}6 & 5 \\
\end{array}$ & \begin{tabular}{l|l}
50 & 6
\end{tabular} & 680 & & \\
\hline 465 & $\begin{array}{l}4 . \\
2\end{array}$ & $\begin{array}{c}\text { Mid- } \\
\text { Channel } \\
\text { Pool } \\
\end{array}$ & 6269 & 967 & 13 & \begin{tabular}{l|l|l}
3 & 11 & 1
\end{tabular} & $17 \mid 1$ & $\left.12\right|_{8} ^{1 .}$ & $\begin{array}{c}1.3 \\
8\end{array}$ & \begin{tabular}{l|l}
2.2 & 0 \\
4 &
\end{tabular} & \begin{tabular}{c|c}
0.2 & 20 \\
1 &
\end{tabular} & d & 3 & & $0 \mid 10$ & 45 & 35 & \begin{tabular}{l|l}
0 & 0 \\
0
\end{tabular} & \begin{tabular}{l|l}
0 & 0
\end{tabular} & 0 & 0 & $20 \mid 2$ & 2020 & 2015 & 155 & 0 & 95 & $109 c$ & \begin{tabular}{l|l}
90 & 0
\end{tabular} & 0 & $\begin{array}{lll}8 & 7\end{array}$ & $70 \mid 6$ & 665 & $\begin{array}{c}2.0 \\
3\end{array} \mid$ & \\
\hline 466 & \begin{tabular}{|l|}
3. \\
4 \\
\end{tabular} & Step Run & $\begin{array}{ll}62 & 72 \\
\end{array}$ & \begin{tabular}{|c|}
11 \\
7 \\
\end{tabular} & 11 & 12 & \begin{tabular}{|l|l|}
7 & 1 \\
\end{tabular} & $14 \begin{array}{l}0 . \\
4\end{array}$ & \begin{tabular}{l|l}
.3 & 1 \\
4 & \\
\end{tabular} & $\begin{array}{l}1.0 \\
3 \\
\end{array}$ & & & 3 & 101 & 015 & 50 & 15 & 00 & \begin{tabular}{l|l}
0 & 0
\end{tabular} & 0 & 0 & 55 & 530 & 3030 & 300 & 0 & 97 & 406 & 500 & 0 & $\begin{array}{lll}6 & 8\end{array}$ & $80 \mid 6$ & 670 & & $\begin{array}{c}3 \text { riffles }\left(6^{\prime}, 7^{\prime},\right. \\
\left.44^{\prime}\right) 2 \text { runs }\end{array}$ \\
\hline 467 & $\begin{array}{l}4 . \\
2\end{array}$ & \begin{tabular}{|c|} 
Mid- \\
Channel \\
Pool
\end{tabular} & \begin{tabular}{|l|l|}
62 & 72 \\
\end{tabular} & $2 \begin{array}{c}15 \\
0\end{array}$ & 19 & 14 & 242 & 202. & \begin{tabular}{l|l}
2.0 & 4 \\
5 &
\end{tabular} & \begin{tabular}{c|c}
4.0 & 0 \\
8 &
\end{tabular} & \begin{tabular}{c|c}
0.2 & 20 \\
1 &
\end{tabular} & d & 2 & $\begin{array}{lll}5 & 3\end{array}$ & 00 & 10 & 30 & \begin{tabular}{l|l}
0 & 0 \\
0
\end{tabular} & \begin{tabular}{l|l}
0 & 0
\end{tabular} & 0 & 30 & 252 & 2525 & 55 & 50 & 15 & $\mid \begin{array}{c}10 \\
0\end{array}$ & 2080 & 300 & 0 & $8 \mid 8$ & $80 \mid 7$ & 710 & $\begin{array}{c}3.8 \\
7\end{array} \mid$ & \\
\hline 468 & $\begin{array}{l}1 . \\
1\end{array}$ & \begin{tabular}{|c|} 
Low \\
gradient \\
Riffle
\end{tabular} & 63 & 27 & 18 & $8|20| 1$ & $18 \mid 1$ & $\left.15\right|_{0 .} ^{0} 9$ & \begin{tabular}{l|l}
0.3 & 0 \\
9 &
\end{tabular} & $\begin{array}{c}0.4 \\
7\end{array}$ & & & 2 & \begin{tabular}{|l|l}
5 & 1
\end{tabular} & 020 & 0 & 10 & 55 & $0 \mid 0$ & 05 & 0 & $0 \mid 0$ & 030 & 3530 & 305 & 0 & 94 & 59 & \begin{tabular}{l|l}
95 & 0
\end{tabular} & 0 & 85 & $50 \mid 6$ & 670 & & \\
\hline 469 & $\begin{array}{l}5 . \\
3\end{array}$ & $\begin{array}{c}\text { Lateral } \\
\text { Scour } \\
\text { Pool - } \\
\text { Root Wad } \\
\text { Enhanced }\end{array}$ & 6377 & 782 & 16 & 5152 & $22 \mid 1$ & $10 \begin{array}{r}1 . \\
1\end{array}$ & \begin{tabular}{l|l}
1.4 & 2 \\
1 &
\end{tabular} & \begin{tabular}{c|c}
2.8 & 0 \\
3 &
\end{tabular} & $\begin{array}{l}0.3 \\
6\end{array}$ & d & 3 & $10: 5$ & 520 & 35 & 35 & 00 & $0 \mid 0$ & 05 & 0 & 202 & 2040 & 105 & 55 & 0 & 91 & $0 \mid \begin{array}{cc}1 c \\
0\end{array}$ & \begin{tabular}{c|l}
10 & 0 \\
0 & 0
\end{tabular} & 5 & \begin{tabular}{|l|l|l}
6 & 8
\end{tabular} & 808 & 850 & $\begin{array}{c}2.4 \\
7\end{array} \mid$ & $\begin{array}{l}\text { Two } 5.3 \text { 's catty } \\
\text { corner to } \\
\text { eachother, } \\
\text { connected by } \\
\text { deep ( }>0.5^{\prime} \text { ') flat } \\
\text { section } \\
\end{array}$ \\
\hline 470 & $\begin{array}{l}1 . \\
1\end{array}$ & \begin{tabular}{|c} 
Low \\
gradient \\
Riffle
\end{tabular} & 6378 & 87 & 10 & 10 & 61 & 130. & \begin{tabular}{l|l}
0.2 & 0 \\
9 &
\end{tabular} & $\begin{array}{c}0.5 \\
2\end{array}$ & & & 1 & $<5$ & 0 & 0 & 0 & 0 & 0.0 & 050 & 0 & $0 \mid 0$ & 020 & 4530 & 305 & 0 & & 2080 & $80 \mid 0$ & 37 & $\begin{array}{ll}8 & 1\end{array}$ & $15 \quad 9$ & $\begin{array}{l}9 \\
9\end{array}$ & & \\
\hline 471 & $\begin{array}{l}4 . \\
2\end{array}$ & \begin{tabular}{|c|} 
Mid- \\
Channel \\
Pool \\
\end{tabular} & 6480 & 50 & 10 & 131 & 11 & $6 \begin{array}{r}1 . \\
3\end{array}$ & \begin{tabular}{l|l}
1.7 & 3 \\
3 &
\end{tabular} & $3.99^{0}$ & \begin{tabular}{c|c}
0.2 & 40 \\
5 &
\end{tabular} & d & 1 & 10 & 0 & 0 & 0 & 07 & 700 & 0 & 20 & 202 & 2030 & 1010 & 100 & 0 & $\begin{array}{c}10 \\
0\end{array}$ & 752 & $25 \mid 0$ & 15 & 86 & $60 \mid 7$ & 710 & $\begin{array}{c}3.6 \\
5\end{array} \mid$ & \\
\hline 472 & $\begin{array}{l}1 . \\
1\end{array}$ & \begin{tabular}{|c|} 
Low \\
gradient \\
Riffle
\end{tabular} & 6478 & 849 & 9 & 6 & 5 & $\left.16\right|_{7} ^{0 .}$ & \begin{tabular}{l|l}
0.3 & 0 \\
7 & 0
\end{tabular} & 0.6 & & & 1 & & 525 & 10 & 40 & 00 & $0: 0$ & 0 & 0 & 00 & 030 & 3030 & 3010 & 0 & $\begin{array}{c}10 \\
0\end{array} \mid$ & 406 & 600 & 0 & 86 & $60 \mid 6$ & 670 & & \\
\hline 473 & $\begin{array}{l}4 . \\
2\end{array}$ & \begin{tabular}{|c|} 
Mid- \\
Channel \\
Pool \\
\end{tabular} & 6480 & 74 & 15 & 5161 & 151 & $15 \begin{array}{r}2 . \\
1\end{array}$ & \begin{tabular}{l|l}
2.0 & 3 \\
1 &
\end{tabular} & \begin{tabular}{c|c}
3.0 & 0 \\
1 &
\end{tabular} & $\begin{array}{c}0.0 \\
8\end{array}$ & d & 3 & 204 & $0 \mid 10$ & 25 & 25 & 0 & 00 & 0 & 0 & 202 & 2045 & 105 & 50 & 0 & 99 & \begin{tabular}{l|l}
5 & 95
\end{tabular} & $95 \mid 5$ & 0 & 86 & 608 & 860 & $\begin{array}{c}2.9 \\
3\end{array} \mid$ & \\
\hline 474 & $\begin{array}{l}1 . \\
1\end{array}$ & \begin{tabular}{|c|} 
Low \\
gradient \\
Riffle
\end{tabular} & 6581 & 177 & 18 & 3152 & 211 & $\left.19\right|_{7} ^{0 .}$ & \begin{tabular}{l|l}
0.2 & 0 \\
7 &
\end{tabular} & $\begin{array}{l}0.6 \\
6\end{array}$ & & & & \begin{tabular}{l|l}
5 & 2 \\
\end{tabular} & 2035 & 0 & 30 & 00 & 0.0 & 0 & 0 & 00 & $\begin{array}{lll}0 & 20\end{array}$ & 3535 & 3510 & 00 & 98 & 595 & \begin{tabular}{l|l}
95 & 0
\end{tabular} & 0 & 87 & $70 \mid 8$ & 885 & & Ford crossing \\
\hline 475 & \begin{tabular}{|l|}
5. \\
1 \\
\end{tabular} & $\begin{array}{c}\text { Corner } \\
\text { Pool }\end{array}$ & 6482 & $\begin{array}{c}10 \\
5 \\
\end{array}$ & 22 & 2191 & 182 & $29 \begin{array}{r}1 . \\
7\end{array}$ & \begin{tabular}{l|l}
1.1 & 1 \\
7 & \\
\end{tabular} & \begin{tabular}{c|c}
1.8 & 0 \\
1 & \\
\end{tabular} & $\begin{array}{ll}0.2 & 30 \\
3 & 30 \\
\end{array}$ & d & 3 & 102 & 515 & 20 & 35 & 50 & 0.0 & 0 & 0 & 252 & 2535 & 105 & 50 & 0 & 94 & 5 & $95 \mid 0$ & 0 & 67 & 70 & 560 & $\begin{array}{c}1.5 \\
8 \\
\end{array}$ & \\
\hline 476 & $\begin{array}{l}4 . \\
2\end{array}$ & $\begin{array}{c}\text { Mid- } \\
\text { Channel } \\
\text { Pool } \\
\end{array}$ & 6481 & 191 & 21 & 1292 & 27 & $7 \begin{array}{rr}1 . \\
3\end{array}$ & \begin{tabular}{l|l}
1.8 & 2 \\
3 &
\end{tabular} & \begin{tabular}{c|c}
2.8 & 0 \\
5 &
\end{tabular} & \begin{tabular}{c|c}
0.2 & 20 \\
4 &
\end{tabular} & c & 3 & 5 & \begin{tabular}{l|l}
5 & 10
\end{tabular} & 25 & 35 & 00 & \begin{tabular}{l|l}
0 & 0
\end{tabular} & 0 & 25 & 202 & 2020 & & 100 & 15 & 94 & \begin{tabular}{l|l}
55 & 45
\end{tabular} & 4535 & 50 & 63 & \begin{tabular}{l|l}
30 & 8
\end{tabular} & 850 & $\begin{array}{c}2.6 \\
1\end{array}$ & \\
\hline 477 & $\begin{array}{l}3 . \\
4\end{array}$ & Step Run & 6681 & $1 \mid \begin{array}{c}27 \\
7\end{array}$ & 11 & 17 & 131 & $12 \begin{array}{r}0 . \\
8\end{array}$ & $\begin{array}{l}0.4 \\
8\end{array}$ & 1 & & & 3 & 103 & 3020 & 15 & 30 & 0 & 0.0 & 05 & 0 & 55 & 525 & 3030 & 305 & 0 & 90 & 158 & $85 \mid 0$ & 0 & 69 & $90 \quad 8$ & 880 & & $\begin{array}{c}5 \text { riffles }\left(7^{\prime}, 11^{\prime},\right. \\
\left.10^{\prime}, 38^{\prime}, 56^{\prime}\right) 4 \\
\text { runs. }\end{array}$ \\
\hline 478 & $\begin{array}{l}4 . \\
2\end{array}$ & $\begin{array}{c}\text { Mid- } \\
\text { Channel } \\
\text { Pool }\end{array}$ & & 42 & 14 & $4 \mid 12$ & & 15 & & \begin{tabular}{c|c}
3.2 & 0 \\
1 & 0
\end{tabular} & 0.340 & c & 3 & 40 & 0 & 60 & 20 & 00 & \begin{tabular}{l|l}
0 & 0
\end{tabular} & 0 & 0 & & & & & & 99 & 595 & $95 \mid 0$ & 0 & 82 & \begin{tabular}{l|l}
20 & 6
\end{tabular} & 635 & $\begin{array}{c}2.9 \\
1\end{array} \mid$ & $\begin{array}{c}\text { Flag, "NMFS fire } \\
\text { pool 19, please } \\
\text { do not distrub." } \\
\text { Log jam. }\end{array}$ \\
\hline 479 & $\begin{array}{l}3 . \\
4\end{array}$ & Step Run & 6680 & 88 & 14 & 415 & 121 & $15 \mid \begin{array}{r}0 . \\
1\end{array}$ & \begin{tabular}{l|l}
.4 & 1 \\
1 & \\
\end{tabular} & $\begin{array}{c}1.1 \\
4 \\
4\end{array}$ & & & 3 & $\begin{array}{lll}5 & 2 \\
\end{array}$ & 525 & 25 & 25 & 00 & 00 & 00 & 0 & 00 & 020 & 3535 & 3510 & 00 & 97 & 1096 & \begin{tabular}{l|l}
90 & 0
\end{tabular} & 0 & 69 & $90 \quad 6$ & 690 & & $\begin{array}{c}2 \text { riffles }\left(40^{\prime}, 17^{\prime}\right) \\
1 \text { run. }\end{array}$ \\
\hline 480 & \begin{tabular}{|l|}
5. \\
1 \\
\end{tabular} & $\begin{array}{c}\text { Corner } \\
\text { Pool }\end{array}$ & 6579 & 988 & 11 & 115 & 15 & $4 \begin{array}{r}1 . \\
5\end{array}$ & \begin{tabular}{l|l}
1.5 & 2 \\
5 &
\end{tabular} & \begin{tabular}{c|c}
2.7 & 0 \\
7 & \\
\end{tabular} & $\begin{array}{l}0.1 \\
9\end{array}$ & & 2 & $\begin{array}{lll}5 & 3 \\
\end{array}$ & 0 & 10 & 40 & 200 & 0.0 & 0 & 0 & 252 & 2530 & 105 & 55 & 0 & 88 & \begin{tabular}{l|l}
55 & 45
\end{tabular} & 4535 & 50 & \begin{tabular}{|l|l}
6 & 4
\end{tabular} & $40 \quad 6$ & 680 & \begin{tabular}{|c|}
2.5 \\
8
\end{tabular} & \\
\hline 481 & \begin{tabular}{|l|}
3. \\
2 \\
\end{tabular} & Glide & 6680 & 018 & 6 & 4 & 6 & \begin{tabular}{l|r}
7 & 0. \\
9
\end{tabular} & \begin{tabular}{l|l}
.5 & 0 \\
9 & \\
\end{tabular} & $\begin{array}{c}0.7 \\
2 \\
\end{array}$ & & & 3 & 252 & 020 & 0 & 20 & 202 & 200 & 0 & 0 & $15 \mid 1$ & 1540 & $151 c$ & 105 & 0 & 88 & 505 & 500 & 0 & 69 & $90 \quad 6$ & 590 & & End $8 / 27$ \\
\hline 482 & $\begin{array}{l}4 . \\
2\end{array}$ & $\begin{array}{c}\text { Mid- } \\
\text { Channel } \\
\text { Pool } \\
\end{array}$ & 6365 & 550 & 15 & 572 & 281 & $\left.11\right|_{8} ^{2 .}$ & $\begin{array}{c}2.9 \\
8\end{array}$ & \begin{tabular}{c|c}
4.2 & 0 \\
3 &
\end{tabular} & $\begin{array}{c}0.1 \\
7\end{array}$ & c & 3 & $90: 5$ & 530 & 40 & 0 & 02 & 200 & 0 & 5 & 202 & 2035 & 105 & $5 \mid 5$ & 5 & 88 & 5 & $95 \mid 0$ & 0 & 68 & 80.8 & 870 & $\left|\begin{array}{c}4.0 \\
6\end{array}\right|$ & $\begin{array}{l}\text { Start 8/28. Log } \\
\text { jam, 29'w x 8'h }\end{array}$ \\
\hline 483 & $\begin{array}{l}1 . \\
1\end{array}$ & \begin{tabular}{|c|} 
Low \\
gradient \\
Riffle
\end{tabular} & 6365 & 547 & 13 & 311 & 11 & $17 \mid \begin{array}{r}0 . \\
1\end{array}$ & \begin{tabular}{l|l}
0.3 & 0 \\
1 &
\end{tabular} & $\begin{array}{c}0.6 \\
2\end{array}$ & & & 3 & $15 \mid 5$ & $5 \quad 40$ & 0 & 0 & $35 \mid c$ & \begin{tabular}{l|l}
0 & 0
\end{tabular} & 020 & 0 & $5 \mid 5$ & 520 & \begin{tabular}{|l|l|l|}
30 & 25 \\
\end{tabular} & 2515 & 50 & 93 & $109 c$ & \begin{tabular}{l|l}
90 & 0
\end{tabular} & 0 & 68 & $80 \mid 7$ & $\begin{array}{l}7 \\
\end{array}$ & & \\
\hline 484 & \begin{tabular}{|l|}
3. \\
2 \\
\end{tabular} & Glide & 6466 & 640 & 16 & 617 & 161 & \begin{tabular}{r|r}
15 & 1. \\
4
\end{tabular} & \begin{tabular}{l|l|}
1.1 & 1 \\
4 & \\
\end{tabular} & \begin{tabular}{c|c}
1.9 & 0 \\
8 & \\
\end{tabular} & \begin{tabular}{|c|}
0.1 \\
1 \\
\end{tabular} & & 3 & \begin{tabular}{|l|l}
5 & 1
\end{tabular} & 040 & 0 & 10 & 40 & 0.0 & 0 & 0 & 10 & $\begin{array}{lll}0 & 25\end{array}$ & 3020 & 205 & 0 & 95 & 1096 & 900 & 0 & 69 & 90 & 675 & \begin{tabular}{|c|}
1.8 \\
7
\end{tabular} & \\
\hline 485 & 2 & $\begin{array}{c}\text { Mid- } \\
\text { Channel } \\
\text { Pool } \\
\end{array}$ & 6365 & 517 & 11 & $1 \mid 15$ & 11 & \begin{tabular}{l|l}
8 & 1.
\end{tabular} & 1.6 & $\begin{array}{c}2.4 \\
2\end{array}$ & 0.8 & & 3 & $75 \mid 1$ & 030 & 35 & 25 & 00 & \begin{tabular}{l|l}
0 & 0
\end{tabular} & 0 & 0 & & 2525 & 50 & \begin{tabular}{l|l|l}
0 & 5
\end{tabular} & 0 & 82 & \begin{tabular}{|l|l|}
5 & 95
\end{tabular} & \begin{tabular}{l|l}
95 & 0
\end{tabular} & 0 & & 806 & 680 & $\left|\begin{array}{c}1.6 \\
2\end{array}\right|$ & Log jam, SDA \\
\hline
\end{tabular}




\begin{tabular}{|c|c|c|c|c|c|c|c|c|c|c|c|c|c|c|c|c|c|c|c|c|c|c|c|c|c|c|c|c|c|c|c|c|c|c|}
\hline 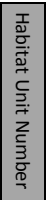 & & 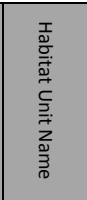 & 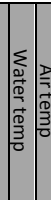 & 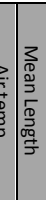 & 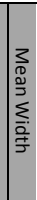 & 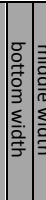 & 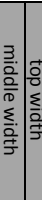 & 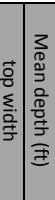 & 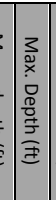 & 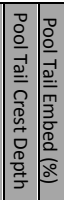 & 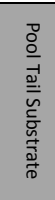 & 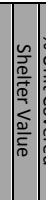 & 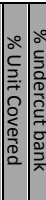 & 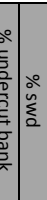 & $\frac{\circ}{\sum}$ & 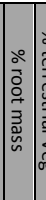 & 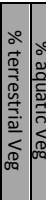 & 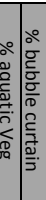 & 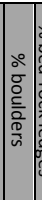 & & 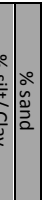 & & 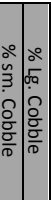 & & 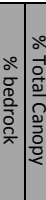 & 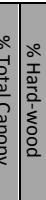 & 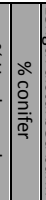 & 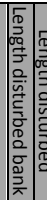 & 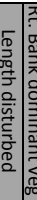 & 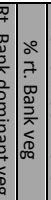 & 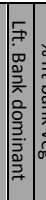 & 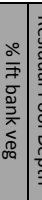 & $\begin{array}{l}\frac{7}{0} \\
\frac{0}{0} \\
\frac{0}{D} \\
\frac{0}{0} \\
0 \\
\frac{0}{D} \\
0 \\
\frac{0}{0} \\
\frac{0}{5}\end{array}$ & 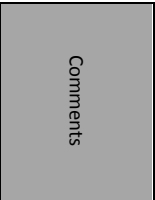 \\
\hline 486 & $\begin{array}{c}1 . \\
1\end{array}$ & \begin{tabular}{|c|} 
Low \\
gradient \\
Riffle
\end{tabular} & & 20 & 11 & 8 & & $14 \mid \begin{array}{c}0.1 \\
9\end{array}$ & \begin{tabular}{l|c}
1 & 0.2 \\
8
\end{tabular} & & & 16 & 600 & \begin{tabular}{l|l}
0 & 0
\end{tabular} & 0 & 0 & $\left|\begin{array}{c}10 \\
0\end{array}\right|$ & \begin{tabular}{l|l}
0 & 0
\end{tabular} & 0 & \begin{tabular}{l|l}
0 & 0
\end{tabular} & 0.0 & 54 & \begin{tabular}{l|l|}
45 & 40
\end{tabular} & 10 & & & & & 6 & 690 & 69 & 90 & & \\
\hline 487 & $\begin{array}{l}4 . \\
2\end{array}$ & \begin{tabular}{|c|} 
Mid- \\
Channel \\
Pool
\end{tabular} & 6268 & $\begin{array}{lll}88 & 47\end{array}$ & 10 & 14 & 107 & \begin{tabular}{l|c}
7 & 1.1 \\
4
\end{tabular} & \begin{tabular}{l|l}
1 & 1.8 \\
1 & 1
\end{tabular} & \begin{tabular}{c|c}
0.0 & 40 \\
7 & 40
\end{tabular} & d & 3 & 1025 & $25 \mid 25$ & 50 & $25 \mid 2$ & $25 \mid 0$ & \begin{tabular}{l|l}
0 & 0
\end{tabular} & 0 & 02 & 020 & 301 & 1010 & 50 & \begin{tabular}{l|l|l|l}
0 & 85
\end{tabular} & \begin{tabular}{l|l|l}
35 & 10
\end{tabular} & 90 & 0 & \begin{tabular}{l|l}
0 & 6
\end{tabular} & $6 \quad 95$ & 69 & 901 & $\begin{array}{c}1.7 \\
4\end{array}$ & $\begin{array}{c}(+10) \text { LB trib with } \\
\text { water and orange } \\
\text { alge }\end{array}$ \\
\hline 488 & $\begin{array}{c}1 . \\
1\end{array}$ & \begin{tabular}{|c|} 
Low \\
gradient \\
Riffle
\end{tabular} & & 23 & 17 & 101 & 1923 & $23 \mid \begin{array}{l}0.1 \\
7\end{array}$ & \begin{tabular}{l|l}
1 & 0.2 \\
8
\end{tabular} & & & 14 & \begin{tabular}{l|l}
40 & 0
\end{tabular} & \begin{tabular}{l|l}
0 & 0
\end{tabular} & 0 & 0 & $\begin{array}{c}10 \\
0\end{array}$ & \begin{tabular}{l|l}
0 & 0
\end{tabular} & 0 & \begin{tabular}{l|l}
0 & 0
\end{tabular} & 0 & & 4545 & 500 & 0 & & & 0 & 0 & \begin{tabular}{ll|l|}
6 & 80
\end{tabular} & 73 & 30 & & \\
\hline 489 & $\begin{array}{l}4 . \\
2\end{array}$ & $\begin{array}{c}\text { Mid- } \\
\text { Channel } \\
\text { Pool }\end{array}$ & 637 & $79 \begin{array}{c}13 \\
6\end{array}$ & 18 & 231 & 171 & \begin{tabular}{l|l}
15 & 1.4
\end{tabular} & $4 \begin{array}{c}2.4 \\
3\end{array}$ & \begin{tabular}{c|c}
0.3 & 30 \\
2 & 30
\end{tabular} & d & 3 & $20 \mid 15$ & $15 \mid 5$ & 5 & 302 & 202 & 200 & 5 & 02 & 020 & & \begin{tabular}{l|l}
15 & 15
\end{tabular} & 510 & \begin{tabular}{l|l}
0 & 87
\end{tabular} & \begin{tabular}{l|l}
37 & 50
\end{tabular} & 50 & 0 & 0 & $\begin{array}{lll}6 & 85\end{array}$ & 69 & $902^{2}$ & $\begin{array}{c}2.1 \\
1\end{array}$ & \\
\hline 490 & $\begin{array}{c}1 . \\
1\end{array}$ & \begin{tabular}{|c|} 
Low \\
gradient \\
Riffle
\end{tabular} & 637 & 434 & 17 & 152 & 2015 & $15 \mid$\begin{tabular}{c|c}
0.3 \\
8
\end{tabular} & \begin{tabular}{l|l}
3 & 0.5 \\
9
\end{tabular} & & & 3 & 1035 & $\begin{array}{ll}35 & 20\end{array}$ & 0 & \begin{tabular}{|l|l}
35 & 1
\end{tabular} & \begin{tabular}{|l|l}
10 & 0
\end{tabular} & \begin{tabular}{l|l}
0 & 0
\end{tabular} & 0 & 0 & 0 & 203 & 3535 & 510 & \begin{tabular}{l|l|l}
0 & 95
\end{tabular} & \begin{tabular}{l|l}
55 & 50
\end{tabular} & 50 & 0 & 0 & \begin{tabular}{l|l|}
7 & 50
\end{tabular} & 69 & 95 & & \\
\hline 491 & $\begin{array}{l}4 . \\
2\end{array} \mid$ & \begin{tabular}{|c|} 
Mid- \\
Channel \\
Pool
\end{tabular} & 637 & $4 \mid \begin{array}{c}12 \\
6\end{array}$ & 14 & 151 & $15 \mid 11$ & \begin{tabular}{l|l}
11 & 1.3 \\
& 8
\end{tabular} & \begin{tabular}{l|l|}
3 & 3.1 \\
3
\end{tabular} & \begin{tabular}{|c|c|c}
0.4 & 60 \\
7 & 60
\end{tabular} & e & 32 & 200 & \begin{tabular}{l|l}
0 & 10
\end{tabular} & 040 & 40 & \begin{tabular}{l|l}
0 & 0
\end{tabular} & \begin{tabular}{l|l}
0 & 0
\end{tabular} & 10 & \begin{tabular}{l|l|l|l|l}
0 & 2
\end{tabular} & 020 & 201 & \begin{tabular}{l|l}
15 & 15
\end{tabular} & 510 & \begin{tabular}{l|l}
0 & 89
\end{tabular} & \begin{tabular}{l|l}
39 & 40
\end{tabular} & 60 & 0 & 0 & \begin{tabular}{ll|l}
6 & 80
\end{tabular} & 64 & $40^{2}$ & $\begin{array}{c}2.6 \\
7\end{array}$ & $\begin{array}{c}\text { Rootwad and log } \\
\text { enhanced }\end{array}$ \\
\hline 492 & $\begin{array}{l}4 . \\
2\end{array} \mid$ & \begin{tabular}{|c|} 
Mid- \\
Channel \\
Pool
\end{tabular} & 647 & 369 & 10 & 11 & \begin{tabular}{l|l}
12 & 7
\end{tabular} & \begin{tabular}{l|c}
7.1 \\
7 & 4
\end{tabular} & \begin{tabular}{l|c|}
1 & 1.5 \\
& 6
\end{tabular} & $\begin{array}{c}0.2 \\
6\end{array}$ & & 3 & $10 \mid 40$ & 400 & 20 & 40 & \begin{tabular}{l|l}
0 & 0
\end{tabular} & \begin{tabular}{l|l}
0 & 0
\end{tabular} & 0 & \begin{tabular}{|l|l|l}
0 & 1
\end{tabular} & $.0 \mid 10$ & & \begin{tabular}{l|l}
25 & 25
\end{tabular} & 510 & \begin{tabular}{|l|l}
0 & 94
\end{tabular} & \begin{tabular}{l|l}
34 & 5
\end{tabular} & 95 & 0 & 0 & \begin{tabular}{l|l|}
8 & 60
\end{tabular} & 68 & \begin{tabular}{|l|l}
80 & 1
\end{tabular} & 1.3 & \\
\hline 493 & \begin{tabular}{|l|}
1. \\
1
\end{tabular} & \begin{tabular}{|c|} 
Low \\
gradient \\
Riffle
\end{tabular} & 647 & $6 \mid \begin{array}{c}18 \\
7\end{array}$ & 10 & 71 & 1112 & \begin{tabular}{l|l}
12 & 0.4 \\
8
\end{tabular} & \begin{tabular}{l|l}
4 & 0.9 \\
3 & 8
\end{tabular} & & & 3 & 535 & \begin{tabular}{l|l}
35 & 10
\end{tabular} & 0 & $35 \mid 1$ & \begin{tabular}{|l|l}
10 & 0
\end{tabular} & \begin{tabular}{l|l}
0 & 0
\end{tabular} & 10 & \begin{tabular}{l|l}
0 & 0
\end{tabular} & 0 & 203 & 3030 & 200 & \begin{tabular}{l|l}
0 & 97
\end{tabular} & \begin{tabular}{l|l}
77 & 5
\end{tabular} & 95 & 0 & 0 & \begin{tabular}{l|l|}
8 & 60
\end{tabular} & 87 & 70 & & $\begin{array}{l}\text { Much more } \\
\text { granite }\end{array}$ \\
\hline 494 & 5. & \begin{tabular}{|c|} 
Lateral \\
Scour \\
Pool - \\
Boulder \\
Formed
\end{tabular} & 647 & $6 6 \longdiv { 4 8 }$ & 13 & 312 & 1315 & \begin{tabular}{l|c}
15 & 1.2 \\
\end{tabular} & \begin{tabular}{|c|c|}
2 & 1.7 \\
7
\end{tabular} & 0.2 & & 2 & 540 & 400 & 0 & 40 & $0 \mid 0$ & \begin{tabular}{l|l}
0 & 0
\end{tabular} & 20 & \begin{tabular}{|l|l}
0 & 1
\end{tabular} & .010 & & 2020 & 1011 & 1094 & \begin{tabular}{l|l}
94 & 5
\end{tabular} & 95 & 0 & 0 & \begin{tabular}{l|l|l|}
8 & 20 \\
\end{tabular} & 66 & 601 & $\begin{array}{c}1.5 \\
7\end{array}$ & $\begin{array}{c}\text { Boulders from RB } \\
\text { bedrock cliff } \\
\text { (mudstone) }\end{array}$ \\
\hline 495 & $\begin{array}{l}4 . \\
2\end{array} \mid$ & $\begin{array}{c}\text { Mid- } \\
\text { Channel } \\
\text { Pool }\end{array}$ & 647 & 461 & 11 & 151 & 108 & \begin{tabular}{c|c}
8.7 \\
6
\end{tabular} & \begin{tabular}{c|c|}
7 & 2.5 \\
6
\end{tabular} & $\begin{array}{c}0.3 \\
9\end{array}$ & e & 1 & \begin{tabular}{l|l}
$<5$ & 0
\end{tabular} & \begin{tabular}{l|l}
0 & 0
\end{tabular} & 0 & 0 & $0 \mid 0$ & \begin{tabular}{l|l}
0 & 0
\end{tabular} & 505 & $50 \mid 1$ & .010 & & 2020 & 1011 & 1099 & \begin{tabular}{l|l}
99 & 0
\end{tabular} & $\begin{array}{c}10 \\
0\end{array}$ & 0 & 0.7 & $\begin{array}{l}7 \\
\end{array}$ & \begin{tabular}{l|l}
8 & 2
\end{tabular} & 20 & $\begin{array}{c}2.1 \\
7\end{array}$ & $\begin{array}{c}5.1>\text { than } 60 \% \text { of } \\
\text { width, so } 4.2 . \text { RB } \\
\text { bedrock. Dry } \\
\text { wash, perched, } \\
\text { RB. } \\
\end{array}$ \\
\hline 496 & \begin{tabular}{|c|}
1. \\
1
\end{tabular} & \begin{tabular}{|c|} 
Low \\
gradient \\
Riffle
\end{tabular} & 657 & $77 \begin{array}{c}10 \\
9\end{array}$ & 16 & 58 & 182 & \begin{tabular}{l|c}
21 & 0.4 \\
21
\end{tabular} & $4 \mid \begin{array}{c}0.8 \\
4\end{array}$ & & & 1 & $<535$ & $35 \mid 30$ & 0 & 35 & $0 \mid 0$ & \begin{tabular}{l|l}
0 & 0
\end{tabular} & 0 & \begin{tabular}{l|l}
0 & 0 \\
0
\end{tabular} & 0.0 & & $30 \mid 30$ & 200 & 0 \begin{tabular}{c|c}
10 & 0 \\
0
\end{tabular} & \begin{tabular}{l|l}
10 & 0 \\
0 & 0
\end{tabular} & $\mid \begin{array}{c}10 \\
0\end{array}$ & 0 & 0 & 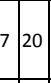 & 6 & 50 & & $\begin{array}{l}(+100) \text { Skid trail } \\
\text { crossing. }\end{array}$ \\
\hline 497 & $\begin{array}{l}4 . \\
2\end{array} \mid$ & \begin{tabular}{|c|} 
Mid- \\
Channel \\
Pool
\end{tabular} & 637 & 663 & 16 & 21 & $17 \mid 11$ & $11 \begin{array}{c}1.8 \\
2\end{array}$ & \begin{tabular}{l|l|}
8 & 3.0 \\
8
\end{tabular} & \begin{tabular}{c|c}
0.2 & 30 \\
1 &
\end{tabular} & e & 1 & $<535$ & \begin{tabular}{l|l}
35 & 0
\end{tabular} & 0 & 30 & \begin{tabular}{l|l}
0 & 0
\end{tabular} & \begin{tabular}{l|l}
0 & 0
\end{tabular} & 0 & 351 & 515 & 201 & 1515 & 5515 & \begin{tabular}{l|c}
15 & 10 \\
\end{tabular} & \begin{tabular}{l|l}
10 & 80 \\
0 & 80
\end{tabular} & 20 & 0 & 0 & \begin{tabular}{ll|}
6 & 60
\end{tabular} & 72 & $20^{2}$ & $\begin{array}{c}2.8 \\
7\end{array}$ & \\
\hline 498 & $\begin{array}{c}1 . \\
1\end{array}$ & \begin{tabular}{|c} 
Low \\
gradient \\
Riffle
\end{tabular} & & 16 & 15 & 14 & & $15 \begin{array}{c}0.4 \\
2\end{array}$ & \begin{tabular}{l|l}
4 & 0.5 \\
1
\end{tabular} & & & & & & & & & & & & & & & & & & & & & & & & & \\
\hline 499 & $\begin{array}{l}3 . \\
3 \\
\end{array}$ & Run & & 34 & 15 & 15 & & $14 \begin{array}{c}0.7 \\
5\end{array}$ & \begin{tabular}{|l|l|}
7 & 1.1 \\
\end{tabular} & & & & & & & & & & & & & & & & & & & & & & & & & \\
\hline 500 & $\begin{array}{c}1 . \\
1\end{array}$ & \begin{tabular}{|c|} 
Low \\
gradient \\
Riffle
\end{tabular} & 657 & $9 \mid \begin{array}{c}13 \\
5\end{array}$ & 18 & 3142 & 2110 & 19 \begin{tabular}{c|c}
0.3 \\
7
\end{tabular} & \begin{tabular}{l|l}
3 & 0.9 \\
5
\end{tabular} & & & 3 & 530 & 3020 & 020 & \begin{tabular}{l|l}
0 & 3 \\
\end{tabular} & $\begin{array}{lll}30 & 0\end{array}$ & \begin{tabular}{l|l}
0 & 0
\end{tabular} & 0 & 200 & 0 & & 3030 & 300 & 0 \begin{tabular}{c|c}
10 & 0
\end{tabular} & \begin{tabular}{l|l}
10 & 90 \\
0 & 90
\end{tabular} & 10 & 0 & 0 & \begin{tabular}{l|l|l|}
6 & 95
\end{tabular} & 69 & 90 & & \\
\hline 501 & $\begin{array}{l}4 . \\
2\end{array} \mid$ & \begin{tabular}{|c|} 
Mid- \\
Channel \\
Pool
\end{tabular} & 657 & $9 \mid \begin{array}{c}10 \\
0\end{array}$ & 18 & 3192 & 201 & $14 \begin{array}{c}2.2 \\
7\end{array}$ & \begin{tabular}{l|l}
2 & 5.4 \\
3
\end{tabular} & $\begin{array}{c}0.1 \\
8\end{array}$ & e & 3 & 1025 & 250 & 0 & 25 & \begin{tabular}{|l|l}
0 & 0
\end{tabular} & \begin{tabular}{l|l}
0 & 0
\end{tabular} & 203 & $30 \mid 2$ & $0 \mid 20$ & 20 & \begin{tabular}{l|l}
5 & 10 \\
\end{tabular} & 10 & $15 \mid \begin{array}{c}10 \\
0\end{array}$ & \begin{tabular}{l|l}
10 & 20 \\
0
\end{tabular} & 80 & 0 & 06 & \begin{tabular}{ll|l|}
6 & 90 \\
\end{tabular} & 8 & $10^{5}$ & $\begin{array}{c}5.2 \\
5\end{array}$ & LB bedrock cliff \\
\hline 502 & $\begin{array}{l}1 . \\
1\end{array}$ & \begin{tabular}{|c|} 
Low \\
gradient \\
Riffle
\end{tabular} & 6678 & $8 \begin{array}{c}11 \\
0\end{array}$ & 14 & $\begin{array}{lll}4 & 14 & 1\end{array}$ & 1415 & \begin{tabular}{c|c}
15 & 0.3 \\
9
\end{tabular} & \begin{tabular}{c|c|}
3 & 0.7 \\
5
\end{tabular} & & & 1 & $<535$ & $35 \mid 0$ & 0 & 35 & \begin{tabular}{|l|l|l|l}
0 & 0
\end{tabular} & \begin{tabular}{l|l}
0 & 0
\end{tabular} & 30 & \begin{tabular}{l|l}
0 & 0 \\
0
\end{tabular} & 0.0 & 252 & $25 \quad 25$ & 5250 & \begin{tabular}{l|c}
10 & 10 \\
0
\end{tabular} & \begin{tabular}{l|l}
10 & 40 \\
0 & 40
\end{tabular} & 60 & 0 & $0 \mid 7$ & $7 \quad 40$ & \begin{tabular}{l|l}
6 & 8 \\
\end{tabular} & 80 & & \\
\hline 503 & $\begin{array}{l}4 . \\
2\end{array} \mid$ & \begin{tabular}{|c|} 
Mid- \\
Channel \\
Pool
\end{tabular} & 6578 & 851 & 15 & 5152 & \begin{tabular}{l|l}
21 & 8
\end{tabular} & \begin{tabular}{c|c}
1.6 \\
3
\end{tabular} & \begin{tabular}{c|c|}
6 & 2.3 \\
6
\end{tabular} & \begin{tabular}{|c|c}
0.1 & 40 \\
9 & 0
\end{tabular} & e & 2 & 1040 & \begin{tabular}{l|l}
40 & 0
\end{tabular} & 0 & $60 \mid$ & \begin{tabular}{|l|l|l|l}
0 & 0
\end{tabular} & \begin{tabular}{l|l}
0 & 0
\end{tabular} & 0 & $\begin{array}{lll}0 & 2\end{array}$ & 020 & 351 & 1010 & 50 & 0 \begin{tabular}{c|c}
10 & 0 \\
0
\end{tabular} & \begin{tabular}{l|l}
10 & 10 \\
0 & 10
\end{tabular} & 90 & 0 & 06 & \begin{tabular}{l|l|l}
6 & 30
\end{tabular} & 83 & $30^{2}$ & $\begin{array}{c}2.1 \\
7\end{array}$ & RB dry wash \\
\hline 504 & $\begin{array}{c}3 . \\
4 \\
\end{array}$ & Step Run & 657 & $9 \begin{array}{c}27 \\
7\end{array}$ & 8 & 8 & \begin{tabular}{l|l}
8 & 9 \\
\end{tabular} & $9 \begin{array}{c}0.5 \\
2\end{array}$ & \begin{tabular}{|c|c|}
5 & 1.8 \\
& 5 \\
\end{tabular} & & & 3 & 525 & 2525 & 525 & 25 & \begin{tabular}{l|l}
0 & 0 \\
\end{tabular} & \begin{tabular}{l|l}
0 & 0
\end{tabular} & 0 & \begin{tabular}{l|l}
0 & 0 \\
0
\end{tabular} & 0 & 252 & 2525 & 5250 & 005 & \begin{tabular}{l|l}
75 & 70
\end{tabular} & 30 & 0 & 06 & \begin{tabular}{l|l|}
6 & 80
\end{tabular} & 68 & 80 & & $\begin{array}{c}3 \text { riffles, } 2 \text { runs } \\
\left(10^{\prime}, 30^{\prime}\right) .\end{array}$ \\
\hline 505 & $\begin{array}{l}4 . \\
2\end{array}$ & \begin{tabular}{|c|} 
Mid- \\
Channel \\
Pool
\end{tabular} & 657 & 7781 & 14 & 49 & 1418 & \begin{tabular}{c|c|c}
18.6 & 2.6 \\
4
\end{tabular} & \begin{tabular}{l|l}
6 & 3.8 \\
9
\end{tabular} & 0.340 & e & 3 & $25 \mid 0$ & \begin{tabular}{l|l|l|l|l|l}
0 & 20
\end{tabular} & 060 & $0 \mid$ & \begin{tabular}{|l|l|l|l}
0 & 0
\end{tabular} & \begin{tabular}{l|l}
0 & 0
\end{tabular} & 0 & $10 \mid 1$ & .515 & 545 & \begin{tabular}{|l|l|}
5 & 5
\end{tabular} & 515 & 1593 & \begin{tabular}{l|l}
33 & 40
\end{tabular} & 60 & 0 & \begin{tabular}{l|l}
0 & 6 \\
-1
\end{tabular} & \begin{tabular}{l|l|l}
6 & 50
\end{tabular} & 7 & 0 & $\begin{array}{c}3.5 \\
9\end{array}$ & SDA creating Pool \\
\hline 506 & $\begin{array}{c}1 . \\
1\end{array}$ & \begin{tabular}{|c|} 
Low \\
gradient \\
Riffle
\end{tabular} & 6678 & $8 \mid \begin{array}{c}12 \\
7\end{array}$ & 10 & \begin{tabular}{l|l|l}
6 & 1
\end{tabular} & 149 & \begin{tabular}{c|c}
0.3 \\
9
\end{tabular} & \begin{tabular}{l|l}
3 & 0.7 \\
5
\end{tabular} & & & 2 & $<5 \mid 0$ & \begin{tabular}{l|l}
0 & 50
\end{tabular} & $\begin{array}{lll}0 & 5\end{array}$ & $0 \mid 3$ & $30 \mid 0$ & \begin{tabular}{l|l}
0 & 0
\end{tabular} & 15 & \begin{tabular}{l|l}
0 & 0
\end{tabular} & 0.0 & 253 & 3030 & 150 & 0.89 & $39 \mid 5$ & 95 & 0 & 06 & $6 \mid 80$ & 68 & 80 & & $\begin{array}{c}\text { More granite } \\
\text { than mudstone } \\
\text { cobbles }\end{array}$ \\
\hline 507 & $\begin{array}{l}. \\
2 \\
\end{array}$ & Glide & 657 & 9443 & 12 & \begin{tabular}{|l|l|l|}
2 & 9 & 1 \\
\end{tabular} & 1512 & \begin{tabular}{c|c}
12 & 0.4 \\
& 6 \\
\end{tabular} & \begin{tabular}{|c|c|}
4 & 0.7 \\
1 & \\
\end{tabular} & & & 1 & $<550$ & 500 & 0 & 50 & $0 \mid 0$ & \begin{tabular}{l|l}
0 & 0
\end{tabular} & 0 & 02 & 525 & 355 & 55 & 50 & \begin{tabular}{l|l}
0 & 98 \\
\end{tabular} & \begin{tabular}{l|c}
8 \\
0
\end{tabular} & 0 & 00 & 0 & $\begin{array}{lll}6 & 70\end{array}$ & 71 & 10 & & \\
\hline 508 & $\begin{array}{l}4 . \\
2\end{array} \mid$ & $\begin{array}{c}\text { Mid- } \\
\text { Channel } \\
\text { Pool }\end{array}$ & 657 & 7948 & 12 & 2121 & 1410 & \begin{tabular}{l|c}
10 & 1.1 \\
9
\end{tabular} & \begin{tabular}{l|l}
1 & 1.7 \\
5
\end{tabular} & \begin{tabular}{c|c|}
0.2 & 65 \\
6 & 65
\end{tabular} & d & 3 & 1530 & 3010 & 0 & 50 & \begin{tabular}{l|l}
0 & 0 \\
0
\end{tabular} & \begin{tabular}{l|l}
0 & 0
\end{tabular} & 10 & 02 & $0 \mid 20$ & 301 & 1010 & 100 & 009 & \begin{tabular}{l|l}
99 & 10
\end{tabular} & 90 & 0 & 06 & \begin{tabular}{l|l|}
6 & 80
\end{tabular} & 82 & $20{ }^{1}$ & $\begin{array}{c}1.4 \\
9\end{array}$ & End $8 / 29$ \\
\hline 509 & $\begin{array}{c}1 . \\
1\end{array}$ & \begin{tabular}{|c} 
Low \\
gradient \\
Riffle
\end{tabular} & 637 & $0 \begin{array}{c}43 \\
8\end{array}$ & 13 & 301 & 1212 & \begin{tabular}{l|l}
18 & 0.6
\end{tabular} & $\begin{array}{lll}6 & 1.6\end{array}$ & & & 3 & 515 & 1515 & 520 & 15 & \begin{tabular}{|l|l|l}
5 & 0
\end{tabular} & \begin{tabular}{l|l}
0 & 0
\end{tabular} & $30 \mid$ & 05 & & 252 & 2025 & 520 & \begin{tabular}{l|l}
0 & 98
\end{tabular} & \begin{tabular}{l|l}
8 & 75
\end{tabular} & 25 & 00 & 0 & 7 90 & 69 & 90 & & $\begin{array}{l}\text { Start } 9 / 3 . \mathrm{BF} \\
\text { taken }\end{array}$ \\
\hline
\end{tabular}




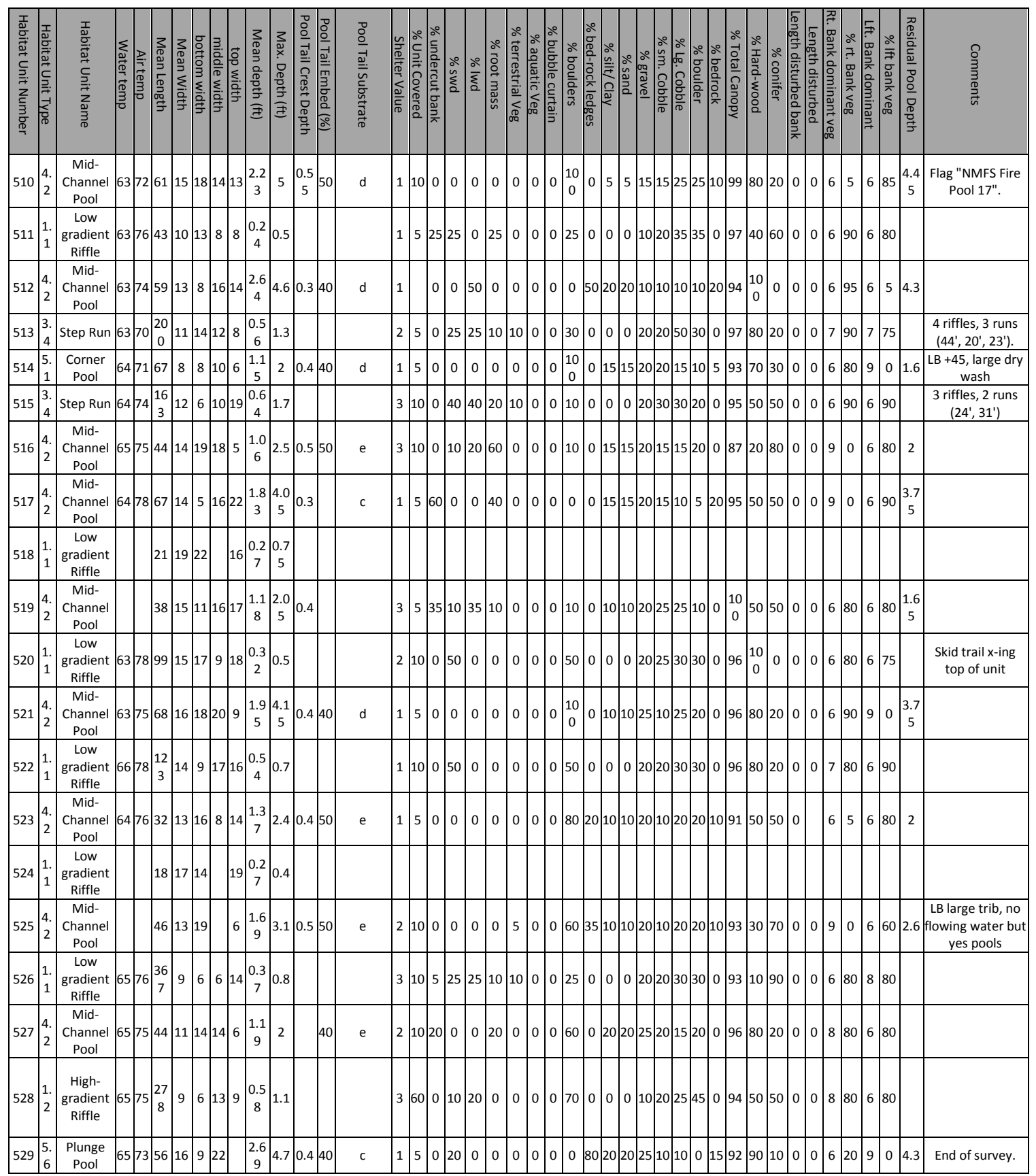

